IMPACT OF EMPIRE • VOLUME I7

\title{
INTEGRATION \\ IN ROME AND \\ IN THE ROMAN WORLD
}

Proceedings of the Tenth Workshop of the International Network Impact of Empire (Lille, June $23^{-2} 5,201$ I)

Edited by

Gerda de Kleijn and Stéphane Benoist 
Integration in Rome and in the Roman World 


\section{Impact of Empire}

Editorial Board of the series Impact of Empire

(= Management Team of Impact of Empire)

Angelos Chaniotis, Ségolène Demougin,

Olivier Hekster, Gerda de Kleijn, Luuk de Ligt, Elio Lo Cascio, Bernhard Palme, Michael Peachin, Christian Witschel and Greg Woolf

Executive Secretariat of the Series and the Network

Lukas de Blois, Olivier Hekster

Gerda de Kleijn and John Rich

Radboud University of Nijmegen, Erasmusplein 1, P.O. Box 9103, 6500 HD Nijmegen, The Netherlands

Academic Board of the International Network Impact of Empire NICOLE BELAYCHE - STEPHANE BENOIST - ANTHONY BIRLEY

LUKAS DE BLOIS - CHRISTER BRUUN - JOHN DRINKWATER WERNER ECK - PETER FUNKE - ANDREA GIARDINA FRANÇOISE VAN HAEPEREN - JOHANNES HAHN - ANNE KOLB FIK MEIJER - ONNO VAN NIJF - MARIE-THERESE RAEPSAET-CHARLIER JOHN RICH - JOHN RICHARDSON - BERT VAN DER SPEK RICHARD TALBERT - WILLEM ZWALVE

VOLUME 17 


\title{
Integration in Rome and in the Roman World
}

\author{
Proceedings of the Tenth Workshop of the \\ International Network Impact of Empire \\ (Lille, June 23-25, 2011)
}

Edited by

Gerda de Kleijn and Stéphane Benoist

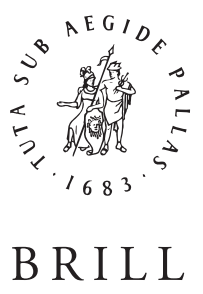




\section{B R I L L OPE N}

This is an open access title distributed under the terms of the CC-BY-NC 4.0 License, which permits any non-commercial use, distribution, and reproduction in any medium, provided the original author(s) and source are credited.

\section{Library of Congress Cataloging-in-Publication Data}

Impact of Empire (Organization). Workshop (10th : 2011 : Lille, France)

Integration in Rome and in the Roman world : proceedings of the Tenth Workshop of the International Network Impact of Empire (Lille, June 23-25, 2011) / edited by Gerda de Kleijn and Stéphane Benoist.

pages $\mathrm{cm}$. - (Impact of empire : volume 17)

English, French, and German.

Includes bibliographical references and index.

ISBN 978-90-04-25598-2 (hardback : acid-free paper) — ISBN 978-9o-04-25667-5 (e-book)

1. Cultural pluralism-Rome-History-Congresses. 2. Rome-History-Republic, 510-30 B.C.-Congresses. 3. Rome-History-Empire, 30 B.C. -476 A.D.-Congresses. 4. Group identity-Italy—Rome-Congresses. 5. Ethnicity-Italy-History-Congresses. I. Kleijn, Gerda de. II. Benoist, Stéphane. III. Title.

DG190.I47 2013

$305.800937^{\prime} 63-\mathrm{dc} 23$

2013023181

This publication has been typeset in the multilingual "Brill" typeface. With over 5,10o characters covering Latin, IPA, Greek, and Cyrillic, this typeface is especially suitable for use in the humanities. For more information, please see www.brill.com/brill-typeface.

\section{ISSN 1572-050}

ISBN 978-90-04-25598-2 (hardback)

ISBN 978-90-04-25667-5 (e-book)

Copyright 2014 by Koninklijke Brill NV, Leiden, The Netherlands.

Koninklijke Brill NV incorporates the imprints Brill, Global Oriental, Hotei Publishing, IDC Publishers and Martinus Nijhoff Publishers.

All rights reserved. No part of this publication may be reproduced, translated, stored in a retrieval system, or transmitted in any form or by any means, electronic, mechanical, photocopying, recording or otherwise, without prior written permission from the publisher.

Authorization to photocopy items for internal or personal use is granted by Koninklijke Brill NV provided that the appropriate fees are paid directly to The Copyright Clearance Center, 222 Rosewood Drive, Suite 910, Danvers, MA 01923, USA.

Fees are subject to change.

This book is printed on acid-free paper. 


\section{CONTENTS}

List of Illustrations .................................................................................. vii

Preface ...................................................................................................... ix

Stéphane Benoist \& Gerda de Kleijn

List of Contributors .................................................................................. Xv

Introduction ............................................................................................

Ségolène Demougin

Pluralisme juridique et intégration de l'empire ..................................... $\quad 5$ Clifford Ando

Gli equilibri della cittadinanza romana, fra sovranità e impatto sociale

Salvo Randazzo

The Evolution of the So-called Provincial Law, or: Cicero's Letters of Recommendation and Private International Law in the Roman World

Hannah M. Cotton

Claude de Lyon, Ancus Marcius et l'âge royal : d'une intégration l'autre

Anne Daguet-Gagey

Das Leben römisch Gestalten. Ein Stadtgesetz für das municipium troesmis aus den Jahren 177-180 n. chr.

Werner Eck

Goths and Romans in the leges visigothorum J.H.W.G. Liebeschuetz

Masters and Freedmen: Junian Latins and the Struggle for

Citizenship Egbert Koops 
Migrant Quarters at Rome?

Roman Citizenship and the Integration of Women into the Local Towns of the Latin West Emily A. Hemelrijk

Du foyer au forum. La place des matrones équestres dans les activités économiques 161 Anthony Álvarez Melero

Integration or Disintegration? The Roman Army in the Third Century A.D. Lukas de Blois

Differentiated Integration Trajectories of the Nomadic Population in Roman North Africa (1st-3rd Cent. A.D.) Wouter Vanacker

Wie integriert man Rom in die polis? Der Kult des Senats in Kleinasien

Günther Schörner

Les marques civiques sur briques et tuiles, témoins de l'intégration des cités dans le monde romain 243 Monique Dondin-Payre

Convergence and Divergence: One Empire, Many Cultures ............. $\quad 263$ Frederick G. Naerebout

Index

Nominum ............................................................................ 283

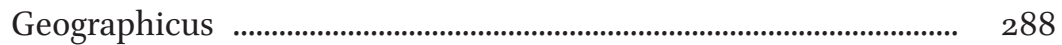

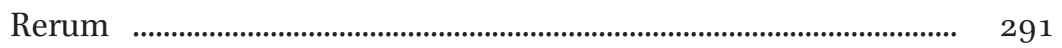

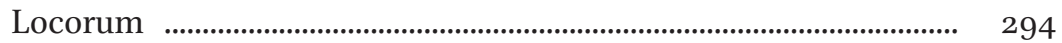




\section{LIST OF ILLUSTRATIONS}

Eck

Tabelle 1. Ordnung innerhalb leges

Tacoma

Table 1. Dimensions and degrees of clustering

Hemelrijk

Fig. 1. Geographical distribution

Fig. 2. Geographical and chronological distribution

Fig. 3. Social status of women in civic roles in the Western

\section{Álvarez Melero}

Table 1. Datation et localisation des manifestations

$$
\text { évergétiques }
$$

Table 2. Edifices financés par des matrones équestres

Table 3. Montants et justification de donations effectuées par des matrones équestres

Table 4. Matrones équestres légataires de leurs proches

Table 5. Matrones équestres propriétaires de domaines fonciers

Table 6. Activités évergétiques des matrones équestres

De Blois

Table 1. Careers in several regions

\section{Schörner}

Abb. 1. AE Smyrna: Synkletos und Livia; Tiberius in Tempel ..... 222

Abb. 2. AE Smyrna: Synkletos ........................................................... $\quad 226$

Abb. 3. AE Perperene: Büste der Synkletos mit Mauerkrone; Demeter

Abb. 4. AE Alabanda: Demos, Synkletos thronend ......................... 230

Tabelle Die Statuenprozession des Vibius Salutaris in Ephesos $\ldots 241$

\section{Dondin-Payre}

Table 1. Les marques civiques sur céramique 



\section{PREFACE}

Stéphane Benoist \& Gerda de Kleijn

The Tenth Workshop of the International Network "Impact of Empire (Roman Empire, $200 \mathrm{BC}-\mathrm{AD}$ 476)" has taken place at the University of Lille-Nord de France, on June $23-25,2011$. It was the first time the Network was received in France for a workshop, after a decision of the Administration and Scientific Boards at Durham in April 2009 to select Lille, New York and Rome for the 10th, 11th, and 12th conferences. Nothing would have been possible without the generous financial support of the Radboud University Nijmegen and of different French institutions: the research laboratory HALMA-IPEL (Histoire, Archéologie, Littérature des Mondes Anciens-Institut de Papyrologie et d'Égyptologie de Lille, UMR 8164, CNRS, Lille 3, Ministère de la Culture et de la Communication), the Institute of Humanities and Social Sciences of the CNRS whose 32nd section of its National Committee has granted the colloquium at the First rank (in October 2010), and the County Council of Nord-Pas de Calais. We wish to thank them for their help in various steps of the preparation and the unfolding of the colloquium, which was the 1oth Impact of Empire meeting as well as the XXXVth international colloquium of the scientific research team Halma-Ipel.

We would like to thank the participants of the colloquium in Lille, either contributors or chairs of the sessions: Anthony Álvarez Melero, Clifford Ando, Stéphane Benoist, Lukas de Blois, Fabienne Burkhalter, Hannah M. Cotton, Anne Daguet-Gagey, Ségolène Demougin, Monique Dondin-Payre, Olivier Hekster, Emily A. Hemelrijk, Christine Hoëtvan Cauwenberghe, Gerda de Kleijn, Egbert Koops, Wolf Liebeschuetz, Frederick G. Naerebout, Salvo Randazzo, Günther Schörner, Daniëlle Slootjes, Laurens Tacoma and Wouter Vanacker. Werner Eck, who was not able to participate, kindly sent us his contribution about the new lex municipalis he had recently edited.

The different sessions of the colloquium took place at the auditorium of the Museum of Fine Arts in Lille and the Research House of the University of Lille 3 at Villeneuve d'Ascq, and we were friendly received at the townhouse of Lille. The organizers of the conference are also grateful for the assistance during the preparation of the workshop of Christine Aubry, 
Jocelyne Casène and Marie-Pierre Fuch. Finally, we are indebted to Brill, and especially Caroline van Erp, Editor Classical Studies and Ancient Philosophy, and Tessel Jonquière, Production editor, for their help in publishing this volume.

The International Network Impact of Empire (Roman Empire $200 \mathrm{BC}-$ $\mathrm{AD} 476$ ) has a dual objective. On the one hand, it aims to explore the dynamics of the Roman Empire in the Mediterranean and beyond for a long stretch of time. On the other hand, the network seeks to create conditions for beginning researchers to meet more advanced scholars, and turn the exchange of ideas to their good advantage. Both objectives applied to the tenth workshop held in Lille, the results of which are presented in this volume.

The main theme of the workshop was "Integration at Rome and in the Roman world". The organizers, Ségolène Demougin, Stéphane Benoist, and Olivier Hekster, have chosen to look at the integration-process in a broad sense in hopes that this would lead to suggestions about the way in which Roman integration was linked to wider dynamics in the Roman Empire. Advanced and beginning scholars from the countries that participate in the network were invited to reflect on juridical, social, religious, and political aspects of the process of inclusion in an empire under the political control of the city of Rome, her princeps, and the different authorities in the provinces and cities. The idea was that it would become possible to consider e.g. changes in the relationships between Romans (however conceived) and non-Romans by looking at developments over a longer period of time. Notions of personal and collective identities might be linked to relevant Roman realia, so that various contents of Romanitas would be defined through contextualization.

Integration is made up of multifaceted processes taking place at various levels in society and at different places, over a long period of time. In this volume, different perspectives underlie the analyses of these processes. Juridical, political, social and religious points of view are articulated, elaborating on epigraphic, literary, juridical and numismatic evidence.

The introduction by Ségolène Demougin infra will give some interpretation clues for the essential integration patterns in Roman social history. Roman citizenship, based on civic and political statutes with juridical statements from the first centuries of the Republic, offered a civic model to the Imperium Romanum, which would, in approximately half a millennium (from the and century $\mathrm{BC}$ to the 3 rd century $\mathrm{AD}$ ), concern the 
great majority of free men living within the limits of the Imperial territory, though not the population as a whole. Two decisive steps in this process of ongoing distribution of citizenship have mainly been studied in the past: the municipalization of Italy at the beginning of the 1st century BC, and the Constitutio Antoniniana of $\mathrm{AD} 212$. This was done through analysis of a wide spectrum of aspects, i.e. the juridical, social, political, cultural and religious ones, or through discussion about what has been named (with some objections) 'Romanization'.

The first part is devoted to juridical approaches of the subject. Clifford Ando investigates the meanings of the jurisdictional pluralism and inquires into the centrifugal and centripetal powers affecting integration processes. Anne Daguet-Gagey tries to discover how the emperor Claudius integrated the history of the origins of Rome into the Roman Empire of his own days. This is shown among other things by the stories about the fourth Etruscan King, Ancus Marcius, which serve as his exempla in the debate about the enrolment of provincial nobles in the Senate. Salvo Randazzo, who is a historian of Roman Law, presents a wide survey of evidence and historiography about ciuitas and its relation to the concept of Imperium Romanum. Hannah Cotton offers a reappraisal of her previous inquiry about Cicero's recommendation letters and studies the concept of the so-called Private International Law. Legal cases in the provinces reveal how imperial and local law systems operated in contrast and in coexistence. Her paper complements Ando's in that way.

The second part is concentrated on the adoption of Roman Law in specific areas and periods of time. Werner Eck presents a new example of a lex municipalis from Troesmis in Moesia inferior, dating from the reign of Marcus Aurelius. It provided for institutional, political, social and religious Roman rules. Striking is that it was written along the same lines as flavian municipal laws from Baetica. It must be assumed that the Roman conception of ciuitas was diffused even at the border of the Roman Empire in the turbulent times of the 2nd century AD. Wolf Liebeschuetz raises the matter of the perennial influence of Roman law in Spain. He wonders how the two peoples, the old rulers and the new ruling people, managed to coexist, and discusses the Leges Visigothorum, the best source to approach personal statutes about Romans and Goths up to the 7th century AD.

Roman perspectives on various aspects of social organization are brought up in part three. This part addresses different integration steps by discussing a series of dynamic alternatives: free-slave, Roman-non Roman, male and female. Egbert Koops examines the personal statute of Junian Latins and other manumitted slaves. He discusses the access to Roman 
citizenship in the process of enfranchisement as an integration factor. Residential segregation and social, economic, and/or ethnic clustering are important factors in modern integration debates. Going into discussions about migrant quarters in Rome, Rens Tacoma makes use of concepts borrowed from these modern debates. Emily Hemelrijk enters into the issue of the integration of women in Roman civic life in the western provinces. She inquires what stimulated or impeded this integration, by using two criteria: the adoption of Roman culture such as dress, customs and values, and participation in activities like civic munificence, priesthoods and patronage. Antony Álvarez Melero also engages in civic roles of women in the western part of the empire. Focussing on women, mothers and daughters of equites Romani, he discusses the evidence for their economic activities and euergetism.

The fourth and last part is chiefly concerned with specific aspects of integration within the army, in cities and in territories belonging to the Roman Empire. Lukas de Blois explores whether replenishments of the legions in the empire's border regions in the third century AD resulted in disintegration, threatening the unity of the only truly "Imperial" community, the Roman army. Wouter Vanacker has chosen an anthropological approach to study the relations between nomadic tribes, semi-nomadic groups and sedentary populations in Mauretania Tingitana. The overall picture is not unambiguous. Each group tried to benefit from agreements and used force if they saw fit. The procedures and activities to shape Roman institutions in poleis in Asia Minor are investigated by Günther Schörner in a case study, i.e. the integration of the senate in a few cities by the introduction of the cult of the Synkletos. To that end, numismatic evidence is essential. Monique Dondin-Payre makes use of epigraphic evidence to examine the concrete meaning of the diffusion of a civic model (i.e. institutions and their counterparts, names of citizens and magistratures) in Italy, the Gauls and Britain.

A number of authors refer to Frederick Naerebout's provocative paper. The editors thought that it would be the best conclusion to this collection of contributions to the concepts of, and ongoing debates on, integration in a Mediterranean Empire.

Was the 1oth conference of the "Impact of Empire" Network in Lille a "success story", if we speak like Frederick Naerebout, about "integration and Romanization"? For the organizers of the meeting and the editors of this volume, the ambition was not to deliver any synthesis about this great and important subject - a task quasi-impossible to achieve in the context 
of a single colloquium -, but to add a few elements to our knowledge about an Empire, which has no equivalent anywhere. ${ }^{1}$ The understanding of Roman integration is definitely linked to our capacity to ask the true questions about ciuitas and Roman citizenship, social mobility within cities and provinces, "universal" conception of law and mass acceptation of it in space and time, relationships between communities of humans and gods, and the proper evolution from a polytheist to a monotheist world. So to speak, these proceedings aim at participating to a large debate by fifteen contributions, which can be considered, each of them, as a new stone to build a common monumentum, our comprehensive way to participate to Romanitas.

February 2013

Paris \& Nijmegen

${ }^{1}$ E.g. the debates about "empires" in global history, after the issue of the book by J. Burbank \& F. Cooper, Empires in world history. Power and the politics of difference (Princeton 2010); see the special volume of the French journal Monde(s) Histoire, Espaces, Relations, $\mathrm{n}^{\circ}$ 2, nov. 2012, dedicated to Empires. 



\section{LIST OF CONTRIBUTORS}

Anthony Álvarez Melero, Ph.D. (2010) in Roman History, Université libre de Bruxelles and Universidad de Sevilla, is currently a Juan de la Cierva post-doctoral researcher at the Universidad de Sevilla. He has published several articles on the women related to Roman knights.

Clifford Ando is David B. and Clara E. Stern Professor as well as Professor of Classics, History and Law at the University of Chicago; and Research Fellow, Department of Classics and World Languages, University of South Africa.

Lukas de Blois is emeritus professor of Ancient History at the Radboud University Nijmegen. His main interests are (the crisis of) the third century $\mathrm{AD}$, and the role of the middle cadre in the Roman army. He initiated the network Impact of Empire.

Professor (emeritus) Hannah M. Cotton, D.Phil. Oxon., Dr h.c. Theol. (Bern), Shalom Horowitz Chair in Classics, Hebrew University of Jerusalem. Corresponding member of the GAI, Member of AE, Honorary Member of the Society for the Promotion of Roman Studies. Author of Masada II, DJD XXVII and "Roman Government and Jewish Life Studies in Documentary Evidence" (collected Papers, forthcoming in Studia Iudaica, De Gruyter). Editor of Corpus Inscriptionum Iudaea/Palaestinae.

Anne Daguet-Gagey is professor of Roman History at the University of Artois-Arras. Her research is focused on the City of Rome and her administration, the opera publica and the aedility, as well as on the 3rd century $\mathrm{AD}$ and mainly the Severans.

Ségolène Demougin is emeritus director of research at the CNRS and emeritus director of study at the EPHE History and philology section (Paris). Her main interests are the ordo equester with the prosopography of equites Romani, as well as the administration of the Roman Empire.

Monique Dondin-Payre is director of research at the CNRS (Paris). Her research is mainly focused on the provincial society of the Western part of the Roman Empire (Gauls, Germanies, Africa), especially the municipal 
institutions and the social implications of Romanization (e.g. onomastical studies, and citizenship).

Werner Eck is emeritus professor of Ancient History at the Universität zu Köln. His research is focused on Roman imperial administration, social history, prosopography, and early Christianity. He is Dr h.c. BabesBolyai University Cluj, Kassel University, and the Hebrew University in Jerusalem, and honorary member of the Society for the promotion of Roman Studies.

Emily A. Hemelrijk is professor of Ancient History at the University of Amsterdam. Her research focuses on Roman women and includes Matrona docta. Educated women in the Roman élite from Cornelia to Julia Domna (London/New York, 1999 / 2004). She is co-editor (with L. de Ligt and H.S. Singor) of Roman Rule and Civic Life: Local and Regional Perspectives (Amsterdam, 2004) and, with Greg Woolf, edited Women and the Roman City in the Latin West (Leiden/Boston, 2013). She is currently preparing a book on Hidden Lives - Public Personae. Women and Civic Life in Italy and the Latin West during the Roman Principate.

Egbert Koops received his $\mathrm{PhD}$ in legal history from Leiden University in 2010 and was afterwards employed in a research project on the Roman slave peculium. He currently is an assistant professor of property law at Groningen University.

Wolf Liebeschuetz is emeritus professor of Ancient History. He has taught at the universities of Leicester and Nottingham. His main interest is in Late Antiquity, focusing on the development of cities, the settlement of barbarians, and religion, both pagan and Christian.

Frederick G. Naerebout is lecturer in Ancient History at the History Department of Leiden University. His research revolves around religion in the Graeco-Roman world and issues of acculturation in the Hellenistic kingdoms and in the Roman Empire.

Salvo Randazzo is professor of Roman Law at LUM Jean Monnet, Bari, where he teaches also Legal Anthropology and European Legal Roots. He has been visiting scholar at NYU, New York, and Maquarie University, Sydney. His publications concern areas of ancient legal experience and the links between Roman Law, European Codes and Common Law. 
Günther Schörner is professor of Classical Archaeology at the Universität Wien, Austria. He is engaged in research into cults, rural settlements, and cultural encounters in the Roman Empire, as well as Greek iconography.

Laurens E. Tacoma is lecturer in Ancient History at Leiden University, The Netherlands, with a special interest in Roman social history. In 2006, he published Fragile Hierarchies. The urban elites of third-century Roman Egypt. Currently he is working on a monograph on Roman mobility and migration as part of the research project Moving Romans. Urbanisation, migration and labour in the Roman Principate, funded by The Netherlands Organization for Scientific Research (NWO).

Wouter Vanacker (Ghent University) is studying patterns of interaction between pastoralists and agriculturalists primarily in Roman North Africa. Through his research, he also aims to improve current knowledge of imperial administration and military organization. 



\section{INTRODUCTION}

\section{Ségolène Demougin}

La dixième réunion du programme international Impact of Empire qui s'est tenue pour la première fois en France, a voulu explorer les voies de l'intégration à Rome, sur la longue durée, du III ${ }^{\mathrm{e}}$ s. av. J.-C. au ve s. ap. J.-C., et d'en cibler les enjeux.

Comme on le sait, les Romains occupent une place singulière dans l'histoire de l'Antiquité ; Rome forme un peuple jaloux de son identité, et qui avantage au maximum ses propres ressortissants, ceux qui participent de la citoyenneté, avec les droits et aussi les devoirs qui y sont attachés. Jamais, durant la construction de son identité, Rome n'a voulu accueillir dans la communauté qu'elle formait les autres, ou devrai-je dire plutôt l'Autre. Entre le citoyen et l'Autre, étranger à la collectivité romaine, il n'y avait pas de choix possible. Il suffit à cet égard de mettre en perspective les débuts de l'expansion romaine, jusqu'aux Guerres Puniques. Dans la conquête des peuples italiens, il n'était pas question d'intégrer les nouveaux sujets, soumis aux exigences du vainqueur. Bien au contraire, à considérer le phénomène de la colonisation, toujours alors de type militaire, il s'agissait d'établir Rome dans les nouvelles possessions, et donc d'en écarter les anciens occupants. Le rejet de la personne de l'autre est donc le fondement premier de la conception romaine du monde. Par là, elle se différencie fondamentalement de l'attitude et des pratiques des cités grecques.

Mais cette rigidité apparente devait être dans les faits tempérée, tout en maintenant le principe d'une stricte hiérarchie : en effet, les différents degrés dans l'appartenance à la citoyenneté apparurent assez tôt, comme le montre la création du droit de cité latin, réservé d'abord aux Italiens, dont on connaît le succès dans tout l'Empire, à partir de la constitution du principat.

Rome fonctionne, comme d'autres systèmes antiques oligarchiques, sur le principe de l'exclusion, qui s'applique d'abord à ses propres citoyens ; il suffira à cet égard d'évoquer l'institution électorale la plus importante, celle des comices centuriates. Les citoyens, romains, tous égaux théoriquement par leur droit de cité, ne peuvent pas tous participer aux choix fondamentaux pour le gouvernement de la cité. Le choix restreint des 
acteurs du champ politique se fonde ici sur le critère censitaire, et adopte donc le principe suivant lequel les plus riches doivent le plus contribuer, mais aussi accéder aux vraies responsabilités. La pratique de l'exclusion s'applique encore plus dans les relations avec les personnes étrangères à la cité.

Il est inutile de rappeler que cette attitude extrême d'un peuple qui dut d'abord se défendre avant de se répandre dans le monde italien, puis dans ce qui devait constituer l'orbis Romanus, fut considérablement amendée et, sur le plan civique au moins, fut abandonnée par la constitutio Antoniniana de 212 ap. J.-C. On a appelé ce phénomène la romanisation. Mais il ne faut pas se tromper, et sortir des clichés faciles. Le concept repose sur un malentendu, et reste équivoque. Trop souvent, l'opinion publique croit que la romanisation a été voulue et imposée par Rome aux habitants de l'Empire. Cette vue simpliste, qui rappelle les motifs et les utopies de la colonisation européenne du $\mathrm{XIX}^{\mathrm{e}}$ s., est totalement contradictoire avec ce que nous savons des pratiques romaines. En effet, ce sont les sujets de Rome - ou du moins certains sujets - qui ont voulu entrer dans la communauté romaine, même en conservant des caractères qui leur étaient propres. N'oublions pas que ce sont les notables de la Gaule « chevelue » qui ont demandé à l'empereur Claude le bénéfice du ius honorum. Par contraste, il n'est pas inutile de mentionner aussi, dans quelques cas, une relative indifférence aux structures romaines : on peut citer le cas des notables éphésiens qui mirent longtemps à accepter, sinon à rechercher, les avantages romains, au contraire de leurs voisins smyrniotes ou pergaméniens. Mais il faut bien dire qu'ils se rattrapèrent par la suite, en abondant les ordres supérieurs romains.

En revanche, on se méfiera, bien évidemment, des tentations de la « résistance à la romanisation », mise à la mode il y a bientôt quarante ans ; il suffit de constater la progression des noms latins, très simples, sur les milliers d'épitaphes retrouvées dans le Maghreb : tous ces pérégrins n’ont pas été contraints de troquer leur nom indigène contre des appellations latines. Ainsi, la notion même de romanisation, vue même sous l'aspect des identités, tout commode qu'il soit, doit être utilisée avec précaution. Il serait d'ailleurs facile, même si ce n'est pas ici le sujet, d'identifier des situations et des comportements comparables dans d'autres époques.

Nous avons préféré parler d'intégration, car l'intégration est à double face : dans un territoire unifié et gouverné par une autorité centrale, dont le contrôle est parfois serré, parfois lâche, appuyé sur une bureaucratie très présente, les individus, les familles, les collectivités sont de plus en plus aspirées par le modèle romain; d'un autre côté, les détenteurs du 
pouvoir romain ont senti les effets néfastes de l'exclusion totale et ont appris aussi l'art des concessions. Pour ne prendre qu'un exemple, on parlera ainsi des octrois individuels du droit de cité, dont les heureux bénéficiaires se vantent dans maintes inscriptions, et cela, dès le $\mathrm{I}^{\mathrm{er}} \mathrm{s}$. av. J.-C., en plein pays hellénophone. Mais il n'y a pas que le domaine du droit et des institutions qui est touché par cette démarche.

Nous savons tous aussi que Rome n'a jamais hésité à assimiler d'autres modèles et à les intégrer dans son propre système, en les répandant dans le monde qu'elle dominait. Ainsi, l'intégration, telle Janus, a deux faces complémentaires Pour ne prendre qu'un exemple, les modèles architecturaux hellénistiques ont été admirés et adoptés par les Romains, et on les rencontre jusque dans la péninsule Ibérique. Sur le plan culturel, les rencontres ont été fécondes, et ont constitué petit à petit un ensemble bien typé, qui n'était pas refermé sur lui-même, mais ouvert sur d'autres cultures.

Cette démarche, il faut bien le dire, fut facilitée par les caractères de certaines institutions, comme l'armée impériale, avec ses milliers d'auxiliaires, engagés non seulement par l'attrait du métier des armes, mais aussi, s'ils arrivaient jusque-là, par l'assurance qu'à la retraite, ils deviendraient citoyens romains. Et on pourra observer que l'armée romaine se rencontre partout, dans tout le territoire formant le monde romain, même dans les provinces théoriquement « civiles »; cette présence constitue en fait un fort facteur d'unité pour les peuples soumis à Rome, et souligne le caractère universel de l'empire.

D'ailleurs, dans le cas présent, cette intégration s'accompagnait aussi de l'adoption des dieux de Rome, sans renonciation d'ailleurs aux dieux ancestraux. En témoignent ces nombreuses marques de piété, offertes, par exemple, par des soldats et anciens soldats palmyréniens, donc dans le dernier cas naturalisés, qui tout en rendant un culte aux dieux romains n'oublient pas ceux de leur lointaine patrie. Et ce phénomène n'apparaît pas seulement dans la sphère militaire. Des exemples encore plus extraordinaires pourront être mis en exergue, comme la dédicace de ce Claudius Mucatra, donc Thrace, à Iuppiter Optimus Maximus assimilé au dieu celtique Cernenus, offerte à la colonie de Carthage (CIL VIII, 12488, Carthage : I(oui) O(ptimo) [M(aximo)] Cer(neno). / Cl(audius) Muc(atra) col(oniae) $d($ ono $) d($ at $))$.

D'autres facteurs ont facilité l'intégration, et l'ont, dans bien des cas, hâtée; et parmi eux, l'un des plus importants est sans aucun doute la figure de la personne impériale, qui rassemble, par son culte, au fur et à mesure de son établissement, autour d'elle, tous ceux qui vivent sous la 
loi romaine. C'est l'empereur qui les fédère autour de lui et qui provoque un consensus universel. Il est remarquable que cette unanimité se soit maintenue, en dépit des nombreuses vicissitudes, pendant quatre siècles. $\mathrm{Au}$ fond, le prince était devenu le garant de l'intégration à l'Empire. L'idéologie impériale avait parfaitement rempli son rôle, avant qu'une autre idéologie, fondée sur le recours à l'éternité, et prétendant elle aussi à l'universalité, ne finisse par la remplacer.

C'est pour mesurer les avances et les limites de cette intégration que la rencontre lilloise nous a réunis voici dix-huit mois, en n'excluant aucun des domaines où elle s'est exercée. Mais ses modes et leurs résultats doivent être scrutés avec attention: dans certains cas, l'influence romaine finit par l'emporter; mais d'autres cas, ce fut Rome qui ne subit pas mais accepta l'apport extérieur. Ce sont ces échanges mutuels, ces acceptations, ces concessions mutuelles qui ont forgé l'identité de ce monde romain, le «monde (qui) finit toujours par vaincre l'histoire», comme le disait A. Camus (Noces, «Le vent à Djémila»), ce monde où nous nous reconnaissons tous. 


\section{PLURALISME JURIDIQUUE ET INTÉGRATION DE L'EMPIRE}

\section{Clifford Ando ${ }^{1}$}

1. Les études consacrées aux empires ont tendance à envisager le droit de la métropole comme un outil uniquement capable de séparer, et jamais d'unir, les habitants de l'empire. A la suite de leur engagement idéologique en faveur des systèmes de statut juridique personnel, les empires des mondes antique et moderne peuvent en effet tous être caractérisés comme juridiquement pluralistes, comme des paysages juridiques dans lesquels plusieurs systèmes de normes et de multiples sources de normes coexistaient. Bien entendu, ce pluralisme était à chaque fois justifié différemment et les conflits de loi qui en résultaient trouvaient des solutions diverses ${ }^{2}$. En droit moderne, ces problèmes sont bien connus, et cette communication s'intègre dans un projet de plus grande envergure ayant pour but - ou peut-être pour objectif principal - d'établir un dialogue plus étroit entre les chercheurs en droit ancien et moderne.

Dans le cadre de notre réflexion commune sur l'« Impact de l'Empire», j'espère contribuer à mieux articuler certains problèmes d'action, de causalité et de mutation, qui reviendront certainement dans les contributions des autres participants.

2. Comme nous le verrons, pour des raisons d'intérêt politique et de philosophie du droit, les distinctions de statut juridique entre les personnes étaient d'une grande importance chez les Romains, comme chez la plupart des peuples impérialistes anciens et modernes, et entraient en corrélation avec des distinctions dans la juridiction. J'utilise le terme «juridiction» dans le sens moderne, comme synonyme de «forum», pour parler à la fois du tribunal dans lequel une affaire est instruite et du cadre juridique dans lequel elle est jugée. Les doctrines qui liaient les systèmes de statut

1 En raison des contraintes de longueur, les notes sont réduites au minimum. Je voudrais remercier Olivier Hekster et Stéphane Benoist pour l'invitation à Lille et Mireille Dobrzynski et Yann Berthelet pour avoir corrigé le texte français.

2 Cf. H.M. Cotton, 'Private international law or conflict of laws', in R. Haensch et J. Heinrichs (eds.), Herrschen und Verwalten. Der Alltag der römischen Administration in der Hohen Kaizerzeit (Köln 2007), 234-255. 
personnel et de juridiction étaient bien réelles, et elles auraient pu poser un obstacle majeur à l'intégration de l'empire, du moins selon la loi.

En dépit, toutefois, de cet engagement envers une forme de pluralisme juridique, le droit fonctionna comme un mécanisme important dans l'intégration de l'empire. Je soutiens que cela se produisit davantage par le biais de procédures juridiques, y compris la procédure civile, que par le droit positif. Cette affirmation se justifie en partie du fait que, comme Birks, Rodger et Richardson l'ont souligné, les questions de droit positif étaient résolues, dans le système formulaire romain, par le biais d'une procédure $^{3}$. Dans un volume publié à l'automne 2011, je souligne ainsi l'importance, dans la littérature jurisprudentielle, d'un petit nombre de procédures légales visant à légitimer l'expansion et le changement dans l'argumentation juridique 4 . Il ne faudrait cependant pas insister démesurément sur l'importance de l'observation de Richardson, car la relation entre le droit positif et la procédure dans les systèmes juridiques locaux fut rarement la même qu'à Rome.

3. On pourrait commencer par donner un aperçu du cadre normatif de base du pluralisme juridique de l'empire romain, tel que les Romains euxmêmes l'ont théorisé 5 . La formulation la plus célèbre et la plus concise, dans un texte classique, est celle fournie par Gaius au début de ses Institutes :

Tout peuple régi par le droit écrit et par la coutume suit en partie un droit qui lui est propre, en partie un droit qui lui est commun avec l'ensemble du genre humain. En effet, le droit que chaque peuple s'est donné lui-même lui est propre et s'appelle droit civil, c'est-à-dire droit propre à la cité (nam quod quisque populus ipse sibi ius constituit, id ipsius proprium est uocaturque ius ciuile, quasi ius proprium ciuitatis) : tandis que le droit que la raison naturelle établit entre tous les hommes est observé de façon semblable chez tous les peuples et s'appelle droit des gens, c'est-à-dire droit dont toute la gent humaine fait usage. C'est ainsi que le peuple romain est régi en partie par un droit qui lui est propre, en partie par le droit commun à tous les hommes. (Gaius Institutes 1.1, trad. Julien Reinach)

3 P. Birks, A. Rodger et J.S. Richardson, 'Further aspects of the Tabula Contrebiensis', Journal of Roman Studies 74 (1984), 45-73, ici 60.

4 C. Ando, Law, language, and empire in the Roman tradition (Philadelphia 2011), 1-18.

5 J. Fournier, Entre tutelle romaine et autonomie civique. L'administration judiciaire dans les provinces hellénophones de l'Empire romain (129 av. J.-C.-235 apr. J.-C.) (Athènes 2010), 19-61. 
Le droit civil est un corps de lois qu'une communauté de citoyens établit pour et sur elle-même; seuls les membres de cette communauté, c'està-dire ses citoyens, ont a priori accès à ses actions du droit. Le fond de l'affirmation de Gaius est exprimé par les pronoms distributif et réfléchi quisque et sibi: ius ciuile dénote ces corps du droit que chaque communauté politique établit pour elle-même.

La même interprétation est présente dans le récit de Tite-Live, par exemple aux lendemains de la guerre contre les Herniques, en 306 av. J.-C. :

Trois cités des Herniques - les Aletriani, les Verulani et les Ferentinati, [qui avaient pris le parti de Rome dans la guerre] -, parce qu'elles préféraient cela à la citoyenneté romaine, obtinrent qu'on leur rendît leurs lois (quia maluerunt quam ciuitatem, suae leges redditae)...(Liv. 9.43.22)

Peu après cet incident, Tite-Live signale que les Romains demandèrent satisfaction de la part d'un autre allié rebelle, les Aequi :

Les Aequi répondirent que la demande était une tentative manifeste de les forcer, sous la menace d'une guerre, à souffrir de devenir Romains : les Herniques avaient montré combien ceci était désirable lorsque, le choix leur étant accordé, ils avaient préféré leurs propres lois à la citoyenneté romaine (cum quibus licuerit suas leges Romanae ciuitati praeoptauerint). A ceux à qui l'opportunité de pouvoir choisir ce qu'ils désiraient n'était pas accordée, la citoyenneté était nécessairement pro poena, semblable à une punition (quibus legendi quid mallent copia non fuerit, pro poena necessariam ciuitatem fore). (Liv. 9.45.6-8)

Plus fondamentalement encore, la même corrélation entre le système de droit et la citoyenneté est à la base de la première loi sur la juridiction dans les provinces, à propos de laquelle on dispose d'un témoignage considérable et plus ou moins contemporain, à savoir la lex Rupilia:

Suivant le droit qui régit les Siciliens, les actions judiciaires qui opposent deux citoyens de la même cité sont jugées dans leur cité, selon leurs propres lois (domi... suis legibus). Pour juger des actions d'un Sicilien avec un Sicilien d'une autre cité, le préteur devait procéder au tirage au sort du juge, conformément au décret de Publius Rupilius, déterminé sur la recommandation de la commission de dix légats: les Siciliens appelaient ce décret la loi Rupilia. Pour juger des poursuites menées par un particulier contre une communauté, ou par une communauté contre un particulier, c'est le sénat d'une autre cité qui devait être juge, avec la garantie que chacune des parties aurait la possibilité de récuser une cité. Quand un citoyen romain poursuit un Sicilien, c'est un Sicilien qui est désigné pour juger : quand un Sicilien poursuit un citoyen romain, un citoyen romain est désigné. Dans toutes les autres affaires, les juges sont habitués à être choisis parmi les citoyens romains résidant dans le district d'assise. (Cic., 2 Verr. 2.32) 
Autrement dit, le paysage juridique de la Sicile - et, pourrais-je ajouter, celui de toutes les autres provinces - était juridiquement pluraliste. Non seulement il était fragmenté en des juridictions multiples, chacune avec son propre code juridique, mais des logiques multiples, en désaccord les unes avec les autres, furent utilisées pour assigner des procès aux fora: certaines relevaient de déterminations géographiques, d'autres de la citoyenneté des parties, et ainsi de suite.

Cette interprétation de la relation entre le statut juridique, l'articulation légale de l'appartenance collective et du système de droit donna lieu à une politique destinée à conserver les ordres législatifs purement locaux, et ces ordres furent à leur tour interprétés comme contribuant à un ordre social explicitement impérial. L'hypothèse opératoire semble être que les ordres sociaux locaux sont les mieux garantis par l'adhésion aux normes localement produites et, comme corollaire, que Rome n'a pas de base épistémique, ni aucune obligation déontologique à remplacer celles-ci ${ }^{6}$. De là, le principe général énoncé à Pline par Trajan, que id ergo, quod semper tutissimum est, sequendam cuiusque ciuitatis legem puto, « il est toujours plus sûr, je pense, que la loi de toute communauté de citoyens soit observée » quand cela relève de sa compétence (Trajan apud Plin., Ep. 10.113). Je reviendrai sur cette question dans un moment.

4. Je souhaiterais faire, auparavant, un aparté. Il m'apparaît de plus en plus évident que la Constitutio Antoniniana a marqué un grand tournant dans l'histoire du droit, comme le remarque Ménandre le Rhéteur dans ses instructions sur l'éloge d'une ville sous le coup de la justice :

De nos jours, le sujet des lois n'a pas d'usage, puisque nous menons nos

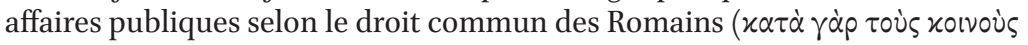

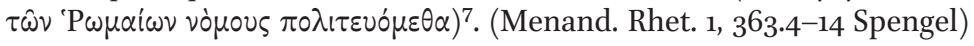

À la documentation déjà présentée, on peut ajouter un papyrus de 223 ap. J.-C., copie d'une pétition relative à un litige sur une question de

6 Cf. C. Ando, Religion et gouvernement dans l'Empire romain (Paris 2012), chapitre 3.

7 Menand. Rhet. 1, 363.4-14 Spengel. Voir aussi Menand. Rhet. 1, 360.10-16 Spengel

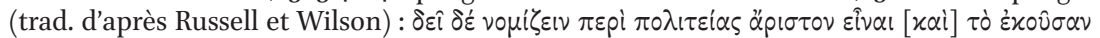

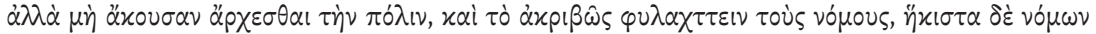

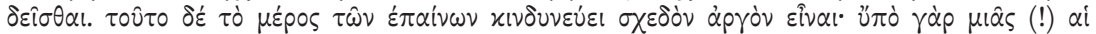

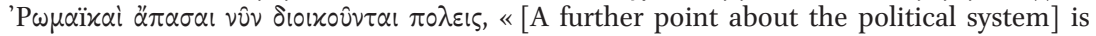
that it is best for a city to be ruled in accordance with its own will, not against its will, and for it to observe the laws with exactness, but not to need laws. This last section of praise, however, is virtually useless today, since all Roman cities are regulated by one < and the same politeia? > ». 
propriété. J'attire en particulier votre attention sur la juxtaposition des termes nomos et politeuomai, termes également employés par Ménandre le Rhéteur :

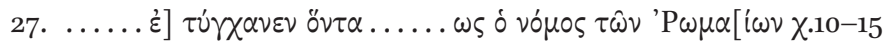

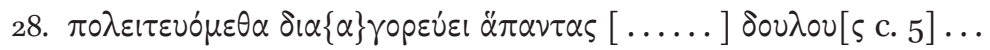

«... happened to be ... the law of the Romans ... we are (Roman) citizens, declares that all the slaves...» (P.Oxy. 4961, 14 novembre 223, ll. 27-28 / $75^{-76}$ : trad. J. David Thomas)

On remarque ici la tentative manifeste d'expliquer le changement de citoyenneté par le changement de cadre légal. Ce texte soutient la comparaison avec d'autres textes qui expliquent le changement social en s'appuyant sur les effets de la Constitutio Antoniniana, les effets les mieux connus et les mieux attestés étant les changements de nomenclature (P.Oxy. 1458, 216-17 ap. J.-C., ll. 2-7) : «...Aurelius Aelurion, cosmêtês en fonction et sénateur d'Athribis, avant qu'il n'obtienne la citoyenneté

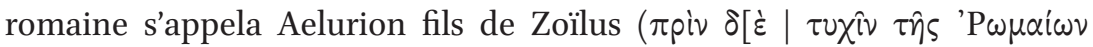

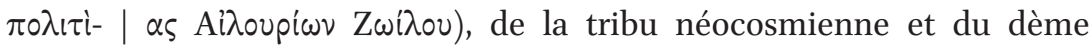
althéen ».

Je voudrais enfin attirer l'attention sur la continuité que l'on observe, sur un quart de millénaire, dans l'interprétation idéologique et philosophique de la citoyenneté et du droit : le même fondement théorique qui avait contraint, justifié et soutenu le pluralisme juridique au début de l'empire a, par la suite, imposé la création d'une culture légale unitaire durant le Haut-Empire.

5. En revenant au texte de Gaius, en particulier aux implications des pronoms distributif et réfléchi quisque et sibi, je souhaiterais faire deux autres remarques.

Premièrement, cette théorie - que l'on trouve, comme je l'ai souligné, chez des auteurs bien plus anciens que Gaius - implique que le désir d'autonomie des communautés provinciales ait coïncidé en grande partie avec un désir des Romains de la leur octroyer: pour adopter la langue du senatusconsultum de Asclepiade, il est préférable de juger les affaires impliquant les étrangers «dans leurs villes en fonction de leurs propres lois » (IGRR 1.118 = IGUR 1.1 = RDGE 22, ll. 7-9 [Latin] : ll. 17-20 [Grec]); ou, pour citer le Stadiasmus de Lycie, les cas seront désormais jugés «en utilisant une juridiction équitable et leurs lois ancestrales » (SEG 51.1832, face A, 11. 20-24). 
Plusieurs raisons expliquent cet engagement romain en faveur des normes locales. J'en citerai deux. La première est celle que je viens de mentionner: une hypothèse opératoire du gouvernement romain à la fin de la République et au début du Principat semble avoir été que les ordres sociaux locaux sont mieux garantis par une adhésion aux normes constituées localement. En conséquence, la persistance nominale des institutions préexistantes dans ces innombrables communautés, sous une direction purement locale, est considérée par les Romains comme devant régir tout à la fois un ordre local particulier et un ordre singulier et impérial. La seconde raison est la réticence des Romains à permettre aux étrangers d'utiliser les actions juridiques romaines : d'où, notamment, les clauses de la lex Iulia iudiciaria, qui restreignaient l'usage formulatoire à la ville de Rome, ou les interdictions imposées par cette loi et par d'autres, qui interdisaient aux étrangers l'accès aux tribunaux romains ou aux actions romaines ${ }^{8}$.

Ma seconde remarque se rapporte au texte de Gaius. Les divers systèmes de droit civil y sont conçus, sinon comme parallèles, du moins comme existant dans une relation non-hiérarchique : non seulement ils sont perçus comme opérant dans des domaines qui ne se chevauchent pas, mais ils sont en outre d'abord définis en termes de territorialité.

Le parallélisme reste toutefois une métaphore en partie inadéquate, pour au moins trois raisons, qui résultent toutes de l'empire et ont toutes une incidence sur le développement et la nature du pluralisme juridique.

Tout d'abord, l'un des principaux effets de l'empire sur le monde méditerranéen fut l'intensification de la mobilité humaine, avec pour conséquence l'essor sans précédent de populations minoritaires au sein des communautés civiques. En outre, comme certaines de ces personnes avaient la citoyenneté romaine, les normes du droit romain pouvaient être invoquées dans des contextes locaux - avec des effets variés, y compris le renvoi systématique d'un procès donné vers un tribunal romain. Les octrois de privilèges légaux, à la fin de la République et à l'époque triumvirale l'illustrent : citons le cas d'Asclépiades ou de Seleukos de Rhosos

8 Ando 2011, op. cit. (n. 4), 2, 10 : Id., 'Law and the landscape of empire', in S. Benoist et A. Daguey-Gagey (eds.), Figures d'empire, fragments de mémoire: Pouvoirs (pratiques et discours, images et représentations), et identités (sociales et religieuses) dans le monde romain impérial ( ${ }^{\text {er }}$ s. av J.-C. $-V^{e}$ s. ap. J.-C.) (Paris 2011), 25-47 ; Id., "Three revolutions in government', dans L. Reinfandt et S. Tost (eds.), Official epistolography and the languages of power (Vienna 2012), 209-218. 
(Asclépiades : cité plus haut: Seleukos : SEG 54.1625). Dans le langage du droit moderne, on pourrait dire qu'Asclépiades et Seleukos ont obtenu le droit de «forum shopping » (on pourrait parler de «surenchère judiciaire »; il n'y a aucun véritable équivalent français pour ce terme ; un tribunal français a utilisé le terme anglais dans une décision publiée en mai 2011).

Deuxièmement, l'intérêt romain était perçu - et compris normativement - comme surpassant tout à la fois l'autonomie locale et l'engagement des Romains eux-mêmes à restreindre le droit romain aux citoyens romains. On trouve une déclaration de principe à cet effet dans le compterendu schématique de Cicéron sur les pouvoirs législatifs des collectivités subordonnées dans son discours pro Balbo ${ }^{9}$. En ce qui concerne la pratique, on observe ses effets principalement dans le droit pénal, par exemple dans l'édit de C. Pétronius Mamertinus, préfet d'Egypte de 133 à 137 de notre ère $(S B$ XII $10929=P$. Yale II 162, col. II-III : l'édit précise les crimes pour lesquels le préfet mènera lui-même une enquête. Les catégories correspondent à celles connues dans le droit pénal romain ${ }^{10}$ ).

En dépit de ces pressions systématiques sur les tribunaux locaux, le pluralisme juridique de l'empire dans son ensemble aurait néanmoins pu devenir un système purement hiérarchique, dans lequel les niveaux supérieur et inférieur fonctionneraient globalement selon des principes tout à fait distincts. Du point de vue pratique, on pourrait qualifier de tels systèmes de pluralistes, non pas simplement pour la raison évidente que des normes multiples, et de multiples sources de normes, coexistaient dans le même espace, mais aussi parce que dans de nombreux domaines d'application du droit dans l'empire, il n'existait aucune obligation de subordination, aucune coordination externe, aucune règle de reconnaissance. En fait, le système décrit voire même suscité par la théorie romaine ressemble beaucoup à ce qu'un théoricien du droit moderne pourrait appeler le pluralisme « institutionnel » ou même «systémique »11.

9 Cic., Balb. 22 (traduction un peu vieillie publiée sous la direction de M. Nisard): «Lorsque le peuple romain a porté une loi, si cette loi est telle qu'on puisse permettre à des peuples fédérés ou libres de décider eux-mêmes de quelle jurisprudence ils veulent se servir pour ce qui les regarde, et non pour ce qui nous intéresse, alors il faut examiner si ces peuples ont donné ou non leur consentement; mais lorsqu'il s'agit de notre république, de notre empire, de nos guerres, de nos victoires, de notre sûreté, on n'a point voulu qu'ils fussent consultés ».

10 N. Lewis, On government and law in Roman Egypt (Atlanta 1995), 157-173, rééditant des essais de 1972 et 1973 .

11 N. Krisch, Beyond constitutionalism: the pluralist structure of postnational law (Oxford 2011), 69-78. 
Dans la pratique, toutefois, ce n'est pas ce que nous observons. Ou plutôt, si le pluralisme systémique a jamais vu le jour, il ne s'est pas avéré durable. Au contraire, l'ajustement ou la relation entre les ordres juridiques - une relation qui a été en théorie si étroitement hiérarchique que les Romains la décrivaient comme parallèle - a été progressivement transformée, de telle façon que les différents ordres juridiques locaux de l'empire dans son ensemble, qui avaient auparavant entretenu une relation purement hiérarchique avec les institutions romaines, ont été progressivement réorientés de façon à entrer avec elles dans une relation de subordination fractale.

Cela s'est fait, comme je l'ai dit, en dépit de l'engagement romain en faveur de principes qui incitaient à un pluralisme institutionnel. Dans ce qui suit, j'examinerai deux des principales sources de pression en faveur du changement, chacune remettant en cause à sa manière les désirs à la fois des Romains et des provinciaux d'entretenir ce pluralisme: en premier lieu, l'usage très répandu de la procédure romaine dans les tribunaux provinciaux, et en second lieu, la pression récurrente sur les institutions locales qui se manifeste par la possibilité de faire appel auprès des tribunaux romains.

6. En ce qui concerne la procédure, on doit tout d'abord se rappeler que les Romains ont plus rapidement développé dans les provinces - ou, peut-être, ont plus rapidement exporté vers ces dernières - un procès en deux étapes, dont la structure dupliquait celle du système formulaire : un magistrat romain, après avoir entendu les données essentielles d'un cas particulier, l'assignait à une personne ou à un conseil jouissant d'un pouvoir de juridiction délégué - probablement après avoir formulé pour cette personne ou ce conseil un exposé des points de désaccord, et leur avoir décrit le cadre légal leur permettant d'arriver à une décision.

Parmi les textes témoignant d'une telle procédure, on doit certainement citer la lex de provinciis praetoriis de 101/100 av. J.-C. et la lex Gabinia de insula Delo de $5^{8}$ av. J.-C. Dans le premier texte, je souhaiterais attirer l'attention sur le pouvoir du magistrat de «donner iudices et recupera-

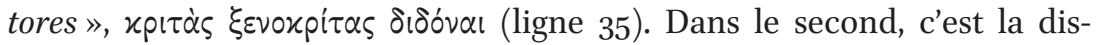
tinction entre iudicium et iudicatio, entre «le jugement et le procès », qui révèle le fonctionnement d'une procédure en deux étapes (1l. 34-35).

Grâce à de fréquentes allusions des juristes, on sait que les gouverneurs des provinces continuaient, sous le Haut-Empire, à déléguer leur pouvoir de jugement, et donc à utiliser une procédure spécifiquement romaine : par exemple, dans le premier livre du Digeste, Julien concéda que le gouverneur d'une province pût décider pour lui-même ipse cognoscere 
an iudicem dare debeat (Julien, D. bk. 1 fr. 5 Lenel = D. 1.18.8); d'une façon similaire, d'après le premier livre du De cognitionibus de Callistratus, quand l'empereur accordait à quelqu'un l'autorisation de parler au gouverneur, le gouverneur pouvait décider utrum ipse cognoscat an iudicem dare debeat (Callistratus, De cognitionibus bk. 1 fr. 1 Lenel = D. 1.18.9).

Bien sûr, il est essentiel de reconnaître que l'utilisation d'une procédure romaine n'entraînait pas l'utilisation du droit positif romain. On pourrait de nouveau citer, à cet égard, une vaste série d'affirmations normatives prononcées pas des juristes et des empereurs - le dicton (???) de Trajan en étant une bonne illustration - ainsi que de procès-verbaux et de comptes rendus sur papyrus, dans lesquels on suivait d'une façon ou d'une autre la procédure romaine, mais dans lesquels le droit ou la coutume locale mettait en place le cadre du droit positif - la pétition de Dionysia est peut-être l'exemple le plus connu ${ }^{12}$. De fait, tout porte à croire que le système fonctionnait souvent dans le but précis de diriger les cas vers les juges et arbitres ayant une compétence locale. On discerne ainsi de nombreux experts d'après les honneurs qui leur ont été octroyés par les communautés reconnaissantes. On se reportera, par exemple, à IGRR 4.618

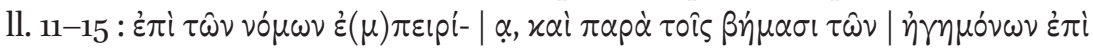

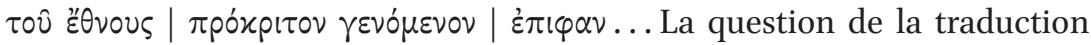

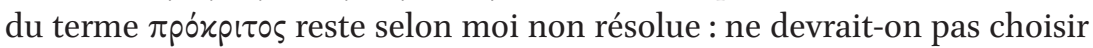
de le traduire, plutôt que par "sélectionné » (ou par « sélecté», l'anglicisme adopté par Paul Veyne), par «officiel qui fonctionne à la place du magistrat qui exerce la juridiction », l'équivalent de xpır̀े $\delta 0 \theta \varepsilon i \varsigma$, traduction grecque, sous l'empire, de iudex datus? Si cette traduction s'avérait exacte, on aurait là un autre exemple d'un terme de l'époque classique dont le sens changea sous la pression des nouvelles réalités sociales et institutionnelles de l'époque.

Les formes culturelles et juridiques romaines purent influencer, toutefois, et influencèrent, de fait, la pratique locale, provoquant un changement culturel sans même utiliser le droit positif romain. Il suffit, pour s'en convaincre, de citer la Contrebiensis Tabula $(A E$ 1984, $586=\mathrm{J} .-\mathrm{M}$. Lassère, Manuel d'épigraphie romaine [Paris : Picard, 2005], $\left.\mathrm{n}^{\circ} 468\right)$ : ici, une procédure romaine fut employée afin d'autoriser les communautés

12 P.Oxy. 237, col. 6, ll. 16-17, col. 7, ll. 27, 31-33, 35, et col. 8, ll. 34-35. C. Kreuzsaler, 'Dionysia vs. Chairemon. Ein Rechtsstreit aus dem römischen Ägypten', in U. Falk et al. (eds.), Fälle aus der Rechtsgeschichte (München 2008), 1-13: C. Kreuzsaler et J. Urbanik, 'Humanity and Inhumanity of Law: The Case of Dionysia', Journal of Juristic Papyrology 38 (2008), 119-155. 
autonomes espagnoles à régler une affaire selon les normes locales ${ }^{13}$. Mais tous les aspects formels du texte - les protocoles, la forme de datation, le médium, la langue - ainsi que les aspects formels de la procédure - la formule, la délégation de compétence, la fiction d'une Sosinestana civitas permettant de postuler l'existence d'un code de droit de ladite civitas sont en contraste absolu avec l'autonomie théorique des communautés espagnoles. Le prestige culturel de la puissance romaine doit avoir doté cet ensemble - le rituel juridique lui-même comme la forme textuelle adoptée par le dossier - d'une légitimité durable.

7. Néanmoins, on a aussi de bonnes raisons de croire que la coexistence de tribunaux romains aux côtés et au-dessus des systèmes juridiques locaux exerçait des pressions considérables sur les institutions locales pour qu'elles se conforment aux normes romaines en ce qui concerne à la fois la procédure et le droit positif. Je souhaiterais donner de brefs exemples de ce processus.

Citons, pour commencer, un édit impérial découvert à Nicomédie et attribué par Oliver au règne d'Hadrien, mais daté par Julien Fournier de l'époque des Antonins ${ }^{14}$ :

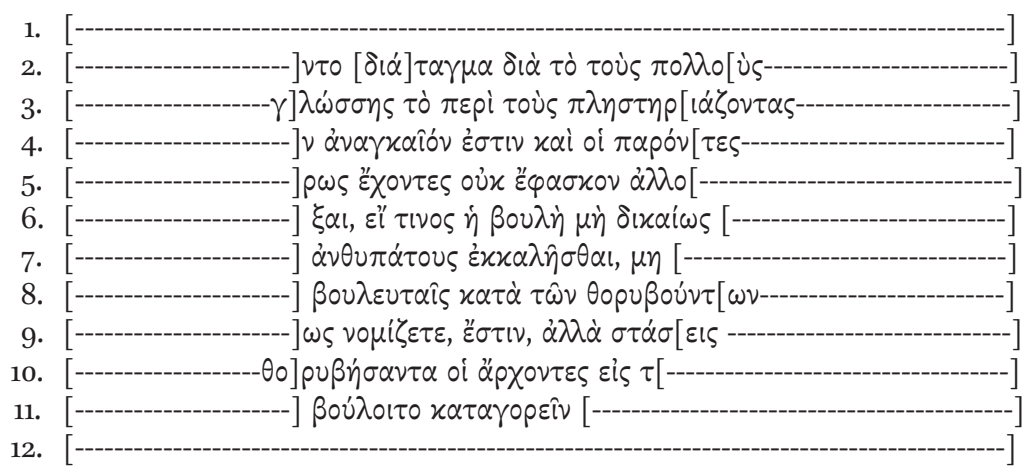

L'état du texte ne permet pas de reconstruire son sens de manière cohérente, mais il semble s'en prendre à ceux qui augmentaient le prix des céréales et permettre les appels auprès du proconsul sous certaines conditions. La justification manifeste utilisée pour autoriser ces appels (à la

13 J.S. Richardson, 'The Tabula Contrebiensis: Roman law in Spain in the early first century BC', Journal of Roman Studies 73 (1983), 33-41: Birks, Rodger et Richardson 1984, op. cit. (n. 3).

14 J.H. Oliver, Greek constitutions of the early Roman emperors from inscriptions and papyri (Philadelphia 1989), $\mathrm{n}^{\circ} 94$; Fournier 2010, op. cit. (n. 5), 521-523. 
ligne 6), à savoir « Si le Conseil injustement... », est digne d'intérêt. Bien que le contexte de cette autorisation à se pourvoir en appel soit très spécifique (quoique inconnu), les autorités locales semblent clairement avoir été informées que leur conduite se devait d'être conforme aux normes de la justice romaine - quelles que soient celles-ci, et de quelque manière qu'elles aient été connues.

On évoquera ensuite un passage du second livre de Marcien de publiciis iudiciis, concernant la procédure à suivre par les autorités locales lors d'interrogatoires de suspects :

Il existe en effet un chapitre des règles que le divin Pius avait publié sous son décret lorsqu'il était gouverneur de la province d'Asie : que les irénarques, lorsqu'ils avaient arrêté des voleurs, devaient les interroger sur l'identité de leurs complices, et de ceux qui les protégeaient, ils devaient mettre leurs interrogatoires sous pli, les sceller, et les envoyer à l'attention du magistrat. Ainsi, ceux qui sont envoyés [devant le tribunal] avec un compte-rendu [de leur interrogatoire], leurs affaires devraient être examinées à nouveau, bien qu'ils aient été envoyés avec des documents à charge, même s'ils ont été amenés par les irénarques. Le divin Pius et d'autres empereurs ont laissé des révisions à cet effet : que même dans le cas de ceux qui sont répertoriés comme étant recherchés, si quiconque semble poursuivre l'un [d'eux] en justice, ils ne devraient pas être traités comme des condamnés mais comme si une nouvelle accusation avait été déposée. En conséquence, lorsqu'un examen est mené, l'irénarque doit recevoir l'ordre d'y assister et d'examiner ce qu'il a écrit. S'il agit ainsi soigneusement et fidèlement, il doit être loué : sans habileté adéquate et sans raisonnement approfondi, [le juge] notera simplement que l'irénarque a produit un compte-rendu insuffisant: mais si [le juge] conclut que son interrogatoire avait d'une quelconque façon l'intention de nuire, ou qu'il avait rendu compte de choses qui n'avaient pas été dites comme si elle l'avaient été, il devrait imposer une punition exemplaire pour empêcher n'importe qui d'autre de tenter une action semblable à l'avenir. (Marcien, De iudiciis publicis bk. 2 fr. 204 Lenel = D. 48.3.6.1)

Dans ce cas, les normes des tribunaux romains sont directement imposées aux autorités locales, parce qu'un tribunal romain est le tribunal en premier ressort pour les affaires criminelles.

Mais plus complexes et plus intéressants sont les effets qui furent exercés par les procès romains en dehors de la nécessité de se conformer au droit positif romain. La documentation la plus révélatrice à cet égard résulte des appels provinciaux auprès de tribunaux romains. Par exemple, une célèbre inscription découverte à Athènes conserve une série de réponses offertes par Marc Aurèle aux recours en appel de cette ville. Il rejette deux appels du fait que les demandeurs ont présenté des documents à charge qui étaient d'une manière ou d'une autre erronés (Oliver, op. cit. [n. 14], $\mathrm{n}^{\circ} 184$, plaque II) : 


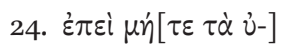

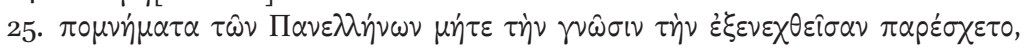
$\dot{\varepsilon} \pi i \tau \hat{\omega} \nu \dot{\varepsilon} \mu \hat{\omega} \nu \mathrm{Kv} v \tau \tau i \lambda \dot{i} \omega[\nu \dot{\alpha}]$

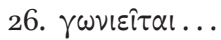

«Puisqu'il n'a présenté ni le compte-rendu des Panhelléniens, ni les conclusions publiées, il plaidera son affaire devant mes Quintilii ...»

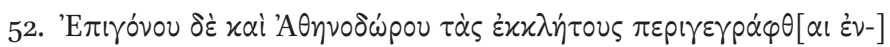

53. $\delta \varepsilon \hat{\imath} \nu \varphi \dot{\alpha} \nu \omega \pi \rho \circ \varepsilon เ \pi \omega \dot{\nu}$.

«J'ai déjà annoncé que les renvois en appel d'Epigonus et d'Athenodorus avaient été mis de côté avec les annotations précisant qu'ils avaient été préparés de façon incomplète. »

À mon avis, de tels cas exerçaient une pression sur les plaideurs à venir, ainsi que sur les institutions non-romaines, afin d'aligner leur conduite en dehors des tribunaux romains - et antérieurement à l'action romaine sur les normes observées à l'intérieur des tribunaux romains, du moins dans les cas où l'on pouvait éventuellement faire appel aux autorités romaines.

Pour finir, je désirerais attirer l'attention sur les règles précisées par Marc Aurèle dans la clause globale qui termine le rescrit omnibus d'Athènes. Il y établit la procédure à suivre dans tous les cas pertinents pour lesquels il n'a rendu aucune décision et, en réalité, pour lesquels il n'avait même offert aucune réponse (Oliver, op. cit. [n. 14], $\mathrm{n}^{\circ} 184$, plaque II) :

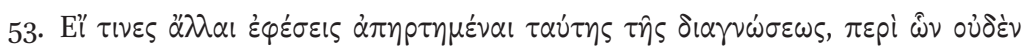
$\dot{\varepsilon} \delta[\dot{\eta} \lambda \omega-]$

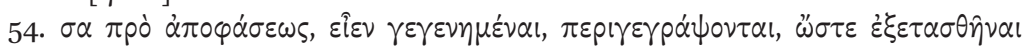
$\pi \alpha \rho \dot{\alpha} \tau \hat{\omega} \iota \delta เ x \alpha \sigma \tau \hat{\imath} \iota x \alpha \tau \dot{\alpha} \tau \dot{\alpha} \alpha \hat{v}[\tau \dot{\alpha}]$

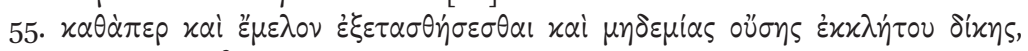

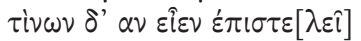

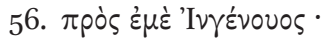

«Si n'importe quelle autre demande de procès ayant dépendu de cette session du tribunal a eu lieu, à propos de laquelle je n'ai fait aucune déclaration qui tiendrait lieu de décision, elle aura été mise de côté pour être examinée devant un juge spécial - même lorsqu'il ne s'agit pas d'une affaire en appel - avec exactement la même procédure selon laquelle elle allait être examinée : quant à leur appartenance éventuelle, Ingenuus me l'enverra par écrit. »

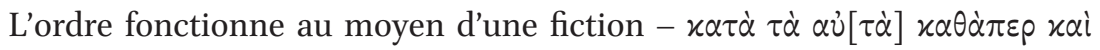

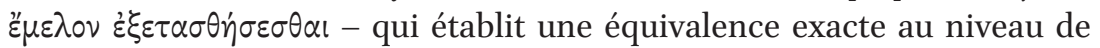
la procédure entre le tribunal présidé par Marc Aurèle et le tribunal qu'il a délégué pour exercer la juridiction à sa place. 
8. J'ai mis de côté quatre questions que je voudrais seulement décrire et non pas traiter dans la présente communication. Il conviendrait de les examiner dans le cadre d'une histoire plus complète des cultures juridiques des provinces sous le Principat.

(1) Certaines communautés ont entamé des actions ostentatoires et spectaculaires d'auto-réorganisation en matière de droit public ou privé, ou des deux. L'exemple le plus célèbre est l'acte enregistré sur la Table d'Héraclée. Certains de ces actes, dont le cas d'Héraclée, ont devancé les instructions de la métropole. D'autres ont été motivés par la flagornerie en cela, ils ressemblaient à la pétition adressée au Sénat par Cumes, qui demandait le droit d'utiliser le latin dans les affaires publiques, peut-être en lien avec une demande de suffrage, et ainsi un changement de la loi (Liv. 40.43.1).

(2) En ce qui concerne l'influence de Rome sur le développement de systèmes juridiques purement locaux, l'histoire comparative du droit nous incite à croire qu'il devait $\mathrm{y}$ avoir des communautés provinciales constituées pour la première fois comme des communautés unitaires à la suite de l'action des Romains. Il existe des équivalents historiques importants de la découverte - autrement dit de l'invention - par les populations colonisées de codes de lois à la lumière d'une telle épistémè impériale. La citation d'un code de loi écrit pendant les procès antérieurement enregistrés, dont les comptes rendus eux-mêmes ont été cités par les plaideurs dans la pétition de Dionysia, pourrait être un exemple de ce type d'influence. Elle est sans doute un exemple unique de l'engagement envers la textualité de la culture juridique romaine.

(3) Je ne veux pas suggérer que les Romains s'intéressaient à toutes les affaires juridiques. Mais dans les textes normatifs, du moins, ils employaient généralement deux critères pour identifier les cas pour lesquels l'impact potentiel sur l'ordre social méritait l'attention romaine : les cas impliquant la peine capitale et les cas où la valeur financière de la question en litige atteignait une certaine limite. Ces cas sont bien connus et il n'est pas nécessaire de les discuter ici ${ }^{15}$.

(4) Enfin, une histoire complète du droit provincial doit prendre en compte l'effet sur la société provinciale de la pratique de la pétition. J'ai abordé ces textes il y a 11 ans : à l'époque, j'avais surtout traité des effets

15 A. Rodger, 'Jurisdictional limits in the Lex Irnitana and the Lex de Gallia Cisalpina', Zeitschrift für Papyrologie und Epigraphik 110 (1996), 189-206: Ando 2011, 'Law, landscape', op. cit. (n. 8) : Ando 2011, Law, language, op. cit. (n. 4), 22-24; Fournier 2010, op. cit. (n. 5). 
que les pétitions provinciales avaient eus sur la doctrine romaine du précédent ${ }^{16}$. Cependant, les pétitions témoignent aussi d'un désir clair de la part des individus d'acquérir et d'exploiter la connaissance du droit romain, chaque fois qu'ils perçurent comme étant dans leur intérêt de le faire - mais pour cela, les étrangers dans les provinces devaient aussi se conduire en conformité avec les normes qu'ils citaient eux-mêmes.

9. Je reviens, pour conclure, à la fiction juridique employée par Marc Aurèle. La fiction était manifestement un outil essentiel de la procédure civile à Rome. Certaines fictions furent aussi employées dans les lois de juridiction et dans le droit public, afin d'établir des formes d'équivalence entre les tribunaux à Rome et ceux d'ailleurs. L'exemple le plus connu est probablement la fiction de la lex Flavia municipalis (Lex Flavia municipalis ch. 91, traduction de l'Année épigraphique 1986, 333 [P. Le Roux] en partie revue):

R(ubrica). Quo iure in tertium denuntietur, dies diffindatur diffi<s $>$ susue sit, res iudicetur, lis iudici\{i\} damni sit, res in iudicio esse desinat.

... et si intra it tempus, quod legis Iuliae, quae de iudici<i $>$ s priuatis proxime lata est, kapite XII senatusue consultis ad it kaput legis pertinentibus conpr $<$ e $>$ hensum est, iudicatum non sit, uti res in iudicio non sit, siremps lex $\{r\}$ i(us) [c](causa)que esto adque $\{m\}$ uti esset si eam rem in urbe Roma praetor $p$ (opuli) R(omani) inter ciues Romanos iudicari iussisset...

Si un jugement n'a pas été rendu durant la période arrêtée par le chapitre XII de la Lex Iulia - qui a été récemment votée concernant les procès privés - et les décrets du sénat relatifs au chapitre de cette loi, que l'affaire ne soit plus en jugement, que les affaires soient soumises aux règles, au droit et soient dans la situation qui prévaudrait si le préteur du peuple romain avait ordonné dans la ville de Rome et entre des citoyens romains de juger cette controverse.

J'ai soutenu que les communautés étrangères de l'empire avaient longtemps revendiqué et cherché à conserver le droit d'utiliser leurs propres lois, et que c'était en outre un droit que les Romains, pour des raisons qui leur étaient propres, cherchaient eux-mêmes à leur octroyer. J'ai aussi soutenu que des individus étrangers, ainsi que les tribunaux locaux, se trouvaient confrontés à des formes de pression diverses pour aligner non seulement leur conduite juridique, mais aussi leur conduite sociale, sur

16 C. Ando, Imperial ideology and provincial loyalty in the Roman Empire (Berkeley 2000), 378-379. 
les normes romaines. Cela s'est produit malgré l'autonomie formelle des systèmes légaux locaux, malgré l'absence évidente de règles de reconnaissance entre juridictions, magistrats et autorités législatives, et malgré l'engagement de principe de la part des Romains en faveur d'un pluralisme juridique radical et systémique. J'ai souligné que cette pression put résulter de certains plaideurs, qui citaient les précédents romains lorsque ces derniers leur donnait un avantage dans un cas donné. Je voudrais surtout insister sur le caractère à la fois centripète et centrifuge de ces pressions intégratrices, qui s'exerçaient non seulement d'en haut mais aussi d'en bas. Longtemps avant que Caracalla n'efface la plupart des types de différences juridiques formelles, les tribunaux locaux en étaient venus à fonctionner à bien des égards comme si les procès étaient en fait soumis aux règles, au droit, et étaient en fait dans la situation qui prévaudrait lorsque le préteur du peuple Romain ordonnerait de juger une controverse dans la ville de Rome, entre des citoyens romains. 



\title{
GLI EQUILIBRI DELLA CITTADINANZA ROMANA, FRA SOVRANITÀ E IMPATTO SOCIALE*
}

\author{
Salvo Randazzo
}

In memoria di Ignazio Maria Marino

\begin{abstract}
1. Nel 214 a.C. Filippo V di Macedonia indirizza ai cittadini di Larissa un'epistola ${ }^{1}$ in cui, mostrando ammirazione per la lungimiranza di Roma, contro la quale stava combattendo una guerra lacerante ${ }^{2}$, ricorda la concessione agli schiavi manomessi della cittadinanza ${ }^{3}$ :
\end{abstract}

....se i cittadini di pieno diritto saranno il numero più alto possibile, la vostra pólis sarà forte e i vostri campi non rimarranno incolti, come sono ora per vostra vergogna. Questa la meta cui dovete mirare, e io penso che nemmeno fra voi si udirà una sola voce contraria. Voi avete avuto modo di osservare altre comunità che seguono una politica liberale nell'estensione della cittadinanza. Un buon esempio è quello di Roma: quando i Romani affrancano i loro schiavi li ammettono in seno alla loro cittadinanza e consentono loro di accedere alle cariche pubbliche. Grazie a questa politica,

* The editor of the online journal Teoria e Storia del diritto privato has kindly given permission to publish this contribution. Il testo riproduce, mantenendone volutamente lo stile colloquiale, salvo l'aggiunta di alcune note, l'intervento svolto a Lille il 23 giugno 2011, in occasione del Convegno su Les voies de l'intégration, à Rome et dans le monde romain. Alcuni aspetti sono poi stati ulteriormente discussi nell'ambito di una relazione tenuta il 19 gennaio 2012 presso l'Università di Salerno nel corso di un Convegno su Flussi migratori, diritti civili e politiche di integrazione. Colgo l'occasione per ringraziare Stéphane Benoist, Ségolène Demougin, Gerda de Kleijn, Laura Solidoro, Lucio De Giovanni, Onorato Bucci e Massimo Panebianco per la duplice opportunità di confronto e di dialogo.

1 W. Dittemberger, Sylloge Inscriptionum Graecarum, con F. Hiller von Gaertringen, J. Kirchner, H. Pomtow, E. Ziebarth (Lipsiae 1915-1924³), 543, ll. 29-34. Riporto il testo nell'efficace traduzione di V. Marotta, La cittadinanza romana in età imperiale (secoli I-III d.C.). Una sintesi (Torino 2009), 33 s. Cf. T. Mommsen, König Philipp V und die Larisäer (1882), Gesammelte Schriften IV, Historische Schriften I (Berlin 1906), 49-55.

2 Che, fra alterne vicende, avrebbe visto soccombere la temibile falange di Filippo nel 197 a.C. - ponendo così fine alla seconda guerra contro i macedoni, nello scontro decisivo presso Cinocefale, in Tessaglia (località collinare oggi nota come Karadagh) - ad opera delle legioni romane guidate da Tito Quinzio Flaminino. Cf. Plut., Vita T.Q. Flam. e N.G.L. Hammond, 'The Campaign and the Battle of Cynoscephale in 197 BC', Journal of Hellenic Studies 108 (1988), 6o-82.

${ }^{3}$ Il problema della cittadinanza romana è stato affrontato da romanisti e storici di Roma in una serie sterminata di ricerche che è impossibile, in questa sede, riepilogare. Mi limito a rinviare (a mo' di repertorio ragionato) alle oltre 40 pagine di fitte note di G. Crifò, Civis. La cittadinanza tra antico e moderno (Bari 2000), 87 ss. e, più recentemente, a Marotta 2009, op. cit. (n. 1), passim. 
essi non hanno soltanto reso più grande la patria, ma sono anche riusciti a inviare colonie in poco meno di settanta località.

Al di là delle informazioni del sovrano macedone, non del tutto esatte ${ }^{4}$, e dalla sua valutazione sulla generosità romana nell'ammissione alla cittadinanza ${ }^{5}$, emerge dal passo un dato evidente: Roma fa della concessione della cittadinanza uno strumento politico funzionale al proprio sviluppo ed al consolidamento del proprio potere, sia in Italia che nelle prouinciae. Una funzione politica dunque che, almeno nell'opinione generale, la cui eco affiora nella riflessione di Filippo, appare utilizzata già quattro secoli prima della Constitutio Antoniniana che concede la cittadinanza a tutti gli abitanti dell'impero ${ }^{6}$, suggellando così un itinerario storico diffusamente seguito nei secoli precedenti.

Ma facciamo un passo indietro e consideriamo l'istituto nei suoi percorsi generali.

Il diritto romano incentra il quadro della cittadinanza romana, in età arcaica e repubblicana ${ }^{7}$, sulla distinzione fra ciues, da un parte, hostes e peregrini (almeno a partire dal III-II secolo) dall'altra. Una distinzione capitale nel disegno politico della ciuitas: solo i ciues $^{8}$ fruiscono di specifiche

4 Cf. Crifò 2000, op. cit. (n. 3), 109 nt. 43 e Marotta 2009, op. cit. (n. 1), 34, che giustamente sottolineano le imprecisioni del testo, sia nel numero delle colonie, sia nella possibilità che il liberto potesse assumere le magistrature, eventualità esclusa ancora sotto Tiberio da una lex Visellia (CJ 9.21.1) relativa ai municipia.

5 Cf. Ph. Gauthier, 'Générosité romaine et avarice grecque', in Mélanges d'histoire ancienne offerts à W. Seston (Paris 1974), 207-215.

6 D. 1.5.17 (Ulp. 22 ad ed.): In orbe romano qui sunt ex constitutione imperatoris Antonini ciues Romani effecti sunt.

7 Un efficace percorso ricognitivo su cittadino e cittadinanza in Roma sino alla Constitutio Antoniniana è sviluppato da A.N. Sherwin-White, The Roman Citizenship ${ }^{2}$ (Oxford 1973). V. pure G. Crifò, 's.v. Cittadinanza', Enciclopedia del diritto 7 (Milano 1960) e G. Luraschi, La questione della cittadinanza nell'ultimo secolo della Repubblica, 'Res publica' e 'princeps', in F. Milazzo (ed.), Atti Copanello (Napoli 1996), 35-99. Mi permetto di rinviare, per alcune mie idee relative a questa fase, a S. Randazzo, 'Lo statuto giuridico dello straniero e l'hospitium nel diritto romano arcaico', in R. Astorri e F.A. Cappelletti (eds.), Lo straniero e l'ospite. Diritto. Società. Cultura (Torino 2003), 51 ss.

8 Come è noto sono tali i nati da iustae nuptiae, con anche soltanto il padre cittadino romano. In assenza di tali nozze il figlio segue la condizione giuridica della madre al momento del parto. Così anche il peregrino cui sia stata concessa la cittadinanza durante la gestazione della moglie, previo matrimonio legitimum, fa assumere al figlio la cittadinanza romana. Altre ipotesi di acquisto della cittadinanza, con varia configurazione e graduazione, fanno seguito a concessioni ad personam o collettive, ovvero ancora, oggettivamente, come a seguito dell'esercizio di ius migrandi di un latino a Roma. Analogamente, se un Latino avesse messo in stato di accusa un magistrato macchiatosi di concussione poteva ricevere la cittadinanza romana, così come la otteneva (con varia configurazione, sino alla piena cittadinanza acquistata soltanto dai discendenti di un liberto) lo schiavo manomesso. Su questa piattaforma giuridica si innesteranno poi varie concessioni gene- 
posizioni di vantaggio, possono contrarre iustae nuptiae ed avere il ius commercii; solo ad essi compete il ius suffragii, ovvero l'insieme dei diritti politici. La concezione politica ed istituzionale della ciuitas definisce così in via esclusiva ed escludente la condizione di coloro i quali, per la loro veste di ciues, ne sono ricompresi, segnandone di volta in volta diritti e, progressivamente, doveri, da coloro i quali ne sono, invece, esclusi.

È dunque su un doppio registro di inclusione/esclusione che le fonti ${ }^{9}$ sembrano delineare la condizione delle persone a Roma. Inclusione come appartenenza ad un gruppo, sul piano sociale (ma prima ancora etnicoreligioso) e giuridico, esclusione come collocazione del soggetto "oltre" e "fuori" il gruppo. Una logica elementare che contraddistinguerebbe il senso di appartenenza dei romani, gelosi custodi di una cittadinanza vittoriosa, da cui tenere fuori gli altri, gli estranei.

Se dunque sviluppiamo l'analisi sul rapporto sovranità/sudditanza, la posizione di preminenza sociale cui è connesso il compito di attribuire o negare l'appartenenza allo stato risiede nello stesso corpo sociale, che difende saldamente le proprie prerogative e ne disciplina l'apertura all'esterno. L'impressione è che la ciuitas, come istituzione astratta, detenga il potere pubblico e dunque regolamenti la più elementare delle condizioni, quella di chi a tale potere è soggetto, e che siano i ciues, l'insieme dei ciues, attorno ai quali è disegnato, in termini tendenzialmente individualistici ${ }^{10}$, il sistema giuridico, in quanto tali, a legittimare il potere pubblico, ad essere in certa misura custodi della sovranità della ciuitas, in un intreccio sincreticamente espresso nell'endiadi senatus populusque ${ }^{11}$,

rali, da quella della lex Iulia del go per Latini ed Italici rimasti leali a Roma e poi alla lex Calpurnia dell'89 che concesse la cittadinanza ai militari, appartenenti a ciuitates foederate, che avevano servito Roma ed alla lex Plautia Papiria che nello stesso anno la riconobbe agli alleati italici che l'avessero invocata, sino a quella cesariana ai Cisalpini del 49 ed alle misure nei confronti dei veterani o alle concessioni imperiali di Claudio ai Galli, di Marco Aurelio ai Cirenei, di Vespasiano alle città iberiche, per culminare, nel 212, nella generale Constitutio Antoniniana de ciuitate peregrinis danda dell'imperatore Antonino Caracalla che dunque chiude il cerchio di un percorso avviato da tempo. Non è questa certo la sede per discutere dei gravi problemi interpretativi, tanto sul piano giuridico che politico, del provvedimento di Caracalla. Ai nostri fini basta segnalare come esso rappresentò un punto di arrivo di un lungo percorso e non un evento isolato e dirompente.

${ }^{9}$ Menzioneremo quelle attinenti al nostro limitato ambito. Più in generale una raccolta ragionata delle fonti rilevanti per la ricostruzione della cittadinanza romana sino alla Constitutio di Caracalla è in P. Donati Giacomini, G. Poma, Cittadini e non cittadini nel mondo antico. Guida ai testi e ai documenti (Bologna 1996).

10 Sullo spirito individualista del diritto romano cf. R. Orestano, Introduzione allo studio storico del diritto romano ${ }^{2}$ (Torino 1963), 224.

11 Non è possibile in questa sede neppure sfiorare i problemi interpretativi in ordine all'espressione. Mi limito a rilevare come il riferimento successivo della sigla S.P.Q.R., 
in cui patres e populus sono due aspetti del medesimo corpo sociale ed entrambi contribuiscono allo sviluppo dell'esperienza costituzionale romana. Così la stessa esistenza della ciuitas segna la costruzione e l'affermazione di un potere sovrano che incide sulle posizioni soggettive di vantaggio seppure, come vedremo, senza modificarne, ma anzi in certa misura garantendola, l'autonomia. Un equilibrio elaborato in quei termini semplici ed empirici che avrebbero caratterizzato, secoli dopo, anche l'esperienza inglese ${ }^{12}$.

Un'esperienza, quella romana, in cui il profilo individualistico appare però caratterizzante e tale da rappresentare il centro anche dell'elaborazione pubblicistica che, proprio muovendo dal ciuis, costruisce, come in un sistema che si allarga a spirale, poteri e situazioni giuridiche che, delineandosi gradualmente, involgono gli altri ciues, disciplinano i rapporti con i peregrini e caratterizzano giuridicamente la comunità fondando con semplici percorsi politici, per riprendere Gerber ${ }^{13}$, quel "tutto organico" da

riferimento preceduto dalla $\mathrm{Q}$, resa generalmente come enclitica (senatus populusque), rimonti, con molta probabilità, ad un originario ${ }^{*}$ co-virites, da ${ }^{*}$ co-virium da cui, ancora e successivamente Quirites, mostrando dunque un senso collettivo, richiamando l'idea di concittadini, come idea che prevale su quella di cittadini. Per una sintesi dei problemi cf. L. Labruna, 's.v. Quirites', Novissimo Digesto Italiano, XIV (Torino 1967) 708 ss.

12 L'intreccio suggestivo fra l'empirismo inglese e il modello di sviluppo politico romano colto da Carl Friedrich Wilhelm von Gerber (Ueber öffentliche Rechte [Jena 1852] e Grundzüge eines Systems des deutschen Staatsrecht [Jena 1865], quest'ultimo scritto, poi pubblicato come Gründzuge des deutschen Staatssrechts ${ }^{3}$ [Jena 1880] è alla base della trad. ital. Diritto pubblico, cur. P.L. Lucchini [Milano 1972]) viene ripreso finemente da M. Nigro, 'Il "segreto" di Gerber', Quaderni Fiorentini per la storia del pensiero giuridico moderno 2 (1973), 293-333. Un intreccio che non sembra abbia avuto evidenti riflessi nell'opera pubblicistica di Mommsen (l'avvio della pubblicazione del Römisches Staatsrecht [Leipzig 1871-1888] è di sei anni successivo alla prima edizione dei Gründzuge) che invece all'opera di Gerber si ispira certamente: cf. Y. Thomas, 'Mommsen et l'“'Isolierung" du droit (Rome, l'Allemagne et l'État)', Préface à Th. Mommsen, Le droit public romain (Paris 1984), 1 ss. e V. Mannino, Questioni di diritto (Milano 2007), 72 ss. e pass. Più in generale sull'opera pubblicistica di Gerber cf. C. Kremer, Die Willensmacht des Staates. Die gemeindeutsche Staatsrechtslehre des Carl Friedrich von Gerber (Frankfurt am Main 2008). Per altro una visione romanistica dell'opera di Gerber non può prescindere dalla valutazione degli influssi reciproci scaturenti dallo stretto rapporto con Jhering e resi evidenti dal ricchissimo carteggio fra i due studiosi, capillarmente indagato da Mario Losano, fra le cui opp. cf. M. Losano (ed.), Carteggio Jhering-Gerber (1849-1972) (Milano 1977); M.G. Losano, E. Bonazzi, Bibliografie di Jhering e Gerbe (Milano 1978), 1 ss. e M. Losano, 'Ricerche d'archivio su Jhering e Gerber', Quaderni Fiorentini per la storia del pensiero giuridico moderno 10 (1981), 149-167.

13 Un percorso agevole, dunque, uno "sviluppo tranquillo" delle istituzioni in relazione "sempre e soltanto ad esigenze concrete", per usare le categorie di Gerber (cf. Nigro 1973, op. cit. [n. 12], 299) che guarda criticamente alla filosofia politica francese, marcandone "irrequietezza" e "mediocrità". 
cui derivare un immediato (starei per dire "automatico") senso di appartenenza. Dunque un meccanismo in cui, muovendo dalla "città" si crea, gradualmente, la "cittadinanza".

Tuttavia la concezione individualistica e il rapporto esclusivo ed escludente fra il ciuis e le cose di sua proprietà non rappresentano una pre-condizione per il diritto di cittadinanza che a Roma ha radici eticoreligiose più salde e generali ${ }^{14}$, in cui il giuridico nasce come "etico" e si intreccia con il vincolo religioso, ma in un senso tutto romano, evidente nell'hospitium, lo stesso senso per cui Seneca scriverà duo...sacratissima inter homines acceperunt, hospitium et adfinitas (Contr. 8.6.17) e in questo senso prescinde dallo status proprietario e dalle sue modificazioni. Ciò a differenza del modello culturale, prima che giuridico, che lega la proprietà e la sua difesa all'appartenza al gruppo e che troviamo invece nell'esperienza ebraica che esclude dalla cittadinanza i proprietari spossessati il cui incremento numerico finiva per creare alterazioni sociali non indifferenti e tali da richiedere che questi soggetti venissero periodicamente reintegrati nella loro "capacità" dalle leggi del Giubileo ${ }^{15}$.

14 In un contesto di sincretismo etico e giuridico come attesta la liturgia della vocatio in hospitium e della consegna della tessera hospitalis, posta sotto la protezione di Iuppiter Hospitalis (Cic., ad Quint. fratr. 2.10-12; Tac., Ann. 15.52) e che ricorda l'analoga cerimonia dello xenos greco posto sotto la tutela di Giove Xenio (Erod. 3.39). E' atto antichissimo, lo attesta già Plauto, Pseud. 5.1.25; 5.2.87 e 89; v. pure Cist. 2.1.27, accompagnato dallo scambio di doni, uno scambio reciproco, in cui il dono assume caratteristiche sinallagmatiche, non è né un dono gratuito, unilaterale e slegato da reciprocità, né questa volta è un dono "remuneratorio", come i doni studiati da Marcel Mauss; è un dono che si salda alla consegna di un altro dono, è un elemento di suggello dell'accordo che è reciproco, obbligatoriamente reciproco, posto dunque a conferma di un atto che vorrei definire contrattuale e formalizzato in tabulae e tesserae. La tessera è divisa in due parti; spesso con l'effigie di Giove hospitalis, frequentemente costituita da due mani congiunte (ne possediamo molte) in cui una recava il nome dell'ospitante e l'altra il nome dell'ospite, un accordo che poteva avere proiezioni (questo mi sembra un dato di rilievo) anche oltre le persone direttamente coinvolte, potendosi estendere, lo ricorda Livio (27.16) liberis posterisque. Una situazione che poteva anche modificarsi, sino a segnare la fine del patto, la fine dell'ospitalità, con la distruzione delle tesserae, quel tesseram confrigere, di cui ci parla già Plauto: Hic apud nos, iam, Alesimarche, confregisti tesseram (Cist. 2.1.27).

15 Cf. L. Campos Boralevi, 'Libertà e proprietà: la politica dello spazio nell'Europa moderna', in B. Consarelli (ed.), La politica e gli spazi. I giornata di studio "Figure dello spazio, politica e società" (Firenze, 25 ottobre 2002) (Firenze 2003) 35: "In questo modo la proclamazione del Giubileo ogni cinquant'anni riproduceva periodicamente il paradigma della storia biblica della liberazione dall'Egitto, a beneficio degli schiavi che avevano perduto la libertà personale, dei debitori insolventi, ma anche e soprattutto dei proprietari che avevano perso la terra loro originariamente assegnata per eredità, secondo la linea: schiavitù liberazione - patto - legge - proprietà - diritti di cittadinanza - libertà". 
Francesco de Martino ha ricordato ${ }^{16}$, muovendo da un passo di Gellio che riferisce di provvedimenti di Catone, che la trascuratezza del ciuis nella manutenzione del suo campo ${ }^{17}$, spintasi al punto da farlo ricoprire da erbacce (sordescere) o da lasciarlo in stato di abbandono, sottoponeva il soggetto al biasimo dei censori i quali "lo facevano erario", cioè gli faceva assumere lo status di cittadino cui non spettavano i diritti politici. Una conseguenza di speciale significato, dunque, che presupponeva un vincolo sociale saldissimo, un obbligo, quello di coltivare e non trascurare la terra, che, osserva lo studioso:

... annullava interamente il carattere assoluto della signoria del proprietario, il quale in teoria poteva fare quello che voleva della sua cosa, trascurarla, perfino distruggerla o renderla comunque improduttiva. In questo contrasto profondo di norme si rivela uno dei tratti più caratteristici del sistema giuridico romano, che non si può comprendere avendo di mira soltanto le norme arcaiche del diritto privato, ma considerando l'esercizio dei diritti alla luce del costume e dei doveri politici del cittadino, che erano determinanti per il suo stato politico ${ }^{18}$.

Prescindendo dai profili più nettamente politici, se non ideologici ${ }^{19}$, variamente indagati, in ordina a caratteri e manifestazioni di questo senso di appartenenza, la mia impressione, tuttavia, è che una massimizzazione

16 Diritto e società nell'antica Roma (Roma 1979), XVII ss. Cf. il caso delle limitazioni all'esperibilità dell'actio aquae pluuiae arcendae, nell'ipotesi in cui il deflusso delle acque fosse stato modificato dal vicino per esigenze di coltivazione, ma anche le regole relative al divieto di immissione di fumi dalla taberna casearia, che non venivano tollerati, a differenza di quelli provenienti dal focolare domestico.

17 Aul. Gell., Noct. Att. 4.12: Si quis agrum suum passus fuerat sordescere eumque indiligenter curabat ac neque arauerat neque purgauerat, siue quis arborem suam uineamque habuerat derelictui, non id sine poena fuit, sed erat opus censorium censoresque aerarium faciebant... Cuius rei utriusque auctoritates sunt, et $M$. Cato id saepenumero adtestatus est.

18 de Martino 1979, op. cit. (n. 16).

19 Nel senso in cui, ad es., di recente, ne parla Marotta 2009, op. cit. (n. 1), 11 ss., sottolineando un ruolo (a mio avviso attentamente costruito ma forse sovradimensionato) delle élites di governo dell'impero: "nel II secolo DC - osserva lo studioso - le élites di governo dell'Impero, fortemente integrate fra loro... manifestavano la convinzione di aver costruito un solido organismo unitario che le accomunava, impegnandole, di conseguenza, a difendere e sostenere Roma, la patria di tutta l'umanità civilizzata". In realtà le ragioni per le quali si incontrano, nel tessuto politico dell'impero, gli interessi delle élites dominanti sono legate all'esigenza di mantenere un'interlocuzione privilegiata col potere centrale e conservare così, se non accrescere, potere e ricchezza. Proprio queste finalità, se girate sul registro della cittadinanza, ne caratterizzano in termini nuovi la natura, oramai sempre più vista come un viatico verso il riconoscimento politico completo e gratificante, come nota per altro, richiamando M. Goodman, Iudaea capta. Il ruolo dell'élite ebraica nella rivolta contro Roma (66-7o DC), trad. it. (Genova 1995), 30 ss., lo stesso Marotta (ib. 13). 
di posizioni, fra ammessi ed esclusi alla cittadinanza, non risulti del tutto agevole né utile, data una poliedricità di situazioni soggettive di vantaggio, in certa misura connesse alla cittadinanza (fruita pleno iure dai ciues, ma oggetto anche di specifiche e varie concessioni) che le fonti ci testimoniano. Ho avuto per altro modo, già in altra occasione ${ }^{20}$, di rilevare come una siffatta distinzione non sembri assumere un carattere di contrapposizione assoluta fra soggetti ammessi e soggetti esclusi dal gruppo. Già muovendo dal dato terminologico è possibile constatare come le fonti parlino non solo di hostis, di nemico, ma anche di hospes, di ospite. La parola hospes, che supera semanticamente il senso più netto di hostis, assume infatti un significato che esula dal conflitto. L'ospite è dunque tendenzialmente ammesso nella Roma arcaica e repubblicana e fruisce di una posizione di vantaggio giuridicamente significativa. Ammesso, a condizioni di reciprocità, nella familia romana, come ho altrove cercato di dimostrare ${ }^{21}$, ma non integrato.

Tuttavia il policentrismo cittadino ingloba ciues Romani di pieno diritto, come anche ciues imminuto iure: abitanti di un municipium o di una colonia. Così la comunità politica allarga il proprio alveo, ricomprendendovi anche i peregrini considerati in generale $\mathrm{o}$ in una situazione di particolare rilievo, come i Latini.

È dunque un filo preciso - sottile ma forse non adeguatamente valutato - quello che distingue il romano dagli "altri", un filo che per essere seguito richiede il superamento della secca contrapposizione inclusione/ esclusione e la valorizzazione di situazioni intermedie, sfumate nella prassi ospitale, occasioni di accoglienza che connotano l'esperienza giuridica romana in questa materia ${ }^{22}$ e preparano un evento, come quello della

20 Cf. ntt. precedenti.

21 Randazzo 2003, op. cit. (n. 7), 57 ss.

22 Tuttavia, secondo una diversa opinione fondata sulla grande autorità di Theodor Mommsen (Römisches Staatsrecht III [Leipzig 1887], 590 ss.) ed espressa nell'ambito della sua nota teoria della guerra come "stato naturale" nei rapporti fra ciues e stranieri, lo stato ordinario sarebbe quello della "inimicizia naturale". Anche quando l'hostis non veniva ucciso o reso schiavo poteva essere ostaggio e alla fine ricevere l'hospitium. Ma tutto questo come conseguenza di uno stato bellico, di una situazione di guerra, di ostilità quasi fisiologica che la dottrina di Mommsen e gli studiosi a cavaliere fra 800 e primi decenni del goo consideravano scontata. Questa prospettiva che considera l'inimicizia come situazione standard nei rapporti fra Roma e gli stranieri credo possa essere definitivamente superata (sul piano dell'approccio culturale) e non soltanto in età repubblicana, come ho altrove creduto di sostenere, ma anche in età imperiale. Su tutto ciò cf. Randazzo 2003, op. cit. (n. 7), passim. 
concessione della cittadinanza a tutti gli abitanti dell'impero nel 212, che certamente è l'epilogo di un percorso ben più risalente e articolato.

È un percorso importante, quello che si snoda fra fine della repubblica ed i primi secoli dell'impero ${ }^{23}$, utile indubbiamente per la ricostruzione del nostro istituto, ma anche per capire l'essenza politica e costituzionale del nuovo regime, dal principato all'impero assoluto, e valutarne in termini di continuità o di discontinuità la natura rispetto all'età precedente.

E tuttavia un percorso di questo genere, che privilegia la cittadinanza come elemento di valutazione di un sistema politico, della sua concezione di sovranità e dei suoi percorsi evolutivi - ma traendone di riflesso elementi di valutazione del dato strettamente giuridico - non è sempre ritenuto significativo per i giuristi moderni.

Hans Kelsen (e cito volutamente un grande teorico generale del diritto, non un romanista) si chiedeva - lo ricorda, efficacemente, Giuliano Crifò ${ }^{24}$ - se la cittadinanza sia "un istituto necessario"25, concludendo col ritenere che esso è, in realtà, "privo di importanza", tale da presentare all'attenzione del giurista un profilo più burocratico che tecnico e per questo di scarso interesse.

Io non penso sia così, almeno non in assoluto e non per l'esperienza giuridica romana. Credo viceversa che l'analisi della cittadinanza sia una preziosa chiave di lettura dell'esperienza politica e giuridica di Roma. Certo, negli stati moderni il concetto è semplice e il rapporto statocittadino che sottostà alle regole che disciplinano la cittadinanza (regole interne e regole di diritto internazionale privato) assume il senso intuitivo e, in effetti, quasi burocratizzante di "Staatsangehörigkeit".

Non così a Roma e non così sino all'avvento dello stato in senso moderno. E tuttavia il pessimistico accenno di Kelsen, seppure troppo categorico per essere sostenuto in quanto tale, fa riflettere anche lo storico: perché la cittadinanza viene vista - da uno studioso della lucidità di Kelsen - come un istituto privo di importanza? Io tenterei una spiegazione della valutazione di Kelsen.

23 Cf. G. Luraschi, 'La questione della cittadinanza nell'ultimo secolo della Repubblica', in F. Milazzo (ed.), Res publica e princeps: vicende politiche, mutamenti istituzionali e ordinamento giuridico da Cesare ad Adriano. Atti del Convegno internazionale di diritto romano (Copanello, 25-27 maggio 1994) (Napoli 1996), 35-99.

${ }^{24}$ Crifò 200o, op. cit. (n. 3), 34 ss. Il compianto studioso muove dal dubbio kelseniano per una valutazione generale dei problemi relativi al dialogo concettuale fra "antico" e "moderno" sul tema della cittadinanza, seguendo un criterio di utilizzazione esemplare dell'esperienza giuridica romana per la valutazione del nostro presente.

${ }^{25}$ H. Kelsen, Teoria generale del diritto e dello Stato ${ }^{2}$, trad. it. S. Cotta e G. Treves (Milano 1954), 246. 
Forse la cittadinanza viene ritenuta priva di importanza perché è un elemento così tanto fisiologico rispetto all'appartenenza al gruppo da potersi ritenere scontato.

E tuttavia, in termini di concreta applicabilità di regole giuridiche, stabilire chi appartenga ad un gruppo per nascita e chi vi appartenga per effetto di forme di accoglienza è essenziale ed è tutt'altro che scontato. E questo a me sembra un dato giuridico elementare.

Stabilire chi appartenga ad un gruppo è infatti imprescindibile per l'esistenza stessa di regole che disciplinino quel gruppo. Così non ci può essere un diritto di famiglia se non si disciplina l'appartenenza alla famiglia. Se non si stabilisce previamente chi siano i coniugi, chi siano i figli, ed ancora oltre, quali siano i gradi di parentela con avi e discendenti, se non si fissano regole per cui ciascuno venga ritenuto componente della famiglia e soggetto al diritto; se non si stabilisce tutto ciò non si potranno disciplinare rapporti ed istituti giuridici familiari, sia inter uiuos che mortis causa. In poche parole, senza una chiara nozione sociale di "famiglia" non ci sarebbe nessun "diritto di famiglia"26.

Torniamo a Roma e scegliamo le nostre categorie di riferimento, i nostri strumenti di lavoro. Sullo sfondo si colloca un dubbio, almeno quello che per me è ancora un dubbio. L'alternativa esclusione-inclusione nel gruppo è un'alternativa rigida?

In altre parole, è possibile andare oltre questo schema e pensare a piani paralleli, a situazioni diversificate che coesistono nell'esperienza giuridica di Roma?

E se cosi fosse, sarebbe possibile ripensare la categoria stessa di cittadinanza, nella ricostruzione giuridica che ne possiamo fare oggi, specie per la fase che va fra la fine della repubblica e la costituzione di Caracalla? Ripensarla, aggiungerei, non soltanto nella sua evoluzione ma anche nelle sue caratteristiche e nelle sue motivazioni sociali.

E, ancora, l'evoluzione dell'istituto depone a favore di una continuità di esso fra repubblica e impero, ovvero possiamo parlare per quest'ultimo di cittadinanza in termini diversi, segnandone la discontinuità, pensando

26 Dico spesso ai miei studenti del primo anno - mi si perdoni la digressione personale - che il giurista è come un sarto, un artigiano che, per quanto avveduto e competente, per realizzare un abito ha bisogno del modello cui l'abito è destinato, senza le cui misure non potrà raggiungere il suo obbiettivo. Se il modello, così come il corpo sociale, anche il più piccolo, come la famiglia nucleare, non è esattamente "misurabile" neppure il più geniale dei sarti e neppure il più competente dei legislatori sarà in grado di confezionare l'abito "su misura", sarà cioè in grado di confezionare regole giuridiche adeguate al corpo sociale cui sono destinate. 
ad un istituto nuovo, nelle logiche sociali, nei presupposti giuridici, nei destinatari, nelle prospettive, prevalentemente inclusive?

Al fondo, dunque, l'idea di una valutazione della cittadinanza in bilico fra una gestione strategica del potere sovrano e l'impatto che, attraverso la concessione del diritto ad essere considerati cittadini, fruendo di benefici e assumendo oneri, condiziona e caratterizza la composita società imperiale.

Non ho certo la pretesa di dare risposte a dubbi così stringenti, ma voglio proporre soltanto ipotesi di lavoro. In questa prospettiva mi limiterò pertanto ad offrire alcune modeste riflessioni, consapevole che questi problemi richiedono, più di altri, la convergenza degli sforzi ricostruttivi di storici e giuristi, secondo il modello di interrelazione scientifica che stiamo sperimentando proprio in questa occasione.

2. Per affrontare il problema della cittadinanza e del trattamento giuridico dello straniero a Roma occorre muovere da alcuni semplici presupposti.

Il primo. In tanto dovrebbe esserci "cittadinanza", come categoria avente rilevanza giuridica, in quanto ci sia uno "Stato", a Roma una ciuitas, una res publica ciuium Romanorum, come aggregazione politico-istituzionale che identifichi con certezza chi è cittadino e chi no. È dunque normale che sia cittadino di uno stato chi nasce all'interno dei confini di quello stato. Dunque è la ciuitas che caratterizza i suoi componenti denominandoli come ciues.

Ma è proprio così a Roma?

Isidoro, nelle sue etimologie (15.2.1) dice che ciuitas non saxa sed habitatores uocantur. Dunque una prospettiva opposta rispetto a quella moderna, per cui la ciuitas non è esclusivamente (o almeno non appare come tale) un ente territoriale, ma un aggregato organizzato a base personale, la cui giuridicità si incentra sui componenti, che sono appunto ciues e sono retti da un comune diritto civile: quid est ciuitas, si chiedeva già Cicerone, nisi iuris societas? (de Rep. 1.49). La mente va alla Cité antique di Fustel de Coulanges: "Cité et ville n'étaient pas des mots synonymes chez les anciens. La cité était l'association religieuse et politique des familles et des tribus; la ville était le lieu de réunion, le domicile de cette association"27.

27 N.D. Fustel de Coulanges, La cité antique (Paris 1900), 181 = La città antica, trad. ital. a cura di G. Perrotta e G. Pasquali (Firenze 1924, rist. Firenze 1972), 156. Sulla valutazione dell'opera di Fustel e specialmente della sua metodologia, tutta orientata sulle fonti e refrattaria a considerare la letteratura mi permetto di rinviare al mio 'Emile Durkheim e 
Una prospettiva ben differenziata, aggiungo, anche rispetto alla polis greca $^{28}$, che è solido presupposto di un'appartenenza ad essa, laddove la ciuitas è specchio riflesso, conseguenza strutturale dell'unione fra ciues, seppure caratterizzata da una sovranità indiscutibile.

Il secondo. Posta l'esistenza di uno "Stato" occorre valutare come l'ordinamento accolga o escluda gli altri. Roma non è stato in senso moderno e non è città-stato in senso greco. Roma è respublica ciuium Romanorum, è la res-publica dei ciues. La specificità dell'esperienza romana mette in crisi le nostre categorie definitorie, tanto di città che di stato, e rende necessaria l'utilizzazione di parametri romani, siano essi coincidenti o meno (come credo sia il caso) con parametri e categorie moderne.

Il terzo. Fatte queste premesse bisogna capire se l'accoglienza dello straniero, occasionale nella repubblica e sempre più generalizzante, sino alla costituzione di Caracalla, segni un momento di evoluzione del sistema statuale oppure una involuzione di esso. Se ne segni cioè un miglioramento, oppure se sia l'anticamera del suo declino identitario che, annunciato nella tarda repubblica, esplode nell'età imperiale. Del suo declino dunque rispetto all'entità che era prima, perché la nuova entità che nell'impero involge persone e popoli nuovi, potrebbe anche essere considerata più valida della prima, specie nel catalizzare, sul piano delle strategie politiche e militari, gli interessi dei provinciali. Forse più valida, più moderna, più "globalizzata" e "globalizzante", ma certamente una cosa diversa, una nuova e diversa entità, un nuovo e diverso stato. Dunque un'entità con caratteristiche nuove, tali da richiedere una differente analisi, in termini giuridici e costituzionali.

In questa logica è possibile che la difficoltà di visione che ha affannato generazione di studiosi derivi, probabilmente, dalla tendenza a forzare la concezione della cittadinanza imperiale nell'ottica di un'evoluzione armonica rispetto alla cittadinanza di età più antica, cercando una continuità necessaria del medesimo istituto. E tuttavia a me sembra che siamo di fronte a fenomeni diversi, sia pure terminologicamente legati e

gli storici del diritto romano. Le radici di un'incomprensione', Index 28 (2000), p. 53-67, specc. §§ 2 e 3. V. altresì, in senso critico e con ulteriore lett., Crifò 2000, op. cit. (n. 3), 10 ss., 18 ss., 95 ss., 100 ss.

${ }^{28}$ Sul punto cf. G. Pugliese Carratelli, Dalla 'polis' all'urbs', in AA. VV., Principi e forme della città (Milano 1933), 1 ss. Più in generale v. H.J. Wolff, 'Polis und civitas', Zeitschrift der Savigny-Stiftung für Rechtsgeschichte (Rom. Abt.) 96 (1978), 1-14. 
storicamente connessi, nel quadro di una continuità formale e terminologica che cela una discontinuità sostanziale.

Una cosa sembra essere la cittadinanza come vincolo fra persone populus come aggregato di quirites, di co-uirites ${ }^{29}$, di concittadini che "insieme" danno spessore e peculiarità giuridica alla ciuitas e ne fondano il potere sovrano - altra cosa una condizione soggettiva di vantaggio, concessa a singoli o a gruppi, elargita come interessato strumento di accreditamento rispetto all'impero.

Sono concezioni, prima ancora che istituti giuridici, assimilabili?

Tentare una risposta soddisfacente su questi interrogativi non si concilierebbe con lo spirito e con i tempi di questo convegno, oltre ad essere ben al di là delle possibilità di chi vi parla. Dunque io darò soltanto alcune impressioni, fermando la mia attenzione sui primi due aspetti.

Naturalmente il ragionamento che farò sarà prevalentemente tecnico. Cercherò di escludere condizionamenti ideologici o culturali che possono derivare dalla nostra esperienza attuale, in cui "cittadino" e "straniero" sono due categorie giuridiche in crisi nell'Europa dei cittadini che si sta formando, in un sistema in cui la ciuitas sembra cedere il passo ai ciues ed in cui il termine "cittadino" è forse da abbandonare, perché troppo sganciato dal contesto semantico originario.

Soltanto un altro richiamo a Fustel de Coulanges ed alla sua Cité antique ${ }^{30}$.

Sono in Francia, ho citato Mommsen ed ora cito Fustel. La mia mente va al vivace (per usare un eufemismo) scambio epistolare fra i due grandi studiosi proprio in occasione della guerra franco-prussiana ed alla lettera (pubblicata sulla Revue des deux mondes) con cui Fustel, brandendo la penna quasi come fosse una spada, risponde a Mommsen: "Monsieur... vous avez quitté vos études historiques pour attaquer la France; je quitte les miennes pour vous répondre". La tensione è altissima, ma il problema dell'Alsazia offre spunti per ripercorrere una differente visione della dominazione romana e del rapporto fra romani e peregrini. Dietro le polemiche del tempo, in cui si muoveranno anche tanti altri

${ }^{29}$ Cf. F. de Martino, 'Intorno all'origine della repubblica romana e delle magistrature', Aufstieg und Niedergang der Romischen Welt, I.1 Von den Anfängen Roms bis zum Ausgang der Republik. Politische Geschichte (Berlin-New York 1972), 217-249, p. 219. Che sia più corretto parlare in termini di concittadini per le origini è anche frutto della migliore riflessione etimologica per cui ciuis "signifie à proprement parler non pas citoyen, mais concitoyen": così E. Benveniste, Le Vocabulaire des institutions indo-européennes I (Paris 1969), 334 s., 367. Cf. anche L. Labruna, 'Quirites', Labeo 8 (1962), 340-348.

30 Supra nt. 27. 
studiosi che in Francia gravitano attorno alla Revue des deux mondes ${ }^{31}$, da George Sand al grande storico Camille Jullian emerge, sullo sfondo, una Weltanschuung complessa, in cui il diritto e la storia di Roma assumono un nuovo ruolo per leggere il presente, anche quando il presente è, come è ovvio, diverso dall'antico.

Penso (e l'ho anche scritto ${ }^{32}$ ) che Fustel abbia ragione quando osserva che nella città antica il diritto nasce dalla religione e che la religione è il presupposto della cittadinanza.

La religione a Roma è dunque equivalente al ruolo che oggi, rispetto allo Stato moderno, assolve il concetto di Nazione? Un meccanismo di identificazione ideale: religione come appartenenza? Forse per l'età arcaica e per la Repubblica è un po' così. I confini della città sono sacri, la città nel suo insieme è un luogo sacro, lo sappiamo, come sappiamo che il debitore insolvente poteva anche essere venduto come schiavo, ma solo trans Tiberim, al di là del Tevere, in un luogo esterno alla cittàsantuario che non poteva essere profanata dalla riduzione in schiavitù di un suo componente.

Certo un "escamotage". Ma un escamotage reso possibile non dalla preponderanza della religione sul diritto, perché in quel caso il prigioniero per debiti non sarebbe mai stato sottratto alla sua comunità cittadina, bensì dalla distinzione fra religione e diritto che a Roma occupano piani diversi ed autonomi, anche quando sembrano confondersi fra loro.

La religione ha invece un fortissimo "added value", in termini di coesione sociale, di appartenenza ad un gruppo, di "nazionalità" nel senso romano del termine. Ma non sarà così per il periodo successivo, non sarà così per l'impero cristiano - nonostante il frequente richiamo terminologico,

31 La celebre Revue des deux Mondes, vivacissima rivista di informazione e dibattito su vari argomenti, nacque a Parigi nel 1831 e rappresentò l'anima degli intellettuali che propugnavano la fine della restaurazione, rivendicando la libertà di critica. Ebbe, fra gli altri, come collaboratori, oltre a Numa Denis Fustel de Coulanges, George Sand, Elisée Reclus, Albert de Broglie, Adolphe Hippolyte Taine ed Ernest Renan. Durante il conflitto Franco-Prussiano (1870-1871), Fustel de Coulanges, Ludovic Vitet and Eugène Aubry-Vitet useranno La Revue des Deux Mondes, in funzione patriottica. I loro interventi avrebbero esaltato specialmente la storia medievale, attualizzando in chiave ideologica la nascita della Francia moderna. Nei loro propositi il convincimento che porre in luce questo periodo storico potesse offrire un esempio di forza e indipendenza politica che consentisse ai francesi di reagire con orgoglio e determinazione alle lacerazioni di una guerra dolorosa. La lettera di Fustel de Coulanges che menziono è tratta dalla seconda edizione delle Questions historiques (1923). E. Emery, "The "truth" about middle ages: La Revue des Deux Mondes and Late Nineteenth-Century French Medievalism', Prose Studies. History, Theory, Criticism 23.2 (2000), 99-114.

32 Randazzo 2000, op. cit. (n. 27). 
specialmente nei testi patristici, soprattutto agostiniani, alla ciuitas $D e i^{33}$, che ha però il diverso senso teologico di "Gottesstaat" - in cui religione e appartenenza alla romanità ${ }^{34}$ non solo non coincideranno, nonostante gli sforzi degli imperatori cristiani, ma creeranno un "gap" di diversificazione fatale sul piano giuridico. E i giuristi sanno bene quanto abbia inciso ad esempio, nello stravolgimento del diritto romano, l'accoglimento di principi frutto dell'etica cristiana, come la pietas o la benignitas ${ }^{35}$.

Negli stati nazionali la religione ha infatti una dimensione che va oltre lo spazio. Il culto non identifica quasi mai uno stato; è raro che uno stato ed un suo culto coincidano, almeno in sistemi che non siano dichiaratamente confessionali, come ancora oggi, ad esempio, nei paesi islamici fondamentalisti. Raro ma non impossibile. Basti pensare alla chiesa anglicana, religione cristiana ma nazionale, nei suoi presupposti storici, nella sua peculiare indipendenza dal papato, nella sua stessa denominazione.

Ma è così a Roma?

La religione romana è elemento identitario, di appartenenza, al punto di considerarlo elemento cardine della cittadinanza? Non credo sia così in assoluto, almeno non credo sia così, lo ripeto, dalla media repubblica in avanti.

Una cosa è certa. Roma, come abbiamo già detto, non è una pólis, Roma è una res publica; il cittadino romano vede la città come una proiezione di sé, non come un'entità caratterizzante, come una realtà, cioè, che lo caratterizza come cittadino, in un senso assorbente ed onnicomprensivo che non troviamo nella Roma repubblicana. È esattamente il contrario: e proprio lui, il cittadino, che caratterizza la ciuitas ed è l'insieme dei ciues che la rende "nostra", con una estensione del senso di appartenenza anche al diritto:

D. 1.1.11 (Paul. 14 ad Sab.): Ius pluribus modis dicitur: uno modo, cum id quod semper aequum ac bonum est ius dicitur, ut est ius naturale. altero modo, quod omnibus aut pluribus in quaque ciuitate utile est, ut est ius civile. nec minus ius recte appellatur in ciuitate nostra ius honorarium.

33 Cf. A. Nesti, 'Multiculturalismo e pluralismo religioso', in AA. VV., La nozione di "Romano" tra cittadinanza e universalità [Da Roma alla terza Roma, Studi, II] (Roma 1984), $53-66$.

34 Sui mutati percorsi del rapporto fra cittadinanza e impero, anche in funzione del concetto di "estraneità" del cristiano, che sente come propria la patria celeste, cf. le sottili considerazioni di V. Neri, 'Cives e peregrini nella Roma tardoantica: l'esaltazione dell'origo romana', in A. Barzanò et al., Identità e valori fattori di aggregazione e fattori di crisi nell'esperienza politica antica, Bergamo, 16-18 dicembre 1998 (Roma 2001), 257-282.

35 Cf. C. Sanfilippo, Istituzioni di diritto romano, a cura di A. Corbino e A. Metro (Soveria Mannelli $\left.2002^{10}\right)$, 44 . 
e ciò in una tensione costante fra ciuis e ciuitas, fra ciuis e "ius ciuile":

D. 1.2.2.12 (Pomp. lib. sing. enchir.): Ita in ciuitate nostra aut iure, id est lege, constituitur, aut est proprium ius ciuile, quod sine scripto in sola prudentium interpretatione consistit.

Noi tutti sappiamo come il percorso verso lo stato sovrano sia un percorso difficile a Roma ${ }^{36}$. Roma nasce come aggregazioni di gruppi parentali, gentilizi, familiari che con sofferta gradualità cedono, in un processo irreversibile, frazioni di potere allo stato. La composizione dello stato è un puzzle difficile a Roma, in cui il potere del pater familias, del ciuis sui iuris è, in origine, un potere solido, un potere che nasce da un fitto fascio di diritti e posizioni giuridiche di vantaggio sulle cose come sulle donne conuentae in manum, sui figli come sugli schiavi.

Un potere che subisce l'erosione derivante dall'appartenenza ad una comunità, un'erosione graduale ma già irreversibile se guardiamo alle origini della comunità politica romana in cui la "sovranità" si identifica con la Ciuitas, nel senso di solida aggregazione politica - policentrica e complessa - che si afferma come tale nei confronti delle altre comunità ${ }^{37}$ e dei singoli che ad essa appartengono. Una sovranità considerata inviolabile e sacra, come sacrilego è l'attentato ad essa, l'attentato alla maiestas populi Romani (D. 48.4.1 pr., Ulp. 7 de off. procons.: Proximum sacrilegio crimen est, quod maiestatis dicitur) inteso come un gravissimo crimine commesso aduersus rem publicam, cioè, in sintesi saldissima fra istituzioni

36 Cf. A. Corbino, 'Governo e sovranità nella res publica', Festschrift für Rolf Knütel zum 7o. Geburtstag (Heidelberg 2009), 229-237.

37 In termini per altro peculiari ed escludenti e che non trovano riscontro nelle varie forme di incidenza sul potere dello stato che oggi provengono dalla comunità internazionale. Si pensi infatti a come la sovranità dello stato nazionale oggi sia limitata sensibilmente ed in campi delicati, dalla politica estera a quella militare o energetica, da parte dell'Organizzazione delle Nazioni Unite e, soprattutto, si consideri la posizione dello stato europeo aderente all'Unione Europea che si sta formando, non a caso, non tanto attraverso la via maestra della scelta popolare, ma attraverso una presenza sempre più pervasiva delle Corti Europee che di fatto stanno creando un diritto dei giudici, senza averne avuto mandato popolare. Un esempio, che si svolge sotto i nostri occhi, di sovranità sostanzialmente autoreferenziale che per altro, per una singolare deformazione dei percorsi storici del diritto, nasce proprio dalla tutela dei diritti umani. Tutela che, giustamente salutata come la più importante conquista giuridica dell'Europa post-bellica, rischia di essere oggetto di un ampliamento concettuale esasperato, che sta portando la Corte di giustizia dell'Unione europea e la Corte europea dei diritti dell'uomo a ricomprendervi (garantendosi, per questa via, una sostanziale superiorità rispetto alle Corti nazionali) anche diritti che lambiscono soltanto le ragioni ideali della tutela dei diritti umani, come, ad es., la proprietà, che la Costituzione italiana tutela e limita (art 42 Cost.; vedi pure i limiti generali già posti dall'art. 832 c.c.), ma al di fuori dei principi fondamentali. 
e collettività politica, aduersus populum Romanum uel aduersus securitatem eius ${ }^{38}$.

In questo contesto i piani della sovranità pubblica e delle situazioni giuridiche di vantaggio dei privati non si sovrappongono né si confondono. Questo consente al diritto privato romano di assumere una latitudine ed un rilievo considerevole nelle relazioni fra i ciues, occupando una sfera di significatività giuridica autonoma, rispetto al potere pubblico ed alla sovranità cittadina da cui è espresso e garantito, ma con cui non confligge né si confonde.

Così troviamo riferimenti a leges privatae, accordi fra privati, con una terminologia simmetrica al più tipico atto di sovranità vincolante i privati che sia espresso da uno stato, la legge, a Roma la lex publica. Il diritto privato romano si svolge su un terreno di sostanziale autonomia rispetto allo stato che tale autonomia garantisce senza ingerirsi in essa. Così nel contrarre non bisogna avvalersi dell'intermediazione di un organo pubblico o di un soggetto privato qualificato, come oggi avviene per certi atti, come la vendita di beni immobili, con l'intervento del notaio, e non bisogna trascrivere l'atto in pubblici registri. Nella mancipatio, forma solenne dell'acquisto del dominium sulle res mancipi, le cose pretiosiores, come le definisce Gaio, il formalismo è assolto con la presenza di testimoni, ciues romani puberi che assistono come elementi qualificati (appunto perché ciues) della comunità, non come espressione di un potere di controllo. Il mancipio dans nella mancipatio tace. E' il mancipio accipiens che dice "hanc rem meam esse" (Gaius 1.11939, 3.16 $7^{40}$ ) ed è lo stesso accipiens

38 D. 48.4.1.1 (Ulp. 7 de off. procons.): Maiestatis autem crimen illud est, quod aduersus populum Romanum uel aduersus securitatem eius committitur. Quo tenetur is, cuius opera dolo malo consilium initum erit, quo obsides iniussu principis interciderent: quo armati homines cum telis lapidibusue in urbe sint conueniantue aduersus rem publicam, locaue occupentur uel templa, quoue coetus conuentusue fiat hominesue ad seditionem conuocentur: cuiusue opera consilio malo consilium initum erit, quo quis magistratus populi Romani quiue imperium potestatemue habet occidatur: quoue quis contra rem publicam arma ferat: quiue hostibus populi Romani nuntium litterasue miserit signumue dederit feceritue dolo malo, quo hostes populi Romani consilio iuuentur aduersus rem publicam: quiue milites sollicitauerit concitaueritue, quo seditio tumultusue aduersus rem publicam fiat.

${ }^{39}$ Est autem mancipatio, ut supra quoque diximus, imaginaria quaedam uenditio: Quod et ipsum ius proprium ciuium Romanorum est; eaque res ita agitur: Adhibitis non minus quam quinque testibus ciuibus Romanis puberibus et praeterea alio eiusdem condicionis, qui libram aeneam teneat, qui appellatur libripens, is, qui mancipio accipit, rem tenens ita dicit: HVNC EGO HOMINEM EX IVRE QUIRITIVM MEVM ESSE AIO ISQUE MIHI EMPTVS ESTO HOC AERE AENEAQVE LIBRA; deinde aere percutit libram idque aes dat ei, a quo mancipio accipit, quasi pretii loco.

40 Communem seruum pro dominica parte dominis adquirere certum est, excepto eo, quod uni nominatim stipulando aut mancipio accipiendo illi soli adquirit, uelut cum ita 
che pronuncia clausole nuncupative che ne precisino qualche profilo $\left(\right.$ Gai. 2.104) ${ }^{41}$. Un formulario che si spiega soltanto come schema rappresentativo, nell'età storica, di quella che in età pre-civica, era l'occupazione con la forza di una cosa.

Lo stato qui sembra essere sullo sfondo ma ciò è espressione di un parallelismo di ruoli e poteri fra entità pubblica e privati, cui viene riconosciuta autonomia nella disciplina dei propri rapporti.

Una rappresentazione di questo equilibrio pubblico-privato la si trae dal processo civile. Sino al principato il processo civile si svolge in due fasi.

Una fase in iure davanti al pretore, organo pubblico, organo dello stato ed una seconda davanti ad un giudice-arbitro privato. Il pretore non giudica, ma si limita a impostare la controversia e metterla, direi costringerla, nei binari della legalità. E si ferma a quel punto, non va oltre. Sarà il giudice a giudicare. Sarà un privato a giudicare altri privati. Solo il giudizio arbitrale è accettato dai romani ma, si badi bene, un tale giudizio si instaura sotto l'egida dello stato e del suo rappresentante nel processo, il pretore, e si svolge anche attraverso una nomina, quella del giudice, in cui un ruolo essenziale viene svolto dal magistrato, nella scelta del iudex e nell'attribuzione del potere di condannare o di assolvere.

Dunque le prerogative del pater familias si stemperano in un potere oramai appannato e contratto, espressione di prerogative diverse rispetto alla vera sovranità, che non può che essere "una" e "piena" e che non potrebbe tollerare né frazionamenti né contrazioni. Circostanza che non incide sulla rilevanza del ruolo del pater familias che agisce o che resiste in giudizio, un ruolo di riguardo, rispettato dalla ciuitas, e che si concilia con l'affidamento al giudizio dell'arbiter, anch'egli un pater familias, dunque un soggetto "pari" a coloro che si sottopongono al suo giudizio.

Un ruolo di riguardo che non esprime alcun profilo di "sovranità" già nel momento della subordinazione al giudizio da parte del convenuto dunque della soggezione del privato ad un potere eminente - intervenuta contestualmente all'instaurazione del iudicium, in un itinerario che si dipana con il consenso dell'autorità di governo, del magistrato, e che colloca così la vicenda processuale su un piano pubblicistico, dando alla

stipuletur: TITIO DOMINO MEO DARI SPONDES? aut cum ita mancipio accipiat: HANC REM EX IVRE QVIRITIVM LVCII TITII DOMINI MEI ESSE AIO, EAQVE EI EMPTA ESTO HOC AERE AENEAQVE LIBRA.

${ }^{41}$ Sul rapporto fra nuncupationes e leges mancipio dictae mi permetto di rinviare a S. Randazzo, Leges mancipii. Contributo allo studio dei limiti di rilevanza dell'accordo negli atti formali di alienazione (Milano 1998). 
successiva nomina pubblica del giudice (che avviene tuttavia, si noti, con la partecipazione del privato alla sua scelta) ed alla sua sentenza un rilievo pubblicistico, acquistando essa valenza pubblica di "giudicato" eseguibile con l'assistenza pubblica.

Un equilibrio, dunque, fra ciues e potere sovrano che conferma come la sovranità sia escludente e non frazionabile.

Ma è una sovranità tuttavia non assoluta, nel senso letterale di $a b$-soluta, sciolta da vincoli esterni che la condizionano. Non è già tale quando viene disciplinata, quando le regole disciplinano, in primo luogo, la successione nel potere sovrano. Non è un caso che la successione al potere imperiale sia il principale enigma del sistema costituzionale imperiale romano.

Perché la sovranità non si trasmette sempre con le medesime regole? Perché bisogna ricorrere all'acclamazione dei militari e non ad un chiaro sistema di scelta da parte del sovrano precedente o ad un meccanismo successorio generale ed analogo a quello civilistico, come nella legge salica, in cui si fisserà la discendenza in linea maschile per cui Clodoveo nel 510 potrà stabilire "De terra uero nulla (salica) in muliere hereditas non pertinebit, sed ad uirilem sexum qui fratres fuerint tota terra pertineat"?

La spiegazione è forse più semplice di quanto si pensi. Non vi è una legge che regola la successione in termini generali perché non vi è un potere realmente autoreferenziale e $a b$-solutus che possa identificarsi in una magistratura svincolata da qualsiasi condizionamento, neppure se suprema. Non è così per il rex, che richiede, per l'investitura, la lex curiata de imperio, non è così neppure per l'imperatore, che richiede, per l'imperium, l'acclamazione, il consenso del senato o una lex de imperio, come avviene per Vespasiano.

Lo stesso tentativo di Augusto di costruire un potere nuovo e assoluto si poggia così, fatalmente, sulle più importanti "referenze" costituzionali repubblicane: la tribunicia potestas, l'imperium proconsolare, sino alle funzioni sacerdotali, tutte attribuzioni e poteri destinati così a dilatarsi esponenzialmente per il combinarsi di esse in capo allo stesso soggetto. E' una logica inevitabile, tanto nella fisiologica continuità costituzionale, quanto nei momenti di apparente "rottura" costituzionale, una logica talvolta considerata ipocrita ${ }^{42}$, ma è la logica romana della sovranità che in Roma è radicata nella ciuitas e nelle sue prerogative.

42 Impressionano ancora, nel loro scetticismo illuminista, le parole di E. Gibbon, History of the Decline and Fall of the Roman Empire (London 1776-1789), quì riprese dal I vol. dell'ediz. a cura di J.B. Bury (New York 1906), 90: “The tender respect of Augustus for a free constitution which he had destroyed can only be explained by an attentive 
Una sovranità che sul piano teorico (direi genealogico) subisce modificazioni concettuali, limiti, ma che non scompare del tutto nella sua matrice ideologica. Quel che cambia profondamente, nel principato, è la dimensione della sovranità, che da pubblica e collettiva (maiestas populi Romani), trasformata fatalmente dall'ipertrofia costituzionale dell'imperatore, diventa maiestas principis. Potere tuttavia pubblico, collettivo, per quanto inglobato nelle prerogative di un uomo solo che lo stato rappresenta o ha la pretesa di rappresentare, nella sua interezza.

Il cittadino repubblicano assume una posizione diversa prima dell'avvento del princeps, una posizione in cui diritti attinenti alla sfera privata e posizioni pubblicistiche di partecipazione politica sono due facce della stessa moneta, specularmente legate nella posizione di privilegio del ciuis, espressione che per altro non ha una valenza onnicomprensiva, posto che il soggetto viene in considerazione dell'ordinamento, di volta in volta, non tanto come cittadino, ma in quanto titolare di posizioni giuridiche di vantaggio esattamente individuate, come l'essere titolare di commercium o di connubium.

Una posizione di privilegio che tuttavia non è espressione di sovranità, tanto da dovere accettare il ciuis che la regolamentazione dei suoi rapporti privati con altri ciues (e, ad un certo punto, anche inter ciues et peregrinos) vengano disciplinati da un meccanismo processuale che si instaura davanti al pretore, magistratus populi Romani.

Nella formula che Gaio ci trasmette dell'agere sacramento il pretore pronuncia tre parole solenni, con cui impone alle parti del processo di fermarsi ed interrompere la dialettica processuale, con un ordine che è specchio riflesso di un potere che può intervenire e bloccare la controversia sul limite dello scontro. Ordina infatti: mittite ambo hominem, lasciate entrambi la cosa controversa (si trattava di uno schiavo) su cui avete compiuto i gesti simbolici di appartenenza ${ }^{43}$.

Mittite ambo hominem: tre parole che sintetizzano la fine della dialettica fra autonomia privata e vincoli sovra-familiari e sovra-gentilizi che provengono dallo stato e che segnano la supremazia della sovranità statuale sull'autonomia privata. Una supremazia che si rafforzerà allorché lo stato si strutturerà burocraticamente anche nel processo. Non è un caso che il

consideration of the character of that subtle tyrant. A cool head, an unfeeling heart, and a cowardly disposition, prompted him at the age of nineteen to assume the mask of hypocrisy, which he never afterwards laid aside".

43 Gaius 4.16: cum uterque uindicasset, praetor dicebat: MITTITE AMBO HOMINEM, illi mittebant. 
processo extra ordinem, nell'impero, non sarà più bifasico ma si svolgerà dall'inizio alla fine davanti al giudice-funzionario, espressione dello stato, o meglio, espressione del principe e del suo crescente potere.

3. Questo è il quadro di sviluppo in cui si colloca anche la cittadinanza. Questa cittadinanza ha una peculiarità che va messa in luce. Essa non è limitata dalla contrapposizione radicale fra cittadini totalmente capaci e non cittadini, totalmente estranei alla capacità.

Esiste infatti una gamma di situazioni intermedie in cui uno straniero può beneficiare di un trattamento favorevole, pur rimanendo privo di cittadinanza.

E' questo il caso della clientela: uno straniero può essere cliente del capo di una gens e ricevere da questi protezione giuridica. Oppure a singoli stranieri può essere concesso l'hospitium; dunque l'hostis non è lo straniero qualsiasi, ma il peregrinus, lo straniero a cui vengono offerti diritti, condizioni giuridiche di tutela. Una valutazione individuale, anche se Roma repubblicana si pone un problema di generale considerazione dei peregrini, sino alla creazione nel 242 a.C., di fronte ad una multa turba peregrinorum ${ }^{44}$, di un praetor peregrinus cui affidare l'esercizio della giurisdizione nelle controversie tra cittadini romani e peregrini, o tra peregrini.

Si noti come le posizioni cambino fra il peregrinus, che occasionalmente risiede a Roma ed entra in relazione con Romani, e l'hospes, che invece viene accolto dalla comunità romana e viene accolto a condizione di reciprocità.

Quando pensiamo alla categoria della reciprocità però rischiamo di utilizzare modelli giuridici e concettuali moderni, per i quali la reciprocità esiste quando lo stato a cui lo straniero appartiene assicura il medesimo trattamento di accoglienza al cittadino dello stato che accoglie l'ospite.

Ma a Roma non è così. L'hospitium è un fatto privato. Dunque in questo contesto l'offerta dell'ospitalità è dono, ma è dono nel senso in cui lo intendeva Marcel Mauss, è dono remuneratorio: dono che obbliga alla reciprocità di un altro dono, dono come primo polo di un sinallagma sociale il cui secondo polo è la garanzia che il Romano che ospita sarà

44 D. 1.2.2.28 (Pomp. lib. sing. enchir.): Post aliquot deinde annos non sufficiente eo praetore, quod multa turba etiam peregrinorum in ciuitatem ueniret, creatus est et alius praetor, qui peregrinus appellatus est ab eo, quod plerumque inter peregrinos ius dicebat. 
trattato in pari modo dallo straniero ospitato e nel suo paese $\mathrm{e}^{45}$. Come qualsiasi situazione su base pattizia privata, presuppone il consenso. Se questo finisce, finisce il legame, finisce l'ospitalità. Si noti come questo atto sia formale e probabilmente veda la presenza di quaestores a segnare la presenza (direi notarile, di presa d'atto) della ciuitas.

Dunque un hospitium privato che si lega ad un hospitium pubblico, frutto di un trattato, di un foedus. Ma sono ipotesi meno frequenti di quanto si pensi. Le fonti parlano spesso di amicitia, raramente di hospitium: una traccia la troviamo nella lex Malacitana, connessa al patrocinium.

Però, a ben vedere, anche l'hospitium privato, che di certo è la forma più antica e direi naturale di ospitalità affonda le sue radici in una dimensione pubblica, nel senso che la collettività si fa garante dell'accoglienza e si adegua all'atto di concessione che il ciuis ha fatto nei confronti dello straniero.

Come vediamo facilmente tutte le nostre categorie di riferimento entrano in crisi a Roma.

La categoria di cittadino, la categoria di straniero, la categoria di stato, la categoria di dono. In questo contesto multiforme il sistema dell'accoglienza non ha niente a che fare con una scelta di integrazione. Il romano è romano, lo straniero è straniero.

Quando i trattati svilupperanno l'hospitium publicum saremo di fronte ad un fenomeno completamente differente. Caracalla consegna l'impero ad un regime giuridico di parificazione che stravolge l'istituto dell'hospitium, lo fa divenire un fatto politico, un riconoscimento forzato dall'interesse pubblico.

Questo modello di cittadinanza assume così un significato profondamente diverso, nei pressupposti etici e nelle valenze identitarie frutto della comune religione. L'appartenenza diventa un profilo burocratico della condizione del soggetto, suddito e non più cittadino ${ }^{46}$, o meglio, concittadino. Caracalla sancisce un sistema che lascia sullo sfondo, appannato e

45 Così si spiega la liturgia della consegna della tessera hospitalis, consegna che avviene sotto l'alta protezione di Iuppiter Hospitalis (Cic., ad Quint. fratr. 2.10-12; Tac., Ann. 15.52) e che ricorda l'analoga cerimonia dello xenos greco posto sotto la tutela di Giove Xenio, di cui ci parla Erodoto (3.39). La tessera è divisa in due parti; spesso due mani congiunte (ne possediamo molte) in cui una recava il nome dell'ospitante e l'altra il nome dell'ospite. Una situazione che poteva anche modificarsi, sino a segnare la fine del patto, la fine dell'ospitalità, con la distruzione delle tesserae, quel tesseram confrigere, di cui ci parla già Plauto.

46 Cf. T. Spagnuolo Vigorita, 'Cittadini e sudditi tra II e III secolo', in A. Schiavone (ed.), Storia di Roma III, L'età Tardoantica, I, Crisi e trasformazioni (Torino 1993), 5 ss. e Id., Città e Impero (Napoli 1996), 98 ss. 
svuotato di significato, non soltanto l'antico "dono" privato dell'ospitalità, intessuto di valenze mistiche e posto sotto la protezione di Iupiter hospitalis, ma la stessa religione romana che si avvia al ruolo, ben differente da quello originario, di religione "di stato".

Essere cittadino diventa, di certo dal 212 in avanti, ma sostanzialmente ben prima di questa data, un fatto sempre più estraneo ai valori coesivi romani e comincia a rappresentare uno di quegli elementi disgreganti che consegneranno l'impero al suo declino morale e, fatalmente, alla sua dissoluzione politica.

La chiusura del cerchio si colloca, né potrebbe essere diversamente, con Giustiniano ${ }^{47}$ che completa in termini generali la concessione di Caracalla, rimuovendo le diversificazioni fra le molteplici categorie di riferimento: ciues, Latini (nelle varie situazioni), peregrini, appartenenti a città riconosciute o stati non ancora tali (come i Germani, o i Parti), a tutti si riconosce lo status di cittadino/suddito:

Nov. Iust. 78.5 (a. 539): Facimus autem nouum nihil, sed egregios ante nos imperatores sequimur. Sicut enim Antoninus Pius cognominatus, ex quo etiam ad nos appellatio haec peruenit, ius Romanae ciuitatis prius ab unoquoque subiectorum petitus et taliter ex eis qui uocantur peregrini ad Romanam ingenuitatem deducens ille hoc omnibus in commune subiectis donauit, et Theodosius iunior post Constantinum maximum sacratissimae huius ciuitatis conditorem filiorum prius ius petitum in commune dedit subiectis, sic etiam nos hoc uidelicet regenerationis et aureorum anulorum ius unicuique petentium datum et damni et scrupulositatis praebens occasionem et manumissorum indigens auctoritate omnibus similiter subiectis ex hac lege damus. Restituimus enim naturae ingenuitate dignos non per singulos de cetero, sed omnes deinceps qui libertatem a dominis meruerunt, ut et hanc magnam quandam et generalem largitatem nostris subiectis adiciamus.

Il testo, direi una sorta di "manifesto" giustinianeo, chiude il cerchio della nostra vicenda: il cittadino è suddito, anello di una catena rigidamente verticistica del potere assoluto. Della cittadinanza romana, delle sue originarie motivazioni, ideali e giuridiche, non c'è più traccia e Giustiniano consegna alle epoche successive un principio, nuovo e diverso, specchio di una storia nuova, preparata in età dioclezianea-costantiniana ${ }^{48}$ e solennemente acquisita dal grande imperatore bizantino.

47 Cf. F. Goria, 'Romani, cittadinanza ed estensione della legislazione imperiale nelle costituzioni di Giustiniano, La nozione di "Romano" tra cittadinanza e universalità', in $D a$ Roma alla terza Roma 1984, op. cit. (n. 33), 277-342.

48 Lo nota, in una pagina lucidissima, E. Betti, Diritto romano I (Padova 1935), 100. 


\title{
THE EVOLUTION OF THE SO-CALLED PROVINCIAL LAW, OR: CICERO'S LETTERS OF RECOMMENDATION AND PRIVATE INTERNATIONAL LAW IN THE ROMAN WORLD*
}

\author{
Hannah M. Cotton
}

The sub-title to this paper, "Cicero's Letters of Recommendation and Private International Law in the Roman World", combines two pieces of autobiography. My unpublished doctorate was devoted to Latin letters of recommendation by Cicero, Pliny the younger and Fronto. ${ }^{1}$ It was much later that, quite by chance, I came across the concept of Private International Law and realized that it could be applied to the Roman Provincial scene, exploring this for the first time in a paper delivered in 2005 in a conference in honour of Professor Werner Eck. As befitted the honorand, this concentrated on the "High Empire". ${ }^{2}$ It was only recently that I have come to see that the concept could be usefully applied to the early imperial period as well, the Republican Empire so to speak.

I. The letter of recommendation - litterae commendaticiae as it is called in the Ciceronian Corpus ${ }^{3}$ - is by no means an exciting literary genre; nor are the formulae and the attempts to manipulate and challenge them so as to distinguish between routine and mechanical letters of recommendation and the unusual one which is meant in earnest, particularly arousing, nor do they ever surprise. Nevertheless these formulae are sometimes quite useful: I got into the habit of ending my letters of recommendation

\footnotetext{
* I am very grateful to Stéphane Benoist for his inexhaustible patience, and to Nicholas Horsfall and Alexander Yakobson for improving my text and my arguments.

1 H.M. Cotton, 'Letters of recommendation: Cicero-Fronto', unpublished DPhil. Dissertation, University of Oxford, 1977, supervised by Professor Sir Fergus Millar.

${ }^{2}$ It was published as 'Private International Law or Conflicts of Laws: Reflections on Roman Provincial Jurisdiction', in R. Haensch et J. Heinrichs (eds.), Herrschen und Verwalten. Der Alltag der römischen Administration in der Hohen Kaizerzeit (Köln 2007), 234-255.

${ }^{3}$ Fam. 13.26.3 with H.M. Cotton, 'Cicero, Ad Familiares XIII 26 and 28: Evidence for Revocatio or Reiectio Romae/Romam', Journal of Roman Studies 69 (1979), 48-50. See however P.Mich. 8.468, col. II ll. 39-40 with H.M. Cotton, Documentary Letters of Recommendation in Latin from the Roman Empire (Beiträge zur klassischen Philologie Heft 132), (Königstein/ Ts. 1982), 1 ff. and S. Strassi, L'archivio di Claudius Tiberianus da Karanis (Archiv für Papyrusforschung und verwandte Gebiete 26) (Berlin-New York 2008), 178-179.
} 
for outstanding students with a variation on Cicero's formula in a letter to Caesar (Fam. 13.15.3): genere novo sum litterarum ad te usus ut intellegeres non vulgarem esse commendationem.

However, their interest for me had nothing to do with any theory of genre. Cicero's letters of recommendation are mostly appeals to Roman officials in the provinces on behalf of people whose interests fell within the officials' sphere of competence (imperium; potestas). Yet surprisingly the letters often fail to refer to the official authority vested in the recipient, and this paucity of references to it contrasts sharply with the multitude of appeals to the man's humanitas, liberalitas, uoluntas, integritas, mansuetudo, clementia, studium, officium, etc. These are all patently personal qualities, not derived from the man's official position. Likewise few recommendations explicitly ask for an official act on the part of the addressee. Generally they are requests to extend his friendship (fides, amicitia, necessitudo and similar terms) to the person or persons recommended.

Where the vagueness and obscurity characteristic of such letters resolves to some detail, a matter of litigation or legal dispute is generally at hand. This is hardly a cause for surprise. Jurisdiction, after all, was the main duty of the governor in a peaceful province. It is precisely because these letters contain appeals to officials in matters concerning the latter's judicial competence that they may justly be held to reflect Roman attitudes towards the administration of justice in their empire. One is surely entitled to suspect, if not condemn, these letters, as blatant attempts to flout justice and make gratia, personal influence, tilt the balance in favour of a protégé. Or as J.M. Kelly put it in a nutshell: "are not justice and favour mutually exclusive ideas, even in Latin?" Consequently he dismissed the saving clause, usual in such letters, "so far as your dignity and justice will permit", as a stock phrase, "which probably meant as little to the recipient as to the sender, as the broad wink of gratia passed between them". 4 I have tried to save Cicero's reputation in an analysis of the realities and principles underpinning Roman provincial administration of justice. ${ }^{5}$ David Daube was left unconvinced by what, in a private communication, he described as "special pleading", meaning sophistry, perhaps naïveté.

4 J.M. Kelly, Roman Litigation (Oxford 1966), 58 and 61.

5 H.M. Cotton, 'The Role of Cicero's Letters of Recommendation: Iustitia versus Gratia', Hermes 114 (1986), 443-46o. 
My main line of defence was that these letters fulfilled an essential function in a system of government, which left the governor with wide discretionary powers on the one hand, and on the other without the machinery needed to carry out judicial and administrative transactions on a regular and impersonal basis. Hence we may regard these letters as part and parcel of the Roman political system as opposed to the ancillary role, which they have in modern bureaucracy. ${ }^{6}$ I also suggested that there were other safeguards, such as the need to preserve decorum on the recommender's part, and to protect his own existimatio on the governor's. Finally, I supplied plenty of extenuating circumstances. I claimed that the legal disputes mentioned in these letters do not necessarily imply court proceedings, and that more often than not Cicero's letter is aimed at something quite different than obtaining the governor's favour at the trial stage. Hence these letters should not be seen as attempts to bias the judge and influence the verdict itself. Furthermore, court proceedings were distasteful to the Romans and they tried to avoid them: the recommendation was one way of doing so. This may take the form of a direct appeal to prevent litigation, as Cicero does in the case of his translator's son's mother in-law: Q. Minucius Thermus proconsul in Asia in 50 BC is requested to help and bring about ut socrus adulescentis rea ne fiat (Fam. 13.54). On other occasions the governor's influence is enlisted as an alternative to legal remedies, i.e. to induce the other party to settle out of court (we are not told whose court of law: the governor's or a local court?). For example P. Silius, governor of Pontus-Bithynia in $5^{\circ} \mathrm{BC}$ is asked to urge the Greek cities to conclude their pactiones with the promagister of the public company which collects the scriptura in the province, P. Terentius Hispo (Fam. 13.65). ${ }^{7}$ In the case of the succession of Cicero's former quaestor, L. Mescinius Rufus, to the inheritance left to him by his cousin, M. Mindius, Servius Sulpicius Rufus, governor of Achaia in 46 вС (Fam. 13.26) is requested to threaten Mindius' widow, Oppia, with a remittal of the case to Rome as means of swiftly concluding the affair, presumably out of court. ${ }^{8}$ An expeditious solution is desired in the case of Cicero's legate, whose services are indispensable to his superior (Fam. 13.57.1). If legal proceedings have already begun by the governor, Thermus, we may

\footnotetext{
${ }^{6}$ For its survival into the imperial period see H.M. Cotton, 'Military Tribunates and the Exercise of Patronage', Chiron 11 (1981), 229-238.

7 H.M. Cotton, 'A Note on the Organization of Tax-Farming in Asia Minor (Cicero, Fam. 13. 65)', Latomus 45 (1986), 367-373.

8 Cotton 1979, op. cit. (n. 3).
} 
assume that Cicero attempts to prevent them from dragging on, but he could be asking for a swift solution out of court. Finally, in his recommendation of T. Claudius Nero's protégé Pausanias of Alabanda, Cicero is trying to convince Silius that he should delay legal proceedings pending Nero's arrival (Fam. 13.64.1). ${ }^{9}$

When Cicero requested a governor to refrain from action, he was not denying anyone his right - at least this is not how the Romans would have seen it. For it is well known that classical law never formulated any idea of an individual's right to an action. The presence of an actio does not necessarily imply that the man had a claim, but rather that there was a formula that could be adapted to his case, and the formula was a matter of agreement between the praetor (governor) and the two parties. This was another way in which the governor could, if he chose, remain passive without, however, depriving anyone of an inherent right.

Some letters have a prophylactic and provisory nature, as in the case of L. Genucilius Rufus, where Cicero requests Thermus to hear in the assize centre of the area any dispute which might arise in the future between this man and a provincial (Fam. 13.53.2). Similarly in the case of L. Manlius who inherited his brother's estate in Sicily, although no complications were foreseen at the time, it may safely be assumed that the recommendation was intended to provide for such an eventuality (Fam. 13.30). The same may be said about the recommendation of Lyso's son's right to inherit from the Roman exile, C. Maenius Gemellus, who adopted the young man in accordance with the local laws of Patrae (Fam.13.19.2). The last two cases have a bearing on the issue of 'double citizenship', which will be discussed later.

The variety of the requests surveyed here suggests a corresponding variety of means at the disposal of the governor. Whether these putative means are to be called judicial, administrative, extra-judicial, authoritative, or by any other name is often a matter of definition. These letters call on the governor to employ his discretion in the preliminaries, the procedure, the pace of the proceedings and so forth. Interference in these, as it might be, 'phases' clearly implies that the discretion of the governor was

9 For an imperial example of the delicate interplay between the governor's discretionary powers, the rights of cities and the influence of individuals see Ch. Kokkinia, 'Aphrodisias's "rights of liberty": diplomatic strategies and the Roman governor', in Chr. Ratté and R.R.R. Smith (eds.), Aphrodisias Papers 4. New research on the city and its monuments (Portsmouth, Rhode Island 2008), 51-60. 
wide, the system was flexible, and the use of discretion need not entail a reversal of justice, to go back to Kelly's doubts (above text to n. 4).

Although I have never published my doctoral dissertation but merely despoiled and looted it in a few articles, my interest in administration and jurisdiction - inseparable in ancient governments - remained a lifetime preoccupation.

However, as one sheds one's youth, the 'noble' principles governing a legal system seem to matter less than its operation: wie es eigentlich gewesen ist. And this new phase coincided by a happy serendipity with the re-distribution in 1992 of the documents from the Judaean Desert by the Advisory Committee for the Dead Sea Scrolls of the Israel Antiquities Authority, some of which now turned up, so to speak, on my desk, and invited me to the study of case law. I was back where I had started, but now equipped with authentic documents from real life - not that the letters assembled in Book 13 of the Ad Familiares are not authentic: they may have been published already in Cicero's own lifetime. ${ }^{10}$ However, this time I had documents written by the litigants themselves, or their lawyers, and in some cases the protocol of court proceedings. I was admitted into the ancient courtroom.

II. This is where my second topic, private international law, or 'conflict of laws' enters the scene, and it seems necessary at this point to repeat here almost verbatim my introduction to the topic in the earlier article. ${ }^{11}$

I became acquainted with private international law almost by accident when one day my eyes fell on the opening anecdote in an article by a close friend, who is a well-known expert on the subject. ${ }^{12}$ The expression 'private international law' suggests at first sight - quite wrongly as turns out - that there are two kinds of international law, private and public. But in fact 'private international law' does not mean 'international law' in the sense of a law common to all nations, but rather that part of the national law of a country which deals with cases where a foreign element intrudes in one form or another: e.g. one side to the contract or suit is a foreigner; or when the property in question is outside the country where the court

10 L. Gurlitt, 'De M. Tulli Ciceronis Epistulis Earumque Pristina Collectione', Diss. Göttingen, 1879 .

11 Cotton, op. cit. (n. 3), 234-235.

12 C. Wasserstein Fassberg, 'On Time and Place in Choice of Law for Property', International and Comparative Law Quarterly $5^{1}$ (2002), 386-400, to whom I wish to thank for letting me read the general introduction to her forthcoming book, in Hebrew, on the subject. 
operates; or if the contract is signed in a third country; or when all these and other factors come together - with many other variations. It is that set of rules which deals with what are known as 'conflicts of laws', although its purpose is to resolve rather than to analyse the conflicts between legal systems. Thus both terms, private international law and conflicts of laws, are to a certain extent misnomers.

Broadly speaking, cases of conflict of laws revolve around two issues: 1) which court has jurisdiction in the matter; and 2) what law applies. It does not follow automatically - nor would it be fair and just that it should - that the court which has jurisdiction in the matter, will apply its own law. A third but closely related issue is the enforcement of a judgement of a foreign court by the national court. Finally, there is no 'harmony of laws' between private international laws of different countries, i.e. in the rules concerning conflicts of law - perhaps it is not even so desirable or beneficial to achieve such harmony.

Notwithstanding the reservation of one of the foremost authorities on the subject, ${ }^{13}$ I believe that it is both legitimate and profitable to apply the conceptual framework called forth by private international law to the Roman Empire as early as republican times, even if is it true that local legal institutions owed their continued existence and their regulation to Roman toleration of their existence. On the other hand, nowhere do we witness that Rome, once she became the sovereign, tried to impose her own legal system, her own law and jurisdiction on the subject populations: ${ }^{14}$ the ius civile was intended for cives; at most Rome extended to foreigners some provisions of its ius civile (e.g. by using the fiction si ciuis Romanus esset). Wolff's argument that 'private international law can only establish itself where respect is shown for foreign law', which he takes to be the sine qua non for the development of private international law, is untenable.

13 H.J. Wolff, 'Nicht Gleichordnung, sondern Über- und Unterordnung bestimmte die Stellung der Normenkreise zu einander', in Das Problem der Konkurrenz von Rechtsordnungen in der Antike (Heidelberg 1979), 7 and 66. I thank Professor Lucia Fanizza for discussing the issue with me, and drawing my attention to some pertinent bibliography. See also Hans Lewald's 'Conflits de lois dans le monde grec et romain', published for the first

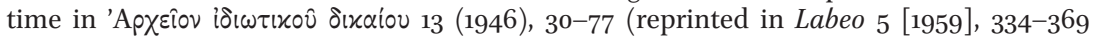
and Revue critique de droit international privé 57 [1968], 419-440 and 615-639), for an early systematic attempt to analyse the legal evidence from the Graeco-Roman world from the point of view of 'private international law' or 'conflict of laws'.

${ }^{14}$ H. Galsterer, 'Roman Law in the Provinces: Some Problems of Transmission', in M. Crawford (ed.), L'Impero Romano e le Strutture economiche e sociali delle Province (Como 1986), 13-27. 
Theoretically, with the exception of some communities who had a special treaty with Rome, all of Rome's provincial subjects were de iure under the jurisdiction (iuris dictio) of Roman officials. However, judicial autonomy, that is the use of local courts of law and of local law, especially in civil cases, may well have been taken for granted, or at least need not have called for a specific grant by the Roman government - at any rate in the case of self-governing communities, i.e. cities. Later, it seems to have become a tralatician part of the provincial edict, as we know from Cicero's provincial edict in Cilicia where he was governor in $50 \mathrm{BC}$ (Cic. Att. 6.1.15), and it is this wholesale, perhaps formerly implicit, grant of local autonomy, that introduced 'conflict of laws' into the scene; no more than the tacit admission of the foreign in legal contexts is needed for the private international law to become part and parcel of a legal system. The legal situation prevailing in Sicily as described by Cicero (2Verr. 2.31-32) adumbrated regulations which modern jurists would have recognised as belonging to the sphere of 'private international law' or 'conflict of laws', as does Augustus' fourth Cyrene edict (FIRA I², 68, p. 409). It is remarkable that with the exception of cases between citizens of the same city in Sicily who are to be tried suis legibus in the city's court, both Cicero and Augustus only refer to courts, i.e. the jurisdiction, but fail to specify the law to be exercised in the court. However, the principle of 'inalienability' of personal legal status (see more below) taken together with the absence of any sign for the imposition of a single juridical framework for the whole empire, ${ }^{15}$ renders the question of the extent of local legal autonomy of secondary importance: limited and curtailed though local jurisdiction might have been, peregrines continued to enjoy the use of their personal law either in their own courts or in those provided by the Romans. A man could not have a choice between the personal laws of one legal system and another. ${ }^{16}$ In republican times, the question concerned Roman citizens living in the provinces but even more so newly made citizens, those who certainly after 212, but also before, received the citizenship with the rider saluo iure gentis. True, with the spread of citizenship and the rapid increase in the number of mixed communities, civitas, citizenship, did change its character, but so far as personal law was concerned, it is hard to believe that the principle enunciated by Cicero in the Pro Balbo 28 suffered any derogation: Duarum ciuitatum ciuis noster esse iure ciuili

15 See Galsterer 1986, op. cit. (n. 14).

16 See on "double citizenship" below. 
nemo potest: non esse huius ciuitatis qui se alii ciuitati dicarit potest. The restriction implied by saluo iure gentis did not apply to personal status: it inserted a wedge between public duties and private status. The possession of Roman citizenship was inextricably bound up with the ius civile in matters concerning the law of persons, and denotes personal status in contrast to public duties. ${ }^{17}$

This is why one can, or rather should, look at these cases through the prism of the principles underlying modern Private International Law, which as we saw were not unknown, even if never formulated as such, in the Roman World. ${ }^{18}$

III. It is my intention to discuss a few cases taken from Cicero's letters of recommendation to republican provincial governors. A good starting point would be Cicero's letter to M. Acilius Glabrio, governor of Sicily in 46-45 BC (Fam. 13.30), where one witnesses the kind of 'conflict of laws' resulting from the acquisition of Roman citizenship by one member of the peregrine family: ${ }^{19}$ "L. Manlius Sosis was formerly a citizen of Catina. He became eligible for Roman citizenship together with the rest of the Neapolitans, since his attachment to that town had preceded the grant of franchise to aliens and Latins. He also served as a town councillor there."20

17 Cf. A.N. Sherwin-White, The Roman Citizenship ${ }^{2}$ (Oxford 1973), 273: 'in the second century $[\mathrm{CE}]$ the status of a Roman citizen was, in private law, as clearly fixed and defined as ever it had been'.

18 One should take account now of the first two chapters of Clifford Ando, Law, Language, and Empire in the Roman Tradition. Empire and After (Philadelphia 2011). As the reader of the present volume will realize, the themes raised in the present article intersect with his "Pluralisme juridique et intégration de l'empire". I would subscribe to all the conclusions reached and summarized at the end of his article, but use his own words to insist: "sur le caractère à la fois centripète et centrifuge de ces pressions intégratrices, qui s'exerçaient non seulement d'en haut mais aussi d'en bas" (emphasis mine). For a salient example of the latter see T.J. Chiusi, 'Zur Vormundschaft der Mutter', ZSav 111 (1994), 155196 and Ead., 'Babatha vs. the Guardians of her Son: A Struggle for Trusteeship - Legal And Practical Aspects of P. Yadin 12-15, 27', in R. Katzoff and D.M. Schaps (eds.), Law in the Documents from the Judean Desert (Leiden 2005), $105 \mathrm{ff}$. In both she demonstrates the legal measures proposed by Babatha (or rather, her lawyer) in the document to support resurface in third-century Roman legal writings.

19 For imperial examples and their interpretation see F. Millar, The Emperor in the Roman World (London 1977), 484, referring to Pliny, Pan. 37-40.

20 Is fuit Catinensis, sed est una cum reliquis Neapolitanis ciuis Romanus factus decurioque Neapoli; erat enim adscriptus in id municipium ante ciuitatem sociis et Latinis datam. Unlike the poet Archias, who was a non-resident adscriptus of Heraclea, L. Manlius Sosis did reside in his adoptive patria, namely Naples, and became a citizen under the lex Iulia: see Sherwin White 1973, op. cit. (n. 17), 151-152, on this letter in conjunction with Cicero, Pro Archia 7. 
His brother had recently died at Catina, but Cicero did not expect any dispute to arise over his title to the estate, and indeed he was in possession of it at the time the letter was written:

However, since he has some old affairs of business in his part of Sicily, I beg to recommend to your notice this matter of his brother's estate and all other concerns of his, above all the man himself. He is an excellent person and a familiar friend of mine, conversant with the literary and scholarly pursuits in which I chiefly delight. Let me ask you (whether or not he goes to Sicily) to be aware that he is one of my closest and most intimate friends, and to let him understand by the way you treat him that my recommendation has been of substantial assistance to him. ${ }^{21}$

Cicero stresses that no dispute is hanging over the inheritance (nullam omnino arbitramur de ea hereditate controuersiam eum habiturum), and under Roman law the fact that "he is in possession" - est hodie in bonis means that "if anyone wishes to disturb (it) ... he can only do so by means of a regular judicial proceeding in which he himself proves a title". ${ }^{22} \mathrm{How}-$ ever, if a dispute arose, could a remedy be sought under Roman law? The testament, if it was a testate succession, or the remedies, if it was an intestate succession, would have been in both cases foreign instruments, and, as pointed out before (text to nn. 16 and 17 above), it is inconceivable that a Roman citizen could inherit (as well as disinherit, or marry, divorce, bequeath, adopt, etc.) according to one system or another, depending on the prospective jurisdiction, in accordance with his own free choice. L. Manlius was subject by the principle of personality to one legal system whereas his inheritance fell under another. How could he have realized it if a dispute arose, and which court of law would take cognizance of the case, a Roman court or a local court? This is a perfect case to which in modern times we would look for the provisions laid down by the private international law of each one of the two countries involved, which may or may not be in harmony with each other, e.g. one law may refer to the lex situs whereas the other to the personal law of the parties involved. ${ }^{23}$

21 sed, quoniam habet praeterea negotia uetera in Sicilia tua, et hanc hereditatem fraternam et omnia eius tibi commendo in primisque ipsum, uirum optimum mihique familiarissimum, iis studiis litterarum doctrinaeque praeditum, quibus ego maxime delector. Peto igitur abs te, ut eum, siue aderit siue non uenerit in Siciliam, in meis intimis maximeque necessariis scias esse itaque tractes, ut intelligat meam sibi commendationem magno adiumento fuisse. Shackleton Bailey's translation in the Penguin edition (1978).

22 H.F. Jolowicz and B. Nicholas, A Historical Introduction to the Study of Roman Law ${ }^{3}$ (Cambridge 1972), 259.

23 See Fassberg 2002, op. cit. (n. 12). 
In contrast to this case, the adoption of Lyso of Patrae's son by C. Maenius Gemellus (Fam. 13.19), ${ }^{24}$ attracted the attention of modern jurists who dealt with the implications of the possession of 'dual citizenship'. It became a bone of contention between them, and thus received two mutually exclusive interpretations.

During the civil wars, C. Maenius Gemellus received the local citizenship of the city of Patrae where he spent his exile. As a citizen of Patrae he adopted Lyso's son in accordance with local law: [tibi commendo] filium eius (i.e. Pisonis) quem C. Maenius Gemellus, cliens meus, quum in calamitate exsilii sui Patrensis ciuis factus esset, Patrensium legibus adoptauit (ibid. \$3). Cicero requests from the governor of Achaia at the time, the celebrated jurist, S. Sulpicius Rufus, to take care of the adopted son's claims to the heritage: eius ipsius hereditatis ius causamque tuear.

Loyal to the principle of incompatibility, Arangio-Ruiz maintained that C. Maenius Gemellus, having returned to Rome after his exile, by regaining his Roman citizenship must have lost his Patrensian citizenship - at least so far as the Romans were concerned - and this resulted in the invalidation of the adoption made in accordance with the local law of Patrae: "e non sappiamo se la grande abilità di un Servio bastasse a venire a capo". ${ }^{25}$ Schönbauer seizes triumphantly on this note of despair in his rival to assert that if one accepts the principles propounded by ArangioRuiz, Cicero's appeal to Servius would be futile: "Ciceros Ersuchen wäre einfach unverständlich, wenn es keine Möglichkeit der Erfullung seines Wunsches gab". ${ }^{26}$ Accordingly he suggests that Gemellus should be treated as a citizen of Patrae who had just received (or been awarded) the Roman citizenship, rather than as a citizen of Patrae who had once held Roman citizenship and had lost it until regaining it, postliminio, when permitted to return to Italy; only in the first case, that of a newly-made Roman citizen, would legal transactions performed in accordance with the laws of his previous polis have remained valid after his having received Roman citizenship. ${ }^{27}$

24 This letter written in 46 BC, like others written after the civil war, adumbrates the advent of a new era: a third party intrudes into the egalitarian relationship between recommender and recipient, that of the dictator, whose good will must be secured in advance, see Cotton 1977, op. cit. (n. 1), chapter 4.

25 V. Arangio-Ruiz, 'Sul problema della doppia cittadinanza nella repubblica nell' impero', in Scritti giuridici in onore di F. Canelutti (Padova 1950) IV, 59-77.

26 E. Schönbauer, 'Zur Entwicklung des "ius publice respondendi”', Iura 4 (1953), 227234 (reply to V. Arangio Ruiz 1950, op. cit. [n. 25]).

27 Schönbauer 1953, op. cit. (n. 26), 393-394. However, even F. De Visscher, who generally shares Schönbauer's views on the principle of double citizenship, holds that compatibility 
Be this as it may, Schönbauer is quite right to observe (n. 27) that it was obviously too early, in any case, for 'positive norms' to have emerged. I would even go further and claim that the very attempt to detect such norms is probably misconceived: by that time the Roman State (whatever we may mean by that) could hardly have formulated principles and made provisions to close the gap between the new reality and traditional theory and practice; under these circumstances the wide discretionary powers of the governor could legitimately be called upon (see text to nn. 4-6 above).

Furthermore: it would seem that even in cases where norms did exist, the concrete evidence we have in Cicero's letters of recommendation does not unequivocally imply that they were followed. Thus in Fam. 13.37, also addressed to the governor of Sicily at the time, M. Acilius, Cicero recommends Hippias, son of Philoxenus, from the city of Calacte, "whose property ... is being held by the state under a different name in violation of the laws of Calacte". 28

This is an instance of a dispute between a private citizen and a city. The Lex Rupilia provides for such a case: "When an individual sues a community or a community sues an individual, the Council of some city is appointed to try the case, each party being entitled to challenge one council thus proposed" (Loeb translation). ${ }^{29}$ Why was the governor's assistance sought when the law (of procedure) was unambiguous? Is the governor expected merely to enforce those provisions, or accelerate the process or should we detect here an appeal for 'illegal' intervention? The cautious and courteous language suggests to me that Cicero was aware of the existence of norms, which did not permit a specific request which might compromise the governor's integrity (existimatio); he could ask only what was commensurate with the latter: Id si ita est, etiam sine mea commendatione

applied only as long as the newly made citizen remained in his home-town, whereas a mutatio ciuitatis would have resulted in its loss, see 'La dualité des droits de cité et la Mutatio Civitatis', Bulletin de l'Acad. Royale de Belgique, 5e sér., 40 (1954), 49; see SherwinWhite 1973, op. cit. (n. 17), 292-336.

The assumption shared by both Schönbauer and Arangio-Ruiz, that C. Maenius Gemellus returned to Rome, albeit reasonable, cannot be proved; it may be based on his being described by Cicero as cliens meus - a designation rarely used in Cicero's letters of recommendations, but which may well imply physical closeness. Nevertheless, C. Maenius Gemellus could hardly be the moving force behind this recommendation which is devoted entirely to promoting Piso's interests and his household's.

28 Hippiam, Philoxenifilium, Calactinum ... tibi commendo ... eius bona, quem ad modum ad me delata res est, publice possidentur alieno nomine contra leges Calactinorum.

${ }^{29}$ quod priuatus a populo petit aut populus a priuata, senatus ex aliqua ciuitate qui iudicet datur, cum alternae ciuitates reiectae sunt. (2Verr. 2.13.32). 
ab aequitate tua res ipsa impetrare debet ut ei subuenias, and later on: tantumque ei commodes in hac re et in ceteris quantum tua fides dignitasque patietur. ${ }^{30}$

I shall conclude this survey with the case of Pausanias, a citizen of the free city of Alabanda ${ }^{31}$ in the province of Asia, ${ }^{32}$ which Cicero, acting for the patrician T. Claudius Nero, brings to the attention of the governor of the province of Bithynia, P. Silius Nerva, in 50 BC: de Pausania Alabandensi sustentes rem, dum Nero ueniat (Fam.13.64.1). Is Pausanias on trial before the governor, who could be requested to delay the case, or is it a request to interfere unofficially in legal proceedings held before another court? Be that as it may, I would suggest that the question as to what legal procedure was available in the case of a citizen of a free city sojourning in another province, would certainly have taxed the skills of a modern expert on private international law. In the Roman Empire, I would maintain, it meant that legal cases tended to gravitate towards the Roman courts, which would account for the long queues and the long delays attested in our sources. ${ }^{33}$ Needless to say, this was not the sole reason for resort to the governor's court: Roman justice was more effective and could be more rigorously imposed on the antagonists. Consequently, even cases calling for the recourse to personal law may come before the governor, who could apply local law, as in the famous 'Complaint of Dionysia' (P.Oxy. 237, AD 186), or failing that, Roman civil law, as in P.Oxy. $706 .^{34}$

The foregoing discussion has by no means attempted to do more than skim the surface of the issues raised in it. In conclusion, I wish to emphasize where precisely there is need for re-thinking and further investigation:

30 For a full discussion of the significance of the terms used here (existimatio, aequitas, fides and dignitas) and elsewhere in letters of recommendation see examples in Cotton 1986, op. cit. (n. 5).

31 For Alabanda see Plinius, nat. 5.108.

32 Unless we accept Shackleton Bailey's conjecture that "the heading is wrong and that this letter was really addressed to the governor of Asia", in Cicero: Epistulae ad Familiares I (Cambridge 1977), 476.

33 E.g. P. Euphr. 1, l. 7 (= SB XXII 15496, AD 245): "spending eight months in attendance at your court" (to the governor of Koile-Syria); see D. Feissel et Jean Gascou, 'Documents d'archives romains inédits du Moyen Euphrate ( $\mathrm{III}^{\mathrm{e}}$ s. après J.-C), I. Les pétitions (P. Euphr. 1 à 5)', Journal des Savants (1995), 3 ff. On the queues see above all G.P. Burton, 'Proconsuls, Assizes and the Administration of Justice under the Empire', JRS 65 (1975), 92 ff.; cf. Cotton 2007, op. cit. (n. 2), 244 ff.

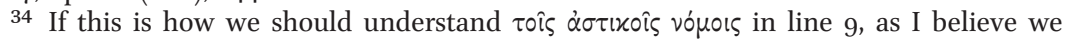
should; cf. N. Lewis, 'Nouveau Texte sur la juridicion du préfet d'Egypte', RHDE 50 (1972), 10 citing D. 1.3.32 pr. I intend to discuss this papyrus elsewhere. I am obliged to Uri Yiftach and Egbert Koops for discussing it with me. 
1) As in my earlier article (Cotton 2007, op. cit. [n. 2], passim) I would like to insist on the distinction between choice of courts and choice of law, procedure as distinct from the substance. A Roman court of law may well use and enforce local law.

2) For our purpose, the Empire could be conceived as an extension of Italy: the first 'conflicts of laws' arose and were solved when Rome entered into various treaties with the cities of Italy. Whatever rules, or rather rules of thumb, had evolved in Italy up to $90 / 89 \mathrm{BC}$, would be applied in the provinces by the very same magistrates who later on went overseas. However, the evidence for Italy is mainly epigraphic, and contains statutes rather than court cases. ${ }^{35}$

3) One cannot a-priori assume that the judicial autonomy of the more privileged communities (Roman and Latin) in a province was wider than that enjoyed by peregrine communities, which stood, so to speak, altogether outside the entire framework of the Roman legal system; the contrary may prove to be true. ${ }^{36}$

4) Above all, it seems to me, one must be fully conscious of the crucial distinction between the statutes and the law-books on the one hand and the courtroom, the litigants and the lawyers on the other. ${ }^{37} \mathrm{We}$ must, at all costs, avoid a schematic legal approach which takes legal writings as a faithful depiction of what happened in the day to day 'to and fro' of litigants and litigators: such an approach removes us from the truth, and from reality.

35 Cf. M. Crawford, Roman Statutes I-II (London 1996). Not exclusively though; for wax tablets recording legal transactions, see e.g. G. Camodeca, Tabulae Pompeianae sulpiciorum (TPSulp.) (Rome 1999) with the review article by G. Rowe in Scripta Classica Israelica 20 (2001), 225-245.

36 H.M. Cotton, 'Jewish Jurisdiction under Roman Rule: Prolegomena', in M. Labahn and J. Zangenberg (eds.), Zwischen den Reichen: Neues Testament und Römische Herrschaft. Vorträge auf der ersten Konferenz der European Association for Biblical Studies (TANZ 36) (Tübingen, 2002), $16 \mathrm{ff}$.

37 I suppose this could be summed up, perhaps anachronistically, as case law. Needless to say, legal papyri constitute our best evidence. A huge amount of work has been done, and is being done by the papyrologists, but the historians of the Roman Empire are not fully cognizant of it. 



\title{
CLAUDE DE LYON, ANCUS MARCIUS ET L'ÂGE ROYAL : D'UNE INTÉGRATION L'AUTRE ${ }^{1}$
}

\author{
Anne Daguet-Gagey
}

En lisant, dans le cadre d'un séminaire, le De republica de Cicéron, et plus particulièrement le passage où l'orateur tardo-républicain traite du règne d'Ancus Marcius², quatrième roi de Rome, j'ai été frappée par le rapprochement que l'on pouvait être tenté d'opérer entre les réalisations mises au crédit de ce monarque et certaines de celles que l'on sait, par Suétone, Tacite ou Dion Cassius notamment, avoir été celles de l'empereur Claude $^{3}$, quatrième empereur romain, pour les Modernes que nous sommes, ou cinquième si l'on suit le même Suétone. Jusqu'à présent, pourtant, c'est plutôt de Servius Tullius, l'avant-dernier roi de Rome, que l'on avait coutume de rapprocher Claude $^{4}$, mais j'ai souhaité montrer, lors des journées lilloises de juin 2011, qu'il n'était pas incongru de relier ce prince au personnage d'Ancus Marcius. Ma réflexion s'est depuis étoffée et il m'apparaît aujourd'hui que c'est plus globalement à l'ensemble de l'âge royal que Claude s'est intéressé et dans ce passé qu'il a puisé, à l'occasion, des arguments destinés à servir ses propres options politiques. On sait avec

1 Je tiens à remercier S. Demougin, S. Benoist et $\mathrm{O}$. Hekster de m'avoir conviée à intervenir dans le cadre de cette rencontre. Ma communication, qui a porté sur les rapprochements susceptibles d'être opérés entre Ancus Marcius et l'empereur Claude, s'est enrichie des discussions qui ont suivi.

2 P.M. Martin, L'idée de royauté à Rome I. De la Rome royale au consensus républicain (Clermont-Ferrand 1982), 253-263 ; J. Martinez-Pinna, 'La Roma de Anco Marcio', Gerión 6 (1988), 55-67 ; D. Briquel, 'Le règne d'Ancus Marcius ; un problème de comparaison indoeuropéenne', Mélanges de l'École Française de Rome 107 (1995), 183-195; T. Camous, Le Roi et le fleuve (Paris 2004).

3 Voir A. Momigliano, Claudius, the Emperor and his Achievement (Cambridge 1961, $2^{\mathrm{e}}$ éd.) ; AA. VV., Die Regierungszeit des Kaisers Claudius (41-54 n. Chr.). Umbruch oder Episode? Internationales interdisziplinäres Symposion aus Anlaß des hundertjährigen Jubiläums des Archäologischen Instituts der Universität Freiburg in Br., 16-18 Februar 1991, V.M. Strocka Hsg. (Mayence 1994).

4 Cf. D. Briquel, 'Claude, érudit et empereur', Comptes rendus de l'Académie des Inscriptions et Belles Lettres 132 (1988), 217-232 ; L. Chioff, 'Ferter Resius. Tra l'augurium di Romolo e il pomerium di Claudio', Rendiconti della Pontificia Accademia Romana di Archeologia 65 (1992-1993), 127-153. Cette dernière étude (voir les p. 141-150) tend à prouver que Claude fit ériger, sur le Palatin, à l'emplacement de l'Auguraculum de Romulus, un sacellum appelé, dans un premier temps, Roma Quadrata, puis, à compter du règne d'Hadrien, Auguratorium. Cet édifice pourrait figurer sur le fr. 469 de la FVR. 
quel mélange de fascination, de suspicion, voire de répulsion la royauté était regardée à Rome. Pour autant, les références aux rois ou à certains de leurs actes, militaires notamment, abondent dans la littérature ancienne, preuve que ces précédents ne constituaient nullement un sujet tabou, du moins pour ce qui est des rois dont on ne pouvait que louer le bilan, ce qui fut le cas d'Ancus Marcius, point de départ de ma réflexion. Paul-Marius Martin a d'ailleurs bien montré la manière dont les Romains, dès le $\mathrm{III}^{\mathrm{e}} \mathrm{s}$. av. J.-C., avaient assumé leur passé royal, récupéré et mis au service de leurs ambitions nationales ou familiales avant d'être instrumentalisé, au dernier siècle de la République, par les factions aux prises les unes avec les autres $^{5}$. Auguste, par la suite, ne devait pas hésiter à exploiter le mythe des origines et les figures de Romulus et de Numa, à des fins de propagande personnelle visant à faire de lui le nouveau fondateur de l'Vrbs et le restaurateur des sacra. Recueillant l'héritage monarchique, il fut cependant suffisamment habile pour ne pas répéter les erreurs de son père adoptif et pour lier son destin à celui de la Cité.

Je défendrais volontiers l'idée selon laquelle Claude, cet empereur érudit et novateur, qui n'était en rien le benêt décrit par une tradition annalistique hostile, pourrait avoir opportunément trouvé dans les acta d'Ancus Marcius, et plus largement dans le passé royal des Romains, des arguments et autant d'exempla destinés à conforter certaines de ses décisions, qu'il semble avoir eu du mal à imposer. Je pense, tout particulièrement, à son souhait d'ouvrir les rangs du Sénat romain à certains provinciaux, notamment aux Gaulois et parmi eux aux Éduens, projet dont on sait qu'il suscita d'âpres débats. Mais, c'est également pour d'autres questions, qui ne touchent pas toutes au thème de l'intégration, que Claude pourrait avoir recouru au précédent des rois de Rome et notamment à celui d'Ancus Marcius, le bonus Ancus du poète Lucrèce ${ }^{6}$, dans le but de convaincre ses contemporains du bien-fondé de ses projets ou de ses décisions. C'est, en définitive, de multiples intégrations qu'il sera question dans les lignes qui suivent : celle réalisée par Ancus Marcius avec les Latins récemment soumis, celles de l'empereur Claude vis-à-vis des provinciaux romanisés et des habitants d'une des collines les plus fameuses de Rome (l'Aventin). Plus largement, c'est à l'intégration du passé royal dans le présent de la

5 P.-M. Martin, L'idée de royauté à Rome II. Haine de la royauté et séductions monarchiques (du $I^{e}$ siècle av. J.-C. au principat augustéen) (Clermont-Ferrand 1994), 212 sv.

6 Lucr., 3, 1025. Horace, pour sa part, devait saluer les figures de Numa et d'Ancus Marcius ; cf. Ep. 1, 6, 25-27 (Cum bene notum / porticus Agrippae et uia te conspexerit Appi, / ire tamen restat, Numa quo deuenit et Ancus). 
Rome impériale du milieu du I ${ }^{\mathrm{er}} \mathrm{s}$. de notre ère que l'on s'intéressera, une intégration à laquelle l'empereur Claude contribua, dans le droit fil de la politique augustéenne.

\section{La geste d'Ancus Marcius}

Ancus Marcius fut un roi particulièrement actif, si l'on en croit les auteurs anciens, tous évidemment très postérieurs au règne considéré. Trois points sont régulièrement retenus par l'annalistique ancienne, qui constituent en quelque sorte et pour une bonne part la geste d'Ancus Marcius. S'arrêter sur les réalisations mises au crédit de ce souverain permettra de mieux souligner les rapprochements que l'on est tenté d'opérer avec certaines initiatives de l'empereur Claude.

Des principales données littéraires à la disposition de l'historien ${ }^{7}$, il ressort qu'Ancus Marcius fut un souverain, pacifique par nature, mais contraint par la nécessité de se montrer belliqueux, une sorte de synthèse de Romulus, figure du roi guerrier, et de Numa Pompilius, réputé avoir donné à la Cité ses règles religieuses. Tite Live devait conclure à son sujet qu'il avait « un caractère moyen, qui rappelait autant Romulus que Numa » (medium erat in Anco ingenium, et Numae et Romuli memor) ${ }^{8}$.

Tous les auteurs évoquent ses guerres, menées notamment contre les Latins. Ancus Marcius semble même des rois pré-étrusques l'un de ceux qui livrèrent le plus de batailles, toutes à l'avantage du peuple romain. Ces guerres victorieuses aboutirent à la soumission et à la destruction d'un certain nombre de villes du Latium ${ }^{9}$. Elles eurent également une autre conséquence, non sans rapport avec mon propos. Ancus Marcius procéda, en effet, aux dires des auteurs anciens, à des transferts de populations, qui lui permirent d'augmenter celle de Rome. Certains habitants des cités vaincues furent conduits à Rome et installés sur la colline de l'Aventin et dans la dépression qui séparait cette colline de celle du Palatin

7 Les principaux auteurs sur lesquels se fondent tant nos connaissances du règne d'Ancus Marcius que notre réflexion sont: Cicéron (rep. 2, 18, 33-34), Strabon $(5,3,5$ et 7), Tite Live $\left(1,3^{2-34}\right)$, Denys d'Halicarnasse (3, 36-45), Florus $(1,1,4$ et 8$)$, l'auteur du $D e$ uiris illustribus $(5)$ et, secondairement, Eutrope $(1,5)$ et Zonaras $(7,7)$, qui reprend Dion Cassius $(2,8)$.

8 Liu., 1, 32, 4 .

9 Politorium, Tellènes, Ficana, Medullia. Si cette tradition a un fondement historique, il faut conclure que les Romains pourraient avoir cherché à attirer certains habitants des cités vaincues, qui avaient sans doute tout perdu dans les combats. 
(à l'emplacement du futur Circus Maximus) ${ }^{10}$. L'Aventin fut donc ajouté à la ville à cette occasion, sans être pour autant et au sens strict du terme intégré à l'Vrbs ${ }^{11}$. De fait, la colline devait demeurer encore longtemps à l'extérieur du pomerium, cette ligne fortifiée enserrant un espace sacré12.

L'ajout de l'Aventin à Rome et l'installation sur cette colline d'une $\mathrm{mul}$ titudo de Latins vaincus (ce sont les propres termes de Tite Live ${ }^{13}$ ) valurent à Ancus Marcius ce jugement de Virgile énoncé dans l'Énéide : «déjà, ici même trop sensible à la faveur populaire $»^{14}$. Le poète augustéen était au fait de l'étroitesse des liens qui unissaient depuis des temps reculés la plèbe romaine à l'Aventin ; j'y reviendrai plus loin à propos de Claude.

Ancus Marcius est donc considéré comme à l'origine d'un long processus ayant conduit les populations soumises du transfert à l'insertion ${ }^{15}$, de l'insertion à l'intégration ${ }^{16}$, et de l'intégration à l'assimilation ${ }^{17} \mathrm{ou}$, dit autrement, de l'installation plus ou moins forcée au « sentiment du semblable », pour emprunter à Tocqueville l'une de ses belles formules. Tite

10 Cicéron et Strabon font très vraisemblablement une erreur en attribuant à Ancus Marcius l'occupation du Célius, traditionnellement imputée à Tullus Hostilius. Sans doute auront-ils confondu l'occupation de l'Aventin et celle du Célius. Voir sur ce point, Camous 2004, op. cit. (n. 2), 181.

11 A. Merlin, L'Aventin dans l'Antiquité (Paris 1906), 36-41.

12 Une réfection pourrait être contemporaine des dates attribuées par la tradition au règne d'Ancus Marcius ( $c a$ 640-617 av. J.-C.) ; cf. A. Carandini, P. Brocato, G. Ricci, N. Terrenato, 'Lo scavo delle mura palatine. La sequenza delle fasi', Bollettino di Archeologia 16 (1992), 117-122 ; Camous 2004, op. cit. (n. 2), 175-176. Datation différente donnée par P. Fontaine, 'Des « remparts de Romulus » aux murs du Palatin. Du mythe à l'archéologie', in P.-A. Deproost et A. Meurant (eds.), Images d'origines. Origines d'une image (Louvain-laNeuve 2004), 35-54; accessible sur le site : http://bcs.fltr.ucl.ac.be/FE/15/FontaineMurs.pdf (700/675 av. J.-C.). Cf. en outre Claud., Gild., 108-109: Vtinam remeare liceret / Ad ueteres fines, et moenia pauperis Anci. "Ah! s'il m'était permis de revenir / Aux anciennes frontières, aux murs du pauvre Ancus!" Sur les débats portant sur l'origine du pomerium, voir l'état de la question dans M. Andreussi, 'Roma : Il pomerio', Scienze dell'Antichità 2 (1988), 220-227.

13 Liu., 1, 33, 1-2 :... multitudinem omnem Romam traduxit... Auentinum nouae multitudini datum.

14 Virg., En. 6, 815-816 : Quem iuxta sequitur iactantior Ancus, / nunc quoque iam nimium gaudens popularibus auris. Pour G. Dumézil, Tarpéia, essai de philologie comparée indoeuropéenne (Paris, 1947), 182, Ancus Marcius « a des rapports spéciaux avec la plèbe ».

${ }^{15}$ L'insertion caractérise le fait pour un étranger de devenir partie intégrante d'une communauté d'accueil tout en conservant son identité d'origine; l'insertion suppose de respecter les normes collectives d'une société, les règles du «bien vivre ensemble ».

${ }^{16}$ L'intégration suppose de se sentir concerné par une communauté de destin et soucieux de transmettre à ses descendants les fondamentaux qui constituent le noyau identitaire de la société d'accueil.

17 L'assimilation implique la pleine adhésion aux normes et aux valeurs de la société d'accueil et la totale appartenance à celle-ci ; l'expression de l'identité et des spécificités socioculturelles d'origine demeurent cantonnées à la seule sphère privée. 
Live parle d'ailleurs des Latins accueillis sur l'Aventin comme de noui ciues $^{18}$ et l'on ne peut exclure que leur installation dans Rome, pour effective qu'elle ait été, ait répondu à une visée stratégique d'Ancus Marcius, désireux de contrer l'influence de l'aristocratie romaine et des gentes ${ }^{19}$.

Plusieurs auteurs, à commencer par Denys d'Halicarnasse ${ }^{20}$, mettent encore au crédit d'Ancus Marcius l'annexion et la fortification du Janicule, réalisées dans des perspectives aussi bien commerciales que défensives; il s'agissait de garantir la sécurité fluviale contre les menées ennemies, celles des Étrusques notamment. Le Janicule fut relié à la Ville par le Pont Sublicius, souvent appelé par les auteurs anciens le Pont de bois. Si l'on ajoute la réunion du Janicule à celle de l'Aventin, évoquée plus haut, on doit reconnaître qu'Ancus Marcius est le roi qui a le plus contribué à étendre le territoire urbain. C'est peut-être pour cette raison que Cicéron ${ }^{21}$ et Strabon ${ }^{22}$ lui attribuent aussi, à tort semble-t-il, l'ajout du Célius, traditionnellement plutôt rapporté à Tullus Hostilius. Ancus Marcius aura par ailleurs été l'initiateur d'une politique de grands travaux, qui devait permettre à Florus de parler de lui comme de l'aedificator Ancus ${ }^{23}$.

A ce propos, il faut rappeler qu'Ancus Marcius est tenu par l'ensemble de la tradition littéraire pour le créateur de salines et le fondateur du port d'Ostie, appelé à devenir le débouché de Rome sur la mer ${ }^{24}$. Denys d'Halicarnasse souligne les avantages d'un site ${ }^{25}$, dont Strabon ${ }^{26}$ pour sa part paraît douter. Il reste qu'aux yeux des auteurs anciens, Ancus Marcius est le souverain qui contribua à métamorphoser Rome et à en faire d'une cité continentale une cité maritime, ouverte sur l'outre-mer, sur l'étranger et sur ses productions. Il façonna en quelque sorte la bouche de ce qui allait

18 Liu., 1, 33, 2: Additi eodem haud ita multo post, Tellenis Ficanaque captis, noui ciues...

19 Camous 2004, op. cit. (n. 2), 279-285.

20 Dion. Hal., 3, 45 ; mais aussi Liu., 1, 33, 6 ; uir. ill., 5 ; Eutr., 1, 5 .

21 Cic., rep. 2, 18, 33.

22 Strab., 5, 3, 7.

${ }^{23}$ Flor., 1, 1, 8. Outre le pont sur le Tibre, la muraille du Janicule et le fossé des Quirites, Ancus Marcius fit encore construire une prison et agrandir le temple de Jupiter Férétrien.

${ }^{24}$ A. Grandazzi, La fondation de Rome (Paris 1991), 109-116; F. Zevi, 'Les débuts d'Ostie', in Ostia, port et porte de la Rome antique (Genève 2001), 3-9, avec la bibliographie antérieure.

25 Dion. Hal., 3, 44.

${ }^{26}$ Strab., $5,3,5$. 
devenir le « ventre de l'humanité ». L'ouverture à l'Autre et à ses richesses (certes mue par le désir de le soumettre et de se les approprier) constitue le point central du récit de Denys d'Halicarnasse et, selon cet auteur, la caractéristique essentielle des Romains ${ }^{27}$. Ancus Marcius apparaît ainsi comme un roi désireux d'animer sa cité d'un double mouvement respiratoire : inspiration, en réalité aspiration, par voie d'intégration du Vaincu - habile moyen pour tenter de le neutraliser ou d'en faire un contre-pouvoir - ; et dans le même temps, mouvement inverse, expiratoire, destiné à propulser le peuple romain jusqu'à la mer, lui offrant, depuis Ostie, des rêves de conquêtes, militaires et commerciales. Ancus Marcius contribua donc à faire de Rome, simple ville ou agglomération des bords du Tibre une véritable cité, dotée d'un ager s'étendant jusqu'à la mer. Sous l'Empire (II ${ }^{\mathrm{e}} \mathrm{s}$. ap. J.-C.), une inscription et une statue érigées en son honneur à Ostie devaient d'ailleurs saluer en lui le fondateur de la colonie ${ }^{28}$.

\section{Les res gestae de l'empereur Claude}

Six siècles plus tard environ, Rome devenue capitale d'empire dominait l'ensemble du bassin méditerranéen. La République qui avait succédé à la royauté n'avait pu supporter cette extraordinaire dilatation du territoire de la Cité. Le régime républicain n'avait pas survécu à cette métamorphose d'une ville en capitale. Le Principat avait pris sa place, Auguste posant les fondations du nouveau régime, ses successeurs consolidant peu à peu l'édifice. Parmi eux figure Claude, qui, comme l'on sait, régna sur l'empire romain entre 41 et 54 de notre ère. Si ce prince a longtemps été tenu pour un sot, jouet de ses femmes et de ses affranchis, il n'est plus question aujourd'hui d'accréditer cette vision du personnage, dont on ne doit pas oublier qu'il fut le deuxième des empereurs à bénéficier après sa mort d'une apothéose. De l'ensemble de l'œuvre de Claude, trois points retiendront dans un premier temps l'attention : la question de la concession du ius honorum et de l'accès des Gaulois au Sénat, celle de l'extension du pomerium et de l'adjonction à l'Vrbs de l'Aventin, enfin, celle des grands travaux engagés à Ostie.

27 Au début du premier livre, Denys d'Halicarnasse insiste sur la grandeur de l'empire des Romains et sur la durée de leur hégémonie.

28 CIL XIV, 4338 (= AE 2000, 266): A[nco] / Mar[cio] / reg[i Rom(ano)] / quart[o a R]omul[o],/quiabu[rbec]ondit[a]/[pri]mum colon[iam/c(iuium)Rom(anorum]/dedux[it]. L'inscription daterait de l'époque d'Hadrien. Cf. M. Cébeillac-Gervasoni, M.L. Caldelli, F. Zevi, Épigraphie latine (Paris 2006), 73-74. 
L'intégration des Gaulois au Sénat est une question bien connue grâce au récit de Tacite $^{29}$ et à la célèbre Table Claudienne ${ }^{30}$, conservée à Lyon, au Musée Gallo-romain de Fourvière ${ }^{31}$. On ne s'y arrêtera que pour rappeler les faits : en 48 ap. J.-C., alors que Claude exerçait sans doute encore la censure et s'apprêtait à opérer une lectio senatus afin de regarnir les rangs de l'Assemblée, de vives discussions s'engagèrent pour déterminer s'il convenait de permettre aux notables des Trois Gaules de disposer du ius honorum et partant d'entrer au Sénat. Deux partis débattirent vigoureusement sur cette affaire, à coup d'arguments contradictoires rapportés tant par Tacite que par la Table.

On sait que l'empereur prit la parole devant ses pairs du Sénat - avec lesquels les relations étaient loin d'être toujours détendues ${ }^{32}$ - pour tenter de les convaincre du bien-fondé de la requête des Gaulois et de l'intérêt pour l'Assemblée d'ouvrir ses portes aux provinciaux. C'est dans le passé de Rome que Claude alla chercher les arguments à opposer à ses détracteurs.

Par une leçon d'histoire, cohérente quoique quelque peu laborieuse si l'on s'en tient au témoignage de la Table Claudienne ${ }^{33}$, Claude entreprit de convaincre les sénateurs. L'empereur remonta jusqu'aux origines de la Cité, souhaitant démontrer que des rois étrangers avaient un jour régné sur Rome, tels Numa le Sabin ${ }^{34}$ ou encore Tarquin l'Ancien, né de l'exilé corinthien, Démarate, et de l'Étrusque Tarquinia. Tel aussi son successeur,

29 Tac., Ann. 11, 23-25. Voir A. de Vivo, Tacito e Claudio (Naples 1980).

30 CIL XIII, 1668 (ILS $212=$ FIRA, I², 43). Voir l'édition de P. Fabia, La Table Claudienne de Lyon (Lyon 1929) et A. Giardina, 'L'identità incompiuta dell'Italia romana', in L'Italie d'Auguste à Dioclétien. Actes du colloque international, Rome, 25-28 mars 1992 (Rome 1994), 1-23. La traduction a été révisée par F. Bérard, 'Claude, empereur né à Lyon', in H. SavayGuerraz (ed.), Rencontres en Gaule romaine (Gollion 2005), 40-41.

31 Les deux documents ont généré de très nombreuses études; voir, en dernier lieu, W. Riess, 'Die Rede des Claudius über das Ius honorum der gallischen Notablen: Forschungsstand und Perspektiven', Revue des Études Anciennes 105 (2003), 211-249, notamment les références bibliographiques données dans les notes. Sur l'attitude de Claude, favorable à l'extension de la citoyenneté romaine et à l'ouverture du Sénat aux provinciaux, voir S. Demougin, 'Claude et la société de son temps', in AA. VV. Die Regierungszeit 1994, op. cit. (n. 3), 11-22, particulièrement 14. Sur les références faites par Claude au passé, voir D. Briquel, 'Le témoignage de Claude sur Mastarna/Servius Tullius', Revue Belge de Philologie et d'Histoire 68 (1990), 86-108, particulièrement, 88 et 107-108.

32 B. Levick, Claude (Gollion 2002), 123-136 et 140.

33 Sur ce point, voir Giardina 1994, op. cit. (n. 30), 3 ; Bérard 2005, op. cit. (n. 30 ), 42.

34 Dans la version tacitéenne du discours de Claude, l'empereur tint, semble-t-il, à rappeler ses propres origines sabines: Tac., Ann. 11, 24, 2. 
Servius Tullius, alias Mastarna (son nom étrusque) ${ }^{35}$. Au passage, Claude cite sans s'y attarder Ancus Marcius, petit-fils de Numa par sa mère, et par conséquent à demi-sabin, mais il omet assez logiquement de la série des rois Tullus Hostilius, d'origine latine. Ce passage de l'oratio impériale offre donc un cas parfait d'exemplum, « moment où l'orateur insère un appel au passé dans une stratégie de la persuasion ${ }^{36}$. Ce faisant, l'empereur énonçait et posait le principe du bon comportement, marqué par l'ouverture à l'Autre et par l'acceptation de cette altérité.

A l'issue du discours impérial, un sénatus-consulte fut effectivement voté, autorisant dans un premier temps les primores des Éduens, alliés traditionnels de Rome, à siéger au Sénat. Tacite laisse implicitement entendre que la mesure fut, dans un second temps, élargie aux notables d'autres cités des Trois Gaules. Il est probable que le Sénat ne s'ouvrit, sous le règne de Claude, qu'à un nombre limité de notables gaulois ${ }^{37}$. Il n'en reste pas moins que la décision alors votée et obtenue de haute lutte permit à l'empereur de poser le principe de «l'élargissement futur du Sénat aux meilleurs des provinciaux $»^{38}$.

L'extension du pomerium est une autre initiative de Claude, qui mérite d'être mentionnée puisqu'il y est question de l'Aventin, déjà évoqué à propos d'Ancus Marcius. Elle est habituellement l'occasion de rapprocher Claude de Servius Tullius, ce royal prédécesseur réputé avoir étendu les limites de l'enceinte sacrée ${ }^{39}$. L'extension claudienne du pomerium intervint, si l'on en croit Tacite et sa datation consulaire, l'année qui suivit le discours de Claude au Sénat, soit en $49^{40}$. La mesure est attestée par des cippes retrouvés en divers points de Rome ${ }^{41}$; elle est également signalée

35 Concernant Servius Tullius/Mastarna, Claude expose, sans prendre parti, la tradition romaine, qui voyait en lui le fils d'une captive, élevé à la cour de Tarquin l'Ancien, et la leçon étrusque selon laquelle il s'agissait d'un prince originaire d'Étrurie, nommé Mastarna.

36 J.-M. David, 'Présentation', Mélanges de l'École Française de Rome, Moyen-Âge, Temps Modernes 92 (1980), p. 9. Nous remercions Monique Dondin-Payre de nous avoir indiqué cette référence.

37 Voir J. Devreker, 'Claude et le Sénat: signification et portée de l'oratio claudiana', in Y. Burnand, Y. Le Bohec, J.-P. Martin (eds.), Claude de Lyon empereur romain. Actes du Colloque Paris-Nancy-Lyon (novembre, 1992) (Paris 1998), 129-135.

38 Demougin 1994, op. cit. (n. 31), 20.

39 Chioffi 1992-1993, op. cit. (n. 4), 127-153, part. 147 sv.

40 Tac., Ann. 12, 23-24. A cette date, l'empereur n'exerçait plus la censure ; c'est donc en vertu de son imperium qu'il procéda à cette extension.

41 CIL VI, 1231a (ILS 213); CIL VI, 1231b-c. Le texte est le suivant: Ti(berius) Claudius / Drusi f(ilius) Caisar / Aug(ustus) Germanicus, / pont(ifex) max(imus), / censor, p(ater) p(atriae), / auctis populi Romani finibus pomerium / ampliauit terminauitq(ue). 
par Aulu Gelle ${ }^{42}$. La question de l'extension du pomerium sous l'Empire, de ses mobiles, de sa mise en œuvre dans l'espace urbain, est infiniment délicate et objet de nombreux débats sur lesquels il n'y a pas lieu de revenir ici en détail ${ }^{43}$. On retiendra seulement le fait, au demeurant bien connu, qu'en étendant le pomerium, Claude inclut dans l'Vrbs, au sens strict du terme, la colline du Pincio, une étroite bande de terre longeant le Tibre sur le Champ de Mars méridional, peut-être ${ }^{44}$, et l'Aventin ${ }^{45}$. Or, la symbolique qui s'attachait à cette colline était lourde. L'Aventin était, comme on l'a vu, la hauteur sur laquelle Ancus Marcius était réputé avoir installé les Latins qu'il venait de soumettre ; les transférant sur cette colline, il avait été mieux à même de les contrôler et de les utiliser; c'est là un point qui ne dut pas échapper à Claude. L'Aventin était plus encore la hauteur sur laquelle la plèbe s'était retranchée en 449 av. J.-C., où elle avait fait sécession et avait frondé les patriciens ${ }^{46}$. Enfin, c'était sur sa pente que se dressait le temple de Cérès, Liber, Libera, ces divinités qui garantissaient dans leur prouidentia le ravitaillement de la Ville et de sa population ${ }^{47}$. Or, c'est un point depuis toujours mis au crédit du règne de Claude que l'attention portée par ce prince à l'approvisionnement de la cité. Mais l'Aventin était aussi la colline dont C. Sempronius Gracchus,

42 Gell., 13, 14, 1.

43 E. Lyasse, 'Auctis finibus populi Romani? Les raisons de l'extension du pomerium sous le principat', Gerión 23 (2005), 169-187. Voir aussi M.T. Boatwright, 'Tacitus on Claudius and the pomerium, Annals, 12, 23.2-24', The Classical Journal 8o (1984), 36-44; J.P. Poe, 'The Secular Games, the Aventine, and the Pomerium in the Campus Martius', Classical Antiquity 3 (1984), 57-68 ; M.T. Boatwright, 'The Pomerial Extension of Augustus', Historia 35 (1986), 13-27 ; Chioffi 1992-1993, op. cit. (n. 4), 127-153 ; G. De Sanctis, 'Solco, muro, pomerio', Mélanges de l'École Française de Rome 119 (2007), 503-526.

44 Le désaccord sur ce point découle de ce que l'on considère ou non que le cippe claudien CIL VI, 31537d a été retrouvé in situ; voir sur cette question Andreussi 1988, op. cit. (n. 12), 233-234, qui établit que la pierre a bien été retrouvée à son emplacement originel et conclut logiquement à une intégration de cette petite partie du Champ de Mars dans le pomerium.

45 Merlin 1906, op. cit. (n. 11), 298-299; M. Labrousse, 'Le pomerium de la Rome impériale', Mélanges de l'École Française de Rome 54 (1937), 174-178; Poe 1984, op. cit. (n. 43), 57-68; Andreussi 1988, op. cit. (n. 12), 227-230 ; Lyasse 2005, op. cit. (n. 43), 170 et 179-181.

46 Sur cette colline, voir l'étude non encore renouvelée de Merlin 1906, op. cit. (n. 11), 266 sv.

47 Sur ce sanctuaire, voir F. Coarelli, 'Ceres, Liber, Liberaque, aedes ; aedes Cereris', Lexicon Topographicum Urbis Romae I (Rome 1993), 260-261. C'est aussi sur l'Aventin que se dressait le sanctuaire de Diane, divinité dont Seruius Tullius était réputé avoir introduit le culte à Rome, en le transférant d'Aricie ; sur ce temple, voir L. Venditelli, 'Diana Aventina, aedes', Lexicon Topographicum Urbis Romae II (Rome 1995), 11-13. Voir en outre K. Sandberg, 'Isis Capitolina and the pomerium. Notes on the augural topography of the Capitolium', Arctos 43 (2009), 149. 
champion de la plèbe, avait fait sa base, en 121 av. J.-C. 48 ; plus récemment encore, en 31 ap. J.-C., c'est sur l'Aventin que le peuple avait ratifié l'élection de Séjan au consulat, et non comme c'était la règle sur le Champ de Mars, où se réunissaient habituellement les comices centuriates ${ }^{49}$.

La mesure de Claude était donc politiquement et symboliquement importante : le prince pouvait se poser à son tour en champion de la plèbe et apparaître de ce point de vue comme «le dernier des populares ${ }^{50}$ ». Mais la mesure ne doit pas tromper. En intégrant l'Aventin dans le pomerium, l'empereur empêchait le peuple de s'y réunir désormais en armes, interdit pesant strictement sur la limite sacrée de la Ville. On retrouve ainsi une motivation qui fut peut-être celle de son royal prédécesseur, installant les Latins dans une Rome agrandie de l'Aventin: intégrer pour mieux contrôler. Le fait qu'Ancus Marcius ait installé les Latins sur l'Aventin et que Claude ait intégré cette colline à l'intérieur du pomerium est significatif; Claude poussa en quelque sorte à son terme l'initiative de son lointain prédécesseur. Ce dernier avait étendu les limites de la Ville ; Claude, pour sa part, prit l'initiative d'étendre le pomerium et d'agrandir ainsi le périmètre sacré de la Cités1.

La transformation du port d'Ostie, enfin, est un autre point important du règne de Claude. Les travaux titanesques engagés par l'empereur le furent, si l'on en croit Dion Cassius, au lendemain d'une disette survenue en $42^{52}$. Cet épisode, semble-t-il, détermina le prince à engager des travaux, qui aboutirent à la construction d'un nouveau bassin, de nombreux entrepôts ainsi que de canaux reliant le port au Tibre et permettant de réduire les effets désastreux des débordements récurrents du fleuve ${ }^{53}$. Claude devait

\footnotetext{
48 App., $B C_{1} 1,25$.

49 CIL VI, 10213 (= ILS 6044). Voir AE 2007, 214. Cf. R. Syme, 'Seianus on the Aventine', Hermes 84 (1956), 257-266.

50 Courtenay Edward Stevens cité par B. Levick, 'Claudius, Antiquarian or Revolutionnary', American Journal of Philology 99 (1978), 89 et Levick 2002, op. cit. (n. 32), 140.

51 De multiples raisons purent inciter Claude à étendre le pomerium et à y inclure l'Aventin; voir les références supra, n. 43.

52 Dio Cass., 6o, 11, $1-5$.

53 R. Chevallier, 'Les ports d'Ostie : pour une relecture des sources', in Ostia, port et porte de la Rome antique (Genève 2001) 20-29, avec la bibliographie antérieure; F. Zevi, 'Inquadramento storico relativo ai porti di Roma', in Le strutture dei porti e degli approdi antichi, (Rome 2004), 211-220 ; M. Millett, S. Keay, K. Strutt, 'Portus Romae : recent survey work at the ports of Claudius and Trajan', ibid., 221-231; S.J. Keay, M. Millett, L. Paroli, K. Strutt, Portus: An Archaeological Survey of the Port of Imperial Rome (Rome 2005), 271281 et 319-321 (sources littéraires).
} 
également perfectionner l'administration et la protection du port. L'œuvre accomplie par Claude à Ostie ne régla pas tous les problèmes, mais elle témoigne du réel souci que le prince prit du ravitaillement de Rome et de l'acheminement des denrées jusqu'au cœur de la capitale. Les réalisations effectuées à Ostie par Ancus Marcius et par Claude peuvent à bon droit être mises en regard: Ancus Marcius passe pour avoir été le fondateur du port ; ce faisant, il rendit réalisable le fabuleux destin promis à Rome : ayant désormais un accès à la mer, l'Vrbs put s'ouvrir sur l'extérieur et sur l'étranger; dans cette optique, Ancus Marcius est bien à l'origine de la domination de Rome en Méditerranée; tout du moins est-il regardé comme celui qui la rendit possible. Plusieurs siècles plus tard, alors que Rome était devenue caput mundi et qu'elle s'était rendue maîtresse de l'ensemble du bassin méditerranéen, Claude engagea d'énormes travaux à Ostie, afin d'accroître la capacité d'accueil du port et d'améliorer ainsi le ravitaillement et l'alimentation de la capitale.

\section{Les leçons de l'histoire ou le miroir royal}

Le parallèle qui vient d'être esquissé entre ces deux figures que sont Claude et Ancus Marcius, n'est pas, me semble-t-il, dénué de tout fondement et je me risquerai à suggérer que l'empereur ait pu lui-même et à dessein utiliser la figure d'Ancus Marcius et recourir à l'histoire pour justifier certaines de ses décisions. La preuve que Claude trouva dans le passé de Rome des arguments destinés à les conforter a déjà été apportée plus haut à propos de l'ouverture du Sénat aux Gaulois. Sur cette question, Claude s'est heurté à un fort parti conservateur, résolument hostile à l'ouverture et lui préférant le statu quo. Tacite ne cache pas, et la Table Claudienne le laisse aussi entendre, que les débats au Sénat ont été passionnés et même houleux. Il est manifeste que l'histoire est venue à la rescousse de l'empereur, l'essentiel des arguments mis en avant par Claude pour défendre sa position étant puisés dans le passé de Rome.

Nous n'avons que peu d'échos des débats que les deux autres sujets abordés - l'extension du pomerium et les travaux engagés à Ostie - ont suscités. Une extension du pomerium n'était pas une opération anodine, a fortiori lorsqu'il s'agissait d'y inclure l'Aventin, colline dont j'ai rappelé qu'elle était associée, dans la mémoire collective des Romains, aux sécessions de la plèbe. On peut supposer dans ces conditions que cette question suscita quelque opposition au sein du milieu sénatorial. Sénèque, 
si l'on en croit notamment A. Giardina ${ }^{54}$, pourrait d'ailleurs avoir glissé dans un bref passage du De breuitate uitae une allusion aux critiques formulées à l'encontre de la mesure que Claude avait prise ou s'apprêtait à prendre ${ }^{55}$. Ainsi ne serait-il pas surprenant que l'empereur ait de nouveau appelé l'histoire de Rome à l'aide afin d'emporter la décision, si ce n'est l'adhésion, de ses pairs du Sénat. C'est à un tel rappel historique que se livre Tacite dans les Annales ${ }^{56}$, en remontant jusqu'au pomerium romuléen et il n'est peut-être pas à exclure totalement qu'il ait repris certains des arguments mis en avant par Claude lui-même, une source dont il lui arriva par ailleurs de s'inspirer, comme a pu le montrer D. Briquel, dans son article, «Claude, érudit et empereur ${ }^{57}$.

Quant aux travaux engagés à Ostie, on doit considérer qu'ils ont également suscité nombre de débats au Sénat, qu'il se soit agi de discuter d'aspects techniques ou financiers, voire même de leur supposée nécessité. Dion Cassius le laisse entendre, lorsqu'il signale que « Claude, comprenant ces difficultés, entreprit de construire un port, sans se laisser détourner de son projet par les architectes, qui, lorsqu'il leur demanda à combien monterait la dépense, lui répondirent: "Tu ne le feras pas", tant ils espéraient, par la grandeur de la dépense, s'il en était informé à l'avance, le forcer de

54 Sen., breu. uitae 13, 8: Sed, ut illo reuertar unde decessi et in eadem materia ostendam superuacuam quorundam diligentiam, idem narrabat Metellum, uictis in Sicilia Poenis triumphantem, unum omnium Romanorum ante currum centum et uiginti captiuos elephantos duxisse ; Sullam ultimum Romanorum protulisse pomerium, quod numquam prouinciali sed Italico agro adquisito proferre moris apud antiquos fuit. Hoc scire magis prodest quam Auentinum montem extra pomerium esse, ut ille affirmabat, propter alteram ex duabus causis, aut quod plebs eo secessisset aut quod Remo auspicante illo loco aues non addixissent, alia deinceps innumerabilia quae aut farta sunt mendaciis aut similia? ; « mais pour revenir au point d'où je suis parti et montrer dans la même matière l'inutile minutie de certains : le même reconnaît que Metellus, dans le triomphe qu'il remporta après sa victoire sur les Carthaginois en Sicile, conduisit, seul de tous les Romains, devant son char, cent vingt éléphants capturés ; que Sylla, le dernier parmi les Romains, agrandit le pomérium, qui, dans l'ancienne coutume, n'était agrandi qu'après des conquêtes faites en Italie et jamais sur les provinces. Est-ce plus utile à savoir que la raison pour laquelle l'Aventin est en dehors du pomérium (c'est, affirmait ce docte personnage, ou parce que la plèbe s'y était retirée, ou parce que les auspices, quand Rémus les prit, n'ont pas été favorables) et une série de balivernes de ce genre qui est bourrée de mensonges ou en ont tout l'air? »; A. Giardina, 'Seneca, Claudio e il pomerio', in C. Nicolet (ed.), “Alla Signorina”. Mélanges offerts à Noëlle de La Blanchardière (Rome 1995), 123-140 ; cf. 137 : « La polemica dell'erudito senechiano si configura dunque come parte di una contesa sui rapporti tra Roma, l'Italia e l'impero aperta tra gli anni 48 e 49 dalle iniziative dell'imperatore Claudio. »

55 Cf. Lyasse 2005, op. cit. (n. 43), 171-172: « On aurait donc opposé territoire italien et territoire provincial pour contester au prince le droit de justifier l'extension du pomerium par la conquête de la Bretagne. »

56 Tac., Ann. 12, 24.

57 Briquel 1988, op. cit. (n. 4), 224-226. 
renoncer à son dessein ${ }^{58}$. Précédemment et par deux fois, Quintilien s'était directement fait l'écho des débats qui ne manquèrent pas d'agiter les Romains, et parmi eux sans doute les sénateurs : «Lorsqu'on délibéra sur la construction du port d'Ostie, l'orateur ne dut-il pas exposer ses vues? Et, pourtant, c'était affaire d'architectes ${ }^{59}$ »; «il arrivera souvent, en effet, que nous soutenions d'abord qu'une chose n'a pas à être faite, même si cela est possible, et ensuite qu'elle ne peut être faite. Dans un autre cas, quand on cherche, comme, par exemple, si l'on peut couper l'Isthme ou assécher les marais Pontins, ou construire un port à Ostie, ou si Alexandre va découvrir des terres au-delà de l'Océan, c'est un état de conjecture $^{60}$.»

Le choix d'engager ou non ces énormes travaux, celui du site, du plan, de la taille donc de la capacité du nouveau bassin, le chiffrage des travaux, la nature des revenus à leur consacrer, tous ces points furent d'autant plus discutés au sein de l'Assemblée qu'un certain nombre de sénateurs, par ailleurs propriétaires de villas suburbaines, pouvaient se trouver directement concernés par cette affaire ${ }^{61}$. On ignore malheureusement tout $\mathrm{du}$ détail des discussions auxquelles ces questions donnèrent lieu. Pour autant, on ne saurait exclure que, pour imposer ses choix, Claude ait une nouvelle fois eu recours à l'histoire, mettant en avant les conséquences, imprévisibles dans leur ampleur, de la fondation du port d'Ostie par Ancus Marcius. Sur ce sujet, l'empereur pouvait aisément se poser en successeur et continuateur du lointain roi de Rome ; transformant de fond en comble le port d'Ostie, il parachevait en quelque sorte l'œuvre initiée par son illustre prédécesseur.

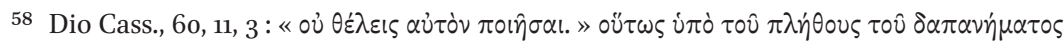

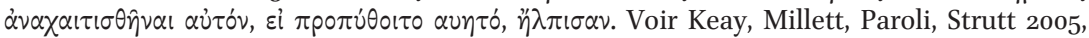
op. cit. (n. 53), 297.

59 Quint., 2, 21, 18 : Ergo cum de faciendo portu Ostiensi deliberatum est, non debuit dicere sententiam orator? Atqui opus erat ratione architectorum. La figure de Claude se cacheraitelle derrière l'orator évoqué par Quintilien et l'un de ses discours derrière la sententia?

60 Quint., 3, 8, 16 : Saepe enim accidet ut prius dicamus, ne se possit quidem fieri, esse faciendum, deinde, fieri non posse. Cum autem de hoc quaeritur, coniectura est: an Isthmos intercidi, an siccari palus Pomptina, an portus fieri Ostiae possit, an Alexander terras ultra Oceanum sit inuenturus.

61 On a notamment justifié le choix du site (au nord du Tibre) par le nombre de propriétés suburbaines situées au sud du fleuve et qui appartenaient à des aristocrates romains, certainement peu désireux d'être expropriés ; sur ce point, Keay, Millett, Paroli, Strutt 2005, op. cit. (n. 53), 299, reprenant les propos de M.G. Lauro et A. Claridge, 'Litus Laurentinum : carta archeologica della zona littoranea a Castelporziano', in M.G. Lauro (ed.), Castelporziano III : campagne di scavo e restauro 1987-91 (Rome 1998), 39-62. 
Dans tous les cas étudiés, on le voit, Claude mit ou fut en mesure de mettre en avant des arguments tirés de l'histoire de Rome et du règne $d^{\prime}$ Ancus Marcius plus particulièrement ${ }^{62}$. J'ai bien conscience du caractère audacieux de ma démarche et du danger qu'il y a à prêter à Claude des arguments dont on ignore, à l'exception significative de la question des Gaulois, s'il les a effectivement utilisés; du moins le parallèle établi entre ces deux figures de l'histoire romaine que sont Claude et Ancus Marcius me semble-t-il avoir une certaine pertinence.

Pour terminer, je souhaiterais montrer qu'au-delà de la figure d'Ancus Marcius, c'est vers l'ensemble de l'âge royal que Claude fut, à l'occasion, tenté de se tourner et dans les acta des bons rois du temps jadis qu'il alla chercher des arguments destinés à conforter ses décisions. Ce faisant, c'est à une intégration du passé royal dans le présent de Rome que le prince se livra, fidèle en cela à l'orientation suivie par Auguste, qui n'avait pas hésité à se présenter en nouveau conditor de $\mathrm{l}^{\prime} \mathrm{Vrbs}^{63}$. Il convient, à ce propos, de rappeler que Claude, empereur érudit et féru d'histoire ${ }^{64}$, s'était luimême lancé dans la rédaction, aussi bien en grec qu'en latin, de plusieurs ouvrages à caractère historique, l'un sur les Étrusques, un autre sur les Carthaginois, un troisième, encouragé dans cette entreprise par Tite Live, sur l'histoire de Rome depuis la mort de César ${ }^{65}$. L'examen des res gestae de Claude invite à conclure que ces travaux et l'érudition alors acquise

62 Il faut rappeler encore qu'Ancus Marcius est, si l'on en croit Tite Live $\left(1,32,5^{-14}\right)$, le roi qui emprunta aux Équicoles les rites de déclaration de guerre, pratiqués par les Fétiaux. Or, Suétone signale que Claude « fit alliance avec des rois dans le Forum, en immolant une truie, et en faisant lire par les fétiaux l'antique formule des serments » (Suet., Cl. 25, 5).

Sous son règne, d'autre part, quatre cippes, copies d'originaux plus anciens, pourraient avoir été mis en place à proximité de l'arc de Titus, au pied du Palatin. Ces quatre inscriptions, qui mentionnent respectivement Anabestas (CIL I2 , 969), Marspiter (CIL I $\left.{ }^{2}, 970\right)$, Remureine $\left(C I L \mathrm{I}^{2}, 971\right)$ et Fertor Resius, rex Aequeicolus (CIL I ${ }^{2}, 202=$ InscrIt. XIII, 3, $\left.\mathrm{n}^{\circ} 66\right)$, pourraient appartenir à un monumentum augustéen, destiné à commémorer la transgression de Rémus, punie par Romulus en vertu du droit fétial. La quatrième inscription dit ensuite : Is preimus ius fetiale parauit : / inde p(opulus) $R$ (omanus) / discipleinam excepit. Le goût d'antiquaire de Claude, développé dans la ligne d'Auguste, n'en est que plus évident. Là encore, on le voit, c'est le passé royal qui fut mis en valeur. Voir M. Ver Eecke, 'De l'Aventin au Palatin : le nouvel ancrage topographique de Rémus au moment du passage de la République à l'Empire', Dialogues d'Histoire Ancienne 32 (2006), 85-86.

63 Martin, 1994, op. cit. (n. 5), 411-418.

64 Momigliano 1961, op. cit. (n. 3), particulièrement le premier chapitre, "The Man of Learning", 1-19; Briquel 1988, op. cit. (n. 4), 217-232 ; Levick 2002, op. cit. (n. 32), 30 ; P.L. Schmidt, 'Claudius als Schriftsteller', in AA. VV. Die Regierungszeit 1994, op. cit. (n. 3), 119-131 ; J. Dion, 'Les publications littéraires sous le principat de Claude', in Burnand, Le Bohec, Martin 1998, op. cit. (n. 37), 69-73.

65 Suet., $\mathrm{Cl}$. 41 et 42,5 . C'est de cette histoire que Tacite pourrait s'être inspiré, si l'on en croit Briquel 1988, op. cit. (n. 4), 226-227. 
par ce prince antiquaire nourrirent les débats qui l'opposèrent en diverses circonstances aux membres de la Haute Assemblée, voire fondèrent certaines de ses décisions. J'ai plus haut mis l'accent sur la personne d'Ancus Marcius, mais l'analyse des sources invite à conclure que c'est également à d'autres figures de la Royauté que l'empereur fit à l'occasion référence. La question de l'accès des Gaulois au Sénat l'a déjà prouvé : dans le cadre de son discours, Claude cita, en effet, outre Ancus Marcius, Romulus, Numa, Tarquin l'Ancien et Servius Tullius, dont on a vu qu'il présenta, au sujet de son origine, le status quaestionis. Quelques décennies plus tard, d'ailleurs, Tacite devait, à propos de la légende de Caeles Vibenna et de son compagnon Mastarna, utiliser, non le discours de Claude, mais son histoire romaine, ainsi que $\mathrm{D}$. Briquel a cru pouvoir le démontrer.

La question du pomerium et de l'intégration de l'Aventin invite à rapprocher Claude, non seulement de la figure d'Ancus Marcius, mais également de celle de Servius Tullius, tout comme invite aussi à le faire l'intérêt porté par l'empereur à l'étrusculogie ${ }^{66}$. Concernant le pomerium, c'est en premier lieu à Tite Live qu'on doit de savoir qu'il fut agrandi par Servius Tullius, une information que l'historien glana à n'en pas douter chez un « antiquaire ${ }^{67}$, et dont Claude eut assurément connaissance ${ }^{68}$. En procédant à son tour à une extension de l'enceinte sacrée de l'Vrbs, l'empereur ne pouvait pas ne pas avoir en tête le souvenir du roi étrusque. Ainsi, cette opération majeure le renvoyait-il immanquablement à la période royale de l'histoire de Rome, qui lui offrait opportunément deux précédents, outre celui de Romulus, le premier à avoir tracé le sillon sacré : celui d'Ancus Marcius, accueillant les Latins vaincus sur l'Aventin, et celui de Servius Tullius, alias Mastarna, auteur d'un élargissement du pomerium. Tacite devait d'ailleurs présenter l'extension de Claude comme la reprise d'un « usage ancien » (more prisco), dont E. Lyasse a bien montré, il y a quelques années, qu'il avait fait l'objet de diverses interprétations ${ }^{69}$.

Sur un autre point, non évoqué jusqu'à présent, l'intérêt porté par Claude à la période de la Royauté est patent: il s'agit des rites des Fétiaux qu'il

66 Voir sur ce point l'étude de J. Heurgon, 'La vocation étruscologique de l'empereur Claude', Compte rendus de l'Académie des Inscriptions et Belles Lettres (1953), 92-97 = Scripta Varia (Bruxelles 1986), 427-432. On rappellera, à ce propos, que la première femme de Claude, Vrgunilla, appartenait à une famille originaire d'Étrurie. J. Heurgon en conclut que «c'était tout le monde étrusque qui lui était offert en dot» (p. 96).

67 Liu., 1, 44, 3-5.

68 Voir en outre Tac., Ann. 12, 23, 2-24; Gell., 13, 14.

69 Lyasse 2005, op. cit. (n. 43), 174 sv. 
remit à l'honneur à l'occasion de la conclusion de traités ${ }^{70}$. Or, la tradition annalistique attribue aux trois rois «nationaux », Numa Pompilius, Tullus Hostilius et Ancus Marcius, un rôle majeur dans la définition et l'élaboration du rituel fétial, à Numa revenant l'initiative d'avoir instauré le collège des Fétiaux et leur fonction de médiateurs diplomatiques, à ses successeurs celle d'avoir fait des Fétiaux les garants de la validité religieuse des déclarations de guerre et des traités ${ }^{71}$. Les travaux que Claude avait consacrés aux Étrusques ne l'empêchaient donc pas, on le voit, de marquer son attachement à des traditions - ici religieuses - qui avaient pour origine les rois non étrusques de Rome. En revanche, le goût de l'empereur pour l'étruscologie et ses liens avec l'Étrurie ne sont pas étrangers à la proposition qu'il présenta aux sénateurs - et qui resta apparemment sans suite - de réunir les haruspices en collège, afin de renforcer leur position et leur rôle au sein de la religion publique ${ }^{72}$.

Sur un ultime point, enfin, il est possible d'ancrer la politique claudienne dans le passé royal de Rome : il s'agit des célébrations $\mathrm{du} 800^{\mathrm{e}}$ anniversaire de la fondation de l'Vrbs, que l'empereur présida en 47 ap. J.-C. et qu'il présenta comme de nouveaux jeux séculaires, fidèles au modèle augustéen. Suétone, dans un passage bien connu, rappelle que cette initiative valut au crieur public, si ce n'est au souverain lui-même, de s'attirer les moqueries de la foule, puisque, contrairement à ce qu'impliquait le délai habituel de cent ou cent dix ans, des Romains, ayant participé aux jeux augustéens de 17 av. J.-C., étaient toujours en vie soixante-trois ans plus tard ${ }^{73}$.

Comme l'a suggéré $\mathrm{S}$. Benoist, «l'historien Claude n'a-t-il pas tenté de réconcilier célébration cyclique des temps et histoire linéaire de Rome depuis la fondation de la cité, et ainsi de rattacher symboliquement Romulus, Auguste et sa descendance, achevant ainsi, métaphoriquement, la légende troyenne que sa famille incarnait? $»^{74}$. Au-delà du respect

\footnotetext{
70 Suet., $\mathrm{Cl}$. 25, 14: Cum regibus foedus in foro icit porca caesa ac uetere fetialium praefatione adhibita.

71 Sur les Fétiaux, voir en dernier lieu l'étude de C. Auliard, 'Les Fétiaux, un collège religieux au service du droit sacré international ou de la politique extérieure romaine', in M.-M. Mactoux et E. Geny (eds.), Mélanges P. Lévêque, VI, Religion (Paris 1992), 1-16.

72 Tac., Ann. 11, 15. Cf. M.-L. Haack, Les haruspices dans le monde romain (Bordeaux 2003), 93-98; l'auteur souligne l'attention particulière portée par Claude aux signes divins.

${ }^{73}$ Suet., $\mathrm{Cl}$. 21, 5 .

74 S. Benoist, La Fête à Rome au premier siècle de l'Empire. Recherches sur l'univers festif sous les règnes d'Auguste et des Julio-Claudiens (Bruxelles 1999), 191. Voir en outre S. Benoist, Rome, le prince et la Cité (Paris 2005), 288-290.
} 
de la tradition augustéenne, c'est bien au retour et à la mise en valeur des origines de la Cité que Claude se livra, deux ans avant de procéder à l'extension du pomerium. L'originalité de son initiative n'allait pas échapper à Domitien, qui ne devait pas hésiter, lorsque son heure serait venue, à rayer de la liste des ludi saeculares les célébrations claudiennes et à rattacher directement celles advenues sous son propre règne aux jeux d'Auguste $^{75}$ : «le grand cycle écarte donc les jubilés de Rome » ${ }^{76}$, avec lesquels les jeux de Claude avaient été à dessein identifiés. Sur ce sujet, on le voit, la politique impériale s'était montrée tout à la fois novatrice et respectueuse de la tradition; désireux d'honorer les origines de la Cité tout en se plaçant dans la ligne augustéenne, l'empereur « choisit ... une nouvelle origine à des rites qu'il inscrit néanmoins dans la continuité »77, ouvrant ainsi la voie aux célébrations jubilaires des siècles suivants ${ }^{78}$.

Le goût développé de Claude pour l'histoire et pour le passé de Rome l'incita donc, tout au long de son règne, à se tourner vers les périodes reculées de l'histoire de la Cité et à se référer à plusieurs figures de l'âge royal, y trouvant utilement des précédents et autant d'exempla susceptibles de le conforter dans des positions qu'il eut souvent de la peine à faire admettre. L'intérêt porté par le souverain aux Étrusques, et plus largement à l'histoire, le conduisit ainsi à puiser dans ce passé, de moins en moins sulfureux, afin d'y trouver des arguments destinés à servir ses options politiques. Auguste ayant «asséché les sources profondes du "complexe" que nourrissaient depuis cinq siècles les Romains à l'égard de la Royauté »79, Claude pouvait dès lors s'engouffrer dans la brèche, pour s'inspirer, invoquer et revendiquer, sans risque de choquer, le précédent royal. Ce faisant, il contribua à alimenter le mouvement qui permit qu'après avoir aboli la royauté, Rome retournât à une forme de monarchie.

75 Suet., Dom. 4, 7 : Fecit et ludos saeculares, computata ratione temporum ad annum non quo Claudius proxime, sed quo olim Augustus ediderat ; «il célébra aussi les jeux séculaires, en se reportant pour le compte des années non pas aux derniers, qui dataient de Claude, mais à ceux qu'avait autrefois donnés Auguste »; cf. aussi Tac., Ann. 11, 11.

76 Benoist 1999, op. cit. (n. 73), 177, n. 70.

77 Benoist 2005, op. cit. (n. 73), 289.

78 L'intérêt de Claude pour les débuts de Rome explique encore que l'empereur ait accordé une exemption fiscale aux Troyens ; cf. Suet., Cl. 25, 10 : Iliensibus quasi Romanae gentis auctoribus tributa in perpetuum remisit recitata uetere epistula Graeca senatus populique $R$. Seleuco regi amicitiam et societatem ita demum pollicentis, si consanguineos suos Ilienses ab omni onere immunes praestitisset.

79 Martin 1994, op. cit. (n. 5), 417. 
Quoiqu'attaché aux origines de l'Vrbs et à ses traditions, Claude ne fut en rien un prince archaïque et passéiste ; il apparaît même à l'historien contemporain, confronté comme ses semblables aux questions d'intégration et d'assimilation de l'étranger, d'extension territoriale ou encore de mise en valeur du passé, d'une singulière modernité. 


\title{
DAS LEBEN RÖMISCH GESTALTEN. EIN STADTGESETZ FÜR DAS MUNICIPIUM TROESMIS AUS DEN JAHREN 177-180 N. CHR. ${ }^{1}$
}

\author{
Werner Eck
}

Dass Stationierungsorte der römischen Armee, seien es die Lager von Legionen, seien es die Kastelle von Auxiliareinheiten, den Anstoß zur Entstehung von zivilen Siedlungen unterschiedlichster Art gegeben haben, ist eine in allen Provinzen wohl bekannte Tatsache. ${ }^{2}$ Der Typ von Siedlung reicht dabei von den canabae einzelner Einheiten über mehr oder weniger große vici in der etwas weiteren Umgebung bis zu römisch organisierten Städten munizipalen oder kolonialen Charakters. Stets ist dabei zu beobachten, dass die Organisationsformen römisch geprägt sind. In canabae erscheinen z.B. neben magistri auch aediles, wie man sie etwa im niedermösischen Troesmis nachweisen kann, ebenso auch ein ordo als "Gemeinderat". 3 In municipia und coloniae sind

${ }^{1}$ Der von mir für das Kolloquium in Lille angekündigte Beitrag konnte aus persönlichen Gründen damals weder geschrieben noch dort vorgetragen werden. Auf Bitten der Herausgeber des Bandes wird hier in einer Art Kurzform das Wichtigste zu dem neuen Fragment eines Stadtgesetzes zusammengefasst. Es muss jedoch darauf verzichtet werden, den gesamten Text der beiden Bronzetafeln hier vorzulegen, da dafür ein detaillierter Kommentar erforderlich wäre, der erst später an anderer Stelle nachgeholt werden kann. Ebenso ist es mir auch nicht möglich, in diesem Beitrag näher auf die wissenschaftliche Literatur einzugehen, da dies wohl den Rahmen sprengen würde. Für den Text der spanischen Stadtgesetze sei auf folgende Editionen verwiesen: FIRA I2 , 21, 23, 24; Th. Spitzl, Lex municipii Malacitani (München 1984); J. González, 'The Lex Irnitana', JRS 76 (1986), 147 ff.: F. Lamberti, Tabulae Irnitanae (Neapel 1993); A. Caballos Rufino, El nuevo bronce de Osuna y la política colonizadora romana (Sevilla 2006); J.G. Wolf, Die Lex Irnitana. Ein römisches Stadtrecht aus Spanien (Darmstadt 2011). Allgemein zu den Stadtgesetzen siehe den Sammelband: L. Capogrossi Colognesi, E. Gabba (eds.), Gli Statuti Municipali (Pavia 20o6); darin M. Humbert, 'Municeps et Municipium: définition et histoire', 3-30; H. Galsterer, 'Die römischen Stadtgesetze', 31-56.

${ }^{2}$ Siehe z.B. Fr. Vittinghoff, 'Die Bedeutung der Legionslager für die Entstehung der römischen Städte an der Donau und in Dakien', in Id., Civitas Romana. Stadt und politisch-soziale Integration im Imperium Romanum der Kaiserzeit hg. W. Eck (Stuttgart 1994), 89-105; Id., 'Die Entstehung von städtischen Gemeinwesen in der Nachbarschaft römischer Legionslager - Ein Vergleich Leóns mit den Entwicklungslinien im Imperium Romanum', ibid., 106-123; Id., 'Die rechtliche Stellung der canabae legionis und die Herkunftsangabe castris', ibid., 140-159; Id., 'Zur römischen Municipalisierung des lateinischen Donau-Balkanraumes. Methodische Bemerkungen', ibid., 16o-209.

${ }^{3}$ CIL III, 6162 = Inscr. Scyth. Minoris V, 156; CIL III, $6166=$ ILS $2474=$ Inscr. Scyth. Minoris V, 154; AE 1957, 266 = Inscr. Scyth. Minoris V, 155; AE 1960, 337 = Inscr. Scyth. Minoris V, 158. Auch Inscr. Scyth. Minoris V, 143-145 bezieht sich auf den ordo der canabenses. 
notwendigerweise die internen Strukturen nach dem römischen Städtemodel gestaltet worden - so muss man dies auf jeden Fall auf Grund der Benennungen, die überall für Magistrate und Institutionen verwendet wurden, schließen. Wie weitgehend freilich die innere Ausgestaltung der Gemeinden durch das römische Modell geprägt war, ließ sich außerhalb der iberischen Halbinsel im Detail kaum erkennen, da uns dafür ausführlichere und direkte, auf einzelne Städte oder Siedlungen bezogene Quellen fehlen.

Ein Inschriftenfund aus der römischen Provinz Moesia inferior, und zwar aus der Stadt Troesmis, lässt hier einen bisher unbekannten, höchst interessanten und recht tief gehenden Einblick zu.

In Troesmis hatte bis zur Regierungszeit Marc Aurels das Lager der legio $V$ Macedonica gelegen; doch sie wurde abgezogen, vielleicht zunächst zur Teilnahme am Partherkrieg. Von dort kehrte sie aber nicht mehr nach Troesmis zurück, sie wurde vielmehr in die Provinz Dacia verlegt, wo sie noch unter Marc Aurel in Potaissa ihr neues Lager bezog. ${ }^{4}$ Ihre früheren castra in Troesmis mit all den umliegenden Versorgungseinheiten waren damit für andere Nutzung frei. Dort entstand die neue, im römischen Rechtssinn organisierte Stadt Troesmis. ${ }^{5}$ Wie weit dabei vielleicht die canabae legionis, die, wie sich aus den Inschriften ergibt, bereits in einer gemeindeartigen Form für die Angelegenheiten ihrer Bewohner gesorgt hatten, oder ein nahegelegener vicus in das neue Gemeinwesen einbezogen wurden, könnte vermutlich die Archäologie klären. Nicht wenige Inschriften sind aus der städtischen Phase des Ortes bekannt; sie lassen eine dem Üblichen entsprechende römische Gemeindestruktur vermuten. Diese ist mit Hilfe des neuen Dokuments nunmehr in einigen Details in bisher unbekannter Klarheit zu erkennen.

Vor mehr als einem halben Jahrzehnt wurde auf dem Antiquitätenmarkt in England die Abschrift einer Inschrift bekannt. Dieser Text stand, wie sich bald danach herausstellte, auf zwei zusammengehörigen Bronzetafeln. Denn für kurze Zeit wurden zwei Bronzetafeln, auf denen man diesen Text in Ansätzen erkennen konnte, im Internet zum Kauf angeboten; ${ }^{6}$

4 Dazu I. Piso, 'Legiones Daciae', in Y. Le Bohec (ed.), Les Légions romaines sous le Haut-Empire, Actes du Congrès de Lyon (17-19 septembre 1998) (Lyon 2000), 204-225, bes. $213-215$.

$5 \mathrm{Zu}$ den canabae dieser Legion siehe ausführlicher Vittinghoff 1995, a.a.O. (Anm.2) $111 \mathrm{f} ., 164 \mathrm{f}$.

6 eBay Inscribed bronze plaque, city law, 2nd c_A_D_ (item 7369084492 and item 8356609348, end time Nov-30-05 073649 PST).htm. Der Preis sollte 48.000 Britische Pfund = rund 82.00o Dollar pro Stück betragen. 
doch verschwanden sie schnell wieder, vermutlich als diejenigen, die die Stücke in Händen hatten und verkaufen wollten, realisierten, dass deren Herkunftsland, nämlich Rumänien, eindeutig durch Teile des Textes selbst zu bestimmen war. Denn große Fragmente oder wie hier zweier fast vollständiger Tafeln eines Stadtgesetzes auf Bronze können mit allergrößter Wahrscheinlichkeit nur aus der genannten Stadt selbst stammen. Später sollen die Tafeln nochmals in London und Bath aufgetaucht sein.

Die Maße sowie die Gewichtsangabe für die beiden Bronzetafeln folgen den Angaben, die bei der Präsentation der Tafeln im Internet zu sehen waren. Die Tafeln repräsentieren zwei Ausschnitte aus einem Stadtgesetz; sie werden im Folgenden mit den Buchstaben A und B bezeichnet.

Tafel A: $67 \mathrm{~cm}$ hoch, $54 \mathrm{~cm}$ breit. Die Dicke der Tafel ist nicht bekannt; doch dürfte sie bei ungefähr 6,5 mm liegen, da das Gewicht der Tafel 21 $\mathrm{kg}$ beträgt; bei einer spezifischen Dichte von Bronze von etwa 8.97 ergibt das die errechnete Dicke. An den Rändern sind kleine Teile abgebrochen, was jedoch nicht zu einem Textverlust geführt hat.

Tafel B: $59 \mathrm{~cm}$ hoch, ${ }^{8} 50 \mathrm{~cm}$ breit; die Dicke dürfte bei ungefähr 6,9 mm liegen, da das Gewicht der Tafel $18 \mathrm{~kg}$ betragen soll. Ein Teil der rechten unteren Ecke ist abgebrochen, wodurch bei den letzten Zeilen ein kleiner Teil des Textes verloren ist.

Jede Tafel war von einem Rahmen eingefasst, der verloren ist, der jedoch auf den Tafeln ringsum seine deutlichen Spuren hinterlassen hat. Zudem lassen einzelne Löcher an den Rändern und ein an Tafel A noch erhaltener Nagel oder eine Art Niete erkennen, wie die Rahmen angebracht waren.

Da der Text auf beiden Tafeln in sehr klaren Buchstaben eingraviert ist, sind die Buchstaben fast überall ohne größere Probleme zu lesen. Lediglich am Ende von Tafel B ist die Entzifferung einiger Zeilen erschwert bzw. fast unmöglich, weil offensichtlich dieser Teil lange den Einflüssen von Wasser und Erdreich ausgesetzt war, wodurch die Oberfläche schwer beeinträchtigt wurde. Ob diese Zeilen durch sachgemäße Restaurierung wieder lesbar würden, muss offen bleiben. Derjenige oder diejenigen, die die Tafeln gravierten bzw. den Text mit Farbe auf den Tafeln vorschrieben,

\footnotetext{
7 Es ist klar, dass das genaue spezifische Gewicht von der Legierung des Metalls abhängt. So groß, dass dadurch die Berechnung der Dicke wesentlich beeinflusst würde, dürfte die Variationsbreite aber nicht sein.

8 Dass die beiden Tafeln nicht gleich hoch, aber fast gleich breit waren, lässt sich auf einem der zugänglichen Photos sehr deutlich erkennen.
} 
waren offensichtlich nicht besonders geübt in diesem Metier; das lassen zumindest die nicht gerade wenigen Schreibfehler vermuten.

Tafel A: Zeile 1, die ausgerückt ist, beginnt mit einem neuen Kapitel, was durch $k X I=k$ (aput) $X I$ angezeigt ist; darauf folgt die Überschrift dieses Kapitels:

\section{DE LEGATIS MITTENDIS EXCVSATIONIḄ OMNIBVSQ ACCIPIENDIS.}

Dabei ist eine Art Dittographie eingetreten, da ONIḄ nochmals als OMNIB wiederholt wurde. Vermutlich geriet der Fehler in den Text, als dieser von der Vorlage (zunächst mit Farbe) auf die Bronzetafel geschrieben wurde. Tatsächlich muss der Text lauten:

de legatis mittendis excusationib\{omnib\}usq(e) accipiendis,

so wie er auch in der lex Irnitana am Anfang von Kapitel 45 erscheint. Der Text auf dieser Tafel umfasst 28 Zeilen.

Tafel B: Sie beginnt in der ersten Zeile mitten in einem Satz. Nach 18 Zeilen setzt, nur durch einen sehr kleinen Zwischenraum vom Vorausgehenden getrennt, ein neues Kapitel ein. Auch hier ist der Beginn des neuen Kapitels zusätzlich dadurch markiert, dass die Zeile ausgerückt ist:

K XXVIII DE MVNICIBVS AD SVFFRAGIVM VOCANDIS CVSTODI BVSQVE TERNIS PONENDIS AD SINGVLAS CISTAS QVAE SVFFRAGIORVM CAVSA POSITE ERVNT =

k(aput) XXVIII: de munici<pi>bus ad suffragium uocandis custodibusque ternis ponendis ad singulas cistas quae suffragiorum causa posit<a>e erunt.

Der Text auf dieser Tafel ist, obwohl die Höhe um acht Zentimeter geringer ist als Tafel A, um zwei Zeilen länger; er umfasst insgesamt 30 Zeilen.

Diese wenigen Hinweise machen es klar, dass wir hier Teile eines Munizipalgesetzes vor uns haben, wie wir sie schon in großer Zahl aus der iberischen Provinz Baetica kennen. Diese Texte und das neue Stadtgesetz unterscheiden sich in manchen äußeren Details. So sind zwar in der Lex Ursonensis die Kapitel wie auch hier mit Ziffern markiert, dort jedoch ohne weitere Bezeichnung mit $k$ (aput) oder $r$ (ubrica) und auch ohne darauf folgende Überschrift. In den erhaltenen Exemplaren des flavischen Munizipalgesetzes werden die einzelnen Kapitel mit r(ubrica) eingeleitet, worauf nur die Überschrift folgt; die Kapitel selbst sind nicht durch eine Ziffer benannt. In allen Stadtgesetzen wie auch in diesem Fall wird jedoch ein neuer Paragraph mit einer neuen Zeile begonnen, so dass der Inhalt der Tafeln auch eine äußerlich sichtbare Struktur aufweist. 
Dieses neue Munizipalgesetz ist für die Stadt Troesmis ausgestellt worden. Das zeigt der Name der Stadt, der an insgesamt drei Stellen erscheint:

A 2f.: munic(ipii) M(arci) Aureli Antonini Aug(usti) Troesmens(ium).

B 15f.: municipi(i) M(arci) Aureli Antonini et L(uci) Aureli Commodi Aug(usti) Troesm(ensium).

B 25f.: municipi(i) M(arci) Aureli Antonini et [L(uci)] Aureli Commodi Aug(usti) Troesm(ensium).

Der Name der Stadt war bisher nur als municipium Troesmensium bekannt, ohne weiteren Beinamen. Der volle Name aber lautet, wie sich nun zeigt: municipium Marcum Aurelium Antoninum et Lucium Aurelium Commodum Augustum Troesmensium. In der nicht gerade armen epigraphischen Überlieferung der Stadt findet sich von diesen zahlreichen Beinamen nichts. Es wird stets nur kurz vom municipium Troesmensium gesprochen. ${ }^{9}$ Angesichts dessen, was wir nun von dem Namen wissen, ist das vielleicht nicht so sehr verwunderlich; denn der neue Name war außergewöhnlich lang und umständlich. So hat man wohl, und zwar sogar in den offiziellen Inschriften der Stadt, auf die vielen Beinamen völlig verzichtet, zumindest so weit dies die bisher bekannten Zeugnisse erkennen lassen.

Nach der ersten Erwähnung des Namens in A 2f. könnte man annehmen, das municpium sei eine Gründung von Marc Aurel alleine; doch muss es sich an dieser Stelle wohl um eine Verkürzung des Textes handeln, da der Name der Stadt nicht unterschiedlich geheißen haben kann. Das Municipium erhielt, nach dem Namen zu schließen, unter Marc Aurel und seinem Sohn Commodus das hier in Teilen greifbare Gesetz für das neue Gemeinwesen und sollte damit auch unter beiden gegründet worden sein.

Die beiden Tafeln enthalten den Text von zweieinhalb Kapiteln der für das Municipium erlassenen lex. Auf Tafel A steht der Text von $k$ (aput) XI, das sich mit der Wahl von städtischen Gesandten befasst, ein Sachverhalt, der auch in der lex Irnitana erscheint, dort in rubrica 45. Von diesem Kapitel steht auf der Bronzetafel nur ein Teil; das zeigen der erhaltene

${ }^{9}$ CIL III $6172=$ Inscr. Scyth. Minoris V 152; CIL III $6173=$ Inscr. Scyth. Minoris V 153; CIL III 6177 = Inscr. Scyth. Minoris V 150; CIL III 6188 = Inscr. Scyth. Minoris V 183; CIL III $6199=12481=$ Inscr. Scyth. Minoris V 149; CIL III $6235=7599=$ Inscr. Scyth. Minoris V 163; CIL III $7504=$ ILS $7184=$ Inscr. Scyth. Minoris V 148; CIL III $7509=$ Inscr. Scyth. Minoris V 166; CIL III 7560 = Inscr. Scyth. Minoris V 244; AE 1980, 820 = Inscr. Scyth. Minoris $\mathrm{V} 164$. 
Text selbst und noch mehr ein Vergleich mit der lex Irnitana. Der zweite Teil des Kapitels, nämlich die in der Überschrift angeführten Excusationsgründe, stand erst auf der nächsten Tafel, die aber verloren ist. Partiell ist der Text mit dem in Irni wortgleich, doch sind auch viele Partien deutlich unterschieden, was vor allem durch die zusätzlichen Detailregelungen geschieht, die hier eingefügt sind. Worauf das vermutlich zurückzuführen ist, muss an anderer Stelle erörtert werden.

Tafel B enthält den Schluss von [k(aput) XXVII] und den Anfang von k(aput) XXVIII. Kaput XXVII handelt, soweit der erhaltene Teil das erkennen lässt, offensichtlich von Zulassungsvoraussetzungen bei Wahlen. Der Text, dessen Anfang freilich fehlt, lautet nach der Abschrift von der Tafel folgendermaßen:

[ - cuius] patri auoue paterno proauoue paterni aui patri, cuius in potestate est, minor $<r>e s$ si $\{n\} t^{10}$ quam ut eum ad legendum numero dec(urionum) conscriptorumue esse inue eum numer(um) legi oporteat. eum, qui sacerdotium petet, quot minor ann(orum) XXXV est, rationem annorum habendam, quae utiq(ue) legis Iuliae de maritandis ordinibus lata<e> kap(ite) VI cauta conprehensaque sunt, ${ }^{11}$ quae utiq(ue) commentari, ex quo lex P(apia) P(opaea) lata est, propositi Cn(aeo) Cinna Magno Vol(eso) Val(erio) Caeso co(n)s(ulibus) IIII kal(endas) Iulias kap(ite) XLVIIII cauta conprehensaque sunt et confirmata legis P(apiae) P(opaeae) k(apite) XLIIII, conseruanda, qui quaeq(ue) comitia habebit, curato. qui aliter quam hac lege licebit creatus erit, is neque annu<u>s IIuir neque q(uin)q(uennalis) neque aedilis neque quaestor neque sacerdos esto. quique eorum quem scies d(olo) m(alo) creauerit is singulas res $s$ (upra) s(criptas) ${ }^{12} X^{13}$ (milia) $n$ (ummum) munici<pi>bus municipi $M$ (arci) Aureli Antonini et L(uci) Aureli Commodi Aug(usti) Troesm(ensium) d(are) $d$ (amnas) esto eiusque pecuniae deque ea pecunia munic $<$ pi $>$ ibus municipi eius, qui uolet cuique per hac lege licebit, actio petitio persecutio esto.

Der erste noch vorhandene Rest der Regelung von Kapitel 27 ist nicht so recht klar; doch geht es sicher um die Qualifikation einer Person, eine Qualifikation, die auch schon beim Vater, Großvater und Urgroßvater

10 Hinweis von Dario Mantovani.

11 Zu dem Ausdruck vgl. CIL V $7637=I L S 5065$ (Pollentia): [ - ] diuisiones reliq[u]am consentiente pleb(e) in munus gladiat[o]rium [e]t saepta lignea inpendere ita ut dedicat [i]one statuae Imp (eratoris) Antonini Aug(usti) Pii p(atris) p (atriae) edi[t]io inchoetur et eodem die omnibus ann [i]s celebretur, dum ea quae legibus plebisue scitis senatusque consultis cauta comprehensaque sunt, seruentur.

12 SS ist jeweils durchgestrichen, womit die Abkürzung gekennzeichnet ist. Die beiden Buchstaben stehen wohl eher für s(upra) s(criptas) und nicht für sestertium. Allerdings ist bisher unklar, worauf sich singulas res s(upra) s(criptas) beziehen soll.

$13 \mathrm{X}$ ist überstrichen, wodurch die Tausenderqualität bezeichnet ist. 
väterlichereits gegeben gewesen sein muss. ${ }^{14}$ Darauf folgt jedenfalls eine Altersregel für jemanden, der sich um ein Priesteramt bewirbt. Jeder, der eine Wahl leite, müsse, wenn jemand (der sich um ein Priesteramt bewerbe) jünger als 35 Jahre sei, dafür sorgen, dass die ratio annorum beachtet werden müsse, wie sie in $k a p(u t) V I$ der lex Iulia de maritandis ordinibus vorgesehen sei; und ebenso die Regeln, die in kap (ut) XLVIIII des commentarius, der unter den Konsuln Cn. Cinna Magnus und Volesus Valerius Caesus (= 5 n. Chr.) am 28. Juni (IIII kal(endas) Iulias) vorgelegt wurde, vorgesehen seien und durch kap (ut) XLIIII der lex Papia Poppaea, die auf Grund dieses commentarius bestätigt wurden. Wer anders, als dieses Gesetz erlaube, gewählt worden sei, der könne weder ein jährlicher IIuir noch quinquennalis noch aedilis noch quaestor noch sacerdos sein; dabei wird durch die Hinzufügung von $a n n u<u>s$ überflüssigerweise betont, dass diese Ämter Jahresämter seien. ${ }^{15}$ Wer dennoch in böser Absicht eine solche Wahl durchführe, der solle den Bürgern von Troesmis 10.00o Sesterzen Strafe bezahlen. Und diese Summe könne jeder Bürger von Troesmis einklagen.

Soweit ersichtlich gibt es in den bisher bekannten Stadtgesetzen keine Passage, die inhaltlich dem entspräche, was hier vorgesehen ist, obwohl natürlich Altersbeschränkungen für bestimmte Funktionen bekannt sind. In der tabula Heracleensis ist das 30. Lebensjahr für alle Magistraturen als Mindestalter vorgesehen, es sei denn ein Bewerber habe bestimmte Leistungen im Heer erbracht. ${ }^{16}$ In der lex Malacitana ist dafür das 25 . Lebensjahr festgeschrieben. ${ }^{17}$ Doch speziell für ein Priesteramt kennt man bisher in der römischen Welt keinen entsprechenden Hinweis, weder während

14 Eine fast gleichlautende Formulierung findet sich in rubrica 86 der Lex Irnitana; dort geht es um die Bestimmung von Richtern, wobei die Vorfahren eines Richters über ein bestimmtes Vermögen verfügt haben müssen. Ein solcher Hinweis könnte auch hier vorliegen; allerdings ist dann der Text so wie oben zu verändern. Ob dann hier die Summe ausgefallen ist oder damit nur auf die schon vorher erwähnte Summe verwiesen wird, muss allerdings offen bleiben.

15 Von einem annuus magister wird beispielsweise in den Arvalakten gesprochen, siehe etwa J. Scheid (ed.), Commentarii Fratrum Arvalium (Rom 1999), n ${ }^{\circ}$ 55, 57, 65, 76, 79; ferner CIL VIII 17167; ILAlg. II 2, 3, 7680; CIL XIII 8772: flamen annuus. Dass die Charakterisierung mit annuus bei Ämtern, die mit dem Kult verbunden waren, erscheint, ist nicht weiter verwunderlich; denn Priesterämter werden üblicherweise lebenslänglich übertragen, nicht aber nur für ein Jahr. Bei den normalen munizipalen Ämtern versteht sich die Dauer von einem Jahr von selbst, war damit an sich unnötig.

16 Tab. Heracl. Z. $89 \mathrm{ff}$.

17 Lex Malacitana, rubrica 54. 
der Republik noch für die Kaiserzeit. ${ }^{18}$ Zudem ist das Alter von 35 Jahren erstaunlich hoch, zumal man jedenfalls bei Senatoren während der Kaiserzeit genügend Fälle nachweisen kann, dass Priesterämter längst vor dem 30. Lebenjahr übernommen wurden. Die Frage, warum es diese Bestimmung gibt, von der wir sonst nirgendwo einen Reflex haben, muss damit offen bleiben. ${ }^{19}$

Wichtig aber ist, dass hier noch in der Spätzeit Marc Aurels auf sehr spezielle Regelungen aus augusteischen Gesetzen verwiesen wird: Zunächst wird auf Kap. 6 der lex Iulia de maritandis ordinibus verwiesen, in dem offensichtlich Altersbestimmungen für die Bewerbung um bestimmte Ämter genannt waren. Gleichartige Bestimmungen aber waren auch im Kapitel 49 des commentarius vorhanden, der am 28. Juni unter den Konsuln Volesus Valerius Messala ${ }^{20}$ und Cn. Cornelius Cinna Magnus, den beiden ordentlichen Konsuln des Jahres $5 \mathrm{n}$. Chr., vorgelegt worden war, nur zwei Tage, bevor die beiden Konsuln von Ihrem Amt zurücktraten. Dieser commentarius war wie ein Gesetz in Kapitel gegliedert und war mit einem konkreten Datum verbunden, dem Datum der propositio, also der Bekanntmachung des Textes. Wenige Jahre später war dieser Text, wie in diesem Abschnitt ausdrücklich betont wird, die Grundlage für die lex Papia Poppaea, die in der zweiten Jahreshälfte $9 \mathrm{n}$. Chr., also etwas mehr als vier Jahre später verabschiedet wurde. Auffallend ist dabei, dass es sich in beiden Texten, im commentarius und in der lex, um ein Kapitel mit einer recht hohen Nummer handelt, im commentarius ist es kaput XLVIIII, in der späteren lex sodann kaput XLIIII.

Wie all das miteinander zusammenhängt und welche Schlüsse daraus für die augusteische Gesetzgebung gegenüber den ordines insgesamt zu ziehen sind, ${ }^{21}$ dafür gibt es aus unserer Überlieferung heraus keine sich

18 Siehe J. Timmer, Altersgrenzen politischer Partizipation in antiken Gesellschaften (Berlin 2008), $261 \mathrm{ff}$. (nur für die Zeit der Republik). Siehe auch J. Scheid, 'Le prêtre et le magistrat. Réflexions sur les sacerdoces et le droit public à la fin de la République', in C. Nicolet (ed.), Des ordres à Rome (Paris 1984), 243-280, bes. 254-266.

19 Auffällig ist bei genauer Betrachtung der Stelle, dass die Zahl in etwas kleineren Buchstaben und wesentlich weniger tief eingraviert zu sein scheint als der übrige Text. Die Zahl wirkt fast wie ein wenig nachgetragen. Doch auch für diese Beobachtung fehlt eine Erklärung.

20 Im Namen dieses Konsuls liegt offensichtlich ein Fehler vor. Möglicherweise hat man den mehr als seltenen Vornamen Volesus nicht mehr gekannt und hat daraus auch ein Cognomen Caesus konstruiert (dankenswerter Hinweis von Matthäus Heil). Angsichts der im gesamten Text recht zahlreichen Fehler bei der Gravur des Textes ist dieser Fehler nicht weiter verwunderlich.

21 Siehe vorerst allgemein und speziell J.-L. Ferrary, 'L'iter legis, de la rédaction de la rogatio à la publication de la lex rogata, et la signification de la législation comitiale dans 
unmittelbar aufdrängende Erklärung. Doch lässt sich vielleicht eine Vermutung formulieren. Wir wissen, dass sich gegen diese Gesetze, die die soziale Ordnung und die freie Lebensweise speziell der höheren ordines erheblicher Widerstand erhoben hatte. Ein Reflex davon findet sich bei Cassius Dio zum Jahr 9 n. Chr. Bei den Spielen, die die beiden consules ordinarii dieses Jahres aus Anlass der Rückkehr des Tiberius aus Illyricum gaben, kam es zu Demonstrationen der Ritter gegen das Gesetz, das den Zwang zur Heirat festschrieb und im Fall der Nichtbefolgung entsprechende Strafen vorsah. Wegen dieser Demonstrationen hielt nach Cassius Dio Augustus eine Rede, in der er die Gesetzgebung verteidigte. Das aber heißt, dass der Inhalt des Gesetzes, das dann von den beiden Suffektkonsuln Papius Mutilus und Poppaeus Sabinus in der zweiten Jahreshälfte 9 vorgelegt und deshalb nach der Verabschiedung auch nach ihnen benannt wurde, bereits vorher als Text vorgelegen haben muss. ${ }^{22}$ Cassius Dio sagt das nicht direkt, doch aus seiner Schilderung dessen, was sich in Rom abspielte, ist dies zwingend. Die Gesetzesinitiative ist nicht die der beiden consules suffecti, geht vielmehr auf andere Personen zurück. Wenn man diesen Schluss mit den Informationen verbinden darf, die sich aus diesem kaput XXVII des Munizipalgesetzes ergeben, verbinden darf, dann liegt es nahe anzunehmen, dass die Gesetzesinitiative bereits unter den beiden consules ordinarii des Jahres $5 \mathrm{n}$. Chr. ihren Anfang genommen hat, und zwar in einem elaborierten Gesetzestext, der mindestens 49 kapita umfasst haben muss. Warum es dann so lange gedauert hat, bis der commentarius auch zu einer lex umgewandelt wurde, ist zum einen wohl mit dem Widerstand, der sich gegen den Gesetzesvorschlag erhoben hat, zu erklären; die von Cassius Dio berichteten Demonstrationen der Ritter sind ein Hinweis darauf. Doch kam wohl anderes hinzu, was die Verzögerungen erklären kann. Denn im Jahr 6 n. Chr. brach der höchst bedrohliche Aufstand in Pannonien aus, zu dessen Bewältigung Notstandsmaßnahmen nötig waren, die von vielen nicht willkommen geheißen wurden. Hinzu kam eine Lebensmittelknappheit in Rom, die sogar die Ausweisung von ganzen Bevölkerungsgruppen aus Rom zur Folge hatte. In dieser angespannten Situation hätten weitere gesetzgeberische Aktionen, die tief in gewachsene Lebensformen eingriffen, nur zu einer Verschärfung der inneren Lage geführt. Das konnte Augustus aber nicht brauchen. Im Laufe des

le système politique de la Rome républicaine', in J.-L. Ferrary (ed.), Leges publicae. Le legge nell'esperienza giuridica romana (Pavia 2012), 3-38; Id., 'La législation augustéenne et les dernières lois comitiales', ibid., 569-592.

22 Cassius Dio 56.10.3. 
Jahres 8 n. Chr. aber hatte sich die Lage entspannt, Tiberius hatte den Aufstand niedergeschlagen und einen deutlichen Sieg errungen. Er kehrte noch vor Ende Juni 9 n. Chr. aus Illyricum zurück. Da konnte sich bei Augustus die Ansicht durchsetzen, es sei an der Zeit, den Vorschlag des Jahres 5 endlich in ein offizielles Gesetz umzuformen. Das Ergebnis war die lex Papia Poppaea.

Wie auch immer der commentarius in die augusteische Gesetzgebung einzuordnen ist, es bleibt die Frage, weshalb wir von diesem Gesetzesvorschlag des Jahres 5 in der gesamten sonstigen Überlieferung zu den augusteischen Gesetzen nichts hören. Noch weit auffallender aber ist, dass diejenigen, die am Ende der Regierungszeit Marc Aurels das Gesetz für Troesmis abfassten, auf diesen commentarius zurückgekommen sind und sich nicht damit begnügten, auf den entsprechenden Paragraphen der lex Papia Poppaea zu verweisen. Eine Antwort auf diese Frage scheint es beim gegenwärtigen Stand unseres Wissens nicht zu geben.

Auf Tafel B folgt nach dem Rest von [k(aput) XXVII] noch der Anfang von $k$ (aput) XXVIII:

de municipibus ad suffragium uocandis custodibusque ternis ponendis ad singulas cistas quae suffragiorum causa posit<a>e erunt.

Diese Überschrift ist klar und eindeutig. Man würde angesichts der sonstigen Kenntnisse zu den Überschriften einzelner Paragraphen eines Gesetzes davon ausgehen, dass dies die gesamte Überschrift wäre. Doch scheint es, dass auch noch zwei weitere Tatbestände in die Überschrift aufgenommen wurden, wodurch eine außerordentlich lange Überschrift entstanden ist. Denn der Text fährt fort:

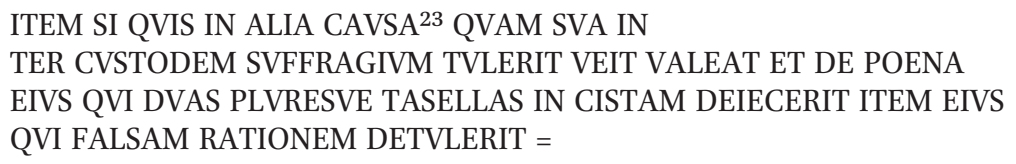

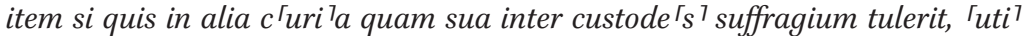
ualeat, et de poena eius, qui duas pluresue tabellas in cistam deiecerit, item eius qui falsam rationem detulerit.

23 CAVSA kann hier nicht zutreffen. Vielmehr ist das Wort wohl zu $c^{\top} u r i{ }^{7} a$ zu korrigieren, in Analogie zu den Bestimmungen in der lex Malacitana, rubrica 55: item ab his positi, qui honorem petent, in ea curia quisque eorum suffragi ${ }^{\top} \mathrm{m}^{7}{ }^{7}$ ferto ad cui $\{i\}$ us curiae cistam custos positus erit e ro $^{7}$ rumque suffragia perinde iusta rataque sunto ac si in sua quisque curia suffragium tulisset. 
In diesem Kapitel werden somit über die Einberufung zu den Wahlen hinaus auch noch Regelungen getroffen über die Stimmabgabe deren, die in einer curia, der sie selbst nicht angehören, als custodes eingeteilt sind und dort abstimmen dürfen, ferner über die Strafe, die gegen jemand verhängt wird, der bei der schriftlichen Stimmabgabe zwei oder sogar mehr Stimmsteine, tabellae, in die Wahlurne werfe und schließlich über die Strafe für denjenigen, der ein falsches Ergebnis der Wahl in einer curia beim wahlleitenden Magistrat melde.

Erst nach dieser langen Überschrift setzt der Text damit ein, dass auf den Wahlleiter verwiesen wird, der - so ist wohl der Text zu verstehen Bürger, municeps, des municipium sein müsse. Anschließend ist noch ein kurzer Abschnitt über die Wähler erhalten, die nach diesem Gesetz in Troesmis die suffragi latio haben. ${ }^{24}$ Was folgt, betrifft wohl erneut den Wahlleiter, der das Recht zur vocatio oder convocatio der Wähler hat; doch ist dort der Text wegen der in den letzten Zeilen sehr zerstörten Oberfläche und des fehlenden rechten Eckstücks der Tafel nicht mehr genauer zu verstehen und zu rekonstruieren.

Der erhaltene Teil dieser lex umfasst also nur zwei sachliche Komplexe: Einmal in Paragraph 11 die Frage der Absendung von städtischen Gesandten; zum zweiten aber Aussagen zur Wahl von Amtsträgern in der Stadt: in dem partiell noch erhaltenen Paragraphen 27 Altersbestimmungen für die Wahl von Magistraten und Priestern und schließlich den Anfang von Paragraph 28 mit den Regeln, die bei der konketen Wahl einzuhalten waren - insgesamt ein sehr kleiner Teil des gesamten Gesetzes.

Der verlorene Teil lässt sich partiell genauer bestimmen: Am Anfang fehlen zehn volle Paragraphen, da Tafel A mit kaput XI beginnt. Zwischen Tafel A und Tafel B fehlen fünfzehneinhalb Paragraphen, nämlich vom verlorenen Ende von kaput XI bis zum ebenfalls fehlenden Anfang von kaput XXVII. Wieviele Paragraphen nach dem begonnenen kaput XXVIII bis zum Ende verloren sind, lässt sich nicht sagen. Vom Textumfang her gesehen stehen also auf den beiden Tafeln nicht einmal zwei ganze Paragraphen. Nimmt man an, dass bei der Präsentation dieser lex auf Bronzetafeln jeder Paragraph, wie es scheint, mehr als eine Tafel eingenommen hat, dann muss man allein bis zum Ende des Paragraphen 28 mit mindestens ebensovielen Tafeln rechnen, vermutlich aber sogar mit deutlich mehr.

${ }^{24}$ Vgl. lex Malacitana, rubrica 53. 
Die beiden Tafeln lassen eine partielle sachliche Ordnung innerhalb der lex erkennen. Nimmt man die flavischen Stadtgesetze aus der Baetica als Modell, dann wird unmittelbar deutlich, dass man in der lex für Troesmis, soweit freilich die wenigen Kapitel eine Aussage zulassen, im Wesentlichen die gleiche Ordnung antrifft. Das zeigt folgende Gegenüberstellung:

Tabelle 1: Ordnung innerhalb leges

\begin{tabular}{ll}
\hline Lex Irnitana + lex Malacitana & lex Troesmensium \\
\hline rubrica 45: de legatis mittendis & kaput 11: de legatis mittendis \\
excusationibusque accipiendis & excusationibusque accipiendis \\
(lex Irnitana) & \\
rubrica 54: quorum comitiis rationem & {$[$ kaput 27] Altersvorschriften bei den } \\
haberi oporteat (lex Malacitana) & Wahlen \\
rubrica 55: de suffragio ferendo & kaput 28: de municipibus ad suffragium \\
(lex Malacitana) & $\begin{array}{l}\text { uocandis custodibusque ternis ponendis } \\
\text { usw. }\end{array}$ \\
\hline
\end{tabular}

Die Anzahl der Paragraphen zwischen rubrica 45 und rubrica 54, wie sie aus der lex Irnitana bzw. Malacitana zu erkennen ist, beträgt neun, in der lex Troesmensium dagegen 15. Das ist eine deutlich höhere Zahl. Hier standen somit wohl auch Regelungen, die uns aus den spanischen Stadtgesetzen nicht bekannt sind. Die lex Troesmensium scheint insgesamt ausführlicher gewesen zu sein, was sich auch in mehr Einzelregelungen innerhalb von $k$ (aput) XI zeigt. Ein deutlicher Unterschied besteht auch im Inhalt von rubrica 54 der lex Malacitana und kaput 27 der lex Troesmensium: Im spanischen Modell wird nur darauf hingewiesen, dass die Magistrate aus dem Kreis der Freigeborenen zu wählen seien und dass sie nicht jünger als 25 Jahre und auch nicht in einer persönlich-rechtlichen Situation der Art sein dürften, dass sie, wenn sie römische Bürger wären, nicht zu den Dekurionen gehören könnten. In der lex für Troesmis aber wird im Zusammenhang mit anderen Bestimmungen, die nicht erhalten sind, auf die Regeln augusteischer Gesetze verwiesen, der lex de maritandis ordinibus und der lex Papia Poppaea einschließlich des commentarius aus dem Jahr 5 n. Chr., die die passive Wahl von einer Altersbestimmung abhängig machen. Diese Differenz ist bemerkenswert; der Grund könnte im unterschiedlichen Rechtsstatus der Gemeinden gelegen haben. Malaga und Irni waren Gemeinden latinischen Rechts, in denen die Regeln der römischen Gesetze, die unter Augustus erlassen wurden, nicht notwendigerweise angewendet werden mussten. Anders war das aber bei den 
Bürgern von Troesmis. Dieses war ganz offensichtlich als Munizipium römischer Bürger konzipiert, in dem auch alle Regeln, die durch römische Gesetze vorgeschrieben waren, beachtet werden mussten.

In den Fragmenten, die uns durch die beiden Tafeln zugänglich sind, werden alle Institutionen, die eine römische Bürgergemeinde kennzeichnen, erwähnt: Die decuriones conscripti, die einzelnen Magistrate: quinquennales, IIuiri, aediles, quaestores, ferner sacerdotes. Auch die Volksversamlung wird genannt. Dabei ist weniger überraschend, dass sie noch existiert, wichtiger ist vielmehr, dass sie offensichtlich zusammentritt und das Vorkehrungen getroffen werden, dass bei den Wahlen einzelne Leute bei der Stimmabgabe nicht zwei oder mehr Stimmsteine, tabellae genannt, ${ }^{25}$ in die Stimmurne werfen. Gewählt wird offensichtlich nach curiae, in die die Bürger eingeschrieben sind, nicht anders als auch nach den spanischen Stadtgesetzen. Das ergibt sich aus der Bestimmung, die zwar durch Korrektur des Textes von B 21 entstanden ist:

item si quis in alia $c^{\Gamma}$ uri $^{7}$ a quam sua inter custode $^{\Gamma} s^{7}$ suffragium tulerit

Diese Textverbesserung ist aber sicher, weil derselbe Tatbestand mit Bezug auf die curia als Wahlkörper und der Frage der Kontrolleure der einzelnen curiae bei der Abstimmung in rubrica 55 der lex Malacitana enthalten ist. ${ }^{26}$

Um ein kurzes, vorläufiges Fazit zu ziehen: Das Gesetz für das municipium Troesmis zeugt davon, dass auch in einer so unruhigen Zeit wie der Marc Aurels unmittelbar an der Grenze des Imperiums eine Gemeinschaft römischer Bürger geschaffen wurde, in der das öffentliche Leben streng und detailreich nach römischen Rechtsregeln gestaltet werden sollte. Dieses Gemeinwesen trägt den umständlichen Namen der beiden regierenden Augusti. Dieses lange Regelwerk wird in einer imponierenden Zahl von Bronzetafeln öffentlich ausgestellt, so dass jeder Bewohner und Bürger der Stadt sich darüber informieren kann, wonach er und seine Mitbürger sich bei der Gestaltung des Lebens in der Stadt zu richten haben.

Sind aber diese Regeln dort auch alle Realität geworden? War diese lokale Gesellschaft, zu der wohl nicht wenige Soldaten nach ihrer Entlassung gehörten, überhaupt in der Lage, diese Regeln alle zu verstehen und anzuwenden? Hatten sie z.B. die Texte der lex de maritandis ordinibus und

25 Auch sonst findet sich dafür der Terminus tabella, etwa in rubrica 55 der lex Malacitana.

26 Siehe oben Anm.23. Auch in rubrica 15 der lex Ursonensis werden die curiae als Wahlkörper angeführt, ebenso in der lex Irnitana. - Für kritische Hinweise danke ich Jean-Louis Ferrary, Dario Mantovani, Roberto Dalla Rosa und John Scheid. 
der lex Papia Popaea in ihrer „Stadtbücherei"? Auf den zitierten commentarius konnten sie vielleicht verzichten, da dessen Regelungen von der lex Papia Poppaea übernommen worden waren, obwohl Juristen in Rom das wohl anders sahen; sonst hätten sie den Hinweis nicht in das Stadtgesetz aufgenommen. Man kann gewisse Zweifel daran haben, dass dieses doch sehr ausgefeilte Regelwerk in allen seinen Nuancen angewandt werden konnte.

Das bleiben freilich eher Fragen, die man kaum wird beantworten können, da uns die Realität, nach der in dem Municipium gelebt wurde, nicht genauer bekannt ist. Doch trotz gewisser Zweifel, wird man doch davon ausgehen dürfen, dass das Leben näherungsweise doch nach diesen Normen gestaltet wurde, da sonst nicht einsichtig wäre, warum die Gemeinde dieses Regelwerk in solch aufwendiger Form präsentieren lies. Eine lange Wand von mindestens 25 Metern muss von den mindestens 50 wenn nicht weit mehr Bronzetafeln bedeckt gewesen sein. Solches tut man nur, wenn man damit etwas Bedeutsames verband und wenn man damit auch nach außen etwas aussagen wollte. Vielleicht liegt man nicht gänzlich falsch, wenn man darin zumindest den Stolz erkennen darf, an der Grenze zur barbarischen Außenwelt zur Welt Roms auch im unmittelbaren Rechtssinn zu gehören, eben Römer zu sein. Die beiden Tafeln werden im kommenden Jahr in einem Band über Troesmis, der in Rumänien von Christina Alexandrescu herausgegeben wird, publiziert werden. Dort wird dann auch ein entsprechender Kommentar erscheinen, auch zu dem längeren Passus, der oben S. 80 schon im Wortlaut zitiert ist. 


\title{
GOTHS AND ROMANS IN THE LEGES VISIGOTHORUM
}

\author{
J.H.W.G. Liebeschuetz
}

\begin{abstract}
A Minimal View of Importance of the Entry of the Barbarians into the Roman Empire
\end{abstract}

There has recently been a tendency to minimise the part of the barbarians in the fall of the Roman Empire. Can the drastic transformation of the Roman world really be attributed to invasion by Germanic tribes (gentes), or are they perhaps the results of internal developments?

Walter Goffart minimises the role of the gentes: "In this panorama of relentless shrinkage, barbarians as recruits or suppliants for peace could consistently be perceived as the desirable lower-cost alternative (to traditional regular army units. The cost-benefit equation of their request offered an opportunity not to be missed. Vandals and Goths introduced no alternative to the nomen Romanum; they were not rivals, proposing a different way of life or competing for loyalty. The barbarians could be recruited into the imperial enterprise because they asked for nothing more than to carry it forward and share its blessings."

Contributors to a volume edited by A. Gillett largely defend this view. ${ }^{2}$ Bowlus describes the gentes as "the 'imagined' communities Wenskus and Wolfram presume existed", ${ }^{3}$ Gillett argues that ethnicity is a circumstantial not a shaping identity. ${ }^{4}$ Kulikowski uses the inadequacy of our sources as an argument to deconstruct the gentes, concluding that when sources label individuals ethnically we cannot be sure what this means, but "what we can do is ask in every single instance what that ethnic identification meant in context". ${ }^{5}$ Kulikowski's scepticism is excessive. In the opinion of

1 W. Goffart, Barbarian Tides: The Migration Age and the Later Roman Empire (Philadelphia 2006), 238 (shortened).

2 A. Gillett (ed.), On Barbarian Identity. Critical approaches to Ethnicity in the Early Middle Ages (Turnhout 2002).

3 C.R. Bowlus, 'Ethnogenesis: the tyranny of a concept', in Gillett 2002 op. cit. (n. 2), 241-256, on 256 .

4 A. Gillet, 'Was ethnicity politicized in the earliest medieval kingdoms?', in Gillett 2002 op. cit. (n. 2) $85^{-121}$, on 86 .

5 M. Kulikowski, 'Nation versus army: a necessary contrast?', in Gillett 2002 op. cit. (n. 2), 69-84, on $83-4$. 
this writer, and of generations of earlier scholars, not all of them blinkered by rabid German nationalism, the sources do adequately show that the gentes were real, and historically important.

The complex of duties and privileges that constituted Roman citizenship had been an essential instrument in the making of the Roman Empire. By spreading of Roman citizenship the Romans won the cooperation of provincial elites, and secured the stability of the Empire. Citizenship conferred enormous advantages to individuals who possessed it. When Paul faced a beating and torture at the hand of a Roman official it was enough to say that he was a Roman citizen and "I appeal unto Caesar", 6 for his case to be withdrawn from the local official to be transferred to Rome and the emperor.

The spread of Roman citizenship was accompanied by Romanization. Although this term is now banned, we have no better alternative to describe the transformation in territories under Roman rule. Prominent among the changes were the introduction of the Latin language, and of Roman law. Roman law and citizenship were closely linked, because Roman citizens used Roman law. After the Constitutio Antoniniana of $\mathrm{AD} 212$ all inhabitants of the Empire were Roman citizens, and all used some kind of Roman law.

But in Hispania in the 6 th and 7 th centuriesAD the situation had changed totally. Every Hispano-Roman was still in law a Roman citizen, but there were also some very important inhabitants who were not Roman citizens: the Goths. Their status was that of 'federates', that is allies of the Empire, and their identity was ethnic, i.e. Gothic, but they were the ruling people of the country, and their leader was its King.

How did this coexistence of two peoples, the old rulers and the new ruling people, work? By far the best source is the law book of the Gothic kings. Historians have traditionally given both law book and kings the epithet Visigothorum, i.e. of the Visigoths, and so conveniently distinguish them from the Ostrogoths in Italy. However the name Visigoths was not used by writers earlier than Cassiodorus. It is a creation of the age of Theoderic. ${ }^{7}$ In this paper the convention of describing the western Goths as Visigoths is maintained, but that gens and its kings identified themselves simply as Goths. The oldest completely preserved edition of the Visigothic Code

6 Acts 22.28 and Acts 25.11.

7 It is used by Procopius, Bell. $3.2 .7 ; 8.5 \cdot 5$. 
dates from $\mathrm{AD} 654 .{ }^{8}$ This includes many older laws, but some of these have certainly been edited, and a good deal of the legislation of the earlier kings is likely to have been omitted as no longer relevant. The Leges Visigothorum (or more correctly Liber Iudiciorum) therefore represents the condition of Spain not when the Goths first entered the country in the fifth century, but more than two hundred years later.

\section{Goths and Romans in the Leges Visigothorum}

All the laws are in good Latin and both the structure of the Code, and the legislation, are based on Roman models. The laws themselves probably include some Germanic elements, ${ }^{9}$ but these are not conspicuous, and even their identification is controversial. Scholars are agreed that the law issued and interpreted by the Gothic kings was essentially Roman. ${ }^{10}$ For our theme it is an important fact that the vast majority of the laws make no distinction between Goths (Gothi) and Romans (Romani), and what is more, only a very small proportion even mention either name. ${ }^{11}$

8 The latest codification was that of Erwig, issued in 683, revising the Code of Chindasuinth and Reccesuinth. The Code of Erwig has been preserved in two copies. The Code of Chindasuinth and his son Reccesuinth, the Liber Iudiciorum, was issued in 654. It also has been preserved in two manuscripts, and is thus the earliest Visigothic Code to have been preserved entirety. It greatly expanded the Code of Leovigild of 586 , of which no copy has been preserved, but 300 of its laws, labelled as Antiquae have been taken over into the later Codes, not necessarily unedited. The first Visigothic Code of which some laws have survived in the later Codes, is that of Euric (Cod.Eur.), probably of 476. A few fragments of this Code have been preserved on a palimpsest. The standard text is K. Zeumer (ed.), Leges Visigothorum (MGH Legum sectio 1) (Hannover \& Leipzig 1892) (=LV).

9 I. Velazquez, 'Jural relations as an indicator of syncretism from the law of inheritance to the dum inlicita of Chindasuinth', in P. Heather (ed.), The Visigoths from the Migration Period to the Seventh Century (Woodbridge 1999), 225-259, traces the development of the Visigothic law of inheritance, concluding that the Kings' legislation is basically the adaptation of Roman law to current conditions, but of Roman law which was compatible with Gothic, i.e. Germanic custom. The list of fixed payments of compensation for injury to different parts of the body in LV 6.4.3 looks Germanic to P.D. King, Law and Society in the Visigothic Kingdom (Cambridge 1972), 91; so does the taking of theft more seriously than robbery (ibid., 254-58) and the punishment of decalvatio (scalping or perhaps merely shaving off of hair; ibid., 90 n. 5); perhaps also the 'Germanic' dowry LV 3.1.5; Form. Vis no. 20.

10 "Neither feud nor self-help - the two inextricably connected in Germanic practice was accorded a regular and legitimate place in the legal scheme of things.", King 1972, op. cit. (n. 9), 86. "There is precious little trace in Ervig's Code of the all-important position which, at an earlier Germanic stage, the kindred had held in society." (ibid., 222).

11 I have argued that the legislation of Gothic kings was territorial and addressed to Goths and Romans alike from the beginning, in 'Citizen status and law in the Roman 
Astonishingly, there are only three topics which have produced legislation required by the coexistence of two peoples: 1. A certain category of estates; 2. Intermarriage between Goths and Romans; 3. The status of manumitted Christian slaves of Jews. The majority of these laws relate to disputes arising over the two thirds of an estate assigned to a Goth and the third left to the Roman owner at the time of the original settlement, or perhaps settlements. Most of these laws even go back to the Code of Euric compiled $c$. AD 470, in Gaul, before the establishment of the Gothic kingdom in Spain, ${ }^{12}$ showing that the Visigoths were settled in Spain on the same terms as they had been in Gaul. The laws not found in the fragments of the Code of Euric are however labelled 'Antiquae', so they go back at least to the Code of Leovigild of $c$. AD $580 .{ }^{13}$ Later laws on disputes about landed property do not distinguish between lands of Romans and lands of Goths. ${ }^{14}$ It looks as if the distinction had become irrelevant for disputes over the ownership of most kinds of land, as indeed for legal disputes of almost any kind.

On the other hand the fact that the laws with reference to respectively sortes Gothorum and tertiae Romanorum were taken into the latest of the Codes, the Code of Erwig of $\mathrm{AD} 683$, suggests that they had not been abolished, and that these estates continued to have a distinct legal status, and this irrespective of whether they were still owned by a Goth or a Roman, and that they kept this status to the end of the Visigothic kingdom. One of the Antiquae which retained a place in the latest edition of the Code lays down that if a Goth seizes the 'third' that had been left to a Roman, ${ }^{15}$ he must give it back. The reason for this law is to avoid loss to the fiscus. The tertiae Romanorum therefore still paid tax, while the sortes Gothorum remained exempt. ${ }^{16}$ This is confirmed by the previous law, which lays down that in the case that a portion of a Roman's tertia has been alienated to somebody else (presumably a Goth), and the new owner has placed a tenant on it, that tenant will become liable for the tax, if the land was returned to Roman ownership. ${ }^{17}$ Presumably the tenant had not been

Empire and the Visigothic Kingdom', in W. Pohl (ed.), Strategies of Distinction: The Construction of Ethnic Communities, 30o-8oo (Leiden, Boston, Köln 1998), 131-152, esp. 146-49.

12 LV 10.3.5 = Cod.Eur. 276; 10.2.1-3 and 10.3.1 = Cod.Eur. 277; 5.4.20 = Cod.Eur. 312.

$13 L V$ 10.1.7-9; 10.1.16; 10.2.1; 10.3.5.

14 LV 10.1.17-19, already in Cod.Eur. 312: Romanus qui Gotho donauerit rem reappears, in $L V$ 5.4.20 without the ethnic qualification.

15 LV 10.1.16: Ut si Goti de Romanorum tertiam quippiam tulerint (...).

16 LV 10.1.16 (Antiqua, Recc. Erv.): ut nihil fisco debeat deperire.

17 LV 10.1.15 (Antiqua, Recc. Erv.). Both laws are obviously very much shortened summaries of much longer laws. 
liable while the land was in possession of the Goth. One might compare this to the status of land that has once belonged to a curialis (decurion), which continued to be taxed to pay for certain services irrespective of who was the present owner. ${ }^{18}$

Up to the reign of Leovigild (568-586) Goths were divided from Romans by a marriage ban. ${ }^{19}$ Leovigild abolished this ban on the ground that it was not right to split up a marriage of partners who were equal in dignity and lineage. ${ }^{20}$ Intermarriage was therefore permitted, subject only to consent of the girl's family. ${ }^{21}$ It is worth noting that Leovigild maintained the distinction between Goths and Romans, but merely asserted that they were equal in status - at least as far as eligibility for marriage was concerned. The law must nevertheless have accelerated the fusion of the two peoples. A further step in the same direction was taken by Leovigild's son and successor Reccared. In 587 he converted from Arian to Catholic Christianity. In the following year he had the lands and churches of the Arian Church transferred to that of the Catholics, and went on to summon the bishops of his kingdom to meet at the Third Council of Toledo over which the king himself presided. The assembled bishops were duly induced to denounce Arianism and adopt Catholicism, which became the established religion of the Visigothic kingdom. ${ }^{22}$ Not all Goths accepted this revolution peacefully, but it is probably correct to assume that henceforth the majority of Goths and Romans were no longer divided from each other by religion. One might have expected these measures of Leovigild and his son Reccared to have ended the dualism of Goths and Romans. ${ }^{23}$ But I will argue that this was not so.

18 LV 5.4.19 (Recc. Erv.): curialibus uel priuatis inter se uendendi, donandi uel commutandi ita licitum erit, ut ille, qui acceperit, functionem rei accepte publicis utilitatibus inpendere non recuset.

19 G. Haenel, Lex Romana Visigothorum (Berlin 1849), 92-3: CTh 3.14.1.

20 LV 3.1.1: personas quas dignitas conpares exequabit in genere.

${ }^{21}$ LV 3.1.1: Premissa petitione (...) consultum perquirendo, prosapie sollemniter consensu comite, percipere coniugem. There is some uncertainty about the text, above all the relevance here of comite. This is sometimes taken require consent of the comes civitatis, but $L V$ 3.1.2, insisting on the necessity of the girl's father's permission for any marriage offers the key to the interpretation of the passage.

22 J.D. Mansi, Sacrorum Conciliorum nova, et amplissima Collectio, vol. 9 (Paris 19o1), 977 ff.; E.A. Thompson, The Goths in Spain (Oxford 1969), 94-101.

${ }^{23}$ But anthropological evidence suggests that the mixing of different ethnic groups living in the same surroundings is often a slow process: G. Ausenda, 'Mixed society', in P. Heather (ed.), The Visigoths from the Migration Period to the Seventh Century (San Marino 1999), 501-503. 
Two laws assign the status of Roman citizenship to the manumitted Christian slaves of the Jews. This would seem to imply that though freed they did not become Goths. ${ }^{24}$

\section{Goths and Romans in the mid Seventh Century, the Age of Isidore of Seville}

It would seem that by the end of the 6th century, as far as law was concerned, there was little distinction between the status of Goths and of Romans, and as we have seen, rules for the settling of disputes between Goths and Romans are conspicuously absent from the great majority of the laws. Moreover as the laws are written in Latin, Goths were expected to understand the Roman language. Indeed there is very little evidence that they still used Gothic. ${ }^{25}$

Documents of many different kinds, inscribed on slate, have been found in a number of places in the Meseta of central Spain. These confirm that the use of written documents in legal processes, and in much of the business of estate management, was widespread even in the countryside. The nomenclature found in these documents includes both Latin and Gothic names, with the Latin names in the majority. The language is invariably Latin, if Latin of a very vulgar character. ${ }^{26}$ Goths and Romans evidently used the same courts and the same laws, and the same language.

So a number of scholars, notably Claude and Teillet, ${ }^{27}$ have provided plausible arguments that there no longer was any distinction between Goths and Romans. But I don't think that that interpretation is compatible with the evidence of the literary texts which, as I will argue, preserved

24 The only references are $L V 12.2 .13$ and 14, both of Sisebutus AD 612-621; also Recc. Erv. $L V$ 12.2.14 explains the legal significance of Roman citizenship in this case as that the liberated slaves will have no continuing obligation towards former owners: nulli scilicet Hebreo nec cuilibet obsequio reseruato. The liberated slaves would be taxed. LV 12.2.13: et prenotati in polipticis publicis adque secundum eorum peculium iustissima aderatione censiti, but this was probably true of all slaves both before and after manumission.

25 On possible remnants of Gothic vocabulary still in use see I. Velasquez, 'The Visigoths', in H.-W. Goetz, J. Jarnut, \& W. Pohl (eds.), Regna and Gentes (Leiden, Boston 2003), 161217 , on 184 .

26 See I. Velázquez Soriano, Las Pizarras Visigodas. Entre el Latin y su disgregacion. La lengua hablada en Hispania, siglos VI-VIII (Burgos, 2004).

27 D. Claude, 'Remarks about relations between Visigoths and Hispano-Romans in the seventh century', in Pohl 1998 op. cit. (n. 11), 117-130. S. Teillet, Des Goths à la nation gothique: les origines de l'idée de nation en Occident du V $V^{e}$ au VII siècle (Paris 1984), 553: "la distinction entre Gothi et Romani tend à disparaître, et le vocable Gothi, sauf exception, en vient à désigner, déja depuis Jean de Biclar, les populi qui constituent la gens Gothorum, indifféremment Goths et Hispano-Romains." 
the distinction between Goths and Romans at least to the mid seventh century, ${ }^{28}$ so that both in literary writings and canons of ecclesiastical councils Gothus still designated a member of the Gothic ruling people, as opposed to a Hispano-Roman. This is clear in the first place from the Lives of the Fathers of Merida of perhaps $633-638 \mathrm{AD},{ }^{29}$ whose author when introducing the principal characters tells us whether they were of Greek, Gothic or of Roman origin. ${ }^{30}$ It clearly still made a difference. Isidore, bishop of Seville c.6oo-636, a man of Roman descent and great learning, who exercised very great influence at the Gothic court, wrote a history of the Goths ending in the year 625, presenting them as a people, with a history and a warlike character of its own. He established the Goths as a historical people, very much as Jordanes had done at Constantinople about sixty years earlier. There is for him as yet no question of a Gothic people incorporating all inhabitants of Spain irrespective whether of Gothic or of Roman descent. The beautiful land of Spain has been 'betrothed' successively to Romans and to Goths; as he states in the conclusion to the preface of his history: "And although this same Romulean power, initially victorious, betrothed you to itself, now it is the most flourishing people of the Goths, who in their turn, after many victories all over the world, have eagerly seized you and loved you." ${ }^{31} \mathrm{He}$ concludes with the statement that the Romans were now serving the Goths. "Subjected, the Roman soldier serves the Goths, whom he sees being served by many peoples and by Spain itself." 32 The merging of the two peoples has not yet happened, and near contemporary texts referring to Goths must be taken to mean Goths as opposed to Hispano-Romans.

The evidence that the distinction between Goths and Romans was maintained at least well into the seventh century is unambiguous. We

28 So also Velasquez 2003, op. cit. (n. 25), passim.

29 A.C. Macías (ed.), Liber Vitas Sanctorum Patrum Emeretensium, (Merida 1988), 29-30 $(=V P E)$.

30 Of the bishops of Merida, Paulus 530-56o (IV.I) and Fidel 56-571 (IV.III) are said to have been Greeks, i.e. from the Greek speaking East, Masona 571-605 was a Catholic Goth (V.I), his Arian rival Sunna was a Goth (V.X). Another rival Nepopis was an Egyptian to judge by his name (V.VI). Certain counts of cities were Goths (V.X), but Claudius the duke of Merida was a Roman and a Catholic (V.X); bishop Renovatus, c. AD 633, was a Goth (V.XIV).

31 Th. Mommsen (ed.), Historia Gothorum, MGH, AA 11, Chronica minora saec.IV.V.VI. VII. (II), 267-295, on 267, lines 22-25. English translation: Isidore of Seville, History of the Kings of the Goths, preface \& transl. by K.B. Wolf in Conquerors and Chroniclers of Early Medieval Spain (Liverpool 1990), 81-110 at p. 82.

32 Wolf 1990, op. cit. (n. 31), 110. 
have already seen that a freed slave gains the status of a Roman citizen, and therefore by implication does not become a Goth. ${ }^{33}$ In 655 , the ninth council of Toledo declared that freedmen and women manumitted by the Church had conubium with neither Goth nor Roman. ${ }^{34}$ As late as AD 693 a law confirming the canons of Toledo XVI states that the king's sword bearer, one Theudemund, clearly a Goth, must no longer hold the position of numerarius at Merida since this was contra generis uel ordinis sui usum. The numerarius was a civic official. He was elected by the bishop and citizens. ${ }^{35}$ It looks as if Goths were not supposed to hold this nor perhaps any position in local government. ${ }^{36}$

Notwithstanding the Roman character of its laws, it is clear that the identity of the kingdom was unambiguously Gothic. Its name proclaims it as the kingdom and the land (patria) of the Gothic people - and not only its name. For the king and the great majority of his councillors had Gothic names, and were clearly Goths, even though there were a few Romans among them too. ${ }^{37}$ At the same time, Romans, or at least men with Latin names, were very prominent among the bishops, ${ }^{38}$ outnumbering Goths by almost two to one up to the middle of the 7 th century. Subsequently the proportion of Gothic bishops attending the national synods increased to thirty-four and forty-two per cent, so that Goths came to form a much higher proportion of bishops than they can have formed of the population as a whole. ${ }^{39}$ So judging by the names of grandees (and names are practically the only evidence we have), secular government was in the hands of Goths, and the church too was becoming gothicized, though more gradually. Goths clearly remained the top people.

The importance of Gothic ethnicity in the mid seventh century is clearly shown in the canons of the 4 th, 5 th and 6 th Councils of Toledo. ${ }^{40}$ Canon

\footnotetext{
${ }^{33}$ LV 12.2.13; 12.2.14; Formulae manumissionis, Form Vis $\mathrm{n}^{0} 2,3,4,5,6$.

34 C. Toletana IX, c.13. In 657, LV 4.5.7 of Wamba confirmed denial of conubium omitting reference to Goths and Romans.

35 LV 12.1.2.

${ }^{36}$ Assuming that here genus is equivalent to gens, cf. C. Toletana VI, c.17 nisi genere Gothus.

37 Zeumer 1892, op. cit. (n. 8), 485-486.

38 J.N. Hillgarth, The Visigoths in History and Legend (Toronto 2009), 46. Among bishops the highest proportion of Germanic names is found in the Meseta, in the dioceses of Palencia and Salamanca, where it is likely that many Goths were settled.

39 Thompson 1969, op. cit. (n. 22), 289-296.

40 The synods were national institutions, working in close association with the king and his leading men. The constitutional phraseology of their canons ought not therefore to be dismissed as derived from "Old Testament models: as salvific, not an ethnic discourse" as it is by Gillett 2002, op. cit. (n. 2), 121.
} 
75 of the fourth Council of $663 \mathrm{AD}$ threatens with anathema anybody who breaks his oath of allegiance to the king. The purpose of this oath is to strengthen the position of the king and the stability of the Gothic people (pro robore nostrorum regnum et stabilitate gentis Gothorum) and to prevent a division between land and the gens (nullum patriae gentisque discidium per uim atque ambitum oriatur). That the gens and their land in this law are precisely the Goths, and not the entire population of Spain, can be seen from a subsequent clause which makes a distinction between the peoples inhabiting the whole of Spain on the one hand, and on the other the land and people of the Goths and the monarchy on behalf of whose security the oath has been taken. ${ }^{41}$ The canon goes on to state that on the death of the king, his successor is to be elected by the leadership of the whole gens (totius gentis primatus) together with the bishops in common assembly. The ethnic character of the monarchy is stated even less ambiguously in the canons of the fifth and the sixth Councils of $\mathrm{AD} 636$, which insist that the king must be of noble Gothic extraction. ${ }^{42}$

Ethnicity clearly did matter. The Goths did substitute an alternative to the nomen Romanum. The tribal cohesion of the Goths was a powerful 'shaping' factor in the establishment and functioning of the Visigothic kingdom. The Roman inhabitants evidently acquiesced in this situation. A system of government which used so many Roman institutions, above all Roman language, law, and administration based on writing, could only have functioned with the cooperation of the Roman population, but we still do not really know how the system worked.

We know practically nothing about local government at city or village level. Romans were certainly neither oppressed nor discriminated against. We have seen that the Church was for a long time essentially a Roman institution. Cooperation of the Visigothic kings with the Church, and use

41 J. Vives (ed.), Concilios visigóticos e Hispano-Romanos (Barcelona etc. 1963), C. Toletana IV, c.75: Quiquumque igitur a nobis (the bishops and royal councillors) uel totius Spaniae populis (Romans and Goths) qualibet coniuratione (...) sacramentum fidei sua, quod pro patriae gentisque Gothorum statu (the land and people of the Goths) uel obseruatione regiae salutis (the monarchy) pollicitus est, temeraverit... Gens and patria are distinct from populi also among electors of king Wamba in $\mathrm{AD} 672$ : Wamba (...) quem sacerdotalis unctio declarauit, quem totius gentis et patriae communio elegit, quem populorum amabilitas exquisiuit. B. Krusch \& W. Levison (eds.), Historia Wambae, MGH SRM 5 (Hannover 1910), 501, lines 16-18.

${ }^{42}$ C. Toletana $\mathrm{V}, \mathrm{c} .3$, of $\mathrm{AD}$ 636: nobody to be king quem nec electio omnium prouehit, nec Gothicae gentis nobilitas ad hunc honoris apicem trahit. So also C. Toletana VI, c.17 of AD 638: nullus (...) uel extraneae gentis homo, nisi genere Gothus (...) prouehatur ad apicem regni. 
of Church councils to legitimise royal authority were central features of the system of government. ${ }^{43}$ The Church controlled education, and thus was responsible for the maintenance of a skill essential for the working of an administration based on literacy. The national synods of the Church were a kind of parliament, even though they met only once or twice in a reign, Bishops had a routine role in the sanctioning of taxation. ${ }^{44}$ The Roman character of the Church therefore gave the Romans, or at least the Roman elite, a real say in the government of the country. It was even occasionally possible for Romans to hold important administrative posts. ${ }^{45}$

Nevertheless the practical significance of the difference between Goths and Romans steadily diminished. The fact that both people shared the lands of Hispania, and owed loyalty to the same king, brought them ever closer together. In the 6th and early 7 th centuries, Goths supplied the army of the kingdom, ${ }^{46}$ as they had done in the Ostrogothic kingdom of Theoderic. But by the reign of Erwig (AD 680-687) Goths and Romans were equally liable for military service when the kingdom was at war. ${ }^{47}$ In Julian of Toledo's Historia Wambae, written soon after 673, Hispania appears to be the unified nation. Julian can now describe Wamba's army as exercitus Hispaniae. ${ }^{48}$ When king Egica $(687-702)$ published an edict cancelling tax arrears, he addressed all his subjects alike, making no distinction between Goths and Romans. ${ }^{49}$ But this did not mean that

43 Thompson 1969, op. cit. (n. 22), 275-289; J. Arce, Esperando a los árabes: Los visigodos en Hispania (507-711) (Madrid 2011) in his chapter Concilios (pp. 233-259), summed up at p. 258: "Es una forma de cesaropapismo que consolida una alianza indisoluble entre Iglesia y el poder civil."

44 C. Toletana III, c.7; De fisco Barcinonensi: J.D. Mansi, Sacrorum conciliorum nova et amplissima collectio, vol. 10 (Paris 1901), 473-4.

45 Eg. Claudius, Dux of Lusitania, VPE, op. cit. (n. 29), 10-11. Leading men of the court included a few men with Roman names: Vitulus, Paulus (3 times), Severianus, Severinus, David are among secular grandees subscribing to the minutes of synods $653-693$. But the overwhelming majority of the names are Gothic (Zeumer 1892, op. cit. (n. 8), 485-486); Thompson 1969 op. cit. (n. 22), 255 .

46 LV 9.2.2 (Antiqua, Recc. Erv.): Serui dominici (...) quando Gotos in hostem exire conpellunt.

47 LV 9.2.9: (.. ) seu sit Gotus siue Romanus, necnon ingenuus quisque uel etiam manumissus (...) decimam partem seruorum suorum secum in expeditione bellica ducturus accedat.

48 Historia Wambae op. cit. (n. 41), 513. The Story of Wamba, Julian of Toledo's Historia Wambae Regis, translated with introduction and notes by J.M. Pizarro (Washington 2005), 200 (Historia), 227-229 (Insultatio).

49 Edictum Ervigii Regis de tributis relaxatis in Zeumer 1892 op. cit. (n. 8), 479-80. The concession is made omnibus populis regni nostri, tam priuatis quam etiam fiscalibus seruis, uiris seu etiam feminis, (...). 
'Gothicity' had been totally abandoned even then. The dux Paulus, who rebelled against Wamba in 673 , may have been a Roman, ${ }^{50}$ but the great majority of his leading followers had Gothic names, ${ }^{51}$ as had most of the grandees of king Wamba. ${ }^{52}$ The overwhelming proportion of the top jobs was surely still filled with Goths. Among the secular grandees signing the 16th Council of Toledo of 693, the predominance of Goths was as overwhelming as ever. Among the bishops, 25 had Gothic names and thirtyfive Roman. Roman bishops were still in a majority, but the proportion of Gothic bishops is much higher than the proportion of Goths in the total population as a whole. ${ }^{53}$ So even in this ecclesiastical council, taking clerical and secular members together, Goths were in a slight majority. ${ }^{54}$ The Goths were still the ruling people.

I hope to have shown that Goths had a strong sense of ethnic identity from the time that they entered Hispania to the time of the Arab conquest, that the Visigothic kingdom was in a real sense a kingdom of the Goths. This leaves an important question unanswered: Who exactly were these Goths? Who decided that somebody was now a Goth? How could somebody become a Goth? It is clear - to our generation at least that Gothicness cannot have depended entirely, or even mainly, on biology. The descendants of the immigrants from Gaul after the destruction of their kingdom in 507, must always have been a small minority in Hispania. ${ }^{55}$ It would have been impossible for the descendants of Alaric's Visigoths to have survived as a powerful military power for three centuries, the losses through battle and disease, the disruption of repeated migration, and of settlement in separate areas spread over the vast lands of Hispania, losses through intermarriage and through assimilation, unless they regularly received reinforcements from outside the gens. We lack evidence how exactly this happened. We can only speculate. It is likely enough that Romans who helped the Gothic king run his administration at court, and in much greater numbers at city level, might eventually simply be accepted as Goths or even formally admitted into the gens by the

\footnotetext{
50 Thompson 1969 op. cit. (n. 22), 214 n. 5.

51 Historia Wambae, op. cit. (n. 41), 506, 507, 512, 513, 531-533.

52 Thompson 1969, op. cit. (n. 22), 226. Zeumer 1892 op. cit. (n. 8), 486.

53 Thompson 1969, op. cit. (n. 22), 295.

54 Goths sometimes took Christian / Roman names (Thompson 1969, op. cit. (n. 22), 289).

55 G. Ripoll Lopez, 'The Arrival of the Visigoths in Hispania', in Pohl 1998 op. cit. (n. 11), $153^{-187}$, on $160-162$ on the very rough estimates of the Gothic population. Her very hypothetical figure of 130,000 individuals, or some 20,500 families is probably an overestimate.
} 
king. Clients, ${ }^{56}$ buccellarii, ${ }^{57}$ and tenants of Gothic landlords might conceivably be treated as if they were Goths, at least as Goths of the lower order. ${ }^{58}$ Though we cannot observe the process by which Romans became Goths, we can be certain that the Goths who entered Spain from Gaul after 507 represented only a small proportion of the ancestors of the Goths who were defeated by the Arabs after 711 .

\section{Goths after the Arab Conquest ${ }^{59}$}

The Arab invasion ended the Gothic kingdom for ever. This is clear from the Chronicles. ${ }^{60}$ Up to the Islamic conquest the Spanish kingdom is regularly referred to as 'the kingdom of the Goths. ${ }^{\prime} 1$ The army defeated by the Arabs is the army of the Goths. ${ }^{62}$ But, after the defeat and death of king Roderic, both the army and the kingdom of the Goths simply disappeared from the narrative. In the regions under Islamic rule Gothicization was replaced by Islamization. Gothic nobles cooperated with the new masters of Hispania during the early years of the Arab take-over. ${ }^{63}$ Local nobles play a leading part in troubles in the early days of Arab rule. They now

56 LV (Antiqua, Recc. Erv.) 5.3.1-4.

$57 L V$ 5.3.1; Saiones ( $L V$ 5.3.2) seem to have been a superior kind of dependent, since weapons given to a saio by his patron became his permanent property, while a buccellarius would have to return them if he changed patron.

58 The Visigothic kings adopted the Roman legal privileging of honestiores over humiliores. On Roman practice see P. Garnsey, Social Status and Legal Privilege in the Roman Empire (Oxford 1970) 222-233. Gothic examples: $L V$ 2.4.3; 8.3.12; 9.3.3.

59 R. Collins, The Arab Conquest of Spain, 710-797 (London 1989).

60 J. Gil Fernández, J.I. Ruiz de la Peña (eds.) \& J.L. Moralejo (tr.): Crónicas asturianas (Oviedo 1985) includes both versions of the Crónica Alfonso III and the Crónica Albeldense. On the chronicles see Hillgarth 2009, op. cit. (n. 38), 66-73. The chronicle of Alfonso III is transmitted in two versions, both early 1oth century, of which the one preserved at Roda (abbreviated Rot.) is thought closer to the original and more reliable than the other, abbreviated Seb. (because preceded by a letter to Sebastianus). English translation of Roda version in Wolf 1990, op. cit. (n. 31), 159-177, of Chronicle of 754, 111-158. The Crónica Albeldense is not included.

61 Chronicle of Alfonso III (Rot.), Wolf 1990, op. cit. (n. 31), c.4, 6, 7. Chronicle of $754=$ Continuationes Isidorianae, Th.Mommsen (ed.), MGH, AA 11, Chronica minora saec. IV.V.VI. VII. (II), 349 line 29, $35^{0}$ line 29, 35 lines 5 and $11=$ Wolf 1990, op. cit. (n. 31), 111-158, in c. $44,51,52,54$.

62 Wolf 1990, op. cit. (n. 31): Chronicle of Alfonso III (Rot.), c. 7, 9; Chronicle of 754 c. 52.

63 On Theodemir (Theudimer): Chronicle of $754=$ Continuationes Isidorianae, op. cit. (n. 61), 354; tr. Wolf 1990, op. cit. (n. 31) c. 87; P. Guichard, Structures sociales 'orientales' et 'occidentales' dans l'Espagne musulmane (Paris 1977), 241-290. 
have Arab names and have converted to Islam, ${ }^{64}$ though they maintained, perhaps correctly, that they were descendants of Gothic families. ${ }^{65}$

A Christian kingdom in the North, a region not conquered by the Arabs, was established, but it was no longer a kingdom of the Goths. The text of a donation by King Alfonso II (791-842) to the church of Oviedo tells us that "in the era 749 (AD 711) the glory of the kingdom (of the Goths) was lost together with its king Roderic. From this disaster thy right hand, O Christ, rescued thy servant Pelagius, who being raised to the power of prince (princeps)... struck down the enemy and defended the gens of the Christians and Asturians." ${ }^{\prime 6}$ The oldest of the northern chronicles, the Chronicle of Albelda, which originally ended in $\mathrm{AD}$ 881, states that: "The kingdom of the Goths perished and with it by fear or the sword all the flowers of the Gothic people", ${ }^{67}$ and, as we have seen, even the later so-called Chronicle of Alfonso III is explicit that the Kingdom of the Goths had come to an end. The Christian kings of the North were kings of Asturia. They are never described as 'kings of the Goths'. Their identity was not ethnic but territorial, and above all Christian. But they did claim to be heirs of Gothic kings. The dynasty may well have had a Gothic origin, though in a chronicle closely associated with the dynasty, their links with the Gothic royal family, people and institutions are clearly exaggerated. In the more reliable version of the Chronicle of Alfonso III, Pelayo (Pelagius), who won a decisive victory over the Arabs and became founder of a Christian realm in the North, is said to have been, as indeed he probably was, a noble Goth, and Alfonso I, his son in law, the founder of the Asturian dynasty, is said to have been of 'the royal line'. ${ }^{68}$ According to the less reliable version of the Chronicle, Pelagius was elected to be their prince

64 On these mulawads see Wolf 1990, op. cit. (n. 31): Chronicle of Alfonso III, c. 22, $25-26$.

65 D.J. Wasserstein, 'Inventing tradition and constructing identity: the genealogy of Umar ibn Hafsūn between Christianity and Islam', Al-Qantara 23 (2002), 269-297. R. Marín-Guzmán, 'Rebellion and political fragmentation of al-Andalus: A study of the revolt of Umar ibn Hafsūn in the period of the Amīr abd Allāh (888-912)', Islamic Studies 33.4 (1994), 419-473. E. Manzano Moreno, La frontera de al-Andalus en época de los Omeyas (Madrid 1991), 110-129. On the Banu Qasi: Chronicle of Alfonso III (Rot.), 25-26. A. Christys, Christians in al-Andalus 711-1000 (Richmond 2002), 158-183, about Ibn al-Qutiyya.

66 A.C. Floriano, Diplomática española 1, 118-141, on 120, cited by Hillgarth 2009 op. cit. (n. 38), 65. Its authenticity has been doubted but according to Hillgarth it is now generally thought be dependable.

67 Crónica Albeldense, 17.3 = Gil Fernández 1985, op. cit. (n. 6o), 183.

68 The story: J. Gil Fernandez (ed.), Crónica Rotensis, in Id. 1985, op. cit. (n. 6o), 122-130, transl. Wolf 1990, op. cit. (n. 31), c. 8-11. Pelayo (Pelagius) the son of a Gothic duke killed by king Wittiza, wins a great victory over the Arabs, and becomes king of the Asturians. 
(princeps) by Gothic refugees who had fled from the Arabs to Asturia. ${ }^{69}$ Peter of Cantabria, the brother in law of Pelagius, and father of Alfonso I, is described as a descendant of the Gothic Kings Leovigild and Reccared. ${ }^{70}$ According to the Chronicle of Abelda, king Alfonso II (791-842) of Asturia "restored the whole Gothic ordo at Oviedo, as it had been at Toledo."71 It was not only members of the royal family who felt it worth while to preserve a link with the lost Gothic past. Early documents from Asturia show that the king's landed subjects preferred Gothic to Latin i.e. Roman names. The same was true in neighbouring Catalonia, which did not become an independent kingdom, but, for a time at least was part of the Frankish world. ${ }^{72}$ In the region of Santillana, charters still refer to Goths and Romans as separate peoples in the eleventh century. ${ }^{73} \mathrm{~A}$ mixture of historical facts and of legends about the Goths has helped to shape Spanish identity until to day.

\section{Conclusion}

The Leges Visigothorum show that, except in respect of the special category of land created by the division of estates between Goths and Romans at the time of the original Gothic settlements, Goths were not privileged over Romans legally. The royal legislation applied to both peoples, yet a distinction between Goths and Hispano-Romans was maintained right until the end of the kingdom. The Goths remained the ruling people, and the king's principal advisers were overwhelmingly Goths. In a situation like that the historians look for 'strategies of distinction' and 'markers of ethnic identity'. ${ }^{74}$ In the case of the Goths in Spain these are not all obvious, or at least they become progressively less conspicuous. The Goths adopted the language and religions of the Romans, and their legislation was largely based on principles of Roman law. Judging by the development of the broaches and belt buckles buried with Gothic dead, their dress or at least its ornaments became assimilated to Byzantine-Mediterranean

His daughter marries Alfonso, son of Peter, leader of the Cantabrians, 'of the royal (i.e. Gothic?) line', who became king of Asturia and founder of a dynasty.

69 Gil Fernandez 1985, op. cit. (n. 6o), Crónicas Asturianas, 123.

70 Crónica Rotensis 13 = Crónicas Asturianas, 131.

71 Crónica Abeldense, $15 \cdot 9$ = Crónicas Asturianas, 174.

72 Hillgarth 2009, op. cit. (n. 38), 78.

73 Hillgarth 2009, op. cit. (n. 38), 79.

74 See Pohl 1998 op. cit. (n. 11), passim. 
fashion. ${ }^{75}$ They also seem to have abandoned distinct burial-customs. We cannot know whether they retained distinctions in dress. While royal officials had Roman titles, we do not know whether these titles disguise positions which were perhaps more ethnic than Roman. E.A. Thompson has argued that in the reign of Reccesuinth (649-672) the Latin title iudex territorii was replaced by the Gothic thiufadus. ${ }^{76}$

Kulikowski is right to emphasize how badly informed we are about Gothic ethnicity, but what we know is enough to show that it was of fundamental importance. It was ethnic solidarity that enable Goths to win power first in Gaul and then in Spain, and to preserve the unity of their kingdom. Subsequently, it was the prestige and patronage of power which enabled the gens to maintain and enlarge its numbers. The story of the Goths is one of continuous transformation, involving both continuities and discontinuities, but to quote Heather, "without continuities there would have been no story", without an active Gothic sense of ethnic solidarity there could have been no Gothic kingdom in Spain.

Latinization and Romanization too were essential elements in the creation and preservation of the kingdom. Latin language, Roman law and techniques of government, and Catholic religion, became so closely associated with the ruling gens that the kings, who established a kingdom in Asturia after the fall of the Gothic kingdom, proclaimed themselves the heirs not of the Romans but of the Goths.

This development reflected Late Roman culture. Roman language, religion and literature were honoured and transmitted in Gothic Spain, as in other parts of the late Roman world, but Roman culture no longer provided an emotional basis for a sense of Roman identity, or to use an anachronistic concept, for Roman patriotism. The wars which Leovigild (566-586) fought to unify Spain under Visigothic rule were fought against people who in law still were Roman citizens. But John of Biclaro and Isidore of Seville defined them not as Romans, but geographically and territorially by where they lived. ${ }^{77}$ In his Etymologies, Isidore explains the

75 G. Ripoll López, 'Symbolic life and signs of identity in Visigothic times', in P. Heather (ed.), The Visigoths from the Migration Period to the Seventh Century (San Marino 1999), 403-431, esp. 414-424; Idem, Toreutica de la Bética (siglos VI y VII D.C.) (Barcelona 1998). S.A. Barney et al., The Etymologies of Isidore of Seville (transl. introd. \& notes, Cambridge 2006) 19.23.7: 'Moustaches and red paint of the Goths'.

76 Thompson 1969, op. cit. (n. 22), 213.

77 John of Biclaro, transl. Wolf 1990, op. cit. (n. 31), c. 27, 32, 36, 40, 47, Isidore, History c. 49. Only the wars which Sisebut and Suinthila fought to expel the Byzantines from their last possessions in Spain are said to have been fought against Romans (Isidore, History 
term ciuitas as the collective description of the inhabitants, as opposed to the buildings, of a city. He does not mention Roman citizenship, which had been one of the principal bonds of the earlier empire, and whose possession had defined an individual as a Roman. ${ }^{78}$ The concept of citizenship had lost its power to unite and to confer identity, and Roman culture had become separated from Roman identity. The Goths could appropriate a great deal of Roman culture without feeling that they had become Romans. Roman culture became Gothic culture. So it came about that a mixture of historical facts and of legends about the Goths has helped to shape Spanish identity until today.

c. 61-62). But this does no mean that 'Romans' in Hispanic sources of the time invariably means 'Byzantines'.

78 Isidore, Etymologies, Barney 2006 op. cit. (n. 75), 15.2.1. 


\title{
MASTERS AND FREEDMEN: \\ JUNIAN LATINS AND THE STRUGGLE FOR CITIZENSHIP
}

\author{
Egbert Koops
}

\section{Citizenship and Problems of Integration*}

Grants of citizenship have long been recognized as an important factor in the integration of the Roman empire. ${ }^{1}$ The state was continually seeking to replenish and increase its citizen body by offering membership as something of value. Citizenship provided status and entry into patronage networks as well as more tangible benefits. It gave the somewhat theoretical right to vote, at least for men, and to pursue a career in the legions. But more importantly, it allowed access to the full extent of the Roman legal system. From access to the courts and the capacity to own land in Italy, to marry other citizens in recognized marriage, to pass citizenship on to children, to make wills and inherit, all the way to the right of appeal to the emperor - citizenship meant full participation in the empire. Nominally, any Roman citizen could take part in law, commerce and politics on an equal footing to any other citizen. And though citizenship alone did not ensure access to power, which also depended on birth, wealth, opportunity and ability, it was advantageous enough to be desirable.

Ciuis Romanus sum is the famous expression that ought to, and in theory would ensure favorable treatment throughout the empire. ${ }^{2}$ Yet it did not detract from other identities. An example is offered by the apostle Paul. Born in Tarsus, on the southern coast of Turkey, in a Jewish community, he addressed a crowd in Jerusalem in Aramaic after asking permission to do so in Greek. ${ }^{3}$ But when he was arrested, he claimed the status

\footnotetext{
* Parts of this chapter, particularly par. 1-2 and 5, expand on a previous article: E. Koops, 'Second-rate citizens: Junian Latins and the Constitutio Antoniniana', Maastricht Journal of European and Comparative Law 19 (2012), 223-239.

1 See, inter alia, J.A. Crook, Law and life of Rome (New York 1967), 38-43; A.N. SherwinWhite, The Roman citizenship (Oxford $1973^{2}$ ), $167 \mathrm{ff}$; C. Williamson, The laws of the Roman people (Michigan 2005), 227-229. For a sociological perspective, F. Gross, Citizenship and ethnicity (Westport 1999), 29-32.

2 Cic., 2 Verr. 5.147, 5.162 and 5.168.

${ }^{3}$ Acts 22.23-29. B. Witherington, The acts of the apostles (Grand Rapids, Carlisle 1998), $680-683$.
} 
of a Roman citizen and impressed upon the local commander that he was born as one, while the commander had paid a large amount of money for the privilege. In a bronze tablet found at Banasa (Morocco), ${ }^{4}$ citizenship is bestowed on a tribal leader and his family at his express request. The emperor, Marcus Aurelius in this case, stresses that although such requests are not normally granted, a reward for the leader's prominence and loyalty may inspire others to emulate his example. Even so, the citizenship is awarded saluo iure gentis, without prejudice to tribal law. ${ }^{5}$ Whether or not such Roman citizens in the provinces felt themselves 'Roman' is an interesting question, but a moot point here. As members of their tribe, they may have been ruled by tribal law; but as Roman citizens, they were by necessity integrated into the machinery of empire.

A modern analogy may be useful. In 1992, the Treaty on the European Union was signed at Maastricht, creating a single European polity that embraces the national citizens of many Western European countries. Article G sub C states: "Citizenship of the Union is hereby established. Every person holding the nationality of a Member State shall be a citizen of the Union." ${ }^{6}$ This phrasing led to such antagonism, including the rejection of the Maastricht Treaty by Denmark, ${ }^{7}$ that a further clause was added five years later: "Citizenship of the Union shall complement and not replace national citizenship." ${ }^{8}$ All citizens of EU countries are also European citizens, which entitles them to work and travel freely, and to vote and stand for municipal or European office throughout the EU. ${ }^{9}$ They may feel themselves German, French or Dutch, and remain so at law, but they exercise a

$4 A E$ 1971, 534. W. Seston and M. Euzennat, 'Un dossier de la chancellerie romaine: la Tabula Banasitana', Comptes Rendus de l'Académie des Inscriptions et Belles Lettres (1971), 468-490; A.N. Sherwin-White, 'The tabula of Banasa and the constitutio Antoniniana', Journal of Roman Studies 63 (1973), 86-98.

5 Dual citizenship was well established by the reign of Claudius. A.N. Sherwin-White, 'The Roman citizenship. A survey of its development into a world franchise', ANRW I.2 (Berlin/New York 1972), 46-53; but also see D. Nörr, s.v. 'Origo', RE Supp. 10 (1965), 431-473. The Banasa grant was notably also made without prejudice to the tributes and vectigals due to the aerarium and the fiscus.

6 Art. G sub C of the Treaty of Maastricht, [1992] C 191/1.

7 The Edinburgh Agreement of December 1992 (C 348, 31 December 1992) granted Denmark an exception, following its rejection of the Maastricht treaty by referendum, to the effect that European citizenship in no way replaced national citizenship (section A).

8 Art. 2 sub 9 of the Treaty of Amsterdam, [1997] C 340/173.

9 Art. 20-22 of the consolidated Treaty on the Functioning of the European Union, $2008 \mathrm{C} \mathrm{115/47.} \mathrm{On} \mathrm{the} \mathrm{ease} \mathrm{of} \mathrm{travel} \mathrm{within} \mathrm{the} \mathrm{Roman} \mathrm{empire,} \mathrm{see} \mathrm{Arist.,} \mathrm{Or.} \mathrm{26.100-101}$ (ed. Keil). 
right inherent in their European citizenship when they wish to work in, or travel to, another EU country; a right, moreover, which no national state can provide. In the same way, a tribal leader in Mauretania was no less a Roman citizen were he to move to Rome, even saluo iure gentis.

The Banasa tablet shows that citizenship grants were instruments in a policy that was designed to spread and consolidate Roman rule in the provinces. Veteran soldiers and members of the provincial elite were rewarded with citizenship for their services, which came at little cost, served as a badge of distinction, and drew them closer to Rome. The tribes of Latium as well as certain other allies had been granted this favor to ensure their loyalty during the Social War. ${ }^{10}$ But not all tribes and tribal leaders were treated equally: in $89 \mathrm{BC}$, the transpadani, non-Romans living between the southern Alps and the river Po, were invested with the so-called ius Latii, ${ }^{11}$ the right to acquire full Roman citizenship if they fulfilled an additional requirement, in this case a term as a magistrate at home. ${ }^{12}$ This type of intermediate citizenship was referred to as Latinitas, ${ }^{13}$ though it had little to do with the ancient tribes of Latium. ${ }^{14}$ Unlike Roman citizenship, which was vested primarily in individuals, ${ }^{15}$ the (coloniary) Latin status was granted to communities and became available to individuals by being a citizen of such a community. Some coloniae with Latin status expanded from veteran settlements, others already existed but were granted the status as an honorific once they had proven to be 'Roman' enough. ${ }^{16}$ Curiously, Vespasian even granted Latin status to the whole of Spain. ${ }^{17}$

10 G. Rotondi, Leges publicae populi Romani (Milan 1912, repr. 1990), 338-341. On the unification of Italy by imposing the Roman model at the municipal level, see SherwinWhite 1972, op. cit. (n. 5), 33-40.

11 L. Pompeia de transpadanis (89 BC). Rotondi 1912, op. cit. (n. 10), 342. The transpadani were later granted citizenship by Julius Caesar: l. Roscia de Gallia cisalpina (49 BC), Rotondi, op. cit., 416; Sherwin-White 1972, op. cit. (n. 5), 40-45.

12 Under the 'minor' Latin right, magistrates could acquire citizenship; the 'major' Latin right, possibly introduced by Hadrian, gave the same option to decuriones. Gaius, Inst. 1.96. Cf. rubrica 21 of the Flavian municipal laws.

13 'Latinitas': Cic., Att. 14.12. 'Latio donati': Plin., nat. 3.20.135. 'Latium dedit': HA, Hadr. 21.7. 'Ius latii': Gaius, Inst. 1.95.

14 Gaius, Inst. 1.79.

15 With the exception, perhaps, of the ius italicum, the right to be considered Italian soil, which was conferred by the emperors on some communities. Sherwin-White 1972, op. cit. (n. 5), 53 .

16 Sherwin-White $1973^{2}$, op. cit. (n. 1), 59-61, 114 f; 222 f.

17 'Latium tribuit': Plin., nat. 3·3.30. 
Latin status was, in essence, a second-class or intermediate form of citizenship. It offered many of the legal rights enjoyed by Roman citizens, but not all, and none of the more important political rights. ${ }^{18}$ In consequence, it was attractive enough, to some, to seek improvement of the Latin status, which was quite possible although it may have been costly. A main mode of gaining the franchise has already been mentioned: by taking on the (expensive) munus of a city councillor, inhabitants of Latin communities could become full citizens at the end of their term. ${ }^{19}$ Latins also became citizens by financing the all-important grain deliveries to Rome for a set period of time, through shipping or milling facilities. ${ }^{20}$ And after the great fire of $64 \mathrm{AD}$, Nero offered full citizenship to those willing to spend half their wealth, with a minimum of 100.000 sesterces, on construction work. ${ }^{21}$ Such grants tapped into the willingness Latins presumably showed to spend their wealth in exchange for civic recognition. ${ }^{22}$

Other avenues were open as well. Children originally followed the status of the 'lowest' parent, in accordance with the lex Minicia (passed before $90 \mathrm{BC}$ ), and would only acquire full citizen status if born out of legally recognized marriage (conubium). ${ }^{23}$ In consequence, mixed marriages would generally lead to a deterioration of status. However, the imperial policy of extending both the Latin and Roman citizenship led to an enactment of the Senate, by which a citizen-parent who had demonstrably erred as

18 Latins could employ the Roman forms of contract (Ulp. Epit. [=UE] 19.4-5) and have them enforced, own Quirite property, make wills (UE 11.16) and take under wills (UE 22.1), and retain their status if they moved to a different Latin community. It is doubtful in imperial times whether they could vote in the comitia tributa if resident at Rome (Liv., 25.3). They could not, in any case, enter into a legally recognized marriage with a Roman citizen unless the right of conubium was explicitly granted to them (UE 5.4). Nor were they allowed to hold Roman magistracies. Sherwin-White 1972, op. cit. (n. 5), 43: "only of practical value for the man who was prepared to leave his native municipium and follow a career in the service of Rome".

19 Gaius, Inst. 1.96; L. Irnit. 21.

20 An edict by Claudius offered full rights to Latins who shipped grain to Rome for six years with a vessel that could carry at least 10.000 modii (Gaius, Inst. 1.32c; UE 3.6, roughly $67.000 \mathrm{~kg}$ ). Trajan offered Roman citizenship to those who operated a mill in Rome for three years, grinding 100 modii of flour a day (Gaius, Inst. 1.34; UE 3.1). Major shippers, that is those shipping over 50.000 modii, were also excused from public duties: D. $50.5 \cdot 3$ (Scaev., 3 Reg.).

21 Gaius, Inst. 1.33.

22 Crook 1967, op. cit. (n. 1), 45. But also see paragraph 5 , below. In a recent contribution Morley seems to apply these texts to slaves, which misses the point (N. Morley, 'Slavery under the Principate', in K. Bradley and P. Cartledge (eds.), The Cambridge world history of slavery I: the ancient Mediterranean world [Cambridge 2011], 279).

23 Gaius, Inst. 1.56 and 1.78-79; UE 5.8. Rotondi 1912, op. cit. (n. 10), 338. 
to the status of his or her spouse, and had married 'down', could gain full citizenship both for the spouse and for the child. ${ }^{24}$ A decree, possibly the same, which the Senate passed under Hadrian, stipulated that the child of a Roman woman would always be a Roman citizen. ${ }^{25}$ Taken together, these two rules imply that the lex Minicia was overturned, leading to an amelioration of children's status and a growing reservoir of Roman and Latin citizens. Grants and births divorced Roman citizenship from any geographical, 'national' or ethnic connotation, turning it into a tool for integration and a matter of birth, merit or wealth. The end of the story is familiar: in $212 \mathrm{AD}$, Caracalla awarded all - or many - of the peoples within the borders of the empire Roman citizenship through a block grant, the constitutio Antoniniana. ${ }^{26}$

\section{Freedmen and Citizenship}

Slavery was an everyday reality in the classical world. Yet the Roman system of slavery differed in some key aspects from that of other civilizations. First, the gates to freedom were never closed. ${ }^{27}$ Many urban slaves acquired freedom in their lifetime, and it seems a fair number, not in the last place members of the familia Caesaris, could reasonably expect manumission from the age of thirty. ${ }^{28}$ It should be noted, though, that

24 Gaius, Inst. 1.67-72; UE 7.4. The sources do not mention what a probatio causae erroris entailed. J.F. Gardner, 'Legal stumbling-blocks for lower-class families in Rome', in B. Rawson and P. Weaver (eds.), The Roman family in Italy (Oxford 1997), 51-53. Only former slaves who were dediticii were barred from acquiring citizenship in this manner: Gaius, Inst. 1.67.

25 Gaius, Inst. 1.30 and 1.80; UE 3.3. Gardner 1997, op. cit. (n. 24), 38-39.

26 D. 1.5.17 (Ulp. 22 Ed.); Dio Cass. 78.9.5. See, instead of others, C. Sasse, Die Constitutio Antoniniana (Wiesbaden 1958) and P.A. Kuhlmann, Die Giessener literarischen Papyri und die Caracalla-Erlasse (Giessen 1994). The grant may have been a mere affirmation of the status quo: Sherwin-White $1973^{2}$, op. cit. (n. 1), 22; 287. The traditional date of $212 \mathrm{AD}$ has been questioned by F. Millar, 'The date of the constitutio Antoniniana', Journal of Egyptian Archaeology 48 (1962), 124-131.

27 The reasons adduced for the social practice of manumission are manifold. However, this is not the place to discuss such motives.

28 G. Alföldy, 'Die Freilassung von Sklaven und die Struktur der Sklaverei in der römischen Kaiserzeit', in Die römische Gesellschaft (Stuttgart 1986), 286 ff.; P.R.C. Weaver, Familia Caesaris (Cambridge 1972), 98-102; G. Alföldy, Römische Sozialgeschichte (4th ed., Stuttgart 2011), 188-189. More cautious: K. Bradley, 'Slavery in the Roman Republic', in K. Bradley and P. Cartledge 2011, op. cit. (n. 22), 256; Morley 2011, op. cit. (n. 22), 281-282. Contra P. Garnsey, 'Independent freedmen and the economy of Roman Italy under the Principate', Klio 63 (1981), 359-371; W. Scheidel, 'Quantifying the sources of slaves in the early Roman 
the expectations and social status of slaves in the cities differed markedly from that of those working the mines and country estates. There was probably little hope for the latter. But in the cities, freedmen formed a large and economically active segment of the population, as evidenced in a wealth of inscriptions. ${ }^{29}$ Though free, they remained at a social and legal disadvantage, since the 'stain' of slavery never disappeared entirely. Social exprobation aside, in practice this meant that they were barred from certain offices ${ }^{30}$ and were forbidden to marry into the senatorial order. ${ }^{31}$ They also owed duties to their patrons, varying from deference to the more burdensome obligation to put part of their labor at a patron's disposal. ${ }^{32}$ Even so, their children or grandchildren bore no such stigma ${ }^{33}$ and could enter the upper ranks of Roman society. Finley rightly called them "evanescent" as a status group. ${ }^{34}$

Secondly, although manumission did not release the freedman from duties towards his former master, the manumission itself was an irrevocable act, ${ }^{35}$ as can be seen from the so-called accusatio liberti ingrati. Under

empire', Journal of Roman Studies 87 (1997), 156 ff; W. Scheidel, 'Human mobility in Roman Italy, II: the slave population', Journal of Roman Studies 95 (2005), 76. Also see the discussion by H. Mouritsen, The freedman in the Roman world (Cambridge 2011), 131-136.

29 Morley 2011, op. cit. (n. 22), 268-269. For a discussion of several difficulties of interpretation, see H. Mouritsen, 'Freedmen and decurions: epitaphs and social history in imperial Italy', Journal of Roman Studies 95 (2005), 38-63; Mouritsen 2011, op. cit. (n. 28), $123^{-131 .}$

30 CJ 10.33(32).1 (Diocl./Max.).

31 D. 23.2.44 pr. (Paul., 1 l. Iul. et Pap.). Rotondi 1912, op. cit. (n. 10), 443-445; J.F. Gardner, Being a Roman citizen (London/New York 1993), 39.

32 Gardner 1993, op. cit. (n. 31), $20 \mathrm{ff}$. Operae were probably less of a burden than they are commonly made out to be. After the Rutilian edict of 118 BC (D. 38.2.1 pr.-1, Ulp., 42 ad ed.; D. 38.1 .2 pr., Ulp., 38 ad ed.), the imposition of operae was only enforceable if the freedman had sworn to perform them (D. 38.1.7.2, Ulp. 28 ad Sab.; D. 40.4.36, Paul., 7 Plaut.) or promised them by stipulation after his release. See D. 38.1.31 (Mod., 1 reg.). They could not consist of the obligation to pay money (D. 38.1.32, Mod. 1 reg.). Moreover, neither those manumitted by testament, whether conditionally (statuliber) or unconditionally (orcinus), nor those freed and instituted as heirs (heres necessarius), nor those who had been freed per fideicommissum (after Hadrian, D. 38.1.7.4, Ulp., 28 ad Sab.; D. 38.1.42, Pap. 5 resp.; D. 38.2.29 pr., Marc. 9 inst.), nor those who had bought their freedom suis nummis (after Marcus Aurelius, D. 38.1 .13 pr., Ulp., 38 ad ed.), could be required to perform operae at all either because they had no patron, or because their patron had little rights over them. Cf. W.W. Buckland, The Roman law of slavery (Cambridge 1908, repr. 1970), 425; 525.

33 B. Rawson, 'Family life among the lower classes at Rome in the first two centuries of the empire', Classical Philology 61 (1966), $72 \mathrm{f}$. The children of freedmen citizens were freeborn Roman citizens if born in iustae nuptiae. The children of Junian Latins probably became freeborn Latins. P.R.C. Weaver, 'Children of Junian Latins', in B. Rawson and P. Weaver (eds.), The Roman family in Italy (Oxford 1997), $57 \mathrm{ff}$.

34 M.I. Finley, The ancient economy (2nd ed., Berkeley 1985, repr. 1999), 77.

35 Gardner 1993, op. cit. (n. 31), 20. It could not be limited in time either. Any clause to the contrary was invalid. D. 40.4.33 (Paul., 12 quaest.); D. 40.4.34 (Paul., 74 ad ed.). 
the lex Aelia Sentia of $4 \mathrm{AD}$, the most important of Augustus' manumission laws, a complaint could be brought before the praefectus urbi or the provincial governor against a freedman who had acted wrongly against his patron. The matter was then investigated and punishments were pronounced and administered by the State. The freedman ran the risk of being reprimanded, caned or temporarily banished, of forfeiting part of his patrimony, of being returned into patria potestas or, in extreme cases, sentenced to the mines. ${ }^{36}$ What is notably missing, is the possibility of a return to slavery under the former master.

From this range of punishments it may appear freedmen had no more than a liberty that was conditional on keeping their patrons happy. It should be noted, though, that for a successful accusatio worse was needed than a mere lack of deference. ${ }^{37}$ The jurists' examples run from dereliction in need, through slander and physical violence, to informing on the patron. No specific norms for punishment were developed, other than a general notion that the punishment should fit the crime, and the matter is usually discussed in advisory manuals written for the benefit of proconsuls and urban prefects, rather than as a point of substantive law. ${ }^{38}$ Thus, the jurists' treatment of the accusatio need not be construed as evidence of a strong patronal interest, but is more likely inspired by the highly factual nature of the cases, by the fact that they were judged by magistrates cum imperio, and also by their relative scarcity. Patrons were not necessarily arraigned against freedmen, since the two categories could and often did overlap. ${ }^{39}$ As such, a private right to discipline freedmen at will, going beyond mild chastisement, would have been a dangerous weapon indeed.

The matter is illustrated by a famous Senate debate in 56 AD. ${ }^{40}$ The lack of gratitude and deference, which many freedmen allegedly displayed, gave rise to a motion empowering patrons to withdraw liberty at will. It was argued in council ${ }^{41}$ before the emperor, that many persons of

36 See the range mentioned in D. 1.16.9.3 (Ulp., 1 off. proc.); D. 1.12.1.10 (Ulp., sing. de off. praef. urbi); D. 25.3.6.1 (Mod., sing. de man.); D. 37.14.1 (Ulp., 9 off. proc.); D. 37.14.7.1 (Mod., sing. de man.). Buckland 1908, op. cit. (n. 32), 423; Gardner 1993, op. cit. (n. 31), 45-48.

37 Gardner 1993, op. cit. (n. 31), 41-43.

38 Buckland 1908, op. cit. (n. 32), 423; Gardner 1993, op. cit. (n. 31), 43.

39 Gardner 1993, op. cit. (n. 31), 25.

40 Tac., Ann. 13.26-27. K. Hopkins, Conquerors and slaves (Cambridge 1978), 130; Gardner 1993, op. cit. (n. 31), 44; Mouritsen 2011, op. cit. (n. 28), 55-56; K. Harper, Slavery in the late Roman world, Cambridge 2011, 487-488.

41 The text is corrupt here, and 'past restoration' according to Jackson, The Annals vol. 5 (Loeb: London 1937), 45 . 
distinction descended from the freedman class, and that if freedmen were set aside, the dearth of freeborn would become evident. Nero decided that the rights of the freedman class should not be reduced. In classical Roman law, the gift of liberty, once granted, could not be revoked. This remained true until Constantine overturned the rule in $320 \mathrm{AD}$, giving patrons the right to withdraw or diminish the citizen status of their freedmen after a successful accusatio. ${ }^{42}$ Though there appear to be classical predecessors of such a right, notably in decisions by Claudius ${ }^{43}$ and Commodus, ${ }^{44}$ it should be remembered that in these exceptional cases it was still the State, and not the patron, who was depriving a freedman of his liberty. This is borne out by a number of rescripts from the chancery of Diocletian, in which the emperor asserts that there is absolutely no right to private reuocatio by the patron. ${ }^{45}$ Roman authors themselves saw this as a typically Roman institution. 46

Thirdly, the Roman law of manumission differed from that of other slave systems by granting full citizenship to freedmen. This is remarkable. ${ }^{47}$ The release of a slave by a Roman citizen effected a transition from one status group to another. Property became a person and the slave became a Roman citizen, in some ways equal to his former master. Such a transition required clear rules which reflect the State's interest in the newly

42 CJ 6.7.2 pr. ( = CTh 4.10.1); Inst. 1.16.1. A diminution of the freedman's status to Junian Latin gave the patron rights against the inheritance of his freedman: CTh 2.22 sing. Buckland 1908, op. cit. (n. 32), 423; Harper 2011, op. cit. (n. 40), 487-488.

43 D. 37.14.5 pr. (Marc., 9 reg.). The freedman had persuaded informers to open a case regarding the liberty of his own patron. Claudius ordered that he should be returned to slavery. The decision may have been motivated by the consideration that if the informers had been successful, the manumission of the freedman would have been invalid in any case. Also see the somewhat conflicting accounts in Suet., Cl. 25.1; Dio Cass., 60.13.2 and 6o.28.1. Buckland 1908, op. cit. (n. 32), 423; Gardner 1993, op. cit. (n. 31), 43-44; Mouritsen 2011, op. cit. (n. 28), 55. This is probably an isolated case: if the right were general after Claudius, there would have been no need for the Senate debate under Nero.

44 D. 25.3.6.1 (Mod., sing. de manumiss.). The freedman is auctioned as a slave and the patron is handed the purchase price, and then only in second instance, after he has already been placed under patria potestas of the patron. Buckland 1908, op. cit. (n. 32), 424; Gardner 1993, op. cit. (n. 31), 48; Mouritsen 2011, op. cit. (n. 28), 56.

45 CJ 7.16.20 (a. 293); CJ 7.16.23 (a. 293); CJ 7.16.26 (a. 294); CJ 7.16.30 (a. 294); CJ 7.16.33 (a. 294). Gardner 1993, op. cit. (n. 31), 48-49.

46 Writing in the first century $\mathrm{AD}$, Valerius Maximus (2.6.6-7) expressly contrasts Roman practice to that of Athens and Marseille. Gardner 1993, op. cit. (n. 31), 44.

47 Cf. Dion. Hal., 4.24.1-3; Tac., Ann. 13.27. Gardner 1993, op. cit. (n. 31), 7; Weaver 1997, op. cit. (n. 33), 55; Bradley 2011, op. cit. (n. 28), 254; Mouritsen 2011, op. cit. (n. 28), 66-70. 
created citizen. Thus, formal modes of manumission existed: ${ }^{48}$ only those slaves acquired freedom and citizenship who were released in front of a magistrate (vindicta); enrolled in the citizens' roster kept by magistrates (censu); or freed in their owner's will ${ }^{49}$ (testamento) - which was originally passed before the comitia or the gathered army, and later by a formal act in front of witnesses. Magistrates represented the citizen body or the State at manumissions, but they never functioned as the gatekeepers of citizenship. No doubt owing to the number of requests, and in an effort to relieve magistrates of unnecessary work when in session, slaves could validly be released vindicta if a magistrate was at the games, or on his way to a bathhouse, and even in the absence of lictors. ${ }^{50}$ The system ensured deliberation and publicity, but left the final decision to manumit (and grant citizenship) to the Roman citizen who owned the slave. ${ }^{51}$

This system may have sufficed under the early Republic, but in the wake of Rome's military victories and conquests came mass enslavement. ${ }^{52}$ By the end of the first century BC, slaves had flooded into Italy on an unprecedented scale, making up anywhere between fifteen and thirty percent of the population. ${ }^{53}$ Socially, the manner and extent of manumission began to be seen as problematic, ${ }^{54}$ as slaves from many different regions and cultural backgrounds were released into Roman citizenship. How could citizenship remain an attractive proposition to free non-Romans, if it were

48 Cic., Top. 10; Gaius, Inst. 1.17; UE 1.6-9; Fr. Dositheanum (= Fr. Dos.) 5. On manumissio censu, which fell into disuse under the empire, see Fr. Dos. 17. Buckland 19o8, op. cit. (n. 32), 439-444 and 451-465; Gardner 1993, op. cit. (n. 31), 8.

49 A slave who was released and instituted as heres necessarius became a Roman citizen in any case. He could not refuse the doubtful honor of freedom but was forced to take on the insolvent estate of the deceased. Gaius, Inst. 1.21; UE 1.14. Buckland 1908, op. cit. (n. 32), 505-508; P.R.C. Weaver, 'Where have all the Junian Latins gone? Nomenclature and status in the early Empire', Chiron 20 (1990), 276.

50 Gaius, Inst. 1.20; D. 40.2.7 (Gaius, 1 cott.); D. 40.2 .8 (Ulp., 5 ad ed.). Buckland 1908, op. cit. (n. 32), 452; Gardner 1993, op. cit. (n. 31), 9-10.

51 Buckland 1908, op. cit. (n. 32), 438-439; Gardner 1993, op. cit. (n. 31), 7 and 14; Mouritsen 2011, op. cit. (n. 28), 69.

52 S. Joshel, Slavery in the Roman world (Cambridge 2010), 54-56.

53 Scheidel 1997, op. cit. (n. 28), 75-77; Bradley 2011, op. cit. (n. 28), 250-251; W. Scheidel, 'The Roman slave supply', in K. Bradley and P. Cartledge 2011, op. cit. (n. 22), 294-296; Joshel 2010, op. cit. (n. 52), 8 and 56. For late Roman slavery figures, now see Harper 2011, op. cit. (n. 40), 59-6o.

54 Suet., Aug. 40.3-4. Also see the tirade in Dion. Hal., 4.24.4-8. W.L. Westermann, The slave systems of Greek and Roman antiquity (Philadelphia 1955), 89-9o; K. Bradley, Slaves and masters in the Roman empire (Brussels 1984), 84-87 and 148-149; Gardner 1993, op. cit. (n. 31), 49-50; Bradley 2011, op. cit. (n. 28), 256; H. Mouritsen 2011, op. cit. (n. 28), 80-87. 
cheapened by granting it readily to non-Roman ex-slaves? The answer was provided by express legislation enacted under Augustus. ${ }^{55}$

\section{Junian Latins}

Of old, the praetors had protected the liberty of slaves who were not manumitted formally ${ }^{56}$ but had been, for instance, granted release papers or freed inter amicos. These persons were not legally free, and if they died their property would return to their owner. This protection of informal or praetorian manumissions was given a more secure footing by the lex Iunia (Norbana), ${ }^{57}$ probably of 17 BC. $^{58}$ An informally released slave became free at law, but his liberty was fettered and his status modelled after that of the coloniary Latins. ${ }^{59}$ Hence: Latini Iuniani. Next, the lex Fufia Caninia of 2 BC set limits on the number of slaves freed by testament and required them to be indicated by name. ${ }^{60}$ And finally, crowning these

55 Called a "radical innovation" by P. Lopez Barja de Quiroga, 'Junian Latins: status and number', Athenaeum 86 (1998), 133; and a "watershed" by Gardner 1993, op. cit. (n. 31), 39. Also see Mouritsen 2011, op. cit. (n. 28), 89-92.

56 Gaius, Inst. 3.56; Fr. Dos. 4-5. There were no requirements for 'informal' manumissions. All that mattered was an expression of the master's will for the slave to be free (Fr. Dos. 7; Quint., Decl. 340; 342). Buckland 1908, op. cit. (n. 32), 445-446; A.J.B. Sirks, 'The lex Junia and the effects of informal manumission and iteration', Revue Internationale des Droits de l'Antiquité 30 (1983), 216-227. Nevertheless, if contested, informal manumission naturally required proof, so that public performance of a private manumission act remained important. Cicero (Att. 7.2.8) announced that he would simply deny the informal manumission of a slave as there were no relevant witnesses, referring to a case in which a praetor, no less, had done the same. Under Diocletian, patrons were required to present freedmen with a manumission deed: $C J$ 7.16.26 (a. 294).

57 Gaius, Inst. 1.22 and 3.56; UE 1.10; Fr. Dos. 6. Rotondi 1912, op. cit. (n. 10), 463-464.

58 The date is in dispute (the other possibility being $19 \mathrm{AD}$, under Tiberius) and largely depends on whether the lex Iunia was enacted before or after the lex Aelia Sentia, which again depends on the perceived relationship between the two, and on whether Justinian was right in referring to it as the lex Iunia Norbana (only once, Inst. 1.5.3). Since the group is named after the Junian law, it seems likely this came first. Buckland 1908, op. cit. (n. 32 ), 534-537; A.J.B. Sirks, 'Informal manumission and the lex Junia', Revue Internationale des Droits de l'Antiquité 28 (1981), 250-251; Weaver 1997, op. cit. (n. 33), 58-6o; Lopez Barja de Quiroga 1998, op. cit. (n. 55), 137-138.

59 Gaius, Inst. 1.22 and 3.56; Fr. Dos. 6. Sirks 1981, op. cit. (n. 58), 252; Lopez Barja de Quiroga 1998, op. cit. (n. 55), 136 and 146-149.

60 Gaius, Inst. 1.42-46, 2.228 and 2.239; UE 1.24-25; Paul., Sent. (= PS) 4.14.1-4; D. 40.4 .24 (Gaius, 1 cott.); CJ 7.5.1.1 (Just., a. 531). Rotondi 1912, op. cit. (n. 10), 454-455. The law was abrogated by Justinian (CJ 7.3 sing., $a .528$; Inst. 1.7), not because of any favor libertatis but because it made no sense to treat testamentary manumission different from manumission among the living. In any case, the antics described by Gaius imply that the law was continually being circumvented. Gardner 1993, op. cit. (n. 31), 40-41. 
two, the important and complicated lex Aelia Sentia of $4 \mathrm{AD}^{61}$ put further restrictions in place. Manumissions with the intent to defraud creditors or patrons were invalid. ${ }^{62}$ Slave owners under twenty years of age had to prove due cause before a consilium ${ }^{63}$ or the manumission would have no effect at all. ${ }^{64}$ And if a slave was released under the age of thirty, a formal manumission was only valid if it took place before a magistrate (vindicta) and due cause was shown. ${ }^{65}$ In all other cases, as long as the owner was over twenty years of age, the released slave became a Junian Latin - whether manumitted informally, or by a bonitary owner, or by a Quiritary owner testamento but under the age of thirty, or by a Quiritary owner vindicta but under the age of thirty and without due cause. An exception, finally, was made for those freedmen who were considered too much marked by the experience of slavery. ${ }^{66}$ If they had been incarcerated or branded by their masters, tortured and convicted of a crime by the State, or had fought in the arena, they could never hope to acquire any form of citizenship, Latin or Roman. ${ }^{67}$ They were forbidden to reside within a hundred miles from Rome, ${ }^{68}$ and their position mirrored ${ }^{69}$ that of the dediticii.

61 Rotondi 1912, op. cit. (n. 10), 455-456.

62 Gaius, Inst. 1.37; UE 1.15; Fr. Dos. 16; D. 40.9 R. It would make little sense to apply this rule to manumission into Roman citizenship, but not to manumission into Latinity, the ending of Fr. Dos. 16 notwithstanding - cf. Gaius, Epit. 1.1.5. Buckland 1908, op. cit. (n. 32), 544 .

63 The consilium consisted of five senators and five equites in Rome, and of twenty Roman citizens as recuperatores in the provinces. Gaius, Inst. 1.20; UE 1.13a.

64 Gaius, Inst. 1.40-41; Fr. Dos. 13; D. 40.9.7.1 (Iul., 2 Urs. Fer.). It made no difference whether the owner wished to release the slave into Roman citizenship or Junian Latinity. Buckland 1908, op. cit. (n. 32), 537-538; Sirks 1983, op. cit. (n. 56), 241.

65 Gaius, Inst. 1.18; UE 1.12-13. Due cause is present if under a legal obligation to release the slave (D. 40.2.15 pr., Paul., 1 l. Ael. Sent.; D. 40.2.16.1, Ulp., 2 l. Ael. Sent.), if a family member is freed, but also if a slave is released in order to appoint him procurator, or to marry her (Gaius, Inst. 1.19 and 1.39; D. 40.2.11-14, Ulp., 2 off. proc. and 2 l. Ael. Sent.), or if a slave has rescued his master from danger or infamy (D. 40.2.9 pr., Marc., 13 inst.; D. 40.2.15.1, Paul., 1 l. Ael. Sent.). Buckland 1908, op. cit. (n. 32), 538-542.

66 The decision to 'mark' a slave lay with the master and may have had nothing to do with the 'badness' of the slave. Mouritsen 2011, op. cit. (n. 28), 32-34. Yet, torture itself did not disqualify a slave from iusta libertas if he were innocent, and neither did the mere fact that he was shackled for insanity: PS 4.12.3 and 7 .

67 Gaius, Inst. 1.13; 1.15; 1.26; UE 1.11. Buckland 1908, op. cit. (n. 32), 544-546. An exception existed for the servus heres institutus, see n. 49. Justinian abolished the status of liberti dediticii, but also indicated that it had been out of use for some time: $C J$ 7.5.1 (a. 530) - one of the quinquaginta decisiones (Inst. 1.5·3).

68 Gaius, Inst. 1.27. Offenders were sold back into slavery (Gaius, Inst. 1.16o).

69 Gaius and Ulpian are careful to distinguish the peregrini dediticii from those freedmen qui dediticiorum numero sunt: Inst. 1.13, 1.15, 1.25-26, 1.160; UE 1.11. A difference at law 
The Augustean manumission laws were intended to force slave owners to consider whom they were releasing, ${ }^{70}$ and also to bar slaves from attaining citizenship that were judged unworthy for ideological reasons. ${ }^{71}$ They made citizenship harder to acquire and more worthwhile, and strengthened the position of patrons as a mediating force. But it should also be remembered that before the Junian law was passed, informally released people remained slaves at law, though protected by the praetor. Thus, although Augustus may have created a class of second-rate citizens, the Junian Latins formed a legally recognized class of free citizens nevertheless. As a special status group modelled after the coloniary Latins, they had commercium ${ }^{72}$ but not conubium. ${ }^{73}$ Unlike the Latins they were prohibited from making a testament, nor could they take under a will or acquire a legacy. ${ }^{74}$ Patched and mended by a multitude of enactments and interpretations, ${ }^{75}$ this system survived the constitutio Antoniniana ${ }^{76}$ and was kept largely intact until Justinian abolished the status of Junian Latins in $531 \mathrm{AD} .{ }^{77}$

The obvious question is how common it was to be relegated to the waiting room of Junian Latinity instead of acquiring full citizenship upon manumission. ${ }^{78}$ Weaver, following Sirks and Lopez Barja de Quiroga, has

is indicated by Ulpian (UE 20.14): unlike peregrini dediticii, freedmen numbered among the dediticii cannot make wills in accordance with the laws of their communities, since they do not belong to any.

70 Dio Cass., 55.13.7. Mouritsen 2011, op. cit. (n. 28), 34-35; 51-52.

71 Suet., Aug. 40.4; Dion. Hal., 4.24.4-8. Gardner 1993, op. cit. (n. 31), 39; Mouritsen 2011, op. cit. (n. 28), 86-88.

$72 U E$ 19.4. Gardner's argument (1997 op. cit. [n. 24], 40 n. 9) that this is a later development, since Gaius (Inst. 1.119) says the form is proper to Roman citizens, makes little sense considering that commercium was already extended to Latins under the Republic. Also see Sirks 1983, op. cit. (n. 56), 227-229.

73 Gaius, Inst. 1.80; UE 5.9-10. In consequence, their children were not freeborn Roman citizens, though they probably were freeborn Latins. Sirks 1983 , op. cit. (n. 56 ), $262-265$.

74 Gaius, Inst. 1.23; 2.110; 2.275; UE 20.14; 22.3. Junian Latins could acquire under a fideicommissum: UE 25.7. Weaver 1997, op. cit. (n. 33), 57-58; Sirks 1983, op. cit. (n. 56), 223.

75 See the list provided by Lopez Barja de Quiroga 1998, op. cit. (n. 55), 149-150 and $161-163$.

76 The epitome of Gaius, for instance, does not mention coloniary Latins, who no longer existed after the Antoniniana, and only mentions Latins as former slaves: Gaius, Epit. 1.1. A diptych from Hermopolis (FIRA III, 23-25; $A E$ 1904, 217) contains a manumission deed inter amicos, dated $221 \mathrm{AD}$ : the freedwoman in question would have been a Junian Latin. Also see Gardner 1997, op. cit. (n. 24), 50; Weaver 1997, op. cit. (n. 33), 57.

77 CJ 7.6 sing. (Just., a. 531), preceded in part by $C J$ 7.15.2 (Just., a. 530). Cf. Inst. 1.5.3; 3.7.4. That Junian Latinity still existed at the time, is shown by the need which Justinian felt to legislate without prejudice to existing cases (CJ 7.6.1.13). Harper 2011, op. cit. (n. 40), 467.

78 The old idea (Buckland 1908, op. cit. [n. 32], 442; Garnsey, op. cit. [n. 28], 362) that manumissio testamento, leading to full citizenship, was the most popular or important 
argued that it was very common indeed, even calling the group "a large undetected black hole at the heart of the 'slave' society". ${ }^{79}$ That is a reasonable conclusion. One should consider that if anything goes wrong at all with a manumission, the result is Junian Latinity. This may be intentional or, for instance, stem from a problem with the validity of a testament, ${ }^{80}$ or a lack of Quiritary ownership ${ }^{81}$ on the part of the master because the slave was acquired through traditio instead of mancipatio. Sick slaves who were abandoned by their masters became Latins, according to an edict of Claudius. ${ }^{82}$ Furthermore, the magistrates needed to witness a formal manumission may have been hard to come by in the provinces. Pliny wrote to his wife's grandfather that he was sure he could entice a friend to drop by who was on his way to a proconsular station, if his prosocer still wished to formally manumit those whom he had recently manumitted inter amicos. ${ }^{83}$

Evidence of Junian Latins on the ground is rather thin, except for the case of L. Venidius Ennychus, known from the Herculaneum tablets, who wished to qualify for full citizenship by establishing that he had conceived a year-old child in proper marriage. ${ }^{84}$ Inscriptions do show a large number of manumissions ${ }^{85}$ under the minimum age of the lex Aelia Sentia,

form, has recently been criticized - rightly - by U. Roth, 'Peculium, freedom, citizenship: golden triangle or vicious circle?', in U. Roth (ed.), By the sweat of your brow: Roman slavery in its socio-economic setting (London 2010), 98-99; and Mouritsen 2011, op. cit. (n. 28), $180-183$.

79 P.R.C. Weaver, 'Reconstructing lower-class Roman families', in S. Dixon (ed.), Childhood, class and kin in the Roman world (London, New York 2001), 103. Also see Weaver 1997, op. cit. (n. 33), 55: “a black hole of large but unknown proportions". Sirks 1981, op. cit. (n. 58$), 274$.

80 E.g. D. 40.4.47 pr. (Pap., 6 quaest.); CJ 7.16.7 (Aur.).

81 UE 1.16; Fr. Dos. 9. Buckland 1908, op. cit. (n. 32), 549. Also see Gaius, Inst. 1.167 and $U E$ 11.19: tutela over Junian Latin women and underage men was granted to the (former) Quiritary owner, not to the bonitary owner who had manumitted the slave. Sirks 1983, op. cit. (n. 56), 245-251. It is not clear whether the one-year period for usucapio healed the informal manumission, if all other criteria for a formal manumission had been met, but the answer should be negative since the former bonitary owner lacked possession after the informal manumission.

82 CJ 7.6.1.3 (Just., a. 531). Also see Suet., Cl. 25.2; Dio Cass., 61.29.7; D. 40.8.2 (Mod., 6 reg.).

83 Plin., Ep. 7.16.4. Lopez Barja de Quiroga 1998, op. cit. (n. 55), 157-159; P.R.C. Weaver, 'Where have all the Junian Latins gone? Nomenclature and status in the early Empire', Chiron 20 (1990), 279-281.

$84 A E$ 1978, 119; HT 5 and 89. Gardner 1993, op. cit. (n. 31), 18; Weaver 1997, op. cit. (n. 33), 68-69.

85 Alföldy 1986, op. cit. (n. 28), 286 ff.; Weaver 1990, op. cit. (n. 49), 276-277; L. Mihailescu-Birliba, Les affranchis dans les provinces romaines de l'Illyricum (Wiesbaden 2006), 86-88 and 125-126; Weaver 2001, op. cit. (n. 79), 103; Lopez Barja de Quiroga 1998, op. cit. (n. 55), 152-155. 
leading to Junian Latinity, ${ }^{86}$ though some of that may be due to manumission on the deathbed. Even so, Junian Latin status is never explicitly mentioned in funerary inscriptions, nor can it be deduced or excluded from the onomastics, ${ }^{87}$ leaving the question of its incidence quite open. This should come as no surprise, since a freedman (or his family) would have had little reason to commemorate what amounted to a secondclass citizenship. Importantly though, the Flavian municipal laws do mention slaves freed by Latins, referring explicitly to their status as comparable to that of Junian Latins. ${ }^{88}$ Since a citizen of a Latin community was not a Roman citizen himself, he could not create one by manumitting a slave. ${ }^{89}$ Thus, if the slave was released in front of the municipal magistrates - mirroring the manumissio vindicta - he would become a Junian Latin. ${ }^{90}$ A large number of slaves may have entered into Junian Latinity in this manner.

Finally, the amount of attention Junian Latins received from jurists and lawmakers is extensive, to say the least. The wealth of material is all the more telling when one realizes that Junian Latinity has been struck out of the Digests completely, following Justinian's abolition of that status. ${ }^{91}$

86 Fr. Dos. 14 and $U E$ 3.3, against $U E 1.12$ which is either wrong, or must be restricted for some unknown reason to servi Caesaris. Buckland 19o8, op. cit. (n. 32), 542-543.

87 Rawson 1966, op. cit. (n. 33), 79; Weaver 1997, op. cit. (n. 33), 56; Weaver 2001, op. cit. (n. 79), 102 f.; Lopez Barja de Quiroga 1998, op. cit. (n. 55), 143-144.

88 L. Irnit. 28.11-12: . . . libera esto uti qui optumo iure latini libertini liberi sunt. A.T. Fear, 'Cives Latini, servi publici and the lex Irnitana', Revue Internationale des Droits de l'Antiquité 37 (1990), 152-155; Lopez Barja de Quiroga 1998, op. cit. (n. 55), 149.

89 Conversely, a peregrinus who manumitted a slave could not create a Junian Latin or Roman citizen: Fr. Dos. 12; Plin., Ep. 10.5.2. The praetor would protect the ex-slave's liberty as he did for all informally manumitted slaves before the lex Iunia Norbana. Buckland 1908, op. cit. (n. 32), 534 and 594.

90 L. Irnit. $28.5^{-6}$ and 11-12. A different rule applies to servi publici of a Latin community. In l. Irnit. 72.17-19, they are declared citizens of Irni upon manumission - hence Latins - but in l. Irnit. 72.23-26, the patronal rights to their inheritance (hereditas) and to operae are said to be the same as if they had been released by a municipality with ius italicum. The assumption is warranted that manumission by the magistrates of a community with ius italicum led to Roman citizenship (cf. CJ 7.9.3.1, Diocl./Max., a. 290/93), and thus that the clause gave less patronal rights to the city of Irni than it would have had over a Junian Latin. Weaver 1997, op. cit. (n. 33), 65. Contra Fear 1990, op. cit. (n. 88), 156-159.

$91 C J$ 7.6.1.12-12a, (a. 531). Though the rule changed, the factual content of cases that used to lead to Junian Latinity remains the same: titles 40.7 and 40.8 of the Digests are particularly revealing. An example is the female slave who is sold under the condition 'ne prostituatur'. If she were nevertheless prostituted, she was free and became a Junian Latin by law (CJ 7.6.1.4). Though her freedom is mentioned in the Digests (D. 40.8.6, Marc. sing. form. hyp.; D. 40.8.7, Paul., sing. lib. dand., also see CJ $4.56 \mathrm{R})$, and the forced prostitution is even compared to manumission (tu uideris quasi manumississe, D. 21.2.34 pr., Pomp., 27 ad Sab.), her status as a Junian Latin freedwoman is given no mention at all. 
After treating the complications arising out of freedmen inheritances for fifteen paragraphs, Gaius is content to have just brushed the matter quasi per indicem. ${ }^{92}$ And that is only for freedmen who were Roman citizens: he needs a further twenty-two paragraphs to deal with Junian Latins. In these inheritances lies the best argument for the prevalence of Junian Latins, since the law offered patrons a much stronger position upon the death of a Junian Latin freedman, than upon the death of a freedman who was a full citizen. The difference is made explicit in the phrase that Junian Latins, "though they lived their lives as free men, still lost both life and liberty at their last breath." 93

\section{The Patronal Interest}

The fundamental rules governing the patron's interest in a freedman's estate depend on a fiction. Since all legally recognized ties are destroyed by lawful slavery, ${ }^{94}$ the former slave is considered to be without kin after his manumission. ${ }^{95}$ The closest thing he has to family is his patron, and for this reason he is expected to behave with filial piety. This family duty works reciprocally: patron and freedman must support each other in times of need, ${ }^{96}$ and they cannot be forced to give criminal evidence against each other. ${ }^{97}$ Yet there is, of course, a fundamental inequality of arms. A freedman cannot bring certain suits against his patron at all, for instance infamatory actions. ${ }^{98}$ In any case he has to petition the praetor ${ }^{99}$ for permission to summon his patron to court. ${ }^{100}$ However, it must be stressed

92 Gaius, Inst. 3.54. Weaver 1997, op. cit. (n. 33), 63.

93 Inst. 3.7.4: ... qui licet ut liberi uitam suam peragebant, attamen ipso ultimo spiritu simul animam atque libertatem amittebant. Cf. CJ 7.6.1.1b (Just., a. 531). The same image is used by Salv., Eccl. 3.7.31: ut uiuant scilicet quasi ingenui et moriantur ut serui.

94 D. 4.5.3.1 (Paul., 11 ad ed.); Inst. 1.16.4.

95 Buckland 1908, op. cit. (n. 32), 76-79 and 676; Gardner 1993, op. cit. (n. 31), 19-20; Joshel 2010, op. cit. (n. 52), 40-44; Mouritsen 2011, op. cit. (n. 28), 37-40.

96 D. 25.3.5.18-26 (Ulpian, 2 off. cons.).

97 D. 22.5.4 (Paul., 2 l. Iul. et Pap.). Cf. Aul. Gell., Noct. Att. 5.13.4 (Cato) and 5.13.5 (Sabinus).

98 D. 2.4.10.11 (Ulp., 5 ad ed.); D. 37.15.5.1 (Ulp., 10 ad ed.). Though Ulpian, following the 1st c. jurist Pedius, notes that an infaming action should be allowed if the insult to the freedman has been very great, for instance if the patron has had him whipped.

99 Gaius, Inst. 4.183; D. 2.4.4.1 (Ulp., 5 ad ed.).

100 If he summons his patron without permission, the patron can sue for fixed damages with a praetorian actio in factum. Gaius, Inst. 4.46; D. 2.4.12 (Ulp., 57 ad ed.); D. 2.4.24 (Ulp., 5 ad ed.); D. 2.4.25 (Mod., 1 poen.). Again, the praetor should temper his jurisdiction in this matter: D. 2.4.11 (Paul., 4 ad ed.). 
that none of these rules apply only to freedmen: they are presented as the natural consequence of kinship. ${ }^{101}$ Hence, a natural son cannot sue his father without praetorian leave either. Moreover, the praetor will in principle grant leave to sue if the action is not infamatory, ${ }^{102}$ which made it a mere formality in most cases. ${ }^{103}$

The kinship any freedman was legally bound to feel with his patron, prevented him from bypassing the patron when making a will. Though the XII Tables originally only gave patrons rights to intestate succession, ${ }^{104}$ and then only if a heres suus was lacking, the praetorian edict provided them with a right to half the inheritance if they had received less by testament, ${ }^{105}$ and again to half if the intestate successor would be anyone but a natural child. ${ }^{106}$ Under Augustus, these rights were further strengthened. The complicated lex Papia of $9 \mathrm{AD}^{107}$ entitled patrons to a part equal to that of any child if the inheritance exceeded 100.000 sesterces, even in the presence of natural children, while it took three children to exclude the patron completely, whether by testament or intestate. ${ }^{108}$ As it is part of Augustus' marriage laws, one of its intents may have been to encourage the richer freedmen, Roman citizens no less, to marry and procreate thus dividing the inheritance among more heirs - by holding the sword of a strong patronal interest over their head. ${ }^{109}$ Finally, the conception of

101 D. 2.4.6 (Paul., 1 sent.); D. 2.4.13 (Mod., 10 pand.). Gardner 1993, op. cit. (n. 31), 24; Mouritsen 2011, op. cit. (n. 28), 56-57.

102 D. 2.4.10.12 (Ulp., 5 ad ed.): permissurus enim est, si famosa actio non sit. Cf. Tac., Ann. 13.26.

103 Gardner 1993, op. cit. (n. 31), 24-25.

104 Gaius, Inst. 3.40; UE 29.1; Inst. 3.7 pr. Gardner 1997, op. cit. (n. 24), 41-42.

105 Since the patron was tutor to any freedwoman, he could refuse his auctoritas, were she to make a will which did not declare him heir. The result would be that he was granted the entire inheritance through intestate succession, as a woman could not have a heres suus. Gaius, Inst. 1.192, 3.43; UE 29.2. The only possible escape was through the ius quattuor liberorum, since four children would free her from tutelage, but even that gate was partially closed by the lex Papia. Gaius, Inst. 3.44; UE 29.3.

106 Gaius, Inst. 3.41; Inst. 3.7.1. Gardner 1993, op. cit. (n. 31), 21-22. The reason provided by Ulpian (D. 38.2.1.2, 62 ad ed., referring to the praetors after Rutilius, 118 BC), is instructive: half is awarded to the patron because the freedman would usually have been in societas with his patron while alive. Gardner 1993, op. cit. (n. 31), 27-29. On the advantage to patrons of business dealings with informally relased slaves, see Sirks 1981, op. cit. (n. $5^{8}$ ), 269-271.

107 Rotondi 1912, op. cit. (n. 10), 457-462.

108 Gaius, Inst. 3.42; Inst. 3.7.2. Changed by Justinian: CJ 6.4.4 (a. 531), Inst. 3.7.3. For the effect on adoption strategies, see Gardner 1997, op. cit (n. 24), 41-42.

109 Gardner 1993, op. cit. (n. 31), 23. 
enforced kinship ensured that these patron rights to the inheritance of freedmen, were passed on to their male heirs, though not to extranei. ${ }^{110}$

It is against this background that the rules concerning the estates of Junian Latins must be understood. ${ }^{111}$ As noted earlier, the liberty of informally released slaves was protected by the praetor before their status was legalized by the lex Iunia Norbana. However, such people remained slaves at law. What they acquired would formally belong to their owner ${ }^{112}$ and on their death the entire estate would devolve to him, as the peculium of a slave. Now that they had become free persons at law, Junian Latins, a rule was necessary to compensate their manumissors, since in their new role as patrons these could hope to claim no more than (generally) half the inheritance. For this reason, the lex Iunia Norbana contained the fiction that a Junian Latin's inheritance would be treated as if the deceased had never been released.13 Junians may have lived in liberty, but they died as if they were still slaves. ${ }^{114}$ This fiction brings the rule concerning the power to make a testament into contrast. As Roman citizens, formally manumitted slaves were able to make legally valid wills; but Junian Latins did not have this right. ${ }^{115}$ As a consequence, they could not pass over their patrons at all, nor leave any part of their estate to their (freeborn) children. ${ }^{116}$ In practice, some patrons may have permitted their Junian Latin freedmen to dispose of (part of) their inheritance by testament, as some masters allowed their slaves to do. ${ }^{117}$ If such a practice existed, however, it was born out of benevolence more than any legal principle.

The patronate carried a monetary value with it, as patrons well understood. ${ }^{118}$ A rescript of Antoninus Pius, for instance, mentions the legacy

110 Gaius, Inst. 3.45; 3.48; UE 29.4. Extranei could lay claim to the inheritance of a Junian Latin though: Gaius, Inst. 3.58; 3.64. Gardner 1997, op. cit. (n. 24), 41 n. 12.

111 Gaius, Inst. 3.56. Sirks 1981, op. cit. (n. 58), 255-257; Mouritsen 2011, op. cit. (n. 28), 41-42.

112 Fr. Dos. 5 .

113 The many differences discussed by Gaius, Inst. 3.57-62, between the inheritances of freedmen who are Roman citizens and those of Junian Latins, are all a result of the fact that a Junian Latin's estate already belongs to his patron iure quodammodo peculii (Gaius, Inst. $\left.3 \cdot 5^{6}\right)$.

114 Inst. $3 \cdot 7 \cdot 4$.

115 Gaius, Inst. 1.23; UE 20.14.

116 Weaver 1997, op. cit. (n. 33), 61 f.: "blatantly and uncharacteristically discriminatory".

117 Plin., Ep. 8.16.1. Servi Caesaris had power over half their peculium: UE 20.16; Fr. de iure fisci $6 \mathrm{a}$.

118 See the rubrica 'si quid in fraudem patroni factum sit', D. 38.5. As Gardner 1997 points out (op. cit. [n. 24], 42-43), the existence of rules to invalidate alienations in fraud 
of a Latin to a colonia, ${ }^{119}$ and Pliny received such a legacy as well. ${ }^{120}$ Since the (Junian) Latin would have been a free man, such a legacy implies little else than a transfer of the patronal interest. This awareness of the value of patronate rights also underpins the practice of granting slaves their peculium upon manumission inter vivos, unless explicitly withheld, but not upon release by testament, unless explicitly granted as a legacy. ${ }^{121} \mathrm{~A}$ patron and his family might reasonably expect to see all or part of their investment returned to them after the death of a freedman who had been freed inter vivos. Testamentary release, however, turned the slave into a freedman of the deceased, a libertus orcinus who had no patron, so that the testamentary heirs of the patron would later have no claim to the freedman's inheritance. Manumission of the slave by fideicommissum got around this problem since the trustee would acquire the patronate, ${ }^{122}$ and, unless the fideicommissum stipulated otherwise, could choose to manumit the slave as he wished. Presumably, trustees who stood in a less direct relationship to the prospective freedman would opt for the manner of release that suited them best. Of course many motives for manumission can be identified, varying from the cruel to the affectionate, and Junian Latinity is certainly not the likely outcome if a patron is freeing his brother, or the woman he intends to marry. But if the manumission took place for economic reasons, for instance because a slave bought his freedom out of his peculium, ${ }^{123}$ then it would make sense to

of patrons, particularly the $a^{o}$ Fabiana and $a^{o}$ Calvisiana, warrants the assumption that freedmen were otherwise attempting to siphon away their estates prior to death.

119 Gaius, Inst. 2.195. The legacy was made per vindicationem, which shows that this patronal interest was still connected to the Quiritary ownership which a manumissor would have retained before the lex Iunia Norbana. On the many problems concerning this text, see Sirks 1983, op. cit. (n. 56), 271-290.

120 Plin., Ep. 10.104, asking Trajan to grant them full citizenship. Lopez Barja de Quiroga 1998, op. cit. (n. 55), 142.

${ }^{121}$ Fr. Vat. 261; D. 15.1.53 (Paul., 11 quaest.); CJ 7.23 sing. (Diocl./Max., a. 294); Inst. 2.20.20 (referring to Sev./Ant.). Buckland 1908, op. cit. (n. 32), 189-19o. But also see Roth 2010, op. cit. (n. 78), 99-103, who stresses that 'explicit' grants or ademptions are a matter of construction.

122 Gaius, Inst. 2.266-267; D. 38.2 .29 pr. (Marc., 9 inst.). The patron had a very limited right, according to Papinian (D. 26.5.14, 9 resp.), but the relevant phrase, licet in bonis eius patroni ius exerceat, has been struck from that passage by Justinian: Fr. Vat. 225.

123 Alföldy 1986, op. cit. (n. 28), 97-129; Hopkins 1978, op. cit. (n. 40), 115-132. For an overview, see Roth 2010, op. cit. (n. 78), 91 f.; Mouritsen 2011, op. cit. (n. 28), 159-180. It should be noted that the slave who was bought suis nummis by a third party had no patron at all after his manumission. The slave who was released by his owner after paying him a sum of money, had his manumittor as his patron. $C J$ 6.4.4 pr. (Sev./Ant., $a$. 210). Garnsey 1981, op. cit. (n. 28), 363-364 appears to have missed the latter nicety. 
manumit him as a Junian Latin, or at least to make his prospective status part of the negotiations. ${ }^{124}$

\section{Escape into Roman Citizenship}

As for coloniary Latins, several options stood open to Junian Latins who wished to better their position. To acquire Roman citizenship meant more to them, though, as it offered the right to make wills and take under wills, which coloniary Latins already had as a matter of course, and it lessened their patron's control iure quodammodo peculii over their future inheritance. The patronal interest could not be negated completely, but full citizenship at least provided the chance to pass (all or part of) their estate on to children. Since the incentive to Junian Latins was so much greater, ${ }^{125}$ socio-economic enactments such as the ones cited by Gaius concerning citizenship acquired by providing grain transport, mills, or construction work, were more likely aimed at wealthy Junians than at coloniary Latins. In fact, some paths were only open to Junian Latins. A lex Visellia of $24 \mathrm{AD}$ gave full rights to those who served in the vigiles for six years, later three. ${ }^{126}$ More important is the rule from the lex Aelia Sentia, that Junian Latins ${ }^{127}$ who had declared that they were married to a woman of equal or higher status, in the presence of seven Roman citizens as witnesses, and afterwards had a year-old child, could petition ${ }^{128}$ for citizenship for themselves, and their family if necessary. ${ }^{129}$ It is quite illustrative of Augustus' sociopolitical goals that 'settled' slaves who had proven to be (re)productive were admitted into the citizenship. ${ }^{130}$ The lex Aelia Sentia even did so at

124 Sirks 1981, op. cit. (n. 58), 262-272; Roth 2010, op. cit. (n. 78), 112-115. Such negotiations might also involve a price for iteratio.

125 Weaver 1990, op. cit. (n. 49), 278.

126 Gaius, Inst. 1.32b; UE 3.5. The lex Visellia also made it a criminal offence (CJ 9.31.1.1, Val./Grat./Valent., $a .378$ ) for freedmen to attempt to gain freeborn honours such as entry into the decuriones: CJ 9.21.1 pr. (Diocl./Max., a. 30o?). However, this can hardly be taken as a measure specifically intended to bar Junian Latins from obtaining citizenship, as that avenue (maius Latium) seems to have been instituted by Hadrian. Crook 1967, op. cit. (n. 1), 42; Mouritsen 2011, op. cit. (n. 28), 73-75.

127 Originally only those who had been released under the minimum age of thirty, after a $S C$ under Vespasian also those over thirty. Gaius, Inst. 1.31.

128 Cf. the dossier of L. Venidius Ennychus mentioned in n. 82. Weaver 1990, op. cit. (n. 49), 277-278.

129 Gaius, Inst. 1.29; UE 3.3.

130 Gardner 1993, op. cit. (n. 31), 40, who ascribes it to the lex Iunia contra Gaius, Inst. 1.29. Also see Lopez Barja de Quiroga 1998, op. cit. (n. 55), 156-157; Mouritsen 2011, op. cit. (n. 28), 86-89. 
the cost of a patron's access to freedmen inheritances, since if the freedman or freedwoman had a year-old son, there was a good chance there would also be a heres suus later on, who could bar the patron fully or in part from intestate succession.

This consequence was an exception, though, and one particular to Augustus' marriage politics. Emperors who granted Roman citizenship to Junians were generally careful not to infringe on patron interests. Trajan ruled that imperial grants of citizenship, against the knowledge or will of the patron, would only take effect saluo iure patroni. ${ }^{131}$ Employing yet another fiction, the freedman would be a full citizen while alive, but die as a Junian Latin, explicitly so that the patron was not passed over for the inheritance. ${ }^{132}$ Conversely, whenever Pliny writes to Trajan asking for a grant of full citizenship, he goes out of his way to ensure the emperor that the patron does not object. ${ }^{133}$ Commodus removed the right to be considered freeborn (ius anulorum) from those who received it against the will or without knowledge of their patrons, who, in any case, still had rights to the inheritance as if the freeborn status had not been granted.134 Even in the municipal laws, a Latin patron who manumitted a slave (creating a Junian Latin) retained his existing patronal rights if the freedman acquired full citizenship by the award of municipal honorifics. ${ }^{135}$ In view of these traditions, it is highly unlikely that the biggest imperial grant of citizenship of all, the constitutio Antoniniana, brought any real difference in status to Junian Latins living at the time. ${ }^{136}$ If it had done so, patrons would have been in uproar. The edict in the Giessen papyrus, taken to be the Antoniniana, excepts dediticii from the grant, which may

131 Gaius, Inst. 3.72; Inst. 3.7.4. Also see D. 40.3 .6 (Ulp., 1 l. Iul. et Pap.) for the grant of freeborn status saluo iure patroni.

132 Children of the freedman cannot inherit intestate. The freedman may make a will, but only to appoint his patron heir, though substitutions are possible. This posed a problem, which Hadrian corrected, namely that a freedman would be worse off with an imperial grant than with citizenship acquired under the lex Aelia Sentia. Gaius, Inst. 1.73.

133 Plin., Ep. 10.5.2 (patron long dead); 10.11.2 (uolentibus patronis); 10.104 (Pliny himself is patron).

134 D. 40.10.3 (Marc., 1 inst. citing Commodus) and D. 40.11 .2 (Marc., 1 inst.); D. 40.10.5 (Paul., 9 l. Iul. et Pap.); D. 40.11.5 pr. (Mod., 7 reg.). Ulpian (D. 38.2 .3 pr., 61 ad ed.) is decided: hic enim uiuit quasi ingenuus, moritur quasi libertus.

135 L. Irnit. 97.27-28. In the mirror opposite. a patron who acquired full citizenship could only exercise patronal rights as if the slave had been released by a Roman citizen. Weaver 1997, op. cit. (n. 33), 66.

136 K. Loewenstein, The governance of Rome (The Hague 1973), 385-386. Gardner 1997 (op. cit. [n. 24], 50) argues that the Antoniniana created conubium for freedman families: that is true enough for freedman citizens, but would only be generally true if all Junian Latins were raised to full citizenship by the grant as well. 
refer to the ex-slaves whom the lex Aelia Sentia pronounced unfit for citizenship. ${ }^{137}$ If so, the enigmatic lacuna in the papyrus, containing a salvatory clause that is usually suppleted from the tabula Banasitana as a grant of citizenship saluo iure gentis, ${ }^{138}$ may rather have contained a clause granting citizenship saluo iure patroni.

\section{The Stick and the Carrot}

Whether the Augustean manumission laws achieved their intended goal can be argued both ways, and the answer largely depends on what that goal is perceived to be. If it was to keep the citizen body free from the macula seruitutis, then it quite probably failed, even as signal legislation aiming at greater deliberation on the part of manumitters. But if the aim was to present citizenship as something of value, and therefore as a political tool that could be used to further the integration of the empire, then the manumission laws succeeded in that goal. It is significant that although coloniary Latinity did not survive the constitutio Antoniniana, the slightly younger Junian Latinity of Augustus survived until Justinian's time.

The lex Iunia Norbana brought praetorian manumissions within the framework of the ius ciuile. What had previously been de facto liberty but de iure slavery, became Junian Latinity: a recognized halfway station on the road to citizenship. In this manner, full citizenship remained an attractive honorific for peregrines and coloniary Latins, but also became a material improvement for the newly created class of Junian Latins. This was achieved neither by forcing slave owners to manumit less - there was no limit to the number of slaves they could free inter uiuos -, nor by denying presumably prudent owners of slaves, that is to say, those over twenty, the right to confer Roman citizenship if they followed proper procedure. Instead, an appeal was made to a patron's perceived self-interest. If the easier path of informal manumission was followed, the patronal interest would be stronger and the freedman would remain in a greater state of dependancy.

137 P. Giss. 40.1; FIRA I, 445. H. Benario, 'The dediticii of the constitutio Antoniniana', Transactions and proceedings of the American Philological Association 85 (1954), 193-196; Crook 1967, op. cit. (n. 1), 45; Loewenstein 1973, op. cit. (n. 136), 386. Others have argued that the dediticii referred to were recently subjugated tribes. A.H.M. Jones, 'Another interpretation of the constitutio Antoniniana', Journal of Roman Studies 26 (1936), 227-232; Sherwin-White 1972, op. cit. (n. 5), 56-57.

138 Sherwin-White 1973, op. cit. (n. 4), 86 ff; Id. 1972 op. cit. (n. 5), 56. 
From the perspective of a Junian Latin, the law of Rome was tempting him with a carrot and threatening him with a stick. The stick was the devolvement of his entire estate to his patron upon death; the carrot the possibility of obtaining full citizenship beforehand. In the end, the choice was between slavery and Rome. An upgrade from second-class Junian Latin citizenship to Roman citizenship brought advantages to freedmen that could not be ignored, particularly the right to dispose of (part of) one's inheritance as one pleased. Later emperors attempted to turn this economic incentive into a source of money and patronage.

The best vignette, perhaps, is offered by the Senate debate under Nero which was mentioned earlier. One of the arguments put forward against a private right to reuocatio in seruitutem, according to Tacitus, was that the difference between formal and informal manumission had been introduced so that the option of a new grant remained. Those freed informally were, so to speak, still shackled in slavery. ${ }^{139}$ Masters retained the power to bestow full citizenship by a second manumission (iteratio) in due form, as long as they were the former Quiritary owner of the freedman. ${ }^{140}$ Tacitus calls this a "new benefice", but it is clearly a weapon in the hands of patrons. Iteratio further liberated a Junian Latin from his patron, but if the patron refused to grant a formal manumission, little remained than to employ the methods vouchsafed by law: to either buy one's way into citizenship, or to marry before witnesses and produce children. An act of private law - manumission - was thus aligned to the State's interest in curbing the admission of 'unsuitable' ex-slaves into the citizen body, by providing patrons with an incentive, a carrot, to keep their freedmen in a state of dependancy as second-class citizens - a state of dependancy, furthermore, which might serve patrons as a stick. Though Tacitus does not say so explicitly, the suggestion is that a former slave owner only has himself to blame, if his freedman turns out intractable.

139 Tac., Ann. 3.27: Quin et manu mittendi duas species institutas, ut relinqueretur paenitentiae aut nouo beneficio locus. Quos uindicta patronus non liberauerit, uelut uinclo seruitutis attineri. It is not clear what Tacitus means by a locus paenitentiae which is allegedly afforded through informal manumission. In any case, there is no legal rule otherwise known to that effect, so that it may be a mere figure of speech.

$140 U E$ 3.4; Fr. Vat. 221. Also see n. 119. Pliny references iteratio in a letter to his wife's grandfather, mentioned earlier: $E p$. 7.16.4. Sirks 1981, op. cit. (n. 58), 253 and especially Sirks 1983 , op. cit. (n. 56 ), 246 ff. 


\title{
MIGRANT QUARTERS AT ROME?
}

\author{
Laurens E. Tacoma
}

\section{Introduction}

This article addresses a seemingly simple question: where did migrants live in the city of Rome under the Principate? Underlying this straightforward question is the more complicated issue whether there is evidence for what is technically called residential clustering, which in its turn is relevant for discussions of integration.

In the case of the city of Rome under the Principate, the existence of significant levels of residential clustering is often taken for granted. So, La Piana, in his massive study of immigration to Rome wrote in 1927:

It is to-day, and has always been everywhere, the natural tendency of a body of immigrants from the same nationality in a foreign city to live together as much as possible in the same district, where they can reproduce the main characteristics of the social and religious life of the country from which they came. They form sections of their own, separate to a certain extent from the rest of the population, and keep their own language and customs at least as long as the current of immigration remains active. This is a universal phenomenon, of which we have evidence on a large scale in the numerous communities of immigrants from Europe and Asia in the large cities of America. There are reasons for believing that the foreign populations of ancient Rome were no exception to this rule, and that they yielded to this tendency so far as the social and economic conditions of the city allowed them to follow this instinctive need. ${ }^{2}$

Dated though it is, La Piana's statement is useful because it explicitly and concisely summarizes a type of argument that still pervades the scholarly

\footnotetext{
1 In addition to the participants of the Lille conference, I would like to thank Miriam Groen, Onno van Nijf and Hanna Stöger for comments and suggestions.

${ }^{2}$ G. La Piana, 'Foreign groups in Rome during the first centuries of the empire', Harvard Theological Review 20 (1927), 183-403, at 204.
} 
literature. ${ }^{3}$ The assumption is that residential segregation is a timeless characteristic of immigrant societies. The logical corollary is that it must have also occurred at Rome. Given its universality, it is deemed legitimate to project the modern situation on the ancient one and describe the latter in the terms of the former. Such residential segregation implies a lack of integration: people stick to their own language and customs. ${ }^{4}$

In itself there is nothing wrong with using comparisons from the modern world. But the danger of anachronism looms large in the case where modern imagery is applied unthinkingly. The phenomenon of migrant quarters that La Piana observed in the early twentieth century is of course also well known from our own society, but it is hardly a universal characteristic of all periods in which migration occurred. A recent large-scale historical survey has found that while clustering occurs frequently, it is certainly not a standard feature of all urbanised societies. ${ }^{5}$

It is undeniable that Rome at the time of the Principate was full of immigrants. We may define immigrants for our purposes as people (slave, freed, free) who were born outside the city of Rome and settled in the city on a permanent or semi-permanent basis. We do not know their numbers, but it seems safe to say that a large part of its population originated from elsewhere. They came from Italy and from the provinces, they comprised of men, women and children of all social statuses, from senators to destitute peasants; and people of all legal statuses, from Roman citizens to

3 E.g. C. Ricci, Orbis in urbe. Fenomeni migratori nella Roma imperiale (2005) 47-51, 72. Traces can be found in for example R. MacMullen, 'The Unromanized in Rome', in S.J.D. Cohen and E.S. Frerichs (eds.), Diasporas in Antiquity (Atlanta, Georgia 1993) 47-64; D. Noy, Foreigners at Rome. Citizens and strangers (London 2000), $15^{2}$ (though both with much caution and sophistication). The radically positivistic approach of P. Lampe, From Paul to Valentinus. Christians at Rome in the first two centuries (Minneapolis 2003) is squarely based on the assumption of residential segregation.

4 The argument bears some affinity to the evolutionary model formulated around the same time by the Chicago School of Sociology in which the appearance of migrant quarters was regarded as a stage in the process leading ultimately to the assimilation of migrants. Despite its large influence in migration studies, I have not found traces of such a stagemodel in the literature on Rome.

5 A.M. York, M.E. Smith, B.L. Stark, J. Novic, S.L. Harlan, G.L. Cowgill and C.G. Boone, 'Ethnic and class clustering through the ages: A transdisciplinary approach to urban neighbourhood social patterns', Urban Studies 48 (2011), 2399-2415, emphasizing in addition the diversity of forms and causes. 
peregrini and to slaves. ${ }^{6}$ Rome had become, in every sense of the word, a cosmopolis. ${ }^{7}$

Migration to Rome was substantial, but at the same time, it is clear that migration patterns differed significantly from those of La Piana's New World cities. In the case of Rome, a significant part of the migration was forced. Slaves obviously had no free choice of residence. Migration was also diffuse: we do not see migration waves, in which large groups of immigrants for some time came from a specific region, then from another, and so on. Migration was also socially rather heterogeneous: it certainly was not confined to the poorer segments of society who left home in search of a better life. Instead, many of the migrants might be described as stakeholders in the empire: they went to Rome because they had an interest in being in the centre of the empire. For many migrants, Rome, as capital of the empire, will have formed familiar territory.

Where, then, did Rome's immigrants live? Is it likely that they lived together? And if so, what are the implications for integration? In what follows, I will first discuss some theoretical issues, then move on to a discussion of the surprisingly few pieces of evidence from Rome, and then discuss indirect ways to analyse the issue. My argument is that with the exception of a Jewish enclave in Trastevere, migrant quarters were absent, and I will end by discussing some of its implications.

${ }^{6}$ For free foreigners see Noy 2000, op. cit. (n. 3) and Ricci 2005, op. cit. (n. 3), mainly based on the epigraphic evidence, and more briefly J. Dresken-Weiland, 'Fremde in der Bevölkerung des kaiserzeitlichen Rom', Römische Quartalschrift für christliche Altertumskunde und Kirchengeschichte 98 (2003), 18-34. For the isotopic evidence for immigration T.L. Prowse, H.P. Schwarcz, P. Garnsey, M. Knyf, R. Macchiarelli and L. Bondioli, 'Isotopic evidence for age-related immigration to imperial Rome', American Journal of Physical Anthropology 132 (April 2007), 510-519 with additional critical observations by C. Bruun, 'Water, oxygen isotopes, and immigration to Ostia-Portus', Journal of Roman Archaeology 23 (2010), 133-136 and K. Killgrove, Migration and mobility in imperial Rome (PhD diss., Univ. of N. Carolina, Chapel Hill 2010). In what follows I confine myself to the civilian part of the immigrant population; for military men at Rome, see apart from the work of Noy also the general survey of J. Coulston, "Armed and belted men": the soldiery in imperial Rome', in J. Coulston and H. Dodge (eds.), Ancient Rome: the archaeology of the eternal city (Oxford 2000) 76-118, with Dresken-Weiland 2003, 29-30 for discussion of living quarters.

7 See the volume of C. Edwards and G. Woolf (eds.), Rome the Cosmopolis (Cambridge 2003), esp. N. Morley, 'Migration and the metropolis', 147-157. 
Comparative evidence suggests that the issue should be approached with an open mind. Residential clustering is attested in many societies and many historical periods, but likewise there are many cases where it is absent, and there are also societies where it does occur in some cities, but not in others. ${ }^{8}$

An open mind, however, should not be confused with a lack of theory. There exists an enormous body of scholarly literature on modern forms of residential clustering. Although a significant part of it is devoted to technical issues of measurement that in the absence of quantifiable data are not relevant to Roman history, ancient historians would do well to take at least some of the major principles into account.

Residential clustering refers to the overrepresentation of a particular group in a particular section of the area in which the population lives. There are three important characteristics to such residential clustering: it can occur along several dimensions, it is a matter of degree, and it can concern more than two groups.

Firstly, residential clustering can appear in several forms. For present purposes, four of these dimensions are particularly relevant. Clustering can occur along social lines, with for example marginal groups living in separate areas, or with an elite that fences itself off in a nicer part of the city. Clustering can exist along economic lines, with richer or poorer neighbourhoods, or with different kinds of industries and shops being located in different parts of the city. It can occur along ethnic lines, with people of a specific ethnic identity living together. Clustering might also occur in terms of migration, with locals and newcomers living in separate areas.

To some extent these dimensions are subject to construction. This is best visible in the case of ethnicity, which is not a natural attribute but an ascribed one (self-ascribed, or ascribed by outsiders). It is also wellknown in the case of immigration: not all people that are born elsewhere are regarded as immigrants, or regard themselves as such, to the same degree. ${ }^{9}$

8 York et al. 2011, op. cit. (n. 5).

9 See the helpful summary of notions of 'boundary shift' in X. de Souza Briggs, 'Civilization in color: The multicultural city in three millennia', City \& Community 3 (2004), $311-342$, at $312-314$. 
Clustering can occur along several dimensions, but at the same time in practice they might overlap: clustering often occurs along several dimensions simultaneously. A segregated neighbourhood might comprise a socially marginal group that is relatively poor and consists predominantly of people with a particular ethnic background who have recently immigrated. It is precisely this convergence that can make the stronger forms of residential clustering so problematic in contemporary urban societies: as each factor reinforces the other, it becomes virtually impossible to escape from the neighbourhood. ${ }^{10}$

The second characteristic of clustering is that clustering is a matter of degree, not something that is absolute. On each dimension, clustering shows a spectrum running from complete dispersal (zero-cases) to complete clustering $(100 \%)$. The term clustering might be used as a neutral term for any type of overrepresentation, no matter how small. Although not all scholars use the same terminology, for our purposes the spectrum may be divided into the following broad categories. If the degree of clustering is significant, it can be termed segregation. At the higher end of the scale, one can speak of enclaves. A major characteristic of an enclave is that it remains dually open. Despite a high concentration of a particular group, not every member of the group lives there, and the area also comprises other groups. ${ }^{11}$ If clustering is total, it is termed a ghetto. In contrast to the enclave the ghetto is dually exclusive: all members of a group live in the area, and no other groups live amidst them. Sometimes, the term hyperghetto is also used, especially to characterise the situation in some contemporary U.S. cities. This might be regarded as the $110 \%$ version of clustering. Its major characteristic is that various dimensions of clustering occur simultaneously and reinforce each other.

A schematic presentation of the first two characteristics can be seen in the table.

$10 \mathrm{~J}$. Iceland, Where we live now: immigration and race in the United States (Berkeley 2009), 9-10. In contemporary cities of the U.S., residential segregation correlates directly with a host of additional factors, for example graduation rates at high schools, unemployment rates, income levels, crime levels, health levels, levels of single parenthood, social exclusion and feelings of alienation.

${ }^{11}$ C. Peach, 'Slippery segregation: discovering or manufacturing ghettos?', Journal of Ethnic and Migration Studies, 35.9 (2009), 1381-1395, at 1388. 
Table 1: Dimensions and degrees of clustering

\begin{tabular}{|c|c|}
\hline $\begin{array}{l}\text { Percentage } \\
\text { of clustering }\end{array}$ & social economic ethnic immigrants etc. \\
\hline $0-10$ & random dispersal \\
\hline $10-5^{0}$ & some clustering \\
\hline $5^{0-70}$ & $\begin{array}{l}\text { significant clustering } \\
\text { (segregation) }\end{array}$ \\
\hline $70-90$ & $\begin{array}{l}\text { very strong clustering } \\
\text { (enclaves) }\end{array}$ \\
\hline $90-100$ & $\begin{array}{l}\text { complete clustering } \\
\text { (ghettos) }\end{array}$ \\
\hline [110] & $\begin{array}{l}\text { complete clustering in } \\
\text { most dimensions } \\
\text { (hyperghetto) }\end{array}$ \\
\hline
\end{tabular}

To this figure we have to add a third characteristic. The discussion of the first two properties is based on the clustering of a single group. However, urban societies quite often consist of many different groups. Each of these groups may show different rates and different forms of clustering. Put differently, and quite importantly, a particular group might live mixed with another, but remain aloof of a third. ${ }^{12}$ This means that the matrix of clustering of figure 1 might look different for each group. It goes without saying that this may result in rather complex urban landscapes.

From the preceding discussion it may appear that clustering is simply a matter of definition and measurement. However, the subject is far from a neutral one. It is a highly charged topic in contemporary studies because of its political resonance.

Even in countries that used to be known for their adherence to multiculturalism, like the UK and the Netherlands, residential segregation is now seen as a substantial threat to national unity. The fear is that, as a consequence of residential segregation, minority ethnic group members run the risk of living 'parallel lives', not interacting with people from different backgrounds. ${ }^{13}$

In connection to this political resonance, agency and causation form large issues. Why do people live in separate neighbourhoods? ${ }^{14}$ Clustering might be regarded as the product of free choice ('self-segregation').

12 Iceland 2009, op. cit. (n. 10), 5 .

13 G. Bolt, A.S. Özüekren and D. Phillips, 'Linking integration and residential segregation', Journal of Ethnic and Migration Studies 36 (2010), 169-186, at 175.

14 Bolt, Özüekren and Phillips 2010, op. cit. (n. 13), 174. 
In some cases, segregation may be regarded as a counter-reaction to the arrival of newcomers: members of the host community might fence themselves off - a typical example is the process known as 'White flight', the movement out of the city to the suburbs. Or segregation may be forced upon a group. It may also be produced by institutional constraints, for example by the structure of the housing market. Then, there might also be active involvement of the community authorities, either to foster such separation, or, as nowadays, to counter its effects.

As the quote mentioned above shows, the issue is so politicised because of its connection to discussions of integration. Residential segregation is taken to be a sign that groups live 'parallel lives', and thereby remain aloof of the rest of society. It is therefore often regarded negatively. ${ }^{15}$

In the debates about integration, there is an understandable tendency to take an essentialist approach: to think that residential segregation is segregation itself, and conversely, to take the absence of clustering as a clear sign of integration. Scholars have warned that that is too easy, and have especially pointed out that the reverse pattern, lack of clustering, might not imply a larger degree of integration. Their studies show that government policies aimed at mixing populations that used to be segregated might just as well lead to social withdrawal and a lack of trust, even between members of one's own group. ${ }^{16}$ Forced heterogeneity, then, is not necessarily a good thing either. Government policies fail because ties in existing neighbourhood networks are severed without being adequately replaced by something else. ${ }^{17}$ Whereas it seems clear that there is a connection between residential clustering and integration, the relationship is not straightforward and should be determined on a case by case basis. Residential clustering offers a good approach to the study of integration, but should not be confused with integration itself.

What follows from these debates for ancient Rome is that residential clustering is neither simple nor self-evident. Clustering is a matter of degree and can take various forms. Rome was an extremely large city. Some degree of clustering along some of the dimensions is to be expected, for a completely uniform urban landscape could only occur in Von Thünen's dreams of isotopic landscapes. We should therefore not only ask whether

15 York et al. 2011, op. cit. (n. 5), 2400.

16 R.W. Putnam, 'E Pluribus Unum: diversity and community in the twenty-first century', Scandinavian Political Studies 30.2 (2007), 137-174.

17 Bolt, Özüekren and Phillips 2010, op. cit. (n. 13), 176-177. 
migrant quarters existed, but also to what degree, and along which lines, clustering took place.

\section{Migrant Quarters at Rome: The Evidence}

What evidence do we have for migrant quarters in Rome? In view of the paucity of the evidence, it seems best to cast the net wide and to take into account any reference to ethnic or migrant clustering.

In theory, archaeology would be capable of supplying indications of forms of residential clustering, for particular neighbourhoods might have had a distinct urban architecture. However, nothing of the sort is visible in the archaeological record of Rome. ${ }^{18}$ Nor does anything in the layout of the city as reconstructed from the Forma urbis Romae suggest dramatic differences between different parts of the city, apart from the fact that the parts that were rebuilt after fires showed more regular street plans. However, the lack of archaeological indicators need not mean much, as our knowledge of housing is extremely poor.

One type of argument that has been based on archaeological remains should be rejected. Especially in the case of the Jewish and the Christian catacombs it has been claimed that the location of the burial places indicates segregated residence within the city. ${ }^{19}$ Establishing connections in individual cases between place of burial and place of residence remains hazardous. Even apart from the fact that the catacombs date from a later period than the one under discussion here, the simple fact remains that there is no obvious reason to assume that extramural burial grounds catered solely or even predominantly for specific neighbourhoods. There is no known correspondence between place of residence and place of burial.

It seems better to concentrate on the evidence for the ethnic composition of neighbourhoods. When the regiones and vici were reorganised by Augustus $^{20}$ the districts were certainly not organised along ethnic lines. None of the (later) names of the regiones refers to ethnic traits, and the

18 J.B. Lott, The neighborhoods of Augustan Rome (Cambridge 2004), 23.

19 H. Leon, The Jews of Ancient Rome (2nd ed. C.A. Osiek; Peabody 1995) for the Jews; Lampe 2003, op. cit. (n. 3.), 23-38 for the Christians.

20 For the reorganisation, see O.F. Robinson, Ancient Rome: city planning and administration (London 1992) 9-10; L. Richardson, A new topographical dictionary of ancient Rome (Baltimore 1992), s.v. 'Regiones quattuordecim'. 
rather meagre evidence for ethnic vici-names is unimpressive. ${ }^{21} \mathrm{We}$ do not find a Little Syria, or a Little Greece, neither in official or in informal designations. Given the nature of the satirists' works, one would expect such statements if there was a reason to make them. ${ }^{22}$

Nevertheless, specific regions may have had or acquired a specific flavour. Three parts of the city merit particular discussion, in ascending order of interest: the Campus Martius, the Subura, and Trastevere. The Aventine as a plebeian area would also come into consideration, were it not for the fact that it is normally assumed that gentrification had set in here from the Late Republic onwards. ${ }^{23}$

Firstly, the Campus Martius. Three late Jewish inscriptions refer to a synagogue or Jewish community of the Campesians, which is normally thought to refer to the Campus Martius. Given the name, it seems reasonable to suppose that the synagogue catered for the Jews living in that area. ${ }^{24}$ There is, however, nothing that indicates that they dominated the Campus Martius. Apart from the presence of some foreign cults, there is no evidence for clustering of other groups.

The second area that merits attention is the Subura. This part of regio IV is generally described as a poorer district of the city. ${ }^{25}$ It is an area where cheap and low-quality agricultural products are sold. ${ }^{26}$ It is also strongly associated with prostitution; so, Martial can use magistra Suburana for prostitute. ${ }^{27}$ In none of the references there is a particular ethnic quality visible. There are a number of references to a synagogue (or community) of the Siburesians. ${ }^{28}$ If this name does refer to the Subura, ${ }^{29}$ it seems

21 In general, see Lott 2004, op. cit. (n. 18). A couple of the 120 vici that are known by name carry ethnic names. But the evidence is hardly compelling. For example, the vicus Tuscus derived its name from Etruscan immigrants in a distant past - perhaps even to the Etruscan phase of the regal period. The vicus Capitis Africae has been supposed to form a neighbourhood for people of African descent, but might with more probability be named after an allegorical sculpted head of Africa. See Noy 2000, op. cit. (n. 3), 152 and DreskenWeiland 2003, op. cit. (n. 6), 32-33.

22 Cf. M.-J. Kardos, 'Quartiers et lieux de Rome dans les épigrammes de Martial'. Bulletin de l'Association Guillaume Budé (2002), 119-135.

23 E.g. La Piana 1927, op. cit. (n. 2), 213-215.

24 JIWE II.288; $560 ; 577$.

25 Varro, De L.L. 5.48 for etymology; Mart., Ep. 5.22, for the area having dirty stones and steps never dry; 12.18 for noise (clamosa); Juv., Sat. 11.50-51.

26 Mart., Ep. 7.31; 10.94 .

27 Mart., Ep. 6.66 (quales in media sedent Subura), cf. also $2.17 ; 11.61 ; 11.78$.

28 JIWE II.338; 428; 451; 452; 527; 557.

29 See Noy on JIWE II.338, adding that in non-Jewish inscriptions the spelling with an i does not occur. The problem then is that it is difficult to think of another explanation 
reasonable to suppose that the synagogue served a locally residing Jewish population. But just as in the Campus Martius, it is unlikely that they dominated the area.

Trastevere is normally regarded as a, if not the, migrant quarter. ${ }^{30}$ The evidence for this is a statement found in Philo's Embassy:

How then did he [i.e. Augustus] show his approval? He was aware that the great section of Rome on the other side of the Tiber is occupied and inhabited by Jews (katechomenen kai oikoumenen pros Ioudaion), most of whom were Roman citizens emancipated.

(...)

Yet nevertheless he neither ejected them from Rome nor deprived them of their Roman citizenship because they were careful to preserve their Jewish citizenship also, nor took any violent measures against the houses of prayer, nor prevented them from meeting to receive instructions in the laws, nor opposed their offerings of the first-fruits. ${ }^{31}$

In itself the statement undeniably points to strong residential clustering of Jews in Trastevere. At the same time, there are also reasons for worry. In the first place (and obviously), we are in the world of rhetoric. Philo's claim that the Jews lived clustered was part of the argument that Augustus did not expel the Jews despite the fact that it would have been possible because they could be localised. Secondly, it is somewhat disturbing to find that this is the single mention to clustering in Trastevere that we have - in fact, it is the only mention of any type of migrant quarter of Rome. Thirdly, it is difficult not to think that Philo is influenced by the case of his home-city Alexandria, where a great part of the Jews lived in the Delta quarter and residential segregation between Greeks and Jews seems to have been the norm. Philo's statement is in itself straightforward, but raises the question if it can be taken at face value.

In that respect it is important to note that there is clear evidence that Jews were not exclusively living in Trastevere. They can be found to live elsewhere in the city - as the evidence for the synagogues/communities of the Siburesians and Campesians mentioned above already implies. ${ }^{32}$

for the name - though note that some other synagogue-names have not been explained satisfactory either.

30 L. Collon, 'Remarques sur les quartiers juifs de la Rome antique', Mélanges d'archéologie et d'histoire 57 (1940), 72-94; Leon 1995², op. cit. (n. 19), 136; MacMullen 1993, op. cit. (n. 3), 62-63; Lott 2004, op. cit. (n. 18), 22.

31 Philo, Leg. 155.

32 Collon 1940, op. cit. (n. 30). 
It is also clear that other groups lived in Trastevere as well. Based on the presence of foreign cults in Trastevere it has been argued that many eastern immigrants resided in Trastevere. The relation between foreign cult and ethnic groups is a complex issue that cannot be treated here. The argument that foreign cults served as local community centres for migrants deserves as much scrutiny as the claim that there existed migrant quarters in Rome. The openness of the polytheistic system is one crucial issue, another that the ethnicity of at least some of the supposedly foreign cults was a constructed one, ${ }^{33}$ a third that many foreign cults were located outside the pomerium anyway, ${ }^{34}$ and a fourth that it is not a given that adherents lived in the vicinity of the cult places. Be that as it may, it seems likely that other migrants also lived in Trastevere. At the same time, as such foreign cults can also be found elsewhere in the city, ${ }^{35}$ the presence of these foreign groups cannot have been confined to Trastevere.

The evidence for migrant quarters is thus flimsy. All we have in terms of hard evidence is one passage in Philo. In itself this passage is quite explicit: a great part of the Jews clustered in Trastevere. In their case there is nothing that points to the double exclusivity that is associated with the strongest forms of ethnic segregation: not all Jews lived in Trastevere, and other groups lived there as well; and both the Jews and these others can be found in other parts of the city. In the theoretical terms discussed above, what Philo described should be regarded as an enclave, not a ghetto. Otherwise, evidence of migrant quarters is absent.

In fact, it is amazing to see how large a scholarly edifice has been build on so little evidence - what can only be explained by the fact that scholars have been projecting their own preconceived notions on the city. At the same time, the explicitness of Philo raises a problem. The fact that the evidence is flimsy does not necessarily mean that no residential segregation occurred. Put differently, the evidence of Philo can be made to fit with more than one scenario. As we saw, clustering can also occur on other dimensions. Is there evidence for clustering along social and economic lines in Rome?

33 For brief skeptical remarks, H. Solin, 'Mobilità socio-geographica nell'impero Romano. Orientali in Occidente. Considerazioni isagogiche', in M. Mayer i Olivé, G. Baratta and A. Guzmán Almagro (eds.), Acta XII Congressus Internationalis Epigraphicae Graecae et Latinae (Barcelona 2007), vol. 2, 1363-1380, at 1375.

34 M. Le Glay, 'Sur l'implantation des sanctuaires orientaux à Rome', in L'urbs: espace urbain et histoire ( ${ }^{\text {er }}$ siècle av. J.-C.-III siècle ap.J.-C.) (Rome 1987), 545-562, rightly emphasizing that no single factor can explain the location of all.

35 Dresken-Weiland 2003, op. cit. (n. 6), 32. 


\section{Housing and Social Clustering at Rome}

Let us start with social clustering, the degree to which people of different status lived segregated. Did specific social groups live in specific parts of the city? Do we find better neighbourhoods and backward ones?

Although the archaeological record for elite domus is surprisingly poor, it is quite clear that at least after the Republic the elite domus were distributed over large parts of the city. The distribution seems not to have been completely random. ${ }^{36}$ Quite naturally domus were located in the better (i.e. higher) parts of the city, and those areas may have concerned more the eastern than western part of the city, but within these parts no real concentration of elite houses seem to have occurred, and even less popular areas attracted some elite housing. Ironically, elite housing can also be found near the Subura, precisely the area that is otherwise described as one of the poorer parts of the city. ${ }^{37}$

In addition, elite residential mobility probably was high. As is wellknown, the domus was one of the main venues for the expression of elite identity. We may therefore assume that a high premium was placed on remaining in the same place. But at the same time the sources suggest that there was a high turn-over rate in elite residences. ${ }^{38}$ It need not be forgotten that there was a continuous influx of new members of the elite from outside Rome, and a relatively high attrition rate. ${ }^{39}$

In one sense, the domus themselves did show social clustering: a great part of Rome's slaves lived within them - and even some freedmen remained there after manumission. At the same time, slave households were mixed: they were mixed ethnically, and they were mixed in the sense

\footnotetext{
36 J.-P. Guilhembet, 'La densité des domus et des insulae dans les XIV régions de Rome selon les régionnaires: représentations cartographiques', Mélanges de l'Ecole Française de Rome. Antiquité 108 (1996), 7-26; W. Eck, 'Cum dignitate otium. Senatorial domus in imperial Rome', Scripta Classica Israelica 16 (1997), 162-19o, at 176-183; G. de Kleijn, The water supply of ancient Rome: city area, water, and population (Amsterdam 2001), 224-246; C.F. Noreña, 'Water distribution and the residential topography of Augustan Rome', in L. Haselberger and J.Humphrey (eds.),Imaging ancient Rome.Documentation-visualizationimagination (Porthsmouth 2006), 91-105.

37 T.A.J. McGinn, 'Pompeian brothels and social history', in Id. et al., Pompeian brothels, Pompeii's ancient history, mirrors and mysteries, art and nature at Oplontis \& the Herculaneum 'basilica' (JRS Suppl. 47) (Portsmouth 2002), 7-46, at 17; T.A.J. McGinn, The economy of prostitution in the Roman world: a study of social history \& the brothel (Ann Arbor 2004), 20.

38 Eck 1997, op. cit. (n. 36), 172, 187-190.

39 K. Hopkins with G. Burton, Death and renewal. Sociological studies in Roman history 2 (Cambridge 1983).
} 
that a part of the slaves was homeborn, and a part consisted of imported slaves, that is, immigrants. The elite domus that were distributed over the city may be visualised as islands with a particular social composition, but that social composition was itself not homogeneous.

As for the rest of the population, the great majority of the free (and part of the freed) population lived in insulae. The quality of their housing is open to debate. ${ }^{40}$ What is clear is that the inhabitants of the insulae were not exclusively consisting of the urban poor. One of the most remarkable features is that insulae contained literally a cross-section of urban society, with the poorest tenants living in cellae high up, but wealthier and more respectable people living at the lower levels of the same buildings. ${ }^{41}$ The insulae were both highly stratified and socially mixed.

Scholars of modern forms of clustering emphasise that institutional barriers often help to reinforce if not produce residential segregation, and they point in particular to the structure of the housing market. ${ }^{42}$ No such institutional barriers seem to apply to the Roman insulae. Again, turn-over rates seem to have been high. The openness of the rental market for housing where short-term contracts were quite normal must have been conducive to significant residential mobility. ${ }^{43}$ Evictions could occur easily in the case of non-payment of rent, and people could themselves voluntarily and on short notice decide to look for other living space.

To sum up: there is no evidence for strong clustering along social lines. The evidence points rather to the reverse: social mixing, at all levels. Given the highly stratified nature of Roman society this in itself is an extremely interesting feature that merits closer study. For our current purposes, it is important because strong social mixture decreases the possibilities that migrant quarters arose.

\section{Occupational Clustering and Economic Zoning}

It is also possible to study residential clustering along economic lines. This issue normally comes under the heading of economic zoning: the

40 A. Scobie, 'Slums, Sanitation, and Mortality in the Roman World', Klio 68 (1986), 399-433; R. Laurence, 'Writing the Roman metropolis', in H.M. Parkins (ed.), Roman urbanism: beyond the consumer city (London and New York 1997), 1-20.

41 J.E. Stambaugh, The Ancient Roman City (Baltimore and London 1988), 174-178.

42 Bolt, Özüekren and Phillips 2010, op. cit. (n. 13), 174; A. Kearns and M. Parkinson, 'The significance of neighbourhood', Urban Studies 38 (2001), 2103-2110, at 2105.

43 B. Frier, Landlords and tenants in imperial Rome (Princeton 1980). 
clustering of specific economic activities in specific parts of the city. This is directly relevant for the subject, though not because specific types of work are associated with migrants or ethnic groups - there is hardly such evidence. It is rather because in the case of workshops, residence and place of work were often the same.

Some zoning seems to have occurred, but it was far from complete. Polluting industry might have been located at the margins of the city. An example is formed by the recent find of a very large fullery or tannery at Casal Bertone at the eastern part of Rome outside the walls. ${ }^{44}$ The areas outside the city functioned of course primarily as burial area. In addition, there was a noticeable tendency in Rome to create elite horti around the city. The areas around the city thus functioned in three quite distinct ways: burial, recreation for the elite, and industry. How such a multifunctional space would work remains difficult to envisage, and in the case of Rome the other two functions, burial and elite recreation, are much better attested than the industrial one. But the phenomenon itself is not in doubt. In that sense economic zoning did occur.

At the same time, some of the supposedly polluting industry was also located in the city itself. 45 The location of industry could also depend on transport possibilities and the availability of resources. This could lead to locations both within and outside the city. An obvious example of the former is industry that was directly connected to the harbour facilities of Rome: the marble district in Emporion forms a good example. By contrast, brick industry was situated near the clay beds outside Rome.

Whereas it has become increasingly clear that there existed a variety of scales in production units in the Roman urban economy, there can be no doubt that in the case of Rome the small-scale shop/workplace remained dominant. Normally they were located on the ground floors of insulae. The fragmented form of production suggests in itself that the workplaces/ shops were dispersed all over the city. This is also suggested by the references in the literary sources, which do not allow for pinpointing specific activities to specific parts of the city, but rather show scatter. ${ }^{46}$ The fourth century regionaries indirectly offer corroboration: they show an almost

44 Killgrove 2010, op. cit. (n. 6), 74.

45 J.P. Morel, 'La topographie de l'artisanat et du commerce dans la Rome antique', in L'urbs 1987, op. cit. (n. 34), 127-155, at 131; F. Kolb, Rom. Die Geschichte der Stadt in der Antike (München 1995), 496-497.

46 Kardos 2002, op. cit. (n. 22), 123 on glass production in Martial. 
complete dispersal of warehouses, baths and bakeries over the fourteen regiones. ${ }^{47}$

However, dispersal was probably not complete; and some economic zoning might have occurred. ${ }^{48}$ Two types of evidence have been adduced for some form of clustering of shops/workplaces. Firstly, the evidence from inscriptions where people with a particular occupation state where they work shows that some occupations are mentioned more than once in the same area. ${ }^{49}$ In the second place, some vici were named after trades. ${ }^{50}$ Neither of them is fully convincing in itself: the fact that people mentioned where they worked and the fact that some craftsmen mentioned the same area or street proves little in itself. Vicus names could refer to old qualities - and the simple fact remains that the overwhelming majority of known vicus-names does not refer to economic activities. Some streets and districts were named after the dominant trades, but nothing precluded the presence of other shops there, nor the presence of the same trade elsewhere. ${ }^{51}$ Some clustering rather than exclusive zoning took place.

One last topic merits attention. A somewhat unexpected part is played by the evidence for prostitution. In particular at Pompeii, prostitution has raised discussions not so much of economic zoning, but of moral zoning: the idea that brothels had to be kept out of sight of the more respectable or vulnerable parts of society. The evidence is complicated, because interpretation hinges on the definition and identification of what counts as a brothel. Estimates of the number of brothels vary radically: only one Pompeian brothel has been identified with certainty, while others have been identified with differing degrees of plausibility. Again, some clustering might have taken place, and again this was hardly complete. ${ }^{52}$ This might also help to understand the connection made between prostitution and the Subura at Rome. As McGinn remarks:

47 E.S. Ramage, 'Urban problems in ancient Rome', in R.T. Marchese (ed.), Aspects of Greek and Roman Urbanism: Essays on the Classical City (BAR 188) (Oxford 1984), 61-92, at 88 .

48 Morel 1987, op. cit. (n. 45), 141; Morel in his study noted that where there is much evidence for districts that are "particulièrement industrieuses", at the same time the evidence shows "une dispersion considérable des activités".

49 R. MacMullen, Roman social relations, 5o B.C. to A.D. 284 (New Haven 1974), 134.

50 See MacMullen 1974, op. cit. (n. 49), 130, 132 for a list.

51 Morel 1987, op. cit. (n. 45), 143; Kolb 1995, op. cit. (n. 45), 503.

52 McGinn 2002, op. cit. (n. 37), esp. 15-19; McGinn 2004, op. cit. (n. 37), esp. 14 f., both with further literature. McGinn argues against clustering, and points out that the more brothels are identified as such, the wider their dispersal becomes. 
I do not deny that the Subura had its brothels and its prostitutes. But the brothels were just one element in the urban mix of residential (both upperand lower-class) and commercial buildings. ${ }^{53}$

Overall, in Rome only modest forms of economic clustering seem to occur, and nowhere do we find exclusivist areas dominated by one trade. The dominant pattern is one of random scatter, with a modest degree of clusters of specific shops and industries.

\section{Meet and Greet: Some Implications}

Let us conclude with where we started, with La Piana.

The question which confronts us at the outset is whether these foreign masses were assimilated by the native element, and if so to what extent, or whether they formed a racial mixture in which the native element itself finally lost its identity. ${ }^{54}$

Though nowadays the question would be phrased differently, it is still valid.

With one notable exception the evidence points to the absence of migrant quarters. The exception concerns the case of the Jews, where a form of ethnic clustering occurred: a major share of them lived in Trastevere. This was certainly not exclusive, in both senses: not all Jews lived in Trastevere, and Trastevere did not consist solely of Jews. If anything, it was an ethnic enclave rather than a ghetto. Otherwise, migrant quarters seem absent. We do not hear of a Syrian quarter, or a quarter of the Gauls. We do not hear about groups of North-Africans living together. The absence of other forms of clustering along social and economic lines strongly suggests that in this case the absence of evidence is evidence of absence.

It seems no coincidence that the sparse evidence that we have relates to the Jews. This was the group with the clearest demarcated identity, in

\footnotetext{
53 McGinn 2004, op. cit. (n. 37), 21, who continues: "If any one factor made for a somewhat higher concentration of brothels in the Subura it was the relative absence of public construction, which would have meant more emphasis on residential and commercial establishments overall. Nevertheless, the presence of prostitution in the Subura was likely to have been more a matter of perception than reality. Its proximity to the Forum heightened its profile in the eyes of the male members of the elite who wrote the texts that define for us the nature of the life in the capital'. Cf. $80-81$, where the argument is also turned around: because of the fact that cities were socially homogeneous, "there is simply no rationale supporting the theory of moral zoning."

54 La Piana 1927, op. cit. (n. 2), 200.
} 
which - famously - ethnic and religious traits merged. It was also a very large group, numbering in the thousands if not tens of thousands of persons. In the case of other migrant groups it is clear that ethnicity did not function as a primary marker of identity. Epitaphs show a much greater propensity to record social ties, occupation, or legal status than origin.

In addition, one might not only look at the extent to which ethnicity functioned as a marker of identity, but also to patterns of migration. Migration to Rome was an extremely unruly phenomenon. People of all statuses were involved in it, and it was massive. Particular streams of chain migration cannot be easily identified. It seems likely that the diverse nature of immigration actively prevented the development of stronger forms of residential segregation. The fact that a significant part of the migration consisted of the forced mobility of slaves who ended up anywhere in the city will not have helped either.

Seen from the perspective of residential clustering, immigration thus occurs as a relatively unproblematic issue. Most immigrants seem to have lived amidst the rest of the urban population. But does the absence of residential clustering suggest successful integration?

As we saw above, scholars of modern forms of residential segregation have warned not to take residential clustering as a straightforward index of integration. It is in fact somewhat ironic to find in the very same statement in which Philo claimed the Jews lived in Trastevere also the contention that most of them had obtained Roman citizenship - often regarded as the sign of integration par excellence. ${ }^{55}$ In the case of the other groups residential mixing does not necessarily imply integration. How, then, should we perceive the relationship between clustering and integration in the case of Rome?

At an admittedly much more abstract level, we might take recourse to acculturation theory. ${ }^{56}$ Acculturation theory of the past two decades has offered a model in which people constructed their identity through selective appropriation of elements of available cultural repertoires. In the case of immigrants, we may envisage these repertoires as consisting of their home culture and of Roman culture. Scholars have emphasised that the outcome of such cultural dialogues might lead to rather hybrid results rather than to purely Roman products, and well-demarcated cultural repertoires should be regarded as analytical categories rather than as existing

55 The classic case was A.N. Sherwin-White, The Roman Citizenship (Oxford $1973^{2}$ ).

56 See the paper by F.G. Naerebout in this volume. 
in reality. What is important is that this type of cultural interaction was highly asymmetrical: Roman culture formed the dominant force. At the same time Roman culture was open: it could be joined by outsiders, and it could be redefined in the process. In a presumably deliberately circular definition, Woolf has defined Roman culture as

the range of objects, beliefs and practices that were characteristic of people who considered themselves to be, and were widely acknowledged as, Roman.

But what counted as 'Roman' changed over time.

Becoming Roman was not a matter of acquiring a ready-made cultural package, then, so much as joining the insiders' debate about what that package did or ought to consist of at that particular time. ${ }^{57}$

The process of becoming Roman through joining the "insider's debate" applied to all levels of society - the definition of Roman culture is applicable to culture in the broad sense of the word.

Although this model of cultural interaction has not been applied to the city of Rome, the process of becoming Roman must have been just as important in the heart of the empire as it was elsewhere. Perhaps it was even more important, for the city was one of the crucial places where Roman identity was defined.58 Of course, not everyone will have participated to the same degree, and migrant identities were certainly not completely obliterated. ${ }^{59}$ But the power of Roman culture will have been alluring. Migrants might have been involved to greater or lesser degrees in becoming Roman, but at the same time this process changed what was considered to be Roman. At the heart of the empire, Roman culture became cosmopolitan.

Residential mixing provided a setting for such processes. By meeting and greeting people of various backgrounds the inhabitants of Rome were forced to define themselves in relation to others, at all levels, ranging from the individual household to the level of the Roman state. It is the precisely the humble, everyday quality of it that makes residential mixing so important. Not so much because it brings us back to antiquarian descriptions of Alltagsleben or wie es eigentlich gewesen, but rather because it reintroduces

57 G. Woolf, Becoming Roman: the origins of provincial civilization in Gaul (Cambridge and New York 1998), 11.

58 See Briggs 2004, op. cit. (n. 9), 315 for the notion that cities produce new social identities.

59 MacMullen 1993, op. cit. (n. 3); Noy 2000, op. cit. (n. 3), 10-12. 
a quality to our discussions which is all too often lacking from it. Ancient historians tend to forget that society is defined not only in collective public rituals, but also in its daily routines. Greeting a neighbour might be just as important as going to the games. It is in day-to-day encounters, in small-scale social interactions that individuals define themselves, their social relations and thereby society.

Especially in view of the immense scholarly effort devoted to the reconstruction of the topography of ancient Rome, it is surprising how little has been written on Roman residential clustering. I suggest we place it higher on our research agenda. ${ }^{60}$ Slippery subjects like integration need concrete research questions. It needs to be realised that in debates about integration in contemporary society the question of residential clustering is accorded a very large amount of attention. In the Roman case, the absence of evidence is only relative, and in itself indicative. In any case paucity of sources has never deterred ancient historians from doing research. We ought to shift the topography of our discourse.

60 A good point of reference is offered by the publications of the research group 'Urban organization through the ages: Neighbourhoods, open spaces, and urban life' of Arizona State University; see http://cities.wikispaces.asu.edu/ (consulted Nov. 2011). I am in complete agreement with the remark of York e.a. 2011, op. cit. (n. 5), 2041 that 'deeper empirical analyses of specific cities are required to model processes of social clustering'; the cities of the Roman world would form an excellent case. See also Briggs 2004, op. cit. (n. 9), for a wider comparative framework (and some discussion of Rome). 



\title{
ROMAN CITIZENSHIP AND THE INTEGRATION OF WOMEN INTO THE LOCAL TOWNS OF THE LATIN WEST
}

\author{
Emily A. Hemelrijk
}

To what extent were women in provincial cities of the Latin West integrated into Roman society? Was there a gender barrier to integration and how can we measure integration? These questions are the starting point of my enquiry. In this paper, I shall try to shed some light on women's integration (or lack of integration) into civic life by looking at Romanization and the spread of Roman citizenship. ${ }^{1}$ Let me start with an example of the difficulties that confront us in trying to assess the integration of women. It has often been observed that on many tombstones in the northern and north-western provinces women are portrayed in indigenous dress, while their husbands wear Roman clothing. ${ }^{2}$ This is usually explained as a sign that women were less integrated into Roman society. Their domesticity and exclusion from politics meant - so it is supposed that they were less influenced by Roman culture than men. In support of this view, some scholars point to epitaphs, which in some provinces show

1 Despite pertinent criticisms of this much-debated concept, aptly summarized by D.J. Mattingly, Imperialism, Power, and Identity. Experiencing the Roman Empire (Princeton and Oxford 2011), 38-40 and 204-207, I shall here retain "Romanization" for want of a better term to cover the multifarious processes of interaction within the Roman Empire, leading both to assimilation and integration, and to divergence and local cultures. For a more detailed discussion of these processes, see the article by Frederick Naerebout in the present volume.

2 Examples are found especially in the German provinces, but also in Raetia, Noricum and Pannonia, see J. Garbsch, Die norisch-pannonische Frauentracht im 1. und 2. Jahrhundert (Munich 1965). For discussion see P. Zanker, 'Bürgerliche Selbstdarstellung am Grab im römischen Kaiserreich', in H.-J. Schalles, H. von Hesberg and P. Zanker (eds), Die römische Stadt im 2. Jahrhundert n. Chr. Der Funktionswandel des öffentlichen Raumes. Kolloquium in Xanten vom 2. bis 4. Mai 1990 (Cologne 1992), 346-347; U. Rothe, Dress and Cultural Identity in the Rhine-Moselle Region of the Roman Empire (Oxford 2009, BAR 2038), 65-66, 69-72; M.L. Okun, The early Roman frontier in the Upper Rhine area: assimilation and acculturation on a Roman frontier (Oxford 1989, BAR 547), 112-113 and 135-136, G. Kremer, 'Die norischpannonischen Grabbauten als Ausdruck kultureller Identität?', in A. Schmidt-Colinet (ed.), Lokale Identitäten in Randgebieten des Römischen Reiches: Akten des Internationalen Symposiums in Wiener Neustadt, 24.-26. April 2003 (Vienna 2004), 152 and 154-155. 
more indigenous names for women than for men, ${ }^{3}$ or to a famous saying in Cicero's De Oratore about women's old-fashioned diction, which Cicero attributed to their retired way of life. ${ }^{4}$ Thus, the notion that women were less integrated in Roman society is widespread. But should local dress and indigenous names on tombstones indeed be regarded as proof of women's lack of integration? Moreover, is it legitimate to generalize from these examples to all women?

Before I continue, I should briefly define what I understand by 'integration'. In line with the description in the call for papers for this conference, I see it as a "process of inclusion". My questions, therefore, are: to what extent were women in the Latin-speaking provinces "included" in Roman society or, in other words, how much were they affected by Roman culture and involved in Roman civic life? Secondly, what stimulated, or impeded, their 'integration'? I shall focus on two aspects that may serve as criteria: first, I shall briefly discuss women's adoption of Roman culture, particularly of Roman dress, customs and values. After that, I shall turn to their participation in civic life, paying special attention to activities that were

3 See S. Fontana, 'Leptis Magna. The Romanization of a major African city through burial evidence', in S. Keay and N. Terrenato (eds), Italy and the West. Comparative Issues in Romanization (Oxford 2001), 167-170 on names in epitaphs from Lepcis Magna in northern Africa, who concludes: "the process of Romanization also seems to affect only minimally women, relegated to a domestic life removed from power" (p. 170) and L.A. Curchin, 'Social relations in central Spain: patrons, freedmen and slaves in the life of a Roman provincial hinterland', Ancient Society 18 (1987), 85: "a strong tradition of indigenous names persisted among females (who were, after all, less likely to require a romanized image for career purposes)." On names and Romanization in general, see B. and H. Galsterer, 'Romanisation und einheimische Traditionen', in Schalles, von Hesberg and Zanker 1992, op. cit. (n. 2), 377-379.

4 D. Cherry, Frontier and Society in Roman North Africa (Oxford 1998), 156-157. Cic. De Or. 3.45 (the speaker is the orator L. Licinius Crassus): "For my own part when I hear my wife's mother Laelia - since it is easier for women to keep the ancient way of speaking unspoiled, as they do not converse with great numbers of people and so always retain the vocabulary and accents they heard first - well, I listen to her with the feeling that I am listening to Plautus or Naevius: the actual sound of her voice is so unaffected and natural that she seems to produce no trace of display or affectation. Therefore, I infer that that was how her father and her ancestors used to speak - not harshly, like the person I mentioned, nor with a broad or countrified or jerky accent, but neatly and evenly and smoothly" (Equidem cum audio socrum meam Laeliam - facilius enim mulieres incorruptam antiquitatem conservant, quod multorum sermonis expertes ea tenent semper quae prima didicerunt - sed eam sic audio ut Plautum mihi aut Naevium videar audire: sono ipso vocis ita recto et simplici est ut nihil ostentationis aut imitationis afferre videatur; ex quo sic locutum esse eius patrem iudico, sic maiores, non aspere, ut ille quem dixi, non vaste, non rustice, non hiulce, sed presse et aequalibiter et leniter), see E.A. Hemelrijk, Matrona docta. Educated women in the Roman élite from Cornelia to Julia Domna (London and New York 1999), 77. 
typical for Graeco-Roman towns, such as civic munificence, priesthoods, and patronage, as well as to the public honour paid to female citizens.

Let us first return to the depiction of women in local dress on tombstones in the northern and north-western provinces: do these representations bear out the interpretation that these women wore native dress because they were hardly affected by Roman culture (or at least less so than men) and that this was due to their confinement to domestic life? This interpretation is hard to prove; moreover, there are other explanations that seem equally feasible. For instance, though acquainted with Roman costume, women may have used local dress in order to express their loyalty to their ethnic identity. The display of local costume, with its rich brooches, fibulae and torques, may have served to show their affluence (or that of their families) and their high status in the local hierarchy. Or indigenous dress may have expressed cherished notions of femininity, such as chastity or fertility. Thus, women's dress allows for several interpretations. Rather than women's supposed lack of acquaintance with Roman culture, a variety of reasons - including local prestige, ethnic identity, ideals of femininity, but perhaps also family pressure or a husband's wishes - may have been decisive for the choice of women's dress. ${ }^{5}$

As Ursula Rothe has recently noted, there are considerable regional differences in relation to dress and gender in funerary art within the northwestern provinces. Whereas in the middle Rhine and Ubian areas women often appear in local costume with their husbands in Roman dress, in the Treveran area it is the other way round: women sometimes appear in Roman clothes, while their husbands and sons wear a Gallic ensemble. Rothe also describes various compromises between Roman and native dress: some women are depicted in mixed ensembles or in a local garment

5 For various interpretations of women's dress in the Rhine-Moselle region, see Rothe 2009, op. cit. (n. 2), 37-39, 44-47, 61-62, 65-6, 69-75: "the role of men in deciding female attire has often been overlooked" (p. 75, n. 811). She also discusses women as "guardians of ethnicity" (p. 66) and posits a gendered division of public and private (p. 71) as possible reasons for women's retention of native dress. Obviously, the local costume was better suited to the colder climate. According to Zanker 1992, op. cit. (n. 2), 346-347 women clung to their native tradition ("ein bewusstes Festhalten an den eigenen Traditionen im privaten Bereich"), whereas men, who identified with public life, adopted the image of the Roman citizen. Kremer 2004, op. cit. (n. 2), 152 assumes that there was a gradual change over time in favour of Roman dress for women in the provinces of Noricum and Pannonia; see also Rothe 2009, op. cit. (n. 2), 69: the majority of the stones from the Middle Rhine and Ubian areas showing women in native dress can be dated in the first and early second centuries. However, Galsterer 1992, op. cit. (n. 2), 381-382 and 388-389 notes a revival of (elements of) native dress (and names, deities and burial rites) in the second century. 
draped in a Roman manner. These different types of dress may be worn in the same period and sometimes even within the same family. ${ }^{6}$ Thus, the cultural meaning of dress is more complicated than it may appear at first sight. Apart from a gradual change over time in favour of Roman dress, especially in the cities, dress seems to be a matter of 'cultural choice', both for men and for women, and may have depended on the circumstances in which it was worn. A similar choice may have applied to names. In northern Africa, for instance, both Roman and indigenous names are found within the same family, and we find men using a Latin(ized) or an indigenous name on different (public or private) occasions. ${ }^{7}$ Thus, applying Andrew Wallace-Hadrill's notion of 'code-switching', we may perhaps suppose that some women stuck to local dress - or indigenous names - in private (which includes their portraits on tombs), but used Latin(ized) names and wore Roman dress when in public. ${ }^{8}$

In the provinces of the Mediterranean area - especially in northern Africa and southern and eastern Spain - women of the wealthy classes are represented in public wholly according to Roman fashion. Though most

6 For an example, see Rothe 2009, op. cit. (n. 2), 72 and 124 (T 26), see further 34-37, 42-43 and 69-77.

7 See Mattingly 2011, op. cit. (n. 1), 240, Galsterer 1992, op. cit. (n. 3), 378 and M. Carroll, Spirits of the Dead. Roman Funerary Commemoration in Western Europe (Oxford 2006), 258-259, on persons using a Roman or native name depending on the (public or private) occasion; see also J.N. Adams, Bilingualism and the Latin Language (Cambridge 2003), 213-229, on the use of Latin or Latinized names alongside Punic ones. For Roman and indigenous names alternating within the same family, see D. Cherry, 'Marriage and acculturation in Roman Algeria', Classical Philology 92.1 (1997), 71-83, Cherry 1998, op. cit. (n. 4), 86 and 101-140 and M. Corbier, 'Family and kinship in Roman Africa', in M. George (ed.), The Roman Family in the Empire: Rome, Italy and Beyond (Oxford 2005), 255-285; see also L.A. Curchin, 'Social relations in central Spain: patrons, freedmen and slaves in the life of a Roman provincial hinterland', Ancient Society 18 (1987), 87, on Roman and indigenous names in central Spain.

8 For the application of the linguistic notion of 'code-switching' to issues of Roman culture and identity, see A. Wallace-Hadrill, Rome's Cultural Revolution (Cambridge 2008). On 'code-switching' between Latin and vernacular languages, see Adams 2003, op. cit. (n. 7). For 'cultural choice', see R.R.R. Smith, 'Cultural choice and political identity in honorific portrait statues in the Greek East in the second century A.D.', Journal of Roman Studies 88 (1998), 56-93. Rothe 2009, op. cit. (n. 2), 69, also suggests that men and women wore different dress on different occasions. Tombs were the "most public of private monuments" (M. Koortbojian, 'In commemorationem mortuorum: text and image along the "street of tombs"', in J. Elsner (ed.) Art and Text in Roman Culture (Cambridge 1996), 225), and their portraits and inscriptions were intended mainly for the family and for fellow citizens. For an illuminating study of how tombs served family interests and indigenous agendas, see Mattingly 2011, op. cit. (n. 1), 246-268, on the monumental tombs at Ghirza in northern Africa. The stylized portrait busts depict (men and) women in local dress and jewellery (pp. 261-262). 
of these women were of local descent, their portrait statues and honorific inscriptions portray them as Roman by their full Roman dress and Latin or Latinized names. In the provinces of Spain, their dress often includes the stola, the symbol of the Roman (citizen) matrona, even in periods and statue types for which, in Italy, the stola is atypical. ${ }^{9}$ Though these women may have worn local dress or have spoken their native languages in private, their public statues and inscriptions present them as thoroughly assimilated to Roman culture. Unlike women of the northern and northwestern provinces, whom we mostly know from tombs, these honorific statues were set up in the public areas of the towns. Does this contrast between the northern and north-western regions and the Mediterranean area merely reflect a difference between public and private dress, or may we draw more far-reaching conclusions regarding the integration of women in these regions?

To answer this question, we should consider our second criterion: women's involvement in civic life, especially in those civic roles that were typical for Graeco-Roman towns and may be taken as a sign of their "Romanness". A quick glance at the chronological and geographical distribution of almost 1,200 inscriptions recording women's civic roles and public honour in the cities of Italy and the Latin West shows that they coincide with the pace of Romanization and urbanization in the western provinces (figs. 1 and 2). ${ }^{10}$ Italy provides the bulk of the evidence: forty-six percent of the inscriptions are from towns in Italy (with the almost total exception of Rome, where munificence and public honour came increasingly to be

9 C. Marcks, Formen statuarischer Repräsentation von Privatpersonen in Hispanien zur Zeit der Republik und in der Kaiserzeit (Cologne 2008), 19, 80, 115-118, 119-121, 129-130 and 167 , notes that, in the Spanish provinces, the stola is also found in statues of the early second century (when the stola was out of fashion in portrait statues from Italy) and in statues of the Eumachia-Fundilia type that, in Italy, do not show a stola. Moreover, in contrast to the north-western provinces, Noricum and Raetia, no (partly) indigenous dress is found in statues (whether male or female) in Roman Spain.

10 These figures are based on almost 1,200 inscriptions recording women's civic roles in Italy and the Latin West - as civic benefactresses, priestesses, and patronesses and "mothers" of cities and collegia - and their public honour by means of public portrait statues, honorific decrees, or public funerals granted them by the local council. About 250 additional inscriptions (almost all from Italy in the second and early third centuries) recording women's various other connections with collegia and their social 'networks' have not been taken into account here. All are discussed in E.A. Hemelrijk, Hidden Lives - Public Personae. Women and Civic Life in Italy and the Latin West during the Roman Principate (forthcoming). 
reserved for members of the imperial family). ${ }^{11}$ Yet, a considerable number, amounting to forty-four percent in total, are found in those provincial regions that were most highly Romanized and urbanized, namely Africa Proconsularis and Numidia (25\%), southern and eastern Spain (14\%) and, to a lesser extent, Gallia Narbonensis (5\%). The number of inscriptions increased steadily reaching its highest point in the period of the greatest prosperity, namely the second century and, for northern Africa, also the early third. By contrast, I have found virtually no evidence for women's civic roles in the more thinly urbanized provinces of the north-western part of the Empire, such as Britannia, Gallia Belgica, and Germania Inferior, and very little in neighbouring provinces such as Germania Superior, Gallia Aquitania, and Lugdunensis.

What conclusions may we draw from this distribution? First, how can we be sure that it is not merely the product of the spread of the "epigraphic habit"? Several differences between the distribution of my corpus of inscriptions and the spread of the "epigraphic habit" argue against this suggestion. The most striking are that there is virtually no epigraphic evidence for civic roles or public honour for women from the area with the highest "epigraphic density": the city of Rome, and from the militarized frontier zones. ${ }^{12}$ Furthermore, when compared to their male counterparts, women fulfilling civic roles - such as benefactresses, city patronesses, and (imperial) priestesses - are not only fewer, but also more narrowly limited to the most densely urbanized and Romanized Mediterranean regions. ${ }^{13}$ Of course, in respect of the scarcity of the evidence for women's civic roles

${ }^{11}$ W. Eck, 'Senatorial self-representation. Developments in the Augustan period', in F. Millar and E. Segal (eds), Caesar Augustus: Seven Aspects (Oxford 1984), 129-167; Id., 'Ehrungen für Personen hohen soziopolitischen Ranges im öffentlichen und privaten Bereich', in Schalles, von Hesberg and Zanker 1992, op. cit. (n. 2), 359-376, and G. Lahusen, Untersuchungen zur Ehrenstatue in Rom. Literarische und epigraphische Zeugnisse (Rome 1983), 97-107; see also E.A. Hemelrijk, 'Local empresses: priestesses of the imperial cult in the cities of the Latin West', Phoenix 61.3-4 (2007), 319-323.

12 On the much-debated issues of the "epigraphic habit" and "epigraphic density" (the number of surviving inscriptions per 1,00o square kilometres) see R. MacMullen, "The epigraphic habit in the Roman empire', American Journal of Philology 103 (1982), 233-46; E.A. Meyer, 'Explaining the epigraphic habit in the Roman empire: the evidence of epitaphs', Journal of Roman Studies 80 (1990), 74-96; G. Woolf, 'Monumental writing and the expansion of Roman society in the early Empire', Journal of Roman Studies 86 (1996), 22-39 and Id., Becoming Roman: The Origins of Provincial Civilization in Gaul (Cambridge 1998), 82-105. For the exceptional position of the city of Rome, see the preceding note.

13 E.A. Hemelrijk, 'City patronesses in the Roman Empire', Historia 53.2 (2004), 209-245; Ead., 'Priestesses of the imperial cult in the Latin West: titles and function', Antiquité Classique 74 (2005), 137-170 and Ead. forthcoming, op. cit. (n. 10). 


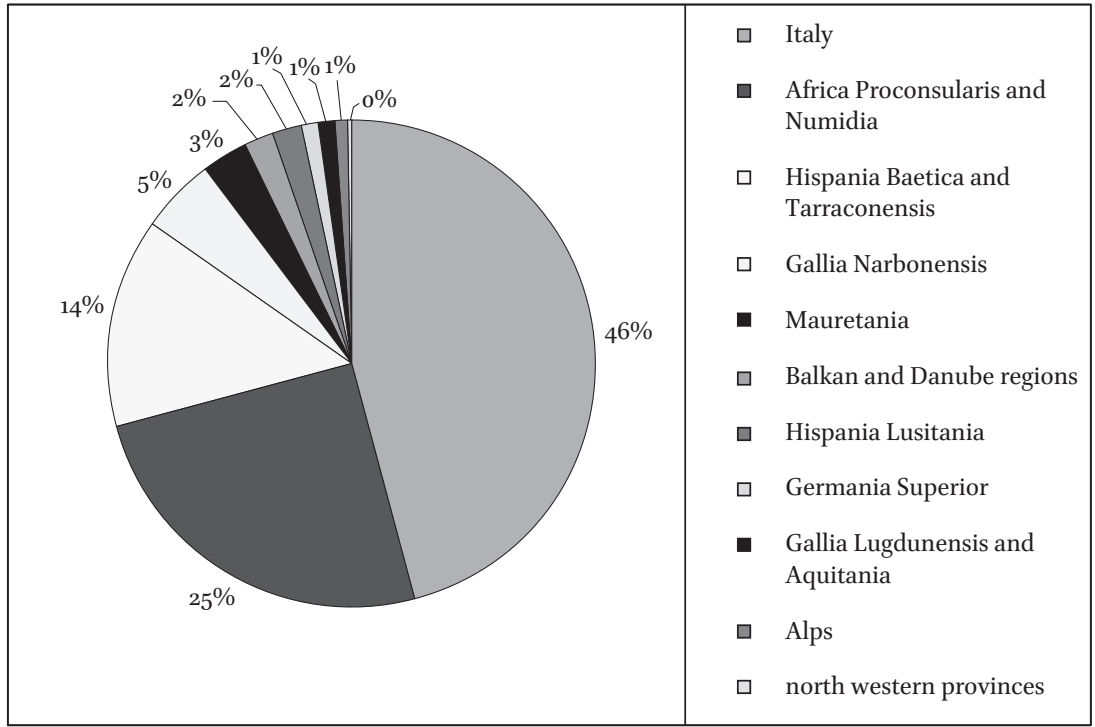

Figure 1: Geographical distribution

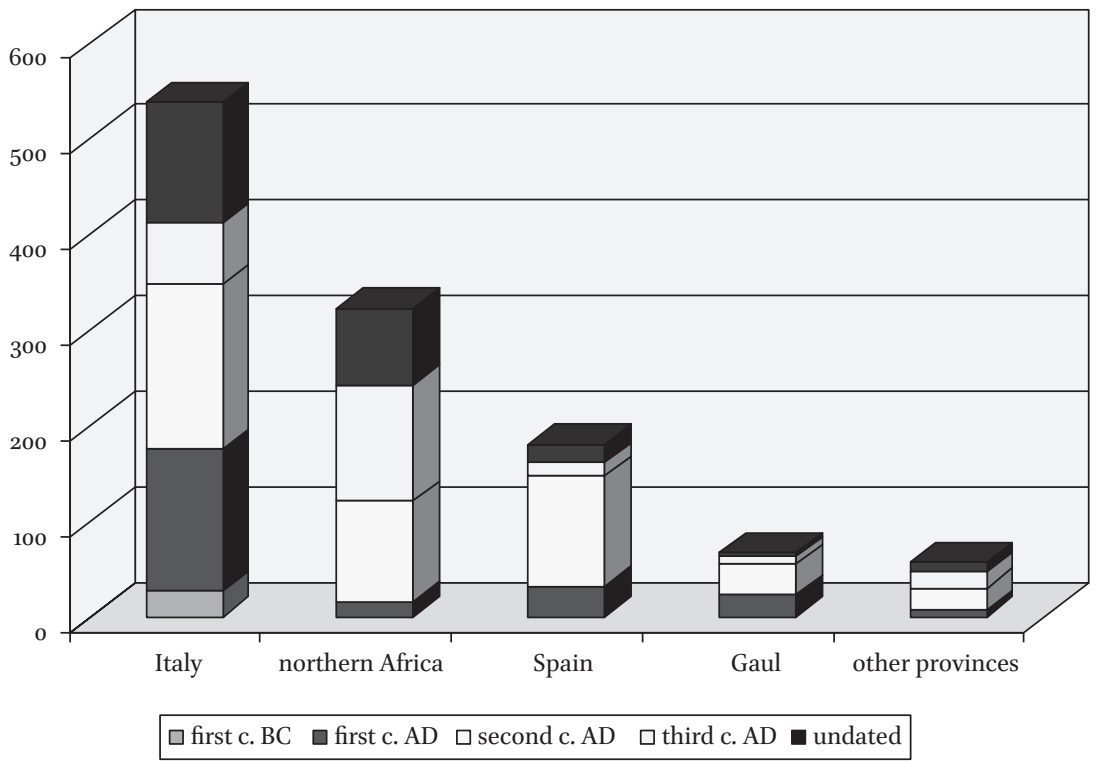

Figure 2: Geographical and chronological distribution 
in the north-western provinces, we must take into account the lower "epigraphic density" of these regions. However, even when offset against the epigraphic totals for each province, the distribution of my corpus shows a particularly low percentage for the north-western provinces. ${ }^{14}$ Taken together, these differences strongly suggest that there must be other reasons for the distribution.

Let us therefore turn to the interrelated processes of Romanization, urbanization, and economic prosperity in the Mediterranean regions between the late first and the early third centuries. To what extent did they encourage the participation of women? Apart from providing the material and mental preconditions for munificence and civic priesthoods, the increase in the number and prosperity of Roman cities and the spread of Roman citizenship and Roman civil law had great consequences for women of the wealthier classes. ${ }^{15}$ This holds especially for the laws of inheritance, which allowed for equal portions for sons and daughters in case of intestacy, and for the separation of property between husband and wife in Roman marriage without manus. Moreover, the Augustan ius liberorum gave female Roman citizens who were sui iuris and had three or more children full legal capacity as property owners. Even when still

14 The numbers of inscriptions in my corpus have been compared to the CIL totals for the same provinces in the Frankfurt database, which also covers recent $C I L$ supplements. They vary between $2.42 \%$ for Baetica and $0.05 \%$ for the north-western provinces (Britannia, Gallia Belgica, and Germania Inferior). If compared to all inscriptions per province in the Frankfurt database (which includes the $A E$ and other epigraphic corpora) the percentages vary between $1.56 \%$ for Baetica and $0.02 \%$ for the north-western provinces. I thank Anna Sparreboom for the calculations.

15 For the spread of Roman citizenship and Roman law - even among civitates that were not compelled to use Roman law - see, among others, A. Lintott, Imperium Romanum. Politics and Administration (London and New York 1993), 129-145 and 154-16o (on Roman and indigenous law), and A.T. Fear, Rome and Baetica. Urbanization in southern Spain c. 50 BC-AD 150 (Oxford 1996), 152-156 and 162-169 (on interpretatio peregrina and various degrees of adoption of Roman law in peregrine communities). On legal pluralism and choice of law in dealings between Roman citizens and foreigners before Caracalla, see the articles of Clifford Ando and Hannah Cotton in the present volume. Whereas these articles are mainly concerned with the use of local law, I am especially interested in the application of Roman law. That Roman family law was actually applied in local cities is apparent, for example, from the Flavian municipal charter of Irni in Spain, ch. 21, $22,29, \mathrm{~B}$ and 56 on potestas, manus, tutela and the ius liberorum, and ch. 86 on the use of patria potestas in non-Roman families; see J. González, "The Lex Irnitana: a new copy of the Flavian municipal law', JRS 76 (1986), 147-243 and J.F. Gardner, 'Making citizens: the operation of the Lex Irnitana', in L. de Blois (ed.), Administration, Prosopography and Appointment Policies in the Roman Empire (Amsterdam 2001), 215-229. Municipal charters, like that of Irni, stimulated non-Roman citizens, too, to live according to Roman law, by dealing with them as if they were Roman citizens, see J.F. Gardner, Being a Roman citizen (London 1993), 188-190 and Ead., 'Making citizens'. 
in tutela, women in the imperial period were not much hampered; or, in the words of Jane Gardner: "where property is concerned, the legal capacity of Roman men and Roman women is virtually the same". ${ }^{16}$ Thus, the spread of Roman citizenship and Roman law gave female Roman citizens of wealthy families unprecedented legal and financial capacities.

The financial independence of married women sui iuris, which is attested in Italy from the late first century вс onwards, gave rise, in the second and early third centuries, to a class of wealthy female landowners and businesswomen in the most densely urbanized and Romanized Mediterranean provinces. ${ }^{17}$ According to a recent estimate, the spread of Roman citizenship and Roman civil law in these regions brought thirty to forty-five percent of private property into the hands of women, mainly through inheritance. ${ }^{18}$ Even if this estimate is too high, the accumulation of wealth in the hands of certain women, and their legal capacity to use it, made them a force to be reckoned with. In comparison to their male peers, women may even have had greater riches, since they did not need money for a political career. This is in agreement with my corpus of inscriptions, where we find women not only among the donors of smaller gifts but, contrary to what is usually believed, especially among the most gener-

16 J.F. Gardner, 'Gender-role assumptions in Roman law', Échos du Monde Classique / Classical Views 39, n.s. 14 (1995), 393; see also R.P. Saller, 'Pater familias, mater familias, and the gendered semantics of the Roman household', Classical Philology 94 (1999), 182-197. For a clear survey of the effects of Roman civil law on women, see J.F. Gardner, Women in Roman Law and Society (London 1990, 3rd edition); Ead. 1993, op. cit. (n. 15), 85-109 and Ead., 'Gender-role'; see also S. Treggiari, Roman Marriage. Iusti Coniuges from the Time of Cicero to the Time of Ulpian (Oxford 1991), on Roman marriage and the ius liberorum.

17 E.W. Haley, Baetica Felix. People and Prosperity in southern Spain from Caesar to Septimius Severus (Austin 2003), 135-170, on wealthy male and female oil merchants, landowners, pottery producers and owners of figlinae (clay-beds) in imperial Baetica; see also Mattingly 2011, op. cit. (n. 1), 146-166 and R.P. Duncan-Jones, 'Economic Change and the Transition to Late Antiquity' in S. Swain and M. Edwards (eds), Approaching Late Antiquity. The Transformation from Early to Late Empire, (Oxford 2004), 20-52, on the economic growth of Africa Proconsularis and Numidia in the 2nd and 3rd centuries AD. See also the article by Anthony Álvarez Melero in this volume on the wealth of women of equestrian rank and the sources of that wealth.

18 A. Arjava, Women and the Law in Late Antiquity (Oxford 1996), 70-71. The much lower percentage (roughly twenty percent) implied by Champlin's discussion of female testators - E. Champlin, Final Judgments. Duty and Emotion in Roman Wills, 200 B.C.-A.D. 250 (Berkeley, Los Angeles and Oxford 1991), 103-130 - is disproved by J. Pölönen, 'The division of wealth between men and women in Roman succession (c.a. 50 BC - AD 250)', in P. Setälä et al. (eds), Women, wealth and power in the Roman Empire (Rome 2002), 147-179, who argues for a more equal division of inherited wealth between men and women, in which women received "at least forty to fifty percent of all private property in inheritance practice in ancient Rome” (p. 179). 
ous benefactors financing public buildings such as temples, bathhouses, amphitheatres and aqueducts. ${ }^{19}$ As a reward, many of them were honoured with one or more public statues, which sometimes included their families. Annia Aelia Restituta, for example, received five public statues because of her promise to build a theatre in her hometown of Calama in Numidia. ${ }^{20}$ And Iunia Rustica, perpetual priestess in Cartima in southern Spain, was granted public statues of herself and her son because of her lavish benefactions. Pleased with the honour, she bore the costs herself and added a statue of her husband, thus forming a family group. ${ }^{21}$

Obviously, my corpus of inscriptions is no random sample. Forty percent of the provincial women mentioned in them belonged to the elite, and all must have been among the most wealthy, and probably most ambitious, women in their towns (fig. 3). This also holds for the small percentage of women of freed families (4\%), many of whom were related to Augustales; their munificence and priestly functions on behalf of their cities furthered the careers and upward mobility of their sons and descendants. ${ }^{22}$ That the social status of a large number of women $(56 \%)$ is unknown to us is partly due to the absence of status indicators for women in epigraphic texts. Apart from the title clarissima femina, which came into fashion for women of senatorial rank in the middle of the second century, there is no clear terminology for female rank. The term stolata femina is rare in inscriptions in the Latin West, and its designation of equestrian rank not

19 Hemelrijk forthcoming, op. cit. (n. 10) contra J.R. Patterson, Landscapes and Cities. Rural Settlement and Civic Transformation in Early Imperial Italy (Oxford 2006), 173.

20 Annia Aelia Restituta (Calama, Num; 161-169): ILAlg. I, 287 = CIL VIII, 5366: ordo univer/sus statua[s] n(umero) quinq(ue) de pu[bl(ico)] faciend[as] / decreuit; ILAlg I, 286 = CIL VIII, 5365 = 17495: or/do ob eam causam sta/tuas quinque de pu/blico pon [i] censuis/set.

21 CIL II, 1956 = ILS 5512 = ILMMalaga 6 (Cartima, Hisp.Baet. late 1st c.): Iunia D(ecimi) f(ilia) Rustica sacerdos / perpetua et prima in municipio Cartimitan[o] / porticus public(as) uetustate corruptas refecit solum / balinei dedit vectigalia publica vindicavit signum / aereum Martis in foro posuit porticus ad balineum / solo suo cum piscina et signo Cupidinis epulo dato / et spectaculis editis d(e) p(ecunia) s(ua)d(edit)d(edicauit) statuas sibi et C(aio) Fabio / Iuniano f(ilio) suo ab ordine Cartimitanorum decretas / remissa impensa item statuam C(aio) Fabio Fabiano uiro suo / d(e) p(ecunia) s(ua) f(actas) d(edit). "Iunia Rustica, daughter of Decimus, first and perpetual priestess in the municipium of Cartima, restored the public porticos that were ruined by old age, gave land for a bathhouse, reimbursed the public taxes, set up a bronze statue of Mars in the forum, gave at her own cost porticos next to the bathhouse on her own land, with a pool and a statue of Cupid, and dedicated them after giving a feast and public shows. Having remitted the expense, she made and dedicated the statues that were decreed by the council of Cartima for herself and her son, Gaius Fabius Iunianus, and she likewise made and dedicated at her own cost the statue for Gaius Fabius Fabianus, her husband".

22 See, among others, S.E. Ostrow, 'The Augustales in the Augustan Scheme', in K.A. Raaflaub and M. Toher (eds), Between Republic and Empire. Interpretations of Augustus and his Principate (Berkeley, Los Angeles and Oxford 1990), 370. 


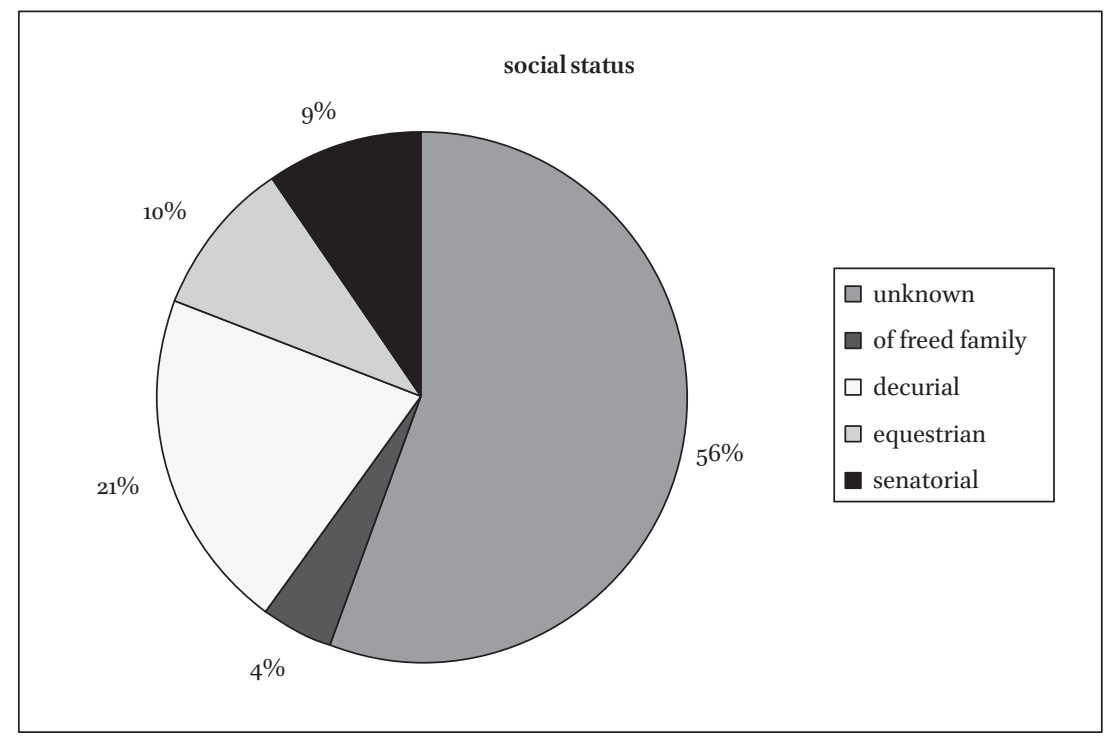

Figure 3: Social status of women in civic roles in the Western Provinces $(n=628)$

always beyond doubt. In the absence of a clear terminology, it is hard to make out whether a woman was of equestrian or decurial family; the surest indication is the career of her husband or father, but this is often unknown. ${ }^{23}$ Thus, more women may have belonged to families of the decurial or equestrian orders than is here recorded. Nevertheless, the majority of the women whose social status is unclear probably did not belong to families of the political elite but to wealthy rising families of what we may somewhat anachronistically call the "upper-middle class". By their benefactions, priesthoods and public statues, these women won public recognition for themselves and furthered the social status of their families.

23 On the title clarissima femina for women of senatorial rank, see M-Th. RaepsaetCharlier, 'Clarissima femina', Revue Internationale des Droits de l'Antiquité 28 (1981), 189212. As an indicator of equestrian rank, the title stolata femina seems restricted to the late second and third centuries, see B. Holtheide, 'Matrona stolata - Femina stolata', Zeitschrift für Papyrologie und Epigraphik 38 (1980), 127-31. J. Edmondson, 'Public dress and social control in late republican and early imperial Rome', in J. Edmondson and A. Keith (eds), Roman dress and the fabrics of Roman culture (Toronto 2008), 24 regards it as the equivalent of the more frequent honesta femina, but honesta does not always indicate equestrian rank; in some cases, it seems a moral qualification rather than an indication of rank. For women's indirect relation to the elite orders, see Hemelrijk 1999, op. cit. (n. 4), 11-12 with further references. 
Virtually all women in my corpus were Roman citizens, but sometimes their local birth shines through in their names and in those of their relatives. The combination of indigenous or Latinized elements in names such as Caecilia Zaba, Fabia Bira, Nahania Victoria, Cornelia Sillibor, and Moccia Silvina reflects their complicated social identity, which was both indigenous and Roman. ${ }^{24}$ This did not prevent them from presenting themselves in public as typically 'Roman' women, conforming in dress and behaviour to Roman custom. They held priesthoods in the cults of 'Roman' deities (including the imperial cult) and embellished their cities according to the Graeco-Roman tradition of euergetism. Especially in the early Empire, when Roman citizens were comparatively rare outside Italy, the display of Roman citizenship and familiarity with Roman culture must have brought great prestige. Its privileges and prizes were desired, and advertised, not only by men but - as is apparent from my corpus also by women. Their honorific statues portray them according to Roman fashion, and (more often than in Italy) these statues were set up in the most prestigious location, the forum. ${ }^{25}$ By contrast, in the northern and north-western provinces, the slower spread of Romanization and Roman citizenship, and the lower level of urban density and prosperity, seem to have had a negative influence, especially on women, who are virtually absent from the public domain. Some scholars believe that their position deteriorated under Roman rule as compared to the pre-Roman period, but the evidence for women in these regions before the Roman conquest is too limited, and regionally too disparate, to allow comparison. ${ }^{26}$

So, what may we conclude as to the integration of women into Roman society and the factors that stimulated, or hampered, their integration? First, that "women" as a category is too broad and undifferentiated to

24 Caecilia Zaba, great-priestess of Ceres(?): CIL VIII, 10575 (Saltus Burunitanus, Afr. Proc. 2nd c.); for Fabia Bira, daughter of Izelta and wife of M. Valerius Severus, son of Bostar, flaminica prima of Volubilis (Maur. Ting.) in the mid first century and Nahania Victoria, flaminica perpetua of Thugga (Afr. Proc. 184-192), see Hemelrijk 2005, op. cit. (n. 13) and Ead., 'Priestesses of the imperial cult in the Latin West: benefactions and public honour', Antiquité Classique 75 (2006), 85-117. Cornelia Sillibor (or daughter of Sillibor): CIL $\mathrm{II}^{2} / 7,5=$ CIL II, $335^{1}=$ CILA 3.1, 339 (Ossigi Latonium, Hisp. Baet., early first c.). Moccia Silvina, benefactress of the local collegium centonariorum: CIL XII, 2824 (Ugernum, Gall. Narb.).

25 Hemelrijk forthcoming, op. cit. (n. 10).

26 See L. Allason-Jones, Women in Roman Britain (London 1989), 190, and R. Hingley, 'Domestic organisation and gender relations in Iron Age and Romano-British households', in R. Samson (ed.), The social archaeology of houses (Edinburgh 1990), 141, on women in Iron Age Britain. 
draw valid conclusions: not only are there considerable regional differences, but there were also other distinctions, such as social status, wealth, age, and residence, that influenced the degree of their integration. Women of wealthy families in the Mediterranean regions seem to have benefited most from the spread of Roman citizenship and law, and from the growing urban prosperity. Though excluded from politics and outnumbered by male benefactors and imperial priests, their wealth, and their legal capacity to use it, gave these women a public face. By spending their money for the embellishment of their cities, and by holding expensive priesthoods, they gained prestige for themselves and for their families. Like their male counterparts, though less frequently, they were granted honorific statues and public funerals, and some were officially co-opted as a patroness or a 'mother' of a city or collegium. ${ }^{27}$ Thus, these wealthy women in Roman cities of the Mediterranean regions may truly be regarded as fully integrated into Roman society. Of course, this does not preclude the possibility that they retained local traditions and used their native languages in private. ${ }^{28}$

By contrast, women of the poorer classes, and women living in rural areas in the north-western regions, may indeed have lagged behind. Before the general grant of Roman citizenship in 212, few of them seem to have been Roman citizens and, being poor, most were unable to benefit from the advantages of Roman marital law. Possibly, as has been suggested by some, Roman rule may even have worked against their integration, since it favoured the men of their class, who entered into direct contact with Romans and Roman habits as craftsmen, traders, or soldiers. ${ }^{29}$ Roman culture and rulings, such as the marriage ban for Roman soldiers before

27 E.A. Hemelrijk, 'Patronesses and "mothers" of Roman collegia', Classical Antiquity 27.1 (2008), 115-162; Ead. 2004, op. cit. (n. 13) and Ead., 'Public Roles for Women in the Cities of the Latin West', in S.L. James and S. Dillon (eds), A Companion to Women in the Ancient World (London 2012), 478-490.

28 See, for instance, Mattingly 2011, op. cit. (n. 1), 239-240, and Adams 2003, op. cit (n. 7), 213-235, on the continued use of Punic, also among members of the local elites.

29 Cf. Cherry 1998, op. cit. (n. 4), 157 on women in northern Africa: "I suspect that the Roman occupation of north Africa may in fact have served to undermine the position of women in indigenous society, because men are likely to have received a much greater share of the favours that the Roman state routinely dispensed to those provincials who collaborated in military and civilian government". See also n. 26 above. Cherry seems to include women of the elite, which - as I hope to have shown - is not justified. The notions "discrepant experience" and "discrepant identities", coined by Mattingly 2011, op. cit. (n. 1), 203-245, seem useful here to understand the different experiences of Roman rule among women of different regions, domicile (urban or rural), wealth, and social standing. 
the reign of Septimius Severus, ${ }^{30}$ may have undermined the position of indigenous women of these classes, who in a changing world may have lost whatever status they had previously enjoyed, without receiving a new one in return. Though this is speculative, it may explain the disparity in our evidence. Whereas women of wealthy urban families in the Mediterranean regions profited from the spread of Roman citizenship and Roman law, women of the poorer, rural classes and regions seem to have been only marginally, or perhaps at first even negatively, affected by Roman influence. Thus, Roman rule may have had opposite effects on different groups of women, depending on differences in wealth, social status, and residence. Though Roman rule did affect all women, it did not have the same effect on all. The resulting diversity in attitudes - ranging from pride in Roman citizenship to loyal adherence to indigenous traditions, or to code-switching between the two depending on circumstances - seems to be reflected in the differences in dress and names on honorific statues and tombstones in the western provinces of the Roman Empire.

30 See S.E. Phang, The Marriage of Roman Soldiers (13 B.C.-A.D. 235). Law and Family in the Imperial Army (Leiden, Boston, Cologne 2001) on the marriage of Roman soldiers and the financial disadvantages of a soldier's family; cf. also B. Campbell, 'The marriage of soldiers under the Empire', Journal of Roman Studies 68 (1978), 153-166, on the problems suffered by soldiers, their 'wives' and their children because of the ban. 


\title{
DU FOYER AU FORUM. LA PLACE DES MATRONES ÉQUESTRES DANS LES ACTIVITÉS ÉCONOMIQUES
}

\author{
Anthony Álvarez Melero ${ }^{1}$
}

L'étude de la place des femmes dans l'économie se révèle en général une tâche peu aisée. En effet, à l'instar de bon nombre d'activités impliquant un certain degré d'interventions en public ${ }^{2}$, les Anciens eux-mêmes, par la bouche de Columelle, par exemple, considéraient que ces dames devaient s'en tenir éloignées pour se contenter de la gestion des affaires domestiques $^{3}$. Toutefois, puisque les sources littéraires, où règnent généralement la partialité et les poncifs négatifs à l'égard des femmes, ne nous sont rarement d'un grand secours et semblent même confirmer leur marginalisation, il faut se tourner vers l'épigraphie et la papyrologie pour tenter de trouver une réponse à nos questions.

Dans le cadre de cet exposé, qui ne prétend nullement épuiser un sujet aux vastes et infinies perspectives, en constant renouvellement, je vais m'intéresser plus particulièrement aux parentes de chevaliers. Sans revenir en détail sur l'historiographie de ce groupe «privilégié » de femmes, il me paraît utile de brièvement rappeler que les matrones équestres, telles que je les ai recensées ${ }^{4}$, sont ces filles, petites-filles, épouses, sœurs, mères et aïeules de chevaliers romains attestées dans nos sources, principalement épigraphiques. Cette définition a pu être établie grâce aux prescriptions

1 Investigador Juan de la Cierva, Departamento de Historia antigua, Universidad de Sevilla et SociAAM-Oikoumene, Université libre de Bruxelles. Je tiens à remercier Mmes S. Demougin (EPHE), E. Hemelrijk (UvA), M.-Th. Raepsaet-Charlier (ULB) et M.G. Chic (U. Sevilla) pour leurs relectures, conseils et avis stimulants. Je demeure seul responsable des lacunes et erreurs qui subsistent. Pour les toponymes les plus connus, j'ai opté pour leur dénomination moderne; pour les autres, j'ai conservé la terminologie latine.

2 Pour un aperçu, voir M.-Th. Raepsaet-Charlier, 'Les activités publiques des femmes sénatoriales et équestres sous le Haut-Empire romain', in W. Eck et M. Heil (éd.), Senatores populi Romani. Realität und mediale Präsentation einer Führungsschicht. Kolloquium der Prosopographia Imperii Romani vom 11.-13. Juni 2004 (Stuttgart 2005), 169-212.

3 La lecture de l'introduction au livre 12 de son ouvrage De l'Agriculture est à cet égard éclairante à plus d'un titre: Colum., pr. 12.

4 Il faut savoir que cette appellation n'apparaît que dans le compte-rendu épigraphique des Jeux séculaires de l'an 204 de notre ère pour qualifier les épouses de chevaliers et mères de famille qui prirent part au sellisterne du 2 juin 204: Cf. I.B. Pighi, De ludis saecularibus populi Romani Quiritium libri sex (Amsterdam 1965² [Milan, 1939]) : Fr V/Va 1. 26-29. 
du sénatus-consulte de Larinum, découvert en 1978, daté de l'an 19 de notre ère ${ }^{5}$, et d'un autre sénatus-consulte de $23^{6}$. En dépit de l'importance numérique de ces dames, peu de recherches leur furent consacrées, étant donné la difficulté qu'il y a à les identifier en tant que telles puisqu'elles ne disposaient pas de titre qui leur soit propre. Malgré tout, quelques études ont paru sur la place des femmes dans l'économie ${ }^{7}$, entre autres de l'ordre sénatorial $^{8}$, mais en revanche aucun travail ne s'est exclusivement concentré sur les matrones équestres, si ce n'est en passant, pour les raisons évidentes que je viens d'évoquer'.

Dans les pages qui suivent, seront présentées quelques activités économiques qui témoignent, entre les règnes d'Auguste et de Gallien, de

$5 A E$ 1978, 145.

6 Plin., nat. 33, 32. Ce sénatus-consulte rappelle l'obligation d'ingénuité pour le père, l'aïeul paternel, et le candidat chevalier pour accéder à l'ordre équestre. Afin de déterminer si les épouses, les mères et aïeules des futurs chevaliers étaient soumises à la même contrainte, je les ai comptabilisées dans mon catalogue prosopographique. Sur tout cela, A. Álvarez Melero, 'Matronae equestres ex provincia Lusitania ortae', Revue belge de philologie et d'histoire 86 (2008), 69-95.

7 Que de chemin parcouru en effet depuis l'article pionnier de S. Treggiari, 'Jobs for Women', American Journal of Ancient History 1 (1976), 76-104. A sa suite, sans prétention à l'exhaustivité et en guise d'introduction, ont paru d'autres études telles celles de N. Kampen, Image and Status. Roman Working Women in Ostia (Berlin 1981); J. Maurin, 'Labor matronalis: aspects du travail féminin à Rome', in E. Lévy (éd.), La femme dans les sociétés antiques. Actes des colloques de Strasbourg (mai 1980 et mars 1981) (Strasbourg 1983), 139-155 ; M. Eichenauer, Untersuchungen zur Arbeitswelt der Frauen in der römischen Antike (Francfort 1988); S.R. Joshel, Women, Identity, and Legal Status at Rome. A Study of the Occupational Inscriptions (Norman-Londres 1992); S. Mratschek-Halfmann, Divites et praepotentes. Reichtum und soziale Stellung in der Literatur der Prinzipatszeit (Stuttgart 1993) (Historia Einzelschriften 70), passim; A.M. Andermahr, Totus in praediis. Senatorischer Grundbesitz in Italien in der Frühen und Hohen Kaiserzeit (Bonn 1998), passim; D. Gourévitch et M.-Th. Raepsaet-Charlier, La femme dans la Rome antique (Paris 2001), 183-204; P. Setälä, 'Women and Brick Production. Some New Aspects', in P. Setälä et al. (éd.), Women, Wealth and Power in the Roman Empire (Rome 2002), 181-201 ; C. BriandPonsart, 'Les dames et la terre dans l'Afrique romaine', Histoire et sociétés rurales 19 (2003), 79-9o ; A. Buonopane et F. Cenerini (éd.), Donna e lavoro nella documentazione epigrafica. Atti del I seminario sulla condizione femminile nella documentazione epigrafica (Faenza 2003). Sur l'évergétisme féminin, voir note 11.

8 M.-Th. Raepsaet-Charlier, 'L'activité évergétique des femmes clarissimes sous le HautEmpire', in M.L. Caldelli et al. (éd.), Epigrafia 2006. Atti della XIVe rencontre sur l'épigraphie in onore di Silvio Panciera con altri contributi di colleghi, allievi e collaboratori, III (Rome 2008), 1029-1045.

9 Une exception, toutefois, celle de Raepsaet-Charlier 2005, op. cit. (n. 2), 202-203 au caractère exemplatif mais non exhaustif. Voir aussi J. Andreau, 'Intérêts non agricoles des chevaliers romains ( $\mathrm{II}^{\mathrm{e}}$ siècle av. J.-C.- $\mathrm{III}^{\mathrm{e}}$ siècle ap. J.-C.)', in S. Demougin, H. Devijver et M.-Th. Raepsaet-Charlier (éd.), L'ordre équestre. Histoire d'une aristocratie (II siècle av.J.-C.III siècle ap. J.-C.). Actes du colloque international organisé par Ségolène Demougin, Hubert Devijver et Marie-Thérèse Raepsaet-Charlier (Bruxelles-Leuven, 5-7 octobre 1995) (Rome 1999), 274. 
la richesse dont disposaient des parentes de chevaliers romains, sur la base des dépouillements effectués dans le cadre de ma thèse de doctorat. L'accent sera porté dans un premier temps sur l'évergétisme ainsi que sur les constructions bâties à l'instigation de ces femmes, puis je vais m'interroger sur les sources de leur fortune, afin de déterminer, autant que faire se peut, par quels biais elle était acquise et/ou transmise.

Enfin, avant d'en venir à l'exposé des résultats, il faut signaler que mes recherches ont permis de rassembler un nombre relativement significatif de témoignages. Cependant, dans le cadre de cette communication et pour davantage de maniabilité, je me suis vu contraint de restreindre le champ de mes investigations à un grand ensemble provincial : l'Occident romain. Ce choix s'explique par le volume et la variété des sources ainsi que des cas de figure qu'elles nous présentent. Toutefois, afin de parvenir à des conclusions exhaustives, je vais y inclure quelques données provenant des provinces de l'Orient hellénophone ${ }^{10}$.

Je commencerai par une expression concrète de la richesse de ces femmes, en l'occurrence l'évergétisme. Cette pratique, surgie dans l'Orient hellénophone, héritée de l'époque hellénistique, ayant été amplement et abondamment étudiée par d'illustres devanciers, je m'abstiens donc de répéter ici ce que d'autres ont écrit avant moi ${ }^{11}$. Toutefois, il convient d'en

10 Il est ensuite un cas spécial sur lequel je ne vais pas trop m'appesantir, celui des matronae stolatae qui, à en croire B. Holtheide, furent toutes des parentes de chevaliers et dans le même temps des propriétaires terriennes. Etant donné que ces assertions sont loin d'être avérées, je ne vais prendre en considération que les femmes clairement et indiscutablement apparentées à des chevaliers. Cf. B. Holtheide, 'Matrona stolata - femina stolata', Zeitschrift für Papyrologie und Epigraphik 38 (1980), 127-131.

11 L'ouvrage pionnier est celui de P. Veyne, Le pain et le cirque. Sociologie historique d'un pluralisme politique (Paris 1976). Par la suite, d'autres chercheurs ont complété son travail : E. Melchor Gil, El mecenazgo cívico en la Bética. La contribución de los evergetas al desarrollo de la vida municipal (Cordoue 1994) et Id., La munificiencia cívica en el mundo romano (Madrid 1999); K. Lomas et T. Collins (éd.), Bread and Circuses. Euergetism and Municipal Patronage in Roman Italy (Londres 2003); J. Andreu Pintado, Munificiencia pública en la provincia Lusitania (siglos I-IV d.C.) (Saragosse 2004). Pour l'ordre équestre en particulier : G. Wesch-Klein, 'Equites Romani und Euergetismus', in S. Demougin, H. Devijver et M.-Th. Raepsaet-Charlier (éd.), L'ordre équestre. Histoire d'une aristocratie (II siècle av. J.-C.$I I I^{e}$ siècle ap. J.-C.). Actes du colloque international organisé par Ségolène Demougin, Hubert Devijver et Marie-Thérèse Raepsaet-Charlier (Bruxelles-Leuven, 5-7 octobre 1995)(Rome 1999), 301-319. Sur l'évergétisme féminin et le rapport des femmes à l'argent: R. Van Bremen, 'Women and Wealth', in A. Cameron et A. Kuhrt (éd.), Images of Women in Antiquity (Londres-Sydney 1983), 223-242 et Ead., The Limits of Participation. Women and Civic Life in the Greek East in the Hellenistic and Roman Periods (Amsterdam 1996); M.T. Boatwright, 'Plancia Magna of Perge: Women's Roles and Status in Roman Asia Minor', in S.B. Pomeroy (éd.), Women's History and Ancient History (Chapel Hill 1991), 249-272 ; M.L. Woodhull, 'Matronly Patrons in the Early Roman Empire. The Case of Salvia Postuma', in F. McHardy 
souligner l'importance pour la vie des communautés locales puisqu'elle résulte souvent, dans leur quête d'honneurs et de prestige social, d'activités publiques détenues par ces femmes (telles que des sacerdoces ou des patronages) qui impliquent de généreuses donations, plus ou moins volontaires (summa honoraria, éventuellement accompagnée d'une ampliatio). Dans le tableau présenté en fin d'article (p. 184-186) ne figurent que les actes concrets d'évergétisme sous la forme de constructions ou de dons en argent, voire aussi en nature, au bénéfice de la communauté civique. Par ailleurs, j'ai pris en considération tous les exemples de munificentia, de merita mais aussi de liberalitates, même s'ils ne sont pas aussi explicites. Je détaille les évergésies, mais aussi leur lieu d'action, leur auteur ou leur nature. J'ai en revanche laissé ici de côté toutes les indications relatives à des dons dans un cadre privé.

Brièvement, on peut constater que seuls 42 cas (pour $75^{0}$ matrones équestres) ont été répertoriés pour les trois premiers siècles de notre ère, soit une proportion légèrement supérieure aux clarissimes ( 56 pour 1200) d'après les travaux de M.-Th. Raepsaet-Charlier qui a également recensé des exemples provenant des provinces orientales de l'Empire ${ }^{12}$. Par ailleurs, la répartition géographique en Occident, plus variée que pour les femmes sénatoriales, octroie un léger avantage aux provinces africaines $(16)^{13}$ sur l'Italie $(15)^{14}$, loin devant la seule province espagnole, la Bétique (6),

et E. Marshall (éd.), Women's Influence on Classical Civilization (Londres 2004), 75-91 ; M. Navarro Caballero, 'L'élite, les femmes et l'argent dans les provinces hispaniques', in L. de Ligt et al. (éd.), Roman Rule and Civic Life: Local and Regional Perspectives. Proceedings of the Fourth Workshop of the International Network Impact of Empire (Roman Empire c. 200 B.C.-A.D. 476), Leiden, June 25-28, 2003 (Amsterdam 2004), 389-400 ; E. Melchor Gil, 'Mujeres y evergetismo en la Hispania romana', in J.F. Rodríguez Neila (éd.), Hispania y la epigrafía romana. Cuatro perspectivas (Faenza 2009), 133-178 ; S. Destephen, 'L'évergétisme aristocratique au féminin dans l'Empire romain d'Orient', in B. Caseau (éd.), Les réseaux familiaux. Antiquité tardive et Moyen-Âge. In memoriam A. Laiou et E. Patlagean (Paris 2012), 71-91.

12 Raepsaet-Charlier 2008, op. cit. (n. 8), 1031 fournit la liste des évergésies réalisées par les clarissimes aux trois premiers siècles de notre ère : 21 en Italie, 16 pour les provinces anatoliennes ( $9+1$ [de Mytilène, comptabilisé sous «Grèce» par l'auteure] en Asie; 2 en Pisidie ; 2 en Galatie ; 1 en Lycie-Pamphylie et 1 en Cilicie), 14 [et non 13 comme elle l'écrit en p. 1031] pour les provinces africaines (11 en Proconsulaire; 3 en Numidie) puis 2 cas pour les Gaules et les Germanies ( 1 en Narbonnaise et 1 en Germanie supérieure) ainsi que pour l'Espagne ( 1 en Bétique et 1 en Lusitanie) et 1 pour la province d'Achaïe. S'il ne fallait prendre en compte que les témoignages issus des provinces occidentales, leur nombre s'établirait donc à 39 .

139 en Proconsulaire, 4 en Numidie, 1 en Maurétanie Tingitane et 1 en Maurétanie Césarienne.

144 exemples en région I; 2 en région III ; 1 dans les régions II, IV, V, VI, VII, VIII, IX, $\mathrm{X}$ et XI. 
suivie de la Dalmatie (3), de la Dacie (1) et de la Pannonie inférieure (1). Quant à la chronologie, elle atteste que la majorité des exemples est à dater du $\mathrm{II}^{\mathrm{e}}$ s. et ils se répartissent comme suit ${ }^{15}$ :

Table 1: Datation et localisation des manifestations évergétiques

\begin{tabular}{|c|c|c|c|}
\hline Datation: & Localisation: & Nombre: & Total: \\
\hline $\begin{array}{l}\text { I }^{\mathrm{er}} \text { s. av.- } \mathrm{I}^{\mathrm{er}} \text { s. ap. } \\
\text { J.-Chr. }\end{array}$ & Italia, regio VII & 1 & 1 \\
\hline $\mathrm{I}^{\mathrm{er}}$ s. ap. J.-Chr. & $\begin{array}{l}\text { Hispania Ulterior Baetica } \\
\text { Italia, regio I } \\
\text { Italia, regio II } \\
\text { Italia, regio IV } \\
\text { Italia, regio IX } \\
\text { Mauretania Tingitana }\end{array}$ & $\begin{array}{l}2 \\
2 \\
1 \\
1 \\
1 \\
1\end{array}$ & 8 \\
\hline $\begin{array}{l}\text { Fin } \mathrm{I}^{\mathrm{er}}- \\
\text { début } \mathrm{II}^{\mathrm{e}} \mathrm{s} \text {. }\end{array}$ & Dalmatia & 3 & 3 \\
\hline II ${ }^{\mathrm{e}}$ s. ap. J.-Chr. & $\begin{array}{l}\text { Africa Proconsularis } \\
\text { Hispania Ulterior Baetica } \\
\text { Italia, regio I } \\
\text { Italia, regio III } \\
\text { Italia, regio V } \\
\text { Italia, regio VIII } \\
\text { Italia, regio XI } \\
\text { Mauretania Caesariensis } \\
\text { Numidia }\end{array}$ & $\begin{array}{l}5 \\
3 \\
1 \\
1 \\
1 \\
1 \\
1 \\
1 \\
1\end{array}$ & 15 \\
\hline $\mathrm{II}^{\mathrm{e}}-\mathrm{III}^{\mathrm{e}} \mathrm{s}$. & $\begin{array}{l}\text { Africa Proconsularis } \\
\text { Hispania Ulterior Baetica } \\
\text { Italia, regio III } \\
\text { Italia, regio VI } \\
\text { Numidia }\end{array}$ & $\begin{array}{l}1 \\
1 \\
1 \\
1 \\
2\end{array}$ & 6 \\
\hline $\mathrm{III}^{\mathrm{e}} \mathrm{s}$. & $\begin{array}{l}\text { Africa Proconsularis } \\
\text { Dacia } \\
\text { Italia, regio I } \\
\text { Italia, regio X } \\
\text { Numidia } \\
\text { Pannonia inferior }\end{array}$ & $\begin{array}{l}4 \\
1 \\
1 \\
1 \\
1 \\
1\end{array}$ & 9 \\
\hline
\end{tabular}

15 Raepsaet-Charlier 2008, op. cit. (n. 8), 1031: pour l'ordre sénatorial, les chiffres se répartissent comme suit : 5 cas au I ${ }^{\mathrm{er}}$ s. ; 30 au $\mathrm{II}^{\mathrm{e}} \mathrm{s}$. ; 12 à la fin du $\mathrm{II}^{\mathrm{e}}$ ou au début du $\mathrm{III}^{\mathrm{e}} \mathrm{s}$. et 9 cas au $\mathrm{III}^{\mathrm{e}} \mathrm{s}$. 
Quant aux évergésies en général, elles sont le fait de femmes seules donatrices, comme le confirment les 27 cas recensés, même s'il convient tout de même de préciser que 3 de ces exemples correspondent en réalité à des donations effectuées par plusieurs d'entre elles: mères et filles à Alba Pompeia, en Italie ${ }^{16}$, et à Seressi, en Proconsulaire ${ }^{17}$, et trois sœurs à Madaure, en Numidie ${ }^{18}$. D'autres cas (9), en revanche, confirment une pratique de l'évergétisme en couple, à l'instar de Cornelia Valentina Tucciana à Timgad, en Numidie ${ }^{19}$, d'Helvidia Priscilla à Chieti, en Italie ${ }^{20}$, ou encore de Succonia Rustica à Ostur, en Bétique ${ }^{21}$. Les exemples restants se réfèrent en général à des dons accomplis en famille.

A titre d'information, car je ne vais pas m'attarder sur ce point, on peut avancer que les motivations de ces dames n'ont pas toutes pour origine leur élection à un sacerdoce, comme dans le cas de Vettia Saturnina ${ }^{22}$ ou de Cantria Longina qui, par exemple, verse 50.000 sesterces à la res publica d'Aeclanum à l'occasion de son accession à la prêtrise ${ }^{23}$. En effet, d'autres causes, quand elles sont explicitées, peuvent être mentionnées, comme lorsqu'il s'agit de remboursements divers.

Venons-en maintenant aux dons effectués par ces matrones équestres qui, mis à part les exemples vagues et imprécis mais nets de munificentia, de merita ou de beneficia, consistent en des statues (15 exemples), souvent remboursées à l'ordo local ${ }^{24}$, mais aussi en des bâtiments imposants ${ }^{25}$, comme le résume ce tableau :

16 CIL V, 7595 (p. 771) et $A E$ 2004, 579.

17 CIL VIII, $937=11216$ (p. 2340).

18 ILAlg I, 2145.

$19 A E$ 1980, 956 ; CIL VIII, 2398 (p. 1693); CIL VIII, 2399 (p. 1693) = ILS 2753 et ILS 5579.

20 CIL IX, $3019=$ ILS 1377 .

21 CIL II, 1267 = CILA Hu, 81 .

22 CIL VIII, $4437=18596$.

23 CIL IX, 1153 = ILS 6487. R. Duncan-Jones, The Economy of the Roman Empire. Quantitative Studies (Cambridge 1974), 107-108 et 216-217 fournit des données comparatives : en Afrique, les montants vont de 90.000 à 1.000 sesterces tandis qu'en Italie, Cantria Longina fait partie du groupe de tête des donateurs qui versent 50.000 sesterces et dont les chiffres minimums sont de 6.000 sesterces. Voir aussi Briand-Ponsart 2003, op. cit. (n. 7), 84-85.

24 Dont les coûts varient, d'après les chiffres de Duncan-Jones 1974, op. cit. (n. 23), 93-99, entre 66.666 sesterces (au total 1 million de sesterces pour 16 statues) à 460 sesterces en Afrique et entre 550.000 sesterces (pour plusieurs statues) et environ 500 sesterces en Italie : $162-163$.

25 Dont les montants s'élèvent, d'après les chiffres de Duncan-Jones 1974, op. cit. (n. 23), 90-93, entre 600.00o sesterces à 500 sesterces en Afrique et entre 2 millions de sesterces et environ 672,5 sesterces en Italie : $157-162$. 
Table 2: Edifices financés par des matrones équestres

\begin{tabular}{|c|c|c|}
\hline Type de bâtiment: & Nom de la matrone équestre: & Lieu: \\
\hline $\begin{array}{l}\text { Aedicula Matris } \\
\text { Magnae }\end{array}$ & Servilia M. f. Copiesilla & Salone ${ }^{26}$ \\
\hline Balineum & $\begin{array}{l}\text { Albucia M. f. Candida et son mari } \\
\text { C. Valerius C. f. Pansa }\end{array}$ & Novare $^{27}$ \\
\hline $\begin{array}{l}\text { Balineum cum } \\
\text { omni ornatu }\end{array}$ & $\begin{array}{l}\text { Cosconia Maluginensis f. Gallitta, } \\
\text { son mari [L. Aelius Strabo] et sa } \\
\text { belle-mère Terentia A. f. }\end{array}$ & Bolsena ${ }^{28}$ \\
\hline Basilica & $\begin{array}{l}\text { Flavia T. f. Tertulla et son mari } \\
\text { M. Flavius T. f. Quir. Fronto }{ }^{29}\end{array}$ & Doclea $^{30}$ \\
\hline $\begin{array}{l}\text { Basilica cum } \\
\text { hypaethro }\end{array}$ & Cornelia Anulla et son fils [- - - $]^{31}$ & Abdera $^{32}$ \\
\hline $\begin{array}{l}\text { Macellum } \\
\text { (+ exornatio), area } \\
\text { et aménagements } \\
\text { au Capitole }\end{array}$ & $\begin{array}{l}\text { Cornelia Valentina Tucciana et son } \\
\text { mari M. Plotius Faustus }\end{array}$ & Timgad $^{33}$ \\
\hline $\begin{array}{l}\text { Porticus cum } \\
\text { statuis et uiridibus }\end{array}$ & Varia Pansina & Nola $^{34}$ \\
\hline Templum & $\begin{array}{l}\text { Antonia Valentina et son mari M. } \\
\text { Cominius Quintus }\end{array}$ & $\begin{array}{r}\text { Sarmizegetusa }^{35} \\
\text { (continued) }\end{array}$ \\
\hline \multicolumn{3}{|c|}{ 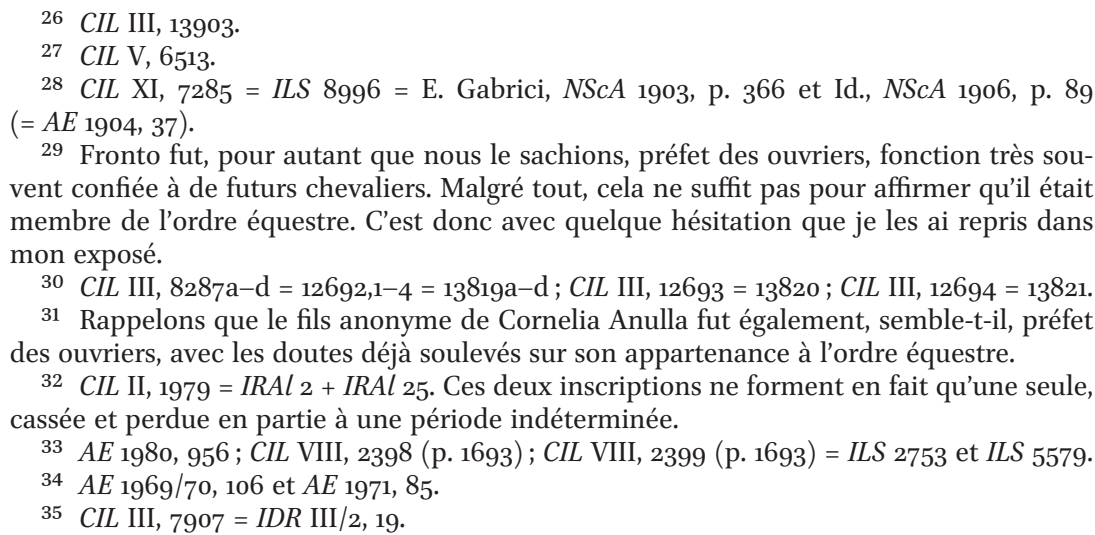 } \\
\hline
\end{tabular}


Table 2 (cont.)

\begin{tabular}{lll}
\hline Type de bâtiment: & \multicolumn{1}{c}{ Nom de la matrone équestre : } & \multicolumn{1}{c}{ Lieu : } \\
\hline$(--)$ & $\begin{array}{l}\text { Aebutia Honorata Agriana et son mari } \\
(\text { Numisius })^{36}\end{array}$ & Uchi Maius ${ }^{37}$ \\
& $\begin{array}{l}\text { Helvidia C. f. Priscilla et son mari } \\
\text { C. Vettius Marcellus }\end{array}$ & Chieti $^{38}$ \\
& $\begin{array}{l}\text { Statia Sex. f. Fida et son fils défunt } \\
\text { Q. Manlius Q. f. Rufus }\end{array}$ & Risinium $^{39}$ \\
\hline
\end{tabular}

Parfois, il peut s'agir de simples ajouts esthétiques comme des ornamenta pour l'arc de Seressi par Armenia Auge et sa fille Bebenia Pauliana ${ }^{40}$, l'exornatio du macellum de Timgad par Cornelia Valentina Tucciana et son mari ${ }^{41}$ ou la décoration avec du marbre du portique de Préneste par Pompeia Mummiana ${ }^{42}$.

Parmi toutes ces manifestations évergétiques, il en est une qui sort du lot, celle de Iunia Rustica, première flaminique perpétuelle du municipe (pré?-)flavien de Cartima, en Bétique, en raison de ses multiples actions. En effet, elle restaure les portiques, offre un espace pour la construction des bains, paie les dettes publiques, érige une statue de Mars et fait bâtir un portique à côté des bains, sur son terrain, avec une piscina et une statue de Cupidon. Enfin, elle rembourse les dépenses que l'ordo a consenties en vue de poser une statue en son honneur et en celui de son fils puis elle organise un epulum et des spectacles pour ses concitoyens ${ }^{43}$.

36 G. Wesch-Klein, Liberalitas in rem publicam. Private Aufwendungen zugunsten von Gemeinden im römischen Afrika bis $284 \mathrm{n}$. Chr. (Bonn 1990), 253-254, $\mathrm{n}^{\mathrm{0}} 11$ et 13. Et tel est également l'avis de E. Ughi, in A. Ibba et al. (éd.), Uchi Maius 2. Le iscrizioni (Sassari 2006), 213-214 où elle se montre plus nuancée que dans son article antérieur 'L'evergetismo cittadino', in M. Khanoussi et A. Mastino (éd.), Uchi Maius 1. Scavi e ricerche epigrafiche in Tunisia (Sassari 1997), 225-226 et 236, nº 16.

37 CIL VIII, $15457=26277=26278=$ Uchi Maius II, 75 .

38 CIL IX, $3019=$ ILS 1377.

39 CIL III, 1717 (p. 1028).

40 CIL VIII, $937=11216$ (p. 2340).

41 CIL VIII, 2398 (p. 1693).

42 CIL XIV, 2995. Duncan-Jones 1974, op. cit. (n. 23), 92-93 et 160-162, nous dévoile que les montants varient en général entre 800.000 sesterces et 672,5 sesterces en Italie et entre 500.000 sesterces et 500 sesterces en Afrique.

43 CIL II, $1956=I L S$ 5512. J.F. Donahue, 'Iunia Rustica of Cartima: Female Munificence in the Roman West', Latomus 63 (2004), 873-891 : coût de 60.000 sesterces selon lui. Sur la formule impensam remisit, voir S. Dardaine, 'La formule épigraphique « impensam remisit » et l'évergétisme en Bétique', Mélanges de la Casa de Velázquez 16 (1980), 39-55. 
Ce dernier exemple m'offre l'occasion d'évoquer la présence de banquets et de sportules dans le cadre de l'évergétisme qui apparaissent dans 12 cas et sont toujours complémentaires d'autres actes évergétiques dont ils matérialisent l'inauguration. Quelquefois, en de rares occasions, des montants nous sont précisés pour des distributions d'argent: Aurelia Calligenia verse ainsi 4 sesterces aux décurions à Rimini ${ }^{44}$ tandis que Varia Pansina, à Nola, remet 30 sesterces aux décurions, 20 sesterces aux Augustales, 12 sesterces aux ministri (desservants du culte de Vénus) et 4 sesterces aux personnes ressortant du populus et aux femmes ${ }^{45}$. Enfin, Aemilia Pudentilla, pour sa part, distribue 50.000 sesterces lors du mariage de son fils aîné Pontianus et de la prise de toge virile de son cadet Pudens ${ }^{46}$.

Pour en finir avec cette section consacrée à l'évergétisme, il convient d'évoquer les sommes investies dans la construction des bâtiments offerts à la communauté. Il n'est, à ce propos, pas toujours possible d'en déterminer le montant avec précision:

Table 3: Montants et justification de donations effectuées par des matrones équestres

\begin{tabular}{llll}
\hline Nom : & \multicolumn{1}{c}{ Lieu: } & \multicolumn{1}{c}{ Motif: } & Montant: \\
\hline $\begin{array}{l}\text { Albucia M. f. } \\
\text { Candida }\end{array}$ & Novare & Legs à la res publica & $200.000 \mathrm{HS}^{47}$ \\
$\begin{array}{l}\text { Septimia Polla } \\
\text { et son frère, }\end{array}$ & Leptis Magna & $\begin{array}{l}\text { Legs pour une statue } \\
\text { d'argent }\end{array}$ & $>115.000 \mathrm{HS}^{48}$ \\
$\begin{array}{l}\text { P. Septimius } \\
\text { Geta }\end{array}$ & & &
\end{tabular}

(continued)

44 CIL XI, 405 .

$45 A E 1969 / 70,106$ et $A E$ 1971, 85.

46 Apul., Apol. 87, 10-11 et Duncan-Jones 1974, op. cit. (n. 23), 105-106 et 188-198 : entre 100 et 1 sesterce par tête en Afrique - la distribution d'Aemilia Pudentilla étant la plus élevée de sa catégorie -, tandis qu'en Italie, les montants s'élèvent de 400 à 1 sesterce par tête. Sur Aemilia Pudentilla en particulier: H. Pavis d'Escurac, 'Pour une étude sociale de l'Apologie d'Apulée', Antiquités africaines 8 (1974), 89-101; A. Gutsfeld, 'Zur Wirtschaftsmentalität nichtsenatorischer provinzialer Oberschichten: Aemilia Pudentilla und ihre Verwandten', Klio 74 (1992), 250-268 et M. Lakhlif, 'La richesse et les stratégies matrimoniales en Afrique au Haut-Empire: Aemilia Pudentilla, épouse d'Apulée de Madaure', in J. González et al. (éd.), L'Africa romana. Le richezze dell'Africa. Risorse, produzioni, scambi. Atti del XVII convegno di studio. Sevilla, 14-17 dicembre 2006 (Rome 2008), 319-326.

47 CIL V, 6513.

$48 A E 1950,158=I R T 607$. Le poids de la statue est de 144 livres et 10 onces et demie (un peu moins de $47 \mathrm{~kg}$ ), volume auquel son frère et héritier ajoute un vingtième $(2,4 \mathrm{~kg})$ et 4 livres, 10 onces et demie $(1,617 \mathrm{~kg})$, pour un coût de plus de 115.000 sesterces selon Duncan-Jones 1974, op. cit. (n. 23), 94, le plus élevé de sa catégorie en Afrique. 
Table 3 (cont.)

\begin{tabular}{|c|c|c|c|}
\hline Nom : & Lieu : & Motif : & Montant : \\
\hline $\begin{array}{l}\text { Pompeia Q. f. } \\
\text { Trebulla }\end{array}$ & Terracine & $\begin{array}{l}\text { Legs avec pour prescription } \\
\text { une restauration du } \\
\text { monument bâti par le } \\
\text { mari, M. Iunius C. f. Gal. } \\
\text { Proculus }\end{array}$ & $100.000 \mathrm{HS}^{49}$ \\
\hline Statia Sex. f. Fida & Risinium & $\begin{array}{l}\text { Ajout au don du fils, } \\
\text { Q. Manlius Q. f. Serg. } \\
\text { Rufus }+5 \% \text { du total }\end{array}$ & $\begin{array}{l}35.000 \mathrm{HS}+ \\
13.250 \mathrm{HS}^{50}\end{array}$ \\
\hline $\begin{array}{l}\text { Armenia Auge et } \\
\text { sa fille, Bebenia } \\
\text { Pauliana }\end{array}$ & Seressi & $\begin{array}{l}\text { Ornements de l'arc } \\
\text { bâti par le fils et le } \\
\text { frère C. Memmius Felix } \\
\text { Armenianus }\end{array}$ & $25.000 \mathrm{HS}^{51}$ \\
\hline $\begin{array}{l}\text { Maria Lucina et ses } \\
\text { deux fils, L. Fulvius } \\
\text { Kastus Fulvianus et } \\
\text { [L.?] Fulvius Felix }\end{array}$ & Mustis & Ob honorem [-- - ] & $>10.000 \mathrm{HS}^{52}$ \\
\hline
\end{tabular}

A titre de comparaison, les chiffres pour les femmes de l'ordre sénatorial sont dans l'ensemble comparables, même si certains montants sont logiquement plus élevés ${ }^{53}$.

Passons maintenant aux quelques témoignages relatifs à des matrones dont les dépenses ne peuvent être mises sur le compte de l'évergétisme. Je pense à Aurelia Flavia qui nous indique explicitement qu'à Lambèse, elle a déboursé 5.000 sesterces pour faire ériger un autel en l'honneur de son défunt mari, l'officier Ulpius Longinus, ce qui rentre dans le dossier des dépenses à des fins «privées» que j'ai mentionnées au début de cet exposé54.

49 CIL X, 6309 (p. 1015). Cf. n. 42 pour les données comparatives fournies par DuncanJones 1974, op. cit. (n. 23).

50 CIL III, 1717 (p. 1028).

51 CIL VIII, $937=11216$ (p. 2340). Les chiffres donnés par Duncan-Jones 1974, op. cit. (n. 23), 92-93 et 160-162, s'élèvent à environ 800.000 sesterces et 672,5 sesterces en Italie et entre 500.000 sesterces et 500 sesterces en Afrique.

52 CIL VIII, 1578 (p. 1501). Duncan-Jones 1974, op. cit. (n. 23), 90-92 et 157-16o, nous informe que les montants varient entre 2 millions de sesterces et 6.000 sesterces en Italie et entre 600.000 sesterces et 3.000 sesterces en Afrique.

53 Raepsaet-Charlier 2008, op. cit. (n. 8), 1037.

$54 A E$ 1989, 872. Duncan-Jones 1974, op. cit. (n. 23), 99-101 et 166-171 : coûts entre plus de 80.000 sesterces et 96 sesterces en Afrique; entre 500.000 sesterces et 120 sesterces en Italie. 
D'autres montants chiffrés peuvent également être dévoilés : c'est ainsi que nous savons que la mère et la sœur de Perse avaient hérité de lui 2 millions de sesterces ${ }^{55}$. Pline le Jeune, lui, relate que son meilleur ami, Voconius Romanus, avait reçu de sa mère, en vue d'intégrer l'ordre sénatorial, un don de 4 millions de sesterces, sans que l'on sache si cette démarche louable fut couronnée de succès ${ }^{56}$. Apulée raconte que son épouse, Aemilia Pudentilla, disposait d'une fortune s'élevant à 4 millions de sesterces, qui suscita bien des convoitises ${ }^{57}$. Quant à sa dot, elle s'éleva à 300.000 sesterces, lorsqu'elle s'unit à son premier mari ${ }^{58}$. Toujours dans l'œuvre d'Apulée, nous apprenons que la grand-mère d'Herennia, épouse de Sicinius Pontianus puis de Pudens, avait reçu de son mari 3 millions de sesterces pour échapper aux dettes qu'il avait contractées ${ }^{59}$. Plus tard, nous lisons que le père d'Herennia a dû emprunter 400.000 sesterces pour son mariage avec les fils de Pudentilla ${ }^{60}$. A titre de comparaison, Apulée lui-même n'avait pas à rougir puisque son père disposait d'un patrimoine de 2 millions de sesterces, dont il hérita la moitié61.

Par ailleurs, nous connaissons un nombre important de femmes qui se déclarent héritières mais pour lesquelles il n'est fait état d'aucun montant. Tout au plus peut-on déduire que le legs a sans doute servi à financer l'érection d'une inscription destinée à commémorer leur parent décédé. Tel est le cas des matrones suivantes:

Table 4: Matrones équestres légataires de leurs proches

\begin{tabular}{lll}
\hline Nom : & \multicolumn{1}{c}{ Nom du testateur: } & \multicolumn{1}{c}{ Lieu: } \\
\hline Aelia Filonice & {$[$ M.?] Aurelius M. f. Romanus, mari } & Lambèse $^{62}$ \\
[Attia M. f. - - ] & {$[$ - Attius M. f. Te]r. Vindex, frère } & $\begin{array}{l}\text { Aix-en- } \\
\text { Provence }\end{array}$ \\
& & (continued)
\end{tabular}

55 Prob., Vita Persi 7-8. A cette somme s'est joint un montant indéterminé qui leur fut reversé par son ami L. Annaeus Cornutus.

56 Plin., Ep. 10, 4, 2-5.

57 Apul., Apol. 71, 6 et 77, 1.

58 Apul., Apol. 92, 1 et 3 .

59 Apul., Apol. 75, 8.

60 Apul., Apol. 92, 2.

61 Apul., Apol. 23, 1.

62 CIL VIII, 2762.

63 CIL XII, 514 (p. 814) = 515 = ILN III, 25. Bien que l'inscription ait été mise au jour sur le territoire d'Aix-en-Provence, les Attii provenaient sans doute d'Arles. 
Table 4 (cont.)

\begin{tabular}{|c|c|c|}
\hline Nom: & Nom du testateur: & Lieu : \\
\hline $\begin{array}{l}\text { Cornelia Eulogia } \\
\text { Romanilla et ses sœurs } \\
\text { Cornelia Romanilla } \\
\text { Postumiana } \\
\text { Cornelia Valentina } \\
\text { Claudiana }\end{array}$ & $\begin{array}{l}\text { M. Cornelius Fronto Gabinianus, } \\
\text { père }\end{array}$ & Madaure $^{64}$ \\
\hline Iulia [Pie]ris & Q. Fabius Q. f. Gal. Blandus, mari & Rome $^{65}$ \\
\hline $\begin{array}{l}\text { Laecania Martina et ses } \\
\text { fils }\end{array}$ & M. Acutius PP. f. Acutianus, mari & Cluana $^{66}$ \\
\hline Longinia Saturnina & -[-] Iulius [- - -], mari & Césarée $\mathrm{e}^{67}$ \\
\hline Numitoria C. f. Moschis & L. Cominius L. f. Maximus, mari & Tivoli $^{68}$ \\
\hline $\begin{array}{l}\text { Statilia Crescentilla et } \\
\text { son fils } \\
\text { Munatius Paulinus }\end{array}$ & $\begin{array}{l}\text { Munatius Paul[i]nianus, mari et père } \\
\text { des héritiers }\end{array}$ & Rome $^{69}$ \\
\hline Viatoria Taurina & C. Viatorius [- - -], père & Virunum $^{70}$ \\
\hline Vibia Maria Maxima & $\begin{array}{l}\text { P. Vibius P. f. Marianus, père } \\
\text { Reginia Maxima, mère }\end{array}$ & Rome $^{71}$ \\
\hline $\begin{array}{l}\text { Ulpia Paratiana et sa } \\
\text { fille Ulpia Valeria }\end{array}$ & $\begin{array}{l}\text { M. Val(erius) Valerianus, mari et père } \\
\text { des héritières }\end{array}$ & Brigetio $^{72}$ \\
\hline
\end{tabular}

Vient maintenant une question importante : à la vue de ces chiffres, quelle est l'origine de la fortune des parentes de chevaliers ? Conformément à la tradition aristocratique romaine, ce sont les biens fonciers qui constituent la seule source de richesse acceptable aux yeux de tous. Il ne faut donc pas s'étonner de découvrir que des parentes de chevaliers aient possédé de

64 ILAlg. I, 2145.

65 CIL VI, 3518.

66 CIL IX, 5805.

67 CIL VIII, 21037.

68 CIL XIV, 3626 = ILS $2742=$ InscrIt. IV, 1, 157. Héritière de son premier époux, elle institue à son tour son deuxième mari et le fils né de leur union comme héritiers : CIL XIV, 3628 = InscrIt. IV, 1, 158 .

${ }^{69}$ CEMC $19=A E$ 1990, 64 .

70 CIL III, $4859=$ ILLPRON 608 (cas douteux).

${ }_{71}$ CIL VI, 1636 (p. 3163, 3811 et 4723) = ILS 1361. La famille provient de Dertosa (Regio XI).

72 CIL III, 4327 = RIU III, 637 . 
telles propriétés ${ }^{73}$. Toutefois, il subsiste une difficulté, et non des moindres, l'impossibilité d'établir avec certitude que nos sources fassent clairement allusion à un domaine rural, si aucune mention n'y est faite explicitement. En outre, à la différence des familles sénatoriales, il n'est guère aisé d'identifier les lieux de résidence des chevaliers et de leurs proches, puisqu'ils n'étaient pas tenus à l'obligation de résidence à Rome. L'ordre équestre était tout aussi provincial dans son recrutement que les sénateurs, mais la présence de chevaliers dans leurs cités d'origine fut plus marquée par leur participation aux magistratures et autres sacerdoces ${ }^{74}$. Cela veut-il dire que toutes les matrones équestres attestées dans n'importe quelle cité de l'Empire y aient possédé une propriété ? Cela est fort possible mais dans le cas qui m'occupe, je ne vais prendre en considération que les exemples documentés les plus nets. Pour ce faire, le recours à l'archéologie s'avère ici la clé essentielle pour confirmer l'existence d'un domaine sur lequel a vécu ou qu'a possédé une matrone équestre ${ }^{75}$.

73 La preuve en a été fournie pour les chevaliers romains : S. Demougin, L'ordre équestre sous les Julio-claudiens (Rome 1988), 85-91; G. Paci, 'I cavalieri romani e la proprietà fondiaria dai Flavi ai Severi', in S. Demougin, H. Devijver et M.-Th. Raepsaet-Charlier (éd.), L'ordre équestre. Histoire d'une aristocratie (II siècle av. J.-C.-III e siècle ap. J.-C.). Actes du colloque international organisé par Ségolène Demougin, Hubert Devijver et Marie-Thérèse Raepsaet-Charlier (Bruxelles-Leuven, 5-7 octobre 1995) (Rome 1999), 291-300.

${ }^{74}$ La présence de sénateurs parmi les magistrats municipaux d'une cité provinciale est justement un indice pouvant certifier qu'ils y possédaient une propriété : Andermahr 1998, op. cit. (n. 7), 10. Des sénateurs titulaires de magistratures locales sont attestés durant tout le Haut-Empire en Italie et dans les provinces. Cf. W. Eck, 'La presenza delle famiglie senatorie nelle città dell'Impero romano fino al tardo III secolo', in Id. (éd.), Tra epigrafia, prosopografia e archeologia. Scritti scelti, rielaborati ed aggiornati (Rome 1996), 175-212.

${ }^{75}$ Le pionnier des travaux sur cette thématique est P.-A. Février, 'Villes et campagnes des Gaules sous l'Empire', Ktema 6 (1981), 359-372 qui s'était intéressé à la Gaule et à l'Italie du Nord en fournissant des critères pour l'identification des propriétés. Depuis lors, Andermahr 1998, op. cit. (n. 7), 7-42, a proposé une série d'indices pouvant mener à identifier les propriétés ayant appartenu à des membres de l'ordre sénatorial et qui sont, bien évidemment, valables pour les chevaliers et leurs proches, avec les nuances que j'ai soulevées. Par la suite, sur la base d'études régionales, certains savants ont présenté d'autres critères d'identification: M. Christol, 'Le patrimoine des notables en Gaule méridionale. Apports et limites de l'épigraphie', Histoire et sociétés rurales 19 (2003), 133-150 ; M. Silvestrini, 'Les biens-fonds des élites locales en Italie du sud. L'exemple des Regiones II et III (Apulie et Calabre)', Histoire et sociétés rurales 19 (2003), 51-65; E. Melchor Gil, 'Las propriedades rústicas de las élites hispano-romanas: un intento de aproximación a través de la documentación epigráfica', in J.F. Rodríguez Neila et E. Melchor Gil (éd.), Poder central y autonomía municipal: la proyección pública de las élites romanas de Occidente (Cordoue 2006), 244-252 et Id., 'Sobre las posesiones rústicas de las élites municipales de la Bética y la vinculación de sus proprietarios con determinadas comunidades cívicas', Mélanges de l'Ecole française de Rome. Antiquité 119 (2007), 435-443. 
A ce propos, en premier lieu, quelques textes littéraires sont clairs: nous savons ainsi que la mère du poète Perse, Fulvia Sisennia, possédait des biens en Ligurie, hérités de son second mari ${ }^{76}$. C'est dans les environs de Rome que nous trouvons un cas un peu plus douteux, celui de Pompeia Paulina, dont on sait qu'elle vivait dans une villa suburbaine mais qui aurait pu appartenir à son mari, Sénèque, ou à son père, le préfet de l'Annone arlésien Pompeius Paulinus ${ }^{77}$. Pour sa part, c'est dans son domaine de Vintimille, en Ligurie, que périt assassinée par les soldats d'Othon Iulia Procilla, mère d'Agricola, le beau-père de Tacite ${ }^{78}$. De son côté, Plinia, mère de Pline le Jeune, était possessionnée dans les environs du lac de Côme avec des propriétés que son fils a héritées ${ }^{79}$. Enfin, en ce qui concerne la mère de Voconius Romanus, nous savons qu'elle avait renoncé à la possession de biens fonciers, sans doute dans la région de Sagonte, qu'elle a remis à son fils pour qu'il puisse disposer du patrimoine nécessaire en vue d'accéder au Sénat ${ }^{80}$.

L'épigraphie, pour sa part, nous renseigne non seulement sur l'existence de domaines ruraux mais aussi très souvent sur leur emplacement, confirmé par plusieurs indices convergents. Quelquefois, la mention est explicite, comme pour Roia Titinia, son mari et ses fils à Uchi Maius, où une architrave indique l'entrée des praedia leur appartenant ${ }^{81}$. Le fundus Flauianus aurait pu, quant à lui, appartenir à Aelia Flaviana, originaire de Cordoue $^{82}$ tout comme le fundus Nasennianus appartiendrait à la famille de Nasennia Iusta de Bénévent ${ }^{83}$. Mais là rien n'est moins sûr.

76 Schol. Pers. 6, 6.

77 Tac., Ann. 15, 6o, 7 .

78 Tac., Agr. 7, 2.

79 Plin., Ep. 7, 11, 5 .

80 Plin., Ep. 10, 4, 3 .

81 A. Merlin et L. Poinssot, Les inscriptions d'Uchi Maius d'après les recherches du capitaine Gonduin (Paris 1908), 108-112, nº 173 = ILS 6024 et Add., p. CLXXXVI. L'inscription figure aussi sous le numéro 26415 du CIL VIII, mais les éditeurs y omettent la titulature équestre de (Pullaienus) Celsinius Pupianus, mari de Roia Titinia.

82 M. Gallego Franco, 'Participación de la mujer hispanorromana en la producción y comercio del aceite bético', in AA.VV., Congreso internacional "Ex Baetica amphorae". Conservas, aceite y vino en el Imperio romano (Sevilla - Écija, 17 al 20 de diciembre de 1998), IV (Écija 2000), 1272, n. 37 suggère cette hypothèse. Aelia Flaviana est connue par CIL II, $2224=I L S 6905=C I L \mathrm{II}^{2} / 7,282$.

83 M.R. Torelli, Beneventum (Rome 2002), 327, n. 99 et 335, n. 129. Signalons qu'à l'instar de Cornelia Anulla, Nasennia Iusta est apparentée à un praefectus fabrum, dont l'appartenance à l'ordre équestre est plus que douteuse : CIL IX, 1646 = ILS 6498. 
Ailleurs, ce sont des indices et des données archéologiques qui, combinés aux inscriptions et à la toponymie, nous certifient l'existence de propriétés rurales :

Table 5: Matrones équestres propriétaires de domaines fonciers

Nom : Lieu :

Acilia Plecusa

Albucia M. f. Candida

Atilia L. f. Vera et son mari L. Aemilius L. f. Paternus

Caecilia L. f. Macrina, avec son mari P. Senecius P. f. Fab. Garrulus et son fils homonyme

Carbetania Maxima et son père

P. Carbetanius Rufus

Domitia Sex. f., et ses enfants L. Domitius L. f. Vol. Magu[- - - ], L. Domitius L. f. Vol. Celer et [-] Domitius L. f. Vol. Macer

Iulia [- - - ]a et son mari, dont on ne peut reconstituer le nom
Singilia Barba ${ }^{84}$

Albuzzano (près de Pavie) ${ }^{85}$

Perolet (près d'Aeso) ${ }^{86}$

Rodengo (près de Brescia) ${ }^{87}$

Ferentinum $^{88}$

Rognes (près d'Aix-en-

Provence $)^{89}$

Cuers (près d'Arles) ${ }^{90}$

(continued)

84 CIL II $/ 5,830$ : Melchor Gil 2006, op. cit. (n. 75), 254 et Melchor Gil 2007, op. cit. (n. 75), 436 et 440. Acilia Plecusa fut l'épouse d'un praefectus fabrum.

85 Albuzzano, situé à une dizaine de $\mathrm{km}$ au nord-est de Pavie, peut indiquer la présence d'une propriété des Albucii, gens à laquelle était rattachée Albucia Candida qui y avait détenu le flaminicat de la divine Sabine (cf. CIL V, 6514). E. Gabba, "Ticinum: dalle origini alla fine del III sec. d. C.', in AA.VV., Storia di Pavia, I, L'età antica (Pavie 1984), 233 et M.S. Bassignano, 'Sacerdozi femminili nell'Italia settentrionale', Atti e memorie dell'Ateneo di Treviso 12 (1994-1995), 74, $\mathrm{n}^{\circ} 11$.

${ }^{86} C I L$ II, 4461 (p. 981) = ILS $2661=I R C$ II, 54. Atilia L. f. Vera était sans doute la fille ou la petite-fille du primipile Atilius Verus et l'épouse de L. Aemilius L. f. Gal. Paternus, préfet des ouvriers, centurion dans diverses légions puis primipile. Son rang équestre n'est donc pas avéré.

87 Cf. InscrIt. X/5, 1005. Pour cette hypothèse, S. Mollo, La mobilità sociale a Brescia romana (Milan 200o), 56 et 94 et F. Tassaux, 'Élites locales, élites centrales. Approche économique et sociale des grands propriétaires au nord de l'Italie romaine (Brescia et Istrie)', Histoire et sociétés rurales 19 (2003), 117.

${ }^{88}$ CIL X, $8387=$ CIL VI, 1602 (p. 3163, 4718).

89 ILN III, 243-245.

90 CIL XII, 392 et $A E$ 1999, 1016. 
Table 5 (cont.)

Nom :

Lieu :

Kharisia C. f. Magn[- - - ], son mari

C. Caecilius Victor et ses fils C. Caecilius

Victor et T. Flavius Felix

Nonia M. f. Arria Hermionilla et son mari Sex. Valerius Sex. f. Fab. Poblicola Vettillianus

Numisia Victorina et son mari [-] Furius P. f. Cambrils (près de Tarragone) ${ }^{93}$ Quir. [M]ontanus

Pompeia T. f. Sextina et son père

T. Pompeius T. f. Trom. Albinus

Publicia Pusinna et son mari M. Nonius

Cornelianus

Vettia C. f. Tranquilla, son mari Q. Sextius C. f. Pap. Martialis et ses fils T. Sextius C. f. Pap. Alexander et C. Sextius Martialis

[- - - ]ia Iusta et son mari [-] Salvius T. f. Pap. Rufinus Minicius Opimianus
Anguillara Sabazia (près de

Rome $)^{91}$

Urago Mella (près de Brescia) ${ }^{92}$
Arco (près de Brescia) ${ }^{95}$

Collatia (près de Rome) ${ }^{96}$

Grottaferrata (près de Tusculum $)^{97}$

D'autres types d'inscriptions, votives celles-là, peuvent également servir de preuve pour établir l'existence de propriétés. Dans le cas de Desideratia Desiderata et de son mari C. Iustinius Favor, nous savons que leur dédicace à Jupiter Très bon, Très grand et à Junon Regina fut érigée in $s u o^{98}$. Il en va de même pour la dédicace à Hygie posée à l'instigation de

91 CIL XI, 448* et $A E$ 1977, 259.

92 Cf. CIL V, 4484 = InscrIt. X/5, 275: Tassaux 2003, op. cit. (n. 87), 118.

$93 A E 1987,731=$ IRAT 27.

94 CIL XII, $2327=I L S 6995=I L N$ V/2, 515. Le toponyme Arbin, sis près de Montmélian, semble indiquer la présence d'une propriété ayant appartenu à cette famille. Cf. en dernier lieu, B. Rémy et F. Kayser, Les Viennois hors de Vienne. Attestations (épigraphiques, littéraires et papyrologiques) de l'activité des Viennois(es) hors de leur cité (Bordeaux 2005), 50.

95 Cf. InscrIt. X, 5, 1070 et CIL V, 4993 = InscrIt. X, 5, 1071 : Tassaux 2003, op. cit. (n. 87), 119.

96 CIL VI, 28715; $A E$ 1974, 143a = CIL VI, 41289 et $A E$ 1974, 143b. La famille semble provenir de Mactar, en Afrique Proconsulaire (cf. G. Alföldy, in CIL VI, ad 41289, p. 5038).

$97 A E$ 1906, 80. Le couple devait posséder un bien à proximité du lieu où fut découvert le fragment d'inscription les mentionnant; le souvenir de l'existence de leur propriété semble s'être maintenu dans l'onomastique à travers les siècles : Andermahr 1998, op. cit. (n. 7), 343-344, nº 346 et M. Valenti, Ager Tusculanus (Florence 2003), 83-84 et 289 (Forma Italiae 41).

98 CIL XIII, 7269. 
Cornelia Valentina Tucciana et son mari à leur domicile privé99. A l'inverse, un hommage dressé en l'honneur de Iulia Festilla à Yverdon par les uikani atteste peut-être un domaine ${ }^{100}$. On peut en dire de même pour Arminia Paulina à Timgad ${ }^{101}$ ainsi que pour Tertia Romana et son mari à Maudach (près du castellum de Rufiniana) ${ }^{102}$.

Le contexte archéologique peut également fournir des éléments de confirmation, comme dans le cas de Valeria Rufina et de son fils pour lesquels l'existence d'une villa a été conjecturée à proximité du lieu où le sarcophage de ce dernier a été mis au jour ${ }^{103}$. L'archéologie, combinée à la présence d'épitaphes d'esclaves, révèle l'emplacement de propriétés d'Arrecina Tertulla en Apulie ${ }^{104}$. En revanche, on ne saurait en dire de même pour Claudia Gallitta, originaire de Rusicade, en Numidie, mais qu'une épitaphe en l'honneur d'un oỉxovópos dénommé Philon, provenant des environs de Nicée, en Bithynie, mentionnerait ${ }^{105}$. Rien n'est moins sûr car l'homonymie est possible et d'autres arguments qu'il n'est pas lieu ici de rappeler rendent délicate cette interprétation ${ }^{106}$.

Les fistules de plomb, elles aussi, attestent, pour leur part, des résidences urbaines suffisamment étendues et appartenant à des personnalités fortunées qui ont reçu le droit de posséder une connexion avec le réseau d'approvisionnement en eau. Cela serait le cas, selon W. Eck, d'Aelia Marciana, participante aux Jeux séculaires de l'an 204 mais un problème d'identification soulevé par C. Bruun rend difficile l'interprétation du chercheur allemand ${ }^{107}$.

\footnotetext{
99 R. Cagnat, Bulletin archéologique du comité des travaux historiques (1901), CCX.

100 CIL XIII, $5064=$ ILS 7010 .
}

101 A. Ballu, 'Rapport sur les travaux de fouilles exécutés en 1906 par le service des monuments historiques en Algérie', Bulletin archéologique du comité des travaux historiques (1907), 277 : Briand-Ponsart 2003, op. cit. (n. 7), 82, n. 15.

102 CIL XIII, 11695: W. Spickermann, "Mulieres ex voto": Untersuchungen zur Götterverehrung von Frauen im römischen Gallien, Germanien und Rätien (1.-3. Jahrhundert n. Chr.) (Bochum 1994), 252.

103 AE 1965, 259 : Y. Burnand, Primores Galliarum, I, Méthodologie (Bruxelles 2005), 105 et 300 .

${ }^{104}$ CIL IX, 6183 = M. Chelotti, 'Epigrafi latine monumentali di Ruvo', in M. Pani (éd.), Epigrafia e territorio. Politica e società. Temi di antichità romane, II (Bari 1987), 73-74, $\mathrm{n}^{\circ} 14$ : Andermahr 1998, op. cit. (n. 7), 159-160.

$105 I K-9 / \mathrm{I}, 196$.

106 L'hypothèse de l'identification de la matrone équestre de Numidie avec son homonyme de Bithynie est soutenue par W. Eck, 'Miscellanea prosopographica', Zeitschrift für Papyrologie und Epigraphik 42 (1981), 254-256.

107 CIL XV, 7407. Cf. W. Eck, 'Die Fistulae aquariae der Stadt Rom. Zum Einfluss des sozialen Status auf administatives Handeln', in AA.VV., Atti del colloquio internazionale AIEGL su «Epigrafia e ordine senatorio ». Roma, 14-20 maggio 1981, I (Rome 1982), 210, n. 64 = Id., Die Verwaltung des römischen Reiches in der hohen Kaiserzeit. Ausgewählte und 
Enfin, un terrain appartenant à Viria Rustica, situé à Thugga et sur lequel fut bâti un sanctuaire en l'honneur de Tibère, dénote qu'elle possédait des terres dans ce pagus dépendant de la cité de Carthage ${ }^{108}$.

Avec toutes ces attestations de biens fonciers, il convient maintenant de se demander quelles fonctions premières occupaient ces propriétés rurales? Manifestement, il s'agissait de résidences de villégiature mais aussi de villas d'exploitation. A cet égard, l'exemple d'Aemilia Pudentilla est sans doute le plus clair puisque nous lisons, sous la plume d'Apulée, qu'elle résidait de temps à autre dans une villa suburbaine, dans laquelle d'ailleurs elle se maria avec l'écrivain ${ }^{109}$. Elle possédait en outre une domus à Oea avec vue sur mer ainsi que d'autres biens distants de 100 milles de la cité côtière ${ }^{110}$. En outre, Aemilia Pudentilla qui sait gérer ses biens avec compétence, comme le reconnaît Apulée lui-même ${ }^{111}$, a acquis, avec l'accord de son tuteur, Cassius Longinus, un petit champ à 60.000 sesterces $^{112}$.

A la vue de tous ces témoignages, on peut constater que des femmes disposaient de plein droit des biens fonciers qu'elles acquéraient ou transmettaient par le biais d'héritage, par mariage ou par achat direct. Rien, juridiquement, ne s'y opposait: sans entrer dans les détails, une femme, sui iuris ou bien mariée cum manu ou sine manu, conservant dans ce cas un lien d'agnation avec sa famille paternelle, héritait de son père au même titre que ses frères. Son patrimoine dans le cas d'une union sine manu, était dissocié de celui du conjoint qui ne possédait aucun droit dessus. En revanche, pour transmettre un legs, une femme devait toujours rédiger un testament.

Puisqu'il est clair que les femmes disposaient des biens fonciers en leur nom propre, il convient maintenant de se demander quels types d'activité y ont-elles développés.

Fort logiquement, l'agriculture ainsi que l'exploitation du bétail constituent les premières productions dans ces propriétés. Une nouvelle fois, Apulée le signale pour son épouse, Aemilia Pudentilla, quand elle léga à ses fils une partie de l'héritage de leur père auquel ils avaient droit : celui-ci

\footnotetext{
erweiterte Beiträge, II (Bâle-Berlin 1997), 259, n. 74 et C. Bruun, The Water Supply of Ancient Rome. A Study of Roman Imperial Administration (Helsinki 1991), 94, n. 75.

108 CIL VIII, 26518 = ILTun. 1402 = ILAfr. 519 = DFH 25 .

109 Apul., Apol. 87, 10.

110 Apul., Apol. 72, 6 et 44, 6.

111 Apul., Apol. 87, 7.

112 Apul., Apol. 101, 5 .
} 
consistait en des terres de qualité variable, une vaste maison, une grande quantité de blé, d'orge, de vin, d'huile d'olive et d'autres produits du sol, au moins 400 esclaves et des troupeaux de valeur non négligeable ${ }^{113}$. En Egypte, vers le milieu du $\mathrm{III}^{\mathrm{e}}$ s., Aurelia Demetria, matrona stolata mariée au chevalier Aurelius Appianus a, quant à elle, loué un terrain dans l'Arsinoïte ${ }^{114}$. Par ailleurs, d'autres papyrus appartenant aux archives de Heroninos, détaillent une liste de comptes la concernant et relatifs à ses exploitations ${ }^{115}$. Parmi les autres sources de revenus, la participation dans la production et la commercialisation de l'huile d'olive ne peut être sousestimée $^{116}$. De fait, cette hypothèse a été maintes fois évoquée pour Iunia D. f. Rustica, la riche évergète de Cartima ${ }^{117}$, car elle semble être une proche parente du mercator olearius $\mathrm{D}$. Iunius R[- - ], connu par des marques d'amphores datées entre $120-125$ de notre ère ${ }^{118}$. Plus récemment, a été mis au jour à Séville un autel funéraire de marbre blanc du début du $\mathrm{II}^{\mathrm{e}} \mathrm{s}$., relatif à un D. Iunius Rusticus, contemporain du précédent, à moins qu'il ne s'agisse du même personnage ${ }^{119}$. Dans ce cas-là, ces attestations confirmeraient la participation de cette famille dans la production et la commercialisation de l'huile d'olive à partir de ports et centres économiquement liés ${ }^{120}$. En effet, tant la situation de Cartima sur l'axe routier Cordoue-Málaga que la possible identification de son mari, C. Fabius Fabianus, avec un homonyme d'Acinipo ${ }^{121}$, rendent même plausibles la

113 Apul., Apol. 93, 3-4.

114 P.Flor. I, 16 (23 février 239).

115 P.Flor. I, $100\left(23^{-232}\right)$; P.Laur. I, $11(248-258)$ et plus récemment P.Prag. III, 236 (octobre 259). Sur Heroninos: D. Rathbone, Economic Rationalism and Rural Society in Third-century A.D. Egypt. The Heroninos Archive and the Appianus Estate (Cambridge 1991).

116 Sur ce thème, S. Morretta, 'Donne imprenditrici nella produzione e nel commercio dell'olio betico (I-III sec. d. C.)', Saitabi 49 (1999), 229-245 et Gallego Franco 2000, op. cit. (n. 82), 1269-1279. Pour une liste, G. Chic, Datos para un estudio socioeconómico de la Bética (Écija 2001), 471-493.

117 CIL II, $1956=$ ILS 5512 .

118 CIL XV, 3914. Cf. G. Chic, 'Los centros productores de las ánforas con marcas de L.F.C.', Hispania antiqua 18 (1994), 218-219.

119 S. Ordóñez Agulla et S. García-Dils de la Vega, 'Nuevas inscripciones de Romulenses. Con un apéndice sobre el paisaje periférico septentrional de Romula Hispalis', Romula 3 (2004), 154-162. Ces liens seraient renforcés par la présence à Séville de Fabii Fabiani, porteurs de la même onomastique que le mari de Iunia Rustica.

120 Ordóñez Agulla et García-Dils de la Vega 2004, op. cit. (n. 119), 161-162.

121 CIL II, 1356. 
participation de Iunia Rustica et de ses proches dans des activités commerciales en liaison avec l'annone ${ }^{122}$.

Pour ce qui est plus concrètement des intérêts non agricoles, d'autres sources, d'un type un peu particulier et exclusivement italien dans leur répartition, nous permettent de prendre connaissance de la présence de matrones équestres en tant que dominae documentées par le biais d'estampilles sur briques provenant de biens en général acquis ou transmis au moyen d'héritages à des parents relativement proches ${ }^{123}$. L'étude de ces marques nous confronte à un matériau qui nous est parvenu dans un état pratiquement intact, sans avoir souffert les aléas de la transmission qu'ont généralement subis les autres types d'inscriptions depuis l'Antiquité. Par conséquent, les marques de briques, où apparaît d'ailleurs un nombre relativement important de femmes, permettent d'élaborer des réflexions statistiques ${ }^{124}$. Ces marques, découvertes sur sites archéologiques, posent divers problèmes d'identification en ce qui concerne le statut des personnages qui figurent sur les sceaux : il n'est parfois en effet guère aisé de distinguer entre le dominus/a investisseur ainsi que propriétaire des figlinae ou du domaine (praedia) où a été extraite l'argile et l'officinator, dont les rôles sont variés mais qui est chargé de la production des briques ${ }^{125}$.

122 G. Chic, 'Nuevos datos económicos sobre el senador hispalense Fabius Iulianus', in C. Alonso del Real et al. (éd.), Urbs aeterna. Actas y colaboraciones del coloquio internacional, Roma entre la literatura y la historia. Homenaje a la profesora Carmen Castillo (Pampelune 2003), 394-395 et E. García Vargas et J. Martínez Maganto, 'Fuentes de riqueza y promoción social de los negotiantes salsarii béticos en el alto imperio romano. Una aproximación diacrónica', Archivo español de arqueología 82 (2009), 146.

123 P. Setälä, Private Domini in Roman Brick Stamps of the Empire. An Historical and Prosopographical Study of Landowners in the District of Rome (Helsinki 1977), 230-241. Dans une étude récente, P. Setälä nuance fortement ses assertions relatives aux héritages qu'elle considérait comme le seul moyen d'acquérir des biens fonciers. Elle estime désormais que la part des legs, sans être totalement négligeable, n'est plus aussi prépondérante comme elle le pensait initialement. De même, elle revoit aussi la place dévolue aux domini/ ae dont le rôle fut plus actif dans la production des briques, en ne se contentant pas d'être de simples propriétaires fonciers vivant de leurs rentes : Setälä 2002, op. cit. (n. 7). Pour une présentation succincte de cette thématique, C. Bruun, 'La ricerca sui bolli laterizi presentazione generale delle varie problematiche', in C. Bruun et F. Chausson (éd.), Interpretare i bolli laterizi di Roma e della valle del Tevere. Produzione, storia economica e topografia. Atti del convegno all'Ecole française de Rome e all'Institutum Romanum Finlandiae, 31 marzo e 1 aprile 2000 (Rome 2005), 3-24.

124 Setälä 1977, op. cit. (n. 123), 11. Voir déjà T. Helen, Organization of Roman Brick Production in the First and Second Centuries A.D. An Interpretation of Roman Brick Stamps (Helsinki 1975), 13.

125 Helen 1975, op. cit. (n. 124), 89-130 ; Setälä 1977, op. cit. (n. 123), 13-16 et Setälä 2002, op. cit. (n. 7), 184 ; J.J. Aubert, Business Managers in Ancient Rome. A Social and Economic Study of Instores, 250 B.C.-A.D. 250 (Leyde-New York-Cologne 1994), 224-236 et Id., 'L'estampillage des briques et des tuiles: une explication juridique fondée sur une appro- 
Qui plus est, une autre incertitude, et non des moindres, se fait jour, dans la mesure où il est difficile de certifier que les dames, fort présentes dans cette documentation au point d'être recensées sur un tiers des attestations ${ }^{126}$, étaient apparentées à des chevaliers, eux-mêmes peu nombreux et difficilement identifiables en tant que tels ${ }^{127}$. Dans sa prosopographie, confrontée à ce même dilemme, M.-Th. Raepsaet-Charlier avait pris le parti d'en faire presque toujours des clarissimes, en s'appuyant sur les données statistiques ${ }^{128}$ mais aussi sur l'onomastique, notamment, quand elle paraît conforter cette extraction ${ }^{129}$. Dans certains cas, le doute subsiste $^{130}$. En revanche, quelques femmes sont clairement apparentées à des chevaliers : il s'agit de Claudia Capitolina ${ }^{131}$, fille du préfet d'Egypte Claudius Balbillus ${ }^{132}$ et épouse d'un autre préfet d'Egypte, M. Iunius Rufus attesté à l'époque de Trajan ${ }^{133}$, et de Cusinia Gratilla ${ }^{134}$, documentée entre les années 120 et 146-147, proche parente (fille ?) de L. Cusinius Messalinus, officier et procurateur originaire d'Ephèse ${ }^{135}$.

Dans le cas qui nous occupe, une autre difficulté à laquelle nous sommes confronté est d'établir la place de ces dames «entrepreneurs», pour reprendre l'expression de P. Setälä ${ }^{136}$, dans la production de briques. Etant donné que les femmes disposaient en général d'une indépendance économique (mâtinée toutefois par la présence d'un tuteur) ${ }^{137}$, elles étaient libres de gérer leurs biens et d'en acquérir d'autres. Les biens fonciers étaient fort recherchés et pouvaient, de ce fait, faire l'objet de transactions. De judicieux investissements, pour poursuivre une «tradition»

che globale', in C. Bruun et F. Chausson (éd.), Interpretare i bolli laterizi di Roma e della valle del Tevere. Produzione, storia economica e topografia. Atti del convegno all'Ecole française de Rome e all'Institutum Romanum Finlandiae, 31 marzo e 1 aprile 2000 (Rome 2005), 53-59. Certains de ces officinatores sont des femmes: Helen 1975, op. cit. (n. 124), 112-113 (qui sous-estime leur rôle); Aubert 1994, op. cit. (n. 125), 224-226; Setälä 2002, op. cit. (n. 7), 198-200.

126 Setälä 1977, op. cit. (n. 123), 11 et 211 ; Setälä 2002, op. cit. (n. 7), 184. Voir aussi Helen 1975, op. cit. (n. 124), 23.

127 Setälä 1977, op. cit. (n. 123), 223-224.

128 Setälä 1977 , op. cit. (n. 123), 211 et $25^{0}-256$.

129 Cf. M.-Th. Raepsaet-Charlier, Prosopographie des femmes de l'ordre sénatorial (I ${ }^{\text {er }}-I^{e}$ siècle) (Louvain 1987), 16 et n. 6.

130 Cf. p. ex. Aelia Severa : Setälä 1977, op. cit. (n. 123), 49-5o.

131 CIL XV, 7520 et $P I R^{2} \mathrm{C} 1086$.

$132 P^{2} \mathrm{C}_{13} ; C_{15} ;$ PME $\mathrm{C}_{124}$ et $C J C_{538 .}$

133 Setälä, 1977 op. cit. (n. 123), 94-95 et $P I R^{2}$ I 812.

$134 P^{2} \mathrm{C} 1631$.

135 Setälä 1977, op. cit. (n. 123), 105 et $P^{2} R^{2}$ C 1627 ; CP 80 ; PME C 262.

136 Setälä 2002, op. cit. (n. 7), 181.

137 Gourévitch et Raepsaet-Charlier 2001, op. cit. (n. 7), 66-68. 
familiale acquise par héritage ou bien par volonté propre de celles-ci ${ }^{138}$, contribuaient dans tous les cas à y produire de juteux bénéfices. Pour ce qui est de la production de briques, un contrat devait être passé entre le dominus/a, propriétaire des figlinae (unités de productions) et l'officinator chargé de fabriquer les briques qui restent propriété du dominus/a qui contrôlait cette production ${ }^{139}$. Comme l'affirme P. Setälä, les femmes jouaient un rôle actif, parfois par le biais d'une societas avec leur mari ou leur frère ${ }^{140}$, en ne se contentant pas de poursuivre l'entreprise commencée par leurs pères ou maris mais en investissant elles-mêmes, de leur plein gré, en vue de produire en masse des briques, comme certaines le firent en 123, pour répondre à la demande ${ }^{141}$.

Enfin, dans un registre différent, il convient de signaler que d'autres sources de revenus pouvaient provenir de la location d'immeubles comme ce que firent Cornelia Valentina Tucciana et son mari à Timgad : à côté du marché qu'ils y firent bâtir, le couple fit édifier sur un terrain de $75 \mathrm{~m} \times$ $36 \mathrm{~m}$ la plus luxueuse maison de la colonie à laquelle furent adossées des boutiques dont la location augmentait les rentrées ${ }^{142}$.

En conclusion, on peut affirmer que les femmes apparentées aux chevaliers, en dépit des difficultés d'identification, furent loin de jouer un rôle passif dans la vie économique de leurs cités ${ }^{143}$. Généralement à la tête de leur patrimoine, basé sur des biens fonciers, elles savent l'administrer avec discernement et sagesse pour l'accroître par des acquisitions et en faire bénéficier leurs proches, au moyen d'héritages ou grâce à leur dot ${ }^{144}$. Leurs concitoyens également en jouissent par le biais de l'évergétisme,

138 Setälä 2002, op. cit. (n. 7), 192-195.

139 Setälä 2002, op. cit. (n. 7), 183-184.

140 Setälä 2002, op. cit. (n. 7), 192.

141 Setälä 2002, op. cit. (n. 7), 186-189 qui nuance ainsi les dires d'Aubert 1994, op. cit. (n. 125), 419, qui tend à minimiser la capacité des femmes à pouvoir se livrer à de telles activités.

142 Briand-Ponsart 2003, op. cit. (n. 7), 87.

143 Cette recherche pose aussi en substance la question de savoir quelle était la part du patrimoine de ces dames qui était consacrée à l'évergétisme. L'exemple d'Aemilia Pudentilla, exceptionnel à tous points de vue, permet de se faire une idée puisque nous savons qu'elle disposait d'une fortune estimée à 4 millions de sesterces au moins (Apul., Apol. 71,6 et 77, 1) et qu'elle a distribué 50.000 sesterces lors de la prise de toge virile de Pudens et du mariage de Pontianus (Apul., Apol. 87, 10-11). Autrement dit, Pudentilla a distribué à cette occasion-là $1,25 \%$ de son patrimoine.

144 Cf. Sen., Dial. 12, 14, 3. Cf. aussi Navarro Caballero 2004, op. cit. (n. 11), 392 et 397. En outre, depuis le règne de l'empereur Antonin, il fut permis aux épouses d'accorder un don à leurs maris afin de les aider à accéder au Sénat et à l'ordre équestre ou bien à organiser des jeux: D. 24, 1, 40-42 (extraits des écrits d'Ulpien, de Licinius Rufinus et de Gaius) et Ulp., Reg. 7, 1. 
comme il convient à des personnalités respectables et elles en retirent honneurs et prestige qui rejaillissent sur leurs proches parents ${ }^{145}$. Tout cela est conforme aux pratiques économiques propres aux femmes de l'élite qui tirent profit des revenus de leurs biens sans devoir y travailler de leurs mains ${ }^{146}$. Cela n'est toutefois pas le cas de toutes les matrones équestres, telles que l'aïeule du préfet du prétoire Nymphidius Sabinus, couturière à gages ${ }^{147}$, ou encore Nymphidia, la mère de ce même Sabinus ${ }^{148}$, accusée à tort ou à raison d'être une prostituée, à la différence de Vistilia que le fit publiquement savoir ${ }^{149}$ : elles «gagnent» leur vie et sont « rémunérées » pour ce faire, contrairement à ce qui sied à des dames de la haute société, mais cela confirme pleinement l'hétérogénéité de l'extraction et du statut social des personnes apparentées à des chevaliers, si souvent soulignée par S. Demougin pour les membres de l'ordre équestre. Par ailleurs, les considérants du SC de Larinum (sur le fait de se louer pour monter sur scène ou combattre dans l'arène) semblent refléter une réalité que la Loi ne pouvait accepter, au nom de la respectabilité des ordines supérieurs ${ }^{150}$. Les matrones équestres, par conséquent, s'adonnaient à des pratiques économiques variées, certaines plus répréhensibles que d'autres mais, en fin de compte, très souvent sur la place publique, loin du foyer où elles se devaient d'être confinées.

145 Cf. Melchor Gil 2009, op. cit. (n. 11).

146 Rien de surprenant puisque sénateurs et chevaliers, et a fortiori leurs parentes, bien que généralement plus discrètes dans nos sources, possédaient des intérêts économiques communs et leurs activités publiques étaient identiques. Voir Andreau 1999, op. cit. (n. 9) et Raepsaet-Charlier 2005, op. cit. (n. 2).

147 Plu., Galb. 9, 2.

148 Plu., Galb. 9, 1-4 et Tac., Ann. 15, 72, 1-2.

149 Tac., Ann. 2, 85, 1-4.

150 Sur le sénatus-consulte de Larinum : C. Ricci, Gladiatori e attori nella Roma giulioclaudia. Studi sul senatoconsulto di Larino (s. 1. 2006). Rappelons que les sources rapportent à maintes reprises que des dames apparentées à des sénateurs et à des chevaliers montaient sur scène et combattaient dans l'arène lors de manifestations organisées à l'instigation ou en présence de l'empereur. On peut à bon droit se demander si elles étaient rémunérées pour cela: Tac., Ann. 15, 32 ; Suet., Nero 4, 2 ; Dio Cass. 55, 10, 11 ; 60, 7, 1; 61, 17, 3. 


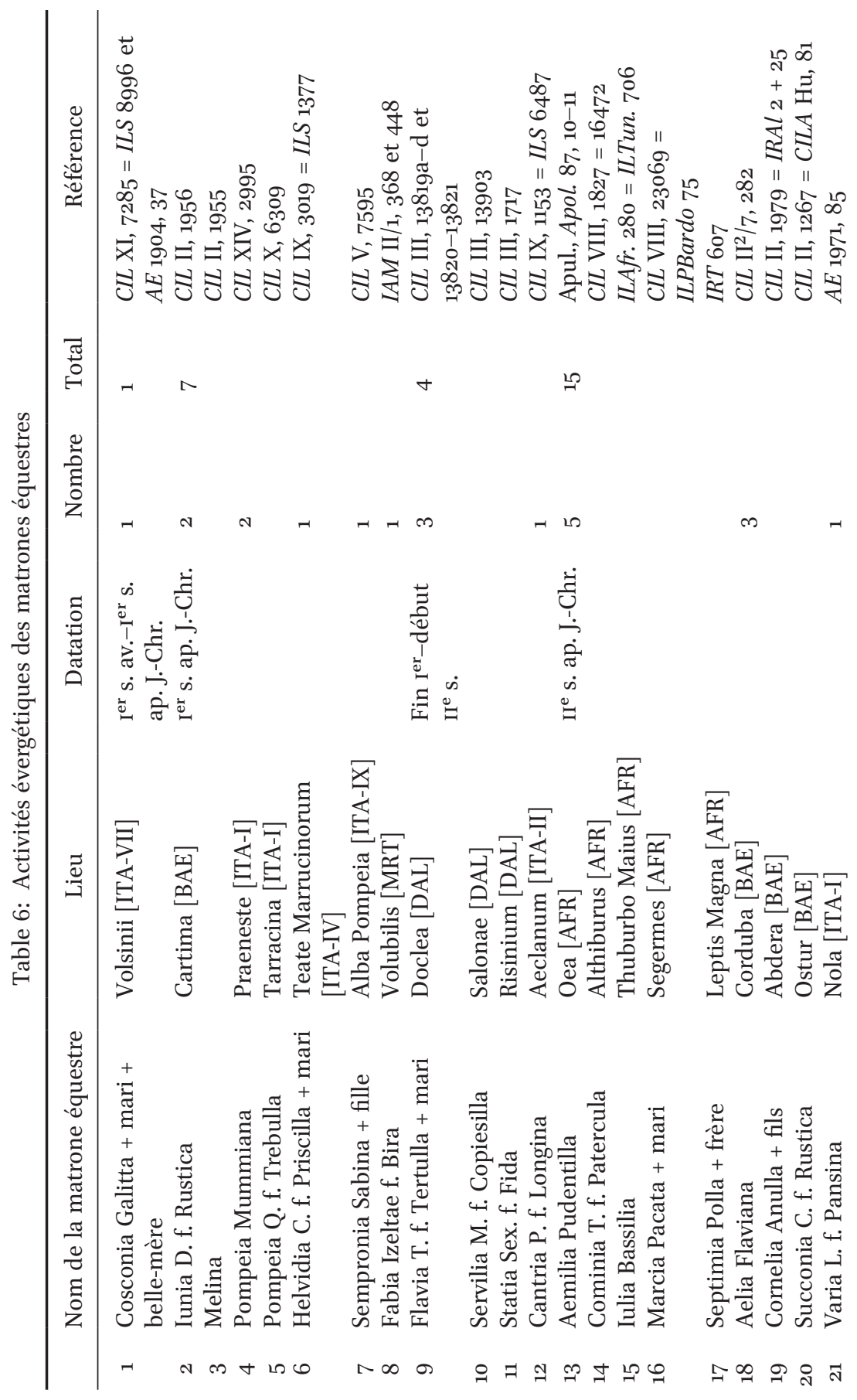




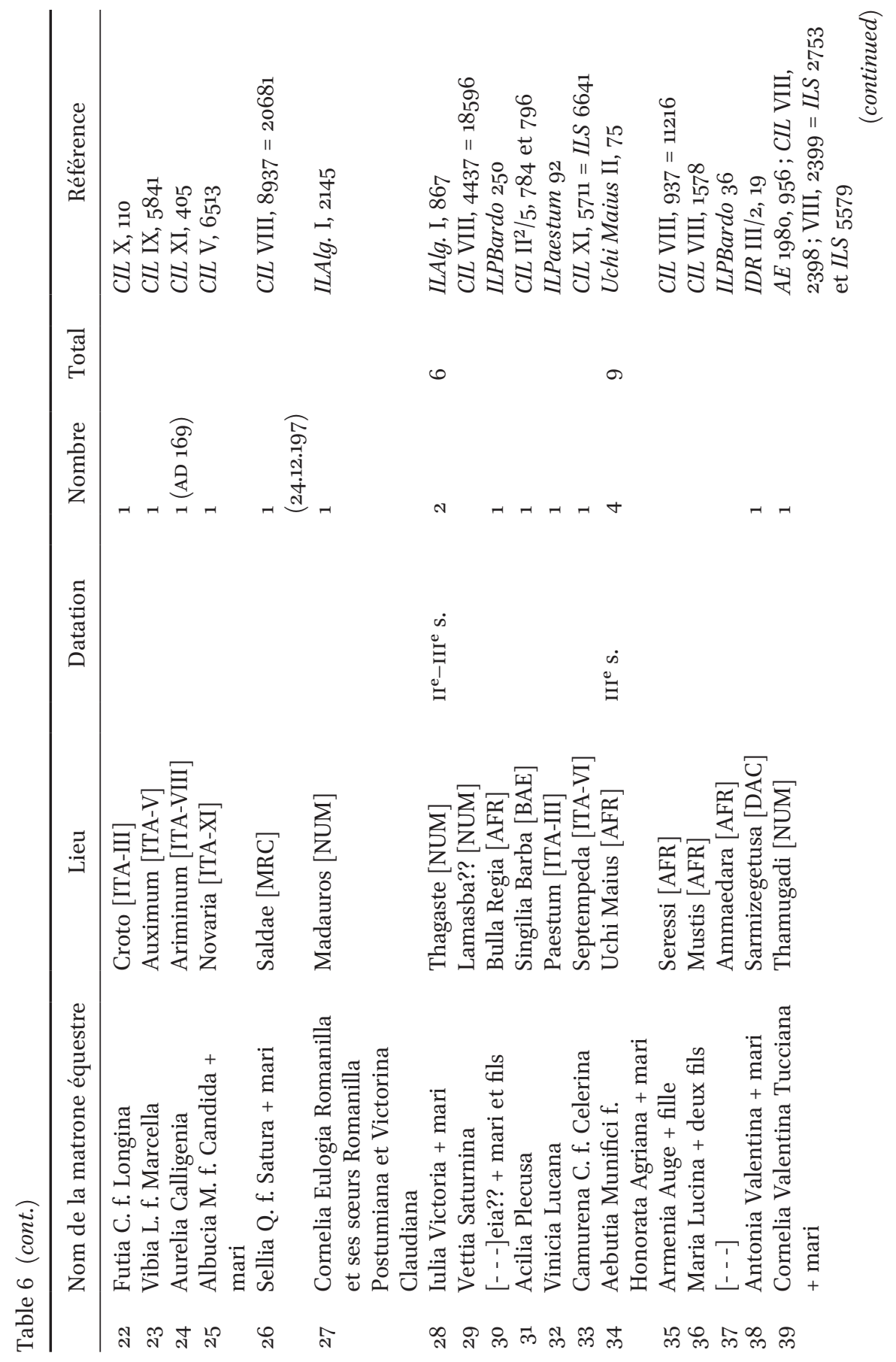




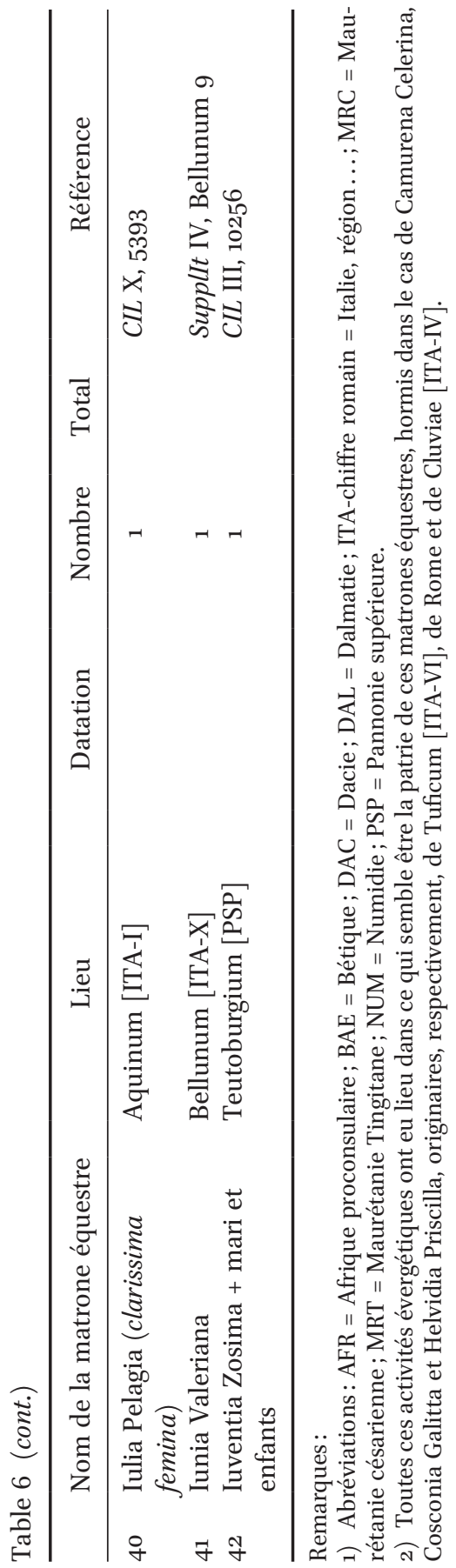




\title{
INTEGRATION OR DISINTEGRATION? \\ THE ROMAN ARMY IN THE THIRD CENTURY A.D.
}

\author{
Lukas de Blois
}

My issue in this paper is: what was the main trend within the Roman military forces in the third century $\mathrm{AD}$ ? Integration, or disintegration into regional entities? This paper is not about cultural integration of ethnic groups in multicultural parts of the Roman Empire, such as the city of Rome, thriving commercial centres, and border regions to which the armies had brought people from various parts of the Empire, and where multicultural military personnel lived together with indigenous groups, craftsmen from different origins, and immigrants from commercially active regions, either in canabae adjacent to castra stativa, or in garrison towns, as in the Eastern parts of the Empire.

In variatio upon an issue raised by Frederick Naerebout in another paper published in this volume, I might ask myself in what sense an army, which in the third century AD was progressively composed of ethnically and culturally different units, kept functioning as an integrated entity, or in actual practice disintegrated into rivalling, particularistic regional forces whose actual or potential competition for money and supplies constantly threatened peace and stability in the Empire, particularly in times of dangerous external wars, when the need for supplies increased.

The discussion should start with Septimius Severus. After his victories over Pescennius Niger, some tribes in northern Mesopotamia, and Albinus in Gaul, Severus had to replenish the ranks of his armies, for example at the Danube frontiers, which had yielded many men to Severus' field armies and his new praetorian guard. Besides he had to compensate for the many losses that Niger's eastern army and Albinus' British troops had sustained, and find recruits for his new legiones Parthicae I, II and III. He had to do so in a post-plague period, in which many mobile young men (soldiers!) were missing because they - as always in times of plague had died first and foremost, prices were rising, workers could demand higher wages, and demographic recovery at best was just taking off. ${ }^{1}$ This

\footnotetext{
${ }^{1}$ On the so-called Antonine plague and its consequences see R.S. Bagnall \& B.W. Frier, The demography of Roman Egypt (Cambridge 1994), 173 f.; R.P. Duncan-Jones, 'The impact of the Antonine plague', Journal of Roman Archaeology 9 (1996), 108-136; E. Lo Cascio,
} 
may be the reason why Severus in A.D. 197 increased the soldiers' pay by $100 \%$. He had to induce good recruits to join the forces but in this way added an enormous burden to state expenditure. ${ }^{2}$ Severus added other inducements to enlist as well. In 3.8.4, Herodian tells us that the soldiers were given - with the increase in pay - a very substantial donative and privileges that they had not had before, such as a permission to wear a gold ring and the right to live at home with their wives. ${ }^{3}$ In this way military personnel started to own property in the region where their units were normally stationed. Its prosperity may have increased, and its status may have been enhanced. ${ }^{4}$ If so, these Severan measures may have rein-

'Prezzi in oro e prezzi in unità di conto tra il III e il V secolo d.C.', in J. Andreau et al. (eds.), Prix et formation des prix dans les économies antiques. Entretiens d'archéologie et d'histoire (Saint-Bertrand-de-Comminges 1997), 168 ff.; W. Scheidel, 'A model of demographic and economic change in Roman Egypt after the Antonine plague', Journal of Roman Archaeology 15 (2002), 97-114. On the three legiones Parthicae see R.E. Smith, 'The army reforms of Septimius Severus', Historia 21 (1972), 485-487; J.-M. Carrié \& A. Rousselle, L'empire romain en mutation des Sévères à Constantin, 192-337 (Paris 1999), 76 f. On young mobile men as first plague victims see S.K. Cohn Jr., The Black Death Transformed. Disease and Culture in Early Renaissance Europe (London 2002), 16-18; $121 \mathrm{f}$. Cf. Duncan-Jones 1996, supra, 112; A.D. Lee, War in Late Antiquity. A Social History (Oxford 2007), 78. On wages, rents and land prices in post-plague periods see Duncan-Jones, 'Antonine Plague', supra, 121-124; Scheidel 2002, supra, $97 \mathrm{ff}$. On the rise in prices that started under Commodus or even earlier see R.P. Duncan-Jones, Money and Government in the Roman Empire (Cambridge 1994), 25-28; Scheidel 2002, supra, 104.

2 On Severus' military reforms in general see J.-M. Carrié, 'Eserciti e strategie', in A. Carandini, L. Cracco Ruggini \& A. Giardina (eds.), Storia di Roma III 1 (Turin 1993), 87-89; Y. Le Bohec, The Imperial Roman Army (London/ New York 1994), 191-194; P. Cosme, L'armée romaine (Paris 2009), 221. On the increase in pay see J.B. Campbell, The Emperor and the Roman Army, 31 BC-AD 235 (Oxford 1984), 185 f.; Duncan-Jones 1994, op. cit. (n. 1), 29; Carrié \& Rousselle 1999, op. cit. (n. 1), 75; M.A. Speidel, 'Roman Army Pay Scales', in M.A. Speidel (ed.), Heer und Herrschaft im römischen Reich der hohen Kaiserzeit (Stuttgart 2009), 380 [this article was published before in The Journal of Roman Studies 82 (1992), 87-106]. Unlike other authors, Speidel comes to the conclusion that Severus doubled the amount paid by Domitian (from $84 \mathrm{AD}$ onwards), and not just augmented it by $50 \%$. Duncan-Jones 1994, op. cit. (n. 1), 45 convincingly argues that about 72 to $77 \%$ of the state budget was spent on the defenses of the empire and the soldiers' pay. So a sizeable raise in the soldiers' pay would have dire budgettary consequences.

${ }^{3}$ See Carrié 1993, op. cit. (n. 2), 87-89. B. Pferdehirt, Die Rolle des Militärs für den sozialen Aufstieg in der römischen Kaiserzeit (Bonn 2002), 212-214 refers to evidence from the Digesta, but unfortunately not very accurately. On the ending of the marriage ban for soldiers see S.E. Phang, The Marriage of Roman Soldiers (13 B.C.-A.D. 235). Law and Family in the Imperial Army (Leiden/Boston 2001), 17-19 who thinks that it was directly meant to encourage recruitment. On p. 382 she rightly remarks: "Severus' military reforms were not merely to indulge the army; he probably sought to increase recruitment after the Marcomannic War, the Antonine plague, and the civil wars of 193-7 had decimated the army."

4 See A. Mócsy, Pannonia and Upper Moesia. A History of the Middle Danube Provinces of the Roman Empire (London/Boston 1974), 236; G. Alföldy, Römische Heeresgeschichte (Amsterdam 1987), 35. 
forced a tendency towards regional particularism among the military in the Rhine, Danube, Eastern, and African frontier zones. As the author of Digesta 50.1.23.1 says: "A soldier seems to live where he serves, unless he has possessions in his homeland."

In this way a growing regional chauvinism may have been an important factor in a process of disintegration within the armed forces. I'll give two examples that seem to confirm this hypothesis. In 6.7.2-3, Herodian relates that in 234 Severus Alexander - who was still in the East after his Persian war - received messages from the governors in the Danube region about Germans invading and devastating Roman territories. In their messages they added that the presence of the emperor and his entire army was needed. Those messages, Herodian continues, caused distress to the soldiers transferred from Illyricum, who were in great anxiety about their families back home, and turned their anger on Severus Alexander, blaming him for his hesitant procrastination over the northern problems. ${ }^{6}$

The other example is the fateful year $\mathrm{AD} 260$, when regional interests, added to a general insecurity, fostered a series of usurpations. A devastating plague had destroyed much of the reigning emperor Valerian's army, so that he lost the battle against the Persians and became their captive, probably in the spring of 260 . His son and colleague Gallienus was pinned down in Italy by raids of Iuthungi and Alamanni, and could not bring relief or rescue to other frontiers, where generals and governors such as Ingenuus, Regalianus, and Postumus took the purple and thus tried to obtain more means, supplies, and military reinforcements for their own regions. In the East something similar was done by Macrianus at Samosata, a high bureaucrat who had apparently been responsible for the logistics of Valerian's army. Together with a capable officer, called Ballista, and the Palmyrene prince Odaenathus, he had assembled remnants of Roman forces, mobilized recruits of his own, and successfully started to fight the Persians. ${ }^{7}$

Did the empire witness a rise of regional particularism among the various frontier armies? I have some doubts. To begin with, the Roman armed forces were no complete strangers to one another, although they were

5 D. 50.1.23.1: miles ibi domicilium habere uidetur, ubi meret, si nihil in patria possideat.

6 Cf. Joannes Antiochenus, in FHG IV 593, 140 who copies Herodian's version.

7 On the history of the year 260 see D.S. Potter, The Roman Empire at Bay, A.D. 180-395 (London, New York 2004), 255-257; J. Drinkwater, 'Maximinus to Diocletian and the "crisis", in $C A H X I I^{2}$ 2005, 42 ff.; A. Goltz \& U. Hartmann, 'Valerianus und Gallienus', in K.-P. Johne, U. Hartmann \& Th. Gerhardt (eds.), Die Zeit der Soldatenkaiser (Berlin 2008) I, 245-265; M. Christol, L'empire romain du III ${ }^{e}$ siècle (Paris 2006² [1997]), 139-145. 
recruited in many places, not only in Illyricum and the Balkans, but also in Italy, North Africa, parts of Asia Minor, Egypt and the East. ${ }^{8}$ Forces from the Danube regions willingly fought Germanic tribes in the West or Persians and other enemies in the East, and only protested or rebelled against east- or westbound imperial war policies when the Danube region lay under imminent threats itself, and many detachments had already disappeared, mainly to the East, and had died of diseases or been rounded up in not very successful warfare. In a similar way troops from the Rhine border and Britain fought on the Danube and the Euphrates, and Easterners or North Africans joined the forces that fought the Germans in the North. A handful of examples. In ILS 2763, we find a military tribune who under Septimius Severus brought Syrian bowmen from Germania Superior to Mauretania, to fight rebellious tribesmen there. ${ }^{9}$ Detachments of all four Rhine legions seem to have fought in Severus' second Parthian campaign. ${ }^{10}$ In 6.7.8, Herodian tells us that in A.D. 234-235, at the Rhine border, the army of Severus Alexander counted in its ranks many North African javelin-men, a force of archers from the East, and Parthian volunteers who had fled from their Persian enemy. ${ }^{11}$ The great army that went

8 On third century recruiting see O. Stoll, Römisches Heer und Gesellschaft. Gesammelte Beiträge 1991-1999 (Stuttgart 2001), 240-242 (most recruits came from Illyricum, but there were new soldiers from other regions as well). Recruiting from Italy: Herod., 2.14.6-7 (under Septimius Severus, cf. Cosme 2009, op. cit. [n. 2], 212); Herod., 6.3.1 f. (under Severus Alexander); $A E$ 1919, 256-257, with CIL V 7889 and 7890 (under Maximinus Thrax; cf. X. Loriot, 'De Maximin le Thrace à Gordien III,' in W. Haase \& H. Temporini, Aufstieg und Niedergang der Römischen Welt II 2 [Berlin, New York 1975], 674). Recruits from North Africa: Cl. Lepelley (ed.), Rome et l'intégration de l'empire: 44 av. J.-C. à 260 ap. J.-C. (Paris 1998), 102-104. Recruits from Asia Minor: S. Mitchell, Anatolia. Land, Men, and Gods in Asia Minor I: the Celts and the Impact of Roman Rule (Oxford 2001²), 136-142, esp.139. Recruits from Syria and neighboring eastern regions: Festus, 23 and Orosius, 7.22.12 (on a so-called agrestis manus called up by Odaenathus, in 26o). Recruits from Egypt: Herod., 6.4.7 (under Severus Alexander), Zos., 1.44.1 f., and BGU 1680 = A.S. Hunt \& C.C. Edgar, Select Papyri I (London, Cambridge MA 1952), $341 \mathrm{n}^{\circ}{ }_{134}$. According to W. Eck in Y. Le Bohec, Les légions de Rome sous le Haut-Empire, (Paris, Lyon 2000), 87-93, legio I Minervia attracted recruits from its own hinterland, but also from other regions, such as Thracia. The army of Severus Alexander (A.D. 234-235) and Maximinus Thrax (235-238) that was fighting at the northern borders and in Germany consisted of troops from Illyricum, many Moorish javelinmen, a force of archers from the East, and Parthian deserters who had fled from their new Persian masters (Herod., 6.7-8; 8.1-3).

9 See Y. Burnand, Primores Galliarum II: Prosopographie (Brussels 2006), 518-520, $\mathrm{n}^{\circ} 234$ E 177, Sex. Iulius Iulianus.

10 M. Reddé in Le Bohec 2000, op. cit. (n. 8), 124.

11 Maximinus Thrax probably used this army to fight Germans and Sarmatians. See I. Piso, 'Maximinus Thrax und die Provinz Dakien', in I. Piso (ed.), An der Nordgrenze des römischen Reiches. Ausgewählte Studien (1972-2003) (Stuttgart 2005), 95-107 (= Zeitschrift für Papyrologie und Epigraphik 49 [1982], 225-238), esp.103-106. Cf. HA, Max. 11.7, where 
to the East under Gordian III contained, among others, a lot of soldiers from the Rhine and Danube borders, together with Germanic volunteers. ${ }^{12}$ In a volume edited in 2000 by Yann Le Bohec, the history of various legions is discussed, for example by Keppie, Eck, Franke, Reddé, and Le Bohec. In the second and third centuries A.D., all of those legions had to send detachments to other frontier zones. ${ }^{13}$ In $I L S$ 546, we meet soldiers of vexillationes from legions serving in Britain and Germany, who are now, under Gallienus, fighting somewhere on the Danube frontier. ${ }^{14}$ In 261 Gallienus' general Aureolus at Mursa in Pannonia conquered the pretender Macrianus, who had come up from the East. According to Aurelius Victor, Caesares 33.1-2, his army comprised quite a few North African unities. The Palmyrene forces of Odaenathus and Zenobia counted many Roman soldiers in their ranks, added to a manus agrestis Syrorum, to use Orosius' words, and Palmyrene cavalry. ${ }^{15}$ After a heavy defeat against the emperor Aurelian, in Syria in A.D. 272, Zenobia seems to have boasted that most of her casualties had been Romans. ${ }^{16}$ In the Res Gestae Divi Saporis, the great inscription of the Persian king Shapur I at Naqsh-i-Rustam, the armies of Gordian III, but particularly of Valerian, are described as consisting of soldiers from practically all provinces, plus Germanic tribesmen. ${ }^{17}$

A second objection is that the military middle cadre in the third century $\mathrm{AD}$ still had empire-wide careers, or at least careers in more than

the author of this work says that "... he [= Maximinus Thrax] crossed over into Germany with the whole army and with the Moors, Osrhoenians, Parthians, and all the other forces that Alexander took when he went to war."

12 See Res Gestae Divi Saporis ll. 6-9, esp. 7. See U. Huttner, 'Von Maximinus Thrax bis Aemilianus', in Johne et al. 2008, op. cit. (n. 7), 185. The Res Gestae Divi Saporis is the great inscription of the Persian king Shapur I at Naqsh-i-Rustam, in which he is broadcasting his victories over three Roman emperors, Gordian III, Philip the Arabian, and Valerian.

${ }^{13}$ Le Bohec 2000, op. cit. (n. 8), 26-35 (L. Keppie, on the legiones Britanniae); 71-73 and 83-85 (Y. Le Bohec, on the legio XXX Ulpia); 87-93 (W. Eck, on the legio I Minervia); 95-104 (Th. Franke, on the legio XXII Primigenia); 120-126 (M. Reddé, on the legio VIII Augusta). On this phenomenon, vexillationes fighting on all frontiers, see also Cosme 2009, op. cit. (n. 2), 210-212.

${ }_{14}$ ILS 546 = CIL III, 3228 .

15 According to Zosimus, 1.39.1-2, Odaenathus' army with which he attacked Shapur I in 26o, when the Persians were on their way home, consisted of some remnants of Roman legions combined with his own Palmyrene forces. He had at his disposal also a band of Syrian country folk, as Festus, 23 and Orosius, 7.22.12 tell us.

16 Anonymus Continuator Dionis, 10.5, in FHG IV 197: after the battle of Immae, where he had beaten the Palmyrenes, Aurelian sent envoys to Zenobia urging her finally to surrender to him. She replied, saying: "I have hardly sustained any serious losses. For of those who had fallen, the majority were Romans."

17 Res Gestae Divi Saporis ll. 6-9 (the army of Gordian III); 1l. 20-23 (the army of Valerian). 
one region, and in this way did not easily become attached to just one frontier zone. 'Military middle cadre' is a viable concept. Centurions and higher officers, such as military tribunes, in earlier days used to come from different social layers in society, but the social distinctions between them progressively became blurred. Some equites voluntarily became centurions, ${ }^{18}$ former centurions became equites, the status of the centurionate was much higher than in republican times, and after 260 senators were barred from the army. Furthermore, in daily practice the lower and higher middle cadre had to work together in many ways, they were not completely separate groups.

The evidence is not plentiful, but we know of just enough careers of centurions who became primi pili, prefects of military camps, praepositi of vexillationes, members of the equestrian officer corps, and even procurators, governors, and high equestrian prefects. Many of them had to go from one corner of the empire to another, and served in several frontier zones, Italy and Rome. ${ }^{19}$ There may be a possibility, however, that the various functions they fulfilled did not convey them to the home bases of the legions, but to vexillationes serving together in one place, but it is very unlikely that this would have reduced the traveling around by the military cadre to zero in all cases we know of.

Table 1: Careers in several regions

\begin{tabular}{|c|c|c|c|c|}
\hline & $\begin{array}{l}\text { Period of } \\
\text { Time }\end{array}$ & Born In & Active In & Reference \\
\hline $\begin{array}{l}\text { L. Artorius } \\
\text { Castus }\end{array}$ & Severan times & & $\begin{array}{l}\text { Syria, } \\
\text { Palestine, } \\
\text { Pannonia, } \\
\text { Moesia, Italy, } \\
\text { Britain, and } \\
\text { Pannonia } \\
\text { again }\end{array}$ & $\begin{array}{l}\text { Dobson 1978, op. } \\
\text { cit. (n. 18), } 267 \text {, } \\
n^{\circ} 15^{1}\end{array}$ \\
\hline
\end{tabular}

18 See B. Dobson, Die primipilares. Entwicklung und Bedeutung, Laufbahnen und Persönlichkeiten eines römischen Offiziersranges (Cologne, Bonn 1978), 269 f., $\mathrm{n}^{\circ} 154, \mathrm{Cn}$. Marcius Rustius Rufinus, from Beneventum (cf. H.-G. Pflaum, Les carrières procuratoriennes équestres sous le haut-empire romain I-II [Paris 1960]; III [Paris 1961], n ${ }^{\circ} 234$ ); Dobson 1978, supra, 306-8, $\mathrm{n}^{\circ} 215$, L. Petronius Taurus Volusianus, also an Italian (cf. Pflaum 1960-1961, supra, $\left.\mathrm{n}^{\circ} 347\right)$.

19 See table. 
Table 1 (cont.)

\begin{tabular}{|c|c|c|c|c|}
\hline & $\begin{array}{l}\text { Period of } \\
\text { Time }\end{array}$ & Born In & Active In & Reference \\
\hline $\begin{array}{l}\text { Cn. Marcius } \\
\text { Rustius } \\
\text { Rufinus }\end{array}$ & Severan times & Beneventum & $\begin{array}{l}\text { Rome, Syria, } \\
\text { Northern Italy }\end{array}$ & $\begin{array}{l}\text { Dobson 1978, op. } \\
\text { cit. (n. 18), } 269 f ., \\
\text { n}^{\circ} 154 \text {; Pflaum } \\
\text { 1960-1961, op. cit. } \\
\text { (n. } 18 \text { ), no } 234\end{array}$ \\
\hline $\begin{array}{l}\text { C. Iulius } \\
\text { Pacatianus }\end{array}$ & Severan times & $\begin{array}{l}\text { Vienna } \\
\text { in Gallia } \\
\text { Narbonensis }\end{array}$ & $\begin{array}{l}\text { Mesopotamia, } \\
\text { the Alps, } \\
\text { North Africa }\end{array}$ & 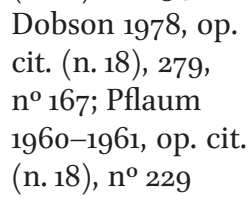 \\
\hline $\begin{array}{l}\text { C. Titius } \\
\text { Similis }^{20}\end{array}$ & $193-238$ & Cologne & $\begin{array}{l}\text { Rome, } \\
\text { North Africa, } \\
\text { Asia Minor, } \\
\text { Eastern } \\
\text { border } \\
\text { regions, } \\
\text { Moesia } \\
\text { Inferior, } \\
\text { Lusitania. }\end{array}$ & $\begin{array}{l}\text { Dobson 1978, op. } \\
\text { cit. (n. 18), } 288 \text {, } \\
n^{\circ} 182 \text {; Pflaum } \\
\text { 1960-1961, op. cit. } \\
\text { (n. } 18), \text { n }^{\circ} 330\end{array}$ \\
\hline $\begin{array}{l}\text { C. Sulgius } \\
\text { Caecilianus }^{21}\end{array}$ & $\begin{array}{l}\text { mid-third } \\
\text { century }\end{array}$ & North Africa & $\begin{array}{l}\text { Rome, } \\
\text { Italy, North } \\
\text { Africa, Spain, } \\
\text { Mesopotamia, } \\
\text { Cappadocia, } \\
\text { Dacia, Britain, } \\
\text { Arabia }\end{array}$ & 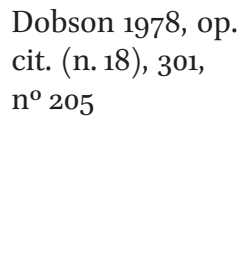 \\
\hline
\end{tabular}

(continued)

20 C. Titius Similis was a centurion in a legion and among the frumentarii, princeps peregrinorum (Rome), primus pilus legionis III Augustae (North Africa), praepositus vexillationum (Asia Minor and Eastern border regions), procurator provinciae Moesiae Inferioris, and procurator provinciae Lusitaniae.

${ }^{21}$ C. Sulgius Caecilianus was optio peregrinorum and a trainer of recruits among the frumentarii (Rome), nauarchus classis Misenatium (Italy), and centurion in the legions III Augusta (North Africa), VII Gemina (Spain), I Parthica (Mesopotamia), XVI Flavia (Cappadocia), and XIII Gemina (Dacia). He was involved in organizing imperial logistics and finances during a campaign, and was praepositus of a detachment of the classis Misenatium, primus pilus legionis XX Valeria Victrix (Britain), and praefectus legionis III Cyrenaicae (Arabia). 
Table 1 (cont.)

\begin{tabular}{|c|c|c|c|c|}
\hline & $\begin{array}{l}\text { Period of } \\
\text { Time }\end{array}$ & Born In & Active In & Reference \\
\hline Ignotus $^{22}$ & $\begin{array}{l}\text { about the } \\
\text { time of Philip } \\
\text { the Arabian }\end{array}$ & & Dacia, Rome & $\begin{array}{l}\text { Dobson 1978, op. } \\
\text { cit. (n. 18), 304, } \\
\text { no } 212^{\circ}\end{array}$ \\
\hline $\begin{array}{l}\text { M. } \\
\text { Septimius ... } \\
\text { lis }^{23}\end{array}$ & Severan times & & $\begin{array}{l}\text { Palestine, } \\
\text { Bonn, } \\
\text { Mesopotamia, } \\
\text { Syria, } \\
\text { Germania } \\
\text { Superior, } \\
\text { Umbria, } \\
\text { Picenum and } \\
\text { Apulia }\end{array}$ & 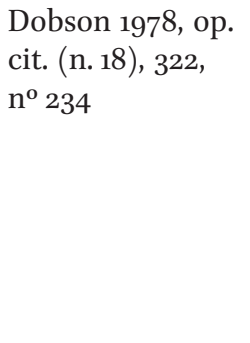 \\
\hline $\begin{array}{l}\text { L.Petronius } \\
\text { Taurus } \\
\text { Volusianus }\end{array}$ & $\begin{array}{l}\text { contemporary } \\
\text { of Gallienus }\end{array}$ & Italy & $\begin{array}{l}\text { Rome, } \\
\text { Pannonia, } \\
\text { Dacia, } \\
\text { Germania } \\
\text { Inferior }\end{array}$ & $\begin{array}{l}\text { Dobson 1978, } \\
\text { op. cit. (n. 18), } \\
306-308, \\
n^{\circ} 215 ; \text { Pflaum } \\
\text { 196o-1961, op. } \\
\text { cit. (n. 18), 901-5, } \\
n^{\circ} 347\end{array}$ \\
\hline $\begin{array}{l}\text { Traianus } \\
\text { Mucianus }\end{array}$ & $\begin{array}{l}\text { contemporary } \\
\text { of Gallienus }\end{array}$ & & $\begin{array}{l}\text { Rome, Italy, } \\
\text { the East, } \\
\text { Illyricum }\end{array}$ & $\begin{array}{l}\text { Dobson 1978, } \\
\text { op. cit. (n. 18), } \\
313-316, n^{\circ} 223 \\
\text { (cf. Pflaum } \\
\text { 196o-1961, op. cit. } \\
\text { (n. } 18 \text { ), 396f.) }\end{array}$ \\
\hline
\end{tabular}

Beyond A.D. 260 the career prospects of military middle cadre personnel became better. From that year onwards senators disappeared from the army and could no longer become tribuni laticlavii or legati legionis. ${ }^{24}$

22 This Ignotus was cornicularius, centurion, dux legionum Daciae, primipilaris, military tribune in the praetorian guard (Rome), and ended up in a procuratorial career.

23 M. Septimius... lis served as a centurion in the legions X Fretensis (Palestine), I Minervia (Bonn), I Parthica (Mesopotamia), III Gallica (Syria), XXII Primigenia (Germania Superior), and as a primus pilus and praepositus in Umbria, Picenum and Apulia, probably against bandits.

24 The last tribunus laticlavius we know of was Publius Balsamius Sabinianus; see Dobson 1978, op. cit. (n. 18), 312 f., $\mathrm{n}^{\circ} 221$ and Pflaum 1960-1961, op. cit. (n. 18), nº 351 . On careers of centurions, grades below the centurionate, primi pili, praefecti castrorum, military tribunes, prefects of cohorts and alae, duces and praepositi of (combinations of) vexillationes see Dobson, Primipilares, 265-323. 
Their places were largely taken by equestrian military middle cadre. This was a change in imperial appointment policies, carried into effect by Gallienus (253-268), who needed militarily experienced administrators and commanders. ${ }^{25}$

Members of the military middle cadre were important, socially as well as politically, ${ }^{26}$ and were in a good position to influence the soldiers and to shape their political preferences. Sensible usurpators and pretenders to the throne would speak with their officers and centurions first, and if these talks went well, they would declare themselves emperors. According to Herodian 2.7.7, Pescennius Niger in A.D. 193 tried to influence his legionary commanders, his tribunes and his more distinguished soldiers before entering the course of an usurpation, and reckoned that positive news would reach his soldiers via his officers. The first thing Septimius Severus did after he had decided to stage a coup against Didius Julianus, in A.D. 193, was to make overtures to small groups of legionary commanders, tribunes, and senior centurions, discussing with them the ruinous state of the empire, brought about because there was no noble or worthy leader to control it, as Herodian tells us in 2.9.7. ${ }^{27}$ In the same author's work, 2.13.1, we read that Septimius Severus, to lure the praetorians into a trap, and disarm and fire them, first approached the military tribunes and centurions, who duly persuaded the praetorian soldiers. In Caesares 20.24-26, Aurelius Victor tells us that the same emperor, Severus, quelled a mutiny by separately speaking with tribunes and centurions. The military middle cadre could also be held responsible for the behavior of their soldiers. In the Historia Augusta, Alexander Severus 53.7, tribunes are blamed for the license of their soldiers at Daphne, outside Antioch, in A.D. 232. This Vita

25 See Aur. Vict., Caesares 33.33: Et patres quidem praeter commune Romani malum orbis stimulabat proprii ordinis contumelia, quia primus ipse metu socordiae suae, ne imperium ad optimos nobilium transferretur, senatum militia uetuit et adire exercitum. See L. de Blois, The Policy of the Emperor Gallienus (Leiden 1976), 39 f.; M. Christol, 'Les réformes de Gallien et la carrière sénatoriale', in Epigrafia e ordine senatorio (Rome 1982), 143-166; K.-P. Johne, 'Die illyrischen Kaiser als Herrscher neuen Typs', in K.-P. Johne, Th. Gerhardt \& U. Hartmann (eds.), Deleto paene imperio Romano. Transformationsprozesse des römischen Reiches im 3. Jahrhundert und ihre Rezeption in der Neuzeit (Stuttgart 2006), 128; Cosme 2009, op. cit. (n. 2), 234 f.

26 B. Rossignol, 'Élites locales et armées: quelques problèmes', in M. Cébeillac-Gervasoni \& L. Lamoine (eds.), Les élites et leurs facettes (Rome 2003), 361, referring to B. Isaac, The Limits of Empire. The Roman Army in the East $\left(\right.$ Oxford $1993^{2}$ ), 137, rightly says: "Le centurion est bien plus puissant que le bouleute et représente l'échelon intermédiaire du pouvoir." See also R. Alston, Soldier and Society in Roman Egypt (London, New York 1995), 86-96 (on important functions of centurions in Roman Egypt).

27 See also Joannes Antiochenus, in FHG IV 587, 126.1. 
may be more of a 'mirror of princes' than an historiographical work, ${ }^{28}$ but even so it is interesting information.

In conclusion, in the third century $\mathrm{AD}$ the Roman military forces were not disintegrating into regional armies that were completely strangers to one another. The military middle cadre, an important factor within the Roman armies, was not a force for disintegration in the third century. On the contrary, having to travel around much more often than in peaceful times, they saw many war zones and parts of the empire, and seem to have had empire-wide careers. Besides, the Roman armed forces came from many parts of the empire and the most important field armies counted detachments from many of those parts, which had to work together through years on end. These factors normally compensated for the material regional interests any troops might have, but not so in A.D. 260-274. In those years serious military situations at practically all borders forced the emperors to accept a continuation of the Imperium Galliarum in the West, and of Palmyrene power in the East. This was survival strategy, more than the outcome of a process of military regionalization and a growing regional particularism among the armed forces. Gallienus' field army comprised detachments from almost all borders. ${ }^{29}$

${ }^{28}$ See C. Bertrand-Dagenbach, Alexandre Sévère et l'Histoire Auguste (Brussels 1990), 189-193.

29 See Christol $2006^{2}$, op. cit. (n. 7), 147-148. 


\section{DIFFERENTIATED INTEGRATION TRAJECTORIES \\ OF THE NOMADIC POPULATION IN ROMAN NORTH AFRICA \\ $\left(1^{\text {ST }}-3^{\text {RD }}\right.$ CENT. A.D. $)$}

\section{Wouter Vanacker}

Along and within the imperial boundaries in North Africa Rome encountered numerous (semi-)nomadic tribes and confederations. The aim of this paper is to give a short overview and evaluation of the different perspectives of modern research on the relations between (semi-)nomads and sedentary communities in North Africa during the Roman period. It is argued that the rise of the symbiosis perspective has offered a valuable counterweight against the traditionalistic view of opposition and antagonism. However, scholars should be aware of the fact that the various sources point to differentiated integration trajectories, both negative and positive. More attention should be paid to the various motives of Roman and indigenous actors which moulded and shaped these integration trajectories, and to the specific contexts in which they appear.

\section{The Nomad Kept Out:}

\section{The Traditional Perspective of Antagonism and Exclusion}

It has long been a truism in studies on relations between sedentary communities and (semi-)nomads in North Africa that Rome had to deal with endemic nomadic insubordination and disinclination to integration. Scholars perceived "ces terribles Maures" as a permanent threat to stability and order. ${ }^{1}$ Such viewpoints were subsequently reinforced by studies

\footnotetext{
${ }^{1}$ R. Cagnat, L'Armée romaine d'Afrique et l'occupation militaire de l'Afrique sous les empereurs (Paris 1912), 98. L. Harmand, L'Occident romain: Gaule - Espagne - Bretagne Afrique du Nord (31 av. J.C. à 235 ap. J.C.) (Paris 1960), 279 (quote); J. Burian, 'Zur Geschichte der nordafrikanischen einheimischen Bevölkerung in den ersten zwei Jahrhunderten u. Z.', Studii clasice 3 (1961), 171; M. Rachet, Rome et les Berbères. Un problème militaire d'Auguste à Dioclétien (Bruxelles 1970); M. Benabou, La résistance africaine à la romanisation (Paris 1976); T. Kotula, 'Les Africains et la domination de Rome', Dialogues d'histoire ancienne 2 (1976), 345. R.I. Lawless, 'Romanization and Berber resistance in Mauretania Caesariensis (western Algeria)', in M. Galley (ed.), Second International Congress of Studies on Cultures of the Western Mediterranean (1978) II (Alger 1978), 166.
} 
emphasizing the complete lack of social, economic and cultural transformation of indigenous societies. ${ }^{2}$ These opinions were inspired both by contemporary colonialist experiences and by new questions and insights put forward in early post-colonial historiography. Certainly, they resulted in part from the extensive focus on literary sources, which tended to mention nomadic groups almost exclusively in the context of conquest, insurgence and resistance. Ancient authors did not hide their negative perception on nomadism in general and displayed poor insights in the social reality of nomadism in the North African interior. ${ }^{3}$

Modern scholars have subsequently constructed extensive overviews of revolts, wars, military expeditions and punitive campaigns, attested by literary, epigraphic and archaeological sources. These overviews incorporated a lot of attestations - albeit indirect - like temporary increases of the number of auxilia in the Mauretaniae or the construction of city walls. Furthermore, it has been understood that the diplomatic negotiations between Roman governors and indigenous tribal leaders of the Baquates at Volubilis suggest periods of chaos and disorder. ${ }^{4}$ Similarly, the view has been held that further conflict indicators can be seen when considering the establishment of special offices. In Mauretania, the procuratores pro

${ }^{2}$ For instance: Benabou 1976, op. cit. (n. 1); D. Cherry, Frontier and Society in Roman North Africa (Oxford 1998).

3 On the Greek and Roman perception of nomadism, cf. P. Trousset, 'Villes, campagnes et nomadisme dans l'Afrique du Nord antique : représentations et réalités', in P.-A. Février, $\mathrm{Ph}$. Leveau (eds.), Villes et campagnes dans l'empire romain. Actes du Colloque organisé par l'U.E.R. d'Histoire (Aix-en-Provence 1980) (Marseille 1982), 198-201; B.D. Shaw, 'Fear and loathing: the nomad menace and Roman Africa', in C.M. Wells (ed.), Roman Africa (Ottawa 1980) (Ottawa 1982), 29-31; B.D. Shaw, "Eaters of flesh, drinkers of milk": the ancient mediterranean ideology of the pastoral nomad', Ancient Society 13/14 (1982-1983), 5-31; G. Devallet, 'Vagi, palantes Afri: quelques réflexions sur l'image romaine du nomade africain', in F. Bejaoui, Histoire des Hautes Steppes. Antiquité - Moyen Âge (Sbeitla 2001) (Tunis 2003), 31-38; M. Sartre, 'Les nomades dans l'Empire romain', in C. Moatti, W. Kaiser, Chr. Pébarthe (eds.), Le monde de l'itinérance en Méditerranée de l'antiquité à l'époque moderne. Procédures de contrôle et d'identification (Bordeaux 2009), 58-60. Nomadic society was perceived as "l'antithèse de la civilisation" according to Y.A. Dauge, Le barbare: recherches sur la conception romaine de la barbarie et de la civilisation (Bruxelles 1981), 620-626. It was situated at "le degré zéro de l'humanité", cf. P. Trousset, "L'image du nomade saharien dans l'historiographie', Production pastorale et société 10 (1982), 101-102.

4 On the rise of troop numbers, for instance during the reign of Trajanus and Antoninus Pius cf. Rachet 1970, op. cit. (n. 1), 185 and 196-200; Benabou 1976, op. cit. (n. 1), 126 and 138-139. On the construction of the city walls of Volubilis Rachet 1970, op. cit. (n. 1), 203; Benabou 1976, op. cit. (n. 1) 147; M.C. Sigman, 'The Romans and the indigenous tribes of Mauretania Tingitana', Historia 26.4 (1977), 431; of Banasa: Sigman 1977, op. cit. (n. 4), 435. On this particular interpretation of the negotiations, cf. Rachet 1970, op. cit. (n. 1), 203; Sigman 1977, op. cit. (n. 4), 429-434. 
legato have been seen as attestations of military confrontations, since they seem to attest the assignation of a special command of legionary units assigned to these procurators. It has been related that also other offices such as a dux exercitus Africae et Mauretaniae (ca. AD 85) and an equestrian subprocurator Mauretaniae Tingitanae (ca. AD 104) also point out the incidence of more serious conflicts in Mauretania. ${ }^{5}$

Generalization and bold overestimations are typical features of publications defending a maximalist reconstruction of insurgence. When the rebels are generally called Mauri in the Historia Augusta it is of course unclear and in fact highly improbable that the insurrection comprised all Moorish tribes and confederations. ${ }^{6}$ Yet this general denomination has often been adopted easily in modern accounts, although the different geographical and chronological contexts of the wars and revolts elucidate the various identities of Rome's opponents. The supposed link between different conflicts is less apparent when the sources receive a closer look. For instance, the connection of Ser. Sulpicius Galba's special mission in Africa (cf. infra) with the Revolt of Aedemon, as postulated by Rachet, seems highly doubtful. ${ }^{7}$ This connection has also been subtly suggested by Benabou's synchronization of both conflicts. ${ }^{8}$ The latter's hypothesis that both revolts of $\mathrm{AD} 118$ and $\mathrm{AD} 122$ should be perceived as one great rebellion assorts to the same category of strategies by which the image of endemic and wide-spread indigenous resistance has been constructed and reinforced without clear-cut hard evidence. ${ }^{9}$ There is no indication whatsoever to argue that every conflict should be perceived as a Roman offensive campaign or an indigenous reaction against Roman oppression.

5 Dux: AE 1903, 368: duci exercitus Africi et/Mauretanici ad nationes quae/sunt in Mauretania comprimendas (1. 8-10). Benabou 1976, op. cit. (n. 1), 110. Subprocurator, cf. CIL III, 14195, 4-9 = $A E$ 1899, 64 .

6 For example, HA, Aur. 21.1 and Com. 13.5.

7 Rachet 1970, op. cit. (n. 1), 129. Similarly: S.L. Dyson, 'Native revolt patterns in the Roman empire', Aufstieg und Niedergang der Römischen Welt II 3 (1975), 165-166.

8 Benabou 1976, op. cit. (n. 1), 93 .

9 As I have argued elsewhere, the first tumultus (HA, Hadr. 5.8) should be connected with the execution of Lusius Quietus and a possibly related uprising of the Baquates: W. Vanacker, 'Aedemon, Lusius Quietus and the Baquates. Ties of resistance and cooperation', Mnemosyne (2013). The second conflict is denoted as "motus Maurorum" in the Historia Augusta (Hadr. 12.7) and was supressed by AD 122. It is probably connected in some way with the military constructions at Igilgili (around AD 128, to the detriment of certain land rights of the Zimises, cf. infra) or more plausibly at Rapidum (CIL VIII, 20833). Laporte has provided an extensive study of the latter camp J.-P. Laporte, Rapidum. Le camp de la cohorte des Sardes en Maurétanie Césarienne (Sassari 1989). 
In many cases, by its nature, the evidence simply does not allow for a precise identification of the conflict's initiators. ${ }^{10}$

Traditional scholarship pointed to the economic antagonism between nomads and sedentary groups as a key primal factor behind the many wars and revolts that continuously marked Roman North Africa. The pastoral nomadic economy has often been perceived as inherently hostile to the agricultural organization of sedentary communities. The belief has been held that groups of pastoral nomads and sedentary agricultural communities were involved in an on-going battle for the sparse fertile lands and that seasonal migrations were fundamental obstacles to the development and growth of the agricultural export economy. Scholars have therefore thought that the termination of pastoral movements was a primordial goal of Roman military strategy, and that this intention repeatedly resulted in rebellion, since "il seminomadismo tribal (...) si trovava oramai inserito in un contesto geopolitico dove la sedentarizzazione, e consequemente la privatizzazione dei suoli, conoscevano un sensibile incremento". ${ }^{11}$ The Revolt of Tacfarinas (ca. AD 15-24) was considered to be a typical example, as the construction of the road between Ammaedara and Tacape seemed to have been the crucial event that sparked the conflict. ${ }^{12}$ It was believed that frontier infrastructure such as walls and ditches were essential tools to keep the nomads out of the civilized sedentary world..$^{13}$ Scholars also related that the nomadic segment of the indigenous population was repulsed to infertile territory or insurmountable and unattainable mountain reservations, for instance to the Aurès massif. ${ }^{14}$ Furthermore,

10 This is clearly so in the case of the special office of Sex. Sentius Caecilianus (cf. infra) and C. Velius Rufus (cf. supra), as well as for the many direct and indirect traces dating to the reign of Trajan and Antoninus Pius, or the fate of L. Flaminius ab hostem in pugna / occisus (1. 7-8) on the Saltus Philomusianus (CIL VIII, 14603).

11 E. Migliario, 'Gentes foederatae. Per una riconsiderazione dei rapporti RomanoBerberi in Mauretania Tingitana', Rendiconti della Classe di scienze morali, storiche e filologiche dell'Accademia dei Lincei 10.3 (1999), 442.

12 T.R.S. Broughton, The Romanization of Africa proconsularis (Baltimore, London 1929), 89-9o; J.-M. Lassère, 'Un conflit "routier": observations sur les causes de la guerre de Tacfarinas', Antiquités africaines 18 (1982), 11-25.

13 L. Leschi, 'Recherches aériennes sur le "limes" romain de Numidie', Comptes Rendus de l'Académie des Inscriptions et Belles-Lettres (1937), 256-262. J. Guey, 'Note sur le limes romain de Numidie et le Sahara au IV siècle', Mélanges d'Archéologie et d'Histoire de l'École Française de Rome 56 (1939), 178-248. J. Baradez, Fossatum Africae. Recherches aériennes sur l'organisation des confins sahariens à l'époque romaine (Paris 1949).

14 Broughton 1929, op. cit. (n. 12), 98-99 and 121-123; T. Frank et al., An economic survey of ancient Rome IV. Roman Africa, Roman Syria, Roman Greece, Roman Asia, (Baltimore 1938), 35; R. Syme, 'Tacfarinas, the Musulamii and Thubursicu', in P.R. Coleman-Norton, F.C. Bourne, J.V.A. Fine (eds.), Studies in Roman Economic and Social History in Honor of Allan 
they believed that Rome pursued an active sedentarization policy, of which centuriatio was an essential tool. ${ }^{15}$

The assessment of the socio-cultural integration of nomadic actors tends to be somewhat ambivalent. Socio-cultural integration implies the abandonment of a life style, of values that distinguish the nomad from the sedentarist. Due to the dearth of sources attesting the socio-cultural transformation of tribal societies, traditional scholarship concluded that social structures and the culture of nomads were hardly affected. Whittaker has argued that in large parts of North Africa Roman occupation changed little to the traditional social, economic and cultural fundaments of the tribe, particularly when considering this at the local clan level. ${ }^{16}$ A few decades earlier, Camps stated that the third century inscriptions attesting the so-called Dii Maurii are indications of the lack of cultural integration in many parts of North Africa. In his view, these tituli were set up by Roman officials or Roman-sided actors to rally support from the indigenous divinities against native rebels. Camps further postulated that the lack of concordance with Roman divinities suggests that these gods were "des dieux et des génies indigènes, des divinités aux caractères fuyants qui n'ont pas trouvé place dans le panthéon romain". ${ }^{17}$ Scholars have connected Roman sedentarization policy with berber insurgence, which in turn has been referred to as a negative illustration of the value of social and cultural continuity for the development of acculturation processes amongst the indigenous society and the durability of Roman dominance. ${ }^{18}$ And while the Tabula Banasitana clearly attests the attribution of Roman

Chester Johnson (Princeton 1951), 124; Burian (1961), op. cit. (n. 1), 168-169; Kotula 1976, op. cit. (n. 1), 345-346; Benabou 1976, op. cit. (n. 1), 431-432. M. Dondin-Payre, 'Recherches sur un aspect de la romanisation de l'Afrique du Nord : l'expansion de la citoyenneté romaine jusqu'à Hadrien', Antiquités africaines 17 (1981), 110.

15 R. Chevallier, 'La centuriation et les problèmes de la colonisation romaine', Etudes rurales 3 (1961), 73 .

16 C.R. Whittaker, 'Land and Labour in North Africa', Klio, 60 (1978), 331-362, at 344-350.

17 G. Camps, 'L'inscription de Béja et le problème des Dii Mauri', Revue africaine 98 (1954), 255. Later it has been argued that these dedications should be linked to the enrolment of Moorish auxiliaries in the Roman army. They may also allude to deliberate efforts of (political and religious) conciliation. Benabou 1976, op. cit. (n. 1), 309-330; P.-A. Février, 'Religion et domination dans l'Afrique romaine', Dialogues d'histoire ancienne 2 (1976), 305-336; E. Fentress, 'Dii Mauri and Dii Patrii', Latomus 37 (1978), 507-516. In reaction to these authors, Camps sustained his main argument in a more recent paper: G. Camps, 'Qui sont les Dii Mauri?', Antiquités africaines 26 (1990), 131-153.

18 P. Le Roux, 'Intervention de P. Le Roux sur l'exposé de Ph. Leveau', in Février, Leveau 1982, op. cit. (n. 3), 92 . 
citizenship to tribal leaders of the Zegrenses, one can still doubt whether the attractions of citizenship could really convince other members of the Zegrenses to affiliate themselves more intensively with the Roman Empire and its cultural traits. The 'colloquium inscriptions' informing us about the aforementioned diplomatic negotiations between Roman procurators and the Baquates indicate how slowly - or rather, how unsuccessfully Roman citizenship took root among the indigenous elites of nomadic tribes. The evidence concerning the Baquates and the Zegrenses seems to suggest that the acquirement of citizenship did not result into nor follow from intense participation in urban communal life. ${ }^{19}$

\section{The Nomad Returns: Symbiosis and Negotiated Integration}

Scholars have reacted against the traditionalistic view of Roman Africa as "un nœud de problèmes complexes aux solutions douteuses et transitoires" by looking for traces of positive relations between nomads and sedentary communities. ${ }^{20} \mathrm{~A}$ close examination of the literary, epigraphic and archaeological sources reveals that the image of endemic resistance against Roman oppression and forced settlement should be nuanced and instead make place for "una visione più sfumata". ${ }^{21}$

Of major importance is the abandonment of the traditionalistic view that the sedentary and nomadic economy are mutually exclusive. Anthropological studies have shown that they are complementary, especially because of the non-autarchic character of nomadic economies. In the view of Khazanov it is the non-autarchic economy, which necessitates that the nomads gain access to and acquire vital commodities from sedentary communities, most frequently through trade or raiding. ${ }^{22}$ Hence,

19 For the 'colloquium inscriptions' cf. CIL VIII, $21826=A E$ 1894, $45=A E$ 1941, 115. $A E$ 1953, 78 = AE 1957, 202. $A E$ 1953, $79=A E$ 1957, 203. $A E$ 1957, 204. $A E$ 1953, 80. $A E$ 1987, 1092. $A E$ 1942/43, $54=A E$ 1946, $5^{2}=A E$ 1946, $275=A E$ 1987, 1093. $A E$ 1952, $42=A E$ 1953, $77=$ $A E$ 1957, $201=A E$ 1987, 1090a $=A E$ 1987, 1094. $A E$ 1952, 43. $A E$ 1954, 110. $A E$ 1920, 44. $A E$ 1921, 23. As Sigman related with respect to the Zegrenses: "this tribe remained remarkably unacculturated. Although they were subject to Roman taxes, and the Juliani were Romanized, the greater part of the tribe seems to have been resistant to Romanization." Sigman 1977, op. cit. (4), 437. For the Tabula, cf. $A E$ 1971, 534.

20 Benabou 1976, op. cit. (n. 1), 250.

21 C. Melani, 'Roma e le tribu della Mauretania Cesariense', Athenaeum 82.1 (1994), 153. A notable work that subscribes to this tendency: A. Gutsfeld, Römische Herrschaft und einheimischer Widerstand in Nordafrika, (Stuttgart, 1989).

22 A.M. Khazanov, Nomads and the Outside World (Madison, 1994), xxxi-xxxii; 70; 202227. This so-called trade or raid paradigm has been tested by other scholars studying the 
imposing or providing regulated access to the market economy could have engendered a two-fold advantage for Rome: in one sense, essential commodities could be obtained by the nomadic actors through trade, thus lessening the chance of raiding. In another sense, such commercial transactions could have provided significant tax revenue. In the light of these perspectives the tax inscription of Zarai of $\mathrm{AD} 202$ becomes all the more interesting. ${ }^{23}$ The tariff details leathers, hides, clothing and riding equipment as well as agricultural products of oasis economies. The tariff not only highlights the value and overall importance associated with the exchange of pastoral goods, but also emphasizes a number of noteworthy tax exemptions. Aside from the regular beasts of burden pequaria were immunia (l. 22), as well as cattle destined for the local market (1. 11: in nundinium immunia). ${ }^{24}$ One should not easily concur with Morizot's suggestion to combine at l. 21-22 maxima pequaria immunia ("very big flocks are exempt from taxes"). ${ }^{25}$ It is important to recognize that such exemptions could ultimately highlight the efforts made by the Romans to devise and adopt a tax policy that was lenient enough for nomads to migrate into the province peacefully and to participate in the market economy. ${ }^{26}$ A similar policy can be observed in Germania, where the Romans seem to

political tensions between the Xiongnu Empire and China, cf. N. Di Cosmo, 'Ancient Inner Asian nomads: their economic basis and its significance in Chinese history', The Journal of Asian Studies 53.4 (1994), 1092-1126.

${ }^{23}$ CIL VIII, $4508=18643$.

24 On the local market as the cattle's destination, cf. P. Trousset, 'Le tarif de Zaraï: essai sur les circuits commerciaux dans la zone présaharienne', Antiquités africaines $38-39$ (2002-2003), 362.

25 P. Morizot, 'Les échanges commerciaux entre la côte méditerranéenne et à l'intérieur du Maghreb au II siècle vus au travers du Tarif Zarai', in J.-R. Gaborit (ed.), Circulation des matières premières en Méditerranée, transferts de savoirs et de techniques. 128e Congrès National des Sociétés Historiques et Scientifiques (Bastia 2003) (Paris 2009), 162.

26 Shaw 1980, op. cit. (n. 3), 47. According to Johannesen, it is unclear whether the commodities were entering the province, or going out. R. Johannesen, "The textile industry in Roman North Africa', The Classical Journal 49,4 (1954), 158. Darmon posed that products were traded in both ways across the frontier and along a southwest-northeast axis. J.-P. Darmon, 'Note sur le Tarif de Zaraï', Cahiers de Tunisie: revue des sciences humaines 47-48 (1964), $19 \mathrm{ff}$. Trousset has argued that the garum mentioned in the tax inscription was produced along the Syrtes: P. Trousset, 'Le franchissement des chotts du sud tunisien dans l'antiquité', Antiquités africaines 18 (1982), 58. Later he posed that the tax records two axes of trade and migration (north-south and east-west): Trousset 2002-2003, op. cit. (n. 24), 368-369. However, Morizot has related that an east-west axis is rather improbable, as trade along the north-south axis could bring along the same products more easily and more quickly: Morizot 2009, op. cit. (n. 25), 160-163. Interestingly, Fentress perceived this tariff as evidence on the progressive development of specialized nomadic pastoralism: E.W.B. Fentress, 'Romanizing the Berbers', Past \& Present 190 (2006), 29-30. 
have made concessions to local economic structures and conditions when levying taxes in kind (ox-hides) among the Frisians. ${ }^{27}$ In this context of Roman engagement in motivating and inducing material exchange within the market (and controlling the security risks connected to this point of assembly) it is useful to recall Janon's assumption that colonists were settled and military camps constructed exactly on locations of contact where seasonal markets existed..$^{28}$ It is possible that the epigraphically attested protection of an annual fair by both Roman and indigenous gods at Bordj Bou Arreridj illustrates and emphasizes the continuous character of commercial exchange. ${ }^{29}$

It has now been acknowledged that the limes in North Africa did not always form a closed barrier, either between the Empire and the unconquered lands, or between two different socio-economic organizations. ${ }^{30}$ As Janon put it: "le fossatum est un signe, il concrétise le passage de l'extérieur à l'intérieur d'un monde que Rome prétend contrôler; cela ne veut pas dire qu'il l'interdise". ${ }^{31}$ By its nature the fossatum does not seem to have been able to hold off an army, even of moderate size. ${ }^{32}$ Particularly troublesome to the traditional archaeologists are the large gaps in these lines, which are difficult to rationalize in a military context. Shaw

27 Tac. Ann. 4.72. J.H.F. Bloemers, 'Relations between Romans and natives: concepts of comparative studies', in V.A. Maxfield \& J.M. Dobson (eds.), XVth International Congress of Roman Frontier Studies (1989) (Exeter 1991), 414. Market control in frontier regions was also influenced by strategic concerns (cf. the reasoning behind barring the Marcomanni from market access during the reign of Marcus Aurelius: Dio Cass. 72.11.3).

28 M. Janon, 'Remarques sur la frontière de Numidie', in Maxfield, Dobson 1991, op. cit. (n. 27), 483. Le Roux noted that in other parts of the Empire the Romans took care to control traditional centres of temporary political, religious as well as commercial/economic importance and to assure their continuity. Le Roux 1982, op. cit. (n. 18), 91-92.

$29 C I L$ VIII, $20627=A E$ 1894, $96=A E$ 1909, $123=A E$ 2002, 1694. Whittaker observed that "larger, regional markets are usually located on the borders of complementary production zones": Whittaker 1978, op. cit. (n. 16), 347. For an in-depth study of various aspects of North African periodic markets (and Roman control of them), cf. B.D. Shaw, 'Rural markets in North Africa and the political economy of the Roman Empire', Antiquités africaines 17 (1981), 37-83.

30 M. Euzennat, 'Recherches récentes sur la frontière d'Afrique (1964-1974), II', in D. Haupt \& H.G. Horn (eds), Studien zu den Militärgrenzen Roms II: Vorträge des 1o. Internationalen Limeskongresses in der Germania Inferior (Xanten 1974) (Bonn 1977), 441. Mattingly has observed that the defence lines did not always converge with the official frontier (although they sometimes did!), though he pointed out that many (thus not all of them!) were located near the limits of the agricultural zone. D.J. Mattingly, Tripolitania (London 1995), 107 and 113 .

31 M. Janon, 'Lambèse et l'occupation militaire de la Numidie méridionale', in Haupt, Horn 1977, op. cit. (n. 30), 477.

32 Cf. the observations of Trousset: P. Trousset, Recherches sur le limes tripolitanus (Paris 1974), 139-141. 
nevertheless proposed an alternative military explanation. In regard to the clausurae, a defence system of walls and ditches often guarded by watchtowers and forts, he posed that they were intended to protect "uniquely exploitable bands or 'belts' of land running along the bases of the Saharan mountain massifs". ${ }^{33}$ The validity of his explanation can be questioned, for instance in the case of the clausurae which clearly had to facilitate the control or restriction of movements towards the lower Oued Zraia, Oued Skiffa and Oued Genini in the sector of Ras el Aïn/Talalati. ${ }^{34}$ Furthermore, an economic rationale has been perceived behind these clausurae. Rather than to obstruct them, the Romans seem to have sought to direct and channel nomadic movements through check-points where the incoming goods could be taxed efficiently. ${ }^{35}$ In this same vein, Janon pointed to a comparable economic role adopted by the army when taking into account the military occupation of the Aurès. ${ }^{36}$ Nevertheless, the view is still defended that the clausurae (also) served as "a labour-saving device in the policing of the region", aimed at preserving symbiotic relationships between arable farmers and nomads. ${ }^{37}$

Yet the idea of opposition between mountain and plain has undoubtedly experienced a significant blow by studies carried out in the Aurès mountains highlighting that agriculture in this region not only pre-dated the Punic era but was ultimately boosted by Roman military presence within and around the massif. ${ }^{38}$ The more traditional view maintains that

33 Shaw 1980, op. cit. (n. 3), 46.

34 On these clausurae, cf.: Trousset 1974, op. cit. (n. 32), 97, 102, 139-140; D.J. Mattingly \& G.D.B. Jones, 'A new clausura in western Tripolitania: Wadi Skiffa South', Libyan Studies 17 (1986), 87-96. Trousset discovered similar clausurae in the sector of Remada/Tillibari: P. Trousset, 'Nouvelles barrières romaines de contrôle dans l'extrême sud tunisien', Bulletin archéologique du Comité des travaux historiques et scientifiques. Série B, Afrique du Nord 24 (1997), 155-163. The clausurae of the region have been discussed at great length in Mattingly 1995, op. cit. (n. 30), 106-115.

35 E.W.B. Fentress, Numidia and the Roman Army: Social, Military and Economic Aspects of the Frontier Zone, BAR. International series (Oxford 1979), 112-113; Mattingly \& Jones 1986, op. cit. (n. 34), 94; C.M. Wells, 'The problems of desert frontiers: chairman's comments on the session', in Maxfield \& Dobson 1991, op. cit. (n. 27), 479; Cherry 1998, op. cit. (n. 2), 62; S. Matz, 'Befestigung im Nirgendwo', Antike Welt 38,1 (2007), 55-59. Matz did not seem to take a clear position in the debate (cf. the construction of a strong defence to ward off the threat of nomadic invasions mentioned on p. 55 vs. a weak border defence with tax and canalization functions p. 59).

36 M. Janon, 'Paysans et soldats', in C.M. Wells (ed.), Roman Africa (Ottawa 1980) (Ottawa 1982), 58; 61f.

37 Mattingly 1995, op. cit. (n. 30), 113-114.

38 Cf. Janon: "Il n'y a donc pas de raisons de voir dans l'Aurès antique une region déshéritée" in Janon 1977, op. cit. (n. 31), 477. And elsewhere: Janon 1982, op. cit. (n. 36), 56-58; Janon 1991, op. cit. (n. 28), 482-483. Formerly, Camps has recognized a substantial 
it was due to the Roman import of irrigation techniques that agriculture took root in the African soils to the south. However, the overriding indigenous character of these techniques has since been recognized. ${ }^{39}$ In his study on land use and agricultural settlement in Mauretania Caesariensis Leveau has discarded the antagonism between mountain and plain. As he stated elsewhere: "dans les campagnes de l'Empire romain, voisinent des formes d'organisation de l'espace rural parfaitement hétérogènes qui n'obéissent à aucun déterminisme physique ou climatique". ${ }^{40}$

A kind of inclusive policy has been noted in respect of the containment strategy, the supposed policy to confine (semi-)nomadic communities to infertile territories or reservations. Contrary to what the adherents of the antagonistic these would believe, Fentress and Shaw have stated that the semi-nomadic Musulamii were definitely not confined to unproductive reservations during the land measurements and allocations in the reign of Trajan. ${ }^{41}$ Nevertheless, it should be admitted that in other cases the Roman advance in the interior clearly engendered the loss in land rights to the indigenous tribes. ${ }^{42}$ But two tituli commemorating land assignations to the gens Numidarum by Hadrian illustrate the attitude of Roman land seizure, which was clearly negotiated and at times compassionate. It should be emphasized, though, that these inscriptions do not mention that the initiative to create them came from the indigenous actors themselves: Ex indulgen/tia Imp(eratoris) Caes(aris) Had/riani Aug(usti) fines / adsignati gen/ti Numidarum / per C(aium) Petroni/um Celerem /

pre-Roman agricultural development in both (!) plains and mountainous areas of North Africa, based on texts (esp. Herodote) and archaeological research: G. Camps 'Massinissa ou les débuts de l'histoire', Libyca 8 (1960), 17-19 and 69-91 (esp. 72-75 on irrigation techniques).

39 B.D. Shaw, 'Lamasba: an ancient irrigation community', Antiquités africaines 18 (1982), 93; D.J. Mattingly, 'New perspectives on the agricultural development of Gebel and pre-desert in Roman Tripolitania', Revue des mondes musulmans et de la Méditerranée 41.1 (1986), 53 .

40 P. Leveau, 'Paysans maures et villes romaines en Maurétanie Césarienne centrale. La résistance des populations indigènes à la romanisation dans l'arrière-pays de Caesarea de Maurétanie', Mélanges de l'École Française de Rome. Antiquité 87.2 (1975), 857-871. Quote: P. Leveau, 'Une ville et ses campagnes : l'exemple de Caesarea de Maurétanie', in Février, Leveau 1982, op. cit. (n. 3), 183 .

41 The land measurements and allocations activities conducted during the reign of Trajan have left many traces in the epigraphic record: $C I L$ VIII, 22787 $=A E$ 1910, 20. $C I L$ VIII, 22788. $C I L$ VIII, $10667=16692$. $C I L$ VIII, $4676=28073 a=A E$ 1898, 39a. $A E$ 1907, 19-21. $A E$ 1923, 26. $C I L$ VIII, $28703 \mathrm{~b}=A E$ 1898, 39b. Fentress 1979, op. cit. (n. 35), 76; Shaw 1980, op. cit. (n. 3 ), 42.

42 For instance the case of the Zimises in CIL VIII, 8369. 
proc(uratorem) Aug(usti) pr[o(uinciae)] / [M(auretaniae) C]aes[ariensis] (CIL VIII, 8814; also see CIL VIII, 8813).

A final argument in favour of the inclusion of nomads within the margins of the sedentary economy is their potential as an utile labour force during harvest. A letter of Augustine can be perceived as a rare attestation of the employment of nomadic workers in agricultural contexts. ${ }^{43}$ The "falcifera turma" mentioned in the inscription of the harvester of Mactar (CIL VIII, 11824, at l. 13) can also allude to a workforce of nomadic origin. According to Whittaker, the double line of defence in several parts of Roman North Africa should be connected with the organization of labour migration. 44

These observations made scholars reconsider the evidence on the military conflicts, and "the long-held notion that indigenous resistance to Roman occupation was ferocious and unremitting". ${ }^{45}$ Especially the indirect attestations have received much attention. The increase of troop numbers is mostly difficult to date. The construction of city walls, it was pointed out, did not necessarily mean a real military threat but should be linked with the need felt to express urban prestige. ${ }^{46}$ Special offices have also been stripped from their military meaning. Defenders of the symbiosis perspective have dismissed the idea that the procuratores pro legato were provided with a legionary command. Frézouls presented a number of inventive but hardly seductive alternative explanations. ${ }^{47}$ Likewise, Spaul argued that the purpose of assigning a procuratorship pro legato was merely to distinguish the regular governmental procurator from a financial or administrative procurator. In my opinion, this view is

43 Aug., Epist. 46.1-6 and 47.1-2. Shaw 1980, op. cit. (n. 3), 47-48.

44 Whittaker 1978, op. cit. (n. 16), 349-350.

45 Cherry 1998, op. cit. (n. 2), 40.

46 R. Rebuffat, 'Enceintes urbaines et insécurité en Maurétanie Tingitane', Mélanges de l'Ecole Française de Rome 86.1 (1974), 513-514; E. Frézouls, 'La résistance armée en Maurétanie de l'annexion à l'époque sévérienne. Un essai d'appréciation', Cahiers de Tunisie: revue des sciences humaines 29 (1981), 55-56. Contra: M. Euzennat, 'Les troubles de Maurétanie', Comptes Rendus de l'Académie des Inscriptions et Belles-Lettres 128.2 (1984), $382-383$.

47 These included the emperor's deliberate augmentation of a favourite's prestige, the lack of equestrians of suitable rank, the increase of the procurator's authority vis-à-vis the indigenous population on both sides of the frontier in order to facilitate administrative measures or road constructions, and even "la volonté (...) de donner plus de réalité à la liaison entre les deux Maurétanies.” Frézouls 1981, op. cit. (n. 46), 51-52. This stands in contrast to the straight adherence to the traditional interpretation he expressed formerly in E. Frézouls, 'Les Baquates et la province romaine de Tingitane', Bulletin d'archéologie marocaine 2 (1957), 109. 
untenable as the office of procurator pro legato appears too irregularly in the Mauretaniae (and beyond). The argument that pro legato implies the extraordinary acquisition of imperium, military power which regular governmental procuratores did not have, is strengthened by the synchronic attestations of a procurator pro legato and military expeditions lead by equestrian officers in Tingitana in the early years of the reign of Claudius. ${ }^{48}$ Similar efforts have been made to question the traditional interpretation of certain special offices as indicators of warfare. For instance, while the most zealous defendants of the antagonistic perspective interpreted Sex. Sentius Caecilianus' assignment as legatus Augusti pro praetore ordinandae utriusque Mauretaniae around $\mathrm{AD} 75$ as an attestation of conflict, it has also been put forward that the office was not a military assignment but comprised administrative activities. ${ }^{49}$ A counterargument may be formed by the literary attestation of a proconsul extra sortem. Around AD 45 this office was given to Ser. Sulpicius Galba ad ordinandam prouinciam et intestina dissensione et barbarorum tumultu inquietam. At least in the latter case, ordinare clearly implicates a military task. ${ }^{50}$ The traditional view is obviously confirmed by precedents, namely by the legati sent to Mauretania to establish direct Roman rule about thirty years before the assignment of Sex. Sentius Caecilianus. ${ }^{51}$

Positive political integration trajectories have been constructed and have received much attention. Political relations in Mauretania do not seem to have been uniformly unstable and antagonistic. In Caesariensis, imperial loyalty becomes apparent in the case of the Maccues, who subscribed to typical negotiation systems of patronage. ${ }^{52}$ Near the empire's

48 J. Spaul, 'The Roman "frontier" in Morocco', Bulletin of the Institute of Archaeology of the University of London 30 (1994), 113. Cf. Plin., nat. 5.11 and $A E$ 1924, 66.

$49 A E$ 1941, 79 (1. 12-13). Rachet 1970, op. cit. (n. 1), 149-153; Benabou 1976, op. cit. (n. 1), 103-104, versus: P. Romanelli, Storia delle province romane dell'Africa (Roma 1959), 296; T. Kotula, 'Culte provincial et romanisation. Le cas des deux Maurétanies', Eos 63 (1975), 403-04; Frézouls 1981, op. cit. (n. 46), 58; Euzennat 1984, op. cit. (n. 46), 375. According to Migliario, the office comprised both administrative and military responsabilities: E. Migliario, 'Gentes foederatae. Per una riconsiderazione dei rapporti Romano-Berberi in Mauretania Tingitana', Rendiconti della Classe di scienze morali, storiche e filologiche dell'Accademia dei Lincei 10,3 (1999), 442. Similarly: A. Marcone, 'Nota sulla sedentarizzazione forzata delle tribù nomadi in Africa alla luce di alcune iscrizioni', in A. Mastino (ed.), L'Africa romana. Atti del IX convegno di studio (Nuoro 1991), 107.

50 Suet., Galba 7.1.

51 M. Licinius Crassus Frugi in AD 40-41 (CIL VI, $31721=A E$ 1973, 16); and Hosidius Geta and Suetonius Paulinus in AD 42 (Dio Cass. 6o.9.1-5).

$52 A E$ 1904, 150. A person of lesser status has been remembered as "defensor gentis" in Numidia, which also points to the possibility to negotiate Roman policy (CIL VIII, 8270). A similar "defensor" named Flavius Geminus appears in CIL VIII, $25902=A E$ 1897, $48=$ 
mountainous periphery in southwest Tingitana, the abovementioned 'colloquium inscriptions' of Volubilis are now understood as commemorations of the reaffirmation of peace between Rome and the Baquates when a new king came into power. ${ }^{53}$ In my view, these formal recognitions of local leadership no doubt weakened local resistance against Roman dominance. At the same time, these colloquia actually show that typical features of nomadic political structures were unaffected by external influences like the absence of a real dynastic sequence and the easy come-and-go of confederate structures. Instead, they point to Roman acknowledgement of their fluidity. ${ }^{54}$ The Tabula Banasitana gives an idea of the deliberate attribution of Roman citizenship to cooperative leaders of the Zegrenses. Such diplomatic efforts unquestionably succeeded in enfeebling tensions by providing formal recognition of local leaders. This approach lessened the importance of a dense military occupation. These observations correspond to Melani's observation that the absence of a large army in North Africa may indicate that the Romans had to deal merely with "conflitti di lieve entità e relativamente ristretto pericolo". 55 The low intensity of the conflicts may have been the outcome of Roman political attempts to tie local kings and kinglets to its cause and to prevent the establishment of large confederations.

In contrast to a number of supporters of the antagonistic train of thought, champions of the symbiosis perspective have argued that the advent of Roman domination did cause socio-cultural changes to indigenous (semi-)nomadic societies. In these studies, the 'survivances' are not longer explained as deliberate acts of traditional "résistance culturelle". Rather, they have been perceived as essential features of the mixed culture, which developed in the absence of a deliberate Romanization policy. ${ }^{56}$ It has been related that the increasing influence of the market and Roman

\footnotetext{
$151=1998,1579$. Cf. Whittaker 1978, op. cit. (n. 16), 354. In the latter case, however, the interests of tenant farmers are at stake. Hamdoune has related that these personal meetings may form part of pre-Roman traditional ways of conferring and recognizing tribal loyalty: C. Hamdoune, 'Un aspect particulier des relations entre les Romains et les gentes: le patronat', Antiquités africaines 37 (2001), 164.

53 E. Frézouls, 'Rome et la Maurétanie Tingitane: un constat d'échec?', Antiquités africaines 16 (1980), 80; Frézouls 1981, op. cit. (n. 46), 49; Spaul 1994, op. cit. (n. 48), 105-108. In fact, these views tend to converge with the opinion of Romanelli expressed long time ago, cf. Romanelli 1959, op. cit. (n. 49), 373 .

54 Only one princeps seems to have been appointed or supported by the Romans (Aurelius Canarta). His son's appearance in Rome may not be coincidental (CIL VI, $1800=$ $A E$ 1941, 118).

55 Melani 1994, op. cit. (n. 21), 173.

56 K.-P. Johne, 'Nordafrika im Widerstand gegen Rom', Klio (Beihefte) 62 (1980), 595.
} 
efforts to regulate and tax indigenous land use sparked processes of economic individualization. These processes went together with a reform of social organization, which became less egalitarian and hence more apt to the Roman social order. ${ }^{57}$ In some instances, the elites of these tribes became eager to adopt Roman socio-cultural traits. An example can be found, again, among the Musulamii. Various inscriptions point out that both the epigraphic habit - of course, not a new socio-cultural phenomenon in North Africa - and the Latin language were adopted by certain members of the Musulamii. ${ }^{58}$ Here, an important impact may have come from mediating communities, which Saddington termed the Empire's semi-periphery, namely the municipia and coloniae. ${ }^{59}$ Interestingly, tribal territories may have been tied to such urban nuclei. Fentress has argued with respect to the Musulamii that they became the attributi of Theveste, since no boundary stones mark a separation between their territory and that of the colonia. ${ }^{60}$ Their leaders were incorporated into the town's elite, as can be seen in the cases of A. Larcius Macrinus and Sextius Victor, both princeps gentis Numidarum. ${ }^{61}$ Hence, this process could have engendered a change of traditional social structure, and the social and cultural values and practices by which it was legitimized. A different situation can be perceived among the Nybgenii. Their lands were far more infertile, and after delimitation they seem to have formed a ciuitas stipendiaria centred on the Civitas Nybgeniorum without any ties with municipia or coloniae nearby. ${ }^{62}$

The advent of Roman control and the connection to the market economy in Tripolitania seems to have sparked the development of agriculture in the pre-desert. ${ }^{63}$ This process may have coincided with the formation

57 Fentress 2006, op. cit. (n. 26), 21-22; 30.

$58 A E$ 1904, 76. AE 1917/18, 39. AE 1980, 971. CIL VIII, 28035. In comparison to the principes gentis Numidarum mentioned below the integration of these tribe members into the local social and political town structures is much more difficult to trace.

59 D.B. Saddington, 'The parameters of romanization', in Maxfield \& Dobson 1991, op. cit. (n. 27), 416.

60 Fentress 1979, op. cit. (n. 35), 76.

61 Among other examples: $A E$ 1905, 10 and CIL VIII, $8826=20628$.

62 Delimitation of the Tiberian period cf. CIL VIII, 22786a-k, during the reign of Trajan: $C I L$ VIII, $22787=A E$ 1910, 20 and CIL VIII, 22788. The civitas was made a municipium by Hadrian: CIL VIII, 83 .

63 R. Rebuffat, 'L'arrivée des Romains en Tripolitaine intérieure', Bulletin archéologique du Comité des travaux historiques et scientifiques. Série B, Afrique du Nord 19 (1983), 249, 252; M. Reddé, 'Occupation humaine et mise en valeur économique dans les vallées du nord de la Libye: l'exemple du Wadi Tlal', Bulletin archéologique du Comité des travaux historiques et scientifiques. Série B, Afrique du Nord 19 (1983), 179-180. 
of a rural aristocracy of tribal origin. Mattingly suggested that Roman land delimitation could have played a crucial role in the deliberate articulation of elite status. ${ }^{64}$ I believe this suggestion cannot remain undoubted since the practice of delimitation of tribal lands has been attested by boundary stones, which identify the indigenous actors collectively, not individually. ${ }^{65}$ The perception of cadastration as a tool to promote social stratification within a tribal society seems more seductive than Whittaker's interpretation of the delimitations as measures to separate and prevent large confederations. The latter's viewpoints on the general continuity of traditional social and economic relations are also difficult to support in this context, although it is unclear to what extent official(ly conferred) landownership differed from indigenous conception and practice, and to what extent the "systematic exploitation of other people in the region" implied social continuity or disruption. ${ }^{66}$

\section{A Quest for Discrepancy}

Some major conclusions can be drawn from this overview. The most important conclusion deals with the apparent impulse to perceive or impose a certain linearity or uniformity upon the relations between nomadic and sedentary populations in Roman North Africa. The view of an ever present threat to Roman rule, of endemic resistance, should be nuanced. At the same time, one should be aware that the eagerness felt in more recent research to reconstruct relationships that were utterly peaceful easily makes one present new truisms based on the highly controversial interpretation or avoidance of important evidence. One of the major setbacks to the devotee of a particular perspective is the fact that the huge amount of assumptions based on generalizations are so easy to adjust for anyone who takes a closer look at the evidence. One should abandon the idea that the Moors or the Romans had shown standard behaviour that did not change over the long time span of the Roman occupation of

${ }^{64}$ Mattingly 1986, op. cit. (n. 39), 52 and cf. 61 for his view on a deliberate Roman policy to encourage "local elites to identify common interests, involving the latter in the consolidation of their local political and social dominance and the concentration of wealth and land in their hands".

$65 A E$ 1940, 70. Similarly, $A E$ 1999, 815. $A E$ 2004, 1878. CIL VIII, $270=11451=23246=A E$ 1907, 17. CIL VIII, $4676=28073 \mathrm{a}=A E$ 1898, 39a. CIL VIII, $10667=16692$. CIL VIII, $28073 \mathrm{~b}=$ $A E$ 1898, 39b; $A E$ 1907, 19-21. $A E$ 1923, 26.

66 Whittaker 1978, op. cit. (n. 16), 350; Mattingly 1986, op. cit. (n. 39), $5^{2}$ (quote). 
North Africa. Instead, one should continue to explore the highly discrepant integration paths of specific tribes. One should take the elements into account that led to the so-called "differenzierende politik" or "rapporti ambigui" of Rome towards the inhabitants, and her motives. ${ }^{67}$ In the same vein, we should at least try to discern which internal and external concerns and impulses influenced indigenous attitudes.

Especially in Mauretania the sources tend to give a highly differentiated perspective on the political relationship between nomads and Romans. While the traditional view of ever bellicose and rebellious Moors should be abandoned, more recent attempts to reconstruct a wholly different image, of persistent tranquillity and peace, should definitely be countered as well. A clear example of such a recent 'minimalist' reconstruction can be found in Spaul's revisionist treatment of the evidence on the character of Roman rule and local resistance in Mauretania Tingitana. In order to defend the argument that the province was hardly struck by internal disorder, the author had to minimize or simply neglect evidence like the Moorish raids in Baetica and the importance of the appointment of the procuratores pro legato. ${ }^{68}$ The same author suggested that the enemies of the Salenses were wild animals, despite the fact that the neighbouring tribe of the Autololes are even literally attested as a serious threat to the town and epigraphic evidence reports endemic injustice and theft of livestock. ${ }^{69}$ The large military presence in the province may indeed have

67 Such a strategy of differentiation may be evoked by Strabo: “... for the divisions of the country have been made in various ways, inasmuch as its occupants have been several in number and the Romans have dealt with them in different ways at different times, treating some as friends and others as enemies, the result being that different parts were taken away from, or presented to, different peoples, but not in the same way." (Strabo 17.3.12). Transl.: H.L. Jones, The Geography of Strabo VII, LCL (London \& Cambridge 1967), 178-181. G. Waldherr, 'Turba Maurorum. Byzantiner und Mauren in Nordafrika', in H. Heftner, K. Tomaschitz (eds.), Ad fontes! Festschrift für Gerhard Dobesch zum fünfundsechzigsten Geburtstag am 15. September 2004 (Wien 2004), 839. Melani 1994, op. cit. (n. 21), 173.

68 On the Moorish incursions, see, apart from archaeological traces: HA, Aur. 21.1. CIL VI, $41140=A E$ 1934, $155=1957,121=1958,26 . C I L$ II2/5, $783=C I L$ II, $2015=A E$ 1961, 340. CIL II, 1120. CIL VIII, 2786. HA, Aur. 22.11-12. HA, Sept. Sev. 2.3-4. On the procuratores pro legato, cf. supra.

69 J. Spaul, 'Across the "frontier" in Tingitana', in W. Groenman-Van Waateringe, B.L.Van Beek, W.J.H. Willems, S.L. Wynia (eds.), XVIth International Congress of Roman Frontier Studies (Kerkrade 1995) (Oxford 1997), 255. AE 1931, 36 and 38. Cf. Plin., nat. 5·5. Frézouls' argument that it was just a minor conflict is more persuasive: Frézouls 1980, op. cit. (n. 53), 89; Frézouls 1981, op. cit. (n. 46), 63. Similarly, Rebuffat: "il s'agit proprement d'un brigandage". Rebuffat 1974, op. cit. (n. 46), 502. The hypothesis of a serious confrontation is defended by Rachet 1970, op. cit. (n. 1), 195; Benabou 1976, op. cit. (n. 1), 137; Sigman 1977, op. cit. (n. 4), 427-429. 
induced the situation that "only a very rash chieftain would have been prepared to face an army of that size", yet its presence as well points out how precarious the attained stability may have been. ${ }^{70}$ Minimalist and maximalist reconstructions tend to contain similar flaws.

How can we explain the discrepancies in the relations between the nomadic and the sedentary communities in Roman Africa? What could have determined the creation of the divergent individual integration trajectories of nomadic tribes?

Firstly, I argue that the indigenous susceptibility to social and cultural changes may have varied according to tribal structure and the specific type of nomadism practiced. The more the nomadic aspect had been developed within the indigenous society, the more the traditional societal organization underwent disruption and the more counter-developments were imminent. Thus the same developments and influences could have been perceived differently among desert nomads and semi-nomads. This assumption makes one doubt the value of Burian's theory concerning the economic and political implications of what he perceived as a Roman settlement policy. He posed that the creation of reservations fostered the settlement of the tribes, who subsequently became fighters for the Roman cause because of their changed economic basis. ${ }^{71}$ But if one acknowledges the observation that these so-called reservations did not exclusively consist of infertile lands and that some tribes already comprised of an extensive agricultural segment before the Roman conquest, then the economic explanation of their changed political affiliation becomes less appealing.

Concerning the political relations between nomads and sedentary groups, the size of the tribes Rome encountered, as well as their potential to form large confederations, could have been important as well. One would expect that smaller tribes were more likely to form ideal targets for Roman expansionist ambitions. On the other hand, large tribes and confederations living on the frontier could be a threat Rome felt necessary to remove. It is noteworthy that whenever ancient sources go beyond general labels to identify the rebels (like Gaetuli, Numidii or Mauri), merely great tribes and confederations have been mentioned (Garamantes, Musulamii). Unfortunately, many attestations of conflict do not give a more precise identification of Rome's enemies. It is to be expected that

70 Quote: Spaul 1994, op. cit. (n. 48), 116.

71 Burian 1961, op. cit. (n. 1), 169. 
small conflicts in which minor tribes were involved, are more likely to remain unattested.

Interactions and influences were less likely developed in the case of tribes living in more remote regions, in areas, which were difficult to access or situated across the frontier. Other tribes with a more permeable socio-economic structure and cultural matrix that enabled interaction between both Roman and other indigenous cultures could have widened the impact significantly by mediating these contacts and influences. ${ }^{72}$ This may have been one cause of different trajectories observed between the tribes of Tingitana and those of Numidia.

The existence, amongst the indigenous elites, of earlier experiences in the mediation between their tribes and other dominant sedentary powers may have played a significant role as well. In part, positive integration paths in North Africa could have been facilitated by earlier contacts with other empires, as had been the case for the semi-nomadic tribes living in the hinterland of Carthage and other Punic settlements. The Roman client kings of Mauretania, Juba II and Ptolemaeus no doubt exercised supreme power on a number of (semi-)nomadic tribes although they were imposed on them. The use of highly mobile Moorish auxilia against Tacfarinas gives evidence of the loyalty they enjoyed by part of the (semi-)nomadic tribes they were expected to control. Just a part of them, however, since both Dio Cassius and Tacitus attest that their rule was not accepted by other tribes who took up arms against them. ${ }^{73}$ The Maccues of Caesariensis also seem to assort to this category of tribes that had connections with preRoman empires, as they provided auxiliaries to the Punic army during the Second Punic War. ${ }^{74}$

Roman political and economic aspirations could have played an important role as well, and this was to some extent related to the geography of the region. Not every part of North Africa paid off the efforts to ensure Roman domination, or could sustain large settlements. The development of an export oriented market economy and its protection could have encouraged a more intensive effort to control and fully subjugate Numidian and Gaetulian tribes in the east. On the other hand, the poor revenue

72 Cf. Bloemers 1991, op. cit. (n. 27), 453.

73 Dio Cass., 55.28.3-4. Tac., Ann. 4.23. On the (unsuccessful) role of the client kings to assure order in the region, and its possible impact on Gaius' decision to dissolve the protectorate, cf. W. Vanacker, 'Conflicts and instability in Roman North Africa and Gaius' Realpolitik', in: Latomus (forthcoming).

74 Plb., 3.33.15. 
and low strategic importance of the western part of Mauretania may have led to a political strategy of laissez-faire and indirect control that suited the Romans better. Ancient military strategists, it has been related, may have looked upon Tingitana as a buffer zone only, defending the province of Baetica against Spanish marauders.

Finally, Roman aspirations of conquest or exploitation should not always be perceived as ever-present and static. Lawless posed that the lack of sources on rebellions and military operations tend to indicate that Caesariensis was marked by relative tranquillity during the first decades of its existence and may well have been the result of Roman laissez-faire and disinterest in the interior of the province, rather than of successful military deterrence. ${ }^{75}$ The silence in the sources can either point to the presence of positive relations, or to the absence of any intense relations at all.

\section{Conclusion}

The nature and availability of the sources inevitably makes any attempt to reconstruct specific integration trajectories of nomadic tribes in Roman North Africa incomplete and susceptible to conjecture. Many tribes have been mentioned occasionally by an ancient cartographer or encyclopaedist but have not been attested otherwise. The question then rises to what extent the specific integration stories of better known tribes are related to those tribes who are less or not traceable in the record. Indirect indications can be provided by the location and density of Roman military constructions or the creation of urban centres, but scholars have argued that their presence could have been motivated by other reasons than military concerns. ${ }^{76}$

It can be sensible to assume that the lack of sources describing social and cultural integration exactly reveals a segregated society. However, in many regions political and economic symbiosis may have predominated over the processes of expulsion or containment of the nomads. One should

75 Lawless 1978, op. cit. (n. 1), 161. However, as it is mentioned above, there may have been some attestations of conflicts.

76 See for instance Janon's views on the military occupation of the Aurès (note 28). Morizot, however, defends a more conservative view on the subject: P. Morizot, 'Deux ouvrages militaires de la province de Numidie révélés par la photographie aérienne: le camp de Bedoura et le fort de Yabous', in Groenman-Van Waateringe, Van Beek, Willems, Wynia 1997, op. cit. (n. 69), 450. 
not derive from its absence in the record - which is essentially the product of an elite that was essentially urban and sedentary - that nomadism was efficiently repulsed.

To conclude, the case studies discussed above clearly show a large diversity of political, juridical, economic and cultural integration patterns. They indicate that nomads were not 'naturally' hostile to integration, and that Roman policy not merely aimed at the inclusion of the sedentary while containing or expulsing the nomad population. 


\title{
WIE INTEGRIERT MAN ROM IN DIE POLIS? DER KULT DES SENATS IN KLEINASIEN*
}

\author{
Günther Schörner
}

\section{Integration: Akteure und Handlungen}

Möchte man Integration im Imperium Romanum untersuchen, so ist nicht nur zu analysieren, auf welche Weise die Provinzen und ihre Bewohner in das Römische Reich integriert wurden, sondern auch, wie Rom selbst in das provinziale Umfeld integriert wurde. ${ }^{1}$ In diesem Fall wird nicht nach reichsweiter Integration, sondern nach lokal begrenzter, je nach politischer Organisation oft nach Integration auf städtischer Ebene gefragt. Dabei sollte man sich nicht darauf beschränken festzustellen, was integriert wurde, da die Frage nach dem Wie mindestens genau so wichtig ist, das heißt welche Aktionen erfolgten und wer diese veranlasste. Im Folgenden sollen Akteure und Handlungen einer solchen Integration an einem konkreten Beispiel aufgezeigt werden, nämlich der Integration der spezifisch römischen Institution 'Senat' in den kultischen Kontext kleinasiatischer Poleis. Es stehen dabei weniger Fragen der Kultausübung im Mittelpunkt, sondern vielmehr die Prozeduren und Aktionen sowohl auf römischer als auch auf kleinasiatischer Seite, die unternommen werden, um für eine dezidiert römische Institution wie den Senat Platz innerhalb der Poleis zu schaffen, d. h. ihn in dieses spezifisch sozio-kulturelle Umfeld einzubinden.

* Ich danke den Organisatoren des Workshops Impact of Empire, vor allem S. Benoist, S. Demougin, und O. Hekster für die Einladung nach Lille. Wichtige Thesen dieses Vortrags wurden bereits in Amsterdam, Erfurt und Würzburg vorgetragen. Für Diskussionsbeiträge danke ich A. Chankowski (Lille), L. de Blois (Nijmegen), S. Demougin (Paris), J. Fabricius (Berlin), M. Meyer (Wien), F. Naerebout (Leiden), J. Rüpke (Erfurt) und Th. Schäfer (Tübingen).

1 Zum Begriff der Integration: G. Alföldy, 'Die Romanisation - Grundbegriff oder Fehlbegriff? Überlegungen zum gegenwärtigen Stand der Erforschung von Integrationsprozessen im römischen Weltreich', in Z. Visy (ed.), Limes XIX. Proceedings of the XIXth International Congress of Roman Frontier Studies (Pécs 2005), 25-56; R. Roth, 'Roman culture between homogeneity and integration', in R. Roth, J. Keller (eds.), Roman by integration: Dimensions of group identity in material culture and text (Journal of Roman archaeology Supplementary Series 66) (Portsmouth, RI 2007), 7-10; zusammenfassend zur Diskussion: D. Mattingly, Imperialism, power, and identity: experiencing the Roman Empire (Princeton, NJ 2010) 3-42; vgl. in diesem Band den Beitrag von F. Naerebout. 
Im Unterschied zu den anderen römischen Institutionen besitzt der Senat als Objekt kultischer Verehrung Eigenheiten, die eine deutlichere Differenzierung zwischen römischer und griechischer Seite zulassen und somit die Funktionsweise der Integration leichter nachvollziehbar machen. ${ }^{2}$ Das wichtigste Charakteristikum ist sprachlicher Natur: Im Unterschied zum lateinischen senatus ist das griechische $\Sigma u ́ \gamma x \lambda \eta \tau o s$ feminin, so dass die

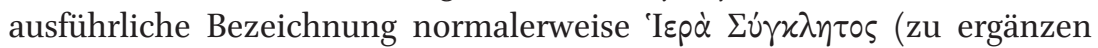
ist: Bov $\lambda$ ) lautet. Der Wechsel des Genus hatte natürlich Konsequenzen für das Erscheinungsbild, das die Senatsgottheit in Kleinasien annahm, da aufgrund des grammatikalischen Geschlechts in Kleinasien an und für sich eine weibliche Personifikation $\Sigma$ YNK $\Lambda H T O \Sigma$ BOY $\Lambda$ H bzw. IEPA $\Sigma$ YNK $\Lambda$ HTO $\Sigma$ zu erwarten ist. ${ }^{3}$ Folglich ist es verständlich, dass trotz der hauptstädtischen Herkunft der Institution nicht die stadtrömische Verkörperung als Genius Senatus übernommen wurde. Der Genius Senatus besitzt spätestens seit flavischer Zeit eine feste Ikonographie:4 Er wird stets als älterer bärtiger Mann in Toga mit idealen Gesichtszügen und langen Locken dargestellt. ${ }^{5}$ Als Personifikationen der res publica tritt der Genius Senatus regelhaft zusammen mit dem deutlich jugendlicher wiedergegebenen Genius Populi Romani auf,, eines der frühesten Zeugnisse sind die so genannten Cancelleria-Reliefs. ${ }^{7}$ Grundsätzlich unterscheidet

2 Zum Kult des Senats/Synkletos in Kleinasien grundlegend: G. Forni, IEPA e $\Theta E O C$ CVNKLETOC. Un capitolo dimenticato nella storia del Senato Romano (Atti della Accademia Nazionale die Lincei, Memorie classe di scienze morali, storiche e filologiche ser. VIII, vol. V.3) (Rom 1953); G. Forni, 'Il culto del Senato di Roma', in Epigrafia e ordine senatorio. Atti del colloquio AIEGL (Roma, 14-20 maggio 1981) (Rom 1982), 14-18; D. Kienast, 'Der heilige Senat: Senatskult und „kaiserlicher" Senat', Chiron 15 (1985), 253-282.

3 Kienast 1985, a.a.O. (Anm.2), 266 f. Vgl. auch A. Chaniotis, 'The dynamics of rituals in the Roman Empire', in O. Hekster, S. Schmidt-Hofner, C. Witschel (eds.), Ritual Dynamics and Religious Change in the Roman Empire. (Proceedings of the Eighth Workshop of the International Network Impact of Empire, Heidelberg, July 5-7, 2007) (Leiden 2009), 4.

4 H. Kunckel, Der römische Genius. 20. Ergänzungsheft der Römischen Mitteilungen (Heidelberg 1974), 37-42.

5 Vgl. auch die Beschreibung des personifizierten Senats, der Traian im Traum erscheint: Dio Cass., 68.5.1.

6 Kunckel 1974, a.a.O. (Anm.4), 33-37, 40-43; J.R. Fears, 'O $\Delta$ HMO $\Sigma$ O P $\Omega$ MAI $\Omega N$ Genius Populi Romani', Mnemosyne 31 (1978), 275-279.

7 Zu den Reliefs allgemein G. Koeppel, 'Die historischen Reliefs der römischen Kaiserzeit II: Städtrömische Denkmäler unbekannter Bauzugehörigkeit aus flavischer Zeit', Bonner Jahrbücher 184 (1984), 5-9, 28-34; N. Hannestad, Roman Art and Imperial Policy (Aarhus 1988), 132-139; H. Herzog, 'Die Cancelleriareliefs', Bollettino. Monumenti musei a gallerie pontificie 21 (2001), 103-147; speziell zu den beiden Genien: J. Béranger, 'Les 
sich der Genius Senatus durch Kleidung und Barttracht signifikant von den meisten anderen Genien, die als junger Mann wiedergegeben sind. ${ }^{8}$

Diese geläufige stadtrömische Erscheinungsform wurde - wie zu erwarten - in Kleinasien nicht verwendet, jedoch wurde auch kein dem griechischen Sprachgebrauch entsprechendes weibliches Bild konzipiert in Analogie zu Verkörperungen von Ratsversammlungen wie Boulê ${ }^{9}$ oder Gerousia. ${ }^{10}$ Geschaffen wurde eine neuartige Personifikation, ${ }^{11}$ anhand deren Ikonographie und Verbreitung gezeigt werden kann, mit welchen Schritten eine römische Institution in das Leben der Provinz Asia integriert wird. ${ }^{12}$

Génies du sénat et du peuple romain et les reliefs flaviens de la Cancelleria', in M. Renard, R. Schilling (eds.), Hommages à Jean Bayet (Brüssel 1964), 76-88.

8 z.B. auch die vielen privaten Genien: Kunckel 1974, a.a.O. (Anm.4) 42 f. (mit Beispielen); allgemein Lexicon Iconographicum Mythologiae Classicae VIII (München-Zürich 1997), 599-607 s.v. Genius (I. Romeo); vgl. auch die Ikonographie der Lokalgenien z.B. L. da Silva Fernandes, 'Genii, Lares e Tutela na provincia da Lusitânia', in J. Cardim Ribeiro (ed.), Religiôes da Lusitânia: loquuntur saxa (Lissabon 2002), 179-188.

9 Zur Ikonographie der Boulê allgemein Lexicon Iconographicum Mythologiae Classicae III (München, Zürich 1984), 145-147 s.v. Boulê (V. Komminos) (mit weiterer Lit.); zur Personifikation in klassischer Zeit: W. Messerschmidt, PROSOPOPOIIA. Personifikationen politischen Charakters in spätklassischer und hellenistischer Kunst (Köln, Weimar, Wien 2003), 48-50; zur Personifikation auf kleinasiatischen Münzen der römischen Kaiserzeit: F. Missere, 'Iconografia della Boulê e del Demos nella monetazione imperiale greca dell'Asia minore', Rivista Italiana di Numismatica 92 (1990), 76-87; Statuen epigraphisch belegt: Th. Pekáry, 'Statuen in kleinasiatischen Inschriften', in S. Sahin, E. Schwertheim, J. Wagner (eds.), Studien zur Religion und Kultur Kleinasiens. Festschrift für Friedrich Karl Dörner zum 65. Geburtstag am 28. Februar 1976 (Leiden 1978), 739.

10 Lexicon Iconographicum Mythologiae Classicae VIII (München, Zürich 1997), 608 s.v. Gerousia (S. Schultz); zur Institution: J.H. Oliver, The sacred Gerusia (6. Supplement Hesperia) (Princeton 1941).

11 Für die Definition einer Personifikation wird gefolgt: A.C. Smith, Polis and personification in Classical Athenian art (Leiden, Boston 2011), 2: „... personification as representation of a thing, place, or abstraction as a person, where person may be human, mythical, and/or divine." $\mathrm{Zu}$ eng und im unseren Kontext nicht anzuwenden ist die Definition von Personifikation durch B. Borg, Der Logos des Mythos. Allegorien und Personifikationen in der frühen griechischen Kunst (München 2002), 49 f.: Personifikationen sind „.... alle anthropomorphen und anthropopsychen Gestalten..., deren Namen gleichzeitig als abstraktes Substantiv oder Bezeichnung für einen unbelebten Gegenstand existiert". Beibehalten wird jedoch ihre Unterscheidung von 'Personifizierung' als der Aktion und 'Personifikation' als dem Produkt: Borg a.a.O., $5^{2}$.

12 Zur Personifikation von Synkletos neben der in Anm.2 genannten Lit. speziell: Lexikon Iconographicum Mythologiae Classicae VII (München, Zürich 1994), 828 f. s.v. Synkletos (F. Canciani). 


\section{Kult und Kultbild: Römischer Entwurf - kleinasiatische Übernahme}

Die kultische Verehrung eines politischen Gremiums erscheint zunächst noch erstaunlicher als die einer einzelnen Person wie eines Kaisers, ${ }^{13}$ doch ist der römische Senat als Kultempfänger zweifelsfrei nachgewiesen. ${ }^{14} \mathrm{Zu}$ berücksichtigen ist dabei, dass vor allem die Handlungsfähigkeit und die Einflussmöglichkeiten entscheidend für die Etablierung kultischer Verehrung waren. ${ }^{15}$ Diese waren im Falle des römischen Senats für die kleinasiatischen Städte aus deren Perspektive sicherlich gegeben: In Asia als einer senatorischen Provinz wurde der Senat als wichtige politische und legislatorische Instanz wahrgenommen, insbesondere erfolgte die Verleihung von Ehrentitel wie Neokoros, um die sich ein wichtiger Aspekt des städtischen Lebens in Kleinasien drehte, durch den Senat. ${ }^{16}$ Es ist also nur verständlich, wenn der Senat noch in der Kaiserzeit sowohl im Verkehr mit Rom als auch unter den Poleis als entscheidende Größe empfunden wurde. ${ }^{17}$

Obwohl der Senat auf städtischer Ebene sicher bereits unter Augustus verehrt wurde, erfolgte die Einrichtung eines provinzweiten Kultes unter Tiberius schließlich in Smyrna, wo für Tiberius, die Kaisermutter Livia

13 Zum Kaiserkult: S.R.F. Price, Rituals and Power: The Roman Imperial Cult in Asia Minor (Cambridge 1984); M. Clauss, Kaiser und Gott: Herrscherkult im römischen Reich (Stuttgart 1999); C. Ando, Imperial ideology and provincial loyalty in the Roman empire (Berkeley 2000); I. Gradel, Emperor worship and Roman religion (Oxford 2002); T. Witulski, Kaiserkult in Kleinasien. Die Entwicklung der kultisch-religiösen Kaiserverehrung in der römischen Provinz Asia von Augustus bis Antoninus Pius (Göttingen 2011²); (jeweils mit weiterer Lit.).

14 Zeugnisse gesammelt bei Forni 1953, a.a.O. (Anm.2), 67-79; Forni 1982, a.a.O. (Anm.2), 14-18; Kienast 1985 a.a.O. (Anm.2) 268 Anm.66, 68.

15 Immer noch grundlegend: E. Bickerman, 'Consecratio', in E. Bickerman, C. Habicht (eds.), Le culte des souverains dans l'empire romain (Entretiens sur l'Antiquité classique 19) (Genf 1972), 1-26; vgl. auch G. Woolf, 'Divinity and power in ancient Rome', in N. Brisch (ed.), Religion and power. Divine kingship in the ancient world and beyond (Chicago 2008), $235^{-252 .}$

16 Zur Neokorie zusammenfassend: B. Burrell, Neokoroi. Greek Cities and Roman Emperors (Leiden, Boston 2004); Senat in dieser Rolle gewürdigt und auf Münze festgehalten: K. Harl, Civic coins and civic politics in the Roman east A.D. 180-275 (Berkeley, London 1987), 61 Taf. 27,6. Grundlegend zur Wahrnehmung des Senats aus Sicht einer kleinasiatischen Polis am Beispiel Ephesos: G.M. Rogers, The sacred identity of Ephesos. Foundation myths of a Roman city (London, New York 1991), $91 \mathrm{f}$.

17 Zum Senat in der Kaiserzeit: R.J.A. Talbert, The senate of Imperial Rome (Princeton, NJ 1984); zur Rolle des Senats in Kleinasien: Harl 1987, a.a.O. (Anm.16), 71-75. 
und den Senat ein Tempel errichtet wurde. ${ }^{18}$ Über die Umstände dieser Kultgründung berichtet vor allem Tacitus: ${ }^{19}$

Dieser Entscheidung halber und, weil auch im vorigen Jahre C. Silanus zur Strafe gezogen worden war, beschlossen die kleinasiatischen Städte, zu Ehren des Tiberius, seiner Mutter und des Senats einen Tempel zu erbauen. Der Bau wurde auch gestattet, und der junge Nero war es, der den Senatoren und seinem Großvater den Dank dafür aussprach - unter freudiger Bewegung der Zuhörer, die Germanicus noch lebhaft im Gedächtnis hatten und jetzt ihn selber zu sehen und hören meinten.

Kienast konnte in überzeugender Interpretation dieser Passage darauf hinweisen, dass der Kult von Synkletos in diesem Kontext auf Tiberius selbst zurückgeht, ${ }^{20}$ dessen Hochachtung des Senats auch durch einen von Sueton überlieferten Ausspruch belegt wird. ${ }^{21}$ Die Kommunikation und Interaktion zwischen Kaiser und Gesandtschaften, das heißt zwischen verschiedenen Erwartungshorizonten ist bekanntermaßen sehr kompliziert, doch ist davon auszugehen, dass man die Spezifikation, d.h. die Bestandteile dieses Kaiserkultkomplexes auf Rom zurückführen kann. Die römische Einwirkung ging jedoch noch weiter: ${ }^{22}$

Bei der Abstimmung des Senats erhielt denn auch Smyrna den Vorzug, und Vibius Marsus beantragte, man möge dem M. Lepidus, dem die Provinz Asien zugefallen war, einen überzähligen Legaten mitgeben der die Aufsicht über den Tempelbau zu führen habe. Lepidus lehnte aus Bescheidenheit ab, diesen Legaten selber zu ernennen; das Los fiel auf den ehemaligen Prätor Valerius Naso.

Die deutliche römische Einflussnahme bei der Konzeption dieser Kultstätte ist $\mathrm{m}$. E. nun auch ikonographisch zu belegen: Die Errichtung dieses provinzialen Kaiserkulttempels wird auch durch eine Münze aus Smyrna memoriert (Abb. 1), die auf der Rückseite einen tetrastylen Tempel zeigt, in dem ein Togatus capite velato, also Tiberius selbst als der erstgenannte Kultempfänger, mit einer Schöpfkelle, einem simpuvium, in der rechten Hand steht. ${ }^{23}$ Auf der Vorderseite sind zwei einander zugewandte Büsten

18 Zur voraugusteischen Verehrung des Senats auf städtischer Ebene: Chr. Habicht, 'Samos weiht eine Statue des Populus Romanus', Athenische Mitteilungen 105 (1990), 262 mit Anm.24. (mit Beleg).

19 Tac., Ann. 4.15; 55 f. (Übersetzung: W. Sontheimer).

20 Kienast 1985, a.a.O. (Anm.2), $261 \mathrm{f}$.

21 Suet., Tib. 29.

22 Tac., Ann. 4.56.3 (Übersetzung: W. Sontheimer): Kienast 1985, a.a.O. (Anm.2), 260.

23 Zur Münze: D. Klose, Die Münzprägung von Smyrna in der römischen Kaiserzeit (Berlin 1987), 212-214 n XXVI 1-35 Taf. 18. 19; A. Burnett, M. Amandry, P.P. Ripollès, Roman 

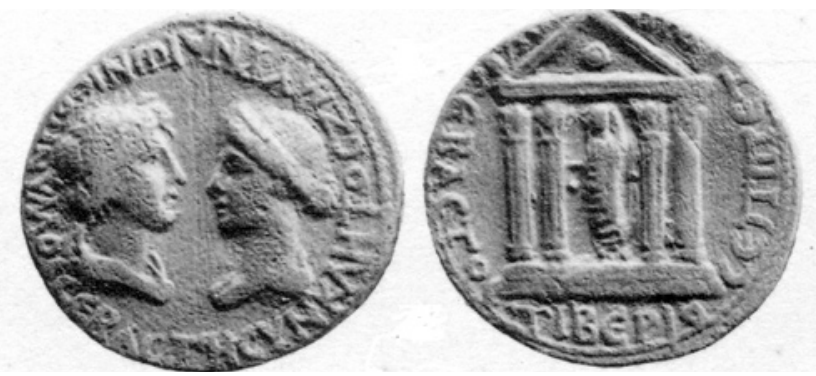

Abb. 1: AE Smyrna: Synkletos und Livia; Tiberius in Tempel

dargestellt, die einer Frau mit Diadem und Nackenknoten und die eines jungen Mannes mit langem Haar. Laut Beischrift handelt es sich um die Darstellungen der Sebaste Livia und der Senatsgottheit Synkletos als die beiden anderen Tempelinhaber, wobei dieser eindeutig nicht weiblich, sondern männlich dargestellt wurde, freilich auch nicht als alter Mann wie der - später bezeugte - römische Genius Senatus. ${ }^{24}$

Die ikonographische Konzeption des Togatus, das heißt der TiberiusStatue, so wie sie auf der Münze wiedergegeben ist, erweist sich eindeutig als stadtrömisch. Dafür sprechen nicht nur die Toga, sondern vor allem die Verhüllung des Hinterkopfes, die velatio capitis, als typisches Zeichen eines nach dem ritus Romanus vollzogenen Kultaktes und das simpuvium. ${ }^{25}$ Dieses Sakralgerät ist charakteristisch für das collegium der pontifices und wurde insignienhaft verwendet, wie Monumente unterschiedlicher Gattungen belegen. ${ }^{26}$ Der Darstellungstyp eines Togatus capite velato mit simpuvium ist für Augustus auf einem stadtrömischen Aureus des Münzmeisters C. Marius aus dem Jahr 13 v. Chr. bezeugt;27 In Kleinasien wurde ein ähnliches Münzbild erstmals in Ilion mit der

provincial Coinage I: From the death of Caesar to the death of Vitellius (London 1992), 419 $\mathrm{n}^{\circ}$ 2469; S.J. Friesen, Twice neokoros. Ephesos, Asia, and the cult of the Flavian imperial family (Leiden 1993), 20 Taf. 3; Burrell 2004, a.a.O. (Anm.16), 39 f.

${ }^{24}$ Hier zu Anm.4.

25 Grundlegend zur velatio capitis: H. Freier, Caput velare (Tübingen 1963).

${ }^{26}$ Zwierlein-Diehl, 'Simpuvium Numae', in H.A. Cahn (ed.), Tainia. Roland Hampe zum 70. Geburtstag am 2. Dezember 1978 dargebracht von Mitarbeitern, Schülern und Freunden (Mainz 1980), 405-422; A.V. Siebert, Instrumenta sacra. Untersuchungen zu römischen Opfer-, Kult- und Priestergeräten (Berlin 1999), 132-134; 236-239.

27 Siebert 1999, a.a.O. (Anm.26), 324 Mü 35. 
Beischrift $\sum \dot{\varepsilon} \beta \alpha \sigma \tau o \zeta$ verwendet, ${ }^{28}$ also in einer Stadt mit bekannt engen Beziehungen zu Rom. ${ }^{29}$

Folgt die Darstellung des Tiberius als togatus capite velato römischwestlichen Vorstellungen, so gilt das dann mit großer Wahrscheinlichkeit ebenso für die Büsten der beiden anderen Kultempfänger auf der Rückseite. So entspricht das Porträt der Livia dem üblichen römischen Typus mit Mittelscheitel. ${ }^{30}$ Die jugendlich-männliche Senatsgottheit dürfte deshalb ebenfalls auf eine römische Konzeption zurückgehen, wie es auch dem lateinischen Sprachgebrauch entsprechend sein Geschlecht nahe legt. Es ist durchaus möglich, dass die Personifikation für die Kultstatue in Smyrna erfunden wurde. Dass es bereits einen jugendlichen Mann als Personifikation von Synkletos in augusteischer Zeit gegeben hat, ist unwahrscheinlich. ${ }^{31}$ Erstaunlicherweise entspricht die Ikonographie aber nicht dem - späteren - Genius Senatus-Typus, sondern nach römischen Begriffen viel eher dem Genius Populi Romani. ${ }^{32}$ Die besten Parallelen für die jugendliche, langhaarige Büste sind somit Darstellungen entsprechender Genii wie auf einem der berühmten Boscoreale-Becher ${ }^{33}$ oder wohl bereits der Ara Pacis. ${ }^{34}$ Die Entstehung des stadtrömischen Genius-Bildes haben H. Kunkel und H. Jucker untersucht: ${ }^{35}$ Jucker konnte zeigen, dass bereits im 1. Jh. v. Chr. gelegentlich unbärtige Genien vorkommen, aber keine durchlaufende Bildtradition nachzuweisen ist. ${ }^{36}$ Der neue, dann schnell übliche Typ des jugendlichen Genius mit schulterlangem Haar

28 Burnett, Amandry, Ripollès 1992, a.a.O. (Anm.23), 391 n $^{\circ} 2311$ (mit weiterer Lit.).

29 D. Hertel, Die Mauern von Troia. Mythos und Geschichte im antiken Ilion (München 2003), 274-284.

30 Zum Typ: P. Zanker in K. Fittschen - P. Zanker, Katalog der römischen Porträts in den Capitolinischen Museen und den anderen Kommunalen Sammlungen der Stadt Rom (Mainz 1983), 3-5 $n^{\circ}$ 3. Livia ersetzt die ansonsten für die Münzprägung Smyrnas übliche Statue der Aphrodite Stratonikis: M. Meyer, 'Die Aphrodite Stratonikis in Smyrna und die Asylie der Stadt', Jahrbuch für Numismatik und Geldgeschichte 60 (2010), 52-54.

31 Lokalprägungen aus Lampsakos in Mysien zeigen eine Kaiserbüste und die Büste

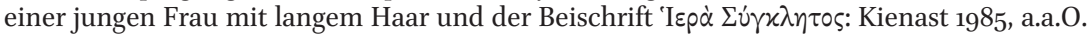
(Anm.2), 257 Taf. I 5 .

32 S.o. Anm.5.

33 A.L. Kuttner, Dynasty and empire in the age of Augustus. The case oft he Boscoreale cups (Berkeley, Los Angeles 1995), passim; zum Genius populi Romani speziell: ebenda 18-22 Abb. 1.

34 G. Koeppel, 'Die historischen Reliefs der römischen Kaiserzeit V: Ara Pacis Augustae Teil 1', Bonner Jahrbücher 187 (1987), $15 \mathrm{n}^{\circ}$ 4, 3 .

35 Kunckel 1974, a.a.O. (Anm.4), 33-37; H. Jucker, 'Ipse suos Genius adsit visurus honores!', in G. Schwarz, E. Pochmarski (eds.), Classica et provincalia. Festschrift Erna Diez (Graz 1978), 93-96; Fears 1978, a.a.O. (Anm.6), 276-279.

36 Jucker 1978, a.a.O. (Anm.35), 94. 
wurde folglich erst unter Augustus ausgebildet, während der Genius Senatus in Rom als Personifikation des Senats eine noch spätere Erfindung darstellt. ${ }^{37}$

Es spricht somit alles dafür, dass die Personifikation von Synkletos für den Tempel in Smyrna erfunden wurde. Allein aufgrund des gewählten Geschlechts ist die Konzeption eindeutig auf römische Vorstellungen zurückzuführen; auch hinsichtlich der Kommunikation ist davon auszugehen, dass senatus der terminologische Ausgangspunkt war. Es liegt deshalb nahe, Valerius Naso eine entscheidende Rolle zuzubilligen. Ob bei der Bilderfindung der jugendlichen männlichen Personifikation rein instrumentell auf griechische Ideen zurückgegriffen wurde, darf bezweifelt werden. Als Anregung kommt nur die Personifikation des Demos in Frage, weil sie in der kleinasiatischen Münzprägung der Kaiserzeit der des jugendlichen langhaarigen Synkletos sehr ähnelt. ${ }^{38}$ Die klassische Bildvorstellung - der Demos als älterer bärtiger Bürger - wird jedoch kontinuierlich bis in die hellenistische und römische Zeit beibehalten, ${ }^{39}$ so dass die Popularität des jugendlichen unbärtigen Demos ebenfalls auf römischen Einfluss in Anlehnung an den Genius Populi Romani zurückzuführen sein dürfte. ${ }^{40}$

\section{Die Personifikation von Synkletos in Kleinasien: ikonographischen Optionen}

Im Hinblick auf die Ikonographie des Synkletos ist somit davon auszugehen, dass speziell für den Export nach Kleinasien von römischer Seite ein Bild des Senates geschaffen wurde, das weder in Rom noch in Kleinasien direkte Vorbilder hatte; jedenfalls wurde die Büste bzw. Kultstatue aus aktuellen römischen Bestandteilen und unter römischer Aufsicht geschaf-

37 So auch H. Wrede, 'Der genius populi Romani und das Fünfsäulendenkmal der Tetrarchen', Bonner Jahrbücher 181 (1981), 115. Wrede geht freilich davon aus, dass frühere Darstellungen des Genius populi Romani als bärtiger älterer Mann das Bild des genius Senatus vorweg nehmen.

38 Missere 1990, a.a.O. (Anm.9), 88-99.

39 Zur klassischen Bilderfindung des bärtigen Demos: Messerschmidt 2003, a.a.O. (Anm.9), 10-21; A.C. Smith, Polis and personification in Classical Athenian art (Leiden, Boston 2011), 96-102 (mit der älteren Lit.); zum Demos allgemein Lexicon Iconographicum Mythologiae Classicae III (München, Zürich 1986), 375-382 s.v. Demos (O. AlexandriTzahou).

40 So z.B. Alexandri-Tzahou 1986, a.a.O. (Anm.39), 382; s. auch die Zusammenstellung bei Missere 1990, a.a.O. (Anm.9), 89. Auch in der Plastik wird in der früheren Kaiserzeit der bärtige Demos-Typ beibehalten: vgl. die Darstellung auf dem Zoilos-Fries: R.R.R. Smith, The monument of C. Julius Zoilos. Aphrodisias I (Mainz 1993), 32 f. Taf. 10. 11. 
fen, wobei anscheinend von den Schöpfern der Personifikation die Diskrepanz zwischen römischen und griechischen Vorstellungen im Genus in Kauf genommen wurde. Die Wahl des Geschlechtes muss auf römische Terminologie zurückgehen, die Nutzung des griechischen Namens $\Sigma u ́ \gamma \chi \lambda \eta \tau \circ \varsigma$ beweist jedoch die Absicht, von griechischer Seite in das eigene Begriffssystem einzubinden und schließlich die Gottheit zu integrieren. Sowohl der Kult selbst als auch die Personifikation weisen jedoch eindeutig auf asymmetrische Machtverhältnisse zwischen Hauptstadt und Provinz hin.

Das aus dem Genuswechsel entstehende Problem besteht in der Rezeption dieser vergöttlichten Institution und ihrer vorgegebenen bildlichen Erscheinung in einer Gesellschaft, die sie - rein sprachlich - weiblich auffasst. ${ }^{41}$ Eine beabsichtigte Entschärfung des Gegensatzes ist vielleicht darin zu sehen, dass auf den Münzen Smyrnas zunächst als Beischrift auch das geschlechtsneutrale $\Theta E O \Sigma \Sigma$ YNK $\Lambda$ HTO $\Sigma$ verwendet wird. ${ }^{42}$ Spätestens seit der mittleren Kaiserzeit wird dann jedoch überwiegend IEPA ¿YNK $\Lambda$ HTO $\Sigma$ als Legende gebraucht, so dass die Diskrepanz zwischen Bild und Text offen zu Tage tritt. ${ }^{43}$ Die Ikonographie von Synkletos wird jedoch unverändert beibehalten, so bleibt auf den zahlreichen städtischen Prägungen Smyrnas das Bild der jugendlichen männlichen Senatsgottheit bis in das 3. Jh. hinein vorherrschend (Abb. 2), wobei sich freilich diese

41 Die Personifikation von Synkletos ist ein besonders eindeutiger Beleg für die gender-Problematik bei Personifikationen. Allgemein, vor allem aber zur Tatsache, dass im griechischen Bereich die meisten Verkörperungen weiblich sind: E.J. Stafford, 'Masculine values, feminine forms: on the gender of personified abstractions', in L. Foxhall, J. Salmon (eds.), Thinking men. Masculinity and self-representation in the classical tradition (London, New York 2006), 43-56; vgl. auch D. Burton, 'The gender of death', in E. Stafford, J. Herrin (eds.), Personification in the Greek world (London 2005), 45-68; für die römische Zeit liegt bisher keine ausführlichere Untersuchung vor, sondern man belässt es bisher bei eher vereinzelten Bemerkungen wie zum ‘männlichen' Aussehen der Gerousia: Schultz 1997, a.a.O. (Anm.10), 6o8. Anscheinend war die Charakterisierung bereits für die Römer nicht immer eindeutig: vgl. Serv., Aen. 2.351, genio urbis Romae sive mas sive femina. Ähnlich gelagert die Bennung von weiblichen Stadtpersonifikationen im Typus der 'Tyche von Antiochia', als Genius auf Münzen römischer Kolonien in Kleinasien, z.B. aus Kremna oder Apameia in Bithynien, sowie andere Frauenfiguren, so aus Antiochia ad Pisidiam oder Antiochia am Orontes (hierzu M. Meyer, Die Personifikation der Stadt Antiocheia. Ein neues Bild für eine neue Gottheit (33. Ergänzungsheft Jahrbuch des Deutschen Archäologischen Instituts) (Berlin 2006), 391-397 Appendices A-D. Frühester Beleg ist der Genius terrae Africae auf Denaren des Qu. Caecilius Metellus Scipio (48-46 v. Chr.) in Gestallt der löwenköpfigen Göttin Tanit/Caelestis: Lexicon iconographicum mythologiae classicae I (München, Zürich 1981), 255 s.v. Africa (M. Le Glay); Meyer a.a.O., 396 App. D 3.

42 Klose 1987, a.a.O. (Anm.23), $130 \mathrm{n}^{\circ}$ II 1-11 (pseudoautonom, neronisch), III 19-61; RPC $420 \mathrm{n}^{\mathrm{o}} 2481$ (pseudoautonom, nach-neronisch); Burnett, Amandry, Ripollès 1992, a.a.O. (Anm.23), $420 \mathrm{n}^{\circ} 2489$.

43 Klose 1987, a.a.O. (Anm.23), 147 no V 1-14 (pseudoautonom, traianisch) 


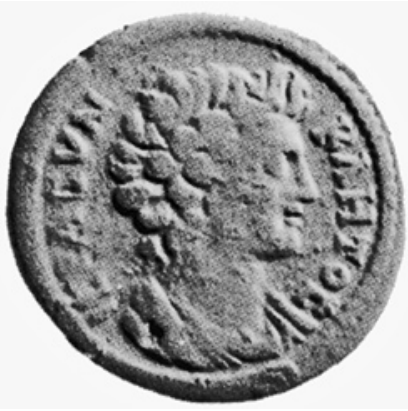

Abb. 2: AE Smyrna: Synkletos

idealen Jünglingsköpfe in Einzelheiten, z.B. der Länge der Haare, unterscheiden können. ${ }^{44}$ An der Tatsache, dass immer junge Männer wiedergegeben sind, ist jedoch nicht zu zweifeln. ${ }^{45}$ Engste Parallelen bestehen während der Kaiserzeit zu Münzen mit der Personifikation des Demos, die eine identische Büste eines jugendlichen, langhaarige Mannes zeigen, so dass grundsätzlich keine ikonographische Möglichkeit besteht, Synkletos und Demos zu unterscheiden. ${ }^{46}$ Dies ist prinzipiell nur möglich, wenn beide Personifikationen als Ganzfiguren wiedergegeben sind, da Demos ein Himation, Synkletos eine Toga trägt. Probleme gibt es bei der Unterscheidung der Personifikationen von Senat und römischem Volk in griechischer Ikonographie: Auf einem der Reliefs vom Sebasteion in Aphrodisias wird Claudius von einer jugendlich männlichen Personifikation bekränzt, bei der nicht eindeutig zu unterscheiden ist, ob es sich um den Demos der Römer oder Synkletos handelt. ${ }^{47}$

Neben Smyrna sind aus vielen kleinasiatischen Städten so genannte pseudo-autonome Münzen erhalten, die anstelle des Kaiserporträts

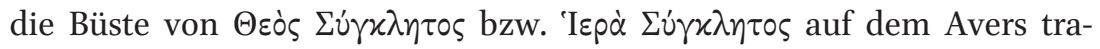
gen, wobei im Normalfall dieselbe Ikonographie wie in Smyrna verwen-

44 Klose 1987, a.a.O. (Anm.23) 184 f. no XIV 1-4; 186-188 no XV 1-10; 189 n $^{\circ}$ XVI 1; 189-192 $n^{\circ}$ XVIII 1-12; 194 f. no XIX 2-6; 196 f. no XX 2-6; 197-203 no XXI 1-44 bzw. $48 ; 327$ f. $\mathrm{n}^{\circ}$ LXXVIII 1-6.

45 Vor allem für die Frisur gibt es keine direkten Parallelen bei weiblichen Idealköpfen. Auffällig und für einen Mann ungewöhnlich ist das stoffreiche, aufwändig gefältelte Gewand, doch auch das ist kein Hinweis auf weibliche Tracht, sondern der Versuch des Stempelschneiders, den Stoffreichtum der Toga zum Ausdruck zu bringen und vom griechischen Himation abzusetzen.

46 So bereits Fears 1978, a.a.O. (Anm.6), 280.

47 R.R.R. Smith, 'The imperial reliefs from the Sebasteion at Aphrodisias', Journal of Roman Studies 77 (1987), 114 f. Taf. 12. 12, 3-4. 
det wurde. ${ }^{48}$ Es liegt somit nahe, in der Vielzahl ähnlicher jugendlicher Senatsgottheiten eine Orientierung an die smyrnäischen Prägungen und letztlich an dem offiziellen Provinzialkult zu vermuten, dessen unter Tiberius errichtetes Kultbild in der Münzprägung reflektiert wurde. Dies gilt sowohl für den 'Prägebezirk' Smyrna als auch weit darüber hinaus. ${ }^{49}$

Trotz dieser weitgehenden ikonographischen Übereinstimmung zeigt sich, dass das Bild des Senats nicht in allen Einzelheiten festgeschrieben und seine Verwendung nicht verbindlich war, so dass generell zwei unterschiedliche Optionen der Darstellung zu scheiden sind:

1.) Beibehaltung der Jünglingsbüste mit Variationen im Detail

2.) grundsätzlich neue Erscheinungsform

Zur ersten Option: Auch wenn der Senat als langhaariger junger Mann gezeigt wird, können die Bilder in Details voneinander abweichen. So kann die Senatspersonifikation auch einen Lorbeerkranz ${ }^{50}$ oder ein Diadem $^{51}$ tragen. Anhand dieser Synkletos-Köpfe mit Diadem wurde versucht, direkte hellenistische Vorläufer zu postulieren; ${ }^{52}$ eine solche Ableitung von Diadochen-Porträts ist jedoch schon aufgrund fehlender hellenistischer Belege für Synkletos nicht zu beweisen. Zudem zeigen die Münzen aus Smyrna, die das gemeinsame Urbild wiedergeben, keine Büste mit Königsbinde. Wesentlich wahrscheinlicher ist es deshalb anzunehmen, dass das Diadem eine spätere Zutat ist, so dass ein traditionelles Zeichen der Autorität auf ein neues Bild übertragen wurde. Grundsätzlich ist Synkletos wesentlich häufiger barhäuptig dargestellt als sein ikonographisches Gegenstück, der griechische Demos. ${ }^{53}$

48 S. Anhang I.

49 Zu den kleinasiatischen Prägebezirken: K. Kraft, Das System der kaiserzeitlichen Münzprägung in Kleinasien (Berlin 1972); zum Prägebezirk Smyrna: Klose 1987, a.a.O. (Anm.23), 86-91. Die Stempelkopplungen anhand von pseudoautonomen SynkletosPrägungen exemplifiziert durch Harl 1987, a.a.O. (Anm.16), Taf. 6, 4-8.

50 z.B. Aphrodisias (gordianisch): M. Spoerri Butcher, Roman provincial coinage VII 1: Province d'Asie (Paris, London 2006): 241 f. no 629-631; Klazomenai (69-79): A. Burnett, M. Amandry, I. Carradice, Roman provincial coinage II: From Vespasian to Domitian (AD 69-96) (London, Paris 1999), $160 \mathrm{n}^{\circ} 1033,1-4$.

51 z.B. Phokaia (Ionia) (235-238): I.A. Carradice, Sylloge Nummorum Graecorum VI: The Lewis Collection in Corpus Christi College Cambridge. Part II: The Greek Imperial coins (Oxford 1992) nº 1440 Taf. 31 1440(1); Aphrodisias: B.V. Head, Catalogue of the Greek coins of Caria, Cos, Rhodes, \& C. (London 1897), 30 f. n 29-32 Taf. V 13.

52 Forni 1953, a.a.O. (Anm.2) 62 f.; K. Harl, Political attitudes of Rome's eastern provinces in the third century A.D. (Yale 1978), 282; Missere 1990, a.a.O. (Anm.9), 98 Anm.171.

53 Vgl. die Zusammenstellung bei Missere 1990, a.a.O. (Anm.9), 89. 
Zur zweiten Option: Interessanter als diese Variationen eines Urbildes sind freilich die Fälle, in denen für Synkletos eine ganz andere, vom Erscheinungsbild der Kultstatue in Smyrna grundsätzlich abweichende Ikonographie gewählt wurde. Aufschlussreich sind sie vor allem deswegen, weil sie zeigen, dass die Ikonographie der Senatsgottheit nicht zentral von Rom vorgeschrieben war und dass die Entscheidung, welches Bild verwendet wurde, auf Seiten der prägenden Poleis, also der provinzialen Führungsschicht lag.

Vergleichsweise häufig sind Münzen, die dem griechischen Sprachgebrauch entsprechend die Büste einer Frau mit oder ohne Schleier zeigen. ${ }^{54}$ Eine Besonderheit bietet eine Prägung des 2. oder 3. Jhs. aus Perperene in Mysien (Abb. 3): ${ }^{55}$ Hier trägt Synkletos eine Mauerkrone ganz in Tradition einer hellenistischen Stadtgottheit, wie sie mit der so genannten Tyche von Antiochia beispielhaft konzipiert wurde. ${ }^{56}$ In all diesen Fällen zeigt sich in der Ikonographie die Abhängigkeit von der griechischen Boulê. Auf Münzen ist die Ratsversammlung nämlich meist ebenso in Form eines Frauenkopfes wiedergegeben, wobei Boulai wie auch Synkletoi selten mit Mauerkrone auftreten. ${ }^{57}$ Auch hierin folgt also die Senatsgottheit der lokalen städtischen Form der Ratsversammlung. Unterschiede bestehen vor allem darin, dass weibliche Synkletoi anscheinend viel seltener Diadem oder Kranz tragen wie dies die Hierai Boulai, vor allem seit der Regierungszeit Caracallas tun. ${ }^{58}$

Eine Besonderheit sind die Münzen, die Synkletos auch mit ikonographischen Mitteln mit dem Kaiserhaus in Bezug setzen. Am einfachsten nachzuvollziehen ist dies im Fall der Prägungen aus Apollonis, die zur Legende IEPA $\Sigma$ YNK $\Lambda$ HTO $\Sigma$ eine weibliche Büste mit typischem flavischen Löckchentoupet zeigen, ${ }^{59}$ so dass deshalb m. E. zu Recht eine Verbindung

54 Darstellungen einer weiblichen Senatsgottheit sind jedoch deutlich seltener als die der jugendlich-männlichen, da viele der von Forni 1953, a.a.O. (Anm.2) und auch noch von Kienast 1985, a.a.O. (Anm.2) als weiblich angesprochenen Senatsdarstellungen auf Münzen nur Varianten des normalen Typus sind.

55 F. Imhoof-Blumer, Kleinasiatische Münzen (Wien 1901), 32 s.v. Perperene Nt. 1; Forni 1953, a.a.O. (Anm.2), $87 \mathrm{n}^{\circ} 22$ Taf II 16; Kienast 1985, a.a.O. (Anm.2), 267 f. Anm.65; M.B. Barth, J. Stauber, 'Die Münzen von Perperene', Epigraphica anatolica 23 (1994) 63 f. nº 17

56 Meyer 2006, a.a.O. (Anm.41), vor allem 175-178; zur Mauerkrone: ebenda, 110-112, 157-164.

57 Vgl. die Tabelle bei Missere 1990, a.a.O. (Anm.9), 101.

58 Ebenda $77 \mathrm{f}$.

59 Burnett, Amandry, Carradice 1999, a.a.O. (Anm.5o), 148 nº 950, 1-8; 951, 1-10; 952, 1-3. 


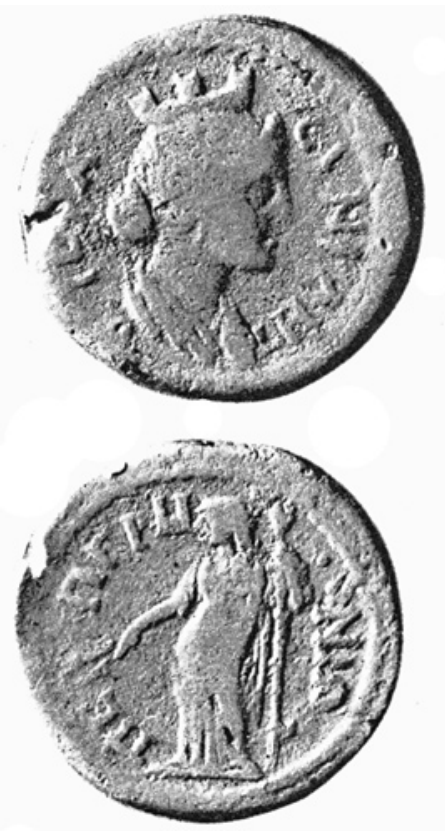

Abb. 3: AE Perperene: Büste der Synkletos mit Mauerkrone; Demeter

zu Domitia oder Iulia Titi gezogen wurde. ${ }^{60}$ Schwieriger zu belegen ist die Verknüpfung von Kaiserporträt und jugendlichem Normaltyp der Personifikation, doch erscheinen die physiognomischen Ähnlichkeiten zwischen Nero und Synkletos auf Münzen aus Sardes ${ }^{61}$ und zwischen Vespasian und Synkletos aus Kyme ${ }^{62}$ überzeugend. Die Personifikation des Senats konnte folglich auf verschiedene Weise 'belebt' werden konnte. In diesen Fällen sollte durch eine Angleichung an Bildnisse des Kaisers und seine Familie der enge Bezug zum Herrscherhaus und somit zu Rom zum Ausdruck gebracht werden, ist der Kult des Senats doch im Kontext des Kaiserkultes in Smyrna etabliert worden. ${ }^{63}$

60 So bereits Forni 1953, a.a.O. (Anm.2), 61; vgl. jetzt auch U. Hahn, Die Frauen des römischen Kaiserhauses und ihre Ehrungen im griechischen Osten anhand epigraphischer und numismatischer Zeugnisse von Livia zu Sabina (Saarbrücken 1994), 241, 359 n 246-247.

61 Burnett, Amandry, Ripollès 1992, a.a.O. (Anm.23), $490 \mathrm{n}^{\circ} 3008,1-19$.

62 Burnett, Amandry, Ripollès 1992, a.a.O. (Anm.23), $15^{2} \mathrm{n}^{\circ}$ 971, 1-9.

63 Zum Phänomen der Angleichung auch über Geschlechtergrenzen hinaus: R. Varner, 'Transcending Gender: Assimilation, Identity and Roman Imperial Portraits', in S. Bell, I. Hansen (eds.), Role Models: Identity and Assimilation in the Roman World (Ann Arbor 2008), 185-205. 


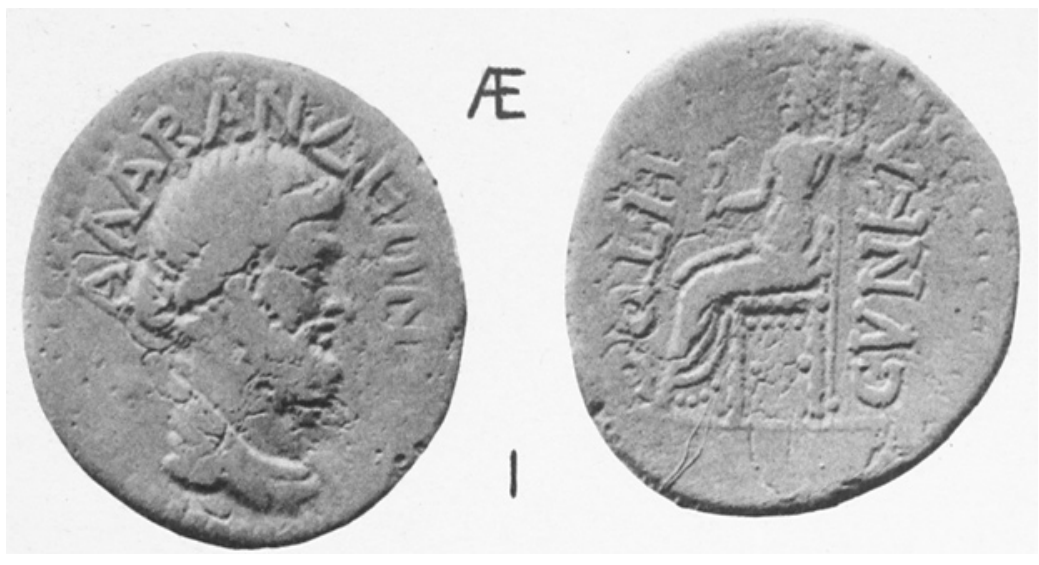

Abb. 4: AE Alabanda: Demos, Synkletos thronend

An einer Münze aus Alabanda lässt sich eine andere Option aufzeigen (Abb. 4), wie Synkletos als Gottheit 'aufgeladen' wurde:64 Dargestellt ist die Personifikation als ganzfigurige Sitzstatue, im Vergleich zu den vielen Büsten eine eher unübliche Form der Wiedergabe. Die - wohl weibliche Senatsgottheit sitzt auf einem Thron und hält in der erhobenen linken Hand ein langes Stabzepter, in der ausgestreckten Rechten einen oben eingekrümmten Stab, einen lituus. Das Sitzmotiv an sich und das Szepter erinnern eindeutig an thronende Gottheiten, den Bewohnern in Alabanda bekannt vor allem von gleichzeitigen Münzbildern des thronenden Zeus. ${ }^{65}$ Anders verhält es sich mit dem lituus, der etwas spezifisch Römisches ist: Bekannterweise ist der Krummstab Signet der Auguren, doch kann er auch Insignie der Befehlsgewalt, des imperium, und somit im wahrsten Sinne des Wortes ein imperiales Zeichen sein. ${ }^{66}$ In diesen Kontext gehört die Darstellung des Augustus auf der Gemma Augustea mit lituus. ${ }^{67}$ In Alabanda selbst ist Vespasian mit einem Krummstab auf einer Münze wiedergegeben. ${ }^{68}$ Die Verbindung Synkletos - lituus kommt bereits früher

64 Burnett, Amandry, Ripollès 1992, a.a.O. (Anm.23), 183 n $^{\circ}$ 1203, 1; vgl. auch Canciani 1994, a.a.O. (Anm.12), $828 \mathrm{n}^{\circ} 5$.

65 Burnett, Amandry, Ripollès 1992, a.a.O. (Anm.23), 183 n $^{\circ} 1202,1$.

66 Siebert 1999, a.a.O. (Anm.26), 130-132.

67 E. Zwierlein-Diehl, Magie der Steine. Die antiken Prunkkameen im Kunsthistorischen Museum (Wien 2008), 98-123; zum lituus: ebenda 101 f., 116 Abb. 40.

68 Es ist dies die Vorderseite der Prägung mit dem thronenden Zeus (hier Anm.59). 
auf Münzen vor, so in iulisch-claudischer Zeit in Pergamon, Metropolis oder Akmoneia, um nur einige Beispiele zu nennen. ${ }^{69}$

Die Prägung aus Alabanda zeigt somit exemplarisch zwei unterschiedliche Optionen, den Senat mit besonderer Befehlsgewalt darzustellen: zum einen durch Angleichung an übliche griechische Götter-Ikonographie, zum anderen durch Anleihen an die offizielle Bildersprache des römischen Herrscherkultes.

Insgesamt lassen sich somit in der kleinasiatischen Münzprägung verschiedene Mittel unterscheiden, die Senatsgottheit ikonographisch als Gottheit zu konstituieren und als entsprechend machtvoll ins Bild zu setzen:

- Herstellung eines Bezugs zum Kaiserhaus in Form der Bildnisangleichung - Übernahme von Elementen der offiziellen römischen Bildersprache

- Übernahme von Elementen der griechischen Götterikonographie

- Übernahme von Elementen der hellenistischen Herrscherikonographie

Die mit Abstand meisten der eben betrachteten Sonderfälle der SynkletosIkonographie stammen aus dem 1. Jh. n. Chr., seit dem 2. Jh. folgt die überwiegende Anzahl der Darstellungen dem jugendlichen Erscheinungsbild des Prototyps in Smyrna mit nur geringen Variationen. Innerhalb einer Zeitspanne von ungefähr 100 Jahren erfolgte eine Integration nicht nur des provinzialen Kultes, sondern auch die Übernahme der durch die Kultstatue in Smyrna fixierten Ikonographie in einen Großteil der asiatischen Städte.

Darin wird deutlich, wie sich nach anfänglicher Experimentierfreudigkeit eine homogenere Vorstellung vom Synkletos unter Verwendung des für den Provinzialkult in Smyrna konzipierten Urbilds als Gegenstück zum Demos und zur Boulê durchsetzte. Konsequente Einheitlichkeit z. B. als Folge zentralen Transports und einer offiziell geregelten Verbreitung von Kultikonographie ist jedoch nicht festzustellen.

\section{Integration von Synkletos in die städtischen Panthea Kleinasiens}

$\mathrm{Zu}$ fragen ist nun, welchen Stellenwert die Senatspersonifikation als Gottheit, aber auch als römische Institution im kaiserzeitlichen Kleinasien

69 Burnett, Amandry, Ripollès 1992, a.a.O. (Anm.23), 404 no 2376, 1-6; ähnlich Münze aus Akmoneia: ibid., $513 \mathrm{n}^{\circ} 3167,1-5$; aus Metropolis: ibid., 426 f. n ${ }^{\circ}$ 2524-2525 usw. 
besaß. Die große Bedeutung wird zum einen dadurch sichtbar, dass Synkletos auf Münzen an Stelle des Kaisers treten kann. Bei den bisher betrachteten Münzen handelt es sich meist um so genannte pseudoautonome Prägungen, die so bezeichnet werden, weil kein Kaiserbild auf die Vorderseite der Münzen gesetzt wurde, so dass der Anschein einer unabhängigen Stadtprägung entstehen kann. ${ }^{70}$

Grundsätzlich lässt sich bei einer übergreifenden Untersuchung der Rückseiten der Synkletos-Münzen feststellen, dass im Motivrepertoire keine Kombinationen besonders häufig vorkommen und sich die Prägungen nicht vom Rest der anderen 'Greek Imperials' unterscheiden. ${ }^{71}$ Mit großem Abstand werden zusammen mit Synkletos herkömmliche Gottheiten des lokalen Pantheon dargestellt. Neben Athena, Zeus, und anderen olympischen Göttern werden auch Kybele, Sarapis und Men bedacht. Synkletos tritt zudem besonders häufig zusammen mit der ephesischen Artemis auf. Diese Vergesellschaftung ist ein Indiz dafür, dass Artemis eben nicht als Zeichen des offenen oder verdeckten Widerstandes gegen Rom interpretiert werden kann, wie dies vor kurzem von J. Elsner behauptet wurde. ${ }^{72}$ Artemis-Verehrung und Verehrung römischer Instanzen sind tatsächlich nur zwei Seiten einer Medaille. Annähernd gleich häufig sind Personifikationen mit Synkletos verbunden, insbesondere die entsprechende Stadtgöttin bzw. Tyche oder Homonoia als Ausdruck besonderer Städtepartnerschaften. ${ }^{73}$ Es folgen in quantitativer Reihung Szenen aus dem lokalen Mythos bzw. Heroen mit dezidiertem Ortsbezug und erst anschließend spezifisch 'römische' Motive wie der Kaiser oder römische Personifikationen, d. h. Roma oder der Demos der Römer. SynkletosMünzen zeigen folglich kein besonderes 'römisches' Spektrum und auch keine abweichende Mengenverteilung, sondern spiegeln das 'normale' Repertoire kleinasiatischer Münzen wider. Die Senatsgottheit wird also in das kleinasiatische religiöse System integriert bzw. ist ein integrales

$70 \mathrm{Zu}$ 'pseudoautonomen' Prägungen: A. Johnston, “The so-called "pseudo-autonomous" Greek imperials', American Numismatic Society Museum Notes 30 (1985), 89-112.

71 Die Angaben beruhen auf einer Suche mit Hilfe der Datenbank „Projekt ISEGRIM Datenbank kleinasiatischer Münzen" des Lehrstuhls für Alte Geschichte der HeinrichHeine-Universität Düsseldorf (Stand 2004); zu Rate gezogen auch: P.R. Franke, W. Leschhorn, A.U. Stylow, Sylloge Nummorum Graecorum Deutschland, Sammlung von Aulock. Index (Berlin 1981).

72 J. Elsner, 'The origins of the icon: pilgrimage, religion and visual culture in the Roman East as "resistance" to the centre', in S. Alcock (ed.), The early Roman Empire in the East (Oxford 1997), 178-199, vor allem 188-191.

73 Zu Homonoia-Prägungen: P.R. Franke, M.K. Nollé, Die Homonoia-Prägungen Kleinasiens und der thrakischen Randgebiete (Saarbrücken 1997). 
Bestandteil dieses Systems, da sie auch einen Anteil an der Ausbildung dieses Systems hat.

Synkletos als Teil städtischer Kultpraxis:

Die Stiftung des Salutaris in Ephesos

Bestätigt wird dieses Ergebnis durch einen Beleg aus einem anderen Kontext, der Stiftung des C. Vibius Salutaris aus trajanischer Zeit in Ephesos: ${ }^{74}$ Salutaris stiftet $3_{1}^{1}$ Statuen aus Edelmetall, die in einer großen Prozession durch die Stadt getragen werden sollen. ${ }^{75} \mathrm{Zu}$ diesen Skulpturen gehören neun Artemis-Statuen, ein Trajan- und ein Plotina-Porträt, Statuen der Stadtgründer Androklos und Lysimachos und zahlreiche Personifikationen, so die Boulê von Ephesos, die Gerousia, der Ritterstand, die Ephebeia, Darstellungen der Phylen, der Demos der Römer und eben der Senat. ${ }^{76}$ Auch wenn es sich bei dem Stifter um einen römischen Ritter gehandelt hat, so wird hier dieselbe enge Verbindung von lokaler Hauptgottheit, den örtlichen politischen Instanzen, Akteuren der lokalen Geschichte und des lokalen Mythos, dem Kaiserhaus und Repräsentanten Roms zum Ausdruck gebracht, wie sie typisch für die Münzprägung ist. Der Stellenwert des Senats innerhalb dieses Kultfestes kann sogar noch weiter bestimmt werden: Zum einen wird er nach Artemis und dem regierenden Kaiser und seiner Frau an dritter bzw. vierter Stelle genannt und bildet zusammen mit der Personifikation des lokalen Rates ein Paar. Zum anderen weist die Gewichtsangabe von 4 Pfund und zwei Unzen hin auf eine Position zwischen den Statuen der Götter, insbesondere Artemis, der allein auch eine Goldstatue zugedacht war, und den übrigen Personifikationen (Anhang II). Das Gewicht der Statuen für Trajan und Augustus ist unbekannt, sicher ist jedoch, dass das Porträt Plotinas leichter war als die Statue von Synkletos. Auch wenn hier der Unterschied darin liegen kann, dass diese eine ganzfigurige Darstellung war, jene jedoch eine Büste, so wird dennoch deutlich, dass die Senatsgottheit zwar nicht die wichtigste Stellung, aber doch eine prominente und auffällige Position innerhalb der Statuenprozession einnahm. Wie bei den Münzen zeigt sich die aktive

74 Grundlegend: Rogers 1991, a.a.O. (Anm.16); zuletzt F. Graf, 'Ritual restoration and innovation in the Greek cities of the Roman Imperium', in A. Chaniotis (ed.), Ritual dynamics in the Ancient Mediterranean (Stuttgart 2011), 105-117 (mit der älteren Lit.).

75 Übersetzung der Inschrift: Rogers 1991, a.a.O. (Anm.16), 152-185.

76 Zur Statue des Senats: Rogers 1991, a.a.O. (Anm.16), $91 \mathrm{f.}$ 
Einbindung in ein lebendiges Kultsystem, die nicht darauf hinweist, dass die Verehrung des Senats eine bloße Pflichtübung gegenüber Rom war. Interessant in mehrfacher Hinsicht ist, dass Synkletos zusammen mit der Personifikation der Boulê genannt wird und nicht mit dem Demos der Römer: Diese Zusammenstellung zeigt erstens, dass man nicht nach römischem Vorbild agierte, sondern eigene Vorstellungen umsetzte, da in der Hauptstadt Genius Senatus und Genius Populi Romani regelhaft kombiniert wurden. Zweitens wird auch klar, dass die römischen Instanzen nicht als einheitlicher (Fremd-)Körper aufgenommen wurden, sondern dass man sie mit lokalen Einrichtungen zusammennahm. Drittens ist auffällig, dass anscheinend immer Frau-Mann-Paare miteinander verbunden werden, vorausgesetzt, dass auch hier der Senat als männliche Personifikation auftritt, so dass für die ephesische Prozession eine Darstellung von Synkletos in Gestalt eines jungen Mannes vermutet werden kann.

\section{Ergebnis}

Für die Integration des Senats war der römische Kaiser Ausgangspunkt, da nach Tacitus Tiberius ihn als Kultempfänger in Smyrna bestimmte. In kaiserlichem Auftrag wurde auch eine bildliche Umsetzung der römischen Institution geschaffen, die weder griechischen Vorstellungen noch der - späteren - römischen Visualisierung folgte. Dennoch entsprach die Verkörperung der Senatsgottheit dem grammatikalischen Geschlecht des lateinischen Terminus senatus und somit eher der römischen Auffassung. Dies wurde jedoch erst evident dadurch, dass man das griechische $\Sigma u ́ \gamma x \lambda \eta \tau \tau$ als Kultbezeichnung wählte, im Rahmen griechischer Begrifflichkeit blieb und ein - gräzisiertes - Fremdwort vermied. Fakt ist aber auch, dass es keine Anknüpfung an bereits bestehende Ikonographien von Boulê oder auch Gerousia gab. Die Aneignung des Kultbildes auf provinzweiter Ebene erfolgte durch die Poleis, wobei die lokalen Eliten zunächst die treibende Kraft gewesen sein müssen. ${ }^{77}$ Die Integration erweist sich als länger dauernder und mehrsträngiger Prozess, wie sich anhand der Bildfassungen von Synkletos ablesen lässt, wo verschiedene Möglichkeiten

77 Zur Rolle der Eliten in der Münzprägung exemplarisch: B. Weisser, 'Pergamum as paradigm', in C. Howgego, V. Heuchert, A. Burnett (eds.), Coinage and identity in the Roman provinces (Oxford 2005), 135-142; grundlegend, aber für eine andere Provinz: K. Butcher, 'Information, legitimation, or self-legitimation? Popular and elite designs on the coin types of Syria', in ebenda, 143-156. 
zur Verbesserung und Erleichterung der Akzeptanz wie Femininisierung bzw. Angleichung an Kaiserbildnisse erprobt (und im Laufe der Zeit überwiegend wieder verworfen) werden. Als entscheidend erweist sich die provinziale Struktur, die Kulttradition und wohl auch die kaiserliche Autorität bzw. Legitimierung des Kultbildes im Tempel von Smyrna. Als Gottheit werden der Senat und sein Bild schließlich integraler Bestandteil der städtischen Panthea wie Einheitlichkeit und Beständigkeit der Ikonographie bezeugen. Selbst der Widerspruch zwischen grammatikalischem Geschlecht und Visualisierung wird nicht als so gravierend angesehen, dass er unbedingt aufzulösen wäre. Synkletos erweist sich dadurch auch als eine Gottheit mit eigenem griechischem Charakter, was wiederum dem Bild des Kaiserkultes in Kleinasien gleicht. Es wäre jedoch ein Fehler, nur die griechische Komponente zu betonen und in Synkletos nur eine Gottheit zu sehen, die zwar anfangs etwas mit Rom zu tun hatte, später aber dann so perfekt integriert war, dass sie ein vollständig kleinasiatischeinheimisches Eigenleben entwickelte. Ein Blick auf die Verbreitung der Zeugnisse für die Senatsgottheit, im Wesentlichen von Münzen und Inschriften, in Kleinasien belegen die römische Kondition: Mit nur wenigen Ausnahmen, z.B. aus Mallos in Kilikien oder Side in Pamphylien, bleibt die Verehrung der Senatsgottheit auf die Provinz Asia beschränkt. ${ }^{78}$ $\mathrm{Zu}$ erklären ist dieses Muster durch die provinzial gebundene Organisation des Kultes und wiederum deren Abhängigkeit von politischen Verwaltungsgrenzen die von Rom festgelegt wurden. Bei aller Beliebtheit in Asia, Synkletos wird nicht exportiert und besitzt kein wirkliches Eigenleben jenseits der Grenzen. Trotz aller Eigenständigkeit und der gelungenen Integration in ein griechisches religiöses System wird doch deutlich, dass es sich hier um ein provinziales religiöses System im wörtlichen Sinne einer Provinzialreligion handelt, das ohne Rom nicht funktionieren kann. ${ }^{79}$

78 J. und L. Robert, Bulletin épigraphique (1954), 112; Forni 1953, a.a.O. (Anm.2), 11-14: 99 Städten, die Münzen mit Synkletos prägen, stehen drei in Creta et Cyrenaica (einer anderen senatorischen Provinz), drei in Cilicia und einer in Pisidia gegenüber.

79 Zum Begriff der Provinzialreligion: J. Rüpke, 'Römische Religion und „Reichsreligion": Begriffsgeschichtliche und methodische Bemerkungen', in H. Cancik, J. Rüpke (eds.), Römische Reichsreligion und Provinzialreligion (Tübingen 1997), 3-23; zum Thema: M. Beard, J. North, S. Price, Religions of Rome I: A History (Cambridge 1998), 313-363; C. Ando, 'Exporting Roman religion', in J. Rüpke (ed.), A companion to Roman religion (Malden, MA 2007), 429-445. 
Anhang I: Liste der Städte der Provinz Asia, die pseudoautonome Münzen mit der jugendlichen, langhaarigen Synkletos-Personifikation prägen ${ }^{80}$

Verwendete Abkürzungen:

Aulock, Phrygien: H. von Aulock, Münzen und Städte Phrygiens (Tübingen 1980/1987).

BMC: H. Mattingly, Coins oft he Roman Empire in the British Museum (London 1923-1950).

Fitzwilliam: S.W. Grose, Catalogue of the MacClean Collection of Greek coins. Fitzwilliam Museum, Cambridge vol. 3 (Cambridge 1929).

Fritze, Mysien: H. von Fritze, Die antiken Münzen Mysiens (Berlin 1913)

Imhoof, GM: F. Imhoof-Blumer, Griechische Münzen (München 189o).

Imhoof, KM: F. Imhoof-Blumer, Kleinasiatische Münzen (Wien 1901/02).

Imhoof, LS: F. Imhoof-Blumer, Lydische Stadtmünzen (Genf 1897).

Klose, Smyrna: D. Klose, Die Münzprägung von Smyrna in der römischen Kaiserzeit (Berlin 1987).

RPC I: A. Burnett, M. Amandry, P.P. Ripollès, Roman provincial Coinage I: From the death of Caesar to the death of Vitellius (London 1992)

Slg. Lindgren: H.C. Lindgren, Ancient bronze coins of Asia Minor and the Levant from the Lindgren collection (San Mateo/CA 1985).

Slg. Weber: L. Forrer, The Weber Collection vol. 3: Asia minor (London 1925-1929)

SNG: Sylloge nummorum Graecorum

Aiolis:

Aigai (177-192; 193-211): Slg. Lindgren I 387; SNG Kopenhagen XXI 17

Elaia (63-68; 238-244): RPC I 2409; Slg.Weber 5557

Kyme (70-73; 117-192; 238-244; 253-268): SNG Slg. von Aulock 1645; SNG Tübingen 2703; SNG Kopenhagen XXI 126; SNG Kopenhagen XXI 128(1)

Myrina (Kaiserzeit): SNG Kopenhagen XXI 227

Temnos (200-250 bzw. 218-222; 222-235; 238-244; 244-249; 253-268): SNG Kopenhagen XXI 266; SNG Kopenhagen XXI 267; SNG Kopenhagen XXI 270; SNG Slg. Righetti (Bern) 0825/ SNG Kopenhagen XXI 272; SNG Slg. von Aulock 1676

80 Der Katalog erhebt keinen Anspruch auf Vollständigkeit und ist vor allem gedacht als Überblick über lokale und chronologische Verteilung der Prägungen und als Hilfsmittel zur Auffindung von Abbildungen. 


\section{Ionien:}

Erythrai (161-180; 244-249 bzw. 260; Kaiserzeit): SNG Kopenhagen XXIII 749; SNG Kopenhagen XXIII 751; SNG Kopenhagen XXIII 779; BMC XIV 143 Nr.230

Klazomenai 69-79; (Kaiserzeit): SNG Kopenhagen XXII 110; SNG Kopenhagen XXII 111

Kolophon (238-249): Imhoof, KM 72 Nr.16

Magnesia am Mäander (200-276): SNG Kopenhagen XXIII 858

Phokaia (222-235; 235-238; 238-244; 244-249; Kaiserzeit): SNG Kopenhagen XXIII 1051; SNG Slg. von Aulock 2139; SNG Kopenhagen XXIII 1055; SNG Slg. Lewis (Cambridge) 1442; BMC XIV 218 Nr.116

Smyrna (54-68; 68-70; 100-105; 161-169; 182-185; 211-260; 218-222; 222235; 235-244; 238-240; 238 bzw. 241-249; 239-241; 242-244; 244-249 bzw. 255): RPC I 2481; RPC I 2489; SNG Kopenhagen XXIV 1253; SNG Kopenhagen XXIV 1299; SNG Kopenhagen XXIV 1303; SNG Kopenhagen XXIV 1323; Klose, Smyrna Nr. XVI; Klose, Smyrna Nr. XVIII; SNG Tübingen 3243; SNG Kopenhagen XXIV 1322; SNG Kopenhagen XXIV 1325; Klose, Smyrna Nr. XVIII; SNG Kopenhagen XXIV 1314; SNG Kopenhagen XXIV 1318; SNG Tübingen 3205; SNG Kopenhagen XXIV 1326

Teos (Kaiserzeit): SNG Kopenhagen 24, 1492

\section{Karien:}

Antiocheia (238-268; Kaiserzeit): SNG Kopenhagen XXV 42; Fitzwilliam 4671

Aphrodisias (100-276; 161-180; 193-211; 238-244; 250-276; 253-268; Kaiserzeit): SNG Slg. von Aulock 8o61; SNG Kopenhagen XXV 98; SNG Kopenhagen XXV 99; SNG Kopenhagen XXV 104; SNG Slg. von Aulock 8062; Fitzwilliam 4680; SNG Kopenhagen XXV 95

Apollonia (100-150; 161-180; 193-211): J.C. Milne, Numismatic Chronicle 1938, 257; SNG Slg. von Aulock 2481; SNG Slg. von Aulock 8068

Antiocheia (238-268; Kaiserzeit): SNG Slg. Righetti (Bern) o939; SNG Kopenhagen XXV 41

Attuda (180-220; 177-192; 193-211; Kaiserzeit): SNG Kopenhagen XXV 166; SNG Kopenhagen XXV 167; SNG Slg. Lewis (Cambridge) 1648; Imhoof, GM $87 \mathrm{Nr} .3$

Herakleia (161-169; 180-220; 193-211; Kaiserzeit): Imhoof, KM 134 Nr.9; SNG Slg. von Aulock 2544; SNG Slg. Lewis (Cambridge) 1656; SNG Kopenhagen XXV 394

Kidramos (Kaiserzeit): BMC XV 81 Nr.1 
Neapolis (69-96): SNG Kopenhagen XXV 451

Orthosia (180-220; Kaiserzeit): SNG Slg. von Aulock 2646; SNG Kopenhagen XXV $45^{8}$

Sebastopolis (100-276; 200-230; Kaiserzeit): BMC XV 146 Nr.4; SNG Kopenhagen XXVI 462; SNG Kopenhagen XXVI 463

Trapezopolis (180-220): SNG Slg. von Aulock 2738

Lydien:

Akrasos (193-211): BMC XVI 9 Nr.3

Aninetos (Kaiserzeit): Imhoof, KM 168 Nr.1

Apollonis (Kaiserzeit): SNG Kopenhagen XXVII 25

Apollonnoshieron (14-10o bzw. 217; 198-217): BMC XVI XXIII Nr. 4; BMC XVI 23 Nr.5; SNG Slg. Righetti (Bern) 1006

Attaleia (100-276; Kaiserzeit): SNG Kopenhagen XXVII 34(1); SNG Slg. von Aulock 2909

Bageis (98-117; 117-138; 161-192; 193-235; 200-268): SNG Slg. von Aulock 2913; BMC XV 31 Nr.4; Slg. Lindgren III 552A; SNG Kopenhagen XXVII 45; SNG Kopenhagen XXVII 44

Blaundos (100-276; 161-180; 251-253): SNG Kopenhagen XXVII 83; SNG Slg. Lewis (Cambridge) 1521; SNG Slg. von Aulock 2922

Daldis (69-96; 193-211; 198-268; Kaiserzeit): SNG Kopenhagen XXVII 110; BMC XVI 70 Nr.6; Slg. Lindgren III 456A; SNG Slg. von Aulock 1647(1)

Dioshieron (100-200; 138-192 bzw. 268): Imhoof, LS 65 Nr.7; SNG Slg. Righetti (Bern) 1022(1)

Gordos Iulia (117 bzw. 177-192): SNG Slg. von Aulock 2980; BMC XVI 90 Nr.5

Hermokapelia (117-138; 193-211): SNG Kopenhagen XXVII 165; BMC XVI $100 \mathrm{Nr} .13$

Hierokaisareia (54-138; 138 bzw. 177-192): SNG Slg. von Aulock 2957; Imhoof, LS 19 Nr.40

Hypaipa (Kaiserzeit): IMHOOF RSN 1923 S299,312

Hyrkanis (Kaiserzeit): BMC XVI 123 Nr.10

Kilbanioi (82-96): SNG Slg. Righetti (Bern) 1017(1)

Maionia (98-117; 161-180; 193-217; 249-251): Slg. Lindgren III 483; SNG Kopenhagen XXVII 219; SNG Slg. von Aulock 3014; SNG Kopenhagen XXVII 228

Magnesia (-27 bzw. 17-37; 138-268; 238-244): RPC I 2453; RPC I 2452A-C; SNG Kopenhagen XXVII 245; SNG Kopenhagen XXVII 249 
Mosteni (69-79; 117-192): Imhoof, KM 177 Nr.1; SNG Slg. Lewis (Cambridge) 1435

Nakrasa (98-161): SNG Slg. von Aulock 3034

Nysa (200-276): SNG Kopenhagen XXVII 311

Philadelpheia (98-117; 161-180; 193-211 bzw. 250; 198-268): Slg. Lindgren III 496; SNG Kopenhagen XXVII 354; BMC XVI 190 Nr.26; SNG Slg. von Aulock 3063; SNG Slg. von Aulock 3066

Saitta (193-268): SNG Slg. von Aulock 3091

Sala (98-117 bzw. 161; 138-161; Kaiserzeit): SNG Kopenhagen XXVIII 421; Slg. Lindgren III 512A; SNG Slg. von Aulock 3110; Imhoof, LS 132 Nr.3

Sardes (54-68; ca. 65; 70-73; 198-244): SNG Kopenhagen XXVIII 506; RPC I 3008; SNG Tübingen 3801; SNG Slg. Righetti (Bern) 1081

Silandos (98-117; 161-169 bzw. 192): Imhoof, LS 143 Nr.1; SNG Slg. von Aulock 3168; BMC XVI 278 Nr.3

Stratonikeia (98-138): SNG Kopenhagen XXVIII 555

Tabala (190-220): SNG Slg. von Aulock 8270

Thyateira (98-138; 100-200; 161-192; 198-217; 222-235; 235-238; Kaiserzeit): SNG Tübingen 3845(1); SNG Slg. Righetti (Bern) 1098; BMC XVI 296 Nr.28; Imhoof, LS 158 Nr.26; SNG Slg. von Aulock 3210(1); IMHOOF RSN 1923 S308,337; SNG Kopenhagen XXVIII 589

Tmolos (69-161): Imhoof, LS 164 Nr.2

Tomaris (Kaiserzeit): Imhoof, LS 165 Nr.3

Tralleis (161-276; 253-26o): BMC XVI 342 Nr.104; Imhoof, KM 187 Nr.7 Tripolis (98-117; 100-200; 138-192; 193-268 bzw. 200-276): SNG Slg. von Aulock 8295; SNG Kopenhagen XXVIII 721; SNG Slg. von Aulock 3301; SNG Slg. von Aulock 3305; SNG Kopenhagen XXVIII 735(1)

\section{Mysien:}

Attaia (98-117; 117-192; 193-211): SNG Slg. von Aulock 7203; SNG Slg. von Aulock 1070; Fitzwilliam 4139

Germe (100-200; 117-192;138-192; 238-244; Kaiserzeit): SNG Slg. von Aulock 7214; SNG Tübingen 2199; SNG Kopenhagen XXVII 124; SNG Kopenhagen XXVII 129; SNG Kopenhagen XXVII 122

Hadrianeia (117-138 bzw. 161; 193-211; 198-235): SNG Slg. von Aulock 1126; Fritze, Mysien 440; SNG Kopenhagen 19 166; Fritze, Mysien 441 Hadrianothera (117-138; 211-268): SNG Slg. von Aulock 1146; Fritze, Mysien 556 
Pergamon (-27-138; 40-6o; 100-200; 238-268; 258-26o): SNG Kopenhagen 19 446; RPC I 2374-2378; SNG Slg. Righetti (Bern) o748; BMC XII 137 Nr.235; SNG Kopenhagen 19460

\section{Phrygien:}

Aizanoi (-27-38; Chr. Geb.-100; 38 bzw. 41-54; 117-138; 200 bzw. 253-268; 250-276; Kaiserzeit): Imhoof, 190 Nr.7; SNG Slg. Righetti (Bern) 1125; RPC I 3097. 3104-3106; BMC XVII 24 Nr.6; SNG Kopenhagen XXIX 68; SNG Kopenhagen XXIX 66; SNG Slg. von Aulock 8302; SNG Kopenhagen XXIX 67

Akkilaion (238-244): Aulock, Phrygien I 1

Alia (98-117; 161-180): Aulock, Phrygien 1 39; Aulock, Phrygien 1 Nr.61

Ankyra (62/63; 69-96; 100-276; 138-161; 193-211 bzw.235): RPC I 3113A-B; SNG Slg. von Aulock 3425; SNG Slg. von Aulock 3426; SNG Slg. Lewis (Cambridge) 1515; BMC XVII 6o Nr.15; SNG Kopenhagen XXIX 130 Apameia (244-268; 251-253): Slg. Weber 7032; BMC XVII 91 Nr.132 Appia (98-117): SNG Slg. von Aulock 3519 Dionysopolis (221/222): Aulock, Phrygien II 88

Dokimeion (100-276; 193-211): SNG Kopenhagen XXIX 353; SNG München XXIV 177

Eumeneia (190 bzw. 193-230): Slg. Weber 7092; SNG Kopenhagen XXIX 389

Grimenothyrai (97 bzw. 117-138): Aulock, Phrygien II 329; Aulock, Phrygien II 395

Hadrianopolis (117-138): SNG Tübingen 3831

Hierapolis (98-117 bzw. 217; 118-222; 198-268; 218-222): SNG Kopenhagen XXX 413; SNG Kopenhagen XXX 414; SNG München XXIV 227; SNG Kopenhagen XXX 445; SNG Slg. von Aulock 3633

Hyrgaleis (198-235; 221/222; 240/241): Aulock, Phrygien I 333; Aulock, Phrygien I 350; Aulock, Phrygien 1357

Kadi (41-54; 117-138): SNG Kopenhagen XXIX 238; SNG Kopenhagen XXIX 239

Kibyra (81-96; 161-180; Kaiserzeit): SNG Slg. von Aulock 8395; SNG Tübingen 4095; SLG HUNTER S482,2

Kidyessos (178-187): SNG Slg. von Aulock 3758

Kolossai (253-268): Aulock, Phrygien II 543

Kotaion (68/69; 68-79; 253-268): RPC I 3223-3226; Slg. Weber 7071; I. Svoronos, Journal International d'Archéologie Numismatique 12(1909) 41 Nr. 1 
Laodikeia (138-161 bzw. 180; 198-217 bzw. 268): SNG Slg. von Aulock 3826; SNG München XXIV 361; Imhoof, GM S161,6; SNG Kopenhagen XXX 541 Okokleia (177-192; 238-244): Aulock, Phrygien 1 704; Aulock, Phrygien I 707

Peltai (138-192; 251-253; Kaiserzeit): BMC XVII 350 Nr.21; SNG Slg. Lewis (Cambridge) 1592; SLG WALCHER 2805

Prymnessos (79-81; 138-192; 193-238): Aulock, Phrygien II 855; Aulock, Phrygien 2 905; SNG Slg. von Aulock 3935

Sebaste (100-276; 198-268; Kaiserzeit): SNG Slg. von Aulock 3950; SNG Slg. von Aulock 3949; SNG Kopenhagen XXX 676

Synaos (69-192; 100-276; Kaiserzeit): SNG Kopenhagen XXX 700; SNG Slg. von Aulock 3963; Slg. Weber 7178

Synnada (Chr. Geb.-200; 138-161; 150-200): SNG Kopenhagen XXX 715; IMHOOF MG S413,159; SNG Slg. von Aulock 3976

Temenothyrai (161-180; 244-249; 253-268): SNG Slg. Righetti (Bern) 1229; BMC XVII 410 Nr.12; SNG Tübingen 4203

Tiberiopolis (98-117; 117-138): SNG München XXIV 497; SNG Slg. Lewis (Cambridge) 1575

Traianopolis (98-117; 117-138; 198-217): SNG Kopenhagen XXX 758; SNG Kopenhagen XXX 759; Aulock, Phrygien II 1424

Troas:

Abydos (-69): RPC I 2283

Ilion (68-69): RPC I 2317(1-6)

Pionia (117-161 bzw. 217): SNG Slg. von Aulock 7631; BMC XII 77 Nr.1

\section{Anhang II:}

Tabelle: Die Statuenprozession des Vibius Salutaris in Ephesos

\begin{tabular}{clll}
\hline Position & Darstellung & $\begin{array}{c}\text { Gewicht } \\
\text { (in g) }\end{array}$ & Material \\
\hline 1 & Traian & $?$ & Silber \\
2 & Plotina & 982 & Silber \\
3 & Artemis & 982 & Gold \\
4 & Synkletos & 1365 & Silber \\
5 & Boule & 1320 & Silber \\
6 & Artemis & 1965 & Silber \\
7 & Demos Rhomaion & $?$ & Silber \\
8 & Gerousia & $?$ & Silber \\
& & & (continued)
\end{tabular}


Tabelle (cont.)

\begin{tabular}{rlll}
\hline Position & \multicolumn{1}{c}{ Darstellung } & $\begin{array}{c}\text { Gewicht } \\
\text { (in g) }\end{array}$ & Material \\
& & $2101+\mathrm{x}$ & \\
\hline 9 & Artemis & 999 & Silber \\
10 & Hippikon Tagma & $?$ & Silber \\
11 & Ephebeia & $?$ & Silber \\
12 & Artemis & $?$ & Silber \\
13 & Augustus & $?$ & Silber \\
14 & Phyle Sebaste & $?$ & Silber \\
15 & Artemis & $?$ & Silber \\
16 & Demos & $?$ & Silber \\
17 & Phyle Epheseon & $?$ & Silber \\
18 & Artemis & $?$ & Silber \\
19 & Androklos & $?$ & Silber \\
20 & Phyle Karenaion & $?$ & Silber \\
21 & Artemis & $?$ & Silber \\
22 & Lysimachos & $?$ & Silber \\
23 & Phyle Teion & $?$ & Silber \\
24 & Artemis & $?$ & Silber \\
25 & Euonymos & $996+\mathrm{x}$ & Silber \\
26 & Phyle Euonymon & $?$ & Silber \\
27 & Artemis & $?$ & Silber \\
28 & Pion & $?$ & Silber \\
29 & Phyle Bembeinaion & 2315 & Silber \\
30 & Athena Pammousos & 1965 & Silber \\
31 & Sebaste Homonoia Chrysophoros & Silber \\
\hline
\end{tabular}




\title{
LES MARQUES CIVIQUES SUR BRIQUES ET TUILES, TÉMOINS DE L'INTÉGRATION DES CITÉS DANS LE MONDE ROMAIN
}

\author{
Monique Dondin-Payre
}

Un des effets les plus visibles de l'intégration dans le monde romain est l'organisation du territoire en cités, seul échelon administratif permanent à l'intérieur des provinces. Être constitué en cité représentait une innovation dans les régions occidentales, qui avaient connu les tribus, les royaumes, les groupes plus ou moins formels et sédentarisés, mais ce n'en était pas une pour la partie orientale de la Méditerranée. La transformation y fut cependant aussi marquée car, pour les cités grecques, indépendantes les unes des autres comme d'un pouvoir central, autonomes quant à leur choix de gouvernement et de gestion, le terme «cité » ne recouvrait que peu les réalités romaines. L'intégration civique dans l'empire romain y eut donc le même retentissement qu'en Occident. Des documents, anodins pris isolément mais significatifs une fois mis en série, apportent des éclaircissements sur les modes de cette intégration : les briques et tuiles, que qualifie au mieux le terme « civique » adopté par les Anglais, portent des marques, en latin ou en grec, qui, à la différence de la majorité des estampilles ${ }^{1}$, ne mentionnent ni les auteurs ni les intervenants de la production, mais une institution ou des magistrats municipaux, c'est-à-dire des cités. Déclarer ces briques et tuiles «publiques $»^{2}$ n'est pas satisfaisant, car l'adjectif renvoie à toutes les séries émanant de collectivités ou des autorités les représentant, quelles qu'elles soient, civiles ou religieuses ${ }^{3}$, comme on en connaît à toutes les époques; les dire «urbaines » ne convient pas non plus car le mot isole celles qui sont trouvées au chef-lieu de celles du territoire ${ }^{4}$.

1 De la considérable bibliographie, on retiendra, parmi les études les plus récentes, Epigrafia della produzione e della distribuzione. Actes de la VIe Rencontre franco-italienne sur l'épigraphie du monde romain (Rome 1994); P. Boucheron, H. Broise, Y. Thébert (eds.), La brique antique et médiévale. Production et commercialisation d'un matériau (Rome 2000); Chr. Bruun (ed.), Interpretare i bolli laterizi di Roma e della Valle del Tevere: produzione, storia economica e topografia (Rome 2005).

${ }^{2}$ H. Dressel, CIL XV (Berlin 1891), 13-14 : les lateres publici ou publice facti rassemblent les marques militaires, du patrimonium, des castra prétoriens, etc.

3 M.G. Granino Cerere, S.M. Marengo, 'Le tegulae sacrae dell'Italia romana', in Instrumenta inscripta III. Manufatti iscritti e vita dei santuari (Macerata 2012), 156-178, spéc. 170-171.

4 E. Gliozzo, 'La diffusione dei bolli laterizi "urbani" nell'Etruria romana: rapporti tra produzione "urbana" e "municipale"', in Bruun 2005, op. cit. (n. 1), 201-212. 
L'adjectif «civique» adopté par les auteurs des Roman Inscriptions of Britain $^{5}$ est parfaitement approprié. La désignation, comme le formulaire, sont essentiels. Les inscriptions sur briques et tuiles présentent une telle diversité, les abréviations, le contenu, la forme des informations sont si variés qu'il faut faire preuve de rigueur et ne retenir que les citations claires d'institutions ou de responsables civiques; les noms homonymes de magistrats connus par d'autres sources, auxquels aucune charge municipale n'est juxtaposée, ne sont pas significatifs puisqu'ils peuvent renvoyer aux propriétaires de fabriques, et non aux détenteurs du pouvoir. Le phénomène de marquage des productions de briques et tuiles est complexe, et des justifications variées en ont été proposées pour les séries des bolli de fabriques impériales ou de particuliers ${ }^{6}$. Mais les marques « civiques», envisagées en groupe, constituent un ensemble spécifique qui implique une explication propre.

Le dossier documentaire comporte plus de 80 occurrences ; l'identification comme marque civique tient souvent à la présence d'un petit nombre de signes, dont le développement est parfois discuté7. Aux deux premiers ensembles repérés, ceux de Brindisi et de Gloucester, est venu s'ajouter un nombre important de documents, dont beaucoup grâce à l'obligeance de collègues participant au colloque épigraphique de Bari en $2009^{8}$. Cet accroissement a permis d'envisager l'explication du phénomène sous un angle nouveau. Il n'est pas douteux que d'autres attestations seront repérées ${ }^{9}$, mais il est peu probable que les conclusions en soient altérées.

5 R.G. Collingwood, R.P. Wright, S. Frere, R. Tomlin, Roman Inscriptions of Britain II, 5 (Gloucester 1993), 41.

6 D. Manacorda, 'I diversi significati dei bolli laterizi. Appunti e riflessioni', in Boucheron et al. 2000, op. cit. (n. 1), 127-159, analyse les hypothèses et donne la bibliographie antérieure. Après lui, J.-J. Aubert, 'L'estampillage des briques et des tuiles: une explication juridique fondée sur une approche globale', ibid., 53-59 voit dans les estampilles, à la suite d'E.M. Steinby, un contrat résumé.

7 On peut toujours imaginer que les marques réduites à des lettres pourraient être des marques civiques, mais aucun raisonnement solide ne pourrait être fondé sur des suppositions.

8 M. Dondin-Payre, 'Les marques civiques sur briques et tuiles : état du dossier et interprétations', in M. Silvestrini (éd.), Le tribù romane (Bari 2010), 443-450. Je remercie tout particulièrement pour leur générosité $M^{\text {mes }}$ et $M^{\text {rs }}$ les Professeurs Bandelli, de Trieste; Cifarelli, de Segni ; Giorgi, de Bologne ; Luciani, de Venise ; Miranda, de Naples ; Rigato, de Bologne; Silvestrini, de Bari ; Solin, d'Helsinki ; toute ma gratitude va à $\mathrm{M}^{\text {mes }}$ les Professeurs M.G. Granino Cerere, de Sienne et M.L. Marengo, de Macerata, qui ont fait preuve d'une grande compréhension.

9 Beaucoup des attestations sont réunies au CIL XV (Berlin 1891-1899), plusieurs au CIL I2 (Berlin 1918), 113-116, mais aussi incluses dans les parties instrumentum des volumes régionaux et dans des recueils récents. 
Table 1: Les marques civiques sur céramique

N.B.: Les mots laterizi et bollatura, qui font défaut en français, recouvrent des produits que les publications ne permettent pas toujours de distinguer. D'autre part, les marques attestées en un nombre important d'exemplaires pour un seul type (à Gloucester, à Trèves ${ }^{10}$ ) figurent sous un seul numéro.

I. Italie

\section{A. Brindisium/Brindisi}

1) amphores, fabrique Aniniana, Apani, $2^{\mathrm{e}}$ moitié $\mathrm{II}^{\mathrm{e}} \mathrm{s}$. av.

CBAI, c.s. n. $115^{2}$

CIL I ${ }^{2}, 3501$, rétrograde

CIL I ${ }^{2}, 35^{21}$, rétrograde et normal

$C I L \mathrm{I}^{2}, 3502$

$\begin{array}{ll}\text { A(ulus) Caeseli(us) A(uli)f(ilius) q(uaestor) } & 1 \\ P \text { (ublius) Claudius } P \text { (ublii) } n \text { (epos) } & 2 \\ q \text { (uaestor) } & \\ N \text { (umerius) Maccius L(ucii)f(ilius) } & 3 \\ q(\text { uaestor) } & \\ L(\text { ucius }) \text { Corneli(us) L(ucii)f(ilius) } & 4 \\ q(\text { uaestor })^{11} & \end{array}$

2) tuile, fabrique de Giancola, époque d'Auguste

\begin{tabular}{ll}
\hline$A E$ 2000, 355 & L(ucio) Audio L(ucii)f(ilio) (quattuor) \\
& uir $(o)$ L(ucio) Graeceio L(ucii)f(ilio) \\
& quinq(uennale) \\
& grandes dimensions; cartouche: \\
& $29,7 \times 7,4 \mathrm{~cm}, \mathrm{cf} . \mathrm{n}^{\circ} 17$ \\
\hline
\end{tabular}

B. Praeneste/Palestrina et ager Praenestinum

tuiles, du ${ }^{\text {er }}$ s. av. au I ${ }^{\text {er }}$ s. ap.

voir aussi $\mathrm{n}^{\circ} 28$

CIL XIV, 4091, 1 = XV, 2291

P(ublio) Annio Septimo S[ex(to)] Geganio

Fimbriae (duum) [uir(is)

CIL XI, 4091, $2^{\mathrm{a} \text { et } \mathrm{b}=}$

P(ublius) Aquilius Gallus (duum)uir

$\mathrm{XV}, 2292^{\mathrm{a}}$ et b

(continued)

10 P. Steiner, 'Einige Bemerkungen zu den römischen Ziegelstempeln aus Trier', Trierer Jahresbericht 10/11 (1917/18), 15-31, spéc. 18-20.

11 Le nominatif a été préféré au génitif par rapprochement avec les $n^{\text {os }} 1-3$, sans certitude.

12 D. Manacorda, 'Produzione agricola, produzione ceramica e proprietà della terra nella Calabria romana tra Repubblica e Impero', in Epigrafia della produzione 1994, op. cit. (n. 1), 38-40; la tuile témoigne de la reprise de l'activité de la fabrique, après une phase d'abandon; $A E$ 2000, 355 : par erreur, brique. 
Table 1 (cont.)

\begin{tabular}{|c|c|}
\hline CIL XIV, 4091, $3=\mathrm{XV}, 2293^{13}$ & $\begin{array}{l}\text {-] Egnati P(ublii) Scrib(onii) } \\
\text { du[umuir(orum)] }\end{array}$ \\
\hline CIL XIV, 4091, $4=\mathrm{XV}, 2294$ & $\begin{array}{l}\text { T(iti) Long(i?) Prisc(i) et C(aii) Treb(i?) } \\
\text { Plautae / (duum)uir(orum) // P(ublii) } \\
\text { Bet(ilieni) Chresimi }\end{array}$ \\
\hline CIL XIV, 4091, 5 & $\begin{array}{l}\left.\text { marque peinte sur une tuile }{ }^{14}:-\right] \\
\left.\text { Laecanio }^{15} \text { (duum)u[ir(o) ou uiris - }\right] P E[-\end{array}$ \\
\hline $\begin{array}{l}\text { CIL XIV, 4091, } 6^{1-3}=\mathrm{XV} \\
2295,1-3\end{array}$ & $\begin{array}{l}Q(\text { uinti) Mestri C(aii) Tappuri (duum) } \\
\text { uir(orum) }\end{array}$ \\
\hline CIL XIV, 4091, $7=\mathrm{XV}, 2296$ & $\begin{array}{l}\text { M(arcus) Tere(ntius?) Cl(-) et [-] (duum) } \\
\text { [uiri] M. P(ublius) B(etilienius) Chr(esimus) }\end{array}$ \\
\hline CIL XIV, 4091, $8=\mathrm{XV}, 2298$ & $\begin{array}{l}\text { [C(aius) Voluntilius } Q \text { (uinti)f(ilius) Va]rus } \\
\text { (duum)uir iter(um) cur(ator) aed(ium) s] } \\
\text { acr(arum) S P F [II]uir }\end{array}$ \\
\hline CIL XIV, 4091, $9=\mathrm{XV}, 2297,1$ & $\begin{array}{l}\text { C. Voluntilius Q.f. Varus IIuir iter. cur. aed. } \\
\text { sacr. }\end{array}$ \\
\hline CIL XIV, 4091, $10=\mathrm{XV}, 2299$ & Macrin(ius) Nerian(ius) aed(ilis) \\
\hline CIL XIV, 4091, $11=\mathrm{XV}, 2300,2$ & $\begin{array}{l}\text { L(ucius) Vol(-) aed(ilis) } \\
\text { date: absence de surnom, avant le milieu } \\
\text { du } \mathrm{I}^{\mathrm{er}} \text { s., comme les } \mathrm{n}^{\text {os }} 17 \text { et } 18\end{array}$ \\
\hline $\begin{array}{l}\text { CIL XIV, 4091, } 12=\mathrm{I}^{2}, 3477= \\
\mathrm{XV}, 2301=A E 1888,36\end{array}$ & $\begin{array}{l}\text { Ma(nius) Later(ius) }(\text { et non Lateranus })^{16} \\
q(\text { uaestor })^{17} \text { grandes dimensions: } \\
60 \times 47 / 49 \mathrm{~cm}\left(\mathrm{cf} . \mathrm{n}^{\circ} 5\right) \\
\text { date: voir } \mathrm{n}^{\circ} 16\end{array}$ \\
\hline
\end{tabular}

date: voir $n^{\circ} 16$

13 CIL : paléographie ancienne.

14 Sur cette même tuile figure l'estampille Hermetis (CIL XIV, 4091, $43^{\mathrm{b}}$ ).

15 La lecture Maecanio (CIL) est impossible : ce nom n'existe pas; le CIL indexe le document comme faux.

16 Une des erreurs de M.F. Petraccia Lucernoni, I questori municipali dell'Italia antica (Rome 1988), 42-43, nos $37-41$.

17 D. Nonnis, 'Attività imprenditoriali e classi dirigenti nell'età repubblicana. Tre città campione', Cahiers Glotz 10 (1999), 93, pour la famille du magistrat. H. Dessau, 'Un amico di Cicerone ricordato da un bollo di mattone di Preneste', Mitteilungen des deutschen archäologischen Instituts 2 (1887), 293-294 avait proposé de voir dans ce questeur M. Iuuentius Laterensis, ami de Cicéron et questeur à Péneste vers 62/61. 
Table 1 (cont.)

\begin{tabular}{|c|c|c|}
\hline $\begin{array}{l}C I L \mathrm{XIV}, 4091,13^{\mathrm{a} \text { et } \mathrm{b}}=\mathrm{I}^{2}, \\
347^{\mathrm{a} \text { et } \mathrm{b}}=\mathrm{XV}, 2302^{\mathrm{a} \text { et }} \mathrm{b}\end{array}$ & $\begin{array}{l}L \text { (ucius) Mag(ulnius?) } M(\text { arci) } f(\text { ilius }) \\
q(\text { uaestor })^{18} \\
\text { date: voir } \mathrm{n}^{0} 16\end{array}$ & 18 \\
\hline$C I L \mathrm{I}^{2}, 3478$ & $C$ (aius) Minuc(ius) f(ilius) $q$ (uaestor) & 19 \\
\hline
\end{tabular}

Gabii/Castiglione

CIL XIV, 4091, $14=\mathrm{I}^{2}, 3480^{\mathrm{a}}$

M(arcus) Naut(ius) q(uaestor)

20

et b $=\mathrm{XV}, 2303$ et $A E$ 1909,

$76^{\mathrm{a}}$

CIL $\mathrm{I}^{2}, 3476+A E$ 1909, $76^{\text {b }} \quad C$ (aius) Gem(inius) Ruf(us) q(uaestor) 21

C. Autres provenances italiennes

Grand St Bernard, au

sommet, cité d'Augusta

Praetoria/Aoste et un ex. à

Aoste, CIL V, 8110, 399 $9^{\mathrm{a}-\mathrm{d}}$ et

$400=$ InscrIt. XI, 1, $123^{\mathrm{a}-\mathrm{c}}$

Asulum/Asolo, Busana

$2000^{20}$

Bononia/Bologne, CIL XI, $6689,254^{\text {a et } b}+5$ ex., Rigato $2004^{21}$

Ravenna/Ravenne, Bandelli 2008, $46^{22}$ tuiles, 2 séries

* Public = Public(us) ou public (a tegula), cf. $\mathrm{n}^{\text {os }} 23,80$ et $86^{19}$

* R P A = r(es) p(ublica) A(ugustanorum)? voir $\mathrm{n}^{\mathrm{o}} 77$

7 ex., théâtre, fin I ${ }^{\mathrm{er}}$ s. av. : publica (tegula?), cf. $\mathrm{n}^{\mathrm{os}} 22,79$ et 85

C(aius) Vatinius Maximus dec(urio)? Bon(oniae)? ou Bon(oniensium)

muraille de la ville, $\mathrm{III}^{\mathrm{e}} \mathrm{s}$. av. : $\operatorname{Ar}$ (iminensium) plutôt

qu'Ar(iminium ou -nii)

22 5

(continued)

\footnotetext{
18 Nonnis 1999, op. cit. (n. 17), 93.

19 D'autres tuiles, portant les marques ROMA ou SPQR, ont été trouvées au même endroit.

20 M.S. Busana, 'Le iscrizioni lapidarie', in G. Rosada (ed.), Il teatro romano di Asolo. Valore e funzione di un complesso architettonico urbano sulla scena del paesaggio (Trévise 2000), 132-134; 164-165; un exemplaire (fig. $93^{7}$ ) est complet; il est donc certain que la formule n'est pas res publica.

21 D. Rigato, 'Riesame di due documenti dall'agro colonario bolognese', Atti e Memorie della Deputazione di Storia Patria per le Province di Romagna 54 (2004), 105-120.

22 G. Bandelli, 'Epigrafie indigene ed epigrafia dominante nella romanizzazione della Cisalpina. Aspetti politici e istituzionali (283-89 a.C.)', in Epigrafia 20o6, Atti della XIVe Rencontre sur l'épigraphie in onore di Silvio Panciera (Rome 2008), 43-66.
} 
Table 1 (cont.)

Suasa/Castelleone di Suasa,

tuile, maison des Coiedii: VI.VIR.SVAS (se)

26

Campagnoli 2008, $\mathrm{n}^{\mathrm{o}} 11^{23}$; cf.

Rigato 2004, 109

Matilica/Matelica, CIL XI,

$854+A E$ 2003, $593^{25}$

uir( $i$ ?) Suas(anorum)

$1^{\text {ère }}$ moitié du I ${ }^{\mathrm{er}}$ s. ap. J.-C.

voir $\mathrm{n}^{\circ} 81^{24}$

2 briques, écriture du déb. du ${ }^{\mathrm{er}} \mathrm{s}$. av. :

27

Matilicatium sacrum

$C I L$ : considéré comme faux, $A E$ : réhabilité

Tusculum et ager

rei publicae Tusculanor(um)

28

Praenestinum, CIL XIV, 4090,

cf. * marques Tusculanorum sacrum

$1^{\mathrm{a}-\mathrm{c}}$ et $2658 ; \mathrm{XV}, 7^{1-3}$

CIL XIV, 2658 et XV, 2813

(voir n. 3: Granino Cerere, Marengo 2012,

spéc. 161, 170)

* tuyau, CIL XIV, 2658, Tusculum: rei

publicae Tusculanor(um)

* près de Castel Gandolfo, CIL XV, 2 :

O D EX S C lu comme o(pus) d(oliare)

exs(enatus) c(onsulto), le sénat étant la

curie de Tusculum (plutôt que d'Aricie)

Signia/Segni, $A E$ 2007, 345

$M$ (anius) Occius M(ani)f(ilius) T(itus)

Manlius T(iti)f(ilius) IVuir(i) i(ure)

$d$ (icundo) s(enatus) $c$ (onsulto)

80-6o av.

Iuиanum/S. Maria del

Tuiles; 3 textes, le premier en plusieurs

Palazzo, $A E$ 1995, 395,

et son territoire (Anxanum/ versions

a) $r$ (es) p (ublica) Iu(uanensium), $\mathrm{I}^{\mathrm{er}}$ s. av./

Lanciano), CIL IX, 6083, déb. I ${ }^{\text {er }}$ s. ap.

164 (d)

b) Vestigator / (seruus) p(ublicus)

Iuuan(ensium), un peu postérieur

même chose à Anxanum ${ }^{26}$

23 P. Campagnoli, 'L'industria ceramica e laterizia a Suasa', in E. Giorgi, G. Lepore (eds.), Archeologia nella valle del Cesano da Suasa e Santa Maria in Portuno (1996-2009) (Bologne 2010), 99-110, $\mathrm{n}^{\circ} 11$.

24 Suasa est aussi un théonyme, qui apparaît peut-être sur une tuile d'Ariminum, de la $1^{\text {ère }}$ moitié du $\mathrm{I}^{\text {er }}$ s. ap. J.-C., S.M. Marengo, 'Suasa, Suasa Felix e i luci di Ariminium: un'ipotesi', Picus 26 (2006), 173-181.

25 Granino Cerere, Marengo 2012, op. cit. (n. 3), spéc. 161, 173.

26 La date proposée par CIL (1 $1^{\text {ère }}$ moitié du $\mathrm{II}^{\mathrm{e}} \mathrm{s}$. ap.) est trop tardive. 
Table 1 (cont.)

Ferentinum/Ferentino, CIL X, 5865 et $A E 1956$, $152+1982,304$, SupplIt. I, 35

Napoli/Naples (2 ex.) et Puteoli/Pouzzoles (1 ex.), inédits, inf. prof ${ }^{\text {ssa }}$ Miranda et Miranda $2005^{28}$

Silarus/Sele, fleuve, $C I L, \mathrm{I}^{2}$, 3475 , grand temple d'Héra, à l'embouchure, ou petit temple à la source 29 tuile (et non pierre), 3 ex., avec diverses

31 abréviations: Anteros / (seruus) publicus / Ferentini(um) développement Ferentini à corriger à la lumière du $\mathrm{n}^{\mathrm{o}} 3 \mathrm{o}^{27}$

briques, en grec: Sebastou Neôpolitôn époque augustéenne

Tuiles; certaines ont a, d'autres a et b, sur 2 faces

$$
\begin{aligned}
& \text { a }: \operatorname{Mag}() \\
& \text { b }: \text { Popl(icum) = publ(icum) Nucer(inorum) } \\
& \text { date: } v \cdot 5^{0}
\end{aligned}
$$

D. Grande Grèce et îles, Sicile, Lipari fin $\mathrm{III}^{\mathrm{e}}-\mathrm{I}^{\mathrm{er}}$ s. av.

Rhegium/Reggio, IG XIV, 2400, 7

Rhegium/Reggio, IG XIV, 2400, 15, a-f

(b au musée de Naples)

Rhegium/Reggio, IG XIV, 2400, 14, b + Lazzarini 1982, $147-15^{30}$ et à Sorrente, Mingazzini, Pfister, 1946, $187^{31}+$ à Messana/Messine, IG XIV, 2400, 14, a tuiles, en grec : Mamertinou $(m)$; cf. no ${ }_{3} 8$ date : $280-270$ tuiles, en grec, Rhèginôn

nombreuses tuiles, en grec: $R h(\grave{e})$ ginôn 36 Orthôn, non «les vrais Regginiens », mais « de Reggio » + nom propre, D’Amore 1998, $292-293^{32}$ cf. $n^{\text {os }} 34$ et 37

(continued)

27 A. Weiß, Sklave der Stadt (Stuttgart 2004), 133 rapproche de CIL III, 4692 $2^{\mathrm{a}-\mathrm{b}}:$ L. Sauariensis Ioni(us), 2 ex., et $4693^{\mathrm{a}-\mathrm{c}}$, ex of. $L$ (ucii) S(auarensis) I(onii), Sauaria.

28 E. Miranda, San Lorenzo Maggiore: guida al museo e al complesso (Naples 2005).

${ }_{29}$ CIL: in fano Herae ad ostium fluminis Silari; Busana 2000, op. cit. (n. 20): grand Heraion; quoi qu'il en soit, la cité est celle des Nucériens.

30 M.L. Lazzarini, 'I "veri Reggini”', Klearchos 93-96 (1982), 145-157.

31 P. Mingazzini, F. Pfister, Forma Italiae, Sumentum (Florence 1946).

32 L. D'Amore, 'Contributo all'antroponimia di Rhegion: Perkuniun', ZPE 123 (1998), 291-296. 
Table 1 (cont.)

Médma/Rosarno, Cavalier,

tuiles, en grec: Medma

Brugnone 1986, 232,

$\mathrm{n}^{\text {os }} 51^{1-52^{33}}$

Messana/Messine, IG XIV,

briques, en grec: Mamertinôn

2394, 2, a-e

cf. $n^{\circ} 34$

Tauromenium/Taormina,

Campagna, La Torre $2008^{34}$

naumachie et théâtre, briques,

Monte Iato et Endella, en grec: Tauromenitan.

briques, en grec: Iatou

Wilson 1999, $538^{35}$

Lipara/Lipari

nécropole, tuiles, en grec : Liparas

Cavalier, Brugnone 1986,

230-231, n ${ }^{\text {os }} 47-48$

Cavalier, Brugnone 1986,

tuile: PARO + sigle $=$ Paropos ? $236, n^{\circ} 63^{36}$

(cité proche de Termini Imarese)

II. Provinces

A. Gallia Belgica, Augusta Treuerorum/Trèves

\begin{tabular}{ll}
\hline CIL XIII, 12603, 1-4 & 1 tuile et plusieurs briques, nombreux ex. : 43 \\
& rei publ(icae), ou REI PVBL ou REI PVB ou \\
& REI P, fin I ${ }^{\text {er }}$ s. av.-déb. ${ }^{\text {er }}$ s. ap. \\
\hline
\end{tabular}

B. Britannia, Gleuum/Gloucester

71 types de marques, 292 ex., début $\mathrm{II}^{\mathrm{e}}$ s. $-\mathrm{III}^{\mathrm{e}}$ s. ap.

RIB II, 5, 2486, 42 types

RIB II, 5, 2487, 1

2 r(ei) p(ublicae) G(leuensium)

$r($ ei $)$ (ublicae) $G$ (leuensium) + duumvirs

(duum)uir(orum) Perp(etui) et Aprilis

Optati et Saturnini

33 M. Cavalier, A. Brugnone, 'I bolli delle tegole dalla necropoli di Lipari', Kokalos 32 (1986), 181-279.

34 L. Campagna, G.F. La Torre, 'Ricerche sui monumenti e sulla topografia di Tauromenion: una stoà ellenistica nell'area della naumachia', Sicilia Antiqua 5 (2008), 115-146.

35 R.J.A. Wilson, 'Iscrizioni su manufatti siciliani in età ellenistico-romana', in M.I. Gulletta (ed.), Sicilia epigraphica 2 (Pise 1999), 531-555.

${ }^{36}$ Cf. n. 33 . 
Table 1 (cont.)

\begin{tabular}{|c|c|c|}
\hline 6 & $\begin{array}{l}\text { D. Senni } V(i) t(\text { alis }) \text { plutôt que } V t(\text { ilis }) G \text {. [V] } \\
\text { aleri } A n(.)\end{array}$ & 47 \\
\hline 10 & $[-)]$ Atto, ou $A() T.().(e t) T() O.()$. & 48 \\
\hline 4 & $V() M.($.$) et P() G.()$. & 49 \\
\hline 5 & {$[-\operatorname{or}(u m) S($.$) et V()$.} & $5^{0}$ \\
\hline 7 & $T($.$) et G()$. & $5^{1}$ \\
\hline 8 & $L($.$) et A()$. & $5^{2}$ \\
\hline 15 & $Q(.) \operatorname{Sil}().[e] t[-$ & 53 \\
\hline$A E$ 2000, 821 & Naso(nis) et $[\ldots$ & 54 \\
\hline RIB II, 5, 2487, 9 & - ] et Iul. L[.] & 55 \\
\hline Med. arch. 1988, 29, fig.14 & $M S O$ et $\left[-;\right.$ sans doute à lire NASOET ${ }^{37}$ & $5^{6}$ \\
\hline RIB II, 5, 2487, 3 & $\begin{array}{l}\text { r. p. G. + duumvir unique } \\
\text { IIuir(i) P. Ael(ii) Finiti }\end{array}$ & 57 \\
\hline & r. p. G. + nombre incertain de duumvirs & \\
\hline RIB II, $5,2487,12$ & IIuir [- & $5^{8}$ \\
\hline 13 & - II]uir[- $] A L[-$ & 59 \\
\hline 23 & [-] Matern [- & 60 \\
\hline 14 & $\mathrm{Se}[-$ & 61 \\
\hline 19 & $-] V[-$ & 62 \\
\hline 20 & $-] T[-$ & 63 \\
\hline 17 & -]RCCCS [-] VCINI & 64 \\
\hline$R I B$ II, $5,2488,1$ & $\begin{array}{l}\text { r. p. G. + quinquennaux } \\
q(\text { uin }) q(\text { uennalium }) \operatorname{Iul}(i) \text { Flor }(i) \text { ou } \\
\text { Flor }(\text { entini) et Cor(neli) } S(i) m(\text { ilis ?) } \\
\text { ou } S(i) m(\text { plicis })\end{array}$ & 65 \\
\hline 2 & $\begin{array}{l}q(\text { uin) } q \text { (uennales) Se }[-] \text { (Septimius?) et } \\
\text { Varius Van(-) ou Vann(-) }\end{array}$ & 66 \\
\hline 3 & q.q. $C(-)$ et $M(-)$ & 67 \\
\hline 4 & q.q. $C(-)$ et $M(-) r . p . G$. & 68 \\
\hline & magistrats incertains & \\
\hline RIB II, 5, 2487, 11 & $S[-$ & 69 \\
\hline 16 & r.p.G. $[-$ & 70 \\
\hline 18 & $-7 M$ & 71 \\
\hline
\end{tabular}

(continued)

37 P. Isaac, 'Excavations on the site of the early Norman Castle at Gloucester. 1983-84. Small finds', Medieval Archaeology 32 (1988), 28-29, fig. 14 lit MSO ; mais R.S.O. Tomlin, M.W.C. Hassall, 'Roman Britain in 1999. Instrumentum domesticum', Britannnia $3^{1}$ (2000), $439, \mathrm{n}^{\circ} 23$ et $\mathrm{n} .41$ l'assimilent à la marque $\mathrm{n}^{\mathrm{o}} 54$. 
Table 1 (cont.)

III. Attestations complémentaires

A. Proche

Ager Picenus, Spina

sur un fragment d'askos : galicos colonos,

72

$A E$ 1979, 292 puis $C I L, \mathrm{I}^{2}$, cf. $\mathrm{n}^{\mathrm{o}} 74$

$2877^{\mathrm{b}}$

date : assignation viritane de 223 par

G. Flaminius

B. Époques de transition

Samnium, notamment

Bouianum/Bojano,

tuiles en langue osque qui citent le medix,

73

Pietrabbondante, Civitella,

Cumes, Pompéi, liste :

Marchese $1974^{38}$, La Regina

1966 et $1989,329-362^{39}$

magistrat civique; ex. : $m$ t mi heri $u v=$

meddis tuntuiks minis hereius uveis = medix

tuticus Minius Hereius Ofif.

noms principaux: Staius, Papius, Numerius,

Pomponius

fin du III ${ }^{\mathrm{e}}$ s. $-1^{\text {ère }}$ moitié du I ${ }^{\mathrm{er}}$ s. av.

C. Res publica, mentions probables ou possibles

Arretium/Arrezo, CIL XI,

[R(es)] p(ublica) col(onorum) Fid(entiorum)

74

6675,1

sur les Arretini Fidentiores, Plin., nat., 3, 52;

cf. $\mathrm{n}^{\mathrm{o}} 71$

Carsioli/Carsoli (près de -),

$R($ es $)$ p (ublica) C(arsiolorum)

CIL XI, 6675, 2 = IX, 6078, 55

Perusia/Perugia, CIL IX,

6075, 3

$R($ es $)$ (ublica) $N($ arnensium $)$

76

Augusta Praetoria/Aoste, CIL

C.M.M. s(eruus?)

tuiles

$\mathrm{V}, 8110^{400}=$ InscrIt. XI, 1, 124

$R($ es ) p (ublica) A(ugustanorum)?

voir $n^{\circ} 22$

38 M.P. Marchese, 'Sulle nove (e antiche) iscrizioni de Pietrabbondante', Studi Etruschi 42 (1974), 371-378.

39 A. La Regina, 'Le iscrizioni osche di Pietrabbondante e la questione di Bovianum Vetus', Rheinisches Museum 109 (1966), 260-285, et I Sanniti, in Italia omnium terrarum parens: la civiltà degli Enotri, Choni, Ausoni, Sanniti, Lucani, Brettii, Sicani, Siculi, Elimi (Milan 1989), 299-432. Désormais on se référera à M.H. Crawford (ed.), Imagines Italicae (Londres 2011), dont nous n'avons pu disposer. 
Table 1 (cont.)

\begin{tabular}{|c|c|c|}
\hline $\begin{array}{l}\text { Bononia/Bologne, CIL XI, } \\
6675,4\end{array}$ & $\begin{array}{l}\text {...] Vedian. RP lu:...] Vedian (a) } \\
\text { (officina?) r(es) p (ublica), cf. Righini, } \\
\text { Biordi 1993, } 40, \mathrm{n}^{\mathrm{o}} \mathbf{1}^{40}\end{array}$ & 78 \\
\hline $\begin{array}{l}\text { Aquae/Prahovo, Dacie, } C I L \\
\text { III, } 14215\end{array}$ & $\begin{array}{l}\text { DRP AQVIS, lu: } d(e) r(e) p(\text { ublica) } \\
\text { Aquis (toponyme dace et pannonien) }\end{array}$ & 79 \\
\hline $\begin{array}{l}\text { Aquileia/Aquilée, infor. prof. } \\
\text { C. Zaccaria }\end{array}$ & $\begin{array}{l}\text { tuiles : } T P=t(\text { egula }) ? p(\text { ublica }) ? \\
\text { cf. } \mathrm{n}^{\text {os }} 22,23 \text { et } 86\end{array}$ & \\
\hline
\end{tabular}

D. Noms de peuples et de lieux

\begin{tabular}{|c|c|c|}
\hline $\begin{array}{l}\text { Ariminium/Rimini, Righini, } \\
\text { Biordi } 1993,40, \mathrm{n}^{\circ} 2^{41}\end{array}$ & $\begin{array}{l}\text { IIII LVC SVAS, lu: (quattuoruiri) Luc(ani) } \\
\text { (et) Suas(ani?) (Biordi); (quattuoruiri) } \\
\text { Luc(eolanorum) (et) Suas(anorum?) }\end{array}$ & 81 \\
\hline $\begin{array}{l}\text { Salona/Solin, Dalmatie, } \\
\text { CIL III, } 6434^{42}\end{array}$ & $\begin{array}{l}\text { (Manacorda } 2000)^{43} \text {; cf. } \mathrm{n}^{\mathrm{o}} 26 \text {; si le } \\
\text { développement IIII luc }(\text { orum }) \text { Suas }(\text { ae })^{44} \\
\text { est accepté, la référence est à exclure } \\
\text { déb. du I }{ }^{\text {er }} \text { s. ap. }\end{array}$ & \\
\hline $\begin{array}{l}\text { Saepinum/Sepino, CIL IX, } \\
6078,147\end{array}$ & Saepin(atium) & 82 \\
\hline $\begin{array}{l}\text { Portus Augusti ?/Ostie, } \\
\text { Thylander, B } 394^{45} \text {, et } C I L \\
X V, 5^{\mathrm{a}} \text { et } \mathrm{b}=\mathrm{XIV}, 4089,1^{\mathrm{a}}\end{array}$ & $\begin{array}{l}\text { Portus Augusti } \\
\text { Portus Aug. }\end{array}$ & 83 \\
\hline
\end{tabular}

(continued)

40 V. Righini, M. Biordi et al., 'I bolli laterizi romani della regione Cispadana (Emilia e Romagna)', in C. Zaccaria (ed.), I laterizi di età romana nell'area nordadriatica (Rome 1993), 23-92.

${ }^{41}$ Righini, Biordi 1993, op. cit. (n. 40); F. Coarelli, 'Praeneste in età repubblicana. Società e politica', in P. Baglioni (ed.), La necropoli di Praeneste: periodi orientalizzante e medio repubblicano (Palestrina 1992), 253-267, spéc. 260.

42 Un exemplaire du timbre trouvé à Rimini a été découvert à Salone, sur la rive opposée de l'Adriatique, où la brique a pu arriver par mer.

43 Manacorda 1994, op. cit. (n. 12), 3-59 et 2000, op. cit. (n. 6), 129 et n. 15; Marengo 2006, op. cit. (n. 24), 173-181.

${ }^{44}$ Marengo 2006, op. cit. (n. 24): il s'agirait d'une tuile du sanctuaire des 4 bois sacrés de la déesse Suasa.

45 H. Thylander, Inscriptions du Port d'Ostie (Lund 1952). 
Table 1 (cont.)

cf. Centum Cellae/Cività

Vecchia, CIL IX, 6675, 6

Syrie, Palmyra/Palmyre, $A E$ 1941, 80
Port(us) Trai(ani) (tombes de soldats

84 de la flotte $)^{46}$

en grec, sur l'agora: Palmyra; Adriana Palmyra

E. À exclure

Monteleone, Bruttium, CIL

$\mathrm{X}, 8041,37$

tuiles

a) C. Ati Rufi // publicus ${ }^{47}$

b) Public(us) plutôt que public(a)

(tegula), cf. n ${ }^{\text {os }} 22,23$ et 80

Pannonie supérieure

Siscia/Sisak, CIL III, 4671

parmi des noms, plutôt ceux des propriétaires que des magistrats, SISC lu : Siscia

Pannonie supérieure

Sauaria/Szombathely, CIL

III, 4692 et 4693

ex of(ficina) L. S(auarensis) I(oni)

Mésie supérieure

Golubinje, près du castellum

de Praevo, et Lazarevic, $A E$

$\mathrm{a}:$ DRPDIERNA $=d(e) r(e) p($ ublica $)$

1890, 8 et CIL III, 8277, $2^{\text {a et b }}$

Dierna?

(a) et 12677 (b)

$\mathrm{b}$ : DRDI et DKDI

La lecture de cette marque est très douteuse; dès sa découverte, à Golubinje ${ }^{48}$, elle a été interprétée comme la mention de Dierna, agglomération de Dacie citée par Pline, Tierna, figurant sur la Table de Peutinger. Mais les variantes (b) ont été développées de la même façon, ce qui est invraisemblable; de plus, la préposition de devant re publica est absolument inconnue et n'aurait pas de sens. On a aussi rapproché de CIL III, 1559, Slatina,

46 Ces marques au nominatif sont distinctes des nombreuses estampilles sur lesquelles est mentionné le lieu de stockage des briques : la mention de porto, portu (Licini, Parrae...) renvoie à des entrepôts, non à une structure portuaire.

47 Le I final de Rufi est parfaitement visible: puisqu'on ne peut lire Atius Rufus, publicus ne peut être associé au nom.

48 Les ruines y témoignent peut-être de l'implantation d'un castellum, mais la nature administrative de la région et des localités est incertaine. 
Table 1 (cont.)

1. 5 : D INtER feC lu: D(ierna) ou

$D$ (robeta) Rien ne désigne ces marques

comme civiques.

Bretagne

Londinium/Londres, RIB II,

PP BRIT LOND: praeses prouinciae

90 5,2485

Britanniae Londinii

remplace : $r$ (es) $p$ (ublica) Brit(annorum)

Lond(inensium) (lu d'après CIL VII, 1235)

Les marques civiques partagent les caractéristiques suivantes ${ }^{49}$ :

1) les supports sont en majorité des briques, plus rarement des tuiles, très exceptionnellement des amphores $\left(n^{\text {os }} 1-4\right)^{50}$, ou des vases $\left(n^{0} 71\right)^{51}$. Ils s'échelonnent sur une durée longue ( $\mathrm{III}^{\mathrm{e}}$ s. av. J.-C.- $\mathrm{III}^{\mathrm{e}}$ s. ap.), mais l'écrasante majorité s'inscrit dans une chronologie haute: la Grande Grèce ( $\left.n^{\text {os }} 34-37\right)$, la Sicile et les îles ( $\left.n^{\text {os }} 3^{8-42}\right)$, la côte adriatique avec Brindisi $\left(\mathrm{n}^{\text {os }} 1-5\right)$ présentent les exemplaires plus précoces (à partie du $\mathrm{II}^{\mathrm{e}}$ s. av. J.-C.), tandis que les plus tardifs ( $\mathrm{II}^{\mathrm{e}}-\mathrm{III}^{\mathrm{e}}$ s. ap.) se trouvent à Glevum/Gloucester, en Bretagne $\left(n^{\text {os }} 44-71\right)^{52}$. Les réutilisations très fréquentes entraînent une large incertitude, surtout pour les exemplaires isolés, mais le déploiement chronologique prouve que ces marques civiques ne peuvent être associées à un événement d'empire, qu'elles dépendent de circonstances locales, mais répétitives ;

2) elles proviennent en écrasante majorité d'Italie; les provinces représentées sont la Gaule Belgique (Trèves, no 43) et la Bretagne (Gloucester/ Glevum, $\mathrm{n}^{\text {os }} 44-71$ );

3) à la différence des autres bolli, l'intitulé ne comporte ni officina ni terme désignant un atelier, un auteur de fabrication, ou renvoyant à un contrat. À trois exceptions près : à Bologne $\left(n^{0} 78\right)$ comme à Savaria (nº 88), le développement est discutable. À Préneste ( $\left.n^{\circ} 9\right)$, P. Betilienus

\footnotetext{
49 Dondin-Payre 2010, op. cit. (n. 8).

50 http://www.dscc.uniba.it/Anfore/Leanfore.html.

51 La formule Galicos colonos, qu'elle soit une exclamation ou une localisation, implique l'action collective d'une communauté socio-juridique; le point sur la réflexion de G. Bandelli qui a beaucoup étudié la question, avec la bibliographie antérieure, Bandelli 2008, op. cit. (n. 22), 46-47; 64, fig. 5 .

52 T. Darvill, A. McWhirr, 'Brick and tile production in Roman Britain: models of economic organization', World Archaeology 15·3 (1984), 239-261, spéc. 249.
} 
Chresimus, probablement connu par d'autres marques $\left(\mathrm{n}^{\circ}{ }_{12}\right.$; CIL XIV, $4091,23^{2}=\mathrm{XV}, 2312^{2}$ ), pourrait avoir eu un rôle public dans la cité ;

4) elles citent des collectivités civiques, très rarement par leur toponyme seul $\left(\mathrm{n}^{\text {os }} 24-25,31\right.$ et 37 , avec un doute sur le développement des abréviations; $\mathrm{n}^{\text {os }} 40-42,83-85$ ), le plus souvent par l'ethnique au pluriel ( $\left.{ }^{\text {os }} 24-28,31-36,38-39,81-82\right)$. Jamais aucun terme autre que res publica n'est employé, le nom des citoyens complétant ou non ( ${ }^{\text {os }} 43$, 78), celui de la collectivité dont le statut juridique (colonie, municipe ...) n'est jamais précisé; ce fait est capital: seule compte la solidarité civique exprimée par res publica ${ }^{53}$. L'emploi de l'adjectif publicus/ publica ( ${ }^{\text {os }} 22-23,33,80$ ) est du même ordre ;

ou 4) elles citent les représentants officiels de ces communautés, les magistrats. Les nomenclatures onomastiques sont plus ou moins complètement transcrites, des tria nomina au cognomen seul; les contraintes matérielles imposant le laconisme, aucune conclusion chronologique ou juridique ne peut en être tirée, sinon que la mention du prénom fixe une date antérieure au milieu ou à la fin du I ${ }^{\text {er }}$ s. ap. J.-C. ${ }^{54}$. Les magistrats, mentionnés seuls ou à plusieurs, sont, à partir de la fin de la République, en écrasante majorité des duumvirs et des quinquennaux, représentant les communautés civiques au plus haut niveau. $\mathrm{Si}$ l'abréviation a été bien interprétée, le décurion de Bologne, $C$. Vatinius Maximus dec. Bon. ( $\left.\mathrm{n}^{\mathrm{o}} 24\right)$, serait un hapax, très improbable puisqu'un décurion, à la différence de la curie, n'est pas autorisé à décider au nom du corps civique ${ }^{55}$. L'intervention d'édiles ( $\left.\mathrm{n}^{\circ \mathrm{s}}{ }_{15}-16\right)$, chargés des questions économiques, n'est pas étonnante. En revanche, les questeurs, magistrats financiers, peuvent surprendre $\left(\mathrm{n}^{\text {os }} 1-4,17-21\right)$; M. Silvestrini propose un rapprochement convaincant avec les collèges de questeurs dans les colonies latines, orientant vers une époque précoce, cohérente avec le reste de la documentation ${ }^{56}$. Les sévirs $\left(\right.$ Suasa, $\left.{ }^{\circ}{ }_{2} 26\right)$ seraient à placer dans la même fourchette chronologique, pour la même raison ${ }^{57}$.

53 M. Dondin-Payre, M.-Th. Raepsaet-Charlier (eds.), Cités, municipes, colonies. Les processus de municipalisation en Gaule et en Germanie sous le Haut Empire romain (Paris 200o), $134-138$.

54 Plus tôt en Italie que dans les provinces.

55 Favorable à cette exception, Rigato 2004, op. cit. (n. 21).

56 M. Silvestrini, 'Aspetti istituzionali e sociali delle colonie latine de Apulia e Calabria', in O. Dally et al. (eds.), Archeologia della Puglia in memoria di Marina Mazzei (Berlin, c.s.) = Epigrafia e Territorio. Politica e Società IX (Bari, c.s.).

57 M.H. Crawford, 'How to create a municipium: Rome and Italy after the Social War', Bulletin of the Institute of Classical Studies, supp.71 (1998), Modus operandi, 37. 
M' Occius M'. f. et T. Manlius T. f., quattuorvirs de Signia ( $\left.\mathrm{n}^{\circ} 29\right)$, sont particulièrement instructifs: IVuir(i) i(ure) d(icundo) s(enatus) $c$ (onsulto), ils sont en exercice au moment de la mainmise syllanienne sur les cités du Latium; peut-être y ont-ils été envoyés, de Pompéi, pour remplacer l'ancienne aristocratie, dans la $1^{\text {ère }}$ moitié $d u I^{\text {er }} s$. av. J.-C. ${ }^{58}$. Les modes de désignation diffèrent, y compris à l'intérieur des séries: nominatif, génitif ou ablatif. La proposition de la restitution d'un verbe comme curauerunt ou fecerunt succédant au nominatif est satisfaisante; la même solution est valable pour l'ablatif, et on a coutume de restituer anno avant le génitif. En ce cas, comme pour les dates consulaires, les magistrats seraient réduits au rôle de références passives. À cela s'opposent la présence d'édiles et de questeurs et l'articulation très fréquente entre res publica et les magistrats, qui fait de ceux-ci les représentants de celle-là : les magistrats sont mandatés par la cité dont ils doivent préserver les intérêts, exactement comme les officiers figurant sur les marques militaires; certaines de celles-ci portent aussi le génitif, qu'il ne peut être question de faire précéder de anno; on pense à cura ${ }^{59}$.

Comment envisager le contexte matériel et légal de ces fabrications?

Elles ne sont mentionnées par aucun texte législatif qui apporterait des éclaircissements ${ }^{60}$. Les produits sont trouvés sur le lieu de fabrication, dans les bâtiments, en réemploi dans les constructions ou n'importe où, par exemple dans des tombes dont les tuiles forment le toit, de sorte que le lieu et les modalités de fabrication sont en majorité inconnus. Les seuls ateliers identifiés par la découverte de produits marqués sur place sont ceux de Brindes et de Gloucester, et sans doute de Segni. À Brindes, cas le plus soigneusement étudié, deux sites se sont succédé: Apani, à quelques

58 F.M. Cifarelli, 'Magistrati ed élites municipali da Segni in bolli laterizi dal territorio', in G. Ghini (ed.), Lazio e Sabina 4 (Rome 2007), 219-224.

59 R. Kurzmann, 'Soldier, civilian and military brick production', Oxford Journal of Archaeology 24 (2005), 405-414.

60 La lex Tarentina (vers 89 av.), col. 1, 1. 26-31, n'apporte pas d'information sur la question: elle fait obligation aux magistrats de posséder depuis 5 ans au moins un bâtiment de 1500 tegulae $\left(440 \mathrm{~m}^{2}\right)$ sur le territoire civique (M.H. Crawford [ed.], Roman statutes 1 [Londres 1996], 304 et 310); la loi d'Urso, ou lex coloniae Genetiuae Iuliae (2 ${ }^{\mathrm{e}}$ moitié du $\mathrm{I}^{\mathrm{er}}$ S. av.), ch. 76, ibid., 404, 424, 438-439 limite la capacité de production infra-urbaine à 300 tuiles ou objets similaires, et spécifie que ces fabriques doivent être propriété publique. P. Mingazzini, 'Tre brevi note sui laterizi antichi', Bullettino Communale 76 (1956/58), 77-93 est aujourd'hui dépassé. 
$\mathrm{km}$ au nord de la ville, puis Giancola $\left(\mathrm{n}^{\text {os }} 1-5\right)^{61}$. À Gloucester $\left(\mathrm{n}^{\text {os }} 44-71\right)$, la fabrique se trouvait au $\mathrm{S}^{\mathrm{t}}$ Oswald's Priory, près de l'ancienne porte du fort et du port fluvial ${ }^{62}$. À Segni, elle était au Valle Riccia $\left(n^{0} 29\right)^{63}$. Toujours la fabrication se fait à proximité du chef-lieu, et hors de l'espace urbain, conformément aux normes de sécurité connues ${ }^{64}$.

Ces trois cas (Brindes, Segni, Gloucester) présentent les deux types de structures envisageables :

- une manufacture propre. À Gloucester ( $\left.n^{\text {os }} 44-71\right)$, le processus s'éclaire par le contexte historique : la colonie de Gloucester prit, à la fin du $\mathrm{I}^{\mathrm{er}}$ siècle, la suite d'une garnison légionnaire, y compris comme responsable de la production céramique. Les marques civiques y manifestent le transfert d'autorité et de propriété par les mêmes moyens que ceux dont usait l'armée ${ }^{65}$. La fabrique municipale propre présente l'avantage d'adapter la production aux besoins de la collectivité, et l'inconvénient, n'étant pas flexible, d'entraîner une lourde charge financière puisque l'outil de production doit être en état de marche, que des travaux édilitaires soient prévus ou non;

- l'autre possibilité est celle d'un atelier privé auquel la cité accorde une concession correspondant à une partie de sa production ou à la totalité, pour une durée plus ou moins longue. Cette solution est assurée à Segni ( $\left.n^{0} 29\right)$, et à Brindes $\left(n^{\text {os }} 1-5\right)$, où les marques civiques ont été trouvées mélangées à des estampilles variées ${ }^{66}$. Elle permet de moduler les commandes selon les besoins, sans assurer les frais d'un fonctionnement permanent, mais le rôle de la cité est identique, les magistrats municipaux exerçant la surveillance sur les lots commandés et payés par la cité. Les modalités de financement, de la relation économique et administrative entre l'atelier et la cité ne peuvent être déterminées, en raison de l'absence d'informations ${ }^{67}$.

61 http://www.dscc.uniba.it/Anfore.

62 Darvill, McWhirr 1984, op. cit. (n. 52); M. Dondin-Payre, X. Loriot, 'Londres et les colonies de Bretagne', in S. Demougin (ed.), Villes et statut municipal dans l'Empire romain (Paris-Rome 2012), 241-243.

63 Cifarelli 2007, op. cit. (n. $\left.5^{8}\right)$.

64 Ainsi la lex Coloniae Genetiuae, ch. 76, Crawford 1996, op. cit. (n. 6o), 404, 424, 438-439.

65 Kurzmann 2005, op. cit. (n. 59), 401-414.

66 Cifarelli 2007, op. cit. (n. 58), 219-221.

67 Sur la relation entre l'estampillage et le statut des ateliers, F. Charlier, 'Les conditions socio-juridiques du travail dans les tuileries d'après les marques sur les matériaux en Gaule et dans les autres provinces occidentales romaines', in J. Annequin, E. Geny, E. Smadja (eds.), Le travail. Recherches historiques (Besançon-Paris 1998), 186-189. 
Bien que le choix de l'atelier public soit le moins adapté aux contraintes de gestion, les éditeurs de marques civiques isolées ne se posent guère la question : ils considèrent ces documents comme des preuves de l'existence d'un atelier civique ${ }^{68}$. Il nous semble que la situation est inverse : naturelle pour la colonia Gleuensis en raison de l'héritage militaire, probable pour Segni, elle ne doit pas être considérée comme allant de soi ${ }^{69}$. L'emploi d'esclaves publics n'implique pas non plus l'existence d'un atelier municipal $\left(\mathrm{n}^{\mathrm{os}} 3 \mathrm{ob}-31,76,86 \mathrm{a}\right)^{70}$ : propriété de la cité, ces esclaves travaillent à des productions civiques dans des structures privées ${ }^{71}$.

Que, mises à part de rares exceptions, les marques civiques soient apposées sur des tuiles et surtout des briques (plus employées que les tuiles) n'est pas indifférent : ces produits, quoique très banals et utilitaires, occupent une place spéciale dans la vie de la cité. Leur portée emblématique est reflétée par la formule «évergétique » que portent des briques et tuiles réparties autour de Terracine et à Pompéi : $C$ n. Domitius M. $f$. Caluinus coloneis dedit ${ }^{72}$. La nature du lien symbolique n'est pas affectée par l'identité discutée de Domitius Calvinus ${ }^{73}$ : il a contribué à ce que Terracine et Pompéi soient dotées d'équipements urbains correspondant au statut juridique des communautés civiques. Une relation similaire, avec le consul Flaminius à l'origine d'une assignation viritane, explique l'inscription du vase de Spina (galicos colonos, $\mathrm{n}^{\mathrm{o}} \mathrm{72}^{2}$ ). Ces bolli sont donc fermement ancrés dans le contexte civique, mais n'apparaissent pas à n'importe quel moment du processus de municipalisation.

De nombreux libellés en langue osque sur des tuiles d'édifices religieux et publics du Samnium $\left(\mathrm{n}^{\mathrm{o}} 73\right)^{74}$ comportent, jusqu'au $\mathrm{I}^{\mathrm{er}}$ s. av. J.-C., le nom et le titre du meddix, un magistrat. Les cités se trouvaient dans une

68 Ainsi, Steiner 1917/18, op. cit. (n. 10), 26 ; Busana 200o, op. cit. (n. 20), 130-133.

69 Darvill, Mc Whirr, op. cit. (n. 52), 246-249.

70 Le développement du $n^{0} 3$ ob est conforté par le $n^{\circ} 31$, mais, à la lumière du féminin publica ( $\left.\mathrm{n}^{\mathrm{o}} 23\right)$, qui sous-entend tegula, on peut hésiter sur le $\mathrm{n}^{\circ} 8 \mathrm{o}$.

71 Weiß 2004, op. cit. (n. 27), 132-135 n'envisage pas cette éventualité.

72 Circeii, Formiae, et Terracina, Latium ; Pompeii, Campanie: CIL X, $6314=8043,1=\mathrm{I}$, 767 , p. $947=I L L R P 430+A E$ 1991, 424 et 2001, 748.

${ }^{73}$ La réutilisation des briques brouille la chronologie: plusieurs se trouvent dans la villa de Domitien, sur le lac Paola, D. Ronghu, L'area archeologica del Porto-Canale sul Lago di Paola, www.latina24ore.it/wp-content/uploads/Relazione-Diego-Ronchi.doc. Domitius Calvinus est identifié comme le consul de 332, ou comme le cos. II de 40 av. J.-C., la fondation des colonies de Terracine et Formies correspond plus au premier qu'au deuxième.

74 A. La Regina, 'Le iscrizioni osche di Pietrabbondante e la questione di Bovianum vetus', Rheinisches Museum 109 (1966), 260-286; J.-P. Morel, 'Artisanat, importations et romanisation dans le Samnium aux $\mathrm{II}^{\mathrm{e}}$ et $\mathrm{I}^{\mathrm{er}} \mathrm{S}$. av. J.-C.', in La Romanisation du Samnium aux $I^{e}$ et $I^{\text {er }}$ siècles av. J.-C. (Naples, 1991), 187-203. Désormais, Crawford 2000, op. cit. (n. 39). 
période de transition, encore dans le monde italique, et au seuil d'une insertion, très progressive, dans le schéma romain ${ }^{75}$. Il en va de même en Grande Grèce et dans les îles ( $\left.\mathrm{n}^{\mathrm{os}} 34-42\right)$, à la charnière entre les périodes hellénistique et romaine et au début de cette dernière. En Sicile, les caractéristiques sont l'abondance des témoignages et leur lien étroit avec l'épigraphie hellénistique, par la mention des "ethniques civiques", qui font référence à une communauté publique mais pas à son statut officiel. Ces formules ( $\left.\mathrm{n}^{\text {os }} 34-36,38-39\right)$ renvoient à des groupes constitués sur le plan juridique selon un schéma grec, qui vont s'intégrer dans le monde romain; ce marquage civique, inscrit dans la pratique hellénistique, est abandonné une fois les structures romaines en place. Les Neôpolitani ( $\left.n^{\circ} 3^{2}\right)$ mentionnent, conformément à la même tradition, l'ethnique seul, mais maintiennent cette formulation après leur insertion dans la grille civique romaine, que concrétise la construction d'un temple en l'honneur d'Auguste: ils se ressentent comme une entité grecque (formulation par l'ethnique), mais aussi romaine (expression de leur fidélité à Rome à travers son empereur, Sebastou). Alors que les autres cités hellénophones, qui utilisent l'ethnique au génitif, cessent une fois la transition entre les mondes hellénistique et romain aboutie, parce que la structure romaine est en rupture avec le monde antérieur, les Napolitains, accoutumés au cadre civique, ressentent la nouvelle situation comme une évolution, non une scission. Sans rompre avec une identification ressentie comme indissociable des structures grecques, comme le font les cités de Grande Grèce et de Sicile, les Napolitains l'ont conservée et adaptée en continuant à se proclamer Neôpolitani. L'émergence des entités civiques au cours de la période de mise en place des structures administratives romaines s'accompagne d'une affirmation de ces nouvelles personnalités collectives. Que $A R$, sur une brique du mur de Ravenne ( $\left.\mathrm{n}^{\circ} 25\right)$, soit lu comme Ar(iminensium) plutôt que comme $\operatorname{Ar}$ (iminium)/Rimini n'est pas anodin : par le contrôle sur l'édification de la muraille de Ravenne, les Ariminenses auraient, au milieu du III $^{\mathrm{e}}$ s., affirmé leur prééminence de communauté constituée officiellement par Rome sur Ravenne qui n'avait pas ce stade ${ }^{76}$. Les marques civiques de Trèves $\left(n^{\circ} 43\right)$ se rattachent à cette même affir-

75 Crawford 1998, op. cit. (n. 57), 38, lenteur du passage de la domination osque à la domination romaine à Pompéi.

76 Bandelli 2008, op. cit. (n. 22), 46-47 et 63-64, fig. 4 et 5 ; contra, V. Manzelli, 'Le mura di Ravenna repubblicana', in Fortificazioni antiche in Italia. Età repubblicana (Rome 2001), 7-28, fig. 9 : il ne s'agit ni de AR=Ariminium, ni de RA, mais d'une des nombreuses marques de maçons, destinées à différencier les productions des différents ateliers ayant fourni des matériaux. 
mation d'une identité civique nouvellement forgée, soit lors de la fondation de la cité trévire, dans le dernier tiers du I ${ }^{\mathrm{er}}$ s. av. J.-C., soit lors de sa promotion au rang de colonie sous Auguste ou Claude, qui lui conféra une place spéciale dans la province belge ${ }^{77}$; elles constituent peut-être les plus anciens témoignage sur la res publica (Augusta Treuerorum). À plus d'un siècle de distance, la désignation de la res publica Gleuensis comme entité civique intégrée à l'empire relève exactement du même processus : dans un moment de transition entre deux modes d'appartenance à l'ensemble romain (le camp puis la colonie), une communauté ressent la nécessité d'exprimer sa personnalité administrative.

Les lieux de découverte de ces tuiles et briques fabriquées sous l'égide de collectivités civiques devraient coïncider avec des constructions publiques : des temples, des murailles, des sanctuaires, des théâtres, des naumachies, des agoras en ont livré, mais aussi des tombes (Préneste, $\mathrm{n}^{\text {os }} 6-21$ ), des maisons en Italie (e.g. $n^{\circ} 26$ ), de nombreuses villas en Bretagne ${ }^{78}$. La liberté d'utilisation en Italie centrale et en Sicile a paru normale dans la période transitoire entre autonomie grecque et intégration romaine ${ }^{79}$. Ailleurs dans l'empire, le schéma administratif strict semblerait impliquer l'emploi exclusif de ces productions civiques dans des bâtiments civiques édifiés sur le territoire civique ${ }^{80}$; or la dispersion est patente, y compris hors de la cité (Gloucester) ${ }^{81}$. Dans les faits, plusieurs facteurs matériels ont favorisé la diffusion: la coutume de transporter, parfois sur de grandes distances, ces produits, quoique lourds et à faible valeur ajoutée ${ }^{82}$,

77 Les 174 marques différentes figurent toutes sur des matériaux en réemploi dans la basilique et dans le palais ; on ignore donc à quelles opérations urbanistiques elles avaient été liées, Steiner 1917/18, op. cit. (n.10), affirme l'existence d'ateliers publics, peut-être situés au bord du fleuve (26-27). Sur Trèves, M.-Th. Raepsaet-Charlier, dans C. Lepelley (éd.), Rome et l'intégration de l'empire 2 (Paris 1998), 157-158.

78 Dondin-Payre, Loriot 2012, op. cit. (n. 62), 242-243.

79 Gliozzo 2005, op. cit. (n. 4).

80 La question est à rattacher à celle de la diffusion des briques militaires, qui ne fait pas l'unanimité : pour Kurzmann 2005, op. cit. (n. 59), l'armée n'achète ni ne cède à l'extérieur ; Y. Thébert, 'Transport à grande distance et magasinage de briques dans l'Empire romain. Quelques remarques sur les relations entre production et consommation', in Boucheron et al. 2000, op. cit. (n. 1), 341-356, n'exclut pas la vente; pour Y. Le Bohec, 'Les estampilles sur briques et tuiles et l'histoire de la VIII 'égion Auguste', ibid., 273-284, l'implication de l'armée dans l'évergétisme impérial explique l'emploi de briques militaires dans des édifices civils ; cf. aussi discussion, 324-326 : les briques peuvent servir de lest pour les navires et ainsi voyager loin ; mais aussi être utilisées, une fois ayant rempli leur rôle, localement et dans un cercle mal défini.

81 Dondin-Payre 2010, op. cit. (n. 8).

82 Chr. Rico, 'Quand les briques romaines prenaient la mer. Discussion autour d'un article d'Yvon Thébert', Pallas 66 (2004), 97-104. R.J.A., Wilson, 'Brick and tiles in Roman 
la difficulté à prévoir précisément les besoins, qui a entraîné la cession des excédents hors du cadre municipal, et la réutilisation généralisée des matériaux.

Les estampilles civiques ${ }^{83}$ expriment le ressenti de collectivités à un stade fondamental de leur histoire: soit ces organisations pré-romaines déjà existantes sont à une époque de transition, en voie d'intégration dans le cadre romain (Italie et îles), soit elles connaissent une mutation (la colonie civile remplaçant le camp à Gloucester). Ces marques sont rares dans les provinces, parce que les peuples, tribus, royautés pré-romains n'y ayant pas expérimenté un système de type civique, le modèle romain constituait une rupture, sans référence antérieure similaire ${ }^{84}$. La relation avec un moment historique précis, souvent précoce, explique que les témoignages soient en nombre limité ; leur importance n'en est pas minorée: les marques civiques constituent un témoignage sur la municipalisation.

Sicily', in A. McWhirr (ed.), Roman brick and tile studies in manufacture, distribution and use in the Western Empire (Oxford 1979), 11-43; Sicily under the Roman Empire: the archaeology of a Roman province, 36 B.C.-A.D. 535 (Warminster 1990), et Id. 1999, op. cit. (n. 35).

83 Granino Cerere, Marengo 2012, op. cit. (n. 3), pour les marques sacrées.

84 Il en découle que les Trévires les ont utilisées soit lors de la promotion coloniale, la première dans la province, soit, solution qui nous paraît préférable, quand ils furent constitués en cité, en ce cas probablement les premiers de la région. 


\title{
CONVERGENCE AND DIVERGENCE: ONE EMPIRE, MANY CULTURES
}

\author{
Frederick G. Naerebout
}

\section{Integration and Romanization}

The conference that this volume of Impact of Empire is based upon, was dedicated to "Integration at Rome and in the Roman World". Looking at the call for papers for that conference, we read the following: "We are therefore explicitly looking for papers that deal with the juridical, social and political aspects of this process of inclusion... on the following themes: integration and juridical statutes, Roman citizenship and local citizenship, cities and tribes; integration and political history, conception of Imperium Romanum, prouinciae, ciuitates and imperial power; integration and social history, Roman concept of ciuitas, local elites and ordines; integration and 'Romanness', frameworks of social life, cultural and cultual practices." I have quoted this at some length, because this passage shows how much integration is taken for granted. The word "integration" is repeated in the context of many different fields - actually covering all of society except the economy which is not singled out, but which might be considered to fall under the heading of "social life" and "cultural practices". ${ }^{1}$ It also summarizes all of this as a "process of inclusion". The integrative power of the empire is the point of departure. As the French title of the conference, «Les voies de l'intégration à Rome et dans le monde Romain», made clear, contributors were supposed to study the ways in which integration was arrived at, its modalities. Maybe one was also to look at persisting local diversity and at those missing out because they could not or would not be integrated, but it seems fairly obvious that participants were to consider above all the success story of the Roman empire as a progressively more integrated whole - until it starts disintegrating again. ${ }^{2}$

${ }^{1} \mathrm{~J}$. Hoffmann-Salz, Die wirtschaftlichen Auswirkungen der römischen Eroberung. Vergleichende Untersuchungen der Provinzen Hispania Tarraconensis, Africa Proconsularis und Syria (Stuttgart 2011) is an exemplary study of integration in the economic field - with conclusions which can readily be compared with my argument below (see esp. 441-498).

2 About those left out of the integration process, eloquently: R. Hingley, Globalizing Roman culture. Unity, diversity and empire (Abingdon 2005), 116. Also, apparent integration 
"Success story" is a way of putting it that would probably be disputed: integration and inclusion could be used in a neutral sense. Or could they? Let us look at a recent example of a study of integration. In the volume entitled Comment les Gaules devinrent romaines, there are contributions on new administrative structures, urbanisation, innovative burial practices, changing patterns of land use, especially the establishment of a villa economy, and the introduction of "viticulture, oléiculture et fructiculture". ${ }^{3}$ The book is a valuable collection of studies, well-documented and nuanced in its interpretations. Its authors have taken on board the debate on Romanization; it is obvious they have read Greg Woolf: they take care to show that the Gauls were not as barbaric as previous generations thought they were, and that although the Romans may have romanized Gaul, the Gauls had their input into the process. ${ }^{4}$ But even if this is never stated explicitly, it is to be understood that much of what the Romans bring (and which gets accepted by the locals and then takes on its own dynamics and so on) is an improvement upon the previous situation - certainly the vines, olive trees and a range of fruit-bearing shrubs and trees seem to get the attention attributed to them because they are essential for France, at least gastronomically, to come into existence. So it must be all for the best. The importance of a Roman past for the nation state, or, in a more recent ideological turn, the Roman empire as a precedent for, if not forerunner of, a united Europe, seems to imply that being conquered by Rome is "a good thing". Rome as the purveyor of literacy, advanced building techniques and agricultural practices, an infrastructure that in part is still functioning, of Christianity and so on: it could hardly be bad. ${ }^{5}$ So when we speak

can be used as what has been called "transculturation": the mimicry of the dominant culture in order to mock or subvert it.

3 P. Ouzoulias and L. Tranoy (eds.), Comment les Gaules devinrent romaines (Paris 2010).

${ }^{4}$ G. Woolf, Becoming Roman. The origins of provincial civilization in Gaul (Cambridge 1998).

5 Of course, there are several who argue for the opposite view: the bad Roman imperialists - because imperialists and empires are bad altogether. Such "empire bashing" is a fruitless affair, burdened with anachronistic notions. For a recent example, see T.H. Parsons, The Rule of Empires. Those who built them, those who endured them, and why they always fall (Oxford 2010). This is sympathetic in its attack on neo-cons and their imperialist nostalgia - but looking for the villain of the piece, I would tend to end up with the nation state. Parsons sees empires as extractive and exploitative. From the top down, these institutions seem rational and relatively benign, but in reality it took intimidation, naked force, and institutional slavery to produce all the grand monuments and cultural achievements of the ancient world (23). It is intriguing to see how this is undercut by an interesting analysis of the cultural make-up of the empire. The Romans are said to have been more open to easing the line between citizen and subject than their successors in 
of integration into the Roman empire of areas and peoples that are conquered by Rome, that generally is seen as a success story, from at least one of the above perspectives.

I am aware that I do Comment les Gaules devinrent romaines injustice, but its argument reminds me of the famous scene from the movie The Life of Brian, by the Monty Python team, in which Reg, the would-be leader of a Jewish rebellion against Roman rule, rhetorically asks: "what have the Romans ever done for us"? His followers do not recognize it for a rhetorical question and come up with: "The aqueduct. And the sanitation! And the roads". Reg retorts: "Well yes obviously the roads ... the roads go without saying. But apart from the aqueduct, the sanitation and the roads..." His audience goes on to mention irrigation, medicine, education, health, wine and, of course, how could one forget, public baths! "And it's safe to walk in the streets at night now. Yes, they certainly know how to keep order... let's face it, they're the only ones who could in a place like this". Reg, exasperated: "All right... all right... but apart from better sanitation and medicine and education and irrigation and public health and roads and a freshwater system and baths and public order... what have the Romans done for us?" "They brought peace!". How deftly the scriptwriters have moved from material items starting with the aqueduct to the immaterial benefits of being part of the Roman empire: safety, order, peace. They are supposed to have taken their cue from a Talmudic passage criticizing Roman achievements as purely Roman self advancement, of no benefit to the conquered. ${ }^{6}$ But this ancient denial of the beneficial nature of Roman rule (and its modern counterpart) is in fact put under discussion here by having Jewish revolutionaries express a most favourable opinion about Roman rule.

But I do not so much object to the idea of the Roman empire as a success, as to the idea of a Roman success story. Talk about "what the Romans bring", all these "Roman concepts" and "Romanness" in the call for papers seems to bring us back to the discourse of Romanization. Some might not find this objectionable because they never rejected the concept. In fact,

\footnotetext{
later empires, even to have adopted "assimilationist policies... because modern conceptions of race did not apply" (25; cf. 61 on the "local and fluid" identities of Rome's subjects). So Rome appears to be rather different from later empires, even no empire at all (at least at a later date - consequently it did not fall, but it evolved into something else: another fashionable paradigm), but nevertheless it must be bad, like all those other empires. Here the anachronisms surface very openly.

${ }^{6}$ A. Reinhartz, Jesus of Hollywood (Oxford 2007), 61, referring to Bab. Shabbat 33b.
} 
it is vulgar, ugly, anachronistic and misleading. ${ }^{7}$ It calls up the image of Rome on the one side and the conquered society on the other, both more or less static and homogenous. ${ }^{8}$ However, there are not two but multiple parties involved, and not one of these parties is static or homogenous. It also implies a Roman point of view, and does so in its very etymology - no amount of redefinition can sanitize this away. Surely, integration was in part a Roman policy. The much-quoted words of Agricola are not just Tacitean rhetoric, they describe what Romans tried to do to secure their territories. But the way the Romans viewed things, is not necessarily what they really looked like. Besides, policy is normative, and the real world behaves differently. We risk an enormous simplification of the complex processes at work in the creation of the Roman empire. This has all been shown many times over, in decades of debate about Romanization, of debunking of the very concept, laying bare its ideological roots. But I daresay we still have this whole idea of Romanization at the back - or front - of our minds. It resonates in the very notions of integration and inclusion as laid out in the call for papers.

\section{Integration and Homogenization}

But cannot integration be used in a perfectly neutral sense? Yes, I suppose one can use it that way. But still I want to problematize the very notion of integration. I cannot help thinking that the only way we can progress towards a better understanding of the extremely complex issues underlying words like 'integration' or 'inclusion', is by comparison, more specifically by confronting (our interpretations of) the ancient sources with social science models based on empirical evidence from the field of migration studies and studies of culture contact and acculturation. ${ }^{9}$

7 R. Syme, 'Rome and the nations,' in Id., Roman Papers vol. 4 (Oxford 1988; first published in Diogenes $3^{1}$ [1983], 33-46), 62-93, quote from 64.

8 The whole idea of a set of "original cultures", seen as unified, stable, persistent phenomena, which then give rise to hybrids (supposedly less authentic), should be rejected. What we see are new phenomena under new conditions. As E. Shils, 'Roots: the sense of place and past: the cultural gains and losses of migration,' in W. McNeill and R.S. Adams (eds.), Human migration. Patterns and policies (Bloomington 1978), 404-429, 417 has put it: "all cultures are amalgamated cultures" (my italics). Pure unadulterated culture is a "naive romanticism". So-called unitary cultures are not unitary, not in their origin and not in their development.

9 On the importance of an empirical evidence base, see G. Myrdal, Asian drama. An inquiry into the poverty of nations (New York 1968), 24: "Generalizations about reality ... precede specification and verification. They constitute 'theory' in research. Theory... must no 
This comparative effort might easily be lost amongst the minutiae of juridical and political developments, and so on. Not for the first time will I quote my mentor Henk Versnel: "the classicist must allow himself to 'become' a psychologist (or a sociologist, anthropologist, ethologist for that matter) as far as his health, capabilities and efforts will allow him. The alternative is that he refrains categorically from any interpretation in the real sense of the word". ${ }^{10}$ Thirty years on I still find that even with a subject such as "integration" - the concept itself of course derived from the social sciences - the input of these disciplines is overlooked or avoided: Comment les Gaules devinrent romaines has a bibliography at the back of about 600 titles - some four of these are from the field of social science, all the others are by archaeologists, ancient historians, epigraphers. Or, not to limit ourselves to a single example: in the Proceedings of the 1981 Cortone colloquium on acculturative processes in the ancient world, there is an excellent introduction by Giuseppe Nenci who discusses the introduction of the word "acculturation" by J.W. Powell in 1880 (in his Introduction to the study of Indian languages), and its ultimate formulation in the famous 'Memorandum for the study of acculturation' by Redfield, Linton and Herskowits of 1936, and who analyses the debate about the term as supposedly an example of the imperialistic gaze (which it is not). ${ }^{11}$ Nenci took his cue from the XII ${ }^{\mathrm{e}}$ Congrès international des sciences historiques, Vienna 1965, where acculturation had already been a main topic, and from the VI ${ }^{\mathrm{e}}$ Congrès international des études classiques, Madrid 1974, which took it up again, as "Assimilation et résistance à la culture gréco-romaine dans le monde ancien". How surprising that there is no or almost no theoretical background to any of the papers in the over 1100 pages of the Cortone Proceedings (including papers authored or co-authored by Nenci). In fact, at the conference of which this volume is the result, I was struck by the absence of an explicit theoretical and

only be subjected to immanent criticism for logical consistency, but must constantly be measured against reality and adjusted accordingly".

10 J.-P. Vernant, J. Rudhardt and O. Reverdin (eds.), Le sacrifice dans l'antiquité, Vandouvres-Genève, 25-30 août 1980: huit exposés suivis de discussions (VandouvresGenève 1981), 189. On the uselessness of empirical data without theory, see Myrdal 1968, op. cit. (n. 9), 18: "the continuing collection of data under biased notions only postpones the day when reality can effectively challenge inherited preconceptions."

11 G. Nenci, 'Introduction', in Id. (ed.), Modes de contacts et processus de transformation dans les sociétés anciennes: actes du colloque de Cortone (24-30 mai 1981) = Forme di contatto e processi di trasformazione nelle società antiche: atti del convegno di Cortona (24-30 maggio 1981) (Rome, Pisa 1983), 1-4. 
methodological social science framework in most papers. ${ }^{12}$ I am not conducting a polemic along the lines of Moses Finley, saying that there is but one way in which to do ancient history; I am merely advocating that it is profitable to look over the fence, when one is after "interpretation in the real sense of the word".

So what - not considering the Romanization issue, already dealt with above, though that and what follows cannot really be seen apart - would be the problem with "integration"? At first sight, it seems quite obvious that integration is what we see happening within the Roman imperial context: the extension of Roman citizenship, the establishment of Roman forms of government, the creation of a new infrastructure, the spread of Latin, and of cultural norms and forms ultimately derived from a Mediterranean context. ${ }^{13}$ We end up with an empire of which every part has a lot of features in common with any other part. A Roman military presence underlies such convergence: even if the locals are willing partners, it all started with conquest. This is more or less why both now and then the phrase "The Roman Empire" was deemed an appropriate designation for the political unit in question. All this might seem self-evident. Of course, we then can go on to look at the local diversity underlying this imperial unity. Before long we will be speaking of the global and the local which develop hand in hand, and one might even be tempted to use the word "glocalization". ${ }^{14}$ And this seems self-evident as well. Of course, in

12 With the honourable exception of Emily Hemelrijk, Clifford Ando, and especially Rens Tacoma (who also happens to be a pupil of, inter alii, Henk Versnel) and Wouter Vanacker (I base myself on the abstracts and the papers as given at the conference, not on the edited texts in this volume).

13 'Mediterranisation', a concept used in paleoanthropology, in studies of flora and fauna, especially of the Black Sea region, and in studies of diet and of global warming, was recently re-defined and set to work as a cultural concept: see A. Schelfhout, "Mediterranisation" of Celto-Germanic religion, unpublished MA thesis, Leiden 2012 [available online at the Leiden Repository, https://openaccess.leidenuniv.nl/]. This certainly is a big improvement over Romanization; but it still obfuscates the true state of affairs.

14 E. Morawska, A sociology of immigration. (Re)Making Multifaceted America (Basingstoke 2009), 147 defines glocalization as the implanting of outside elements into everyday existence of the inhabitants of receiver-country localities so that they become a natural part of their orientation and practices. Cf. ibid., 2: "This simultaneous process of homogenization and diversification characteristic of the contemporary world whereby international migration plays a crucial role has been called by sociologists glocalization". To the aspect of diversification we will have ample opportunity to return. One should not use "glocalization" when dealing with the ancient world, because global is the wrong word to apply to Antiquity, also to the Roman Empire, where things were not literally global (App., Roman History, Preface 7: "they [i.e. the Roman emperors] wisely wish to preserve what they have rather than to extend the empire endlessly"), and more importantly because globalization is a concept that exclusively addresses recent historical developments. See F.G. Naerebout, 
an empire stretching from Scotland to the Sahara desert, there will be local variations. The local element may be stronger - as when the Roman cultural presence is seen as a mere veneer beneath which the local is alive and well and ready to bounce back as soon as the Roman influence, read military might, wanes; or it may be weaker - as when some parts of the empire are thoroughly integrated into the Roman cultural world (romanized, as it is still called despite this life-time of debate on the concept of Romanization already referred to $).{ }^{15}$ And this seems self-evident again. The ins and outs are debatable, but the main outlines are clear enough. Or are they?

Integration as sketched in the above outline is about homogenization: the constituent parts of the empire, despite different points of departure and different trajectories, are made more similar. We find this notion reiterated left and right: "ordering the disorderly" and "standardizing the multiform", moving towards "a unitary symbolic system" or "a consensual identity". ${ }^{16}$ Even the most careful conclude that there was "a convergence between the desires of certain provincials and the publicized aims of Rome". ${ }^{17}$ One could question whether there was homogenization at all, and if there was (any), whether it was intentional or merely accidental.

'Global Romans? Is globalisation a concept that is going to help us understand the Roman empire?,' Talanta, 38-39 (2008), 149-170. Add the forceful plea by J.A. Scholte, 'Globalization,' s.v. in R. Robertson and J.A. Scholte (eds.), Encyclopedia of globalization (New York 2007), 526-532, 529, to restrict the concept of globalization to "the unprecedented growth of transplanetary connectivity in recent times". Happily, we do not need the word 'globalization', nor globalization theory. A useful concept such as "connectivity" can be put to work without the globalization framework.

15 Cf. F. Millar, 'Local cultures in the Roman Empire: Libyan, Punic, and Latin in Roman Africa,' in Id., Government, society, and culture in the Roman empire (Chapel Hill 2004; first published in Journal of Roman Studies 58 [1968] 125-151), 249-264, 249: "We might conclude for one area that Graeco-Roman culture remained the merest facade, for another that it completely obliterated a native culture. More commonly, we will find a mixture or co-existence of cultures. In such a situation, again, the local element might have been culturally and socially insignificant, or, as it was in Egypt and in Judaea, embodied in a coherent traditional civilization with its own language, literature, customs, religion, and (in Egypt) art forms".

$16 \mathrm{~J}$. Farrell, Latin language and Latin culture, from ancient to modern times (Cambridge 2001), 1; K. Hopkins, 'The political economy of the Roman empire,' in I. Morris and W. Scheidel (eds.), The dynamics of ancient empires. State power from Assyria to Byzantium (Oxford 2009), 186 (who takes care to stress that the Romanness that is the yardstick of unity or consensus is changing in the very process of integration). E.M. Orlin, Foreign cults in Rome. Creating a Roman empire (Oxford 2010), 7-8: in the encounter between Rome and "others", which is not so much "Romanization" as negotiation between two parties, Romanness is taken for granted, but it cannot be taken for granted as it itself is negotiated in the encounter with others.

17 Hingley, op. cit. (n. 2), 63 (he uses the word "acculturation"). 
Burbank and Cooper, in their popular diachronic account of imperial power, maintain that it is the nation state that homogenizes - it proclaims commonality (even where this is untrue). The empire, however, declares the non-equivalence of its multiple populations. But both are incorporative. ${ }^{18}$ Incorporative to me seems to be on the same level as integration. Homogenization is what may follow as a consequence of incorporation, or must follow as a consequence of (a policy of) integration. The main point I want to make, however, is a different one: that the very fact of integration (convergence) carries divergence within itself. ${ }^{19}$

\section{Integration and Current Debate}

The moment we use the word "integration" we enter into an arena of intense scholarly debate by social scientists and social historians, and, not unrelated of course, of equally intense political debate, reacting to but also shaping public opinion. Let us start with the political debate: this has been ongoing ever since minorities, whether indigenous or immigrant, were seen as in some sense problematic; this is of course very much linked to the rise of the modern nation state, especially in its nineteenth-century guise - which is still with us. In fairly recent history, in Western Europe, we have gone through a phase of multiculturalism: the conviction that different strands of culture can exist side by side; cultural diversity is viewed as an asset. Now the pendulum has swung in the opposite direction, and the ideal is that everyone should assimilate to the majority culture of whatever region; cultural diversity is seen as a liability.

18 All foregoing expressions are taken from J. Burbank \& F. Cooper, Empires in world history. Power and the politics of difference (Princeton 2010), 8. Cf. the special issue of the journal Monde(s) Histoire, Espaces, Relations, $\mathrm{n}^{\circ}$ 2, novembre 2012, which presents a discussion of "empires" in general, and offers a debate about the Burbank-Cooper volume in particular (Empires. De la Chine ancienne à nos jours, Paris 2011, in the French translation). There, S. Benoist reviews the Roman part of Burbank-Cooper. In this context one should consider the possibility that only the Roman empire amongst empires is integrative, while other empires want to avoid integration (Clifford Ando, in discussion during the conference).

19 I do not use divergence in the sense of K. Pomeranz, The Great Divergence: China, Europe, and the making of the modern world economy (Princeton 2000), which usage was taken up by W. Scheidel, "From the "Great Convergence" to the "First Great Divergence": Roman and Qin-Han state formation and its aftermath,' in Id., (ed.), Rome and China. Comparative perspectives on ancient world empires (Oxford 2009), 11-23, nor do I mean divergence in the sense of Hingley, op. cit. (n. 2), 71: alternative, deviant or discrepant readings of common cultural traits. 
Both ways of looking at things depend on the presuppositions that, first, diversity is actually or potentially problematic and should be "managed", and, secondly, the erroneous idea that such large-scale and long-term processes as culture contact and its acculturative consequences can be managed at all, by some kind of social engineering. ${ }^{20}$ Consequently, "integration" has become a loaded concept: it is supposed to happen, or not, or to be impossible, it is either a good thing or a bad thing. Popular opinion is usually less variable than political positioning, and tends to reject diversity and prefer unity - but without much consistency. Politicians may either go with or against popular opinion - they may also try to influence popular opinion to further their own ends. ${ }^{21}$

Scholarly debate in part reflects the political debate. Especially social scientists can be called upon to provide the research to prop up political decision making. But in the end the ideologically charged contributions get filtered out, and we end up with a view of societies that is evidencebased. Here we can learn that the presence of minorities or long-term contact with "the other" is not intrinsically problematic - there is no problem for people to worry about or for politicians to solve. Next we learn that integration is a common pattern (with exceptions, no doubt, but common nevertheless). Bring culturally diverse units into a larger whole, whatever way, and in due course nobody will be able to tell the difference: it will all amalgamate. As Hoerder puts it: "much of the immigrants' input into cultures and social systems over time appears neither as 'foreign' nor as deliberately constructed, since, once adopted, innovations become part of the Self and are considered indigenous" 22 A main point is of course that

20 Diversity is negatively associated with segregation, social fragmentation, instability, tension, conflict, even terrorism: J. Stillwell and M. Van Ham (eds.), Ethnicity and integration. Understanding population trends and processes, volume 3 (Dordrecht 2010).

21 I will limit myself to a single example to illustrate the prevailing climate - at least amongst part of the constituency to whom the following document so obviously panders. Donner, Dutch minister of the interior, came out with a government paper in 2011, in which multiculturalism was declared a failure (one should suppose: as a policy; the cabinet of which Donner forms part rejects the relativism implied in multiculturalism) and responsible for the incomplete integration of newcomers. Integration should be pushed forward (but NOT by government: restrictions and sanctions should force individuals to take their integration into their own hands), and it should be clear that one culture - even if it is changing - should be the dominant, national one.

22 D. Hoerder, Cultures in contact. World migrations in the second millennium (Durham 2002), 8; Cf. Morawska, op. cit. (n. 14): 5, on the time- and place-specific contexts of the interaction between individual's or group's volition and structures (i.e., the more or less enduring organizations of social formations and cultural relations); ibid., 114: the process of assimilation is incidental to everyday lives, it occurs by itself, so to speak: a lot of individual 
this implies change all around. This is very much the classic definition of integration (also called assimilation) by Robert Park \& E.W. Burgess of the 1920s Chicago School: "a process of interpenetration and fusion in which persons and groups acquire the memories, sentiments, and attitudes of other persons and groups and, by sharing their experience and history, are incorporated with them in a common cultural life." 23 The so-called "new assimilation theory" also sees such convergence, but distinguishes two stages: first acculturation, followed by socio-cultural integration. ${ }^{24}$ Milton Gordon, who was a founding father of the new theory, saw this essentially as one-way traffic: migrants adapting to the host society; Richard Alba and Victor Nee, more recent protagonists of assimilation theory, however, stress that it is a two-way process. ${ }^{25}$ We will return to their ideas below.

choices add up to the process of acculturation (Morawska uses that word on the same page).

${ }_{23}$ After R. Alba and V. Nee, Remaking the American mainstream: assimilation and contemporary immigration (Cambridge, MA 2003), 19; Alba and Nee stress that this definition leaves room, and was supposed to do so, for the persistence of the specific characteristics of individuals and groups - in fact, Park and Burgess opposed the idea of a total "Americanization".

${ }^{24}$ M.M. Gordon, Assimilation in American life: the role of race, religion, and national origins (New York 1964); R. Alba and V. Nee, 'Rethinking assimilation theory for a new era of immigration', International Migration Review 31 (1997), 826-874; Alba and Nee, op. cit. (n. 23). Opposed is the racial/ethnic disadvantage model, which sees successful integration blocked by both sides (apart from the disadvantages experienced by the migrant, there is also active discrimination by the receiving population). This model deals with the socalled "new" immigrants (post 1965) to the U.S. and seems to have a short term perspective. Hypothesis of persisting ethnicity (the "ethnicity-forever-approach": E. Morawska, "The sociology and history of immigration', in V. Yans-McLaughlin [ed.], Immigration reconsidered. History, sociology and politics [New York 1990], 187-240, 218) is based on a limited time frame and on the study of specific situations. In fact, we see all kinds of affiliations. There may be symbolic ethnicity (also called optional ethnicity), with a lot of invented tradition: this is all part of the assimilation process. For instance, you do not have to stress you are of Dutch descent and join in the "klompendans" in Michigan, unless you do not in fact feel Dutch anymore, in some unquestioning way. This should not be confused with resilient ethnicity: the disadvantaged who rebel by exaggerating their ethnic separateness. But all evidence points to eventual socio-economic mobility and thus to convergence. For an attempt to combine the disadvantage and the convergence models: A. Portes and M. Zhou, 'The new second generation: segmented assimilation and its variants', Annals of the American Academy of Political and Social Science 530 (1993), 74-96, on "segmented assimilation": assimilation and negative assimilation ("structural acculturation").

25 Alba and Nee, op. cit. (n. 23), 25. 


\section{Integration and Migration}

I focus on migration (in the sense of human geographical mobility, even when unfree, involuntary and so on - this is all called "migration" in a social science context). ${ }^{26}$ First of all migration is basic to human societies of all periods: people are constantly on the move and it is this which is one of the important driving forces of human culture and society. The opposing view is that of the myth of autochtony and homogeneity. ${ }^{27}$ But it is migration that is the norm. ${ }^{28}$ That the Roman empire is full of migratory movement needs no further illustration. Walter Scheidel turns this into a main characteristic of the empire: "how did the relentless spread of Roman power change people's lives? From military mobilization, urbanization, slavery, and the nexus between taxation and trade to linguistic and religious change and shifting identities, the most pervasive consequences of empire had one thing in common: population movements on an unprecedented scale."29 People move into cities, the empire is travelled by countless traders, scholars and students, pilgrims, and tourists, soldiers and officials go where they are ordered. All who consider the benefits of moving elsewhere to outweigh the costs of doing so, and who are triggered by the enormous increase in possibilities, are on the move. Such dynamic culture contact is indeed the key to understanding the Roman empire. Here we should note a danger involved in focusing on migration: the danger that the presence of culture traits that are thought to belong to culture $\mathrm{X}, \mathrm{Y}$ or $\mathrm{Z}$ are seen as necessarily indicating the presence of migrants from a particular cultural (and/or ethnic) background. For instance, we

26 Migration is usually discussed completely separate from "Romanization", as if the two have nothing to do with each other. The intimate connection between the one and the other was analyzed by Ylva Klaassen in her excellent "Leaving his native land, he came to this region". Processes of migration, integration and cultural change in the Roman empire (unpublished MA-thesis Leiden 2009), now summarized in Ead., 'Migration and integration in the Roman world. A new approach towards culture and identity', Talanta $42-43$ (2010-2011).

27 Or even wider: the assumption of a static past, "the world we have lost", enracinement. Hoerder, op. cit. (n. 22) states that migrants cannot undermine a stable society, because societies are not stable, and if they are, they are petrified and people do not migrate to them but from them. Strange to have Morawska, op. cit. (n. 14), 1 speak of "oncehomogenous societies".

28 Hoerder, op. cit. (n. 22), xix: "a basic condition of human societies"; P. Manning, Migration in world history (New York 2005); J. Lucassen, L. Lucassen \& P. Manning (eds.), Migration history in world history. Multidisciplinary approaches (Leiden 2010).

29 W. Scheidel, 'Progress and problems in Roman demography', in Id. (ed.), Debating Roman demography (Leiden 2001), 1-81, at 1. 
see what we label a Greek element in a non-Greek context and interpret this as an indication of a Greek presence in that same context. Leaving aside the difficult (but very relevant) issue of how to establish what is Greek, this is obviously untrue, for two reasons: a Greek element can be distributed by non-Greeks; a Greek element need not be distributed at all, but it can be a case of appropriation. Still, migration is a main factor (the "how did it really work" of Fergus Millar) - only one should never jump to conclusions as to the identity and place of origin of any migrant on the basis of supposedly "typical" cultural traits. ${ }^{30}$ Material culture and ideas travel with humans - in the ancient world exclusively so (I think we can safely discount the odd written text - which of course in itself is a material object that has to be handled by someone to get it from A to B). ${ }^{31}$ So it always starts with mobility of some kind. For an item to survive in its new, generally inhospitable surroundings, there even has to be repeated mobility, what is called "ongoing migration". It is a well-known phenomenon that if a migrant group undergoes constant rejuvenation by new arrivals, its original culture and language survive longer. But I am

\footnotetext{
${ }^{30}$ Let us take a concrete example: the Roman province of Britannia. In the epigraphic record, with a few additions from literary sources, we have 845 named individuals who certainly or very likely were born outside the province and migrated there (all types of migration included) - most epigraphically attested, a few from literary sources, or both. These comprise 180 Roman officials, 332 members of the Roman army, and 333 civilians, including slaves. RIB now has $35^{27}$ inscriptions (with the publication of the third volume in 2009). So one quarter (23.95\%) of all inscriptions mention "foreign born" individuals. Of course, only part of the 3527 inscriptions name individuals, so the percentage of foreigners amongst known individuals will be even higher. If we look at civilians only - to get rid of some of the bias caused by the huge overrepresentation of the military - the percentage will still be quite high. There is no need to be very precise, because these figures have to be handled with care anyhow: migrants are much more likely to leave traces than locals (at least in the Roman context), for instance the 43 traders attested (on the Nehalennia altars), the $33 \mathrm{fabri}$, and the 54 slaves and freedmen. Thus, we cannot say anything about the percentage of newcomers in the total population. But in absolute figures the influx must have been quite large in order to leave us with two migrants per annum when only eight individuals per annum are attested at all. The above is based on J. Gruson, Kosmopolitisme in Romeins Britannia. De multiculturele dynamiek van een provincie (unpublished MA-thesis Leiden 2008); cf. several contributions to H. Eckhardt (ed.), Roman diasporas. Archaeological approaches to mobility and diversity in the Roman empire (Portsmouth, RI 2010) (its contents are as much epigraphic as archaeological).

31 This is not to deny the importance of writing as a means of overcoming distance in space and time (see Hopkins, op. cit. [n. 16], 178), but my point is that even if sender and receiver of a letter are at the far ends of the empire, we still need someone to literally carry the letter across that huge space. The telegraph and everything that came after led to a space-time compression that is of a completely new order (cf. n. 14 above on the contemporaneity of globalization).
} 
not interested in survival as such: survival for an increased period of time means an increase in the opportunities for acculturation. ${ }^{32}$

So theory says that we should see integration following from culture contact. Only, the theory is mainly concerned with immigrant minorities in a dominant culture - they get integrated into it (which is not the complete story, we will come back to that, but certainly the main part of it). In the Western half of the Roman empire, we see local cultures integrated into the culture of the migrants: the language and habits of Roman soldiers, officials and traders become more or less dominant, depending on what part of the empire we are speaking of. We could compare many instances of integration in nineteenth- and twentieth-century colonies: it is the culture holding political, military and economic power that can assert some measure of dominance. That is of course the integration we have been speaking of above: integration on an imperial scale, not the integration of migrants into local culture - of which kind of integration there will have been plenty in the ancient world, but most of the time it does not interest us. It is, however, of some interest and relevance to consider questions like the following: does a trader of Palmyrene descent settled in the north of Britannia learn to speak (some) Celtic? Does he turn to indigenous deities? For how many generations will his descendants feel Palmyrians? Or at least outsiders or newcomers?

32 Cf. Chr. Ehret, 'Linguistic testimony and migration histories', in Lucassen, Lucassen \& Manning, op. cit. (n. 28), 113-154, on language: integration is what will take place, however long the survival rate (three centuries at the most, usually far less); word borrowings and pidginization are a continuous process. Interesting in this respect is T.P. Bonfiglio, Mother tongues and nations: the invention of the native speaker (Berlin 2010), who shows that the idea of a mother tongue is absent from the ancient world. He discusses the unbiassed bi- or multilinguism of the Roman empire (alas limiting himself largely to the relationship between Greek and Latin - a much broader view of the interaction of languages in the Empire is offered by M.S. Visscher, Landscape of languages. The position of provincial languages in the Roman Empire in the first three centuries A.D. [unpublished MA-thesis Leiden 2011]). Bonfiglio's notion of unbiassed multilinguism can find support in empirical research into the plurilinguism of contemporary Marocco: see M. Benítez Fernandéz, J.J. de Ruiter and Y. Tamer, Développement du plurilinguisme. Le cas de la ville d'Agadir (Paris 2010). Their study of the use and appreciation of classical Arab, local Arab dialects, Amazighe and French contradicts J.N. Adams, Bilingualism and the Latin language (Cambridge 2003), 755, who states that diglossia implies the setting up of a hierarchical relationship between the languages concerned. 


\section{Migration and Divergence}

Migration drives forward convergence, and as a substantial part of the total number of migrants are carriers of the dominant culture, the empire integrates culturally. As the existence of a common culture fosters immigration, integration leads to more immigration leads to greater integration. ${ }^{33}$ But let us look at it from another perspective: that of the local community where the immigrants come in. That local community is faced with - as long as migration continues - the introduction of new habits, new products. Seen from within their own cultural repertoire this means divergence. Paradoxically, in every constituent part of the empire, convergence means divergence. The cultural repertoire diversifies. ${ }^{34}$ This is what Hoerder calls the "immigrants' input". That input is a relatively neglected element in the social science literature. Debate revolves not so much around the enrichment of the receiving cultures, as about homogenization across cultures (comparable to the integration in the Roman Empire). Current concern about homogenization sees it as resulting from globalization, and usually looks at it in a negative light, using expressions such as Americanization, McDonaldisation, and Disneyfication. ${ }^{35}$ America is considered an imperial or hegemonic power, exporting its culture to every part of the globe, if not actually forcing it on everybody. But also other homogenizing policies by hegemonic powers, such as the sinification of Tibet, are discussed in the same manner. On the one hand, this is nuanced by those who point to local agency: the adoption of foreign cultural elements, which are "domesticized", fitted into local cultural patterns and thus turned into new, but nevertheless native elements. On the other hand, whether this domestication takes place or not, in any case the introduction of new elements into an existing culture will, from

33 Shils, op. cit. (n. 8), 424.

34 Morawska, op. cit. (n. 14), 1-2, puts its succinctly: migration is an important contribution to globalization and at the same time a diversifying sociocultural force in the local communities where the immigrants settle. Cf. the quote from Morawska in n. 14 above. Alba and Nee, op. cit. (n. 23), 25 stress that this is a two-way process (I look at it from only one end here), and that not only x substitutes for $\mathrm{y}$, but that there can also be an extension of repertoire. Cf. ibid., 282: the paradigm of the melting pot implies fusion into a new, unitary culture; but much change occurs as the mainstream expands to accommodate cultural alternatives (often after some reworking to make them less exotic).

35 G. Ritzer, 'Homogenization', s.v. in R. Robertson \& J.A. Scholte 2007, op. cit. (n. 14), 575-579. This encyclopedia has no entries on heterogeneity, pluralism, diversity or divergence. 
the perspective of that culture, not be seen as homogenization, but as diversification. The choices increase in number. This might even be the case when a foreign import replaces (kills off, if you want) an existing bit of culture. If the number of imports is large and consists of several competitive items coming from various sources, one might still end up with a more diversified cultural landscape. Of course, this in its turn can be evaluated negatively as well: there will always be those who consider any possible alternative for local traditions as a threat to an assumed identity. But even though it is obvious that every situation has to be judged on its own merits, the very notion of an unchanging and unadulterated local culture has to be rejected - as was already stated.

As said above, divergence is not an important topic in social science theory or empirical research. A lot of the relevant literature is anecdotal. ${ }^{36}$ Manning speaks of migration as an engine for social change, because of "the dynamics of ideas": "The benefits of migration at the aggregate level are equally important. The exchange of language, customs, and technology leads to innovations... known goods and services are spread among communities ... the movement of people speeds the movement of plants, animals, and minerals ..." ${ }^{37}$ Shils, who adheres to the disadvantage model, stresses the cultural impoverishment that can result from migration (adding that a culture can also work towards impoverishment without any migrants to assist); however, he is one of the few to speak at some length about the gains brought by migration: "the gain lies in the widened scope of reference - or awareness - and appreciation in the hybrid culture to which immigrants contribute... The immigrants gain to the extent that sooner or later their more gifted descendants will acquire something of this (high, host) culture. The host society gains, too... the mental cosmos of a society is extended... a society which acquires immigrants and which in the course of time assimilates them by transmitting its own culture to them also assimilates some of their culture into itself. Not all of

36 Typical of the anecdotal nature is the stress on popular culture: Shils, op. cit. (n. 8), 413: earlier waves of immigrants [to the U.S.] left their imprint on popular culture; cf. Morawska, op. cit. (n. 14), 151: the receiver country has a taste for the popular culture of the newcomers. See also n. 41 below. J. Lucassen and L. Lucassen, Winnaars en verliezers. Een nuchtere balans van vijfhonderd jaar immigratie (Amsterdam 2011) end every chapter in their account of immigration into the Netherlands with a balance sheet, in which they also pay some attention to cultural enrichment, mostly of a culinary or popular culture nature, but also pointing out creative individuals, such as literary authors.

37 Manning, op. cit. (n. 28), 2, 11. 
the growth of a central culture through immigration is 'bastardization' in the pejorative sense." ${ }^{38}$ The Swedish anthropologist Ulf Hannerz sums it up as follows: "There is now a world culture, but we had better make sure what this means: not a replication of uniformity but an organization of diversity, an increasing interconnectedness of varied local cultures." 39 "An organisation of diversity" is exactly what I argue is brought about by the Roman Empire.

Now we can go one step further: the divergence is not merely the other face of integration as described above (i.e. integration looked at from the local perspective). There is a much broader range of divergence - which in its turn can be seen as integration in a wider sense than was here discussed before. Integration is not only conquered territories A, B or C being incorporated into a "Roman" empire. It is also territories A, B and C being integrated with each other. ${ }^{40}$ Rome may be the instigator of the empire and its raison d'être, but Rome's empire is a huge set of entities whose contacts are greatly intensified by being incorporated in the empire - as was discussed above under the heading of migration. "Increased connectivity" is convenient shorthand for this development. That does not mean that everything is directed by, let alone channelled through Rome. Intraempire contacts are free for all. Thus every part of the empire - but the one more than the other - is confronted with an influx of people from several other cultural spheres and thus with an extension of the cultural

38 Shils, op. cit. (n. 8), 419. Shils speaks about acculturation in the light of the presence of a single "high culture" which completely dominates the scene. We may ask (if such cultures exist at all) whether Roman culture is in the same league.

39 Quoted after J. Nederveen Pieterse, Ethnicities and global multiculture. Pants for an octopus (Lanham MD 2007), 198. If we replace "world culture" with "Roman empire", we are not much amiss. For world culture, cf. Shils, op. cit. (n. 8), 421-425: common culture, Weltkultur. Even in globalizing times, we may doubt the concept of a "world culture".

40 R. Häussler, 'Signes de la "romanisation" à travers l'épigraphie : possibilités d'interprétations et problèmes méthodologiques', in Id. (éd.), Romanisation et épigraphie. Etudes interdisciplinaires sur l'acculturation et l'identité dans l'Empire romain (Montagnac 2008), 9-30, 13: "Rome n'est pas nécessairement le point de repère pour les sociétés indigènes." However muddled Häussler's account (where we move from "romanisation", by way of "globalisation culturelle", "interactions, contact culturel ou acculturation" to "articularisation" (as anti-globalization), "ethnogenèse" and "construction identitaire"), it still offers a good theoretical departure; the papers in the volume do not live up to this. Wolfgang Spickermann, 'Romanisierung und Romanisation am Beispiel der Epigraphik der germanischen Provinzen Roms', ibid., 307-320, 307, states that the frontier provinces have "eine sehr heterogene Bevölkerung... die mit Ausnahme einiger weniger Gebiete keineswegs auf eine einheitliche Kultur zurückgreifen konnte". One would have liked him to elaborate on this. 
repertoire that goes far beyond "Roman imports", material and immaterial. Some items can be traced to their origin, many cannot - how would one characterize a bit of Syrian culture imported into Gaul by a NorthAfrican, and re-exported after some re-packaging by a Rhineland trader to Britain? We could say that as soon as an indigenous culture appears to be integrated into the Roman empire as a consequence of conquest, it turns multicultural. To a local observer it is of small or no import that now, having been integrated into the empire, his environment has a number of traits in common with elsewhere in the empire; to that same observer it is of great import that his choice of dress, food, literature, religion, complete lifestyle, even language, is increased manifold. One might readily compare modern cosmopolitism and globalization: a local observer does not care whether some South Sea islander now watches the same television series and buys the same branded articles as he does. What he cares about is his own changing environment - change that will either be seen as beneficial, profitable, pleasurable, or that will instil insecurity and fear. ${ }^{41}$ This will hardly have been different in the Roman empire, although the pace of change may have been slow enough to avoid the strains and stresses so obvious today.

Not many ancient historians have been very explicit about this divergence. Most explicit is Jörg Rüpke who, limiting himself to the religious life of the Empire, in a few well-chosen lines almost manages to summarize much of what I have been saying above. His keyword is "Pluralität", cultural pluralism (here especially cultual pluralism): "Standardisierung heisst nicht Nivellierung oder 'kleinster gemeinsamer Nenner': Religion wurde in diesem Prozess zunehmend komplexer. Wie gezeigt, wuchs die Zahl der religiösen Zeichen, der Götter für die individuelle Auswahl massiv an ... Aber auch die Funktionsvielfalt wurde gesteigert". ${ }^{42}$ Also Barclay

41 Nederveen Pieterse, op. cit. (n. 39), 96 belittles the positive view as "confetti culture", "multiculturalism light", restricted to increased consumer choice. This addresses one of the things wrong with globalization, but some people may be true multiculturalists. It is impossible to make such fine distinctions for the ancient world. More to the point is E. Cashmore, 'Introduction', in Id. (ed.), Encyclopedia of race and ethnic studies (London 2004), xiii-xix, at xvii on plurality and its acceptance versus a "philosophy of the absolute" (and the ethical problems involved in this).

42 J. Rüpke, Von Jupiter zu Christus. Religionsgeschichte in römischer Zeit (Darmstadt 2011), 242. Repeated from: Id., 'Wie verändert ein Reich Religion - und wie die Religion ein Reich? Bilanz und Perspektiven der Frage nach der "Reichsreligion"', in H. Cancik and J. Rüpke (eds.), Die Religion des Imperium Romanum. Koine und Konfrontationen (Tübingen 2009), 5-18. See also: Id., 'Religiöser Pluralismus und das römische Reich', ibid., 331-354. 
discusses this growth of choice - and the need to negotiate one's way through it. ${ }^{43}$ Barclay rightly stresses the many sources contributing to this diversity (even there where an unchanging tradition was claimed to exist): "there were so many kinds of 'Greek' to become. And the Jew-Greek encounter is ... only part of a far more complex cultural pattern, in which Jews in the Diaspora engaged with different native traditions, which were themselves transformed in their encounters with 'Hellenism'".

Did Rome favour diversity? That they favoured integration is obvious: they looked for a modicum of coherence within their empire. But that empire is held together not only by Roman force and Roman benefits in exchange for taxes and so on, it is also an empire that has a real existence because the constituent parts interact. The cultural landscape is thereby diversified. I know of no source where Romans celebrate this diversity (indeed, we have the opposite where this diversity reaches Rome itself: then it is seen as threatening, from a typical xenophobic stance). But there are also no indications of Rome trying to stop this process, in favour of exclusive homogenization - something they could not have done anyhow. There is the possibility that they were not aware of this cultural divergence at the local level (but, as we have just seen, when "at home" they were very much aware of it). However, they may have noticed it, and they may very well have judged that divergence is not necessarily a bad thing, hand in hand with integration as it goes.

I see the Empire as a big machine designed (not knowingly, as an explicit policy, with a grand overall design, but wrought bit by bit) to facilitate movement of people, and thus culture contact and acculturation. The empire enables people - and with people ideas, religions, artefacts, in fact everything - to travel, to migrate, to interact. It produces and furthers connectivity. The empire also produces integration; indeed, that the empire does so ensures its very survival as an empire. Without a certain modicum of integration there would be no empire. But in becoming an integrated part of empire, its constituent parts become "imperial", in the sense of part of an empire, i.e. open to a wide range of outside influences,

43 In the sense of J.M.G. Barclay, 'Using and refusing. Jewish identity strategies under the hegemony of Hellenism', in M. Konradt and U. Steinert (eds.), Ethos und Identität. Einheit und Vielfalt des Judentums in hellenistisch-römischer Zeit (Paderborn 2002), 13-25, at $16-17$ a positive strategy: "conflictual fusion" or "resistant adaptation". One uses and refuses, and what one uses, one uses to one's own ends. Of course, there is a power factor: dominance, hegemony may force others (if only by denying social recognition) to adapt. But the actual power relationships are different all the time. 
and thus multicultural. This is what Rome is all about: it is not about being Roman in some restricted sense, but about being part of a larger whole that for want of anything better we call after its instigators: the Roman empire, but which seen from a cultural point of view is not Roman at all, but, in its own way, as multicultural as any multicultural society of today. 



\section{INDEX NOMINUM}

Acilia Plecusa $\quad 175,185$

Acilius Glabrio, M. 50, 53

Acutius Acutianus, M. 172

Aebutia Honorata Agriana 168,185

Aedemon 199

Aedui 58, 64

Aelia Filonice 171

Aelia Flaviana 174, 184

Aelia Marciana 177

Aelius Strabo, L. $\quad 167$

Aemilia Pudentilla 169, 171, 178, 184

Aemilius Paternus 175

Agricola 174, 266

Alamanni $\quad 189$

Alaric 99

Alba, R. 272

Albucia Candida $167,169,175,185$

Aletriani 7

Alfonso I, king 101-102

Alfonso II, king 101-102

Ancus Marcius, king $\quad \mathrm{xi}, 57-73$

Androklos 233

Antonia Valentina 167,185

Antoninus Pius, emperor 15, 42, 79-80, 121

Apuleius Madaurensis, L. $\quad$ 171, 178

Arabs 100-102

Arangio-Ruiz, V. 52

Armenia Auge $168,170,185$

Arminia Paulina 177

Arrecina Tertulla 177

Artemis 232-233

Artorius Castus, L. 192

Asclépiades 10

Athena 232

Augustine 207

Atilia Vera 175

Augustus, emperor $38,49,58,62$, $70,72-73,83-84,86,111,114,116,120$, 123-125, 134, 136, 162, 220, 222, 224, 230, 233, 260-261

Aulus Gellius 26, 65

Aurelia Calligenia 169,185

Aurelia Demetria 179

Aurelia Flavia 170

Aurelianus, emperor 191

Aurelius Aelurion 9

Aurelius Appianus 179
Aurelius Romanus 171

Aurelius Victor 191, 195

Aureolus 191

Autololes 212

Ballista $\quad 189$

Baquates 198, 202, 209

Barclay, J.M.G. 279-280

Bebenia Pauliana 168,170

Benabou, M. 199

Benoist, S. 72

Betilienus Occius, P. $\quad 255^{-256}$

Birks, P. 6

Bowlus, C.R. 89

Briquel, D. 68,71

Bruun, C. 177

Burbank, J. 270

Burgess, E.W. 272

Burian, J. 213

Caecilia Macrina 175

Caecilia Zaba $\quad{ }_{15}^{8}$

Caecilius Victor, C. $\quad 176$

Caeles Vibenna 71

Caesar (C. Iulius Caesar) 44

Camps, G. 201

Cantria Longina 166, 184

Caracalla, emperor 19, 29, 31, 41-42, 109, 228

Carbetania Maxima 175

Carbetanius Rufus, P. 175

Cassius Longinus $\quad 178$

Cassiodorus 90

Cicero (M. Tullius Cicero) xi, 11, 30, $43-57,61,148$

Cinna Magnus, Cn. $\quad 80-82$

Claude, D. 94

Claudia Capitolina $\quad 181$

Claudia Gallitta 177

Claudius Balbillus $\quad 181$

Claudius Nero, T. 46, 54

Claudius, emperor xi, 2, 57-6o, 62, $63-74,112,117,208,226,261$

Clodius Albinus 187

Clovis, king $3^{8}$

Columella $\quad 161$

Cominius Quintus, M. 167

Cominius Maximus, L. 172 
Commodus, emperor $\quad 79-80,112,124$

Constantine, emperor $\quad 42,112$

Cooper, F. 270

Cornelia Anulla 167, 184

Cornelia Eulogia Romanilla 172,185

Cornelia Romanilla Postumiana 172,185

Cornelia Sillibor $15^{8}$

Cornelia Valentina Claudiana 172

Cornelia Valentina Tucciana $\quad 166-168$, $177,182,185$

Cornelius Cinna Magnus, Cn. $\quad 81-82$

Cornelius Fronto, M. 43

Cornelius Fronto Gabinianus, M. 172

Cosconia Maluginensis Galitta 167,184 , 186

Crifo, G. 28

Cusinia Gratilla 181

Cusinius Messalinus, L. $\quad 181$

Daube, D. 44

Demaratus 63

Demos 224, 226-227, 231-234

Demougin, S. 183

Desideratia Desiderata $\quad 176$

Didius Julianus 195

Dio Cassius $57,66,68,83,214$

Diocletian, emperor 112

Dionysia 13, 17, 54

Dionysius of Halicarnassus $\quad 61-62$

Domitia Longina 229

Domitian, emperor 73

Domitius Calvinus, Cn. 259

Eck, W. $\quad 43,177,191$

Egica, king 98

Elsner, J. 232

Erwig, king $\quad 98$

Etrusci $\quad 61,70,72-73$

Fabia Bira 158, 184

Fabius Blandus, Q. 172

Fabius Fabianus, C. 179

Fentress, E.W.B. 206, 210

Ferentinati 7

Finley, M.I. 110, 268

Flavia Tertulla 167,184

Flavius Felix, T. 176

Flavius Fronto, M. 167

Florus (L. or P. Annius Florus) 61

Fournier, J. 14

Franke, Th. 191

Frézouls, E. 207

Frisians 204

Fulvia Sisennia 174
Fulvius Felix 170

Fulvius Kastus Fulvianus, L. 170

Furius [M]ontanus $\quad 176$

Fustel de Coulanges, N.D. $\quad 30,32-33$

Gaetuli 213-214

Gaius $\quad 6-10,36,39,119,123$

Gallienus, emperor $\quad 162,189,191,194-196$

Gardner, J.F. 155

Gauls $\quad 58,62-64,67,70-71,142,264$

Genucilius Rufus, L. 46

Gerber, C.F.W. von 24

Germani $\quad 42,190-191$

Giardina, A. 68

Gillet, A. 89

Goffart, W. 89

Gordianus III, emperor 191

Gordon, M.M. $\quad 272$

Goths xi, 89-104

Hadrian, emperor $\quad$ 14, 109, 206

Hannerz, U. 278

Heather, P. 103

Helvidia Priscilla 166, 168, 184, 186

Herennia 171

Hernici 7

Herodian 188-190, 195

Hippias, son of Philoxenus 53

Hispano-Roman 90, 95, 102

Hoerder, D. 271,276

Homonoia 232

Hygieia 177

Ingenuus $\quad 16,189$

Isidore of Seville 30, 94-95, 103

Iulia (daughter of Titus, emperor) 229

Iulia Festilla 177

Iulia [Pie]ris 172

Iulia Procilla 174

Iulius Pacatianus, C. 193

Iunia Rustica 156, 168, 179-180, 184

Iunius Rufus, M. 181

Iunius Rusticus, D. 179

Iuthungi $\quad 189$

Janon, M. 204-205

Jews $92,94,105,129,134-137,142-143$, 265,280

John of Biclaro 103

Jordanes 95

Juba II 214

Julian of Toledo 98

Jucker, H. 223

Juno $\quad 176$ 
Jupiter $\quad 176$

Justinian, emperor $\quad 42,116,118,125$

Justinius Favor, C. 176

Kelly, J.M. 44, 46

Kelsen, H. 28

Keppie, L. 191

Kharisia Magn[...] $\quad 176$

Khazanov, A.M. 202

Kulikowski 89, 103

Kunkel, H. 223

Kybele 231

Laecania Martina 172

La Piana, G. 127-129, 142

Larcius Macrinus, A. 210

Latini $\quad x i, 27,42,50,58-61,65^{-66}, 71$, 105-126

Le Bohec, Y. 191

Leovigild, king 93, 102-103

Lepidus, M. 221

Leveau, Ph. 206

Livia 221-223

Livius, T. $\quad 7,59-61,70-71$

Longinia Saturnina 172

Lopez Barja de Quiroga, P. 116

Lucretius (Lucretius Carus, T.) $\quad 5^{8}$

Lyasse, E. 71

Lysimachos 233

Lyso of Patrae 46, 52

Maccues 208, 214

Macrianus 189, 191

Maenius Gemellus, Cn. 46, $5^{2}$

Manlius Rufus, Q. 168

Manlius Sosis, L. $\quad 46,5^{0-51}$

Marcia Pacata 184

Marcius Rustius Rufinus, Cn. 193

Marcus Aurelius, emperor xi, 15-16, 18, $76,79-80,82,84,87,106$

Maria Lucina 170,185

Martial 135

Martino, F. de 26

Martin, P.-M. $\quad 58$

Mattingly, D.J. 211

Mauri 197, 199, 201, 211-213

McGinn, T.A.J. 141

Melani, C. 209

Mescinius Rufus, L. 45

Millar, F. 274

Mindius, M. 45

Minucius Thermus, Q. 45

Moccia Silvina $\quad 158$

Mommsen, Th. 32
Morizot, P. 203

Munatius Paulinus 172

Munatius Paul[i]nianus 172

Musulamii 206, 210, 213

Naerebout, F.G. $\quad$ xii, 187

Nahania Victoria $\quad 158$

Nasennia Iusta 174

Nee, V. 272

Nenci, G. $\quad 267$

Nero, emperor (Nero Claudius Caesar) 108, 112, 126, 221, 229

Nonia Arria Hermionilla 176

Nonius Cornelianus, M. 176

North-Africans 142,190

Numa Pompilius $\quad 58-59,63-64,71-72$

Numidii 206, 210, 213-214

Numisia Victorina $\quad 176$

Numitoria Moschis 172

Numphidius Sabinus 183

Nybgenii 210

Nymphidia 183

Odaenathus 189,191

Oliver, J.H. 14-16

Oppia, widow of Mindius, M. 45

Orosius 191

Ostrogoths 90, 98

Papius Mutillus 83

Park, R. 272

Paul, apostle 90, 105

Paulus, dux 99

Pausanias of Alabanda 46, 54

Pelagius (Pelayo) 101-102

Persians 189-190

Persus 171, 174

Pescennius Niger 187,195

Peter of Cantabria 102

Petronius Celer, C. 206-207

Petronius Mamertinus, C., praef. in Egypt 11

Petronius Taurus Volusianus, L. 194

Philip V, king of Macedon 21-22

Philo Judaeus 136-137, 143

Philo (oikonomos) 177

Plinia 174

Plinius Caecilius Secundus, C. $8,43,117$, 122, 124, 171, 174

Plotina 233

Plotius Faustus, M. $\quad 167$

Pompeia Mummiana 168, 184

Pompeia Paulina 174

Pompeia Sextina 176 
Pompeia Trebulla $\quad 170,184$

Pompeius Albinus, T. 176

Pompeius Paulinus 174

Pontianus $\quad 169$

Poppaeus Sabinus 83

Postumus 189

Powel, J.W. $\quad 267$

Ptolemaeus (Mauretania) 214

Publicia Pusinna 176

Publius Rupilius 7

Pudens 169, 171

Quintilianus 69

Rachet, M. 199

Raepsaet-Charlier, M.Th. 164, 181

Reccared, king 93, 102

Reccesuint, king 103

Reddé, M. 191

Regalianus $\quad 189$

Reginia Maxima 172

Richardson, J.S. 6

Roderic, king 100, 101

Rodger, A. 6

Roia Titinia 174

Romulus $\quad 5^{8-59,71-72}$

Rothe, U. 149

Rüpke, J. $\quad 279$

Sadington, D.B. 210

Salenses 212

Salvius Rufinus Minicius Opimianus $\quad 176$

Sarapis 232

Scheidel, W 273

Schönbauer, E. $\quad 52-53$

Seianus (L. Aelius Seianus) 66

Seleukos of Rhosos 10

Sempronius Gracchus, C. 65

Seneca (L. Annaeus Seneca) 25, 67, 174

Senecius Garrulus, P. 175

Sentius Caecilianus, Sex. 208

Septimia Polla 169, 184

Septimius Geta, P. 169

Septimius Severus, L., emperor 160,187 , 188, 190, 195

Servilia Copiesilla $\quad 167,184$

Servius Tullius, king, alias Mastarna 57 64,71

Setälä, P. $\quad 181-182$

Severus Alexander 189-190, 195

Sextius Alexander, T. $\quad 176$

Sextius Martialis, C. $\quad 176$

Sextius Martialis, Q. $\quad 176$

Sextius Victor 210
Shapur I 191

Shaw, B.D. 204, 206

Shils, E. 277

Sicinius Pontianus 171

Siculi 7

Silanus, C. 221

Silius Nerva, P. $\quad 45-46,54$

Silvestrini, M. 256

Sirks, B. 116

Spaul, J. 207, 212

Statia Fida $168,170,184$

Statilia Crescentilla 172

Strabo 61

Succonia Rustica 166, 184

Suetonius Tranquillus, C. $57,72,221$

Sulgius Caecilianus, C. 193

Sulpicius Galba, Ser. 199, 208

Sulpicius Rufus, Ser. $\quad 45,5^{2}$

Synkletos xii, 221-235

Tacfarinas 200, 214

Tacitus (P. Cornelius Tacitus) 57, 63-64, $67-68,71,126,174,214,221,234,266$

Tarquinius Priscus, L., king 63, 71

Teillet, S. 94

Terentius Hispo, P. 45

Tertia Romana 177

Theoderic, king $\quad$ 90, 98

Theodosius, emperor 42

Thompson, E.A. 103

Tiberius, emperor $83-84,178,220-223$, 227, 234

Titius Similis, C. 193

Tocqueville, A. de 60

Traianus Mucianus 194

Trajan, emperor (M. Ulpius Traianus) 8 , 13, 124, 181, 206, 233

Tullus Hostilius, king 60 n., 61, 64, 72

Tyche 228, 232

Ulpia Paratiana 172

Ulpia Valeria 172

Ulpius Longinus 170

Valerianus, emperor $\quad 189,191$

Valeria Rufina 177

Valerius Naso 221, 224

Valerius Pansa, C. 167

Valerius Poblicola Vettillianus, Sex. $\quad{ }_{176}$

Valerius Valerianus, M. 172

Varia Pansina $167,169,184$

Vatinius Maximus, C. $\quad 256$

Venidius Ennychus, L. 117

Versnel, H. $\quad 267$ 
Verulani 7

Vespasian, emperor (T. Flavius

Vespasianus) 38, 107, 229-230

Vettia Saturnina 166,185

Vettia Tranquilla $\quad 176$

Vettius Marcellus, C. 168

Veyne, P. 13

Viatoria Taurina 172

Viatorius, C. 172

Vibia Maria Maxima 172

Vibius Marianus, P. 172

Vibius Marsus 221

Vibius Salutaris, C. $\quad 233,241$

Virgilius Maro, P. 60

Viria Rustica $\quad 178$

Visigoths 90, 92, 93, 97, 99, 103

Vistilia 183
Voconius Romanus 171,174

Volesus Valerius Caesus $\quad 80-82$

Volesus Valerius Messala 82

Von Thünen 133

Wallace-Hadrill, A. $\quad 150$

Wamba, king 98-99

Weaver, P.R.C. 116

Wenskus, R. 89

Whittaker, C.R. 201, 207, 211

Wolfram, H. 89

Woolf, G. 144, 264

Zegrenses 202, 209

Zenobia 191

Zeus 230, 232

Zoïlus 9 


\section{INDEX GEOGRAPHICUS}

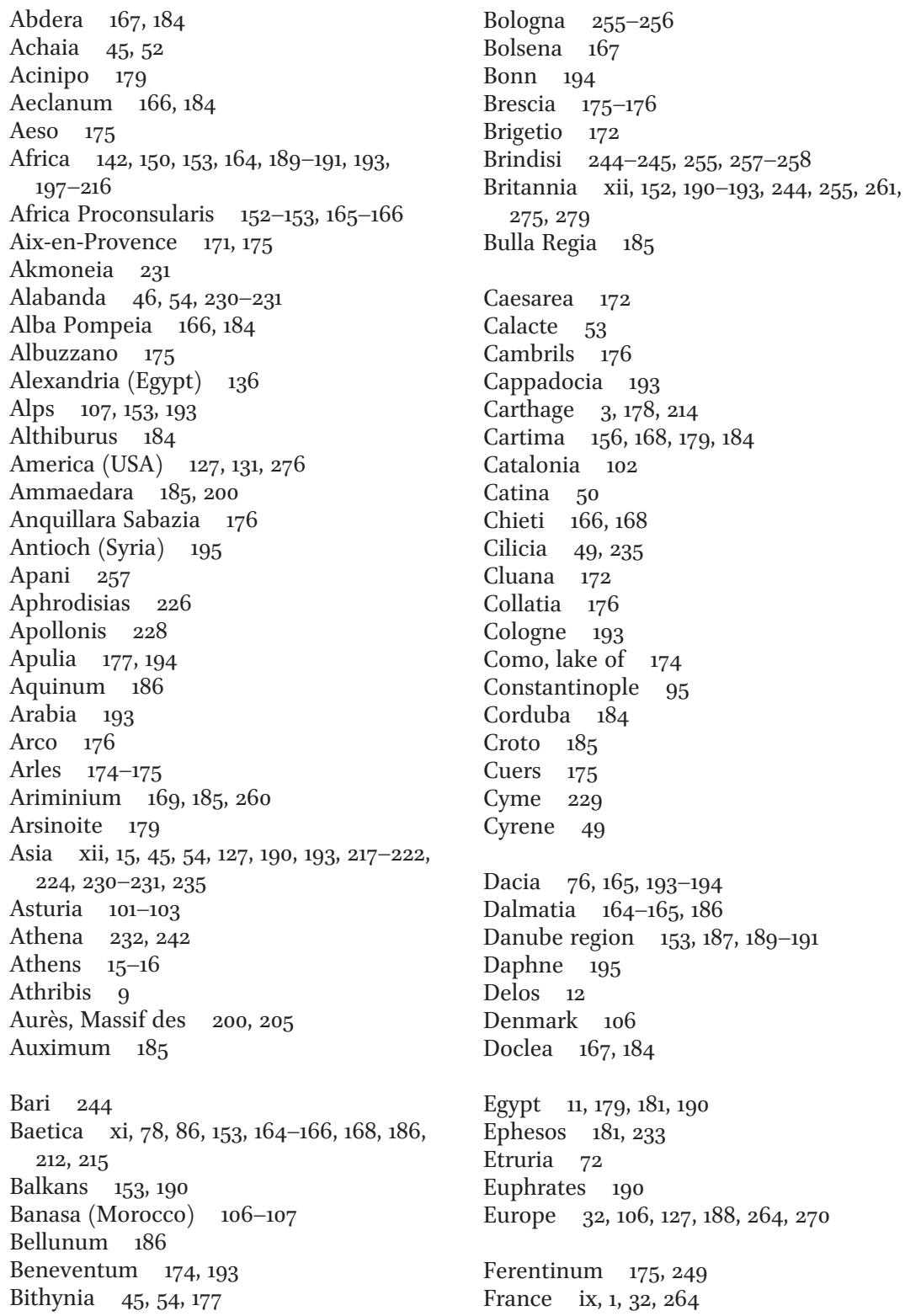


Gallia Aquitania $\quad 152-153$

Gallia Belgica $152,255,261$

Gallia Lugdunensis $\quad 15^{2-153}$

Gallia Narbonensis $\quad 152-153,193$

Gaul 2, 92, 99, 100, 103, 153, 187, 279

Germania 203

Germania Inferior $\quad 152,194$

Germania Superior $\quad 15^{2-153}, 190,194$

Giancola 258

Gloucester $\quad 244-245,257-259,261-262$

Grottaferrata 176

Heraclea 17

Herculaneum 117

Hispania 90, 98-100

Hispania Lusitania $\quad$ 153, 193

Hispania Tarraconensis 153

Ilion 222

Illyricum $83,84,189-190,194$

Irni 80,86

Isthmus 69

Italy xi, xii, 22, 52, 55, 90, 105, 113, 128, $151,153,155,158,164-166,186,189-190$, 192-194, 245, 255, 261-262

Jerusalem 105

Judaea 47

Lamasba $\quad 185$

Lambaesis 170-171

Larinum 162, 183

Larissa 21

Latium 59, 107, 257

Leptis Magna 169,184

Liguria 174

Lugdunensis $\quad \mathbf{1 5}^{2-153}$

Lyon 63

Maastricht $\quad 106$

Mactar 207

Madauros 166, 172, 185

Magna Gracia $\quad 255,260$

Malaga $\quad 86,179$

Mallos 235

Maudach 177

Mauretania $107,153,190,198-199,208$, 212, 214-215

Mauretania Caesariensis $\quad 165,186,206$, 208, 214-215

Mauretania Tingitana xii, 165, 186, 199, 208-209, 212, 214-215

Merida 96

Mesopotamia $\quad 187,193-194$

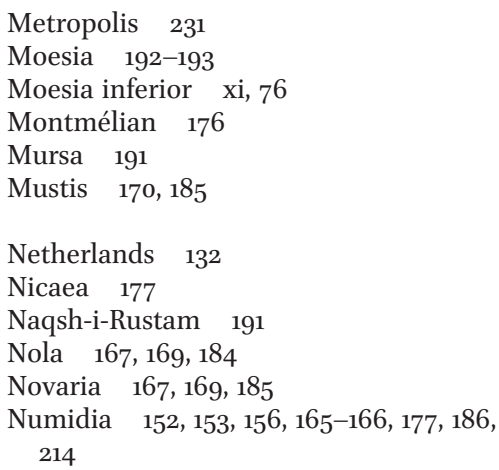

Oea 178,184

Ostia 61-62, 66-69

Ostur 166, 184

Oued Genini 205

Oued Skiffa 205

Oued Zraia 205

Oviedo 101-102

Paestum 185

Palestine 192, 194

Palmyra $\quad 3,189,191,196,254,275$

Palus Pomptina 69

Pamphilia 235

Pannonia 83, 191-192, 194

Pannonia Inferior 164-165

Pannonia Superior 186, 254

Patrae 46, 52

Pavia 175

Pergamum 2, 231

Perolet 175

Picenum 194

Pompeii 141, 257, 259

Pontus-Bithynia 45

Potaissa 76

Praeneste $168,184,255,261$

Ras el Aïn/Talalati 205

Ravenna 260

Rhine 149, 189-191

Rimini 169, 260

Risinium 168, 170, 184

Rodengo 175

Rognes 175

Romania 77

Rome, Aventinus $\quad 58,60-62,64-67,71$, 135

Rome, Caelius 61

Rome, Campus Martius $\quad 65^{-66}, 135^{-136}$

Rome, Casal Bertone 140 
Rome, Emporion $\quad 140$

Rome, Janiculum 61

Rome, Palatinus 59

Rome, Pincio 65

Rome, Pons Sublicius 61

Rome, Subura $\quad 135,138,141-142$

Rome, Trastevere $33,129,135^{-137}$, 142-143

Rufiniana 177

Rusicade 177

Saguntum 174

Sahara 205, 269

Saldae 185

Salonae 167,184

Samnium 259

Santillana 102

Sardes 229

Sarmizegetusa $\quad 167,185$

Savaria 255

Scotland $\quad 269$

Segermes 184

Segni $\quad 257-259$

Septempeda 185

Seressi $\quad 166,168,170,185$

Seville $94-95,103,179$

Sicily $8,46,49,51,53,255,260-261$

Side 235

Singilia Barba 175,185

Smyrna 2, 220-221, 223-229, 231, 234

Spain or Hispania $\quad x i$, 9o, 91-92, 94-95, 97-100, 102-103, 107, 150, 151, 152, 153, 156

Suasa $\quad 256$

Syria $\quad 135,191-194$
Tacape 200

Tarquinia 63

Tarracina 170, 184, 259

Tarragona 176

Tarsus (Turkey) 105

Teate Marrucinorum 184

Teutoburgium $\quad 186$

Thagaste 185

Theveste 210

Thuburbo Maius 184

Thugga 178

Timgad $\quad 166-168,177,182,185$

Tivoli 172

Tres Galliae $\quad 63-64$

Treveran area 149

Trier 159, 245, 255, 260-261

Troesmis (Moesia) $\quad$ xi, $75^{-88}$

Tusculum 176

Ubian area 149

Uchi Maius 168, 174, 185

Umbria 194

United Kingdom 132

Urago Mella $\quad 176$

Vienne 176, 193

Virunum 172

Volsinii 184

Volubilis 184, 198, 209

Yverdon 177

Zarai 203 


\section{INDEX RERUM}

Acculturation $\quad 143,201,266-267,271-272$, 275,280

Administration $\quad 44-45,47,97-99,195$, 208, 243, 260, 264

Aedilis $\quad 75,80-81,87,256-25^{8}$

Agriculture 135, 178, 180, 200, 203, $205^{-207}, 210,213,264$

Antiquae 92

Ara Pacis 223

Arab conquest 99-101

Aramaic, language 105

Army xii, 3, 75, 81, 89, 98, 100, 113, 187-196, 204-205, 209, 213-214, $25^{8}$

Assimilation $60,74,99,102,142,151,267$, 270, 272, 277

Augustales 156, 169

Auxilia 3, 75, 198, 214

Barbarians 89, 208, 264

Benefaction 2, 126, 156-157, 164, 166, 182

Benefactor/ress $\quad 3,152,156,159$

Bishops 93, 95-99

Border xi-xii, 87-88, 109, 152, 187, 189-192, 196, 200, 213-214, 235

Boscoreale cups 223

Boule 219, 228, 231, 233-234

Brick stamps $\quad 180-182,243-264$

Burial 103, 134, 140, 264

Businesswomen 155

Canabae $75,76,187$

Cancelleria-Reliefs $\quad 218$

Career $\quad$ 105, 155-157, 191-194, 196

Castra 76, 187

Catacombs 134

Centuriatio 201

Child 105, 108-110, 117, 120-121, 123, 126, 128, 154

Christianity 264

Christianity, Arian 93

Christianity, Catholic 93, 103

Church 93, 96-98, 101

Church, councils $93,95^{-99}$

Citizenship $\quad \mathrm{x}-\mathrm{xiii}, 1,7-11,49-52,61,86$, $87,90,94,104-126,136,143,147-160$, 201-202, 209, 263, 268

Citizen sui iuris $\quad 35,154-155,178$

Civic life xii, 147-148, 151

Civis $18,23-42,48-49,52,61,105$
Civitas xi, xiii, 14, 22-24, 30-32, 32, $34-35,37-38,41,49,104,210,263$

Clausurae 205

Code of Erwig 92

Code of Leovigild 92

Code of Euric 92

Coins 221-235

Collegium 72, 159, 222, 256

Colloquium inscriptions 202, 209

Colonia $3,22,27,62,75,107,122,182,210$, 256, 258, 261-261

Comitia (Assembly) 63, 69, 8o, 87, 113

Comitia Centuriata 1,66

Commentarius $81-84,86,88$

Commercium 39, 116

Constitutio Antoniniana xi, 2, 8-9, 22, 27, 49, 90, 109, 116, 124-125

Conubium 96, 108, 116

Courts Law $5,10-16,18,45,47-49,5^{1}$, 54-55, 94, 95, 105, 119

Cronicle of Albelda 101

Cronicle of Alfonso III 101

Cult xii, 3, 34, 135, 137, 158, 169, 217-235

Curia 85,87

Dead Sea Scrolls 47

Decuriones/ decurial order $86-87,93$, 157,256

Dediticii $\quad 115,124$

Digest $12,118,189$

Domus 138-139

Dress xii, 102-103, 147-151, 158, 16o, 218, 226, 279

Duumvir $80-81,87,246,250-251,256$

Elite $90,98,107,130,138-140,156-157$, $183,202,207,210-211,214,216,234,263$

Enclave 129, 131-132, 137, 142

Epigraphic habit 152,210

Equites, equestrian order xii, 83, 157, 161-186, 192, 192, 199, 208

Estate $46,51,92,94,102,110,119,121,123$, $126,173^{-174}, 177,180$

Ethnicity/ethnic groups $\quad 89-90,96-97$, 99, 101-103, 109, 130-132, 137, 140, 143, $187,256,260$

Euergetism xii, 158, 163-164, 166, 168-170, 179, 182, 259

European Union 106 


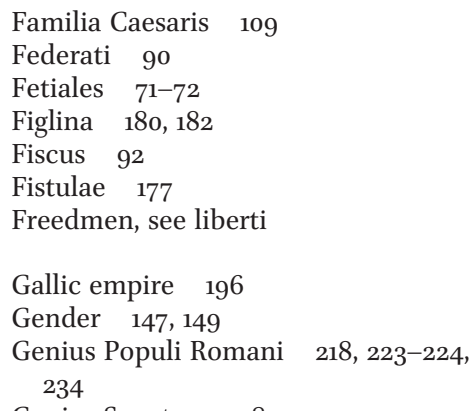

Genius Senatus 218-219, 222-224, 234 gens, gentes 40, 61, 89-90, 97, 99, 101, 103, 206

Germanic tribes $\quad 89,91,190-191$

Ghetto 131-132, 137, 142

Globalization $\quad 31,276,279$

Gods xiii, 201, 204, 218, 222-223, 225, 227-228, 230-235, 279

Grain/annona $108,123,174,180$

Heroninos archive 179

Horti 140

Identity $1-2,4,15,34,41,89-90,96,99$, 101-104, 130, 138, 142-144, 149, 158, 259, 261, 269, 270, 277

Industry $130,140,142$

Inheritance $45,51^{-} 52,58,105,116$, $118-124,126,154-155,178-180,182,259$

Insulae $139-140$

Intermarriage $\quad 92,93,99$

Islamic conquest 100

Islamization 100

Ius civile $\quad 6-7,34-35,48,50,125$

ius honorum $\quad 2,62-63$

Jurisdiction $\quad x i, 5^{-9}, 12-13,16,18-19,44$, 47-49, $5^{1}$

Latin status $86,107-108,114,116,118,123$

Latin, language $\quad 44,90-91,94,102-103$, $148,210,243,268$

Latini Iuniani $105^{-126}$

Latinitas 107

Latinization $103,150,158$

Law of the Twelve Tables 120

law, Civil law 54, 154-155

law, conflict of laws $\quad 47-50,55$

law, international $x i, 43,47-51,54$

law, local $x i, 46,49,52,54-55$

law, municipal xi, $75,77-78,81,86-88$, 118,124 law, Roman xi, 6, 13-15, 18, 51, 86-87, 90, $97,102-103,112,155,160$

law, tribal 106

Legacy 116, 121-122

Legal status $\quad 5,8,49,92,128,143,25^{6}$, 259

legio V Macedonica 76

Legiones Parthicae 187

Legions $\quad 75,105,190-192,199,258$

Legislation $\quad 82-84,91-92,102,114,125$

Lex Aelia Sentia 111, 115, 117, 123, 125

Lex de provinciis praetoriis 12

Lex Flavia municipalis 18

Lex Fufia Caninia 114

Lex Irnitana $78-80,86$

Lex Iulia de maritandis ordinibus $\quad 80-82$, 86-87

Lex Iunia (Norbana) 114, 121, 125

Lex Malacitana $\quad 41,81,86-87$

Lex Minicia 108-109

Lex Papia Poppaea 8o, 81, 82, 84, 86, 88, 120

Lex Rupilia 7, 53,

Lex Troesmensium $\quad 75^{-88}$

Lex Ursonensis 78

Lex Visellia 123

Liberti 94, 96, 105-126, 138, 156-157

Limes 204

Ludi saeculares $\quad 72-73$

Lyon tablet 63,67

Magistrates xii, 12-13, 15, 19, 37, 39, $55,76,85-87,107,111,113,115,117-118$, 243-244, 256-259

Marriage $\quad 35,92-93,105,108,117,120,124$, $154,159,178$

Matrona 151, 161-186

Migration 99, 127-130, 143, 200, 207, 266, 273-278

Military middle cadre $\quad$ 191-196

Minority 99, 132

Monty Python 265

Multiculturalism $\quad 132,187,270,279,281$

Municipium 27, 75, 79-8o, 85, 87-88, 168, 210, 256

Myths $\quad 58,232-233,273$

Names xii, 94, 96, 99, 101-102, 148, $150-151,158,160$

Nomads xii, 197-216

Officina 253,255

Officinator 180,182

Ordo $75,82-83,102,166,168,183,263$ 
Parthian wars 76,190

Patria potestas 111

Patronage, patron/ess xii, 103, 105, 110-112, 115-116, 119-126, 149-150, 152, 159, 164, 208

Peregrini $\quad 2,22,24,27,30-32,39-42$, 49-50, 55, 125, 129

Persian war 189-191

Personification 218-219, 223-226, 229-234

Plague 187,189

Polis xii, 21, 31, 34, 52, 217-242

Pomerium 6o, 62, 64-68, 71, 73, 137

Praetor 18-19, 40, 114, 116, 119-121

Priest/ess $\quad 85,152,156,159$

Priesthood 81, 82, 149, 154, 156-159

Prostitution 135, 141-142, 183

Provincial governor $12-13,15,44-45$, $49-50,5^{2-54}, 111,189,192,198$

Quiritary ownership $\quad 115,117,126$

Regiones (Rome) 134-135, 141

Res publica $30-31,34,166,218,25^{6-257}$, 261

Rescripts 112, 121

Resistance 2, 198-199, 202, 207, 209, 211-212, 267

Romanitas $\mathrm{x}$, xiii

Romanization $\quad x i-x i i, 2,90,103,147,151$, 154, 158, 209, 263-266, 268-269
Sacerdos $\quad 80-81,87,164,166,173$

Sedentary communities xii, 197, 200, 202, $207,211,213,216,243$

Senate $\quad x i-x i i, 7,17-18,38,58,62-64,67$, 68, 71, 108-109, 111, 126, 174, 217-235, 248

Senator (Rome) 82, 128, 192, 194, 221

Senatorial order $110,162,170-171$

Senatus Consultum 9, 18, 64, 162

Slavery, slaves $\quad \mathrm{xi}, 9,92,94,109-126$, 128-129, 138-139, 143, 177, 179, 259, 273

Social science $143-144,266-268,273$, 276-277

Social status $110,128,156-157,159$

Social War 107

Statue $\quad 62,151,156-160,166,168-169$, 222-224, 228, 230-233, 241

Summa honoraria 164

Synagoge $\quad 135^{-136}$

Tabula Banasitana 125, 201, 209

Tabula Heracleensis 17,81

Tax 92-93, 98, 203-205, 210, 273, 280

Tenants 92, 100, 139

Tertiae Romanorum 92

Testament $113-117,120-122,178$

Tile stamps $\quad 243-262$

Urbanization $128,15^{-15}$, 154-155, 264, 273

Veterans 107

Vicus $75^{-76}, 134-135,141$

Will 105, 113, 116, 120-121, 123 


\title{
INDEX LOCORUM
}

\author{
1. Literary evidence
}

Acts 22.23-29: 105, n.3; 22.28: 90, n.6; 25.11: 9o, n.6

Anonymus Continuator Dionis, 10.5 (FHG IV 197): 191, n.16

App., $B C$ 1.25: 66, n.48 Rom., Praef. 7: 268, n.14

Apul., Apol. 23.1: 171, n.61; 44.6: 178, n.110; 71.6: 171, n.57; 182, n.143; 72.6: 178, n.110; 75.8: 171, n.59; 77.1: 171, n.57; 182, n.143; 87.7: 178, n.111; 87.10-11: 169, n.46; 182, n.143; 184; 87.10: 178, n.109; 92.1: 171, n.58; 92.2: 171, n.6o; 92.3: 171, n.58; 93.3-4: 179, n.113; 101.5: 178, n.112

Aug., Epist. 46.1-6; 47.1-2: 207, n.43

Aul. Gell., Noct. Att. 4.12: 26, n.17; 5.13.4, 5 119, n.97

Aur. Vict., Caesares 20.24-26: 195; 33.1-2: 191; 33.33: 195, n.25

Bab. Shabbat 33b: 265, n.6

Cic., Att. 6.1.15: 49; 7.2.8: 114, n.56; 14.12: 107, n.13

Pro Archia 7: 50, n.20

Balb. 22: 11, n.9; 28: 49

Fam. 13.15.3: 44; 13.19: 52; 13.19.2: 46; 13.19.3: 52; 13.26: 45; 13.26.3: 43, n.3; 13.30: 46, 50-51 \& n.21; 13.37: 53 \& n.28; 54 ; 13.53.2: 46 ; 13.54 ; 13.57.1: 45 ; 13.64.1: 46; 54 ; $13.65: 45$

De Or. 3.45: 148, n.4

ad Quint. fratr. 2.10-12: 25, n.14; 41, n.45 de Rep. 1.49: 30; 2.18.33-34: 59, n.7; 2.18.33: 61, n.21

Top. 10: $113, \mathrm{n} .48$

2Verr. 2.13.32: 53 \& n.29; 2.31-32: 49; 2.32: 7 ; 5.147; 5.162; 5.168: 105, n.2

Claud., Gild. 108-109: 6o, n.12

Colum., pr. 12: 161, n.3
Crónicas Asturianas: 100, nn.6o-63; 101, nn.64-65; 67-68; 102, nn.69-71

Dio Cass. 2.8: 59, n.7; 55.10.11: 183, n.150; 55.13.7: 116, n.70; 55.28.3-4: 214, n.73; 56.10.3: 83, n.22; 60.7.1: 183, n.150; 6o.9.1-5: 208, n.51; 6o.11.1-5: 66, n.52; 6o.11.3: 69, n.58; 60.13.2; 6o.28.1: 112, n.43; 61.17.3: 183, n.15o; 61.29.7: 117, n.82; 68.5.1: 218, n.5; 72.11.3: 204, n.27; 78.9.5: 109, n. 26

Dion. Hal. 3.36-45: 59, n.7; 3.44: 61, n.25; 3.45: 61, n.20; 4.24.1-3: 112, n.47; 4.24.4-8: 113 , n.54; 116, n.71

Erod. 3.39: 25, n.14; 41, n.45

Eutr. 1.5: 59, n.7; 61, n.20

Festus, 23: 190, n.8; 191, n.15

Flor. 1.1.4 \& 8: 59, n.7; 1.1.8: 61, n.23

Gell., 13.14: 71, n.68; 13.14.1: 65, n.42

HA, Hadr. 5.8; 12.7: 199, n.9; 21.7: 107, n.13

Com. 13.5: 199, n.6

Sept. Sev. 2.3-4: 212, n.68

Alex. Sev. 53.7: 195

Max. 11.7: 190, n.11

Aur. 21: 212, n.68; 21.1: 199, n.6; 22.11-12: 212, n.68

Herod., 2.7.7; 2.9.7; 2.13.1: 195; 2.14.6-7: 190, n.8; 3.8.4: 188; 6.3.1 f.; 6.4.7: 190, n.8; 6.7.2-3: 189; 6.7.8: 190; 6.7-8; 8.1-3: 190, n.8

Historia Gothorum: 95 \& n.31

Hor., Ep. 1.6.25-27: 58, n.6

Isidor., Ety. 15.2.1: 30; 104, n.78 Hist. c. 49; 61-62: 103, n.77

Iohan. Biclar, c. $27 ; 32 ; 36 ; 40 ; 47$ : 103, n.77 
Ioan. Antiochenus, 126.1 (FHG IV 587): 195, n.27; 140 (FHG IV 593): 189, n.6

Iul. Tol., Historia Wambae: 97, n.41; 506; 507; 512: 99, n.51; 513: 98, n.48; 99, n.51; 531-533: 99, n.51

\section{Liber Vitas Sanctorum Patrum \\ Emeretensium: 95, nn.29-30}

Liv. 1.32.4: 59, n.8; 1.32.5-14: 70, n.62; 1.32-34: 59, n.7; 1.33.1-2: 6o, n.13; 1.33.2: 61, n.18; 1.33.6: 61, n.20; 1.44.3-5: 71, n. 67 9.43.22: $7 ; 9.45 .6-8: 7$ 25.3: 108, n.18 27.16: 25, n.14 40.43.1: 17

Lucr., 3.1025: 58, n.6

Mart., Ep. 2.17: 135, n.27
5.22: 135, n.25
6.66: 135, n.27
7.31: 135, n.26
10.94: 135, n.26
11.61; 11.78: 135, n.27
12.18: 135, n.25

Menand. Rhet. 1, 360.10-16 Spengel: 8, n.7; 1, 363.4-14 Spengel: 8 \& n.7

Orosius, 7.22.12: 19o, n.8; 191, n.15

Philo, Leg. 155: 136 \& n.31; 137; 143

Plaut., Cist. 2.1.27: 25, n.14 Pseud. 5.1.25; 5.2.87 \& 89: 25, n.14

Plin., nat. 3.3.30: 107, n.17; 3.20.135: 107, n.13; 3.52: 252; 5.5: 212, n.69; 5.11: 208, n.48; 5.108: 54, n.31; 33.32: 161, n.6

Plin., Ep. 7.11.5: 174, n.79; 7.16.4: 117, n.83; 126, n.140; 8.16.1: 121, n.117; 10.4.2-5: 171, n.56; 10.4.3: 174, n.8o; 10.5.2: 118, n.89; 124, n.133; 10.11.2: 124, n.133; 10.104: 122, n.120; 10.113: 8; 10.124, n.133

Pan. 37-40: 50, n.19

Plut., Vita T.Q. Flam.: 21, n.2 Galb. 9.1-4: 183, n.148; 9.2: 183, n.147

Plb., 3.33.15: 214, n.74
Prob., Vita Persi 7-8: 171, n.55

Procopius, Bell. 3.2.7; 8.5.5: 90, n.7

Quint., 2.21.18: 69 \& n.59; 3.8.16: 69 \& n.6o Decl. 340; 342: 114, n.56

Salv., Eccl. 3·7.31: 119, n.93

Schol. Pers. 6.6: 176, n.74

Sen., breu. uitae 13.8: 68, n.54

Dial. 12, 14, 3: 182, n.144

Serv., Aen. 2.351: 225, n.41

Strabo 5.3.5 \& $7: 59$, n.7; 5.3.5: 61, n.26; 5.3.7: 61, n.22; 17.3.12: 212, n. 67

Suet., Aug. 40.3-4: 113, n.54; 40.4: 116, n.71 Tib. 29: 221, n.21

Cl. 21.5: 72, n.73; 25.1: 112, n.43; 25.2: 117, n. $82 ; 25.5: 70$, n.62; 25.10: 73, n.78; 25.14: 72, n.70; 41 \& 42.5: 70, n.65

Nero 4.2: 183, n.15o

Galba 7.1: 208, n.50

Dom. 4.7: 73, n.75

Tac., Agr. 7.2: 174, n.78

Ann. 2.85.1-4: 183, n.149

3.27: 126 , n.139

4.15: 221 \& n.19; 4.23: 214, n.73; 4.55 f.: 221, n.19; 4.56.3: 221, n.22; 4.72: 204, n.27

11.11: 73, n.75; 11.15: 72, n.72; 11.23-25: 63 , n.29; 11.24.2: 63, n.34

12.23-24: 64, n.40; 12.23.2-24: 71, n.68; 12.24: $68, \mathrm{n} .56$

13.26-27: 111, n.40; 13.26: 120, n.102; 13.27: 112, n. 47

15.32: 183, n.150; 15.52: 25, n.14; 41, n.45; 15.6o.7: 174, n.77; 15.72.1-2: 183, n.148

uir. ill. 5: 59, n.7; 61, n.2o

Valerius Maximus, 2.6.6-7: 112, n.46

Varro, De L.L. 5.48: 135, n.25

Virg., En. 6.815-816: 6o, n.14

Zon., 7.7: 59, n.7

Zos., 1.39.1-2: 191, n.15; 1.44.1 f.: 190, n.8 


\section{Epigraphical evidence}

$A E$ 1888, 36: 246

1890, 8: 254

1894, 45 = 1941, 115: 202, n.19; 96 = 1909, $123=2002,1694: 204$, n.29

$1897,48=151=1998,1579: 208$, n. 52

1898, 39a; 39b: 206, n.41; 211, n.65

1899, 64: 199, n.5

1903, 368: 199, n.5

1904, 37: 167, n.28; 184; 76: 210, n.58; 150: 208, n.52; 217: 116, n.76

1905, 10: 210, n.61

1906, 80: 176 , n.97

1907, 17: 211, n.65; 19-21: 206, n.41; 211, n. 65

1909, $76^{\text {a }} ; 76^{\text {b }}: 247 ; 123=2002,1694: 204$, n. 29

1910, 20: 206, n.41; 210, n.62

1917/18, 39: 210, n. 58

1919, 256-257: 190, n. 8

1920, 44: 202, n.19

1921, 23: 202, n.19

1923, 26: 206, n.41; 211, n.65

1924, 66: 208, n. 48

1931, 36; 38: 212, n.69

1934, $155=1957,121=1958,26: 212$, n.68

1940, 70: 211, n.65

1941, 79: 208, n.49; 80: 254; 115: 202, n.19; 118: 209, n.54

$1942 / 43,54=1946,5^{2}=275=1987,1093$ : 202, n.19

1946, $5^{2}=275$ = 1987, 1093: 202, n.19

1950, 158 = IRT 6o7: 169, n.48

$1952,42=1953,77=1957,201=1987$, $1090 \mathrm{a}=1094 ; 43: 202$, n.19

1953, $77=1957,201=1987,1090 \mathrm{a}=1094:$ 202, n.19; $78=1957,202 ; 79=1957$, 203; 80: 202, n.19

1954, 110: 202, n.19

1956, 152: 249

$1957,121=1958,26: 212$, n. $68 ; 201=1987$, 1090a = 1094: 202, n.19; 202; 203; 204: 202, n.19; 266 = Inscr. Scyth. Minoris $\mathrm{V}, 155: 75, \mathrm{n} \cdot 3$

1958, 26: 212, n.68

1960, 337 = Inscr. Scyth. Minoris V, 158: $75, \mathrm{n} \cdot 3$

1961, 340: 212, n.68

1965, 259: 177, n.103

1969/70, 106: 167, n.34; 169, n.45

1971, 85: 167, n.34; 169, n.45; 184; 534: 106,

n.4; 202, n.19

1973, 16: 208, n.51
1974, 143a = CIL VI, 41289; 143b: 176, n.96 1977, 259: 176, n.91

1978, 119: 117, n.83; 145: 162, n.5

1979, 292: $25^{2}$

1980, 820 = Inscr. Scyth. Minoris V, 164:

79, n.9; 956: 166, n.19; 167, n.33; 185; 971: 210, n. 58

1982, 304: 249

1984, 586: 13

1986, 333 (Lex Flavia municipalis, c.91): 18

1987, 731 = IRAT 27: 176, n.93;

1090a = 1094: 202, n.19; 1092; 1093:

202, n.19

1989, 872: 170, n.54

1990, 64: 172, n.69

1991, 424: 259, n.72

1995, 395: 248

1998, 1579: 208, n.52

1999, 815: 211, n.65; 1016: 175, n.90

2000, 355: 245 \& n.12; 821: 251

2001, 748: 259, n.72

2002, 1694: 204, n.29

2003, 593: 248

2004, 579: 166, n.16; 1878: 211, n.65

2007, 214: 66, n.49; 345: 248

CBAI, c.s. n. 1152: 245

CEMC $19=A E$ 1990, 64: 172, n.69

CIL I, 767, p. $947=I L L R P$ 430: 259, n.72

CIL I' ${ }^{2}$ 113-116: 244, n.9; 202 = InscrIt. XIII, 3, n 66: 70, n.62; 969; 970; 971: 70, n.62; $2877^{\text {b }}: 252$; 3475: 249; 3476: 247; 3477: $246 ; 3478: 247 ; 3478^{\text {a \& b }}: 247 ; 3480^{\text {a \& b }}$ : 247; 3501; 3502; 3521: 245

CIL II, 1120: 212, n.68; 1267 = CILA Hu, 81: 166, n.21; 184; 1356: 179, n.121; 1955: 184; 1956 = ILS 5512 = ILMMalaga 6: 156, n.21; 168, n.43; 179, n.117; 184; $1979=$ IRAl 2 + IRAl 25: 167, n.32; 184; $2015=$ = AE 1961, 340: 212, n. $68 ; 2224=I L S 6905=C I L$ $\mathrm{II}^{2} / 7,282: 174$, n.82; $4461=I L S 2661=$ IRC II, 54: 175, n.86

CIL II ${ }^{2} / 5,783=$ CIL II, $2015=A E$ 1961, 340: 212, n.68; 784; 796: 185

$\mathrm{II}^{2} / 7,5=$ CIL II, 3351 = CILA 3.1, 339: 158, n. $24 ; 282: 174$, n.82; 184; 830: 175, n.84 
CIL III, 1559: 254; 1717: 168, n.39; 170, n.5o; 184

3228: 191, n.14

4327 = RIU III, 637: 172, n.72; 4671: 254; 4692; 4693: 254; 4692 ${ }^{\mathrm{a}-\mathrm{b}} ; 4693^{\mathrm{a}-\mathrm{c}}: 249$, n.27; 4859 = ILLPRON 6o8: 172, n.7o

6162 = Inscr. Scyth. Minoris V, 156: 75, n.3; $6166=$ ILS $2474=$ Inscr. Scyth. Minoris V, 154: 75, n.3; $6172=$ Inscr. Scyth. Minoris V, 152: 79, n.9; $6173=$ Inscr. Scyth. Minoris V, 153: 79, n.9; 6177 = Inscr. Scyth. Minoris V, 150: 79, n.9; 6188 = Inscr. Scyth. Minoris V, 183: 79, n.9; $6199=12481=$ Inscr. Scyth. Minoris V, 149: 79, n.9; $6235=7599$ = Inscr. Scyth. Minoris V, 163: 79, n.9; 6434: 253

$7504=I L S 7184=$ Inscr. Scyth. Minoris V, 148: 79, n.9; 7509 = Inscr. Scyth.

Minoris V, 166: 79, n.9; 7560 = Inscr. Scyth. Minoris V, 244: 79, n.9; $7907=$ IDR III/2, 19: 167, n.35

$8277,2^{\text {a \& b }: 254 ; ~ 8287 a-d ~=~ 12692,1-4 ~=~}$ 13819a-d: 167, n.30

10256: 186

12677: $254 ; 12693=13820 ; 12694=13821$ : 167, n.30; 184

13819: 184; 13820; 13821: 167, n.30; 184; 13903: 167, n.26; 184

14195, 4-9 = $A E$ 1899, 64: 199, n.5; 14215: 253

CIL V, 4484 = InscrIt. X/5, 275: 176, n.92; 4993 = InscrIt. X, 5, 1071: 176, n.95

6513: 167, n.27; 169, n.47; 185; 6514: 175, n. 85

7595: 166, n.16; 184; $7637=I L S_{5065: 80}$, n.11; 7889; 7890: 190, n.8

$8110,399^{\mathrm{a}-\mathrm{d}} \& 400=$ InscrIt. XI, $1,123^{\mathrm{a}-\mathrm{c}} \&$ 124: $247 ; 252$

CIL VI, 1231a = ILS 213; 1231b-c: 64, n.41; 16o2: 175, n.88; 1636 = ILS 1361: 172, n.71; 1800 = $A E$ 1941, 118: 209, n.54;

3518: 172, n. 65 10213 = ILS 6o44: 66, n.49

28715: 176, n.96

31537d: 65, n.44; $31721=A E$ 1973, 16: 208, n. 51

$41140=A E 1934,155=1957,121=1958$, 26: 212, n.68; 41289: 176, n.96

CIL VII, 1235: 255
CIL VIII, 83: 210, n.62; $270=11451=23246=$ $A E$ 1907, 17: 211, n.65

937 = 11216: 166, n.17; 168, n.40; 170, n.51; 185

1578: 170, n.52; 185; 1827 = 16472: 184

2398: 166, n.19; 167, n.33; 168, n.41; 185; 2399 = ILS 2753: 166, n.19; 167, n.33; 185; 2762: 171, n.62; 2786: 212, n.68

$4437=18596: 166$, n.22; $185 ; 4508=$ 18643: 203 \& n.23; $4676=28073 \mathrm{a}=$ $A E$ 1898, 39a: 206, n.41; 211, n.65 5365 = 17495: 156, n.20; 5366: 156, n.2o 8270: 208, n.52; 8369: 206, n.42; 8813; 8814: 207; 8826 = 20628: 210, n.61; $8937=20681: 185$

10575: 158, n.24; 10667 = 16692: 206, n.41; 211, n. 65

$11451=23246=A E 1907,17: 211$, n.65; 11824: 207

12488: 3

14603: 200, n.10

$15457=26277=26278=$ Uchi Maius II, 75: 168, n.37

16692: 206, n.41; 211, n.65

17167: 81, n.15; 17495: 156, n.20

18596: 166, n.22; 171, n.62; 185

$20627=A E$ 1894, $96=1909,123=2002$, 1694: 204, n.29; 20628: 210, n.61; 20681: 185; 20833: 199, n.9

21037: 172, n.67; $21826=A E$ 1894, $45=A E$ 1941, 115: 202, n.19

22786a-k: 210, n.62; 22787 = AE 1910, 20; 22788: 206, n.41; 210, n.62

$23069=$ ILPBardo 75: 184; $23246=A E$ 1907, 17: 211, n.65

$25902=A E 1897,48=151=1998,1579$ : 208, n. 52

$26277=26278$ = Uchi Maius II, 75: 168, n.37; 26415: 174, n.81; $26518=$ ILTun. $1402=$ ILAfr. $519=$ DFH 25: 178, n.108

28035: 210, n.58; 28073a = $A E$ 1898, 39a: 206, n.41; 211, n.65; 28703b = $A E$ 1898, 39b: 206, n.41; 211, n.65

CIL IX, 1153 = ILS 6487: 166, n.23; 184; 1646 = ILS 6498: 174, n.83 $3019=I L S$ 1377: 166, n.20; 168, n.38; 184 5805: 172, n.66; $5841: 185$

6o78, 55: 252; 6078, 147: 253; 6083, 164 (d): 248; 6183: 177, n.104; 6675, 6: 254 
CIL X, 110: 185

5393: $186 ; 5865: 249$

6309: 170, n.49; $6314=8043,1=\mathrm{I}, 767$,

p. $947=I L L R P$ 430: 259, n.72; 184

8041, 37; 38: 254; 8043, $1=$ I, 767, p. 947

= ILLRP 430: 259, n.72; 184; $8387=C I L$

VI, 1602: 175 , n.88

CIL XI, 405: 169, n.44; 185; 448*: 176, n.91; 854: 248

4091, 2a \& b = XV, 2292a \& b: 245

$5711=$ ILS 6641: 185

6675,$1 ; 2$ = IX, 6o78, 55; 3: 252; 6675,

4: $253 ; 6689,254^{\text {a \& b }}: 247$

7285 = ILS 8996: 167, n.28; 184

CIL XII, 392: 175, n.90; $514=515=I L N$ III, 25: 171, n.63

$2327(=I L S 6995)=I L N \mathrm{~V} / 2,515: 176$,

n.94; 2824: 158, n.24

CIL XIII, 1668 (ILS $212=$ FIRA, I' ${ }^{2}$ 43): 63,

n. 30

$5064=I L S$ 7010: 177, n.100

7269: 176, n.98

8772: 81, n.15

11695: 177, n.102

12603, 1-4: $25^{\circ}$

CIL XIV, 2658: 248; 2995: 168, n.42; 184; $3626=$ ILS 2742 = InscrIt. IV, 1, 157: 172, n. $68 ; 3628$ = InscrIt. IV, 1, 158: 172, n. 68

4089, $1^{\text {a. }}: 253 ; 4090,1^{\text {a-c }}: 248 ; 4091,1=$

$\mathrm{XV}, 2291: 245 ; 4091,3=\mathrm{XV}, 2293: 246$;

4091, 4 = XV, 2294: 246; 4091, 5: 246; 4091, $6^{1-3}=\mathrm{XV}, 2295,1-3: 246 ;$ 4091, 7

= XV, 2296: 246; 4091, 8 = XV, 2298:

246; 4091, 9 = XV, 2297, 1: 246; 4091, 10

=XV, 2299: 246; 4091, $11=\mathrm{XV}, 2300,2$ :

$246 ; 4091,12=\mathrm{I}^{2}, 3477=\mathrm{XV}, 2301=A E$ 1888, 36: 246; 4091, $13^{\mathrm{a} \& \mathrm{~b}}=\mathrm{I}^{2}, 3478^{\mathrm{a} \& \mathrm{~b}}$ $=\mathrm{XV}, 2302^{\mathrm{a} \& \mathrm{~b}}: 247 ; 4089,1^{\mathrm{a}}: 253 ; 4091$, $14=\mathrm{I}^{2}, 348 \mathrm{o}^{\mathrm{a} \& \mathrm{~b}}=\mathrm{XV}, 2303: 247 ; 4091$, $23^{2}=\mathrm{XV}, 2312^{2}: 256$; 4091, 43b: 246, n.14; 4338 (= $A E$ 2000, 266): 62, n.28

CIL XV, 2: 248; 5 a \& b = XIV, 4089, $1^{\text {a: }}$ 253; $7^{1-3}: 248 ; 13-14: 243$, n.2 2291: 245; 2292a \& b: 245; 2293: 246; 2294: 246; 2295, 1-3: 246; 2296: 246; 2297, 1: 246; 2298: 246; 2299: 246 2300, 2: 246; 2301: $246 ; 2302^{\text {a \& b }}: 247$; 2303: $247 ; 2312^{2}: 256$
2813: 248

3914: 179, n.118

7407: 177, n.107

7520: 181, n.131

FIRA I, 445: 125, n.137

$\mathrm{I}^{2}, 21,23,24: 75$, n.1; 68, p. 409 (Cyrene edict): 49

III, 23-25: 116, n.76

$H T$ 5, 89: 117, n.84

IAM II/1, 368 \& 448: 184

IDR III/2, 19: 185

IG XIV, 2394, 2, a-e: 250; 2400, 7; 14, a; 14, b; 15, a-f: 249

IGRR $1.118=$ IGUR $1.1=R D G E$ 22, ll. 7-9 [Latin]: ll. 17-20 [Grec]: 9; 1.618 ll. 11-15: 13

$I K-9 /$ I, 196: 177, n.105

ILAfr. $280=$ ILTun. $706: 184 ;$ 519: 178, n.108

ILAlg. I, $286=$ CIL VIII, $5365=17495: 156$, n.20; 287 = CIL VIII, 5366: 156, n.20; 867: 185; 2145: 166, n.18; 172, n.64; 185

II, 2, 3, 7680: 81, n.15

ILLRP 430: 259, n.72

ILPaestum 92: 185

ILPBardo 36; 75: 184; 250: 185

ILN III, 243-245: 175, n.89

$\mathrm{V} / 2,515: 176$, n.94

$I L S 546$ = CIL III, 3228: 191, n.14 1361: 172, n.71; 1377: 166, n.20; 168, n.38; 184

2661: 175, n.86; 2742: 172, n.68; 2753: 166, n.19; 167, n.33; 185; 2763: 190 5512: 156, n.21; 168, n.43; 179, n.117; 5579: 166, n.19; 167 , n.33; 185

6o24: 174, n.81; 6487: 166, n.23; 184; 6498: 174, n.83; 6641: 185; 6905: 174, n.82 7010: 177, n.100 8996: 167 , n.28; 184

ILTun. 1402: 178, n.108 
InscrIt. IV, 1, 157: 172, n.68; 158: 172, n.68

$\mathrm{X} / 5,275: 176$, n.92; 1005: 175, n.87; 1070: 176, n.95; 1071: 176 , n.95

XI, 1, 123 $3^{\text {a-c }}: 247 ; 124: 252$

Inscr. Scyth. Minoris V, 143-145: 75, n.3; 148: 79, n.9; 149: 79, n.9; 150: 79, n.9; 152: 79, n.9; 153: 79, n.9; 154: 75, n.3; 156: 75, n.3; 163: 79, n.9; 166: 79, n.9; 183: 79, n.9; 244: 79, n.9

IRAT 27: 176, n.93

IRC II, 54: 175, n.86

IRT 6o7: 169, n.48; 184

JIWE II.288: 135, n.24; 338: 135, nn.28-29; 428; 451; 452; 527; 557: 135, n.28; 56o; 577: 135, n.24

Merlin-Poinssot, Les inscriptions d'Uchi Maius 173=ILS 6o24: 174, n.81

Oliver, Greek constitutions of the early Roman emperors, $\mathrm{n}^{\circ} 184$, plaque II, ll. $24-26$; 52-53; 53-56: 16
Pighi, De ludis saecularibus populi Romani Quiritium libri sex, Fr V/Va ll. 26-29: 161, n.4.

Res Gestae Divi Saporis 1l. 6-9: 191, n.12; n.17; 20-23: 191, n.17

RIB: 274, n.30; II, 5, 41: 244, n.5; 2485: 255; 2486; 2487, 1 \& 2: 250; 2487, 3; 9; 11; 12; 13; 14; 16; 18; 19; 20; 23; 2488, 1; 2; 3; $4: 251$

Scheid, Commentarii Fratrum Arvalium, $\mathrm{n}^{\circ} 55,57,65,76,79: 81$, n.15

SEG 51.1832, face A, ll. 20-24: 9 54.1625: 11

SIG 543, ll. 29-34: 21-22 \& n.1

SupplIt. I, 35: 249; IV, Bellunum 9: 186

Uchi Maius II, 75: 185

\section{Juridical evidence}

Callistratus, De cognitionibus bk. 1 fr. 1 Lenel = D. 1.18.9: 13

CJ 4.56 R: 118, n.91

6.4.4: 120, n.108; 122, n.123; 6.7.2 pr. (= CTh 4.10.1): 112, n.42

7.3 sing.; 7.5.1: 115, n.67; 7.5.1.1: 114, n.6o; 7.6 sing.: 116, n.77; 7.6.1.1b: 119, n.93; 7.6.1.3: 117, n.82; 7.6.1.4: 118, n.91; 7.6.1.12-12a: 118, n.91; 7.6.1.13: 116, n.77; 7.9.3.1: 118, n.9o; 7.15.2: 116, n.77; 7.16.7: 117, n.8o; 7.16.20; 7.16.23: 112, n.45; 7.16.26: 112, n.45; 114, n.56; 7.16.3o; 7.16.33: 112, n.45; 7.23 sing.: 122, n.121 9.21.1: 22, n.4; 123, n.126; 9.31.1.1: 123, n.126 10.33(32).1: 110, n.30

Clodoveo: 38

Cod.Eur. 276: 92, nn.12 \& 13; 277: 92, n.12; 312: 92, n.12; n.14

CTh 2.22 sing.: 112, n.42 3.14.1: 93, n.19 4.10.1: 112, n.42
C.Toletana III, c.7: 98, n.44

IV, c.75: 97, n.41

V, c.3: 97, n.42

VI, c.17: 96, n.36; 97, n.42

IX, c.13: 96, n.34

D. 1.1.11 (Paul. 14 ad Sab.): 34 1.2.2.12 (Pomp. lib. sing. enchir.): 35; 1.2.2.28 (Pomp. lib. sing. enchir.): 40, n. 44

1.3.32 pr.: 54 , n.34

1.5.17 (Ulp. 22 ad ed.): 22, n.6; 109, n.26 1.12.1.10 (Ulp., sing. de off. praef. urbi): $111, \mathrm{n} .36$ 1.16.9.3 (Ulp., 1 off. proc.): 111, n.36 2.4.4.1 (Ulp., 5 ad ed.): 119, n.99; 2.4.6 (Paul., 1 sent.): 120, n.101; 2.4.10.11 (Ulp., 5 ad ed.): 119, n.98; 2.4.10.12 (Ulp., 5 ad ed.): 120, n.102; 2.4.11 (Paul., 4 ad ed.): 119, n.100; 2.4 .12 (Ulp., 57 ad ed.): 119, n.100; 2.4.13 (Mod., 10 pand.): 119, n.100; 2.4 .24 (Ulp., 5 ad ed.): 119, n.100; 2.4.25 (Mod., 1 poen.): 119, n.100 4.5.3.1 (Paul., 11 ad ed.): 119, n.94 15.1.53 (Paul., 11 quaest.): 122, n.121 
21.2.34 pr. (Pomp., 27 ad Sab.): 119, n.91 22.5.4 (Paul., 2 l. Iul. et Pap.): 119, n.97 23.2.44 pr. (Paul., 1 l. Iul. et Pap.): 110, n. 31

24.1.40-42: 182, n.144

25.3.5.18-26 (Ulpian, 2 off. cons.): 119, n.96; 25.3.6.1 (Mod., sing. de man.): 111 , n.36; 112, n.44

26.5.14 (Pap., 9 resp.): 122, n.122

37.14.1 (Ulp., 9 off. proc.): 111, n.36; 37.14.5 pr. (Marc., 9 reg.): 112, n.43; 37.14.7.1 (Mod., sing. de man.): 111, n.36; 37.15.5.1 (Ulp., 10 ad ed.): 119, n.98 38.1.2 pr. (Ulp., 38 ad ed.): 110, n.32; 38.1.7.2, 4 (Ulp. 28 ad Sab.): 110, n.32; 38.1.13 pr. (Ulp., 38 ad ed.): 110, n.32; 38.1.31-32 (Mod., 1 reg.): 110, n.32; 38.1.42 (Pap. 5 resp.): 110, n.32; 38.2.1 pr.-1 (Ulp., 42 ad ed.): 110, n.32; 38.2.1.2 (Ulp., 62 ad ed.): 120, n.106; 38.2 .3 pr. (Ulp., 61 ad ed.): 124, n.134; 38.2.29 pr. (Marc. 9 inst.): 110, n.32; 122, n.122; 38.5: 121, n.118

40.2.7 (Gaius, 1 cott.): 113, n.50; 40.2.8

(Ulp., 5 ad ed.): 113, n.50; 40.2 .9 pr. (Marc., 13 inst.): 115, n.65; 40.2.11-14 (Ulp., 2 off. proc. \& 2 l. Ael. Sent.): 115, n.65; 40.2.15 pr. (Paul., 1 l. Ael. Sent.): 115, n.65; 40.2.15.1 (Paul., 1 l. Ael. Sent.): 115, n.65; 40.2.16.1 (Ulp., 2 l. Ael. Sent.): 115, n.65; 40.3 .6 (Ulp., 1 l. Iul. et Pap.): 124, n.131; 40.4.24 (Gaius, 1 cott.): 114, n.6o; 40.4.33 (Paul., 12 quaest.): 110, n.35; 40.4.34 (Paul., 74 ad ed.): 110, n.35; 40.4.36 (Paul., 7 Plaut.): 110, n.32; 40.4.47 pr. (Pap., 6 quaest.): 117, n.8o; 40.8.2 (Mod., 6 reg.): 117, n.82; 40.8.6 (Marc. sing form. hyp.): 118, n.91; 40.8.7 (Paul., sing. lib. dand.): 118, n.91; 40.9 R: 115, n.62; 40.9.7.1 (Iul., 2 Urs. Fer.): 115, n.64; 40.10.3 (Marc., 1 inst.): 124, n.134; 40.10.5 (Paul., 9 l. Iul. et Pap.): 124, n.134; 40.11.2 (Marc., 1 inst.): 124, n.134; 40.11.5 pr. (Mod., 7 reg.): 124, n.134

48.4.1 pr. (Ulp. 7 de off. procons.): 35 \& 36, n. 38

50.1.23.1: 189 \& n.5; 50.5.3 (Scaev., 3

Reg.): 108, n.20

\section{Edictum Ervigii Regis de tributis relaxatis:} 98, n.49

Fr. de iure fisci 6a: 121, n.117
Fr. Dositheanum 4-5: 114, n.56; 5: 113, n.48; 121, n.112; 6: 114, n.57; n.59; 7: 114, n.56; 9: 117, n.81; 118, n.89; 13: 115, n.64; 14: 118, n. $86 ; 16: 115$, n. $62 ; 17: 113$, n. 48

Fr. Vat. 221: 126, n.140; 225: 122, n.122; 261: 122, n.121

Gaius, Epit., 1.1: 116, n.76; 1.1.5: 115, n.62 Institutes 1.1: 6 ; 1.13; 1.15: 115, n.67; n.69; 1.17: 113, n.48; 1.18: 115, n.65; 1.19: 115, n.65; 1.20: 113, n.50; 115, n.63; 1.21: 113, n.49; 1.22: 114, n.57; n.59; 1.23: 116, n.74; 121, n.115; 1.25: 115, n.69; 1.26: 115, n.67; n.69; 1.27: 115, n.68; 1.29: 123, n.129; n.130; 1.30: 109, n.25; 1.31: 123, n.127; 1.32b: 123, n.126; 1.32c: 108, n.20; 1.33: 108, n.21; 1.34: 108, n.20; 1.37: 115, n. $62 ; 1.39: 115$, n. $65 ; 1.40-41: 115$, n.64; 1.42-46: 114, n.6o; 1.56: 108, n.23; 1.67-72: 109, n.24; 1.73: 124, n.132; 1.78-79: 108, n.23; 1.79: 107, n.14; 1.80: 109, n.25; 116, n.73; 1.95: 107, n.13; 1.96: 107, n.12; 108, n.19; 1.119: 36, n.39; 116, n.72; 1.160: 115, n.68; n.69; 1.167: 117, n.81; 1.192: 120, n.105

2.104: 37, n.41; 2.110: 116, n.74; 2.195: 122, n.119; 2.228; 2.239: 114, n.6o; 2.266-267: 122, n.122; 2.275: 116, n.74

3.40: 120, n.104; 3.41: 120, n.106; 3.42: 120, n.108; 3.43, 44: 120, n.105; 3.45, 48: 121, n.110; 3.54: 119, n.92; 3.56: 114, n.56; n.57; n.59; 121, n.111; n.113; 3.57-62: 121, n.113; 3.58, 64: 121, n.110; 3.72: 124, n.131; $3.167: 36-37$, n.40 4.16: 39, n.41; 4.46: 119, n.10o; 4.183: 119, n.99

Inst., 1.5.3: 114, n.58; 115, n.67; 116, n.77; 1.7: 114, n.6o; 1.16.1: 112, n.42; 1.16.4: 119, n.94 2.20.20: 122, n.121

3.7 pr.: 120, n.104; 3.7.1: 120, n.106; 3.7.2, 3: 120, n.108; 3.7.4: 116, n.77; 119, n.93; 121, n.114; 124, n.131

Julien, D. bk. 1 fr. 5 Lenel = D. 1.18.8: 12

Leges publicae populi Romani (L. Pompeia de transpadanis; l. Roscia de Gallia cisalpina): 107, n.10-11

Leges Visigothorum 2.4.3: 100, n.58 3.1.1: 93, n.20-21; 3.1.2: 93, n.21; 3.1.5: 91, n.9 
4.5.7: $96, \mathrm{n} \cdot 34$

5.3.1-4: 100, n.56; 5.3.1.2: 100, n.57; 5.4.19: 93, n.18; 5.4.20 = Cod.Eur. 312: 92, n.12; n.14

6.4.3: 91, n.9

8.3.12: 100, n. 58

9.2.2: 98 , n.46; 9.2.9: 98, n.47; 9.3.3: 10o, n. 58

10.1.7-9: 92, n.13; 10.1.15: 92, n.17; 10.1.16: 92, nn.13, 15-16; 10.1.17-19: 92, n.14; 10.2.1: 92, n.13;

10.2.1-3 and 10.3.1 = Cod.Eur. 277: 92, n.12; 10.3.5 = Cod.Eur. 276: 92, nn.12 $\& 13$

12.1.2: 96, n.35; 12.2.13: 94, n.24; 96, n.33; 12.2.14: 94, n.24

Formula 20: 91, n.9

Formulae manumissionis, Form Vis $\mathrm{n}^{\circ} 2$, 3, 4, 5, 6: 96, n.33

Lex coloniae Genetiuae Iuliae (Urso), ch. $76: 257$, n.6o; 258, n.64

Lex Flavia municipalis, c.91: 18

L. Irnit. 21: 108, n.19; 154, n.15; 22: 154, n.15; 28.5-6: 118, n.9o; 28.11-12: 118, n.88; n.9o; 29, B; 56: 154, n.15; 72.17-19; 23-26: 118, n.9o; 86: 154, n.15; 97.27-28: 124, n.135

Lex Tarentina, col..1, ll.26-31: 257, n.6o
Marcien, De iudiciis publicis bk. 2 fr. 204 Lenel = D. 48.3.6.1: 15

Paul., Sent. 4.12.3, 7: 115, n.66; 4.14.1-4: 114, n.6o

Treaty of Maastricht, C 191: 106, n.6 Edinburgh Agreement, C 348: 106, n.7 Treaty of Amsterdam, C 340: 106, n.8 Treaty on the Functioning of the European Union, C 115/47: 106, n.9

Ulp. Epit. 1.6-9: 113, n.48; 1.10: 114, n.57; 1.11: 115, n.67; n.69; 1.12-13: 115, n.65; 1.12: 118, n.86; 1.13a: 115 , n.63; 1.14: 113, n.49; 1.15: 115, n.62; 1.16: 117, n.81; 1.24-25: 114, n.6o 3.1: 108, n.20; 3.3: 109, n.25; 118, n.86; 123, n.129; 3.4: 126, n.140; 3.5: 123, n.126; 3.6: 108, n.20

5.4: 108, n.18; 5.8: 108, n.23; 5.9-10: 116, n.73

7.4: 109, n.24

11.16: 116, n.72; 11.19: 117, n.81

19.4: 116, n.72; 19.4-5: 108, n.18

20.14: 115, n.69; 116, n.74; 121, n.115; 20.16: 121, n.117

22.1: 108, n.18; 22.3: 116, n.74

25.7: 116, n.74

29.1: 120, n.104; 29.2, 3: 120, n.105; 29.4:

121, n.110

Reg. 7, 1: 182, n.144

\section{Papyrological evidence}

$B G U$ 1680: 190, n.8

P. Euphr. 1, l. 7 (= SB XXII 15496): 54, n.33

P.Flor. I, 16: 179, n.114; 100: 179, n.115

P. Giess. 40.1; FIRA I, 445: 125, n.137

P.Laur. I, 11: 179, n.115

P.Mich. 8.468, col.ii ll.39-40: 43, n.3

P.Oxy. 237: 54; col.6, 1l.16-17, col.7, ll.27, 31-33, 35 \& col.8, ll.34-35: 13, n.12 706: 54
1458, 1l.2-7: 9

4961, ll.27-28 / 75-76: 9

P.Prag. III, 236: 179, n.115

P. Yadin 12-15, 27: 50, n.18

P. Yale II 162, col.II-III: 11

$S B$ XII $10929=P$. Yale II 162, col. II-III: 11 XXII 15496: 54, n.33

Nov. Iust. 78.5: 42 
5. Numismatic evidence

Greek coins of Caria, Cos, Rhodes, 29-32: $227, \mathrm{n} .5^{1}$

Münzprägung von Smyrna, II 1-11; III 19-61: 225, n.42; V 1-14: 225, n.43; XIV 1-4; XV 1-10; XVI 1; XVIII 1-12; XIX 2-6; XX 2-6; XXI 1-44: 226, n.44; XXVI 1-35: 221, n.23; LXXVIII 1-6: 226, n.44

$R P C$ I, 971, 1-9: 229, n.62; 1202, 1: 230, n.65; 1203, 1: 230, n.64; 2311: 223, n.28; 2376, 1-6: 231, n.69; 2469: 222, n.23; 2481; 2489: 225, n.42; 2524-2525: 231, n.69; 3008, 1-19: 229, n.61; 3167, 1-5: 231, n.69
II, 950, 1-8; 951, 1-10; 952, 1-3: 228, n.59; 1033, 1-4: 227, n.50

VII-1, 629-631: 227, n.50

SNG VI, 1440: 227, n.51

Liste der Städte der Provinz Asia, die pseudoautonome Münzen mit der jugendlichen, langhaarigen SynkletosPersonifikation prägen: 236-241 